\title{
Struktur und Umwandlungen von Eisphasen in Gegenwart der Gase Helium, Neon und Argon
}

\author{
Dissertation \\ zur Erlangung des Doktorgrades \\ der Mathematisch- Naturwissenschaftlichen Fakultäten \\ der Georg- August- Universität zu Göttingen
}

vorgelegt von

Frank Gotthardt

aus Göttingen

Göttingen 2001 
D 7

Referent: $\quad$ Prof. Dr. W.F. Kuhs

Korreferent: $\quad$ Prof. Dr. G. Eckold

Tag der Disputation (mündlichen Prüfung): 17.05.2001 
Für meine Familie 


\section{Inhaltsverzeichnis}

1. Vorwort 1

Zusammenfassung 2

1.1. $\quad$ Einleitung $\quad 9$

1.2 Die Wasserstoffbrückenbindung 11

$\begin{array}{lll}1.3 & \text { Das Phasendiagramm von Eis } & 17\end{array}$

1.4 Phasenübergänge im System Eis 26

2. Experimentelle und theoretische Grundlagen

2.1 Experimentelle Grundlagen: Die pVT-Apparatur 30

2.1.1 Differenzdruck-Analyse und Temperaturprofile 33

2.1.2 Wiedergewinnung von Clathrat-Hydraten und Hochdruckeisphasen 36

2.1.3 In-Situ Strukturbestimmungen $\quad 39$

2.1.4 Feldemissions-Rasterelektronenmikroskopie 42

2.2 Theoretische Grundlagen: Rietveld-Strukturanalyse 43

2.2.1 Peakprofilfunktionen und Berechnung physikalischer Parameter 46

2.2.2 Grundlagen und Anwendungen der JMAK-Theorie 49

3. Die Herstellung von Eisphasen

3.1 Präparationsrouten $\quad 52$

3.2 Die Herstellung von Eis Ih 54

3.3 Phasenübergänge von Hochdruckeisphasen in kubisches Eis Ic als transiente Phase 59

3.3.1 Strukturelle Untersuchungen an kubischem Eis Ic $\quad 65$

3.3.2 Kinetik der Phasenübergänge in kubisches Eis Ic 75

3.3.3 JMAK- Fits der Phasenübergänge XII $\rightarrow$ Eis Ic, $\mathrm{V} \rightarrow$ Eis Ic $\quad 83$

3.4 Der Phasenübergang von kubischem Eis Ic in Eis Ih 85

3.4.1 Imperfektionen im Eis Ih $\quad 92$

$3.5 \quad$ Herstellung von Eis II, Eis III 96

3.6 Herstellung der Eisphasen Eis V und Eis VI 99

3.7 Herstellung der metastabilen Eisphasen IV, XII 100 
4. Stabilitätsgrenzen der Eisphasen

4.1.1 Eisverwandte Strukturen: Clathrat-Hydrate 108

4.1.2 Eisverwandte Strukturen: Gas-Hydrate 112

4.2 Das Phasendiagramm von Eis in Wechselwirkung mit Gasen 114

4.3 Experimentelle Untersuchung von Schmelzkurven im System Eis und Gas bis zu Gasdrücken von $\mathrm{p} \sim 6.5 \mathrm{kbar}$

4.3.1 Schmelzkurvenexperimente an Eis Ih 118

4.3.2 Schmelzkurvenexperimente an Eis im Druckbereich von $\mathrm{p} \sim 2.5 \mathrm{kbar}$ bis $\mathrm{p} \sim 6.5 \mathrm{kbar} \quad 124$

4.3.3 Eis V und Eis VI 124

4.3.4 Metastabiles Eis XII und Eis IV 127

\section{Strukturuntersuchungen}

5.1 Argon-Clathrat-Hydrat 133

5.2 Neue Phasen im System Wasser und Neon 136

$\begin{array}{lll}\text { 5.2.1 Neon-Clathrat-Hydrat } & 137\end{array}$

$\begin{array}{lll}\text { 5.2.2 } & \text { Gas-Hydrate } & 144\end{array}$

5.2.3 Eis II-Neon-Hydrat 144

5.3 Strukturelle Untersuchungen an Eis Ih 151

5.3.1 Die mittlere Struktur von Eis Ih 151

5.3.2 Strukturelle Untersuchungen an $\mathrm{D}_{2} \mathrm{O}$-Eis Ih in Wechselwirkung mit Gasen 155

5.4 Verfeinerungsstrategie und Verfeinerungsproblematiken der Strukturbestimmung und des Eis Ih-Gasinhaltes 158

$\begin{array}{lll}5.5 & \text { Experimentelle Ergebnisse } & 164\end{array}$

5.5.1 Gitterkonstanten von Eis Ih unter Gaswechselwirkung als Funktion von $\begin{array}{ll}\text { Druck und Temperatur } & 164\end{array}$

5.5.2 Eis Ih-Struktur als Funktion des Druckes und der Temperatur 178

5.5.3 Eis Ih-Struktur unter Einfluss von Argongas 179

5.5.4 Eis Ih-Hydrat. Wechselwirkung von Eis Ih mit Helium- und Neongas 185

5.5.5 Gasinhalt in Eis Ih für Helium- und Neongas 186

$\begin{array}{lll}\text { 5.5.6 Neon } & 186\end{array}$

$\begin{array}{lll}\text { 5.5.7 Helium } & 191\end{array}$

5.5.8 Struktur von Eis Ih und Eis Ih-Hydrat. Experimentelle Ergebnisse im Vergleich 195

5.6 Sorptionssimulationen von Helium- und Neongas in Eis Ih-Hydrat 198

$\begin{array}{lll}\text { 5.6.1 Ergebnisse der Sorptionssimulationen } & 200\end{array}$ 


\section{Anhang}

8.2.1 Experimentelle und theoretische Grundlagen - Die pVT-Apparatur: Schematische Zeichnung und Erläuterung des HD-Sticks

8.2.1.3.1 GEM (General Material Diffraktometer)

8.2.2.1 Experimentelle und theoretische Grundlagen - Rietveld-Strukturanalyse: Definition des thermischen Auslenkungsparameters U

8.3.2 Die Herstellung von Eis Ih : Rekristallisation von Eis Ih

8.3.3 Phasenübergänge von Hochdruckeisphasen in Eis Ic als transiente Phase: Stapelfehler und lokale Delokationen

8.3.3.1 Strukturelle Untersuchungen an kubischem Eis Ic

8.3.3.1.2 Strukturelle Untersuchungen an kubischem Eis Ic: Rietveld-Anpassungen

8.3.3.1.3 Strukturelle Untersuchungen an kubischem Eis Ic: Peakprofilparameter

8.3.3.1.4 Strukturelle Untersuchungen an kubischem Eis Ic: Quantitative Phasenanteile an kubischem Eis Ic mittels Einzelreflexfits

8.3.3.2 Kinetik der Phasenübergänge in kubisches Eis Ic

8.5.4 Verfeinerungsstrategie und Verfeinerungsproblematiken der Strukturbestimmung und des Eis Ih-Gasinhaltes

\section{Danksagung}

\section{Lebenslauf}




\section{1.}

\section{Vorwort}

Diese Arbeit zur Erlangung des Doktorgrades wurde im Rahmen des DFG- Projektes Ku920/3 durchgeführt. In dem ursprünglichen Projektantrag waren die Bildungsbedingungen sowie die Kristallstruktur von Hochdruck-Gashydraten im System Wasser und Gas als Funktion der Temperatur und des Druckes bis hin zu p $6.5 \mathrm{kbar}$ Gasdruck Ziel der Untersuchungen. Die im allgemeinen als Gas-Hydrate bezeichneten Clathrat-Hydrate koexistieren in diesem Druckbereich mit Hochdruck-Eisphasen. Eine Überprüfung der Existenzbereiche dieser Eisphasen, besonders im Hinblick auf die Bildung von Gas-Hydraten nahe der Schmelzkurve, war daher angebracht. Im Zuge dieser Untersuchungen und zahlreicher Experimente konnte durch den experimentellen Aufbau (pVTGashochdruckapparatur), bestehend aus einer innerhalb des DFG-Projekts bewilligten Hochdruckgasanlage und einer am Mineralogisch-Kristallographischen Institut der Universität Göttingen vorhandenen externen Kühlapparatur, experimentelle Befunde erarbeitet werden, die auf eine bevorzugte Bildung von bisher wenig bekannten metastabilen Eisphasen bei der Verwendung von clathratbildenden Gasen als Drucküberträgermedium hinwiesen. Zum anderen wurde es im Rahmen der Überprüfung und Verifizierung der Existenzbereiche nötig, Präparationsrouten für die gezielte Herstellung von Hochdruckeisphasen als Grundlage für die Gas-Hydrat Bildung festzulegen und durch Schmelzkurven- als auch durch in situ Strukturbestimmung abzusichern. Die im Rahmen dieser Arbeit durchgeführten Untersuchungen beinhalten eine Reihe offener Fragen, die im Zuge der Vorarbeiten zu den ursprünglichen Zielsetzungen des Projektes aufgekommen sind. Die Untersuchungen hatten das Ziel, die Phasengrenzen der unterschiedlichen kristallinen Eisphasen und deren Wechselwirkungen mit den als Drucküberträgermedien verwendeten Gase systematisch $\mathrm{zu}$ vermessen sowie strukturelle Informationen $\mathrm{zu}$ gewinnen. Im besonderen Interesse der Schmelzkurvenexperimente sowie der Bestimmung struktureller Eigenschaften lag jedoch das für die bisherigen Clathrat-Hydrat-Synthesen verwendete Eis Ih. 


\section{Zusammenfassung}

Nach dem Aufbau der pVT-Gashochdruckanlage, einer ausgedehnten Testphase und der Überführung der Anlage in den Routinebetrieb bis zu realisierbaren Gasdrücken von p 6.5kbar, war ein erster Schwerpunkt dieser Arbeit die Durchführung von Schmelzkurvenexperimenten zur Verifizierung der in der Literatur angegebenen Schmelzkurven.

Im Hinblick auf die Koexistenz mehrerer, auch metastabiler Eisphasen sowie eisverwandten Strukturen, den Gas-Hydraten, in einem weiten Druck- und Temperaturbereich war die Kenntnis der Phasengrenzen für die gezielte und reproduzierbare Herstellung einzelner Eisphasen, gerade in dem Stabilitätsbereich von Eis V und Eis VI von besonderer Bedeutung. Im Zuge der Bestimmung der Schmelzkurven in dem Druckbereich von p 3.5kbar bis p 6.5kbar, vornehmlich von Eis $\mathrm{V}$, konnte durch die Ausarbeitung eines neuen Herstellungsverfahrens eine Eisphase, die mit einer hohen Wahrscheinlichkeit später in in situ Experimenten als die metastabile Eisphase XII identifiziert wurde, nukleiert werden. Das gefundene Herstellungsverfahren, das in mehreren in situ Experimenten erarbeitet wurde, ergab eine sehr hohe und reproduzierbare Nukleationswahrscheinlichkeit von Eis XII aus der flüssigen Phase. Im Zuge weiterer Schmelzkurvenexperimente konnte die Schmelzkurve von Eis XII in einem Druckbereich von $\mathrm{p} \sim 4.5 \mathrm{kbar}$ bis $\mathrm{p} \sim 6.5 \mathrm{kbar}$ bestimmt und zudem durch in situ Bestimmung am D2B des ILL sowie am GEM am ISIS des RAL die Schmelzkurve überprüft sowie der Existenzbereich von Eis XII im Rahmen der zur Verfügung stehenden Messzeit abgegrenzt werden.

Weiteres Ziel dieser Arbeit war, Aussagen über das Phasenverhalten der unterschiedlichen Eisphasen in dem Druckbereich von $\mathrm{p} \sim 1$ bar bis $\mathrm{p} \sim 7 \mathrm{kbar}$ unter Wechselwirkungen von atmosphärischen Gasen, zumeist Reinstgasen wie Argon, Stickstoff, Neon aber auch Helium, zu gewinnen. Im besonderen Interesse lagen die Phasenübergänge sowie im speziellen die Schmelzkurven unter Verwendung der verschiedenen als Drucküberträgermedium verwendeten Gase. Vergleichende pVT-Experimente wurden detailliert für das Eis Ih und das Eis V mit Argon, Stickstoff und Neon durchgeführt. In diesen Experimenten zeigten sich zunächst große Verschiebungen in den Schmelzkurven von Eis V zu tieferen Temperaturen 
im Vergleich zu den aus der Literatur bekannten Referenzkurven. Es wurde der Frage nachgegangen, ob dies durch probenbedingte Effekte, z.B. Gas-Eis-Wechselwirkungen, verursacht ist. Dies führte $\mathrm{zu}$ einer systematischen Überprüfung der pVT-Apparatur hinsichtlich der absoluten Genauigkeiten von Druck und Temperatur (unter Verwendung sekundärer und tertiärer Standards). Diese wurden zum Teil durch in situ Neutronendiffraktionsexperimente am D2B des ILL (Institut Laue-Langevin in Grenoble, Frankreich) sowie am GEM am ISIS des RAL (Rutherford-Appleton-Laboratory in Didcot, GB) verifiziert und erlauben zudem auch eine absolute Fehlerabschätzung der an der Göttinger pVT-Apparatur bestimmten Schmelzkurven zu denen der in in situ Experimenten bestimmten Kurven. Nach der Korrektur der systematischen apparativen Fehler zeigten sich weiterhin, wenn auch geringere Abweichungen der Schmelzkurvenverläufe von Eis V unter Verwendung von Gasen. Ob diese durch Gas-Eis-Wechselwirkungen verursacht sind, z.B. durch Schmelzpunkterniedrigung des Eises durch Änderung der relativen Gaslöslichkeiten von Eis und Wasser, konnte aus einem Vergleich der eigenen experimentellen Ergebnisse sowie den in der Literatur beschriebenen nicht endgültig geklärt werden.

Der Tripelpunkt von Eis V- Eis VI- flüssigem Wasser L wurde unter Verwendung der beiden Gase Stickstoff und Argon mit Verwendung von $\mathrm{D}_{2} \mathrm{O}$ zu p=6.64kbar $\pm 132 \mathrm{bar}$, $\mathrm{T}=275.47 \mathrm{~K} \pm 0.5 \mathrm{~K}$ und für $\mathrm{H}_{2} \mathrm{O} \quad \mathrm{zu} \quad \mathrm{p}=6.88 \mathrm{kbar} \pm 137 \mathrm{bar}, \mathrm{T}=265.3 \pm 0.5 \mathrm{~K}$ bestimmt. Weitergehende strukturelle Untersuchungen an Eis $\mathrm{V}$, die auch unter Verwendung der verschiedenen Gase Argon, Stickstoff und Neon durchgeführt werden sollten, konnten aufgrund fehlender in situ Messzeit an Großgeräten nicht nachgegangen werden.

Für das „normale“ Eis Ih konnte unter Verwendung der Gase Argon und Stickstoff die Eis IhSchmelzkurve in einem Druckbereich von etwa $p \sim 200$ bar bis $p \sim 2.7 \mathrm{kbar}$ vermessen werden. Im Rahmen der Fehler konnte keine Verschiebung oder Abweichung zu den Literaturkurven festgestellt werden. Eine Löslichkeit dieser Gase in Eis Ih kann somit mit großer Sicherheit ausgeschlossen werden. Bei der Verwendung von Neon als Drucküberträgermedium zeigte sich hingegen, dass eine Verschiebung der Schmelzkurve $\mathrm{zu}$ höheren Drücken und Temperaturen stattfand. Dieses Verhalten deutet auf eine Löslichkeit des Neons in der Eisstruktur hin, die eine Stabilisierung der Eisstruktur zur Folge hat. Eis Ih ist daher aufgrund des Gasinhaltes eher als Neon-Hydrat zu bezeichnen. Im Zuge dieser Experimente konnte die Schmelzkurve von Eis Ih unter Verwendung von Neon von $\mathrm{p} \sim 1 \mathrm{kbar}$ bis $\mathrm{p} \sim 2$.7kbar mit $\mathrm{H}_{2} \mathrm{O}$ 
sowie $\mathrm{D}_{2} \mathrm{O}$ vermessen werden. Die $\mathrm{D}_{2} \mathrm{O}$-Schmelzkurve zeigte von dem Verlauf des unbeeinflussten „normalen“ Eises eine deutliche Abweichung zu höheren Temperaturen. Ebenso zeigten sich durch die Bestimmung der Schmelzkurven mit Neon Hinweise auf die Ausbildung neuer, bis dahin unbekannter Phasen in dem Stabilitätsbereich von Eis Ih sowie Eis II in Wechselwirkung mit Neon. Diese beiden neuen Phasen konnten mit sehr hoher Wahrscheinlichkeit als zwei bisher unbekannte Phasen, zum einen Neon-Clathrat-Hydrat, vom Stackelberg Typ II, sowie ein dem Eis II verwandtes Neon-Eis II-Hydrat identifiziert werden.

Für das Neon-Eis II-Hydrat konnte in Göttingen an der pVT-Apparatur über einen weiten Druckbereich, von $\mathrm{p} \sim 2.5 \mathrm{kbar}$ bis $\mathrm{p} \sim 4.5 \mathrm{kbar}$ die Schmelzkurve bestimmt werden. Der Tripelpunkt des Neon- Eis Ih- / Neon-Eis II-Hydrat zu flüssigem Wasser L konnte aus den Schmelzkurvenverläufen für $\mathrm{D}_{2} \mathrm{O} z u$ p $=2.40 \mathrm{kbar} \pm 48 \mathrm{bar}, \mathrm{T}=254.1 \mathrm{~K} \pm 0.5 \mathrm{~K}$ bestimmt werden. Die strukturelle Identifikation sowie die Verifizierung der bestimmten Schmelzkurven der Phasen im System Eis und Neon erfolgte in situ durch Neutronendiffraktion am D2B des ILL. Die Existenz von Neon-Clathrat-Hydrat wurde mehrfach in der Literatur ausgeschlossen, eine Aussage, die damit durch die eindeutigen experimentellen Befunde widerlegt, ist.

Von weiterem Interesse war auch die Untersuchung der druck- und temperaturabhängigen Kristallstruktur von Eis Ih, und die Verifizierung von Gaseinflüssen auf die Struktur. Zielsetzung war, zum Einen Aussagen über die Kompressibilitäten und zum Anderen über das Verhalten der Wasserstoffbrückenbindungen von Eis Ih in Wechselwirkungen mit Gasen als Funktion von Druck und Temperatur $\mathrm{zu}$ erhalten. In zwei in situ Neutronendiffraktionsexperimenten am D2B des ILL in Grenoble wurden die im allgemeinen als Funktion von Druck und Temperatur gegebenen strukturellen Eigenschaften von Eis Ih unter Verwendung der Gase Argon, Helium und Neon bestimmt. Die RietveldVerfeinerungen von Eis Ih mit Argongas zeigten, dass die Eisstruktur über den bestimmten Temperaturbereich von $\mathrm{T} \sim 255 \mathrm{~K}$ bis $\mathrm{T} \sim 140 \mathrm{~K}$ sowie im Druckbereich von $\mathrm{p}=1$ bar bis $\mathrm{p} \sim 2.5 \mathrm{kbar}$ isokompressibel ist, d.h. dass das c/a-Kristallachsenverhältnis des Eis IhKristallgitters im Rahmen des Fehlers konstant ist. Bei der Verwendung der Gase Helium und Neon als Drucküberträgermedium zeigten sich im Vergleich zum Eis Ih mit Argongas Abweichungen in dem Verlauf der Gitterkonstanten als Funktion von Druck und Temperatur. Die Änderungen zeigten sich in einem abweichenden c/a-Verhältnis der Gitterkonstanten 
zueinander und deuten damit auf eine Beeinflussung durch Eis-Gas-Wechselwirkungen hin. Signifikante Änderungen in den fraktionellen Koordinaten als Funktion des Druckes oder der Temperatur konnten aufgrund hoher Fehler der Rietveld-Verfeinerungen nicht festgestellt werden. Als Ursache der hohen Fehler sowie der mangelnden Aussagefähigkeit der Experimente ist eine nur eingeschränkte Probengüte, verursacht für die mit Argongas gemessenen Datensätze durch fortschreitende Clathrat-Hydrat-Synthese, für die mit Helium gemessenen Datensätze durch einen verbliebenen Clathrat-Hydrat-Phasenanteil, sowie für das mit Neongas durchgeführte Experiment durch die Probenherstellung bedingte Imperfektionen im Eis Ih, anzuführen.

Aussagen über den Gasinhalt des Eises als Funktion von Druck und Temperatur konnten aus den Rietveld-Verfeinerungen der durchgeführten Eis Ih-Diffraktionsexperimente nicht getroffen werden. Die signifikanten Änderungen in den Gitterkonstanten von Eis Ih bei der Verwendung der Gase Helium und Neon sowie die deutliche Änderung der c/a-Verhältnisse der Gitterkonstanten als Funktion von Druck und Temperatur bestätigen aber die durch die pVT-Experimente vermuteten Gaslöslichkeiten.

Um eine Aussage über den Gasinhalt der Eis Ih-Struktur treffen zu können wurden Sorptionsrechnungen mit molekularen Monte-Carlo-Methoden (MC-Methoden) durchgeführt. Für die Sorptionsrechnungen wurden mehrere hinreichend bekannte Potenziale zur Modellierung der Wasser-Wechselwirkungen verwendet. Als Sorbat wurden die Gase Helium und Neon in die Sorptionssimulationen eingebaut. Unter Verwendung verschiedener Wechselwirkungen in den MC-Modellen und der Potenzialparameter wurden die Gasbesetzungen als Funktion der Temperatur und des Druckes mit den Gasen Helium und Neon simuliert. Der simulierte Gasinhalt zeigte sich mit Helium und Neon vom Besetzungsverlauf als auch vom Besetzungsgrad her unterschiedlich. Für Helium ergaben sich wesentlich höhere Besetzungen als für Neon bei vergleichbaren Druck- und Temperaturbedingungen. Für Neon ergab sich z.B. bei $\mathrm{T} \sim 237 \mathrm{~K}$ und $\mathrm{p} \sim 2 \mathrm{kbar}$ eine Gasbesetzung von $\mathrm{n} \sim 0.091$ Neonatomen auf 96 Wassermoleküle, während sich bei Umgebungsdruck eine Gasbesetzung von n 0.013 Neonatome auf 96 Wassermoleküle ergab. Ein Einfluss der Gasbesetzung auf die Eisstruktur, Änderungen der Bindungsverhältnisse, kann durch das verwendete starre Modell der Eis-Wirtsstruktur nicht ermittelt werden. Die aus den Sorptionsrechnungen ermittelten Gasbesetzungen zeigen sich konsistent zu den 
experimentell gefundenen Änderungen in den Gitterkonstanten als Funktion von Druck und Temperatur. Diese stimmen sowohl für Heliumgas als auch für Neongas jedoch im Vergleich zu den bisher aus der Literatur bekannten Gasbesetzungen nicht überein.

Das für die Experimente benötigte Eis Ih wurde durch die Erwärmung von wiedergewonnenen (,recoverten“) Hochdruckeisphasen über einen Phasenübergang von kubischem Eis Ic in hexagonales Eis Ih hergestellt. Das wiedergewinnen (,recovern“) von Hochdruckeisproben erfolgte durch die Herstellung der gewünschten Eisphase über die Herstellungsrouten unter Verwendung der pVT-Apparatur und sodann durch Abschrecken der Probe, d.h. sehr schnellem Kühlen (quenchen) mit flüssigem Stickstoff auf T $77 \mathrm{~K}$ sowie durch eine Reduzierung des Gasdruckes bei T $77 \mathrm{~K}$ auf Umgebungsdruck.

In dem Neon Eis Ih-Experiment wurden die meisten Messungen zur Bestimmung der Gitterkonstanten nach der Eis Ih-Präparation, d.h. nach dem Phasenübergang aus dem kubischen Eis Ic, unterhalb von T $237 \mathrm{~K}$, bei $\mathrm{T} \sim 215 \mathrm{~K}$ sowie $\mathrm{T} \sim 140 \mathrm{~K}$ durchgeführt. Für diese Datensätze ergaben sich systematische Intensitätsabweichungen der drei stärksten Eis IhPeaks ( [100]-,[002]-, [101]-Reflexe). In dem Eis Ih-Experiment mit Argongas zeigten sich ähnliche Effekte jedoch nur bei Temperaturen bis zu T 215K. Da jedoch bei den mit Argongas gemessenen Eis Ih-Datensätzen zuerst bei höheren Temperaturen gemessen worden ist, zeigten sich die Effekte abweichender Intensitäten im weiteren Verlauf der Messungen nicht mehr. Die Intensitätsabweichungen für die Neon Eis Ih-(Neon)-Messungen näherten sich bei einer Temperatur von über $\mathrm{T} \sim 237 \mathrm{~K}$ relativ sprunghaft an die simulierten Intensitätsverhältnisse eines polykristallinen Eis Ih an und sind ein Indiz für einen letztendlich nicht abgeschlossenen Phasenübergang vom kubischen ins hexagonale Eis Ih.

Durch die in situ Verifizierung konnte zweifelsfrei die Existenz kristallographischer Fehlordnungen, die bei dem Übergang von kubischem Eis Ic ins hexagonale Eis Ih verbleiben, bis zu Temperaturen oberhalb von $\mathrm{T} \sim 237 \mathrm{~K}$, nachgewiesen werden. Dies ist konsistent mit Literaturhinweisen auf einen weiteren Phasenübergangsbereich von kubischem Eis Ic ins hexagonale Eis Ih.

Der Phasenübergang von den „recoverten“ Hochdruckeisphasen in das kubische Eis wurde an den Eisphasen II, III, V, XII und an HDA(LDA)-Eis in kubisches Eis Ic verfolgt. Von 
Interesse hierbei waren sowohl die Kinetik der Phasenumwandlung als auch strukturelle Unterschiede. Die Kinetik- und die Strukturunterschiede zeigten sich für das aus verschiedenen Hochdruckeisphasen hergestellte kubische Eis als deutlich verschieden, wobei die strukturellen Unterschiede durch verschieden stark ausgeprägte kristallographische Fehlordnungen bedingt sind. Die Phasenübergänge der Eisphasen V und XII konnten in situ verfolgt werden und wurden unter Anwendung der JMAK-Theorie (Johnson-Mehl-AvramiKolmogorov-Theorie zur Rekristallisation von Phasen, vergl. Kapitel 2.2.2) ausgewertet. Für Eis XII ergab sich ein flächiges, isotropes, topotaktisches Wachstum. Für Eis V konnte ein anisotropes, topotaktisches Wachstum festgestellt werden. Die Phasenübergänge von Eis IX und Eis II konnten verfolgt werden. Eine Auswertung mit der JMAK-Theorie wurde aufgrund eines zu schnell vollzogenen Phasenüberganges von Eis II in Eis Ic sowie für das Eis IX durch stark ausgeprägte Vorzugsorientierungen nicht durchgeführt. Aus den strukturellen Unterschieden der kubischen Eisphasen wurde versucht durch Reflexprofil-Fits sowie anhand der Anpassung eines einfachen zwei Phasengemisch-Rietveld-Modells die kristallographischen Stapelfehlordnungen der unterschiedlich hergestellten kubischen Eisphasen zu quantifizieren. Im Rahmen der Fehler konnten durch beide Varianten der jeweilige Anteil der Fehlordnungen in der Probe hinreichend quantifiziert werden. Der eigentlich für die Eis Ih-Probenherstellung vermutete relevante Phasenübergang von kubischem Eis Ic in hexagonales Eis Ih konnte in der zur Verfügung stehenden in situ Messzeit nicht detailliert genug verfolgt werden. Konkrete Aussagen bezüglich der strukturellen Vorgänge bei dem Phasenübergang konnten nur qualitativ getroffen werden.

Bedingt durch begrenzt zur Verfügung stehende in situ Messzeit erfolgten Versuche, hergestellte Eisphasen zu „recovern“ und das Phasenübergangsverhalten von „recoverten“ Hochdruckeisphasen in kubisches Eis Ic sowie weiterhin in hexagonales Eis Ih mittels Röntgendiffraktion an Geräten im eigenen Institut zu untersuchen. Es ergaben sich jedoch durch die Art der Konzeption der Apparatur aus physikalischen und technischen Gründen Probleme, die zu keiner zufriedenstellenden experimentellen Untersuchung führten.

Aufgrund der zum Teil schlechten Probenqualität, verursacht durch die Ausbildung von extremen Vorzugsorientierungen, mit zum Teil großen Kristalliten, d.h. nur geringer Feinkristallinität, wurden Herstellungsverfahren und Präprationsrouten ausgearbeitet, die eine reproduzierbare und gezielte Herstellung einzelner Eisphasen mit hinreichender 
Probenqualität (Pulvergüte) erlaubten. Grundlage für diese Experimente war die Herstellung von feinkristallinem, regellosem Eis Ih-Pulver. Es wurden daher Experimente durchgeführt, die sich mit der Frage der Ausarbeitung von Präparationsverfahren zur reproduzierbaren Herstellung von polykristallinem Eis aller Hochdruckeisphasen im allgemeinen beschäftigten. Die hergestellten Eispulver, die zudem als Ausgangsmaterial für die Herstellungsversuche anderer Eisphasen verwendet wurden, wurden mittels elektronenoptischer Methoden an einem FE- REM hinsichtlich der Homogenität der Korngrößen als auch der Korngrößenverteilung qualitativ untersucht. Erste Untersuchungen der Probengüte in Bezug auf die Ausbildung von Vorzugsorientierungen und den Korngrößen wurden am BW5 des HASYLAB am DESY in Hamburg durchgeführt. Die Untersuchung beschränkte sich jedoch nur auf die Charakterisierung der unterschiedlich hergestellten Eisproben, insbesondere auf die Eignung des Herstellungsverfahrens und nicht die detaillierte Auswertung hinsichtlich der Vorzugsorientierungen oder Korngrößen. 


\subsection{Einleitung}

Unser Planet Erde ist zu ca. 70\% von Wasser in den Ozeanen bedeckt. Das Wasser ist in verschiedenen Regionen der Erde wie z.B. in Eisschilden und Gletschern auf den Kontinenten, in der Atmosphäre sowie hauptsächlich in den Ozeanen vorhanden. Aufgrund seiner Häufigkeit und dem Vorkommen in allen drei Aggregatzuständen ist Wasser für das System Erde und für Lebewesen, d.h. in der Geo- und Biosphäre, von fundamentaler Bedeutung und ist nicht zuletzt aufgrund der Anomalien des Wassers, z.B. der Dichtanomalie des Wassers bei $4^{\circ} \mathrm{C}$, seit längerer Zeit Gegenstand chemischer, physikalischer und auch struktureller Untersuchungen.

Das Zusammenspiel von Wasser in Ozeanen mit den Eismassen der Arktis und Antarktis, sowie die möglichen Wechselwirkungen des Wassers bzw. Eises mit seiner Umgebung, z.B. der Atmosphäre, werfen in geophysikalischer und klimatologischer Hinsicht wesentliche Fragen auf. Die Eiskappen auf den Polen unseres Planeten haben im Zusammenspiel mit den Ozeanen und deren Wechselwirkungen einen wesentlichen Einfluss auf die Ausbildung von Meeresströmungen durch Erwärmung der Wassermassen am Äquator sowie der Abkühlung an den Polen. Aufgrund der komplexen Wechselwirkungen zwischen der Atmosphäre und den Wasserströmungen in den Ozeanen ergeben sich gekoppelte Strömungssysteme, die zu einem Wärmetransport aus den äquatorialen in die polaren Bereiche führen. Dieser Wärmetransport hat z.B. eine wesentliche Bedeutung nicht nur für das kontinentale Klima in Europa, sondern stellt im eigentlichen Sinn eine globale geophysikalische Wärmepumpe dar.

Das Eis ist auch in den Permafrostregionen und den Polarregionen bedingt durch den alljährlichen Schneefalls von weiterer Bedeutung. Dem Niedergang des Schnees folgt aufgrund der Kompression darüber liegender Schichten eine Umbildung zu Eis. Durch den stetigen Wechsel von Jahreszeiten ist dieses Eis ein klimatologisches Archiv, aus dem Aussagen über die frühere Zusammensetzung der Atmosphäre und des bei der Eisbildung bestehenden Klimas getroffen werden können. Das Eis enthält, durch den Bildungsprozess bedingt, Luftbläschen, die sich im Laufe der Zeit, d.h. mit zunehmender Stärke bzw. Alters der Eisschilde, in geologischen Zeiträumen in größeren Tiefen zu Clathrat-Hydraten umwandeln. Diese Luftbläschen sowie die Clathrat-Hydrate enthalten im Idealfall repräsentativ die Gaszusammensetzung der Atmosphäre bei der Bildung des Schnees und 
können so Aufschluss über die Anteile von Treibhausgasen wie z.B. Methan, Kohlendioxid und den Sauerstoffgehalt geben.

Das Isotopenverhältnis von Sauerstoff ${ }^{18} \mathrm{O} /{ }^{16} \mathrm{O}$ des Eises lässt Aussagen über die Bildungstemperaturen zu. Verschiedene Eiskernbohrungsprojekte wie GRIP I und NGRIP in Grönland oder DOMEC und VOSTOK in der Antarktis versuchen mittels der Bergung von Eiskernen den klimatologischen Fragestellungen in Zusammenhang mit der Bildung von natürlichen Clathrat-Hydraten nachzukommen und durch Korrelation der experimentellen Befunde Aussagen über das Verhalten des Klimas in früheren Perioden zu erhalten. Ein Überblick über das Klima ist für die Antarktis in einem Zeitraum von 400.000 Jahren und für die Arktis von 200.000 Jahren zu erhalten.

Ein weiteres Gebiet der Eisforschung ist die Glaziologie, die sich der Untersuchung der Gletscher- und Eisschildbildung, dem mechanisch-physikalischen Verhalten von Eis, d.h. dem Fließverhalten, sowie der Wechselwirkung von Eis mit der Atmosphäre bzw. allgemein seiner Umgebung, widmet. Sowohl für das mechanische als auch das physikalische Verhalten von Eis ist die Frage nach den Bildungsbedingungen sowie der Rekristallisation von Eis von großer Bedeutung. Als Grundlage für die Eiskernbohrungen sowie der Beschreibung des mechanischen Verhaltens von Eismassen durch Modellierung des Fließverhaltens sind daher Kenntnisse der Eigenschaften von Eis als Funktion des Druckes und der Temperatur von Bedeutung. Wünschenswert wäre z.B. die Kenntnis der isothermen Kompressibilitäten von Eis, um das tiefenabhängige Fließverhalten genauer beschreiben zu können. Ebenso ist die Protonenordnung als Funktion der Temperatur hinsichtlich des Verhaltens von Defektstrukturen im Eis von Bedeutung. An dieser Stelle ist anzumerken, dass Hochdruckeisphasen auf der Erde nicht vorkommen, jedoch aufgrund der Schichtdicken der auf der Erde befindlichen Eismassen Drücke bis zu einigen hundert bar auf die Struktur des Eis Ih einwirken.

Makroskopische Eigenschaften des Wassers und des Eises sind in vielfältigen Experimenten untersucht worden, jedoch sind wesentliche Fragen hinsichtlich der Anomalien von Wasser und Eis, den Wechselwirkungen mit Gas, z.B. Gas-Löslichkeiten, Clathrat-Hydratbildung sowie der Ausbildung von derzeit 15 strukturell verschiedenen Eisphasen nicht hinreichend geklärt. Eine Vielzahl von theoretischen Modellen des Wassers wurden erstellt, jedoch 
entsprechen diese nicht gänzlich dem Verhalten von Wasser in seinen drei Aggregatzuständen. Ein auf molekularer Ebene basierendes Modell „Wasser“ durchgängig in seinen kristallinen Erscheinungsformen, den unterschiedlichen Eisphasen, zu beschreiben ist noch nicht vorhanden. Sowohl aufgrund der komplexen Wechselwirkungen von Wassermolekülen untereinander, z.B. der Ausbildung von Wasserstoffbrücken und der NichtAdditivität der Wasserpotenziale bei Systemen mit mehreren Wassermolekülen, als auch der Wechselwirkungen von Wasser und gelösten Stoffen, z.B. Gasen, ist die Forschung auf diesem Gebiet schwierig und viele Fragen hinsichtlich der genannten Einflüsse und Wechselwirkungen in vielen Fällen im Detail noch nicht geklärt. Wasser in seinen festen Formen stellt nicht zuletzt aufgrund der unterschiedlichen kristallinen Eisphasen und der vollständigen Ausbildung von Wasserstoffbrücken ein ideales System dar, um Aussagen über die vielfältigen Wechselwirkungen der Wassermoleküle untereinander sowie mit Gasen mittels experimentell ermittelter Strukturdaten zu gewinnen.

\subsection{Die Wasserstoffbrückenbindung}

Das Wassermolekül $\mathrm{H}_{2} \mathrm{O}$ bzw. schweres Wasser $\mathrm{D}_{2} \mathrm{O}$ bestehen aus jeweils zwei leichten (Wasserstoffatomen bzw. schweren Wasserstoffatomen (Deuterium-Atomen) und einem Sauerstoffatom. Das Sauerstoffatom besitzt in seiner äußeren Elektronenschale sechs Valenzelektronen, über die durch eine kovalente Bindung mit den einzelnen Elektronen des Wasserstoffatoms diese chemisch gebunden werden. Die verbleibenden vier Valenzelektronen des Sauerstoffs bilden jeweils paarweise freie Elektronenpaare. Der Aufbau des Wassermoleküls ist in der Abbildung 1.1 dargestellt:

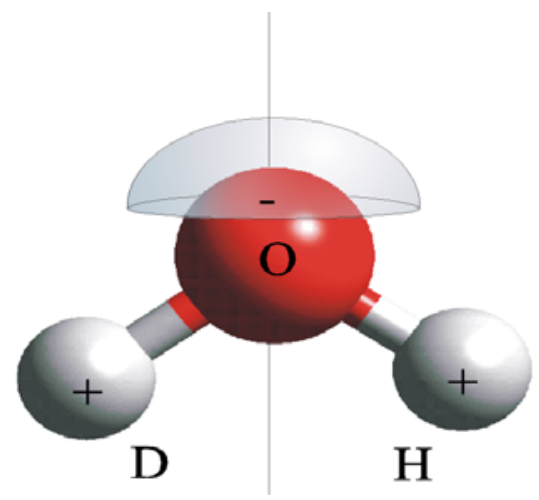

Abbildung 1.1: Dargestellt ist die Geometrie des Wassermoleküls. Die als Schirm dargestellten freien Elektronenpaare des Sauerstoffs stehen bei einem Dimer in einem Winkel von ca. $57^{\circ}$ von der Symmetrieachse in und aus der Blattebene hinein/heraus [Dyke et al. (1977) und Odutola \& Dyke (1980)] 
Bei dem Wassermolekül handelt es sich um ein nichtlineares und polarisiertes Molekül. Die Nichtlinearität wird durch elektrostatische Wechselwirkungen der freien Elektronenpaare mit den in den $\mathrm{O}-\mathrm{D}(\mathrm{O}-\mathrm{H})$-befindlichen kovalenten Elektronenpaaren hervorgerufen. Aufgrund der höheren Elektronegativität des Sauerstoffs werden zudem die Elektronen der Wasserstoffatome in der kovalenten Bindung näher an das Sauerstoffatom gebunden, so dass an dem Sauerstoff eine negative Ladung bzw. an den verbleibenden Protonen eine positive Ladung erzeugt wird. Dies führt zu einem elektrostatischen Dipolmoment, dass die Ursache für die Ausbildung der Wasserstoffbrückenbindung ist. Aufgrund des molekularen Aufbaus fungiert das Wassermolekül durch die $\mathrm{D}(\mathrm{H})$-Protonen zugleich als Protonendonator sowie über die freien Elektronenpaare des Sauerstoffs als Protonenakzeptor. Durch die elektrostatische Wechselwirkung der freien Elektronenpaare und den $\mathrm{D}(\mathrm{H})$-Protonen führt dies zu vier Anlagerungs- bzw. Anbindungsmöglichkeiten weiterer Wassermoleküle. Bei einer Vierfach-Koordination ordnen sich diese tetraedrisch zu einem Pentamer an (Abbildung 1.2).

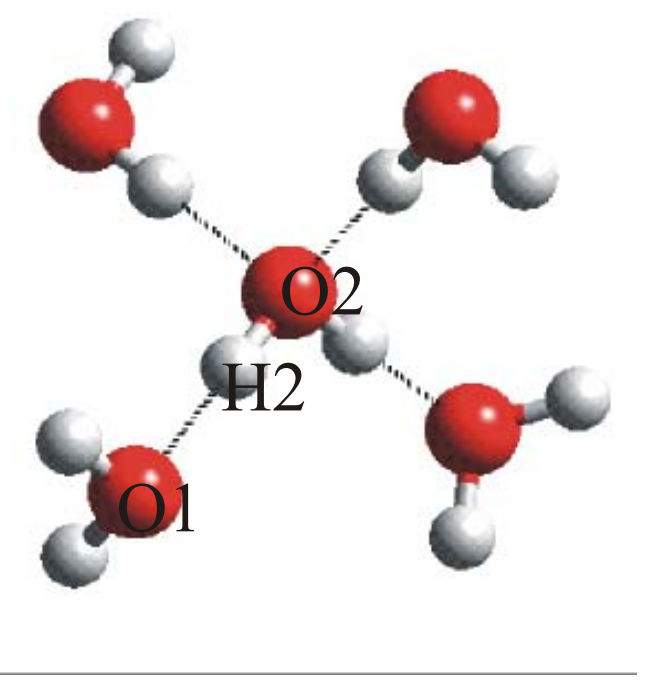

Abbildung 1.2: Tetraedrische Anordnung von Wassermolekülen zu einem Pentamer. Ursache ist die Ausbildung von H-Brücken, z.B. O(1)...H(2)-O(2). Aus dieser Anordnung lassen sich die sogenannten Eisregeln definieren: 1. Eine H-Brücke ist maximal mit einem Wasserstoff besetzt. 2. Ein Sauerstoff ist von vier Wasserstoffen umgeben, d.h. voll wasserstoffbrückengebunden. 3. Geometrie der Wassermoleküle bleibt weitgehende erhalten. Im folgenden wird nur von dem Deuterium ausgegangen. Die Argumentation ist jedoch für das leichte Wasseratom gleichermaßen gegeben und schlüssig. Relevante Unterschiede zwischen Wasserstoff und Deuterium werden gegebenenfalls angegeben und diskutiert.

Im Wasser und im Eis konnte diese Tatsache schon früh durch röntgendiffraktometrische Untersuchungen (Morgan \& Warren (1938); Dennison (1921)) gezeigt werden. Aus der Arbeit von Morgan \& Warren (1938) ergibt sich, dass ein Wassermolekül in der Flüssigkeit einfach bis vierfach gebunden vorliegt. In der Flüssigkeit existieren neben einzelnen 
Wassermolekülen sowie Dimeren, Trimeren insbesondere vierfach-koordinierte Bereiche. Diese sind jedoch nur über einen sehr kurzen Zeitraum stabil und befinden sich daher in ständigem Umbau. Ungefähre Vorstellungen der Struktur und quasikristalliner Bereiche im Wasser existieren zwar, jedoch gibt es derzeit keine experimentellen Aussagen, die z.B. die Vorstellungen von Eis Ih ähnelnden Strukturen untermauern können. Sowohl die Struktur von Wasser als auch das physikalische Verhalten sind Gegenstand aktueller theoretischer und auch experimenteller Untersuchungen. Die tetraedrische Anordnung wurde ebenso früh mittels diffraktometrischer Messungen an Eis Ih gefunden (Dennison (1921)) und ist für bisher alle gefundenen kristallinen Eisstrukturen nachgewiesen. Die Wasserstoffbrücke, mit zwei an dieser Bindung beteiligten Moleküle $\mathrm{D}_{2} \mathrm{O}(1)$ und $\mathrm{D}_{2} \mathrm{O}(2)$, wird z.B. durch die Folge $\mathrm{O}(1)-\mathrm{D}(1) \ldots \mathrm{O}(2)$ gegeben. Hierbei ist die chemische Bindung durch ,-“ und die Wasserstoffbrücke durch „,...““ dargestellt. Pro Wasserstoffbrücke enthält diese jeweils nur ein Deuterium- bzw. Wasserstoffatom. Der Bindungsabstand zwischen O(1)-O(2)Sauerstoffatomen liegt bei einem Wasserdimer bei ca. $d_{b}=2.976 \AA$, in hexagonalem Eis Ih unter Standardbedingungen $\mathrm{d}_{\mathrm{b}} \sim 2.76 \AA$ (Kuhs \& Lehmann (1983)). Die Bindungsstärke der Wasserstoffbrückenbindung liegt mit einer Bindungsenergie von E 20kJ/mol zwischen der van der Waals Wechselwirkung mit etwa $\mathrm{E} \sim 1 \mathrm{~kJ} / \mathrm{mol}$ und der kovalenten Bindung mit etwa E 400kJ/mol. Die Bindungsenergien sind jedoch von der Konfiguration, d.h. den interatomaren Abständen und Bindungswinkeln der an der Wasserstoffbrückenbindung beteiligten Atome in dem Wassertetraeder, abhängig. Die an dem Aufbau des Wasserpentamers beteiligten Wassermoleküle lassen sich in sechs verschiedenen möglichen Permutationen konfigurieren, in denen das Wassermolekül Protonen sowohl „abgeben“ als auch ,akzeptieren“ kann. Jede dieser Konfigurationen ist gleichberechtigt, d.h. 6-fach entartet (vergleiche Abbildung 1.3 links). Aufgrund der sechs verschiedenen Konfigurationsmöglichkeiten der Wassermoleküle können unterschiedliche Anordnungen auftreten, in denen die Wassermoleküle in ihrer Orientierung sowohl geordnet bzw. ungeordnet als auch partiell geordnet/ungeordnet vorliegen können (Abbildung 1.3). 


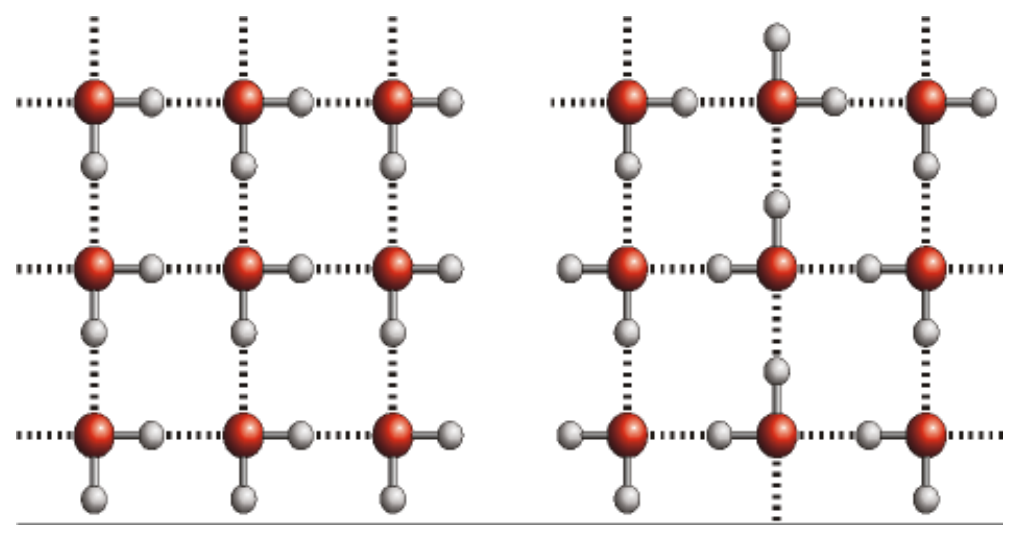

Abbildung 1.3: links: protonengeordnete Darstellung; rechts: protonenungeordnete Darstellung eines hypothetischen ebenen Kristallgitters

Beispiel für eine völlig ungeordnete Konfiguration der Protonen ist Eis Ih (Kuhs and Lehmann (1986)), während Eis II völlig protonengeordnet ist (Kamb (1964)). Beispiele für partiell protonengeordnete Eisstrukturen sind Eis III und Eis V, wobei der Protonenordnungsgrad dieser Eisstrukturen zu tieferen Temperaturen hin zunimmt (Lobban et al. (2000)). Die durch Diffraktion mittels Neutronen-, Synchrotron- und Röntgenstrahlung bestimmbare Kristallstruktur ergibt sich im Falle geordneter, ungeordneter bzw. partiell geordneter Eisphasen als räumlich (durch Konfigurationsanordnung ) und zeitlich (aufgrund thermischer Schwingungen der Atome) gemittelten Struktur über die sechs möglichen Konfigurationen (Abbildung 1.4).

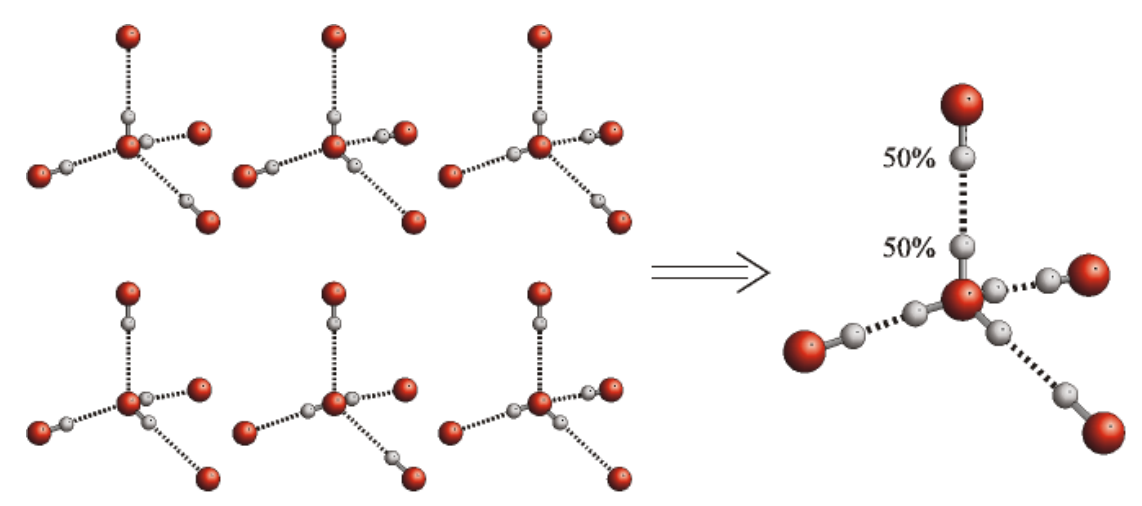

Abbildung 1.4: links: Darstellung der sechs tetraedrischen Anordnungen der Wassermoleküle; rechts: Darstellung der räumlich und zeitlich gemittelten Struktur, für eine Wasserstoffbrückenbindung ergeben bei einer völlig protonenungeordneten Struktur je zwei Besetzungen der Protonen mit einer halben Besetzungswahrscheinlichkeit

Die gemessene mittlere Struktur stellt sich als geordnete Konfiguration der Sauerstoffatome dar. Auf der Verbindungslinie zwischen zwei O-Atomen befinden sich die delokalisierten Protonen des Deuteriums bzw. Wasserstoffs (Bragg (1922), Peterson \& Levy (1957)). In einer 
völlig protonenungeordneten Struktur ist aufgrund der zwei Orientierungsmöglichkeiten des Wassermoleküls in den Brückenbindungen die Besetzungswahrscheinlichkeit für beide Protonen der an der Bindung beteiligten Wassermoleküle lediglich 50\%. Für eine protonengeordnete Struktur ist die Besetzungswahrscheinlichkeit für eine der beiden Wasserstoffbrückenpositionen entweder $0 \%$ bzw. 100\%. Für partiell geordnete Strukturen wie Eis III oder Eis V liegt diese für eine Wasserstoffbrückenbindung zwischen $0-100 \%$, komplementär dazu ist die Besetzungswahrscheinlichkeit des anderen Protons in der Bindung. In einem freien Wassermolekül beträgt der Bindungsabstand von O-H 0.9572 $\AA$ und der Bindungswinkel $\mathrm{H}-\mathrm{O}-\mathrm{H} \sim 104.52^{\circ}$ (Benedict et al. (1956)), der von dem idealen Tetraederwinkel von $109.47^{\circ}$ abweicht. Im Gegensatz zu einem freien Molekül beträgt in einer tetraedrischen Anordnung von brückengebundenen Wassermolekülen wie Eis Ih der Winkel $\mathrm{O}(2)-\mathrm{O}(1)-\mathrm{O}(3)$ über die Wasserstoffbrücken $\mathrm{O}(2) \ldots \mathrm{D}(1)-\mathrm{O}(1)$ und $\mathrm{O}(1) \ldots \mathrm{D}(2)-$ $\mathrm{O}(2) \sim 109.47^{\circ}$. Mittels NMR-Messungen an Eis Ih konnte nachgewiesen werden, dass der ursprüngliche Bindungswinkel im Wassermolekül D-O-D erhalten bleibt. Erste Hinweise wurden hier jedoch auch schon früh von Bernal \& Fowler (1933) gegeben (vergl. Kapitel 5). Von Kuhs \& Lehmann (1983) konnte an Eis Ih nachgewiesen werden, dass eine geringe Aufweitung des Bindungswinkels $\mathrm{D}(2) \ldots \mathrm{O}(1)-\mathrm{D}(1)$, bzw. der Sauerstoffwinkel dreier zueinander verschieden orientierter Wassermoleküle, als Funktion der Temperatur stattfindet. Aufgrund äußerer Einflüsse, z.B. durch Kompression, können die Wasserstoffbrücken durch Verzerrung der Bindung, d.h. durch die Änderung des Bindungsabstandes und des Bindungswinkels oder eine völlige Reorientierung, diesem Einfluss ausweichen. Bei den Hochdruckeisphasen, Eis II und Eis III, die sich durch Kompression von Eis Ih ergeben, werden die Wassermoleküle reorientiert. Die Bindungsabstände sowie die Bindungswinkel der Wasserstoffbrücken weichen von denen des Eis Ih deutlich ab. Aufgrund der Neuorientierung erlaubt dies eine dichtere Packung der Wassermoleküle in der Eisstruktur. Findet keine Neuorientierung, d.h. kein Umbau der Kristallstruktur, statt, reagieren die Wasserstoffbrücken auf einen äußeren Einfluss mit einer Veränderung der Brückenbindungsabstände sowie des H-Brückenbindungswinkels. Die ursprüngliche Anordnung wird verzerrt. Verschiebunegn der Atompositionen des Sauerstoffs und der HProtonen auf den durch die Struktur der jeweiligen Phase kristallographisch festgelegten Positionen treten auf und deuten auf eine lokal deformierte Kristallstruktur hin. Ebenso wie bei der Reorientierung bleibt die Wassermolekülgeometrie wie auch die tetraedrische Anordnung der Moleküle weitgehend erhalten. Die tetraedrische Anordnung ist im 
wesentlichen durch die elektrostatischen Wechselwirkungen innerhalb einer gegebenen Struktur mit den nächsten Nachbarn bestimmt. Diese sind durch die bindenden O-D bzw. nichtbindenden O-O und D-D Wechselwirkungskontakte der nächsten Nachbarn bzw. den daraus resultierenden Wechselwirkungspotenzialen gegeben. Für alle bisher entdeckten und strukturell bestimmten Eisphasen wurde eine tetraedrische Anordnung von Wassermolekülen gefunden, jedoch variieren die Bindungsabstände und Bindungswinkel. Die aus dem elektronischem Aufbau des Wassermoleküls resultierende Eigenschaft Wasserstoffbrücken auszubilden ist sowohl für Wasser in seinem flüssigen Aggregatzustand als auch für Eisstrukturen und deren strukturelle und physikalische Eigenschaften maßgebend. Die tetraedrisch gebundenen Wassermoleküle sind in allen Eisphasen in Ringstrukturen angeordnet, die sich durch die Ringgröße sowie der Anzahl der Wassermoleküle unterscheiden. Eine Aufstellung der zu findenden Ringstrukturen der bisher bekannten Eisphasen ist von Savage (1985) gegeben worden (vergl. Tabelle 1.1). Diese Wechselwirkungspotenziale werden im Allgemeinen in molekularen Computersimulationen durch Lennard-Jones-Potenziale beschrieben.

\begin{tabular}{|c|c|c|c|c|c|c|c|}
\hline Eis & $\begin{array}{c}\text { Anzahl nächster } \\
\text { Nachbarn, } \\
\text { O...O-Abstand } \\
\text { (H-gebunden) } \\
{[\AA ̊]}\end{array}$ & 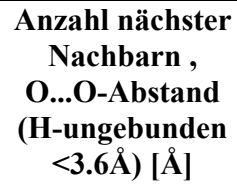 & $\begin{array}{l}\text { Protonen } \\
\text { Ordnung }\end{array}$ & $\begin{array}{l}\begin{array}{c}\text { Bindungs- } \\
\text { Winkel }\end{array} \\
\text { O-O-O }\left[^{\circ}\right]\end{array}$ & $\begin{array}{c}\text { Dichte } \\
{\left[\mathrm{gcm}^{-3}\right]} \\
\mathrm{H}_{2} \mathrm{O}\end{array}$ & $\begin{array}{c}\text { Wasser- } \\
\text { Moleküle / } \\
\text { Elementar- } \\
\text { zelle }\end{array}$ & $\begin{array}{c}\text { Anzahl der } \\
\text { Moleküle } \\
\text { in Ring }\end{array}$ \\
\hline I h & $4,2.76$ & ---- & $\mathrm{D}$ & 109 & 0.931 & 4 & 6 \\
\hline I c & $4,2.75$ & ---- & $\mathrm{D}$ & 109.5 & 0.93 & 2 & 6 \\
\hline II & $4,2.77-2.84$ & $9,3.24-3.60$ & $\mathrm{O}$ & $81-128$ & 1.18 & 12 & 6,8 \\
\hline III & $4,2.75-2.80$ & $1,3.45$ & $\mathrm{D}$ & $87-144$ & 1.16 & 12 & $5,7,8$ \\
\hline IX(III) & $4,2.75-2.80$ & $1,3.45$ & $\mathrm{P}$ & $87-144$ & 1.16 & 12 & $5,7,8$ \\
\hline IV & $4,2.79-2.92$ & $9,3.14-3.29$ & $\mathrm{D}$ & $88-128$ & 1.27 & 16 & 6,8 \\
\hline $\mathbf{V}$ & $4,2.76-2.80$ & $7,3.28-3.49$ & $\mathrm{D}\left(\mathrm{P}^{*}\right)$ & $84-128$ & 1.23 & 10 & $4,5,6,7,8$ \\
\hline VI & $4,2.80-2.82$ & $17,3.44-3.46$ & $\mathrm{D}$ & $76-128$ & 1.31 & 12 & 4,8 \\
\hline VII & $4,2.90$ & $4,2.90$ & $\mathrm{D}$ & 109.5 & 1.50 & 5 & 6 \\
\hline VIII & $4,2.88$ & $5,2.74-3.14$ & $\mathrm{O}$ & 109.5 & 1.50 & 5 & 6 \\
\hline $\mathbf{X I I}^{(a)}$ & $4,2.803$ & $6,2.766$ & $\bar{D}$ & $83.36-132.58$ & 1.437 & 12 & 7,8 \\
\hline
\end{tabular}

Tabelle 1.1: Aufstellung der Ringstrukturen in den Eisphasen, Savage (1985), D: ungeordnete, O : geordnete, $\mathbf{P}$ : partiell protonengeordnete Struktur, * : partielle Protonenordnung für Eis V durch Lobban et al. (2000) nachgewiesen, @ : aus Lobban et al. (1998) sowie O'Keefe (1998). 
Die Wechselwirkungspotenziale legen bei gegebenem Druck und Temperatur die Anordnung und damit die Wechselwirkungskontakte der Struktur fest. Aus den sich daraus ergebenden Wechselwirkungskontakten ergeben sich die physikalischen Eigenschaften der Struktur.

Die freie Gibbs'sche Energie bei gegebenen Druck- und Temperaturbedingungen eines Systems ist gegeben durch

$$
G=E+p \cdot V-T \cdot S
$$

Sowohl die innere Energie E als auch die Konfigurationsentropie S des Systems werden über die druck- und temperaturabhängigen Bindungslängen und Bindungswinkel und somit über die Anzahl der bindenden und nichtbindenden Kontakte bestimmt. Diese haben dadurch einen wesentlichen Einfluss auf die Stabilität der Eisstruktur und deren physikalischem Verhalten. Festzustellen ist, dass die freie Gibbs'sche Energie bei Druck- und Temperaturänderungen auf verschiedene Art und Weise minimiert werden kann. Zum einen kann dies durch die Verkürzung der Wasserstoffbrückenbindungen geschehen wie z.B. bei Eis Ih bei Kompression. Zum anderen wird dies durch eine dichtere Packung der Wassermoleküle, infolge der Ausbildung kleinerer Ringstrukturen bewerkstelligt. Beispiel hierfür ist Eis V. Weiterhin können die Wasserstoffbrückenbindungen verbogen werden, wie bei Eis III und Eis XII, um so eine dichtere Packung zu ermöglichen. Die höchste Packungsdichte jedoch erhält man durch sich selbstdurchdringende Strukturen, bei denen sich, z.B. bei Eis IV Ringe aus Wassermolekülen oder aber auch gleiche Sub-Gitterstrukturen wie bei Eis VI,VII und VIII, selbst durchdringen.

\subsection{Das Phasendiagramm von Eis}

Das Phasendiagramm von Eis ist seit den ersten Arbeiten von Tammann (1900) nun mehr über hundert Jahre Gegenstand physiko-chemischer Experimente. Ebenso ist die Strukturbestimmung der einzelnen Eisphasen als Funktion von Druck- und Temperatur von Interesse. Die Phasengrenzen von Eis Ih zu der Flüssigkeit Wasser (L) und die Phasengrenzen von Eis Ih wurden erstmals für leichtes und schweres Wasser von Tammann (1903) bestimmt.

Das Eis Ih, das häufig in unserer alltäglichen Welt vertreten ist und sich durch Ausfrieren von Wasser unter normalen atmosphärischen Bedingungen bildet, wurde erstmals von Tammann 
(1900) unter Druck systematisch untersucht. Im Interesse der damaligen Experimente lagen die Druck- Volumenrelationen von Wasser und seiner kristallinen Form, dem Eis, als Funktion eines von außen aufgebrachten Druckes. In mehreren Arbeiten untersuchte Tammann den Stabilitäts- bzw. Existenzbereich von Eis Ih. Tammann $(1903,1909)$ entdeckte im Zuge dieser Experimente zwei neue Eisphasen, Eis II und Eis III, durch Kompression von Eis Ih auf Drücke von über p 3 kbar und bestimmte deren Phasengrenzen von Eis $\mathrm{Ih} /$ Eis II sowie Eis Ih / Eis III als auch Eis II / Eis III und Eis III / L.

Eis und Wasser waren in anderen Arbeiten die ersten Materialien, deren Strukturen mittels Röntgendiffraktion untersucht wurden. Röntgenographische Untersuchungen an Eis Ih wurden erstmals von Dennison (1921) durchgeführt, in denen er mit hinreichender Genauigkeit die Struktur des Sauerstoffgerüstes von Eis Ih bestimmte. Aus den Röntgendaten ermittelte Dennison (1921) eine hexagonale Elementarzelle sowie die mittleren Atompositionen der Sauerstoffatome.

Bragg (1922) nutzte diese Erkenntnisse zur Vorhersage einer Eisstruktur aus Sauerstoffatomen, in deren Verbindungslinie sich von Sauerstoff $\mathrm{zu}$ Sauerstoff ein Wasserstoff (Proton) in der Mitte der Bindung befindet und so das Modell zur Aneinanderbindung von Wassermolekülen durch die Ausbildung von Wasserstoffbrückenbindungen nutzte. Barnes (1929) führte an Eis Ih Einkristalluntersuchungen durch und konnte die von Dennison (1921) bestimmten strukturellen Eigenschaften der Elementarzelle sowie den hexagonalen Aufbau des Eiskristallgitters bestätigen.

Bernal und Fowler (1933) führten aus, dass Wassermoleküle in Eis und in Wasser trotz der unterschiedlichen Aggregatzustände dieselbe Geometrie haben wie in der Gasphase. Diese Annahme wurde durch den Vergleich von Ramanspektren von Wasser in den verschiedenen Aggregatzuständen begründet. Konkrete Aussagen über die Bindungswinkel bzw. Bindungslängen im Wassermolekül wurden jedoch nicht getroffen.

Pauling (1935) entwickelte ein einfaches statistisches Modell für die Struktur von Eis Ih. Basis des Modells war die durch Messungen zu vermutende tetraedrische Anordnung von 
Wassermolekülen, aus der er über die möglichen Konfigurationen der Wassermoleküle zueinander die Nullpunktsentropie von Eis Ih berechnen konnte.

Wollan et al. (1949) gelang es erstmals unter Verwendung von Neutronenstrahlen die Protonen in der Kristallstruktur direkt in deuteriertem Eis Ih nachzuweisen. Peterson \& Levy (1957) führten an deuterierten Eis Ih-Einkristallen Neutronendiffraktionsexperimente durch. Die Fourieranalysen der gemessenen Neutronenstreudichten zeigten, dass sich die Vermutung von Bragg (1922), ein Proton befinde sich zwischen zwei O-Atomen, bestätigte. Aus dem Experiment ergab sich jedoch ein weiterer, neuer Aspekt: Die Besetzung entlang einer Wasserstoffbrückenbindung, zwischen zwei O-Atomen zeigte eine Aufspaltung in den Wasserstoffatompositionen. Es ergaben sich entlang einer Bindung zwei H-Positionen mit je einer halben Besetzungswahrscheinlichkeit. Dadurch belegten Sie erstmals eindeutig die Protonenunordnung, d.h. die statistisch verteilte Orientierung der Wassermoleküle in der EisIh-Struktur und bestätigten damit das Modell der Eis Ih-Struktur von Pauling (1935).

Goto et al. (1990) zeigten in sehr präzisen und hochauflösenden Röntgendiffraktionsexperimenten, dass die Lokalisierung der Wasserstoffe nicht nur mittels Neutronen sondern auch über die Elektronendichteverteilung zu bestimmen ist.

Die Struktur von Eis Ih wurde detailliert von Kuhs \& Lehmann (1986) an Eis Ih-Einkristallen mittels Neutronendiffraktion bestimmt. Sehr präzise Untersuchungen hinsichtlich der Gitterkonstanten wurden durch Röttger et al. (1994) erstmals an Eis Ih-Pulver durchgeführt. In diesen Messungen wurden mittels Synchrotronbeugung die Gitterkonstanten als Funktion der Temperatur bestimmt. Weitere Experimente hinsichtlich der strukturellen Eigenschaften als Funktion der Temperatur wurden von Line \& Whitworth (1996) durchgeführt und bestätigten die Ergebnisse von Röttger et al. (1994). Das Modell von Kuhs \& Lehmann (1986) sowie die von Röttger et al. (1994) bestimmten strukturellen Parameter bilden die Grundlage für die Rietveld-Verfeinerungen der mittels Neutronendiffraktion gewonnen Datensätze von Eis Ih als Funktion des Druckes und der Temperatur, ebenso wie für die unter der Verwendung der verschiedenen als Drucküberträgermedien dienenden Gase Argon, Helium und Neon präparierten Eisproben. 
Bridgman (1912) führte die Arbeiten von Tammann (1903, 1909) an Hochdruckeisphasen fort und vermaß zum einen die Phasengrenzen der bis dahin bekannten Eisphasen I, II und III und stellte seine Ergebnisse neben die von Tammann ermittelten experimentellen Befunde. Die Ergebnisse von Bridgman stimmten im Rahmen der damaligen und angegebenen messtechnischen Möglichkeiten überein. In weiteren Untersuchungen fügte Bridgman (1912) dem, zu der damaligen Zeit noch übersichtlichen, Eisphasendiagramm die HochdruckEisphasen V, VI, VII sowie eine metastabile Variante, Eis IV, hinzu. Bridgman bestimmte für $\mathrm{H}_{2} \mathrm{O}$ (1912) sowie $\mathrm{D}_{2} \mathrm{O}$ (1935) alle Schmelzkurven der bis dahin bekannten Eisphasen als Funktion des Druckes. Im Interesse der damaligen Untersuchungen lagen die Druck- und Volumenrelationen der Eisphasen als Funktion des Druckes- und der Temperatur bei Phasenumwandlungen oder dem Schmelzen. Strukturelle Untersuchungen wurden von Tammann (1900, 1903, 1909) sowie von Bridgman $(1912,1935$, 1937) nicht durchgeführt, sondern erst zu einem späteren Zeitpunkt, da Strukturuntersuchungen erst durch das Aufkommen der Röntgenographie möglich wurden.

Die Bestimmung der strukturellen Eigenschaften der von Tammann (1900) entdeckten Eisphasen II und III erfolgte erst sehr viel später und zudem an ,recoverten” Proben. Die erste detaillierte Bestimmung der Struktur von Eis III wurde von Kamb \& Prakash (1968) mittels röntgenographischer Methoden an „recoverten“ Eis III-Proben durchgeführt. Die Autoren lösten die Struktur in der tetragonalen Raumgruppe $\mathrm{P} 4_{1} 2{ }_{1} 2$ mit a=6.73 $\mathrm{a}$ und $\mathrm{c}=6.83 \AA$. Durch die Raumgruppe ergibt sich, dass die Wasserstoffpositionen auf einer Wasserstoffbrücke nicht auf symmetrie-äquivalenten Positionen festgelegt sind, so dass die Eis III-Struktur einen Zustand zwischen völliger Protonenordnung als auch -unordnung annehmen kann. Dieser Effekt wurde von Whalley et al. (1968) durch eine Serie von Messungen der Dielektrizitätskonstanten an Eis III belegt. In einem Temperaturbereich zwischen $\mathrm{T}=-65^{\circ} \mathrm{C}$ und $\mathrm{T}=-108^{\circ} \mathrm{C}$ zeigte sich eine Transformation in eine völlig protonengeordnete Eis IIIStruktur. Diese protonengeordnete Struktur erhielt die Bezeichnung Eis IX. Weitergehende in situ Untersuchungen zur Protonenordnung wurden von Londono et al. (1993) sowie von Kuhs et al. (1998) sowie Lobban et al. (2000) durchgeführt.

Eis II wurde erstmals von Kamb (1964) mittels röntgenographischer Methoden strukturell bestimmt und die Struktur von Kamb et al. (1971) mittels Neutronendiffraktion an „,recoverten“ Eisproben bestätigt. Aus diesen Experimenten ließ sich durch diffraktometrische 
Methoden direkt bestätigen, dass eine vermutete völlige Protonenordnung für Eis II, im Gegensatz zu der völlig protonenungeordneten Eisphase Eis Ih oder der partiell geordneten Eisphase III, vorlag. Der erste Nachweis einer völligen Protonenordnung wurde sowohl durch Untersuchungen der Eisphasengrenze von Eis Ih zu Eis II als auch durch spätere Experimente von Wilson et al. (1965) gegeben. Wilson bestimmte die Dielektrizitätskonstante und konnte durch eine nicht vorhandene Debye-Relaxation im dielektrischen Verhalten auf eine völlige Protonenordnung des Eis II schließen. Kamb et al. (1971) bestimmten über Neutronenbeugung an „recoverten“ Einkristallproben $\left(\mathrm{D}_{2} \mathrm{O}\right)$ eine rhomboedrische Raumgruppe $\mathrm{R} \overline{3} \mathrm{c}$.

Die von Bridgman (1912) gefundene Existenz der Eisphase IV wurde durch Schmelzkurvenexperimente von Evans (1967) sowie Engelhardt und Whalley (1972) als auch von Nishibata (1972) bestätigt. Eis IV wurde über verschiedene Herstellungsverfahren gewonnen. (vergl. Kapitel 3.). Die Struktur von Eis IV konnte erst später von Engelhardt \& Kamb (1981) an einer zuvor „recoverten“ Probe mittels Röntgendiffraktion festgestellt werden. Die Struktur konnte in der rhomboedrischen Raumgruppe $\mathrm{R} \overline{3} \mathrm{c}$ gelöst werden. Während des „quenchens“ der hergestellten Eis IV-Probe konnte keine Phasenumwandlung festgestellt werden. Jedoch konnte aufgrund der Metastabilität dieser Eisphase eine Phasenumwandlung in eine weitere unbekannte Eisphase bzw. die Umwandlung aus einer bei den Herstellungsbedingungen existenten unbekannten Phase in das als Eis IV bezeichnete Eis während des Quenchens nicht ausgeschlossen werden. Erst Lobban et al. (1998) konnten bei in situ Neutronendiffraktionsexperimenten in einem einzigen Fall Eis IV feststellen und bei den Bildungsbedingungen die Struktur als die von Engelhardt \& Kamb (1981) bestimmte kristallographische Struktur identifizieren.

Die Struktur von Eis V wurde erstmals von Kamb et al. (1967) bestimmt. Das Eis V zeigt eine sehr komplexe Struktur in der monoklinen Raumgruppe A2/a mit $a=9.22 \AA, b=7.54 \AA, c=10.35$ $\AA$ und $\beta=109.2^{\circ}$. Die Elementarzelle enthält 28 Wassermoleküle, die in kleinen Anordnungen von Ringen, die sowohl mit jeweils vier Wassermolekülen als auch mit fünf, sechs, sieben und sogar acht Wassermolekülen besetzt sind, gebildet wird (vergl. Tabelle 1.1). Dielektrische Messungen in dem Stabilitätsbereich von und an Eis V von Wilson et al. (1965) zeigten, dass Eis V eine protonenungeordnete Struktur ist. Jedoch zeigten sich schon früh und durch spätere Experimente bestätigt (Neutronendiffraktionsexperimente von Hamilton et al. 
(1969), Kuhs et al. (1998), durch ramanspektroskopische Messungen von Minceva-Sukarova et al. (1986) sowie durch kalorimetrische Messung an „recoverten“ Eis V- Proben von Handa et al. (1987)), dass die Protonenunordnung des Eis V eine Funktion der Temperatur ist. $\mathrm{Zu}$ tieferen Temperaturen hin zeigte sich eine zunehmende Protonenordnung, die ein zu dem Ordnungsverhalten von Eis III / Eis IX ähnliches Verhalten aufweist. Eine vollständige Protonenordnung wurde für das Eis V hingegen nicht gefunden (Lobban et al. (2000)).

Eis VI wurde erstmalig von Kamb (1965) an „recoverten“ Proben strukturell bestimmt. Das Eis VI wurde von Kamb (1965) als eine Struktur beschrieben, in denen zwei gleichartige Gitter in allen drei Raumrichtungen um je die halbe Gitterkonstante verschoben sind (,SelbstClathrat“). Diese wiederum „recoverte” Struktur wurde von Kuhs et al. (1984) an $\mathrm{D}_{2} \mathrm{O}$-Proben unter in situ Bedingungen mittels Neutronendiffraktion bestätigt. Aufgrund des strukturellen Aufbaus und dem Verhalten der Phasengrenzen kann für das Eis VI eine Protonenunordnung angenommen werden. Diese Annahme ist durch experimentell ermittelte dielektrische Relaxationsprozesse durch Johari \& Whalley (1976) bis zu Temperaturen von T 128K bestätigt.

Als weitere metastabile Eisphase neben Eis IV wurde in dem Stabilitätsbereich von Eis V / Eis VI die Eisphase XII von Lobban et al. (1998) während eines in situ Neutronendiffraktionsexperimentes entdeckt. Aussagen von Lobban et al. (1998) über den Existenzbereich konnten erst durch einige wenige Messungen getroffen werden. Koza et al. (1999) fanden bei Herstellungsversuchen von ,amorphen” Eisphasen, dass Eis XII in einem anderen Druck- und Temperaturbereich des Phasendiagramms hergestellt werden konnte. Koza et al. (1999) konnten Eis XII bei schneller Kompression von Eis Ih bei tieferen Temperaturen $(\mathrm{T} \sim 77 \mathrm{~K})$ auf Drücke $\mathrm{p}>12 \mathrm{kbar}$ gewinnen. Weitere experimentelle Hinweise auf eine weitere, eher zufällig gefundene und nicht identifizierte Phase ist in dem nahezu äquivalenten Druck- und Temperaturbereich von Bizhigitov \& Sirota (1987) gefunden worden. Sirota \& Bizhigitov $(1987,1988)$ konnten mehrmals diese unbekannte Phase herstellen und durch Enthalpieänderungen punktuell die Phasengrenze zu Ih, Eis VII sowie Eis VIII bestimmen. Eine strukturelle Identifizierung oder die Angabe von Phasengrenzen wurde nicht gegeben. Ob es sich hierbei um einen Phasenübergang von HDA („High-DensityAmorphous-Ice") in Eis XII oder um eine wiederum unbekannte Eisphase oder eisverwandte Struktur handelt, kann aufgrund der wenigen experimentellen Untersuchungen und der 
letztendlich fehlenden strukturellen Identifizierung nicht ausgesagt werden. Der Frage ob es sich um zwei weit getrennte Existenzenklaven oder gar um einen sehr weitreichenden metastabilen Existenzbereich von Eis XII handelt, wurde im Zuge eigener Eis XIIHerstellungsversuche nachgegangen (vergl. Kapitel 3.7)

In dem Eisphasendiagramm existieren zudem Eisphasen, die durch Diffraktionsexperimente als verschieden identifiziert, deren Struktur letztendlich aber als nicht eindeutig bestimmt gelten muss. Beispiele für diese sind z.B. die ,glasartigen oder amorphen Eisphasen”. Diese sind durch vielfältige Messungen untersucht worden, ohne jedoch definitive Aussagen hinsichtlich deren strukturellen Eigenschaften treffen $\mathrm{zu}$ können. Als Beispiel sind hier sowohl die amorphen Eisphasen HDA („High-density-amorphous-Eis“) sowie das LDA („Low-density-amorphous-Eis“) anzuführen, die erstmals von Mishima, Calvert \& Whalley $(1984,1985)$ gefunden wurden als auch das schon früh entdeckte „,vitrous ice” von Dowell und Rinfret (1960). In Diffraktionsexperimenten zeigte sich, dass diese im allgemeinen als amorphe Eisphasen bezeichnete Strukturen im "Streubild” ein „quasi-Wasseraussehen“ gemein haben. Jedoch ergeben sich für HDA und für LDA Unterschiede hinsichtlich des Diffraktogramms, die auf strukturelle Differenzen hinweisen. Die durch Diffraktionsexperimente bestimmten strukturellen Unterschiede in den „quasiWasserdiffraktogrammen“ lassen auf eine fehlende oder nur verhältnismäßig wenig ausgebildete Fernordnung (Periodiziät) schließen. Es liegt nahe, dass die Unterschiede durch lokale Nahordnungsphänomene der Wassermoleküle in den einzelnen Eisphasen verursacht werden.

Zudem existiert mit dem kubischen Eis Ic eine Eisphase, dessen Eisstruktur mittels Diffraktionsexperimenten mehrfach bestimmt wurde, für die jedoch trotz einer langreichweitigen Ordnung und der damit verbundenen Periodizität keine eindeutige Struktur angegeben werden kann. Kubisches Eis Ic kommt neben Eis Ih als eine metastabile Variante vor und wurde von König (1943) entdeckt. Sowohl Dowell und Rinfret (1960) als auch andere Autoren untersuchten das Phasenverhalten und die Herstellung von kubischem Eis und Kuhs et al. (1987) die strukturellen Eigenschaften. In der Arbeit von Kuhs et al. (1987) wurde die Struktur von kubischem Eis Ic als stapelfehlgeordnete Eisphase diskutiert. Detaillierte Angaben über die Stapelfehlerhäufigkeit und lateraler Fehlordnungen konnten damals jedoch 
nicht getroffen werden, sind jedoch im Kapitel 3.3 Gegenstand von Untersuchungen und Interpretationen.

Beweise für die Existenz weiterer, bis dato völlig unbekannter Eisphasen wurden experimentell gefunden bzw. wurden aufgrund theoretischer Überlegungen vermutet. Experimentelle Beweise für zwei bisher strukturell unbekannte Eisphasen, die ,possible new phases" pnp-XIII und pnp-XIV, sind durch Schmelzkurvenbestimmungen von Mishima \& Stanley (1998) gegeben worden. Chou et al. (1998) fanden, weiterhin durch Schmelzkurvenexperimente, einen Beweis einer vermutlich neuen Eisphase, einfach bezeichnet als „new ice“, die wahrscheinlich nicht mit den vorher genannten identisch ist. In beiden Arbeiten werden keine Aussagen über die Struktur dieser Eisphasen getroffen. Auch sind keine sehr detaillierten Angaben bezüglich der Herstellung oder der experimentellen Vorgehensweise aufgeführt worden. Es kann davon ausgegangen werden, dass die Herstellung dieser Eisphasen nicht ohne weiteres reproduzierbar ist, da seit dem Zeitpunkt der Veröffentlichung keine weiteren Hinweise bezüglich der Herstellung erfolgt sind.

Abschließend ist anzuführen, dass der in diesem Kapitel erstellte Überblick und die Aufzählung der Eisphasen nicht vollständig ist. Es geht lediglich um eine chronologische Aufzählung der mit den experimentellen Mitteln erreichbaren Eisphasen. Die Eisphasen VII, VIII, X und XI sowie die "glasartigen und amorphen Eisphasen" werden aufgrund der im Rahmen dieser Arbeit durchgeführten Experimente im Druck- und Temperaturbereich bis p 6.5kbar Gasdruck als auch den Temperaturen bis T $\sim 77 \mathrm{~K}$ im weiteren nicht diskutiert. Für die Darstellung und die Diskussion der bisher nicht erwähnten Eisphasen sei der Vollständigkeit halber auf das Buch „Physics of Ice” von Victor F. Petrenko \& Robert W. Whitworth (1999) verwiesen. Eine Aufstellung der bisher bekannten und strukturell identifizierten Eisphasen ist in Tabelle 1.2 gegeben. Die genannten Eisphasen finden sich in sehr unterschiedlichen Druck- und Temperaturbereichen, die von Eis Ih unter Standardbedingungen bis hin zu Drücken oberhalb von $\mathrm{p} \sim 100 \mathrm{kbar}$ für Eis $\mathrm{X}$ und Temperaturen von weit unterhalb von $\mathrm{T} \sim 77 \mathrm{~K}$ für Eis XI bis weit über die Schmelztemperatur von normalem Eis Ih geht. Alle Eisphasen, die oberhalb des Existenzbereiches von Eis Ih existieren, werden im allgemeinen als Hochdruckeisphasen (HD-Eisphasen) bezeichnet. Die folgende Abbildung 1.5 stellt das zur Zeit gängige und allgemein akzeptierte 
Eisphasendiagramm dar und vermittelt einen Eindruck über die Lage und Größe der Existenzbereiche der unterschiedlichen Eisphasen.

\begin{tabular}{|c|c|c|c|c|c|c|c|c|c|}
\hline Eis & $\begin{array}{l}\text { Kristall- } \\
\text { Klasse }\end{array}$ & $\begin{array}{l}\text { Raum- } \\
\text { gruppe }\end{array}$ & $\begin{array}{c}\text { Protonen } \\
\text { ordnung }\end{array}$ & $\begin{array}{l}\text { Molek. } \\
\text { pro } \\
\text { EZ }\end{array}$ & $\mathbf{T}(\mathrm{K})$ & $\begin{array}{c}\mathbf{p} \\
(\mathbf{G P a})\end{array}$ & $\begin{array}{c}\text { Dichte } \\
\left(\mathrm{Mg} \mathrm{m}^{-3}\right)\end{array}$ & $\begin{array}{c}\text { Gitterkonstante } \\
\text { (§) }\end{array}$ & Literatur \\
\hline I h & Hex. & $\mathrm{Pb}_{3} / \mathrm{mmc}$ & $\mathrm{D}$ & 4 & 250 & 0 & 0.920 & $\begin{array}{l}a=4.518, c=7.356+ \\
a=4.522, c=7.363^{*}\end{array}$ & Röttger et al. (1994) \\
\hline I c & Kub. & $\mathrm{Fd} \overline{3} \mathrm{~m}$ & $\mathrm{D}$ & 8 & 78 & $0(q)$ & 0.931 & $\mathrm{a}=6.358^{*}$ & Kuhs et al. (1987) \\
\hline II & Rhom. & R 3 & $\mathrm{O}$ & 12 & $\begin{array}{l}123 \\
83\end{array}$ & $\begin{array}{l}0(\mathrm{q}) \\
0(\mathrm{q})\end{array}$ & $\begin{array}{l}1.170 \\
1.342\end{array}$ & $\begin{array}{c}\mathrm{a}=7.78, \alpha=113.1^{\circ}+ \\
\mathrm{a}_{\mathrm{H}}=12.92, \mathrm{c}_{\mathrm{H}}=6.23+(\mathrm{x}) \\
\mathrm{a}_{\mathrm{H}}=12.871, \mathrm{c}_{\mathrm{H}}=6.217^{*}\end{array}$ & $\begin{array}{c}\text { Kamb (1964) } \\
\text { ILL D1B Experiment }\end{array}$ \\
\hline III & Tetr. & $\mathrm{P} 4_{1} 2_{1} 2$ & $\mathrm{P}$ & 12 & $\begin{array}{c}250 \\
\text { vergl. } \\
\text { IX }\end{array}$ & 0.28 & 1.165 & $a=6.666, c=6.936^{*}$ & Londono et al. (1993) \\
\hline IV & Rhom. & $\mathrm{R} \overline{3} \mathrm{c}$ & $\bar{D}$ & 16 & $\begin{array}{l}110 \\
260\end{array}$ & $\begin{array}{l}0(\mathrm{q}) \\
0.50\end{array}$ & $\begin{array}{l}1.272 \\
1.292\end{array}$ & $\begin{array}{c}\mathrm{a}=7.60, \mathrm{cH}=\alpha=70.1^{\circ}+ \\
\mathrm{a}_{\mathrm{H}}=8.74, \mathrm{c}_{\mathrm{H}}=17.05+(\mathrm{x}) \\
\mathrm{a}_{\mathrm{H}}=8.67, \mathrm{c}_{\mathrm{H}}=17.018^{*}\end{array}$ & $\begin{array}{c}\text { Engelhardt and Kamb } \\
\text { (1981) } \\
\text { Lobban et al. (1998) }\end{array}$ \\
\hline $\mathbf{V}$ & Monok. & $\mathrm{A} 2 / \mathrm{a}$ & $P$ & 28 & $\begin{array}{l}98 \\
100\end{array}$ & $\begin{array}{c}0 \\
0(q)\end{array}$ & $\begin{array}{l}1.231 \\
1.390\end{array}$ & $\begin{array}{c}\mathrm{a}=9.22, \mathrm{~b}=7.54 \\
\mathrm{c}=10.35, \beta=109.2^{\circ}+ \\
\mathrm{a}=9.141, \mathrm{~b}=7.538 \\
\mathrm{c}=10.288, \beta=109.31^{*}\end{array}$ & $\begin{array}{l}\text { Kamb et al. (1967) } \\
\text { Lobban et al. (2000) }\end{array}$ \\
\hline VI & Tetr. & $\mathrm{P} 4_{2} / \mathrm{nmc}$ & $\mathrm{D}$ & 10 & 225 & $\begin{array}{l}1.1 \\
-\end{array}$ & $\begin{array}{c}1.373 \\
-\end{array}$ & $\begin{array}{c}a=6.181, c=5.698^{*} \\
-\end{array}$ & $\begin{array}{c}\text { Kuhs et al. (1984) } \\
-\end{array}$ \\
\hline IX & Tetr. & $\mathrm{P} 4{ }_{1} 2_{1} 2$ & $\mathrm{O}$ & 12 & $\begin{array}{l}165 \\
83\end{array}$ & $\begin{array}{l}0.28 \\
0(q)\end{array}$ & $\begin{array}{l}1.194 \\
1.312\end{array}$ & $\begin{array}{l}a=6.692, c=6.715^{*} \\
a=6.687, c=6.80^{*}\end{array}$ & $\begin{array}{l}\text { Londono et al. (1993) } \\
\text { ILL D1B Experiment }\end{array}$ \\
\hline XII & Tetr. & $\mathrm{I} \overline{4} 2 \mathrm{~d}$ & $\bar{D}$ & 12 & $\begin{array}{l}260 \\
83\end{array}$ & $\begin{array}{l}0.50 \\
0(q)\end{array}$ & $\begin{array}{l}1.292 \\
1.458\end{array}$ & $\begin{array}{l}a=8.304, c=4.024^{*} \\
a=8.258, c=4.015^{*}\end{array}$ & $\begin{array}{l}\text { Lobban et al. (1998) } \\
\text { ILL D1B Experiment }\end{array}$ \\
\hline
\end{tabular}

Tabelle 1.2: Aufstellung der Eisphasen und deren Strukturen in dem relevanten Druckbereich von $p=1$ bar bis p 6.5kbar. D: ungeordnete, $\mathrm{O}$ : geordnete, $\mathrm{P}$ : partiell protonengeordnete Struktur. ${ }^{*} \mathrm{D}_{2} \mathrm{O}-,+\mathrm{H}_{2} \mathrm{O}$-Daten, (q) : Probe gequenched, (x) : $a_{H}, c_{H}$ Gitterkonstanten in der korrespondierenden hexagonalen Einheitszelle der Raumgruppe $\mathbf{R} \overline{3} \mathbf{c}$

Die Stabilitätsbereiche, in denen die Eisphasen Ih, II, III, IV und V sowie die metastabilen Eisphasen IV und XII existieren, besitzt gegenüber den Stabiliätsbereichen von Eis VI sowie den weiteren Existenzbereichen der Hochdruckeisphasen VII, VIII, sowie für Eis X bis zu p 100kbar (10GPa) eine nur geringe Ausdehnung. Gerade im Hinblick auf die Vielzahl der koexistierenden Eisphasen und den experimentellen Hinweise der Existenz weiterer unbekannter Eisphasen in dem Stabilitätsbereich von Eis V und im unteren Stabilitätsbereich von Eis VI ist der Bereich von $\mathrm{p} \sim 2.5 \mathrm{kbar}$ bis $\mathrm{p} \sim 8 \mathrm{kbar}$ aber von besonderem Interesse. Die Bestimmung der strukturellen Eigenschaften und der Phasengrenzen der jeweiligen Eisphasen 
sowie der Verifizierung der unterschiedlichen Herstellungsbedingungen kommt daher eine besondere und wesentliche Bedeutung $\mathrm{zu}$.

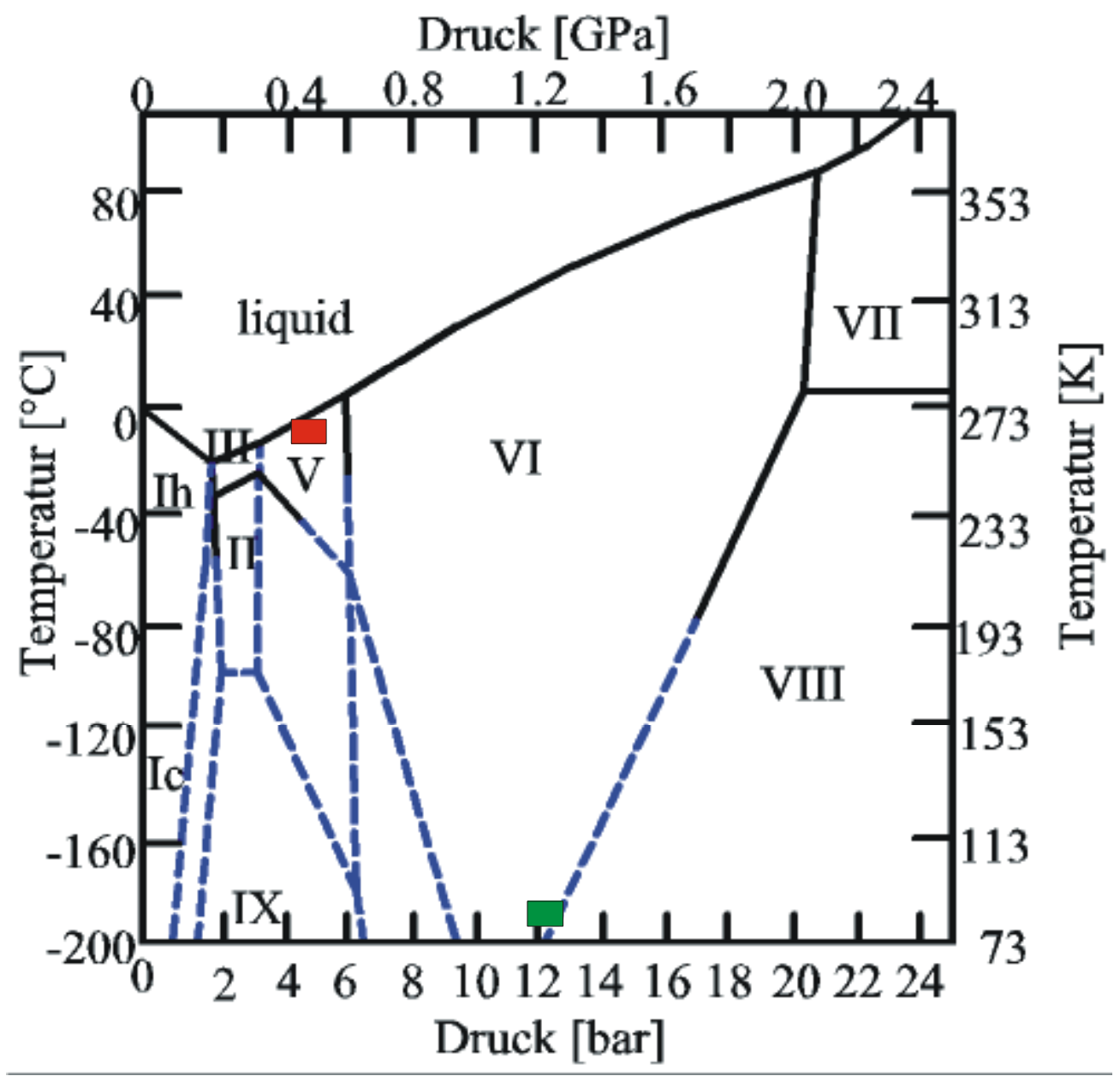

Abbildung 1.5: Schematisches Phasendiagramm von Eis im Druckbereich bis p 24kbar. Die experimentell ermittelten Phasengrenzen sind durchgehend gezeichnet, während die inter- bzw. extrapolierten Verläufe als gestrichelte Linien dargestellt sind; Quadrate : mittels Streumethoden nachgewiesene Existenz von Eis XII, (rot) Lobban (1998); (grün) : Koza et al. (1999).

\subsection{Phasenübergänge im System Eis}

Durch die Ausbildung von Wasserstoffbrückenbindungen der Wassermoleküle untereinander können sich durch die äußeren Einflüsse wie Druck und Temperatur, durch Änderung der Bindungswinkel sowie der Bindungsabstände mehrere Anordnungen der Wassermoleküle ergeben (vergl. Kapitel 1.2). Für die jeweiligen Eisphasen ergeben sich daher unterschiedliche kristallographische Strukturen, die im wesentlichen durch Ringstrukturen darstellbar sind. Die Ringstrukturen unterscheiden sich durch die Anzahl der in dem Ring enthaltenen 
Wassermoleküle sowie durch deren Form. Allen gemein ist jedoch die vollständige VierfachBindung durch Wasserstoffbrücken. Eine Aufstellung über die in den jeweiligen Eisphasen zu findenden Ringstrukturen ist durch Savage (1985) gegeben worden (vergl. Tabelle 1.1).

Die Frage, welche Vorgänge bei einem Phasenübergang von einer Eisphase in eine andere stattfinden, ist von grundlegendem Interesse. Von fundamentaler Rolle sind hier sowohl die Wasserstoffbrückenbindungen der Wassermoleküle untereinander als auch die Mechanismen, die zu einem Umbau von Wassermolekülen sowohl im Bulk, d.h. im Inneren eines Kristalliten als auch auf dessen Grenzflächen führen. Man kann davon ausgehen, dass aufgrund einer geringeren Bindung, die Wassermoleküle in den Grenzflächen der Kristallite eine höhere Beweglichkeit als im Bulk aufweisen. Ein Umbau der Strukturen dürfte daher eher an und in den Grenzflächen durch Reorientierung einzelner oder Wassermolekülegruppen stattfinden. Als weiterer Mechanismus ist nicht auszuschließen, wahrscheinlich jedoch von untergeordneter Bedeutung, dass Sublimation sowie Kondensation von Wassermolekülen eine Rolle bei der Keimbildung und dem Kornwachstum sowie letztendlich bei der Rekristallisation von Eisstrukturen sowie dem Wachstum eisverwandter Strukturen, wie z.B. den Gas-Hydraten in einer Eis-Ausgangsphase spielen kann. Als Ursache ist hier der hohe Dampfdruck von Eis anzuführen. Speziell an dem System Eis wurden bisher nur einige wenige Arbeiten hinsichtlich der systematischen Untersuchung von Phasenumwandlungen durchgeführt. Stern et al. (1997) und Bennett et al. (1997) untersuchten die Phasenumwandlungen von Eis Ih zu Eis II und konnten durch Bestimmung von Texturen (Vorzugsorientierungen) eine Texturverwandschaft von Eis Ih $\mathrm{zu}$ Eis II feststellen. Die Ergebnisse lassen darauf schließen, dass die Kristallite, mit der basalen Ebene der Eis IIElementarzelle mit ihrer c-Achse bevorzugt senkrecht zur c-Achse der hexagonalen Eis IhElementarzelle orientiert sind, topotaktisch wachsen. Eine Art des „Topologie-Recycling” ist damit naheliegend. Hieraus kann gefolgert werden, das es sich bei dieser Art des Phasenüberganges um einen partiell strukturellen Umbau handelt, bei denen die vorhandenen lokalen Ringstrukturen des Eis Ih ,recycelt” werden (Stern et al. (1997), Bennett et al. (1997)). Weitergehende experimentelle Untersuchungen, auch an Phasenübergängen anderer Eisphasen, sind nicht vorhanden. Theoretische Überlegungen und Arbeiten zu strukturellen Verwandschaften wurden von Minceva-Sukarova (1986) durchgeführt. 
Das Phasenübergangsverhalten von Wasser bzw. Eis war im Rahmen dieser Arbeit nicht im eigentlichen Interesse, obwohl einige Effekte wie z.B. das Hysterese-Verhalten bei festflüssig- / fest-fest-Phasenübergängen oder Einfluss der Präparationsrouten auf die Lage von fest-fest-Phasengrenzen im Rahmen der durchgeführten pVT-Experimente festzustellen waren.

Im Zuge von Herstellungsexperimenten für polykristallines Eis Ih über eine Tieftemperaturroute zeigte sich, dass ,recoverte“ Hochdruckeisphasen unterschiedliche Kinetiken der Phasenumwandlung in kubisches Eis Ic aufwiesen. Das jeweils erhaltene kubische Eis Ic zeigte zudem strukturelle Unterschiede. Diese Unterschiede weisen wie die Arbeiten von Stern et al. (1997) sowie Bennett et al. (1997) auf unterschiedliche Umbauprozesse der Kristallstrukturen durch die jeweiligen Strukturunterschiede der Ausgangsphasen sowie durch die unterschiedlichen Kinetiken der Phasenumwandlungen hin.

Weiterhin wurde im Zuge der Herstellungsversuche von Eis XII sowie Eis IV ein wesentlicher Einfluss der Druck- und Temperaturänderungen auf die Bildungs- bzw. Nukleationswahrscheinlichkeit der beiden metastabilen Eisphasen XII und IV aus der flüssigen Phase nachgewiesen. Ob und inwieweit Druck- und Temperaturänderungen einen Einfluss auf die Keimbildung in der Flüssigkeit, z.B. für eine homogene oder heterogene Keimbildung haben, oder aber ob durch die Abkühlung sowie den Druckverlust eine Keimselektion stattfindet, lässt sich bislang nicht aussagen. Ebenso interessant ist die Frage, inwieweit heterogene oder topotaktische Nukleationsbedingungen Einfluss auf die Ausbildung von Vorzugsorientierungen oder Texturen haben. Unterschiede hinsichtlich der Vorzugsorientierung haben sich für Eis III und für Eis V bei Nukleationen aus der flüssigen Phase ergeben. Bei fest-fest-Umwandlungen erscheint die Fragestellung hinsichtlich des Umbaus wesentlich einfacher zu sein als für die Nukleation aus Flüssigkeiten mit einer möglichen heterogenen Nukleation an der Druckzellenwand. Jedoch sind auch hier die Fragen, wie der Umbau stattfindet ebenso schwierig zu beantworten wie auch die Frage nach der Art der Keimbildung, sowie dem Kornwachstum in der als Ausgangsphase dienendem Material.

Theoretische Arbeiten, wie z.B. die von Johnson \& Mehl (1939), Avrami (1939) und Kolmogorov (1937) etablierte JMAK-Theorie (benannt nach den Autoren) zur Beschreibung 
von Phasenumwandlungen durch Rekristallisation, versuchen unter der Voraussetzung sehr enger Annahmen, z.B. nur dem Zulassen von sphärischen Kristallisationskeimen und einer konstanten Keimbildungsrate, hinsichtlich der Art des Phasenüberganges Aussagen über den Umbau der kristallinen Strukturen zu treffen (vergl. Kapitel 2.2.2 sowie Kapitel 3.3.3).

Als Faktoren, die einen ebenso denkbaren Einfluss auf das Phasenumwandlungsverhalten haben könnten, wären z.B. Korngrößeneffekte und Texturen der Ausgangsphase zu diskutieren. Für diese Art der Untersuchungen ist es jedoch wesentlich, sowohl über hinreichend messtechnische Möglichkeit hinsichtlich der räumlichen und zeitlichen Auflösung zu verfügen, als auch Proben vergleichbarer Güte für systematische Untersuchungen gezielt herstellen zu können.

Weitergehende Schwierigkeiten der Interpretation der Mechanismen und Vorgänge bei einer Phasenumwandlung sind für Strukturen zu erwarten, die bei derzeitigem Stand nur annähernd strukturell beschrieben werden können. Beispiele hierfür sind die amorphen Eisphasen, das HDA (High-Density-Amorphous Ice), LDA (Low-Density-Amorphous Ice) sowie die glasartigen Eisphasen (“vitreous ice”). Einen Überblick über die ,,amorphen” Eisphasen ist in dem Buch von Petrenko \& Whitworth (1999) gegeben. Dies gilt ebenso für das bereits erwähnte kubische Eis Ic (König (1943)), der metastabilen Eisvariante von Eis Ih (Dowell \& Rinfret (1960)). Eine weitergehende Diskussion von untersuchten Phasenübergängen und deren Verhalten wird im folgenden nur für Hochdruckeisphasen-Übergänge in das kubische Eis Ic sowie für das Phasenübergangsverhalten von kubischem Eis Ic in die hexagonale Eisphase gegeben.

Einzelne Hochdruckeisphasen-Übergänge von metastabilen Eisphasen wie dem Eis XII in Eis $\mathrm{V}$ und Eis VI konnten in situ verfolgt werden. Als bemerkenswert kann ein in situ beobachteter Phasenübergang von Eis IV in das metastabile Eis XII erwähnt werden, der im Zuge der Nukleationsversuche am GEM beobachtet werden konnte. Aussagen hinsichtlich der Kinetik der Phasenübergänge bzw. eine Beschreibung mittels der JMAK-Theorie konnten hier nicht getroffen werden. 


\section{Experimentelle und theoretische Grundlagen}

\subsection{Experimentelle Grundlagen: Die pVT-Apparatur}

Die in dem DFG-Projekt bewilligte Hochdruckapparatur wurde nach der Testphase mit einem Flüssigkeitstauchbad kombiniert und ermöglichte so die instantane Messung von Druck $\boldsymbol{p}$ und Temperatur $\boldsymbol{T}$ bei bekanntem isochoren Volumen $\boldsymbol{V}$. Die Abbildung 2.1 stellt schematisch den zuletzt erreichten technischen Stand der aufgebauten und hinsichtlich der Experimente benötigten Änderungen und Erweiterungen der Versuchsapparatur dar.

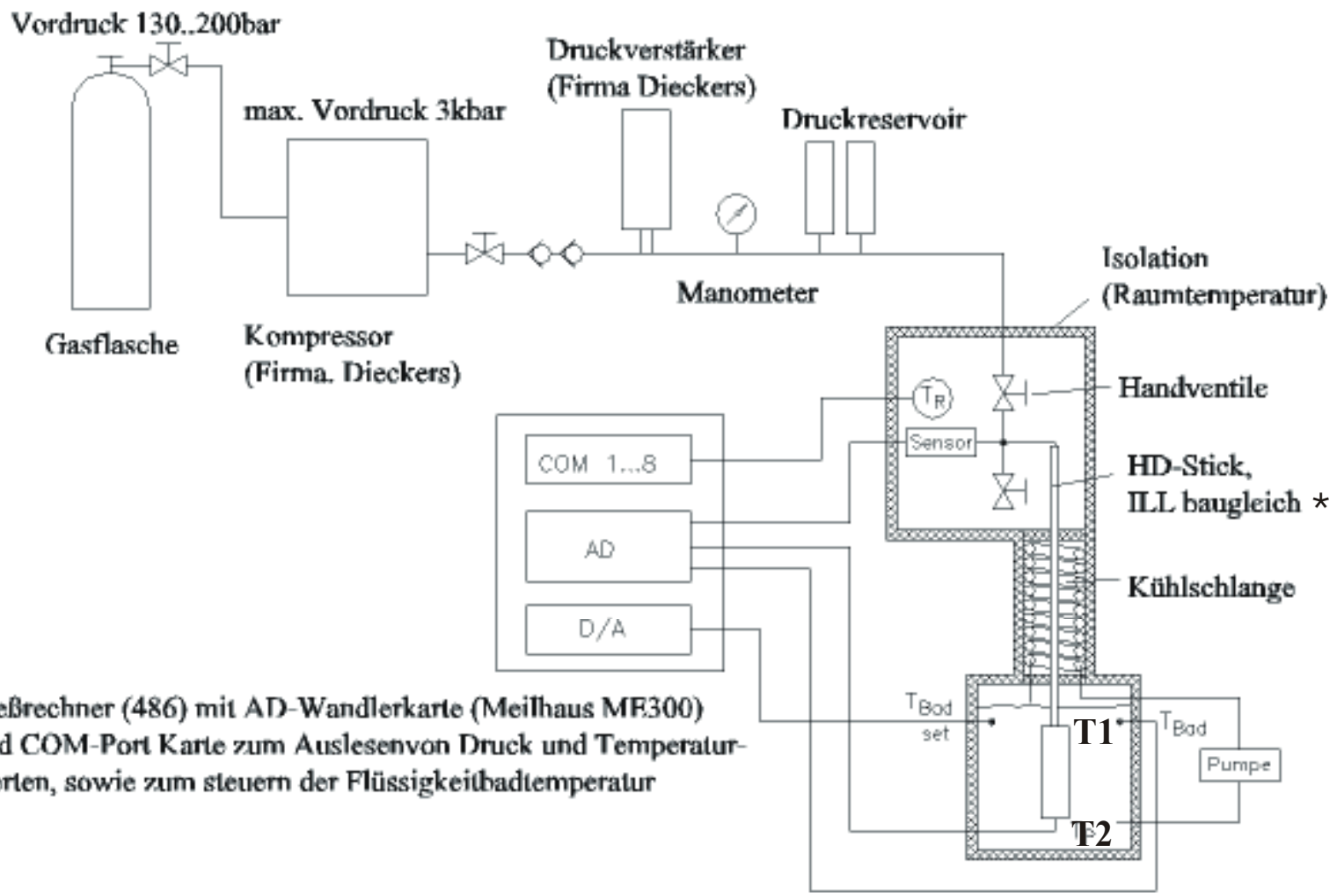

Abbildung 2.1: Schematischer Aufbau der pVT-Apparatur, * : Schematische Zeichnung und Erläuterung des HD-Stick vergl. Abbildung, Anhang 8.2.1)

Mit dieser $\boldsymbol{p} \boldsymbol{V T}$-Apparatur wurden sowohl systematische Messungen von Phasenverläufen im System Wasser und Gas als Funktion von Druck und Temperatur, als auch Experimente zur gezielten und reproduzierbaren Herstellung von Eisphasen durchgeführt. Ebenso wurden mit der Apparatur Proben für Experimente an Großforschungszentren wie dem ILL in Grenoble und dem HASYLAB in Hamburg hergestellt. 
Im Zuge der Experimente wurden systematische Abweichungen zu Großgeräten bei der Bestimmung der Parameter Druck und Temperatur festgestellt, die eine Überprüfung der Apparatur hinsichtlich der Ursachen dieser Abweichungen notwendig werden ließ. Das Ergebnis dieser Überprüfung war, dass apparative Verbesserungen und Erweiterungen sowohl im Hinblick auf die Druck- und Temperatur-Messwerterfassung als auch die Regelung von Temperatur- und Temperaturprofilen der verwendeten Kühlflüssigkeitsbäder notwendig wurden. Die Apparatur ist im Zuge der Experimente auch mit einer automatisierten Messwerterfassung ausgestattet worden. Dies bedingte den Einbau von Hardware sowie die Entwicklung und Implementierung von eigens entwickelter Software zur Datenerfassung von Druck- und Temperaturwerten. Der Arbeitsbereich der dargestellten pVT-Apparatur reicht mit einem Flüssigkeitskühlbad der Firma Neslab (Typ RTE-140) von T $150^{\circ} \mathrm{C}$ bis $\mathrm{T} \sim-40^{\circ} \mathrm{C}$ bzw. mit einem später zur Verfügung gestelltem Tiefstkühlbad (Neslab, Typ ULT-80) von $\mathrm{T} \sim 10^{\circ} \mathrm{C}$ bis $\mathrm{T} \sim 80^{\circ} \mathrm{C}$. Der Aufbau erlaubt einen Arbeitsbereich bis p 6.5kbar Gasdruck. Ein Arbeiten oberhalb dieses Druckes bis p 6.9kbar war möglich, jedoch traten ab Drücken von p $6.5 \mathrm{kbar}$ Undichtigkeiten auf. In ersten Schmelzkurvenexperimenten an Eis $\mathrm{V}$ mit den Gasen Argon und Stickstoff zeigten sich im Vergleich zu den in der Literatur beschriebenen Kurven zum Teil signifikante Abweichungen (vergl. Kapitel 4.3.4, Abbildung 4.7). Diese Abweichungen wurden zuerst probenbedingten Effekten, wie z.B. Löslichkeitseffekte von Gasen und einer dadurch bedingten Schmelzpunkterniedrigung von Eis V, zugeschrieben. Um dieser Frage im Detail nachzugehen und eventuelle apparative Effekte auszuschließen, wurden zuerst die Schmelzkurven für verschiedene Eisphasen an der Göttinger Versuchsapparatur vermessen sowie in späteren Experimenten an Großgeräten, am GEM (ISIS) sowie am D2B (ILL), punktuell in situ verifiziert. Ein Vergleich zu den in situ verifizierten Schmelzkurvenpunkten ergab für die mit der Göttinger pVT-Apparatur bestimmten Schmelzpunkte eine Temperaturabweichung, die systematisch unterhalb der in situ bestimmten Vergleichswerte lagen. Dieser Temperaturoffset betrug im Mittel zwischen dem D2B (ILL) und der Göttinger Apparatur $\Delta \mathrm{T} \sim 1.7 \mathrm{~K}$. Am GEM (ISIS) wurde dieser Temperaturoffset durch eine Teilvermessung der Eis V-Schmelzkurve über einen Druckbereich von $\mathrm{p} \sim 1 \mathrm{kbar}$ wesentlich genauer bestimmt. Die mittlere Abweichung betrug hier $\Delta \mathrm{T} \sim 1 \mathrm{~K}$. Als Ursache für die Abweichung der in Göttingen bestimmten Schmelzpunkte konnte ein Temperaturgradient von $\Delta \mathrm{T} \sim 2 \mathrm{~K}$ zwischen dem HD-Zellenboden $\left(\mathbf{T}_{\mathbf{2}}\right)$ und HDZellenkopf ( $\left.\mathbf{T}_{\mathbf{1}}\right)$ festgestellt werden (vergl. Abbildung 2.1 sowie Anhang 8.2.1). 
Bei einem angenommenen linearen Verlauf des Temperaturgradienten über die Probe ergibt sich im Mittel eine um $\Delta \mathrm{T} \sim 1 \mathrm{~K}$ höhere Probentemperatur. Die mittlere Probentemperatur entspricht in etwa dem am GEM (ISIS) bestimmten Temperaturoffset. Der Vertrauensbereich der Temperatur kann an der Göttinger pVT-Apparatur lediglich zu $\Delta \mathrm{T} \sim \pm 0.5 \mathrm{~K}$ angegeben werden. Die Genauigkeit der Flüssigkeitsbäder ist mit $\Delta \mathrm{T} \sim \pm 0.1 \mathrm{~K}$ angegeben und entspricht durchaus der erreichbaren Genauigkeit.

Die Ursache für den Temperaturgradient ist durch eine ständige Wärmeeinleitung durch den Hochdruck(HD)-Stick auf die Probe gegeben. Der zum Großteil auf Raumtemperatur befindliche Stick wurde nur bis zur Höhe des Flüssigkeitsstandes des Bades auf die eingestellte Badtemperatur gekühlt. Die an dem Stick befindliche HD-Zelle befand sich zwar in der gekühlten Flüssigkeit, jedoch wurde über den Stick Wärme auf die HD-Zelle und somit auf die Probe eingeleitet. Die Isolation, die sich um den Stick befand, brachte in dieser Hinsicht keine wesentliche Verbesserung. Die Isolation verbesserte jedoch die Ablesegenauigkeit des Druckes durch Minimierung der Raumtemperaturschwankungen und den damit verbundenen Gasdruckschwankungen. Die durch Raumtemperaturschwankungen verursachten Ablesegenauigkeiten können bei $\mathrm{p} \sim 2.2 \mathrm{kbar} z \mathrm{zu} 5 \mathrm{bar} / \mathrm{K}$ angegeben werden. Um den Temperaturgradienten an der HD- Zelle und damit an der Probe zu minimieren, wurde ein Kühlschlangensystem um den Stick entworfen und in die Apparatur implementiert. Mit der Stickkühlung konnte der Stick sowie das Gas oberhalb des Kühlflüssigkeitsstandes auf annähernd Badtemperatur gekühlt werden. Der Temperaturgradient zwischen der eingestellten Badtemperatur sowie der Gas- und Sticktemperatur konnte durch diese Implementierung der Stickkühlung oberhalb der Probe auf etwa $\Delta \mathrm{T} \sim 0.5 \mathrm{~K}$ verringert werden.

Die Temperatur der Probe an der GEM-HD-Zelle wurde über zwei Temperatursensoren am Boden sowie am Kopf der HD-Zelle, äquivalent positioniert wie in Abbildung 2.1 dargestellt, gemessen. Die größte Abweichung zwischen diesen Sensoren betrug bei den durchgeführten Experimenten maximal $\Delta \mathrm{T} \sim 0.3 \mathrm{~K}$, so dass von einer Genauigkeit der am GEM bestimmten mittleren Temperatur von $\Delta \mathrm{T} \sim \pm 0.15 \mathrm{~K}$ ausgegangen werden kann. Die am GEM bestimmten Schmelzpunkte wurden aufgrund der hohen Genauigkeit als Referenz für die bis zu diesem Zeitpunkt mit der pVT-Apparatur gemessenen Schmelzkurven verwendet. 
Durch die punktuellen Schmelzpunktbestimmungen am GEM (ISIS) sowie am D2B des ILL konnten gerätebedingte Druckabweichungen der in Göttingen verwendeten Druckmesssensoren (Firma Ashcroft, KXD-7000) festgestellt werden. Als Problem stellte sich nicht die relative Genauigkeit und Reproduzierbarkeit sondern vielmehr die absolute Kalibrierung der verwendeten Druckmesssensoren heraus. Mit zunehmendem Druck zeigte sich eine zunehmende Abweichung zu den Drucken der Schmelzkurven in der Literatur. Eine Überprüfung der Kalibrierung der Druckmesssensoren war daher nötig. Herstellerangaben verwiesen nur auf die relativen Genauigkeiten von etwa 1\% vom Ablesewert. Am ILL wurde mit der dortigen Gasdruckapparatur und einem vorhandenem Heise-Druckmanometer (Typ NG, p=1-7000bar, Genauigkeitsklasse 0.1\%) die Kalibrierung der Druckmesssensoren überprüft. Es zeigte sich, dass der in Göttingen verwendete Messsensor Drücke systematisch zu niedrig erfasste. Die Abweichungen zudem bei steigenden Drücken größer wurden. Mit der am ILL durchgeführten Kalibrierung wurden dann die in Göttingen gemessenen Schmelzkurven sowohl mit der am GEM (ISIS) bestimmten Temperaturkorrektur als auch der D2B (ILL) Druckkorrektur reskaliert.

\subsubsection{Differenzdruck-Analyse und Temperaturprofile}

Für die Bestimmung der Phasengrenzen standen durch die Konzeption und des Aufbaus der pVT-Apparatur, Druck und Temperatur als physikalische Messgrößen zu Verfügung. Durch die bei einer Phasenumwandlung stattfindende Dichteänderung veränderte sich aufgrund des isochoren Gesamtvolumens der Apparatur das für das Gas frei auszufüllende Volumen. Proportional zur Volumenänderungen der Probe ergaben sich Druckänderungen, die über die Druckmesssensoren als Messwert zur Verfügung standen. Es wurde also ein dem DTA(,differential-temperature-analysis“) äquivalentes DPA-Verfahren („differential-pressureanalysis“) zur Bestimmung der Phasengrenzen verwendet. Ein schematischer p,T-Verlauf ist in der Abbildung 2.2 dargestellt und im Detail erläutert.

Bei der Bestimmung von Schmelzkurven wurde die Probe bei einem gegebenem Druck in kleinen Schritten erwärmt und jeweils die Druckänderung als Funktion der Temperatur verfolgt. Die Bestimmung des Schmelzpunktes erfolgte letztendlich durch eine „statische“ DPA, d.h. dem Überschreiten der Phasengrenze und dem Warten auf die darauffolgende neue Gleichgewichtseinstellung bzw. den sich neueinstellenden Gleichgewichtsdruckes an der 
Phasengrenze. Die Druckänderungen wurden automatisch als Funktion der Zeit mit dem Meßrechner (486er-PC mit eingebauter Meilhaus AD-Wandlerkarte ME-300) dokumentiert und als Datei auf Festplatte gespeichert. Zeigten sich keine weiteren Druckänderungen, so konnte man von einer neuen Gleichgewichtslage an der Phasengrenze ausgehen.

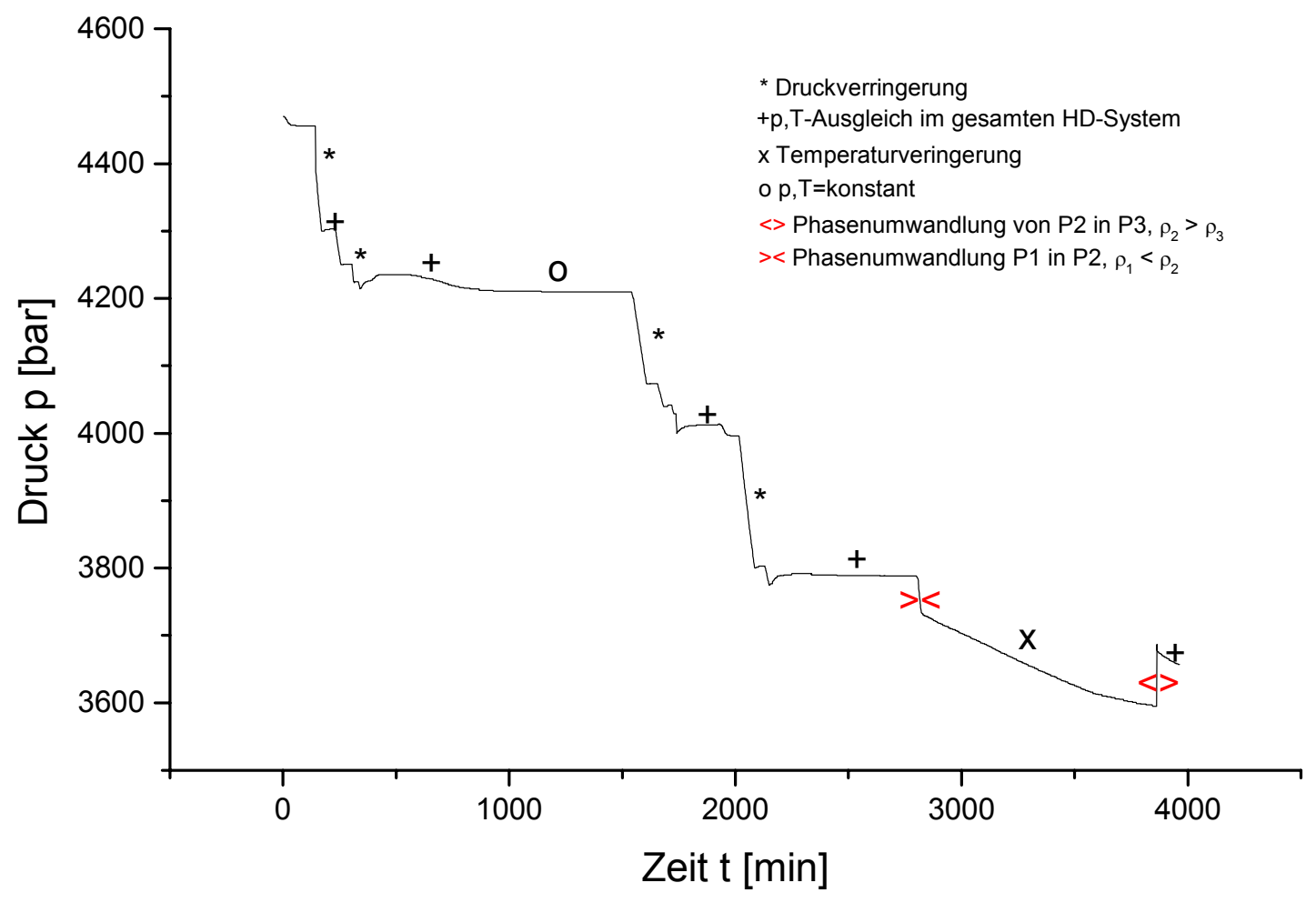

Abbildung 2.2: Druck- und Temperaturverlauf als Funktion der Zeit. Ausgehend von einer kristallinen Phase $P_{1}$ wurde der Druck $p$ in mehreren Schritten verringert (*) bis eine Phasenumwandlung in eine dichtere Phase $P_{2}><$ stattgefunden hat. Eine kontinuierliche und konstante Verringerung der Temperatur (x) führt zu einer Phasenumwandlung von Phase $P_{2}$ in eine weniger dichte $P$ hase $P_{3}<>$

Als wesentlich für die Bestimmung der Phasengrenzen ergab sich das Verhältnis von Gasvolumen der Anlage bzw. des HD-Sticks zu dem Probenvolumen. Durch die bei einer Phasenumwandlung stattfindende Volumenänderung wurde eine, proportional zur Masse der Probe, reziprok proportionale Änderung des Druckes bedingt. Das Verhältnis von Stickvolumen zu Probenvolumen bestimmte daher neben der Genauigkeit des Messsensors im wesentlichen die Auflösung der Druckänderungen. Aufgrund des apparativen Aufbaus war es nicht möglich, zeitgleich den Druck im System zu messen und den Druck während der Phasenumwandlungen konstant zu halten. 
Bei den Experimenten wurde der Stick daher nach Einstellen des gewünschten Druckes bei einer gegebenen Temperatur durch ein Ventil von dem übrigen Gasdruckerzeugersystem getrennt, so dass ein abgeschlossenes Gasvolumen in dem Stick vorlag (vergl. Abbildung 2.1).

Die Änderungsraten, mit denen Druckänderungen durchgeführt wurden, waren im wesentlichen von der manuellen Einstellung der Gasventile anhängig und daher durch den Experimentator bestimmt. Die Druckänderungsraten wurden unter anderem durch die möglichen manuellen Einstellmöglichkeiten als auch durch die mögliche Abfragegeschwindigkeit der Messelektronik bestimmt und lagen daher im Bereich von 12bar/s bis 50-100bar/s. Problematisch war die Realisierung variabler, hoher Kühlraten. Die Flüssigkeitsbäder sind durch ihre Konstruktion bedingt eher als Konstanttemperaturbäder konzipiert. Aufgrund der „hohen thermischträgen Masse“ der Sticks und der HD-Zellen konnten durch den Kühlkompressor der Bäder lediglich Kühlraten von etwa $0.1 \mathrm{~K} / \mathrm{s}$ erreicht werden. Um höhere Kühlraten zu realisieren, wurde das Flüssigkeitsbad auf eine Temperatur voreingestellt, bevor der HD-Stick mit der Zelle in das Flüssigkeitsbad getaucht und damit gekühlt wurde. Aufgrund dieses Verfahrens ist die Kühlrate jedoch mit der Badtemperatur gekoppelt und nicht unabhängig voneinander einzustellen. Unterschiedliche Kühlraten wurden durch die Variation der Badtemperatur bzw. Endtemperatur erreicht, wobei Kühlrate und Badtemperatur miteinander korreliert sind und durch diese Art der Kühlung eine asymptotische Annäherung der Temperatur an die Badtemperaturvoreinstellung vorgegeben ist. Die durch dieses Verfahren realisierbaren Kühlraten lagen bei den durchgeführten Versuchen z.B. bei einer Endtemperatur von $\mathrm{T} \sim 250 \mathrm{~K}$ je nach verwendetem Material und Zellentyp bei anfänglichem Kühlen bei 3-5 K/s. Der Temperatursensor in der HD-Zelle wies eine Genauigkeit von $\Delta \mathrm{T} \sim \pm 0.5 \mathrm{~K}$ auf und entspricht dem Vertrauensbereich, mit denen die Temperaturen auch im Vergleich zu den in situ Schmelzpunkten bestimmt werden konnten. Der Vertrauensbereich zur Bestimmung des Druckes liegt unter Berücksichtigung des normalen täglichen Maßes der Raumtemperaturschwankungen in der Größenordnung von 2\% vom Ablesewert des Druckes. Temperaturänderungen durch endo- oder exotherme Phasenumwandlungen sollten mit Hilfe des an der HD-Zelle angebrachten und nahe an der Probe befindlichen Temperatursensors erfaßt werden. Die Auflösung sowie die Reaktionszeiten des Temperatursensors stellten sich im Zuge der Experimente jedoch als ungenügend heraus, zudem ergaben sich durch den Temperatursensor geringere 
Probenvolumina. Diese Verringerung durch den Sensor betrug durchaus $\sim 35 \%$ des ursprünglichen Volumens und führte daher zu einer Verringerung der Auflösung im Druck aufgrund der Verringerung des Probevolumens zum Totvolumens des Sticks. In den meisten Schmelzkurvenexperimenten wurde daher auf den Einsatz des Temperatursensors zugunsten einer höheren Empfindlichkeit (Auflösung) und Ablesegenauigkeit der Druckänderungen verzichtet.

\subsubsection{Wiedergewinnung von Clathrat-Hydraten und Hochdruckeisphasen}

Aufgrund der nur in beschränktem Umfang zur Verfügung stehenden in situ Messzeiten am ILL zur Untersuchung sowohl von Clathrat-Hydratstrukturen, als auch Hochdruckeisphasen, wurden neue HD-Zellen mit eingebautem Probenhalter zur Röntgendiffraktion im eigenen Hause von ,recoverten Proben“ entwickelt und verwendet. Die HD-Zellen wurden auch unter anderem mit einem zusätzlichen Temperatursensor, der im Hochdruckbereich nahe an der Probe sitzt, versehen. Anfängliche Probleme hinsichtlich der mechanischen Dichtigkeit und Stabilität der verwendeten Bridgman-Dichtungen konnten durch Variation der Dichtpackungen verschiedener Materialien zufriedenstellend gelöst werden. Als die am besten geeignete Dichtpackung stellte sich eine Kombination aus Dichtscheiben aus Kupfer-, Polypropylen-, Polyethylen- und Polypropylendichtscheiben heraus (in dieser Reihenfolge). Diese behielten auch hinsichtlich des „Recoverns“ von Proben ihre Dichtigkeit bis zu flüssigen Stickstofftemperaturen bei, erlaubten jedoch auch das Öffnen der HD-Zelle und Bergen der „recoverten“ Proben bei Temperaturen um die $\mathrm{T} \sim 77 \mathrm{~K}$ für die Experimente an „recoverten“ Proben.

Die Strukturanalysen dieser wiedergewonnenen Proben sowie die Identifikation hergestellter Eisphasen wurden mit einem im Hause zur Verfügung stehenden Röntgengerät (Philips MRD mit Huber-Eulerwiege), ausgerüstet mit einem „,closed cycle“ Helium-Kryostaten (Firma APD), durchgeführt (vergl. Abbildung 2.3). Diese direkte Herstellung von Clathrat-Hydrat und Eisproben in dem für diese Röntgenbeugungsuntersuchungen entwickeltem Röntgenhalter gestaltete sich aufgrund der sicherheitstechnischen Aspekte der Gasdruckanlage allerdings als schwierig. Es ergab sich, dass die Probenmenge etwa nur 1/5 des ursprünglichen Fassungsvermögens der Druckzelle ausmachte (Masse in dem Röntgenprobenhalter maximal $\mathrm{V} \sim 0.3 \mathrm{ml}$ ). 
Dies hatte zur Folge, dass sowohl die über Gasdruckänderungen festzustellenden Phasenänderungen als auch die Adsorptionskurven bei der Clathrat-Hydratbildung mit großen Unsicherheiten behaftet waren. Probleme beim Befüllen des Halters mit frisch gemörsertem Eis führten letztendlich zu dem Versuch, die Proben von der flüssigen Phase ausgehend in dem Probenhalter herzustellen.

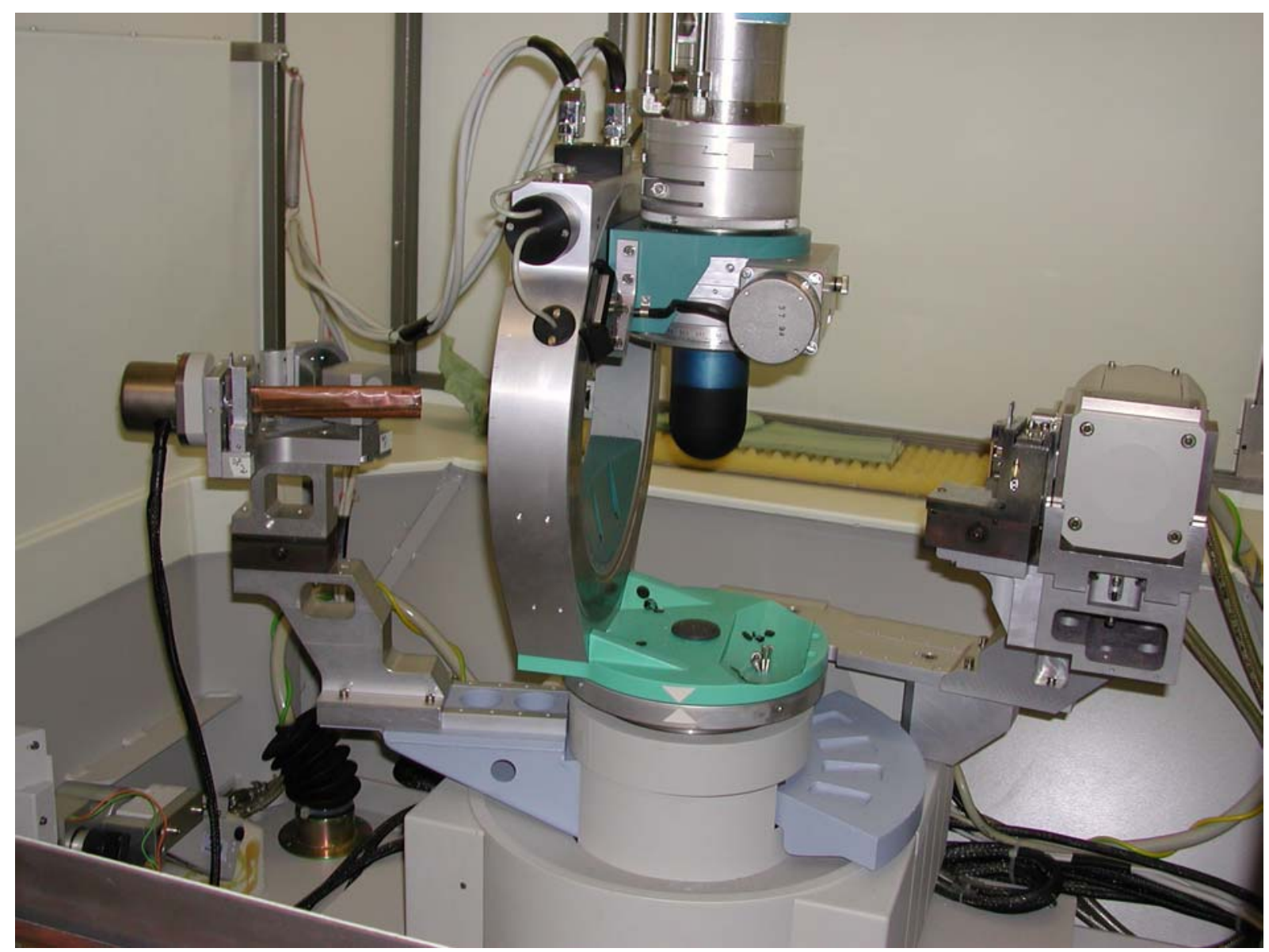

Abbildung 2.3: Philips-MRD Röntgendiffraktometer mit APD-Cryostat. Bild links: Detektorkreis mit Kupferpipe, Bildmitte: Huber-Eulerwiege mit aufgebautem APD-Helium-Kryostaten und aufgeschraubtem Vakuumdom, Bild rechts : Röntgenröhre.

Alle so synthetisierten Eisproben wiesen jedoch starke Texturierungen sowie eine schlechte Kornstatistik auf, die hinsichtlich der röntgenographischen Untersuchung Probleme bereiteten. Die Verwendung von Quarzwolle oder anderer physikalischer Nukleatoren zeigten bedauerlicherweise kaum Einfluss auf die Texturausbildung. Eine weitere Konsequenz der geringen Probenmenge und der kleinen Atomstreufaktoren von Wasser bzw. Eis waren lange Messzeiten zur Gewinnung von Beugungsdaten mit hinreichender statistischer Genauigkeit. 
Für Rietveldstrukturanalysen geeignete Diffraktogramme mit einem Winkelbereich in $2 \theta \sim 10^{\circ}-160^{\circ}$ ergaben sich Messzeiten von mehr als zwei Tagen. Aufgrund des hohen Dampfdruckes von Eis und Clathrat-Hydrat konnte unter dem Isoliervakuum $\left(\mathrm{p} \sim 10^{-3}-10^{-4}\right.$ mbar) des Helium-Kryostaten ein beträchtlicher Massenverlust während der Messungen an der Probe beobachtet werden, der nach etwa $\sim 1.5$ Tagen zu einer völligen Sublimation der Probe führte. Zudem zeigte sich, dass bei den untersuchten Pulverproben bei längeren Vakuumstandzeiten zunehmend mehr grobkörniges Probenmaterial verblieb, die kleineren Körner erwartungsgemäß schneller sublimierten, so dass die Reflexhöhen als auch die Reflexprofile sich über den Zeitraum der Messung änderten. Die Messung war daher nicht mehr repräsentativ für den Ausgangszustand der Probe. Ein großes Problem hinsichtlich der Stabilität und Topologie von Hochdruckeisphasen ergab sich durch die Applikation der Probe auf den Kryostaten. Zu jeder Zeit mußte die Probe, sofern es sich um HD-Eisphasen und Clathrat-Hydrate handelte, bei Temperaturen kleiner als $\mathrm{T} \sim 120 \mathrm{~K}$ gehalten werden, um eine Umwandlung der Hochdruckeisphasen in kubisches Eis Ic zu verhindern. Gleichzeitig mußte aber auch eine Kondensation von Wasserdampf aus der Atmosphäre an dem kalten Probenhalter vermieden werden. Ähnliche Probleme ergaben sich auch hinsichtlich der Versuche, HD-Eisphasen und Clathrat-Hydrate im ,recoverten“ Zustand aus einer HD-Zelle zu bergen und auf einen externen Probenhalter, z.B. für FE-REM Untersuchungen, in HeliumKryostaten des Röngtengerätes oder aber der in Göttingen vorgefertigten Proben in den Orange-Kryostaten am D1B/D2B am ILL zur Strukturanalyse zu applizieren. ClathratHydrat-Proben zeigten sich im Gegensatz zu den Eisproben als wesentlich temperaturstabiler. Topologische Oberflächenveränderungen zeigten sich bei den Clathrat-Hydraten erst bei Temperaturen von T 180K. Vorversuche zeigten, daß bei den verwendeten Probenmassen die mittlere Erwärmungsrate bei diskontinuierlicher Kühlung der Probe mit flüssigem Stickstoff bei ca. $2-3 \mathrm{~K} / \mathrm{s}$ lag. Bei einer Temperaturdifferenz zwischen flüssiger Stickstofftemperatur und des Stabilitätslimits der ,recoverten“ Phasen von T $120 \mathrm{~K}$ ergaben sich somit „sichere Probenhandlingzeiten“ von maximal t $\sim 20$ Sekunden. Der Applikationserfolg der Proben lag lediglich bei $20 \%$. Der geringe Applikationserfolg durch die Erwärmung, den geringen Probenmengen, der Probentexturierung und den daraus resultierenden langen Meßzeiten sowie die aus der Sublimation der Probe auftretenden Problematiken führten zu einer Verlagerung auf in situ Untersuchungsmethoden. Problemlösungen hinsichtlich der röntgenographischen Untersuchung von ,recoverten“ Proben konnten aus Zeitgründen nicht mehr erarbeitet werden. 
Die Verlagerung auf in situ Untersuchungsmethoden, wie Neutronen- und Synchrotronbeugung resultierte daher in einigen wenigen Fragestellungen, die sich im Rahmen dieser Arbeit untersuchen ließen.

\subsubsection{In Situ Strukturbestimmungen}

Am D2B des ILL in Grenoble wurden die meisten der in situ-Strukturuntersuchungen durchgeführt. Für Hochdruckexperimente kam ein dort vorhandenes Druckerzeugersystem, bestehend aus einem Kompressor, Druckverstärker (Intensifier), Druckleitungen, Stick und eine Druckzelle zur Anwendung. Die dortige Ausrüstung ist mit der in Göttingen vorhandenen pVT-Apparatur funktionstechnisch identisch. Der realisierbare Druckbereich dieser Apparatur lag aus sicherheitstechnischen Gründen bei p 6kbar. Die Messungenauigkeit des Drucksensors betrug ca. 2\% vom abgelesenen Wert und ist somit mit der Ableseungenauigkeit in Göttingen vergleichbar. Bei den Experimenten fand eine HD-Zelle sowie ein Stick vom ILL Verwendung. An der Zelle befand sich ein aufgeklebter Dehnungsmessstreifen (DMS), der eine qualitative Aussage und Verifizierung über die stattfindenden Druckänderungen zuließ. Bei dem D2B handelt es sich um ein hochauflösendes 2-Achsen-Diffraktometer. Die Achsen des Diffraktometers bestehen aus einem $\omega$-Winkel, um den die Probe gedreht werden kann, und einem Detektorwinkel 20. Der Detektorring besteht aus 64 einzelnen ${ }^{3} \mathrm{He}$-Detektoren, die mit einem Soller-Kollimator versehen jeweils einen Winkelbereich von $\Delta \theta \sim 2.5^{\circ}$ abdecken, über den in Schritten von $0.05^{\circ}$ gescannt wird. Der Messbereich lag bei allen durchgeführten Messungen zwischen 20 $5^{\circ}$ $160^{\circ}$. Die am D2B für die eigenen Messungen bevorzugte Wellenlänge wurde mit einem Monochromator eingestellt und lag größenordnungsmäßig bei $\lambda \sim 1.59 \AA$. Für jedes Experiment wurde eine Siliziumpulvermessung zur genauen Bestimmung der Gitterkonstanten und zur Festlegung des Nullpunktes durchgeführt. Die Wellenlänge und der Nullpunkt wurden dann durch eine Rietveld-Verfeinerung bestimmt. Zur Kühlung der Eisproben und zu der Realisierung der tiefen Temperaturen wurde ein mit flüssigem Stickstoff und Helium gekühlter Orange-Kyrostat mit einem Temperaturbereich von $\mathrm{T} \sim 1.5 \mathrm{~K}$ bis $\mathrm{T} \sim 300 \mathrm{~K}$ eingesetzt. Es wurden jedoch lediglich Messungen in dem Temperaturbereich von T 77K bis T 265K durchgeführt. An der HD-Zelle (Material: TiZr) befanden sich am Zellenkopf sowie am unteren Ende der Zelle je ein kalibrierter Temperatursensor. In der Regel wurde eine Temperaturdifferenz von $\mathrm{T} \sim 1.5 \mathrm{~K}$ zwischen den Sensoren beobachtet. Die Genauigkeit, mit 
der die mittlere Temperatur zwischen den Sensoren am Orange-Kryostaten eingestellt werden konnte, wird aufgrund des Temperaturoffsets sowie den Gleichgewichtseinstellungszeiten auf $\Delta \mathrm{T} \sim \pm 0.75 \mathrm{~K}$ angegeben und liegt daher in einer vergleichbaren Größenordnung zur Göttinger Apparatur. Die minimale Messzeit, bei denen die Diffraktogramme strukturelle Aussagen ermöglichen, liegen bei $\mathrm{t}=15-30$ Minuten. Strukturelle Untersuchungen wie z.B. die strukturellen Eigenschaften von Eis Ih als Funktion des Druckes und der Temperatur unter Verwendung der Gase Argon, Helium und Neon als auch die strukturellen Untersuchungen der aus den verschiedenen „recoverten“ Hochdruckeisphasen hergestellten kubischen Eisphasen wurden am D2B im mittleren Auflösungsmodus (20' Kollimatoröffnung) gemessen. Die Verifizierung des Nukleationsverfahrens von Eis XII als auch die Bestimmung von in situ Schmelzpunkten als Funktion des Druckes wurden ebenso am D2B durchgeführt. Für weitere Angaben wird zum einen auf Hewat (1986) sowie zum anderen auf die Web-Seite des D2B am ILL verwiesen (http:॥www.ill.fr ).

Die Untersuchungen der Kinetik der Phasenübergänge von „recoverten“ Hochdruckeisphasen in das kubische Eis Ic wurden am D1B in situ verfolgt. Das D1B ist wie das D2B ein ZweiAchsendiffraktometer jedoch mit höherem Neutronenfluß. Das D1B ist mit einem linearen ${ }^{3}$ He-Detektor mit etwa 400 Elementen ausgestattet. Bei einem messbaren Winkelbereich von $2 \theta \sim 80^{\circ}$ ergibt sich somit nur eine Winkelauflösung pro Element von $\Delta 2 \theta \sim 0.2^{\circ}$ in $2 \Theta$ und ist damit um einen Faktor 8 kleiner in der Winkelauflösung als beim D2B. Die Wellenlänge, mit der am D1B die Kinetik der Phasenumwandlung verfolgt wurde, betrug $\lambda \sim 2.52 \AA$. Aufgrund der geringeren Winkelauflösung wurde für die kinetischen Messungen keine Nullpunktssowie Wellenlängenbestimmung durchgeführt, da die Datensätze für eine StrukturVerfeinerung keine ausreichende Güte besitzen. Die Kühlung der „recoverten“ Hochdruckeisproben wurde mit einem weitgehend baugleichen Orange-Kryostaten gewährleistet. Die Temperaturgenauigkeit kann auch hier zu $\Delta \mathrm{T} \sim \pm 0.75 \mathrm{~K}$ angegeben werden. Die kinetischen Messungen wurden in einem Temperaturbereich von $\mathrm{T} \sim 77 \mathrm{~K}$ bis $\mathrm{T} \sim 175 \mathrm{~K}$ durchgeführt. Für weitere Angaben ist ebenso auf die Web-Seite des ILL (http:||www.ill.fr) verwiesen.

An der Beamline BW5 des HASYLAB in Hamburg wurden sowohl Messungen bezüglich der Pulvergüte (Pulverqualität) als auch in situ Experimente zum Phasenübergang von Eis III in kubisches Eis Ic (vergl. Kapitel 3. ff.) und zur Rekristallisation von Eis Ih durchgeführt. Auf 
eine 3-Kreis-Huber-Eulerwiege mit ansteuerbaren Winkeln $\omega, \Phi$ und $\Psi$ wurde ein zum Göttinger Helium-Kryostaten ähnlicher Kryostat (Fa. APD Cryogenics, Typ DF 2020, HC-2) verwendet. Die Proben wurden in einem verschraubbaren und nahezu gasdichten Aluminiumröhrchen in einem speziellen Probenhalter auf den Kühlfinger des Kryostaten aufgesetzt. Nach der Applikation wurde die Probe mit sogenannten Vakuumdomen (Aluminium) des Kryostaten von der Umgebung isoliert. Durch Evakuierung des Gasvolumens in den Vakuumdomen des Kryostaten wurde ein Vakuum als Thermoschild zur Umgebung aufgebaut. Mit einem vorhandenen ImagePlate-Detektor (Typ MAR345), wurden die Beugungsringe der Probe zweidimensional in Transmission vermessen. Aufgrund der hohen Intensität der Röntgenstrahlen mit einer Energie von etwa E 80-100keV betrug die mittlere Meßdauer für ein Diffraktogramm mit hinreichender Meßstatistik lediglich ein bis zwei Sekunden. Aufgrund des Flächendetektors konnte durch die Bestimmung der Beugungsringe eine Aussage hinsichtlich Pulverqualität sowie der Existenz von Vorzugsorientierungen getroffen werden. Für die in situ Messungen des zeitlichen Rekristallisationsverhalten von Eis Ih-Proben wurde diese während der Messzeit in einem Winkelbereich von etwa $\Delta \Phi \pm 10^{\circ}$ in $\Phi$ hin und her gedreht (,gewobbelt"), um eine Verfälschung der Beugungsintensitäten durch eine $\mathrm{zu}$ geringe Zahl von sich in Reflexionsstellung befindlichen Eiskörnern auszuschließen. Durch dieses Verfahren konnte gewährleistet werden, dass sich während der Messung eine hinreichende Anzahl von Eiskörnern in Reflexionstellung befanden. Für weitergehende Informationen zum BW5 ist auf Brückel und Süßenbach (1995) sowie auf die Webseite des Hasylab (http:||www.hasylab.de) verwiesen.

Am GEM am ISIS des Rutherford Appleton Laboratory (RAL) in Didcot stand in der Testphase des experimentellen Aufbaus des GEM Messzeit zur Verfügung, die zur Verifizierung sowohl des Eis XII-Herstellungsverfahrens (strukturelle Identifizierung) als auch zur Überprüfung der in Göttingen gemessenen Eis XII-Schmelzkurve verwendet wurde (vergl. Kapitel 3.7). Bei dem GEM (General Materials Diffractometer) handelt es sich um ein TOF (Time of flight) Neutronendiffraktometer. Für Angaben zur Neutronenquelle bzw. für die Erzeugung der gepulsten Neutronenstrahlung sei an dieser Stelle auf die Web-Seite des RAL (http:||www.isis.rl.ac.uk) verwiesen. Das GEM verfügt über sieben Detektorbänke, die in verschiedenen Abständen zu der Probe liegen und damit die Möglichkeit geben, einen weiten Winkelbereich von der einfallenden Neutronen-Strahlrichtung $\mathrm{zu}$ detektieren. 
Insgesamt verfügt das GEM über 6470 Einzel-Detektoren, die in den sog. Modulen in einzeln auswertbaren Detektorbänken zusammengefasst sind. Die Anordnung der Detektorbänke ist so ausgelegt, dass der Winkelbereich in $2 \theta$ von $2 \theta \sim 5^{\circ}$ bis $170^{\circ}$, ausgehend von der Neutronenstrahlrichtung, erfasst werden kann. Die Strahlgeometrie und damit die Apertur ist über einen weiten Bereich bis max. $40 \mathrm{~mm}$ in der Höhe sowie $20 \mathrm{~mm}$ in der Breite einstellbar. Dazu ist über zwei getrennt regelbare Chopper auch die Wellenlänge der Neutronen sehr variabel einstellbar. Mit dem GEM war es möglich unter Ausnutzung aller Detektorbänke innerhalb von einigen Minuten Strukturen zu identifizieren. Die längste Messzeit belief sich in dem Experiment auf $\mathrm{t} \sim 2.5 \mathrm{~min}$, um eine detaillierte Aussage über die Struktur als auch über die Güte dieser zutreffen und ermöglichte daher in kurzer Zeit sowohl die Durchführung einer Vielzahl von Nukleationsexperimenten als auch deren Beurteilung. Aufgrund des komplexeren Aufbaus des GEM, im Gegensatz zu den Aufbauten des D2B, D1B sowie des BW5, ist zur Verdeutlichung eine schematische Skizze im Anhang 8.2.1.3.1 dargestellt. Eine detaillierte Beschreibung des Instrumentes ist durch Williams et al. (1998) sowie Day (2000) gegeben. Am GEM stand ein $\mathrm{zu}$ der Göttinger pVT-Apparatur funktionstechnisches äquivalentes Druckerzeugersystem zur Verfügung. Aus Sicherheitsgründen konnte dieses jedoch nur einen maximal Druck von p 5.2kbar erzeugen, verfügte jedoch bei einer äquivalenten HD-Stick-und HD-Zellenkonstruktion über eine deutlich bessere Auflösung in der Temperatur.

\subsubsection{Feldemissions-Rasterelektronenmikroskopie}

Um der Frage der Oberflächenbeschaffenheit und der Pulverqualität von hergestellten Eisproben nachzugehen, wurden elektronenoptische Untersuchungen an unterschiedlich hergestellten Eisproben mittels FE-REM (Fa. Zeiss, Typ LEO Gemini 1530 mit Kryopräparationseinheit Oxford Instr.) durchgeführt. Die typischen Elektronenenergien bei diesen Untersuchungen lagen bei $\mathrm{E} \sim 1 \mathrm{keV}$. Diese geringen Elektronenenergien resultierten in einer geringen thermischen Belastung der Proben. Ähnlich der Probenapplikation an das Röntgendiffraktometer mußten die Proben während der Applikation in das Gerät ständig gekühlt und deren Temperatur $\mathrm{T}<120 \mathrm{~K}$ gehalten werden. Es zeigte sich jedoch im Zuge der Applikationen, dass die „recoverten“ Hochdruckeisproben nach dem Transfer in das Gerät innerhalb kürzester Zeit, maximal $\mathrm{t} \leq 1.5$ Minuten, zerfielen. Der Zerfall zeigte sich rein optisch in einem Aufblähen bzw. Aufschäumen der Probenkristallite. 
Dieser Zerfall deutete die Phasenumwandlung der wesentlich dichteren „recoverten“ Hochdruckeisphasen in kubisches Eis Ic an. Als Gründe hierfür kann sowohl die Destabilisierung des Kristallgitters durch das in der Schleusenkammer herrschende Vakuum ( $p \sim 10^{-4}$ mbar) als auch die ungenügende thermische Abschirmung der Vakuumkammer diskutiert werden. Aufgrund einer nicht vorhandenen thermischen Isolierung der Schleusenkammer und durch Kontaktkühlung stattfindende Kühlung der Probe ist von einer unkontrollierten Wärmeeinleitung auf die Probe durch den Probenhalter des FE-REM als Ursache auszugehen. In jedem Falle war eine Untersuchung von „recoverten“ Hochdruckeisproben nicht möglich. Jedoch konnten mit dem Gerät sehr wohl Oberflächenstrukturen von Eis Ih sowie vermutlich kubischem Eis Ic untersucht werden. Die elektronenoptischen Aufnahmen bis zur Nanometerskala erlauben so eine 2-dimensionale Vermessung der Eiskörner.

\subsection{Theoretische Grundlagen: Rietveld-Strukturanalyse}

Wie bei anderen, heutzutage meist computergestützten, Verfahren zur Kristallstrukturanalyse werden bei der Rietveld-Methode die aus dem Strukturmodell berechneten Intensitäten den beobachteten durch Anwendung eines Least-Squares-Verfahren, durch Variation der Kristallstruktur sowie der Instrumentenparameter angepaßt. Die Parameter, die den Verlauf eines Pulverdiagramms charakterisieren, sind durch die Reflexlagen, bestimmt durch die Elementarzellenstruktur und deren Gitterkonstanten, der Reflexform, die durch die Profilparameter beschrieben wird, sowie die Reflexbreite und Reflexhöhe, deren Integralintensität durch die Atomparameter in der Elementarzelle beschrieben werden, gegeben. Die Integralintensität $\mathrm{I}_{\mathrm{Int}}$ läßt sich nach folgender, vereinfachter Formel berechnen:

$$
I_{I n t}=S \cdot L \cdot A \cdot M \cdot O \cdot\left|F_{h k l}\right|^{2}
$$

Dabei ist $\mathrm{S}$ der Skalierungsfaktor, L der Lorentz-Polarisationsfaktor (von der Aufnahmetechnik abhängig), A der Absorptionskorrekturfaktor (Probengeometrie), M die Flächenhäufigkeit sowie $\mathrm{O}$ der Korrekturfaktor für eine bevorzugte Orientierung ("preferred orientation") der Kristallite.

Die wichtigste Größe, die durch die Kristallstruktur-Verfeinerung angepaßt werden soll, ist der komplexe Strukturfaktor $\mathrm{F}_{\mathrm{hkl}}$, der die Beziehung zwischen der Anordnung der Atome in 
einem Kristall (=Struktur) und der Intensität der gebeugten Strahlen (elektromagnetische oder Korpuskelstrahlen) angibt.

Da die Intensität der Beugungsreflexe dem Quadrat des Strukturfaktors proportional ist, ist hieraus nur der Absolutwert $\left|F_{h k l}\right|$ zu bestimmen, da für eine komplexe Größe das Quadrat einer Multiplikation mit der konjugiert komplexen Zahl entspricht $\left(F_{h k l}=\left|F_{h k l}\right| e^{(i ~ p h k l)}\right.$; $\varphi=$ Phase). Dieser Absolutwert wird als Strukturamplitude bezeichnet. Der komplexe Strukturfaktor läßt sich nach folgender allgemeiner Gleichung ermitteln:

$$
F_{h k l}=\sum_{j} f_{j} \cdot e^{-B_{i s o_{j}}\left(\frac{\sin ^{2} \Theta}{\lambda^{2}}\right)} \cdot e^{\left(2 \pi_{j}\left(h x_{j}+k y_{j}+l z_{j}\right)\right)}
$$

Dabei ist $f_{j}$ der Atomformfaktor für Röntgenstrahlung bzw. die Streulänge für Neutronenstrahlung für das j-te Atom in der Elementarzelle. An dieser Stelle ist anzumerken, dass die Neutronenstreulänge von Atomkernen im Gegensatz zum Atomformfaktor der Röntgenstrahlung vom Beugungswinkel unabhängig ist. Der Faktor $B_{\text {iso }}$ ist der atomare isotrope Auslenkungsparameter (engl. ADP [Atomic Displacement Parameter]) für das j-te Atom in der Elementarzelle. Dieser beschreibt unter anderem die Auslenkung der Atome aus ihrer Ruhelage aufgrund thermischer Schwingungen und wird daher auch als thermischer Auslenkungsparameter bezeichnet. Für diese gilt:

(vergl. Definition Anhang 8.2.2.1).

$$
B_{\text {iso }}=8 \cdot \pi^{2} \cdot U_{\text {iso }}
$$

Die Güte der Verfeinerung wird durch verschiedene Residualwerte, R-Werte, angegeben. Am engsten mit der zu minimierenden Summe der Fehlerquadrate hängt der gewichtete Profil-RWert $\mathrm{R}_{\mathrm{wp}}$ zusammen $\left(\Delta \mathrm{i}=\mathrm{y}_{0 \mathrm{i}}-\mathrm{y}_{\mathrm{ci}}\right)$, wobei $\mathrm{y}_{0 \mathrm{i}}$ den beobachteten Intensitäten, bestimmt abzüglich des Untergrundes, $\mathrm{y}_{\mathrm{ci}}$ den berechneten Intensitäten, $\mathrm{w}_{\mathrm{i}}$ der Gewichtung entsprechen. Der Residualwert ist gegeben durch:

$$
w R p=\left[\frac{\Sigma_{i} w_{i} \cdot \Delta_{i}{ }^{2}}{\Sigma w_{i} \cdot y_{0 i}}\right]^{\frac{1}{2}}
$$


Als Gewichte $\mathrm{w}_{\mathrm{i}}$ werden meist die reziproken Varianzen $1 / \sigma^{2}$ der Meßwerte $\left(\mathrm{y}_{0 \mathrm{i}}\right.$ ) benutzt. Durch die Poisson-Statistik des Zählvorganges ist $\mathrm{w}_{\mathrm{i}}=1 / \sigma^{2}\left(\mathrm{y}_{0 \mathrm{i}}\right)=1 / \mathrm{y}_{0 \mathrm{i}}$.

Eine andere Abschätzung der Güte der Verfeinerung ist ,the goodness of fit“", GOF oder $\chi^{2}$ bezeichnet, wobei $\mathrm{N}$ der Anzahl der Meßpunkte in dem Diffraktogramm entspricht:

$$
G O F=\left[\frac{\Sigma_{i} w_{i} \cdot \Delta_{i}{ }^{2}}{(N-n)}\right]^{\frac{1}{2}}
$$

Nach der Aufspaltung in einzelne Reflexe läßt sich auch die Güte der Einzelintensitäten vergleichen, jedoch sind die verwendeten Integralintensitäten von dem jeweiligen Modell abhängig, wobei $\mathrm{I}_{0}$ der beobachteten und $\mathrm{I}_{\mathrm{c}}$ der berechneten Intensität entspricht:

$$
R_{\text {Bragg }}=\frac{\Sigma\left|I_{0}{ }^{\prime}-I_{c}\right|}{\Sigma I_{0}{ }^{\prime}}
$$

Das in dieser Arbeit verwendete Rietveldprogramm GSAS (General Structure Analysis System) ist im Los Alamos National Laboratory in New Mexico (USA) geschrieben worden (Larson \& v. Dreele, GSAS-Manual (1994)) und kann über den ftp-server von Los Alamos abgerufen werden. Die Profilparameter, die sowohl durch apparative als auch durch probenbedingte Größen wie z.B. den Parametern S, L, A, M und O beschrieben werden, können durch unterschiedliche Funktionen angepaßt werden. Verwendung finden hier z.B. Gauß- , Lorentz-, Voigt-, Pseudo-Voigt- oder Pearson VII- Funktionen (Young, (1995)). Welche Funktion für die gemessenen Daten am besten geeignet ist, hängt im wesentlichen von der Quellencharakteristik der Strahlung ab. Für Neutronendaten wird meist die GaußFunktion, für Röntgendaten vielfach die Pseudo-Voigt-Funktion verwendet. Die Abhängigkeit der Halbhöhenbreiten $\Gamma$ (auch bezeichnet als HB oder FWHM (full width half maximum) von $2 \Theta$ wird durch den folgenden Ansatz erfasst (Cagliotti et al. 1958)

mit

$$
\Gamma=2 \cdot \sqrt{2 \cdot \ln 2 \cdot \sigma^{2}}
$$

$$
\sigma^{2}=U \cdot \tan ^{2} \Theta+V \cdot \tan \Theta+W
$$


wobei U, V und $\mathrm{W}$ für eine Gauß-Funktion die zur Verfeinerung freigegebenen Werte darstellen. $\mathrm{Zu}$ den Atomparametern zählen die Atomkoordinaten $\mathrm{x}, \mathrm{y}, \mathrm{z}$, die mittleren Amplituden der Wärmeschwingung und der Fehlordnung um diese Atomlage $\left(\mathrm{U}_{\text {iso }}-\right.$ bzw. $\mathrm{B}_{\text {iso }}{ }^{-}$ Werte) sowie der Besetzungsfaktor (frac) für die betreffende Atomart. Für Röntgen- bzw. Synchrotrondaten sind die $U_{\text {iso }}$-Werte etwas ungenauer zu ermitteln, vor allem für Atome mit niedriger Elektronenzahl, da für die Festlegung der $U_{\text {iso }}$ auch das Quadrat der Elektronenzahl eine Rolle spielt. Je niedriger die Elektronenzahl, desto schlechter die Bestimmung und somit ist es vor allem z.B. für die Sauerstoffatome schwieriger, den $U_{\mathrm{ij}}-$ Wert und auch die Atomparameter $\mathrm{x}, \mathrm{y}, \mathrm{z}$ festzustellen. Zur exakten Bestimmung der $\mathrm{U}_{\mathrm{ij}}$-Werte für leichte Atome werden meist Neutronendaten herangezogen, da bei Neutronenmessungen die Atomkerne die Streuzentren darstellen und die Streukraft unabhängig von $2 \theta$ nur durch die Streulänge bestimmt wird. Dadurch sind die $\mathrm{U}_{\mathrm{ij}}$-Werte und die Atomparameter $\mathrm{x}, \mathrm{y}, \mathrm{z}$ für leichte Atome, wie z.B. Sauerstoff, oft besser zu ermitteln, da unteranderem bei der Verwendung von Neutronenstrahlung für Sauerstoff eine größere relative Streulänge gegeben ist.

In der Regel erfolgt die Verfeinerung einer Struktur in zwei Stufen. In einem ersten Schritt wird eine Anpassung des Profiles durch die Verfeinerung der Reflexlagen, bestimmt durch die Raumgruppe und die Gitterkonstanten, der Reflexform sowie des Untergrundes durchgeführt. In einem zweiten Schritt erfolgt die Anpassung der Atomparameter, die im wesentlichen die Reflexhöhen bestimmen. Dabei gibt es einige Parameter, die für alle in einer Probe enthaltenen Phasen in gleicher Weise gelten, da diese geräteabhängig sind. Die Anpassung dieser Parameter erfolgt meist in mehreren Schritten und kann je nach Verfeinerungsstrategie $\mathrm{zu}$ unterschiedlichen Verfeinerungsergebnissen führen. Angaben zur Festlegung von Verfeinerungsstrategien sowie zur Aussagefähigkeit von Rietveld-Verfeinerungen sind vielfach in der Literatur beschrieben (McCusker et al. (1999)), sollen an dieser Stelle jedoch nicht diskutiert werden.

\subsubsection{Peakprofilfunktionen und Berechnung physikalischer Parameter}

Für die in GSAS durchgeführten Verfeinerungen wurden zumeist Pseudo-VoigtProfilfunktionen auf Basis der Simpson-Rule-Integrationsmethode nach Howard (1982) verwendet (in GSAS Peakprofil-Funktion Nr. 2). Im Gegensatz zu einem einfachen 
Gaußprofil zeichnet sich diese Funktion durch eine bessere Anpassung von asymmetrischen Peakprofilen durch eine variable Anpassung von Gauß- sowie Lorentzanteilen an den gemessenen Intensitätsprofilen aus. Im folgenden wird nur ein grober Überblick über die funktionalen Zusammenhänge der in GSAS verfeinerbaren Profilparameter der Profilfunktionen $\mathrm{zu}$ den daraus berechneten physikalischen Größen gegeben. Für eine detaillierte Darstellung sowie den zugrundliegenden physikalisch-kristallographischen Mechanismen zur Reflexverbreiterung sei sowohl auf das GSAS Technical Manual (Larson \& v. Dreele, GSAS-Manual (2000) als auch auf die im folgenden zitierten Arbeiten sowie Young (1995) verwiesen.

Die totale Halbwertsbreite eines asymmetrischen Reflexes ist für die CW-Peak-Profilfunktion (CW: Continous Wavelength) Nr.2 als Funktion der Gauß'schen Halbwertsbreite $\Gamma_{\mathrm{g}}$ sowie des Lorentz'schen Koeffizienten $\gamma$ nach Thompson et al. (1987) durch die folgende Gleichung gegeben:

$$
\Gamma=\sqrt[5]{\Gamma_{g}{ }^{5}+\kappa_{2} \cdot \Gamma_{g}{ }^{4} \cdot \gamma+\kappa_{3} \cdot \Gamma_{g}^{3} \cdot \gamma^{2}+\kappa_{4} \cdot \Gamma_{g}{ }^{2} \cdot \gamma^{3}+\kappa_{5} \cdot \Gamma_{g} \cdot \gamma^{4}+\gamma^{5}}
$$

Diese Funktion findet zudem ihre Anwendung in der Beschreibung der Peak-Profilfunktionen für TOF-Daten (vergl. GSAS-Manual (2000), Time of Flight S.150 sowie CW S.159). Die

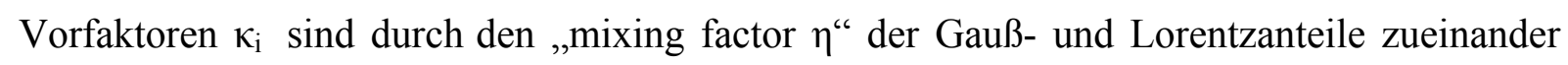
gegeben. Die Gauß'sche Halbwertsbreite $\Gamma_{\mathrm{g}}$ sowie der Lorentz-Koeffizient $\gamma$ sind durch die folgende Gleichungen gegeben:

$$
\begin{gathered}
\Gamma_{g}=\sqrt{(8 \cdot \ln 2) \cdot \sigma^{2}} \text { mit der Peakvarianz nach Cagliotti et al. (1958) mit } \\
\begin{array}{c}
\sigma^{2}=U \cdot \tan ^{2} \Theta+V \cdot \tan \Theta+W+\frac{P}{\cos ^{2} \Theta} \\
\text { sowie für die Lorentzanteile } \\
\gamma=\frac{X+X_{e} \cdot \cos \Phi}{\cos \Theta}+\left(Y+Y_{e} \cdot \cos \Phi\right) \tan \Theta
\end{array}
\end{gathered}
$$

Die Parameter U, V, W, P sowie $\mathrm{X}, \mathrm{X}_{\mathrm{s}}, \mathrm{X}_{\mathrm{e}}, \mathrm{Y}, \mathrm{Y}_{\mathrm{e}}$ stellen die in GSAS verfeinerbaren Profilparameter zur Anpassung der gemessenen Reflexprofile dar. Der Faktor P in Gleichung 
$\Gamma_{\mathrm{g}}$ stellt den Scherrer Koeffizient für die Gauß-Verbreiterung dar. Der erste Term in der Gleichung $\gamma$ stellt die Lorentz-Verbreiterung dar und enthält durch den Parameter $\mathrm{X}$ sowie $\mathrm{X}_{\mathrm{e}}$ einen isotropen als auch anisotropen Anteil. Der Faktor $\cos \Phi$ stellt den Beobachtungswinkel zur Stapelfehlordnungsrichtung der Kristallite dar und ist bei einem regellosem Pulver annähernd $\cos \Phi=1 \mathrm{zu}$ setzen. Der zweite Term mit den Parametern Y sowie $\mathrm{Y}_{\mathrm{e}}$ beschreibt sowohl die isotrope als auch anisotrope Reflexverbreiterungen durch interne Spannungen. Bei Definition z.B. einer zweiten Phase, durch Stapelfehlordnungen beeinflussten Struktur, wird der Parameter $\mathrm{X}_{\mathrm{e}}$ durch $\mathrm{X}_{\mathrm{s}}$ ersetzt und ergibt die folgende Gleichung.

$$
\gamma=\frac{X+X_{s} \cdot \cos \Phi}{\cos \Theta}+\left(Y+Y_{e} \cdot \cos \Phi\right) \tan \Theta
$$

Diese beschreibt allein die für die zweite Phase unterschiedlichen ScherrerReflexverbreiterungen, hervorgerufen durch die Existenz von Stapelfehlordnungen. Konkret für die Bestimmung der Partikelgrößen sowie der Stapelfehlordnungsbereiche aus den gemessenen und peakprofilverfeinerten Diffraktogrammen lassen sich diese durch folgende abgeleitete Relation berechnen (vergl. (Larson \& v. Dreele, GSAS-Manual (2000)):

$$
d=\frac{f \cdot K \cdot \lambda}{\pi \cdot X}[\AA]
$$

mit $\mathrm{f}=18000, \mathrm{~K}:$ Scherrer-Konstanten ${ }^{1}$, Wellenlänge $\lambda$ sowie dem Verfeinerungsparameter $X$, wobei sich die anisotropen Partikelgrößen sowie die mittlere Größe der Stapelfehlordnungsbereiche durch folgende Verfeinerungsparameter gemäß der obigen Gleichung berechnen lassen.

$$
\begin{gathered}
d_{p}=\frac{f \cdot K \cdot \lambda}{\pi\left(X+X_{e}\right)}[\AA] \text { und } d_{s}=\frac{f \cdot K \cdot \lambda}{\pi \cdot X}[\AA] \text { sowie } d_{s f}=\frac{f \cdot \lambda}{\pi \cdot X_{S}}[\AA] \\
\mathrm{d}_{\mathrm{p}}: \text { parallele, } \mathrm{d}_{\mathrm{s}}: \text { senkrechte Partikelgröße, } \mathrm{d}_{\mathrm{sf}}: \text { mittlere Größe der } \\
\text { Stapelfehlordnungsbereiche }
\end{gathered}
$$

\footnotetext{
${ }^{1}$ Die Scherrer-Konstante ist ein Formfaktor, in den die unterschiedlichen Größen der einzelnen Netzebenen eingehen und ist von der annähernden Form der Kristallite der Struktur anbhängig. Für kugelförmige, d.h. sphärische Kristallite ist $\mathrm{K}=0.89$, für würfelförmige $\mathrm{K}=0.94$. Bei unbekannter Kristallitform kann in guter Näherung der Formfaktor zu K=0.9 angenommen werden (vergl. Allmann (1994).
} 
Die Modellierung der Stapelfehler in GSAS unter Verwendung der Peakprofilfunktion CW2 nach Howard (1982) erfolgt durch die Definition von Stapelrichtungsvektoren in der Struktur. In der Anpassung der zur Berechnung der Stapelfehlordnungsbereiche benötigten Reflexprofilparameter, werden in der Rietveld-Verfeinerung dann lediglich die Reflexe berücksichtigt, die eine Reflexprofilverbreiterung in der angegebenen Stapelfehlerrichtung aufweisen. Im Falle des kubischen Eis Ic, mit vermuteten stapelfehlgeordneten „hexagonalen Sequenzen“ (vergl. Kapitel 3.3 ff.) wurde die Stapelrichtung aufgrund eines vermuteten verbreiterten hexagonalen [001]-Reflexes in die bevorzugte hexagonale Stapelrichtung [001] sowie in Richtung der hexagonalen [100]- /[010]-Basisstruktur, d.h. einfachen lateralen Fehlordnungen der hexagonalen Struktur in [001] festgelegt. Versuche der Beschreibung der Imperfektionen im Eis Ih (vergl. Kapitel 3.4.1) bei der Verfeinerung der Gasinhalte von Eis Ih in Gegenwart verschiedener Gase (vergl. Kapitel 5.5 ff.) durch diese Art der Stapelfehlermodelle zu berücksichtigen, führten zu keiner zufriedenstellenden Lösung.

\subsubsection{Grundlagen und Anwendung der JMAK-Theorie}

Die JMAK-Theorie beschreibt das Rekristallisationsverhalten eines Systems beim Phasenübergang von einer festen Phase in eine andere und ist durch Arbeiten von Kolmogorov (1937), Johnson und Mehl (1939) und Avrami (1939) zur Bildung von Kristallisationskeimen und deren Wachstumseigenschaften begründet. Diese Theorie geht bei einer durch Phasenumwandlung stattfindenden Keimbildung einer neuen Phase davon aus, dass das Keimwachstum von sphärischen Keimen in der Ausgangsphase zu jeder Zeit der Phasenumwandlung linear verläuft. Der Verlauf der Phasenanteile der Ausgangsphase sowie der sich neu bildenden Phase $\mathrm{X}_{\Omega}$ wird unter den Annahmen der JMAK-Theorie durch die folgende Gleichung beschrieben:

$$
X_{\Omega}=1-e^{-B \cdot t^{n}}
$$

Der Phasenanteil $\mathrm{X}_{\Omega}$ ist auf den Ausgangsphasenanteil 1 normiert. Die Konstante B ist der reziproke Wert der Zeitkonstanten $\tau$ der Phasenumwandlung und wird durch den zeitlichen Verlauf der Intensitäten bestimmt. $t$ ist die Zeit nach dem Beginn der Phasenumwandlung. Der Exponent $\mathrm{n}$ wird im allgemeinen als Avrami-Exponent bezeichnet und beschreibt unter den Annahmen, z.B. der einer konstanten Keimbildungsrate sowie z.B. eines sphärischen Keimwachstums, die Dimensionalität des Phasenüberganges während des Umbaus der 
Kristallstrukturen von Phase 1 in Phase 2. Mit Dimensionalität ist hier die Anzahl der Kristallisationsrichtungen gemeint. Diese gibt an, ob der Phasenübergang von Struktur 1 in die Struktur 2 mehr isotrop / anisotrop, z.B. überwiegend mehr durch einen z.B. linearen Umbau entlang einer ausgezeichneten Kristallachse oder z.B. flächig, d.h. 2-dimensional in einer Ebene des Kristallsystems oder gar im Volumen vollzogen wird (vergl. Tabelle 2.1).

Geht man von einer konstanten Keimbildungsrate und einem zeitlich linearen Keimwachstum während der Kristallisation aus, so wäre im Falle eines isotropen dreidimensionalen Wachstums der Keime der Avrami-Exponent n=4. Avrami (1939) betrachtete den Fall, dass die Keimbildungsrate nicht konstant, sondern vielmehr eine Funktion der Zeit ist. Er ging davon aus, dass die Keimbildungsrate eine zeitliche Abhängigkeit besitzt und mit zunehmender Bildung der neuen Phase abnimmt. Er bestimmte für einen solchen Fall den Avrami-Exponenten als Wert zwischen $n=3$ und $n=4$. Bei der Anwendung der JMAK-Theorie sind jedoch zwei Grenzfälle von wesentlicher Bedeutung. Der erste Fall, für den der AvramiExponent $\mathrm{n}=4$ beträgt und auf ein dreidimensionales isotropes Wachstum der Keime schließen lässt, und der zweite Fall, bei dem die Keimbildung am Anfang der Phasenumwandlung sehr schnell vonstatten geht. In diesem Fall ist die Keimbildungsrate nicht konstant und fällt nach dem Beginn der Keimbildung bzw. der Phasenumwandlung sehr schnell ab. Das Rekristallisationsverhalten wird dann im wesentlichen durch die Kornrekristallisation der neu gebildeten Phase, d.h. dem isotropen bzw. anisotropen Kornwachstum bestimmt.

Der Avrami-Exponent wäre für eine solche Phasenumwandlung n=3. Cahn (1956) erweiterte die JMAK-Theorie hinsichtlich des anisotropen Kornwachstums. Er fand heraus, dass bei Zulassen von zufälligen, anisotrop dreidimensionalen Wachstumsvorgängen an den Körnern der sich neu bildenden Phase der Avrami-Exponent von $\mathrm{n}=4$ beim Beginn der Phasenumwandlung auf $n=1$ beim Ende der Phasenumwandlung abfällt.

\begin{tabular}{|c|c|c|}
\hline Dimensionen & Wachstumsrichtungen & Avrami-Exponent \\
\hline $3 \mathrm{~d}$ & 3 & 4 \\
\hline $2 \mathrm{~d}$ & 2 & 3 \\
\hline $1 \mathrm{~d}$ & 1 & 2 \\
\hline
\end{tabular}

Tabelle 2.1: Wachstums-Dimensionalität gemäß JMAK-Theorie, Avrami-Exponenten bei möglichen Kritsallwachstumsrichtungen und konst. Keimbildungsrate 
Die Frage, ob eine Phasenumwandlung einem JMAK-Verhalten sowie welcher Dimensionalität der Phasenübergang entspricht, wird durch einen Vergleich der experimentell ermittelten Daten des Rekristallisationsvorganges mit dem JMAK-Modell getroffen. Zu diesem Zweck werden die experimentell ermittelten Intensitätsverläufe durch einen sogenannten JMAK-plot gemäß der folgenden Funktion dargestellt:

$$
\ln \left(\ln \left(\frac{1}{1-X_{\Omega}}\right)\right)=f(\ln (t))
$$

Bei einem JMAK-Verhalten sollte sich in dieser Auftragung ein annähernd linearer Verlauf des zum Phasenanteil proportionalen Intensitätsverlaufes ergeben. Die Steigung entspricht in dieser Auftragung dem Avrami-Exponenten n.Frühe experimentelle Arbeiten hinsichtlich der Rekristallisation wurden von Anderson und Mehl (1945) an Aluminium, von Reiter (1952) an Carbon-Stählen sowie von Gordon (1955) an Kupfer durchgeführt, wobei die verwendeten Proben sehr feinkörnig waren. Der Avrami- Exponent wurde in diesen Experimenten zu n=4 bestimmt. Es ist jedoch anzumerken, dass die Körner bzw. das Kornwachstum und damit die Bestimmung des Avrami-Exponenten bei den Rekristallisationvorgängen von der Probengeometrie und von internen mikrostrukturellen Zwängen, wie interne mechanische Spannungen, Korn- und Kornvorzugsorientierungen der Ausgangsphase, sowie etwaigen Rekristallisationseffekten der Ausgangsphase abhängt. Deutlich werden diese Effekte durch Abweichungen der ermittelten Rekristallisationsverläufe von dem erwarteten JMAKVerhalten. Die Abweichungen von dem JMAK-plot kann sich entweder durch einen nicht linearen Verlauf und / oder durch die Steigung, d.h. einen Avrami-Koeffizienten kleiner als $\mathrm{n}=3$ äußern. Arbeiten diesbezüglich wurden von Hansen et al. (1981) an Kupfer, von Michalak und Hibbard (1961) sowie Rosen et al. (1967) an Eisen durchgeführt. Bei einer sehr detaillierten Betrachtung von kinetischen Rekristallisationsexperimenten, die zum Teil durch die eben genannten Autoren durchgeführt wurden, kann ausgesagt werden, dass eine eher ungenügende Übereinstimmung zwischen dem auf der JMAK-Theorie erwarteten und dem experimentell bestimmten Rekristallisationsverhalten festzustellen ist. Eine ausführliche Beschreibung der Grundlagen der JMAK- Theorie ist in dem Buch "Recrystallisation and Related Annealing Phenomena" von H.J. Humphreys, M. Hatherley (1996), sowie den in diesem Buch zitierten Artikel von Christian (1965) gegeben. 


\section{Die Herstellung von Eisphasen}

Im Hinblick auf die Koexistenz mehrerer, auch metastabiler Eisphasen sowie eisverwandter Strukturen, den Gas-Hydraten (vergl. Kapitel 4. ff.), in einem weiten Druck- und Temperaturbereich war die genauere Kenntnis der Phasengrenzen für die gezielte und reproduzierbare Herstellung einzelner Eisphasen von Bedeutung. Als Grundlage für Pulverdiffraktionsmessungen am D2B und D1B des ILL war es zudem notwendig, die zu untersuchenden Eisphasen mit einer hinreichenden Pulvergüte, d.h. sehr feinkristallines Eispulver herzustellen. Durch die Feinkristallinität wird gewährleistet, dass bei der eindimensionalen Bestimmung der Diffraktogramme in $2 \theta$ Kristallite in hinreichender Anzahl in Reflexionsstellung zum Detektor orientiert sind. Zu diesem Zweck wurden mit der in Kapitel 2 dargestellten pVT-Apparatur die Phasengrenzen der einzelnen Eisphasen vermessen sowie in der Literatur beschriebene Präparationsverfahren und Präparationsrouten für deren Herstellung verifiziert. Im hauptsächlichen Interesse lag die Bestimmung der fest-flüssigPhasengrenzen unter Verwendung verschiedener Gase, die zur Clathrat-Hydrat-Synthese verwendet wurden. Die über die ausgearbeiteten Präparationsrouten hergestellten Eisphasen wurden in den pVT-Experimenten durch die Bestimmung der Schmelzkurven identifiziert. Durch in situ Strukturbestimmungen mittels Neutronen- sowie Synchrotronbeugung wurden die über die Präparationsrouten hergestellten Eisphasen hinsichtlich ihrer Struktur sowie der Pulvergüte untersucht und verifiziert.

\subsection{Präparationsrouten}

Als Grundlage für die Verifizierung von Präparationsrouten wurden die in der Literatur beschriebenen Verfahrensweisen verwendet. Ein Überblick über die in der Literatur aufgezeigten Präparationsrouten ist in der folgenden Abbildung 3.1 dargestellt. Angaben über die Reproduzierbarkeit der Präparation oder bezüglich Pulvergüte hinsichtlich der Untersuchung mittels Pulverdiffraktion werden selten gemacht. Es war daher notwendig, die aufgeführten Präparationsrouten im Detail zu untersuchen und diese mittels der hergestellten Eisphasen in situ zu verifizieren. 


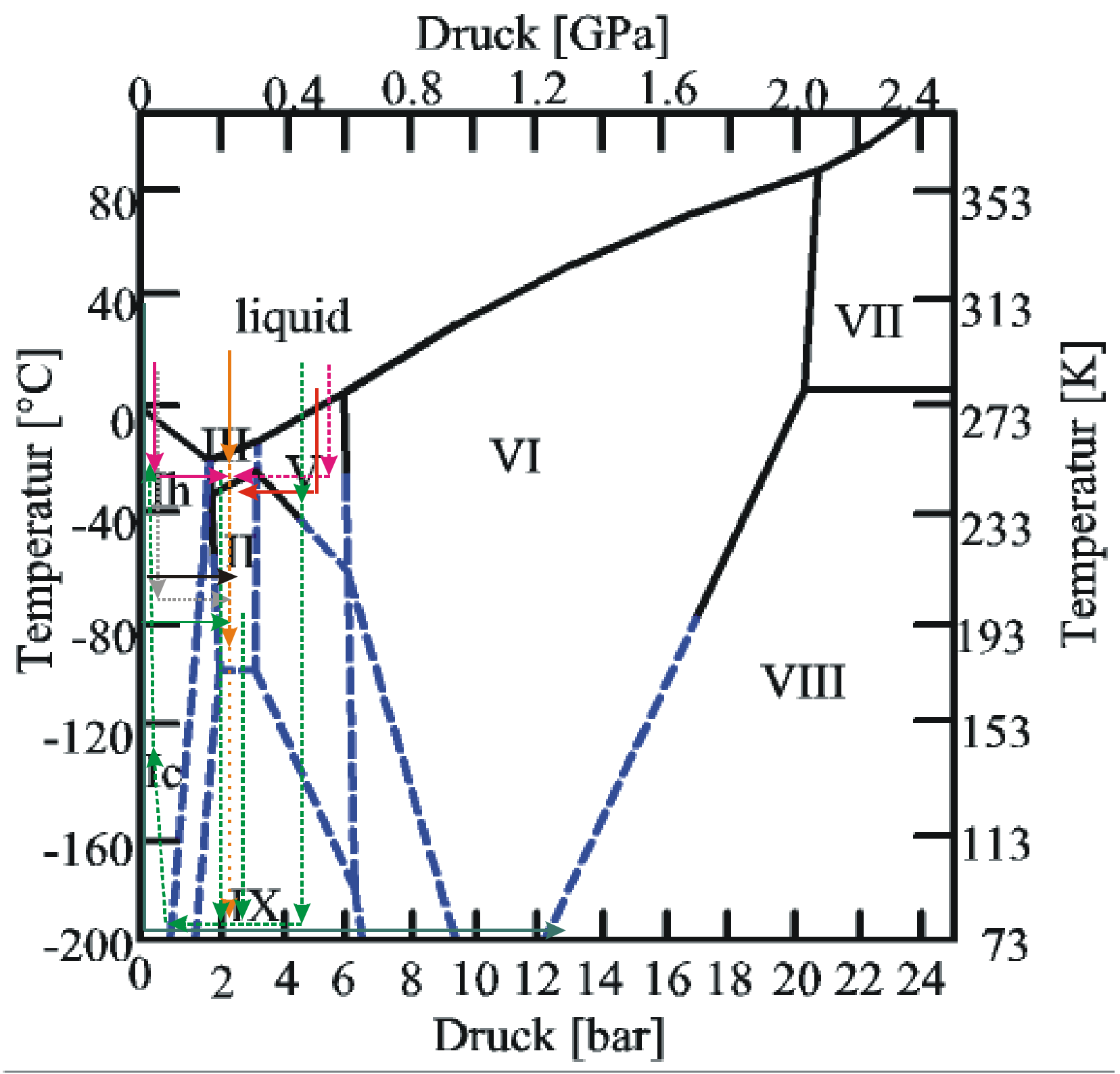

Abbildung 3.1: Auszug aus den in der Literatur beschriebenen Präparationsrouten zur gesicherten Herstellung verschiedener Eisphasen im Stabilitätsbereich von Eis Ih, Eis II, Eis III und Eis V. (- - -) : Eis $\mathrm{V} \rightarrow$ Eis III Bridgman (1912), (-) : Eis Ih $\rightarrow$ Eis III Bridgman (1912), (- - ) Eis Ih $\rightarrow$ Eis II Sukarova (1982), (-) Eis Ih $\rightarrow$ Eis II Tammann (1903), Wilson et al. (1965), (-) Eis V $\rightarrow$ Eis III Wilson et al. (1965), (-) Eis Ih $\rightarrow$ Eis XII Koza et al. (1999), (-) L $\rightarrow$ Eis III Kell \& Whalley (1968), (- - -) Eis III $\rightarrow$ Eis II Londono et al. (1993), (. . .) Eis III $\rightarrow$ Eis IX Whalley et al. (1968), (一) Eis Ih $\rightarrow$ Eis III Bertie et al. (1963), (- - -) Hochdruckpräparationsroute aus Hochdruckeisphasen für kubisches Eis Ic sowie in hexagonales Eis Ih (Bertie et al. 1963), 


\subsection{Die Herstellung von Eis Ih}

Bei der Herstellung von Eis Ih war insbesondere die letztendlich erreichte Pulverqualität sowie deren Reproduzierbarkeit von Bedeutung. Bisherige Erfahrungen der Eispräparation haben gezeigt, dass die Herstellung eines feinkörnigen Eispulvers z. B. durch Mörsern unter Kühlung, zumeist mit flüssigem Stickstoff nur bis zu einem gewissen Grad funktioniert. Häufig verblieben trotz intensiven Mörserns größere Eiskörner. FE-REM Aufnahmen zeigen, dass derartig hergestellte Eispulver eine inhomogene Korngrößenverteilung aufweisen (vergl. Abbildung 3.4). Es zeigte sich, dass die Pulvergüte durch intensives und längeren Mörserns verbessert werden konnte, jedoch nicht in jedem Fall reproduzierbar und genügend feinkristallin wird. Mikroskopische Befunde durch FE-REM Untersuchungen zeigten, das während des fortgesetzten Mörserns eine Vergröberung des Eispulvers stattfand. Diese Vergröberung ist möglicherweise durch eine nicht sehr kontinuierliche Kühlung, d.h. Erwärmung der Probe während des Mörserns und damit durch eine einsetzende Rekristallisation des Eispulvers bedingt (vergl. Abb. 3.2 bis Abb. 3.5).

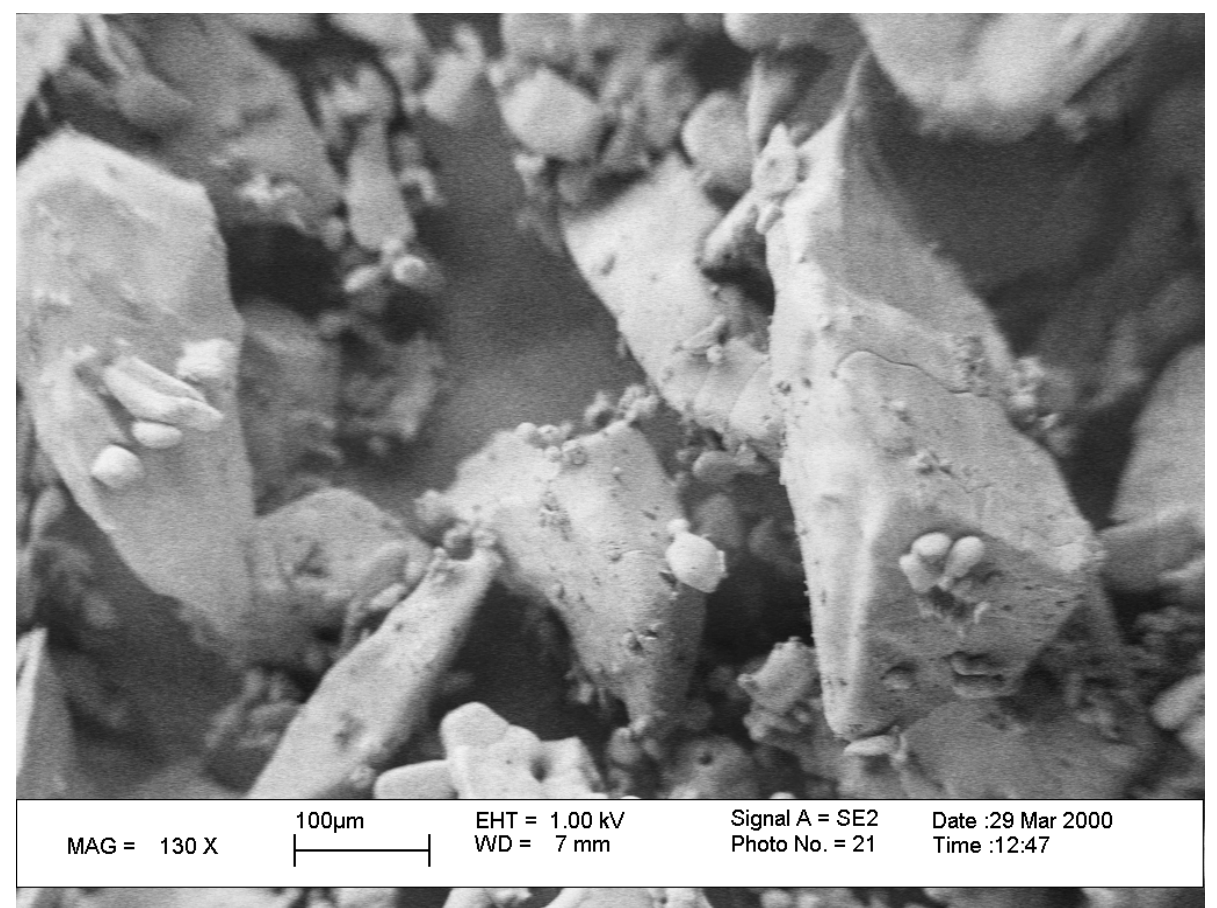

Abbildung 3.2: gemörserte $\mathrm{D}_{2} \mathrm{O}$-Eis Ih-Probe nach einer Temperzeit von $\mathrm{t} \sim 15 \mathrm{~min}$ bei einer Temperatur von $\mathbf{T} \sim 0^{\circ} \mathrm{C}$.

Die durch die Zerkleinerungstechnik bedingte mäßige Pulverqualität wurde während der Präparation der Probe durch die Rekristallisation stetig weiter verschlechtert. Durch Mörsern hergestellte Pulver konnten daher nicht reproduzierbar und genügend fein hergestellt werden. 


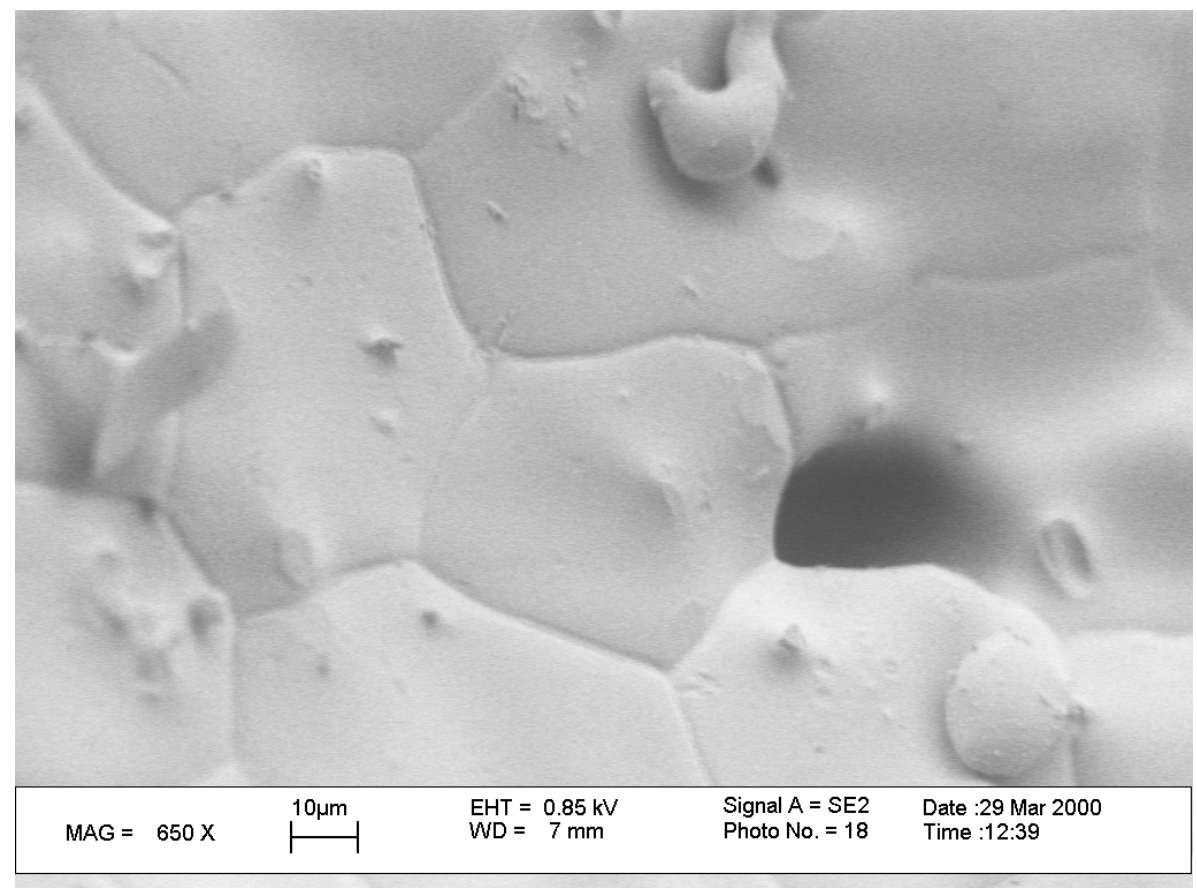

Abbildung 3.3: gemörserte $\mathrm{D}_{2} \mathrm{O}$-Eis Ih-Probe nach einer Temperzeit von $\mathrm{t} \sim 15 \mathrm{~min}$ tempern bei $\mathrm{T}=0^{\circ} \mathrm{C}$. Die Abbildung zeigt im Detail das Zusammenwachsen von kleineren Eiskörnern zu größeren Eiskörnern. Der Effekt der Vergröberung zeigt sich verstärkt mit zunehmender Temperzeit t, vergl. Anhang 8.3.2)

Gerade im Hinblick auf die in situ-Strukturuntersuchungen von Eis Ih als Funktion des Druckes und der Temperatur in zwei zeitlich voneinander getrennten Experimenten am D2B, zum einen Eis Ih mit Argon- und Heliumgas sowie in dem zweiten Experiment Eis Ih mit Neongas als Drucküberträgermdeium, war eine reproduzierbare und hinreichend gute Pulverqualität als Voraussetzung wesentlich. Ebenso sind die Korngrößen des Eis-Pulvers hinsichtlich der Bildung von Clathrat-Hydraten sowie bei der Kinetik der Clathrat-HydratSynthese von großer Bedeutung. Die Synthese von Clathrat-Hydraten ist, durch die Adsorption von Gasen über die Oberfläche des Eises, ein oberflächenbestimmter Effekt. Möglichst große Oberflächen, die durch kleine Korngrößen gegeben sind, sind deswegen erwünscht. Für einen Vergleich von Synthesezeiten ist daher eine reproduzierbare EisOberfläche, z.B. berechnet über die mittlere Korngröße, von Bedeutung.

Es wurden daher mehrere Präparationsverfahren zur Herstellung von Eis Ih verwendet und mittels Neutronenstreuexperimenten in situ verfolgt und in weiteren Untersuchungen durch FE-REM hinsichtlich der Pulvergüte im "recoverten" Zustand qualitativ untersucht und verifiziert. Um eine reproduzierbare Herstellung von Eis Ih-Pulver als Ausgangsmaterial zu gewährleisten wurde die von Bertie et al. (1963) beobachtete Phasenumwandlung aus den Hochdruckeisphasen in das kubische Eis Ic als Grundlage für die Herstellungsexperimente 
verwendet. Zuerst wurde mit der aufgebauten pVT-Apparatur eine Hochdruckeisphase, in den meisten Fällen Eis V, hergestellt. Eis V wurde als Ausgangsmaterial verwendet, da aufgrund bisheriger Erfahrungen die kristalline Struktur des Eis V bei der Herstellung aus der flüssigen Phase sowie bei der Herstellung über die feste Phase nur geringe Unterschiede in der Ausbildung von Vorzugsorientierungen aufweist (Kuhs 2000, pers. Mitteilung). Die Hochdruckeisphase wurde dann mit flüssigem Stickstoff, mit einer Kühlrate von $\sim 1.5 \mathrm{~K} / \mathrm{s}$, auf eine Temperatur von T 77K abgeschreckt (Lobban et al. 2000) und der Gasdruck auf Umgebungsdruck reduziert. Die Eisprobe, das „recoverte“ Hochdruckeis, wurde danach erwärmt. In einem weiten Temperaturbereich von $\mathrm{T} \sim 130 \mathrm{~K}$ bis $\mathrm{T} \sim 200 \mathrm{~K}$ erfolgte dann die Phasenumwandlung in kubisches Eis Ic, der unter diesen Druck- und Temperaturbedingungen zu Eis Ih metastabilen Eisvariante. Die Phasenumwandlung führt aufgrund des Dichteunterschiedes der Hochdruckeisphasen zu kubischem Eis Ic, innerhalb der kurzen Erwärmungsphasen durch das stark zunehmende Volumen zu einer mechanischen Zerstörung des makroskopischen Gefüges. Die Dichte verschiedener „recoverter“ Eisphasen sind in Tabelle 3.1 gegeben. An dieser Stelle sei jedoch nochmals auf die Dichten der Eisphasen unter verschiedenen Umgebungsbedingungen in Tabelle 1.2, Kapitel 1.3) verwiesen.

\begin{tabular}{|c|c|c|c|}
\hline Eisphase & $\mathrm{T}[\mathrm{K}], \mathrm{p} \sim 1 \mathrm{bar}$ & $\rho\left[\mathrm{gcm}^{-3}\right]$ & \\
\hline Ih & 77 & $0.924^{+}$ & \multirow[t]{2}{*}{ Dowell und Rinfret (1960) } \\
\hline Ic & 77 & $0.923^{+}$ & \\
\hline Ic(HDA) & 140 & $1.034^{*}$ & D2B-Experiment (1999) ${ }^{1}$ \\
\hline II & 123 & $1.170^{+}$ & Kamb (1964) \\
\hline II & 83 & $1.342^{*}$ & D1B-Experiment $(1999)^{2}$ \\
\hline $\mathbf{V}$ & 98 & $1.231^{+}$ & Kamb et al. (1967) \\
\hline $\mathbf{V}$ & 84 & $1.390^{*}$ & D1B-Experiment (1999) ${ }^{2}$ \\
\hline IX & 123 & $1.141^{+}$ & Rabideau et al. (1968) \\
\hline IV & 110 & $1.272^{+}$ & Engelhardt und Kamb (1981) \\
\hline XII & 127 & $1.30^{*}$ & \multirow[t]{2}{*}{ Koza et al. (1999) } \\
\hline HDA & 127 & $1.17^{*}$ & \\
\hline
\end{tabular}

Tabelle 3.1: Dichten „,recoverter“ Hochdruck-Eisproben bei Umgebungsdruck von $p \sim 1$ bar, ${ }^{1,2}$ Dichte aus verfeinerten Datensätzen recoverter Hochdruckeisphasen am D2B, D1B; ${ }^{*}: \mathrm{D}_{2} \mathrm{O},{ }^{+}: \mathrm{H}_{2} \mathrm{O}$

Eine mikroskopische Interpretation als denkbare Ursache für die Feinkristallinität der Probe ist eine Mehrfachnukleation von Eis Ic-Körnern auf den Körnern der Ausgangsphase sowie einem begrenzten topotaktischen Wachstum dieser konkurrierenden Eis Ic-Körner. Je nach verwendeter Hochdruckeisphase, als Ausgangsphase für die Umwandlung in kubisches Eis Ic, sollten sich daher diesbezüglich Unterschiede in dem Wachstumsverhalten sowie strukturelle Unterschiede des kubischen Eis Ic verbinden. Hinweise auf strukturelle Unterschiede und 
einem damit verbundenem unterschiedlichen Phasenübergangsverhalten lassen sich in Arbeiten von Stern et al. (1997) sowie Bennett et al. (1997) finden (vergl. Kapitel 1.4).

Der Übergang von den „recoverten“ Hochdruckeisphasen in die Struktur des kubischen Eis Ic wurde für die Eisphasen XII, V, IX (Tieftemperaturphase des Eis III) sowie dem Eis II detaillierter verfolgt. Es wurde versucht sowohl den Phasenübergang mittels der JMAKTheorie als auch die strukturellen Unterschiede des kubischen Eis Ic zu beschreiben und zu quantifizieren.

Bei der weiteren Erwärmung des aus den Hochdruckeisphasen erhaltenen kubischen Eis Ic erfolgte die Phasenumwandlung in das hexagonale Eis Ih (Temperaturbereich $\mathrm{T} \sim 160 \mathrm{~K}$ bis T $220 \mathrm{~K}$ bei Umgebungsdruck). Diese Phasenumwandlung konnte in einem weiteren Temperaturbereich als in der Literatur beschrieben, beobachtet werden und wird später eingehender diskutiert (vergl. Kapitel 3.4.1).

Hinsichtlich des Überganges aus den HD-Eisphasen $\rightarrow \mathrm{Ic} \rightarrow \mathrm{Ih}$ wurden durch in situ Neutronenstreuexperimente eine sehr zögerliche Umwandlung in Eis Ih beobachtet, die zu einem wesentlichen Problem bei den experimentellen Untersuchungen sowie deren Auswertungen mittels Rietveld-Verfeinerungen an Eis Ih führten und die Aussagefähigkeit einiger Experimente sehr stark einschränkte (vergl. Kapitel 3.4.1). Die unterschiedlich hergestellten Eis Ih-Proben wurden mittels FE-REM elektronenoptisch auf ihre Korngrößen verglichen. Die folgenden Abbildungen 3.4 und 3.5 zeigen die durch die verschiedenen Präparationsrouten hergestellten Eis Ih-Pulver im Vergleich. Aus den Bildern ist zu ersehen, dass die Korngrößen des Eispulvers bei beiden unterschiedlichen Präparationen eher einer Korngrößenverteilung als einer homogenen Korngröße entsprechen. Der Vergleich der Bildmaßstäbe erlaubt die Aussage, dass die mittlere Korngröße des über die Hochdruckpräparationroute hergestellten Eis Ih mindestens um den Faktor 5-10 kleiner ist. Auch scheint diese wesentlich homogener zu sein als bei einer manuellen Präparation durch Mörsern. Größere Körner sind ebenso zufinden, jedoch ist deren Anteil an dem Volumen aufgrund der Aufnahmen als gering anzusehen. In mehreren elektronenoptischen Untersuchungen verschiedener Probenherstellungen hat sich gezeigt, dass die in Abbildung 3.5 dargestellte Korngrößenverteilung für das über die HD-Eis $\rightarrow \mathrm{Ic} \rightarrow \mathrm{Ih}$ Herstellungsverfahren präparierte Eis Ih als durchaus repräsentativ angesehen werden kann. 
Bei Präparationen einer gleichartigen, über die Hochdruckeisphase hergestellten Probe, kann daher mit sehr hoher Wahrscheinlichkeit von einem polykristallinen Eispulver nahezu gleicher Güte ausgegangen werden.

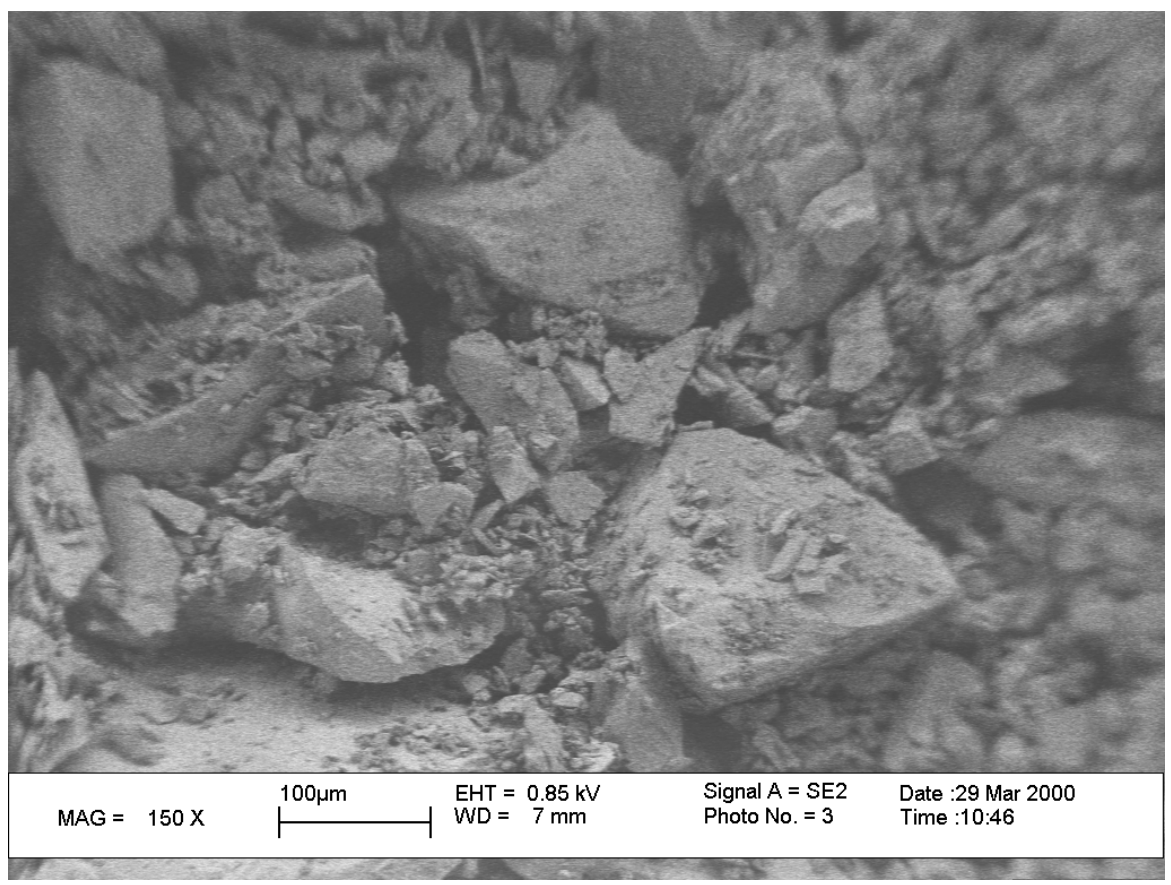

Abbildung 3.4: per Hand frisch gemörsertes Eis Ih, Mörserzeit t 10min., nicht kontinuierliche Kühlung mit flüssigem Stickstoff und zeitweiser Erwärmung während des Mörservorganges.

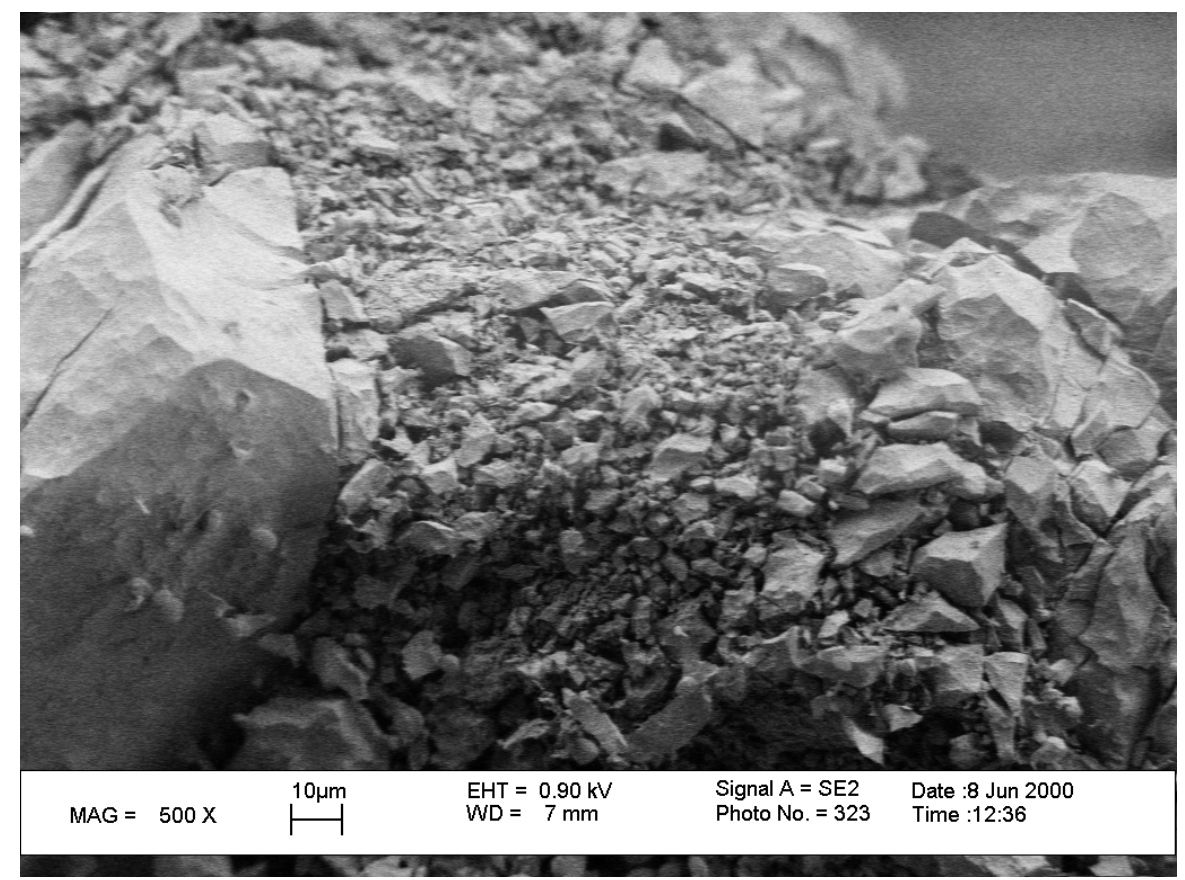

Abbildung 3.5: Eis Ih-Körnung nach dem Phasenübergang der Hochdruckeisphase V in kubisches Eis Ic in hexagonales Eis Ih. 
Bei einem Synthesevergleich an Argon-Clathrat-Hydraten mittels eines über die HDEis $\rightarrow I c \rightarrow I h$ Präparationsroute hergestellten Eis Ih-Pulver zeigte sich bei vergleichbaren Synthesebedingungen eine Synthesezeit von zwei Tagen für eine nahezu vollständige Umsetzung. Bei der üblichen Präparation mit gemörsertem Eis erhielt man ein vergleichbares Syntheseergebnis erst nach etwa einer Woche Synthesezeit.

\subsection{Phasenübergänge von Hochdruckeisphasen in Eis Ic als transiente Phase}

Der Übergang von Hochdruckeisphasen in das kubische Eis Ic als Teil der Eis IhPräparationsroute war zunächst nicht im eigentlichen Interesse der Arbeit, jedoch zeigten sich bei der Herstellung und Verifizierung der Präparationsrouten sowohl Unterschiede hinsichtlich der Kinetik der Phasenumwandlung aus Hochdruckeisphasen in das kubische Eis Ic als auch strukturelle Unterschiede, die weiter verfolgt wurden. Das kubische Eis Ic wurde erstmals von König (1943) durch Elektronenbeugungsexperimente an aus der Gasphase kondensiertem Eis entdeckt und beschrieben. Weitere Untersuchungen $\mathrm{zu}$ den Bildungsbedingungen durch Kondensation aus der Gasphase wurden systematisch von Blackman \& Lisgarten (1958) durchgeführt. Sie stellten fest, dass bei Temperaturen oberhalb von $\mathrm{T} \sim 150 \mathrm{~K}$ hexagonales Eis Ih aus der Gasphase kondensierte. Kubisches Eis Ic bildete sich in dem Temperaturbereich von $\mathrm{T} \sim 130 \mathrm{~K}$ bis $\mathrm{T} \sim 150 \mathrm{~K}$, unterhalb von $\mathrm{T} \sim 130 \mathrm{~K}$ bildete sich amorphes Eis. Dowell \& Rinfret (1960) untersuchten basierend auf der Herstellungsmethode von Blackman \& Lisgarten (1958) gewonnenes kubisches Eis Ic. Sie fanden konsistent zu den Arbeiten von Blackman \& Lisgarten (1958), dass bei tiefen Temperaturen und niedrigen Drücken (Umgebungsdruck $\mathrm{p} \leq 1$ bar) neben dem hexagonalen Eis Ih die kubische Variante sowie eine weitere als ,vitreous ice” bezeichnete Eisphase existiert. Unterschiede in der Struktur der kubischen Eisphasen wurden von Dowell \& Rinfret (1960) hinsichtlich der Herstellung des kubischen Eises gefunden. Ein aus der „vitreous ice”- Eisphase gewonnenes kubisches Eis zeigte ein anderes Intensitätsprofil als ein durch Kondensation hergestelltes. Beide heben hervor, dass es nicht möglich war, eine rein kubische Eisphase herzustellen, sondern die kubische Eisphase bei jedweder Art der Herstellung Kontaminationen mit zum einen „vitreous ice”-Anteilen $(T<130 \mathrm{~K}$ hergestellt) aufweist oder bei Kondensation $(130 \mathrm{~K}<\mathrm{T}<150 \mathrm{~K})$ zusätzliche Streuanteile an den Reflexen des kubischen Eis zu finden waren (vergl. Kuhs et al. (1987)). Bertie et al. (1963) beobachteten, dass „recoverte“ Hochdruckeisphasen bei Erwärmung von $\mathrm{T} \sim 77 \mathrm{~K}$ an aufwärts im Temperaturbereich von 
$\mathrm{T} \sim 120 \mathrm{~K}$ bis $\mathrm{T} \sim 170 \mathrm{~K}$ eine Phasenumwandlung in das kubische Eis Ic vollzogen. Handa et al. (1988) berichteten, dass bei Phasenumwandlungen vom kubischen Eis Ic, hergestellt aus unterschiedlichen Hochdruckeisphasen, ins hexagonale Eis Ih unterschiedliche Enthalpieänderungen, deren Beträge zwischen $\Delta \mathrm{H}=13 \mathrm{~J} / \mathrm{mol}$ und $\Delta \mathrm{H}=50 \mathrm{~J} / \mathrm{mol}$ liegen, gemessen wurden. Diese signifikanten Unterschiede wurden von Handa et al. (1988) als strukturelle Unterschiede der kubischen Eis Ic Ausgangsphase gedeutet. Diese Energieunterschiede sind jedoch im Vergleich $\mathrm{zu}$ den Übergängen der „recoverten“ Hochdruckeisphasen in das kubische Eis Ic sehr gering (vergl. Kapitel 3.4, Tabelle 3.7 und Tabelle 3.8). Erste strukturelle Untersuchungen mittels Neutronendiffraktion wurden von Arnold et al. (1968) an kubischem Eis Ic, hergestellt aus verschiedenen Eisphasen, durchgeführt. Kuhs et al. (1987) untersuchten mittels Neutronenstreuexperimenten die Struktur von kubischem Eis Ic, dass durch die von Bertie et al. (1963) beschriebene Präparation aus „recovertem“ Eis II hergestellt wurde. Ebenso wie bei den Untersuchungen von Dowell \& Rinfret (1960) zeigte sich, dass das kubische Eis Ic nicht als reine kubische Phase vorlag. Die Struktur wurde von Kuhs et al. (1987) durch Least Squares Fits der einzelnen Reflexe bestimmt. Bei der Annahme einer kubischen Elementarzelle, Raumgruppe $\mathrm{Fd} \overline{3} \mathrm{~m}$ und einer bei $\mathrm{T} \sim 78 \mathrm{~K}$ bestimmten Gitterkonstanten von $\mathrm{a}_{0}=6.358 \AA$, konnten die gemessenen Reflexe indiziert werden. Die Atompositionen (fraktionellen Koordinaten) wurden in der Raumgruppe $\mathrm{Fd} \overline{3} \mathrm{~m}$ für $\mathrm{O}$ auf einer speziellen Lage in der Elementarzelle zu $\mathrm{x}=\mathrm{y}=\mathrm{z}=0.375$, für $\mathrm{D}$ auf einer allgemeinen Lage $\mathrm{zu} \mathrm{x}=\mathrm{y}=\mathrm{z}=0.4670(19)$ bestimmt. Die isotropen ADP's (atomic displacement parameter) wurden für Sauerstoff O zu B=1.56(59) $\AA^{2}$ sowie für die Deuteriumatome $\mathrm{D}$ zu $\mathrm{B}=2.02(64) \AA^{2}$ bei einer Temperatur von $\mathrm{T} \sim 78 \mathrm{~K}$ bestimmt.

Eine Beschreibung des Intensitätsprofils der Autoren weist hauptsächlich auf drei Anomalien hin, die durch eine reine kubische Struktur nicht beschrieben werden können. Als Ursache dieser Anomalien wurden von Kuhs et al. (1987) Wachstumsstapelfehler in der Eis IcStruktur angeführt und diskutiert.

Die folgende Abbildung 3.6 stellt exemplarisch ein am D2B des ILL gemessenes Diffraktogramm des aus Eis XII hergestellten kubischen Eis Ic bei Umgebungsdruck und $\mathrm{T} \sim 180 \mathrm{~K}$ dar. 


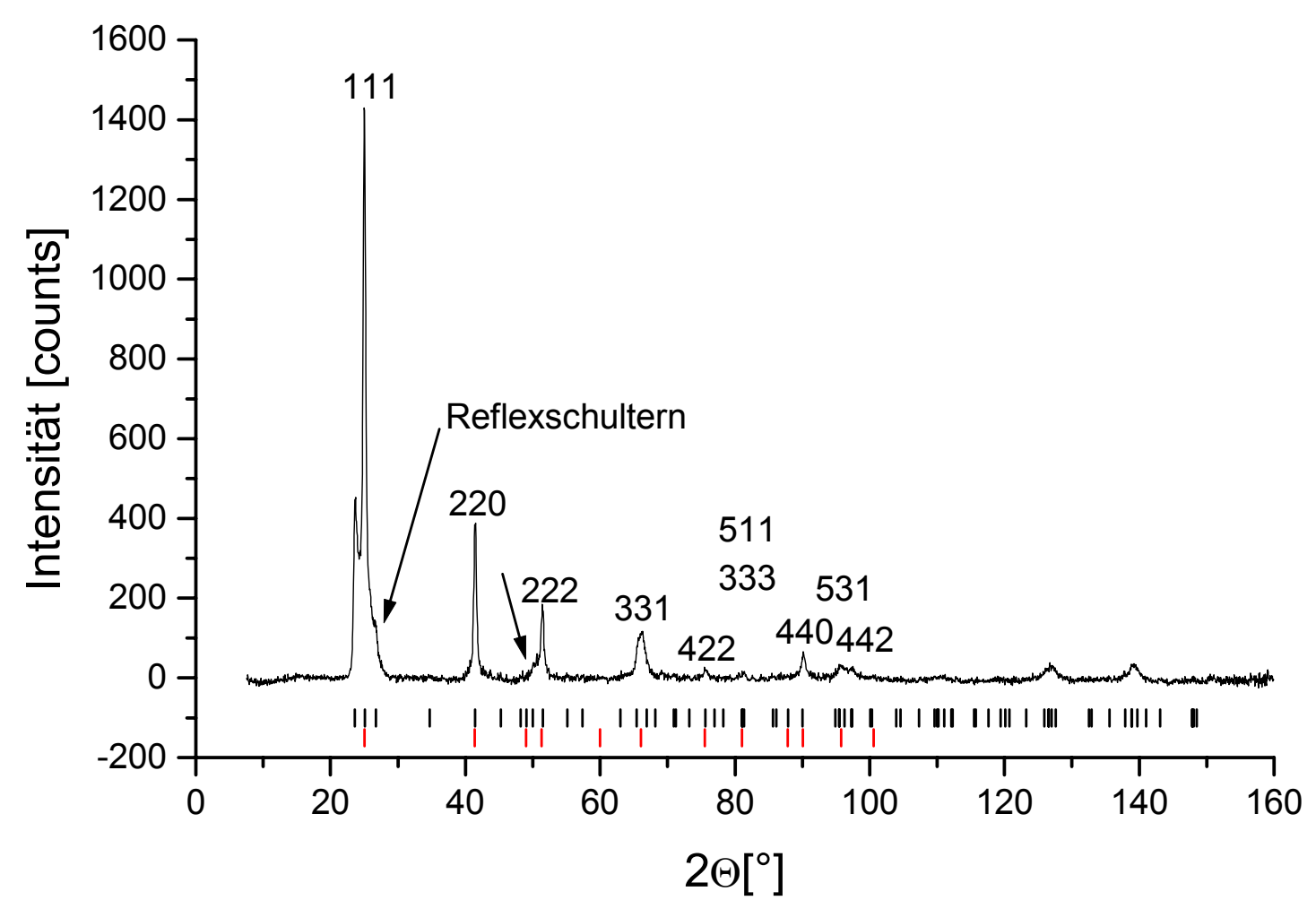

Abbildung 3.6: Kubisches Eis Ic aus der Hochdruckeisphase Eis XII hergestellt, gemessen am D2B bei T 180K. Die Pfeile markieren die von Kuhs et al. (1987) als Stapelfehlordnungen interpretierten Reflexschultern. Die Reflexschultern treten ebenso für das aus den Hochdruckeisphasen V, IX/III, II hergestelltem kubischen Eis auf, sind jedoch unterschiedlich stark ausgeprägt (vergl. Abbildung 3.7, Kapitel 3.3.1), | : markierte Eis Ih-Reflexlagen, | : markierte Eis Ic-Reflexlagen.

Abweichungen in den Intensitätsprofilen sind für die aus anderen HD-Eisphasen hergestellten kubischen Eisphasen zu verzeichnen und werden später diskutiert. Kuhs et al. (1987) beschreiben, dass sich für alle Peaks der kubischen Eisphase mit den Indizes $h+k+l \neq 3 n$ Reflexverbreiterungen zeigen. Am [111]- und am [222]-Reflex der kubischen Eisphase zeigen sich, wie in der Abbildung 3.6 dargestellt, Schultern. Ein zusätzlicher Reflex, der aus dem Intensitätsprofil angenommen werden kann, konnte nicht in der Raumgruppe $\mathrm{Fd} \overline{3} \mathrm{~m}$ der kubischen Eisphase indiziert werden. Zudem weist dieser aufgrund des steilen Intensitätsanstieges sowie eines langsamen Abfalles zu höheren Winkeln des Intensitätsprofils eine ausgeprägte Asymmetrie auf.

Derlei Asymmetrien von Intensitätsprofilen wurden erstmals von Warren (1941) als Folge der Existenz von lateralen Fehlordnungen in Strukturen beschrieben. Erste theoretische 
Untersuchungen zu möglichen Stapelfehlern in Eis wurden von Frank und Nicholas (1953) durch geometrische Betrachtungen durchgeführt und diskutiert. Ein guter Überblick über die Theorie von Stapelfehlordnungen und deren Auswirkungen ist in dem Buch von Drits und Tchoubar (1990) gegeben.

Das Intensitätsmaximum des im kubischen System nicht indizierbaren Reflexes stimmt annähernd mit der bei vergleichbaren Bedingungen simulierten Reflexlage des hexagonalen Eis Ih [100]-Reflexes überein. Geht man von hexagonalen Anteilen an dem Probevolumen aus, so liegen die bei annähernd vergleichbaren Umgebungsbedingungen simulierten hexagonalen Reflexe unter denen von Eis Ic. Hinweise auf hexagonale Reflexe mit den Indizes von $1 \neq 0$ wurden nicht gefunden, so dass die Existenz eines „regulären“ hexagonalen Phasenanteiles ausgeschlossen werden kann. Die an einigen kubischen Bragg-Peaks ausgeprägten Reflexschultern ([111]-, und [222]-Reflex), sowie die Existenz des zusätzlichen Peaks wurden von Kuhs et al. (1987) als Folge der Anordnung von basalen hexagonalen Strukturen in der kubischen Stapelfolge diskutiert. Geht man von solchen Strukturen aus, so ist anzunehmen, dass diese hexagonalen Strukturbereiche unterschiedliche Ausdehnungen aufweisen können. Für diese kann dann zu den Effekten der Art und Häufigkeit dieser Stapelfehlordnungen zudem ein Partikelgrößeneffekt für die hexagonalen Strukturen diskutiert werden. Die experimentell bestimmten Verbreiterungen an den kubisch indizierten Reflexen sowie die starke Asymmetrie des zu vermutenden hexagonalen [100]-Reflexes wurden von Kuhs et al. (1987) durch die Existenz von Stapelfehlordnungen in der kubischen Stapelfolge interpretiert und diskutiert. Kuhs et al. (1987) errechneten durch Anwendung der Debye-Scherrer-Formel über die aus den experimentellen Daten ermittelten Halbwertsbreiten die isotrope, mittlere Partikelgröße für das kubische Eis zu d 160Å. Für die Bestimmung fanden die Halbwertsbreiten des [111]-, [220]-, [222]- und des [440]-Reflexes Verwendung. Der [331/533]-Reflex wurde ausgelassen, da dieser eine starke auffällige Abweichung in den Reflexprofilen aufwies. In den Arbeiten von Warren (1941) und Kuhs et al. (1987) ist auf die Mannigfaltigkeit von Ursachen für Reflexverbreiterungen durch Partikelgrößeneffekte und Stapelfehlordnungen hingewiesen, deren Anordnungen oder Strukturen nicht eindeutig zu beschreiben sind. Mögliche Aussagen können jedoch bezüglich des Phasenanteiles des aus den verschiedenen HD-Proben hergestellten kubisch angeordneten Phasenanteilen getroffen werden um ein mehr qualitatives Maß für die Fehlordnungen sowie die Größe der Fehlordnungsbereiche geben zu können. 
Als einfachste Variante ergab sich eine Rietveld-Modellierung als Phasengemisch aus einer kubischen Phase sowie eines hexagonalen Phasenanteiles, der als stark stapelfehlgeordnet und auch stark partikelgrößenbeeinflusst angenommen wurde.

Die Anordnung der hexagonalen Ringstrukturen für Eis Ih ist in Schichten entlang der bevorzugten [001]-Stapelrichtung durch die Stapelfolge ...ABABAB... gegeben und entspricht der Hexagonal-Dichtest-Packung von Metallen (vergl. Anhang 8.3.3, Abbildung 8.3.3.1 (b)). Die Stapelfolge für die kubischen Anteile ist durch ...ABCABCABC... gegeben (vergl. Anhang 8.3.3, Abbildung 8.3.3.1 (a). Folgt man der Diskussion der Stapelfehlordnungen in der kubischen Struktur, so liegen diese bevorzugt in [111]-Richtung (kubische Stapelrichtung), die im Hexagonalen mit der bevorzugten [001]-Richtung übereinstimmt. Die Ursache von Stapelfehlern ist im einfachsten Fall durch planare Defekte der in Stapelrichtung angeordneten Schichten gegeben. Diese Stapelfehler können z.B. von der Art

\section{...АВСАВСАВ.А.ВСАВСАВС... \\ oder}

\section{...АBCABCAB.A.CBACBACB...}

sein (kubische Stapelsequenz ABC wird unterbrochen). Abbildungen möglicher Stapelfehler sowie daraus resultierender lokaler Gitterdefekte (Gitterfehlordnungen) sind in der Abbildung 8.3.3.2 im Anhang 8.3.3 dargestellt. Diese Art der Defekte sind als lokale Strukturänderungen innerhalb einer bzw. zwischen zwei benachbarten Schichten anzusehen, müssen jedoch für einen bestimmbaren Streubeitrag, d.h. die messtechnische Erfassung durch Beugung, eine Periodizität aufweisen.

Für die Existenz von hexagonalen Basisstrukturen spricht, wie von Kuhs et al. (1987) interpretiert, der zusätzlich zu vermutende Peak an der Stelle des hexagonalen [100]-Peaks. Diese müssten jedoch, um einen scharfen Streubeitrag in der Probe leisten zu können exakt in den ABC Lagen „eingerastet“ sein. Neben der Reihenfolge der Stapelung ist die Ausweitung der jeweiligen Stapelanordnungen von Bedeutung. Bei einer ständig wechselnden Stapelfolge sowie der Anzahl von regulär angeordneten Bereichen zu der Anzahl der Defekte würden sich diese Bereiche durch unterschiedliche Partikelgrößen und damit durch eine Beeinflussung des Reflexprofiles, im speziellen der Reflexbreite, bemerkbar machen. Für kleiner werdende 
Stapelbereiche führt dies zu einer Verbreiterung der Reflexprofile. Lediglich Polytypen, d.h. Überstrukturen, würden sich in Form von Satellitenreflexen bemerkbar machen. Für die D1BMessungen sind diese aufgrund der geringen Winkelauflösung nicht zu erfassen, während hingegen am D2B diese ausreichend sein sollte. Hinweise auf derartige Strukturen sind allerdings aus den gemessenen D2B-Diffraktogrammen nicht zu erkennen.

Geht man von der Existenz fehlgeordneter hexagonaler Anteile in der Probe aus, würde die Existenz von partiellen Verschiebungen, d.h. lateralen Deformationsfehlern (vergl. Anhang 8.3.3, Abbildung 8.3.3.2) zu einer Verbreiterung der hexagonalen Reflexe führen. Für den hexagonalen [100]-Peak ist dies durch die ausgeprägte Asymmetrie gegeben. Ebenso ließe sich unter dem kubischen [111]-Reflex und dessen Reflexschulter ein breiter hexagonaler [002]-Peak annehmen. An der rechten Flanke des kubischen [111]-Reflexes ist für einige Eisphasen, wie auch für Eis XII in Kapitel 3.3.1, Abbildung 3.7, eine Überhöhung angedeutet, die auf einen verschobenen hexagonalen [101]-Peak schließen lässt, der auf einen hexagonal fehlgeordneten Anteil hinweisen könnte. Jedoch müsste aufgrund der im Diffraktogramm fehlenden hexagonalen [102]-Reflexe und aller anderen hexagonalen Reflexe mit $1 \neq 0$ ein starker Abfall der Intensitäten durch eine sehr starke Unordnung, d.h. großen atomaren Verschiebungen der in den Ringen befindlichen Atomen und damit des Debye-Waller-Faktors vorliegen. Die Nichtexistenz eines hexagonalen [102]-Reflexes steht einem regulär angeordnetem hexagonalen Phasenanteil

\section{ABCABC.AB....AB.ABCABCABC}

entgegen. Geht man von hexagonalen Sequenzen der Art

$$
\text { ...AB...AB...AB...AB... }
$$

aus, d.h. Strukturen die in der basalen hexagonalen Ebene angeordnet sind (z.B. ebene ringförmige Strukturen), so ließe sich die Existenz des [100]-Reflexes erklären. Bei kleinen kohärenten Domänen in Stapelrichtung würde sich zudem eine extreme Verbreiterung aller Reflexe mit $1 \neq 0$ aufgrund eines Partikelgrößeneffektes erklären lassen. 
Laterale Verschiebungen der Schichtpakete in der basalen Ebene würden letztendlich zu einer asymmetrischen Aufweitung des Reflexprofiles des zusätzlichen hexagonalen [100]-Peaks führen. Wie in den Arbeiten von Kuhs et al. (1987) und in der Arbeit von Warren (1941) angeführt, haben Reflexverbreiterungen vielfältige Ursachen, die durch Partikelgrößeneffekte und Stapelfehlordnungen bedingt sind. Diese Anordnungen oder Strukturen sind bei begrenzter Geräteauflösung oft nicht eindeutig zu beschreiben. Für die Rietveld-Modellierung des Phasengemisches aus kubischen und hexagonalen Phasenanteilen wurde von einer kubischen Stapelung, die durch hexagonale Sequenzen der Art

\section{ABCABCAB.ABABABABABAB.ABCABCABC.......}

unterbrochen wird, ausgegangen. Dabei darf die Anzahl der hexagonalen Stapelungen in [001]- Richtung aufgrund der in den Diffraktogrammen nicht festzustellenden hexagonalen Reflexe mit $1 \neq 0$ und senkrecht dazu lediglich kleine Domänen, unter einigen hundert $\AA$, bilden. Diese wurden in den Rietveld-Verfeinerungen durch die Anpassung bzw. Verfeinerung der Peakprofil-Parameter und dem damit korrelierten Partikelgrößeneffekt sowie den ADP's der als hexagonal angenommenen Strukturen in dem Modell angepasst (vergl. Kapitel 2.2.1).

\subsubsection{Strukturelle Untersuchungen an kubischem Eis Ic}

Im Rahmen der Überprüfung der Präparationsrouten und der Pulvergüte wurden die Phasenübergänge der Hochdruckeisphasen II, V, XII und III bzw. der Tieftemperaturphase IX in kubisches Eis Ic in situ am D1B hinsichtlich der Kinetik der Phasenumwandlung verfolgt. In einem weiteren Experiment wurden am D2B die strukturellen Unterschiede bestimmt. Die Abbildung 3.7 zeigt die gemessenen D2B-Diffraktogramme des kubischen Eises Ic, hergestellt aus den Hochdruckeisphasen II, V, XII und III bzw. der Tieftemperaturphase IX sowie einer amorphen Hochdruckeisphase (HDA), in dem Bereich von $20^{\circ}$ bis $30^{\circ}$ in $2 \theta$. Die Eisphase XII wurde über die von Koza et al. (1999) angegebene Präparation am ILL ebenso hergestellt wie die Eisphase HDA. Die Eisphasen II, III und V wurden über die Hochdruckpräparationsroute Eis $\mathrm{V} \rightarrow$ Eis Ic $\rightarrow$ polykristallines Eis Ih $\rightarrow$ Phase $\mathrm{X}$ unter der Verwendung der im vorherigen Kapitel 3.2 beschriebenen Druck- und Temperaturbedingungen in Göttingen mit der pVT-Apparatur hergestellt. 


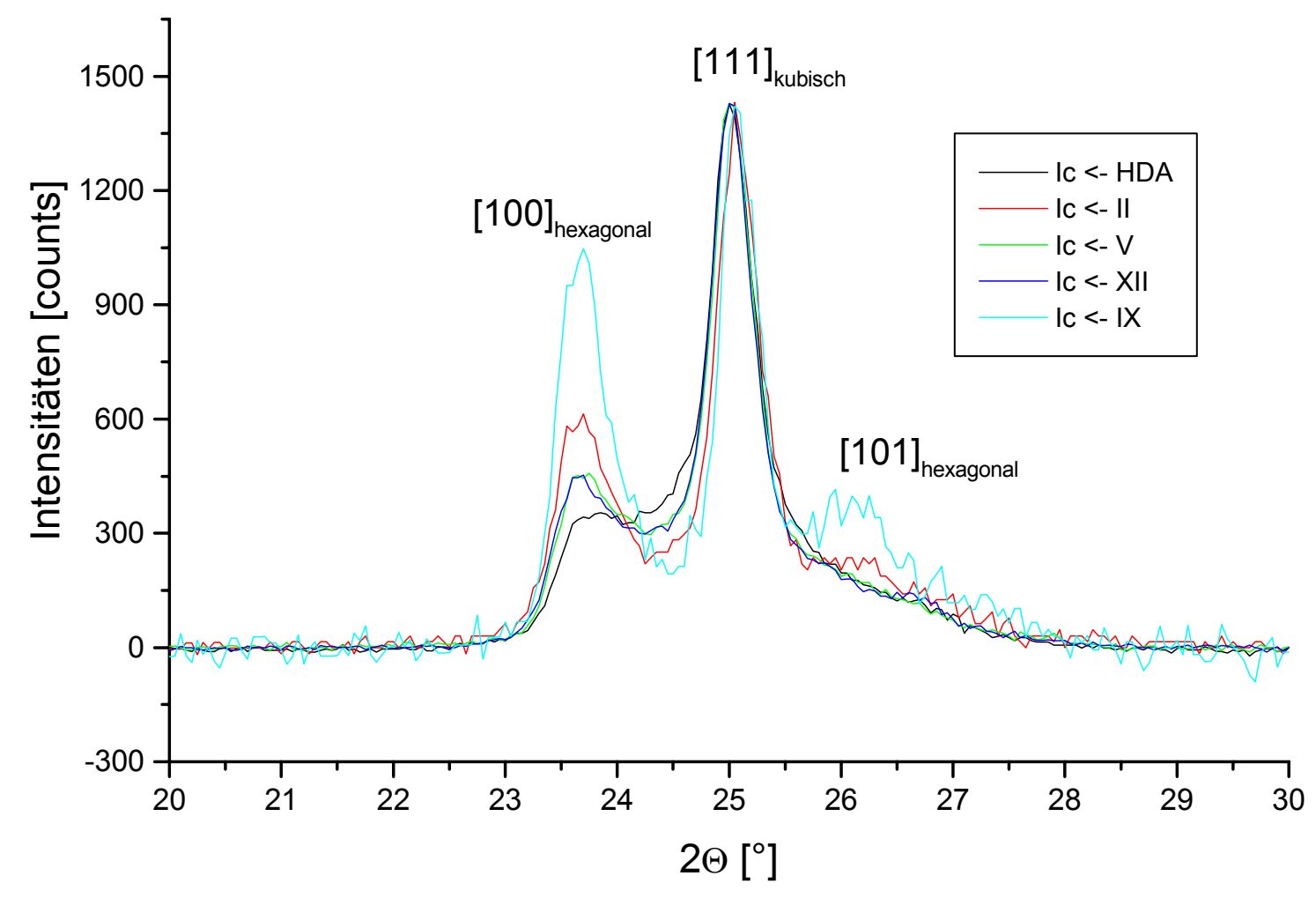

Abbildung 3.7: Vergleich der Diffraktogramme von kubischem Eis Ic, hergestellt aus verschiedenen Hochdruckeisphasen, gemessen am D2B des ILL bei T 180K.

Die Diffraktogramme von Eis II sowie Eis IX/III weisen aufgrund einer zu geringen Probenmasse eine deutlich erhöhte Schwankung der Intensitäten auf. Eine Gesamtabbildung der Diffraktogramme in dem gemessenen Winkelbereich in $2 \theta \sim 7.5^{\circ}$ bis $2 \theta \sim 160^{\circ}$ ist im Anhang 8.3.3.1, in der Abbildung 8.3.3.1.1 dargestellt. Die Intensitätsprofile in dem angegebenen Winkelbereich sind für die einzelnen Eisphasen in ihrem Verlauf sehr unterschiedlich. Abweichungen zeigen sich durch die verschiedenen Intensitätsverhältnisse des als hexagonal vermuteten [100]-Peaks in Relation zum kubischen [111]-Peak, ebenso wie Unterschiede in dem Verlauf der Intensitätsprofile. Diese sind je nach Ausgangsphase durch strukturelle Unterschiede bedingt.

Ausgehend von dem kristallographischen Modell von Eis Ic von Kuhs et al. (1987) wurde ein Rietveld-Modell für ein Zweiphasen-Gemisch aus einer kubischen Eisphase und einem „extrem durch Partikelgrößen sowie Stapelfehler gestörtem“ hexagonalen Eisanteil erstellt und verfeinert. 
Ziel dieser Verfeinerungen war es, das hexagonale Eis durch Stapelfehler, durch richtungsabhängige Partikelgrößen sowie Vorzugsorientierungen hinsichtlich der weiteren fehlenden hexagonalen Reflexintensitäten, des vorhandenen hexagonalen [100]-Reflexes sowie der Anpassung des Warrenanteiles zu modellieren, um erste Aussagen bezüglich des prozentualen Phasenanteiles einer als rein kubisch angenommenen Phase zu erhalten und einen qualitativen Vergleich hinsichtlich der Quantität von Stapelfehler-Anteilen sowie von Partikelgrößeneffekten des als hexagonal angenommenen gestörten Eisanteiles treffen zu können. Zuerst wurde für den kubischen Phasenanteil eine rein kubische Eisphase in die Rietveld-Verfeinerung eingebaut und hinsichtlich der Gitterkonstanten und der ADP's verfeinert. Im Zuge der Verfeinerungen wurde für den kubischen [111]-Reflex der gemessenen Eisproben eine Reflexverbreiterung zum simulierten polykristallinen kubischen Eis Ic (vergl. Kuhs et al. (1987), bedingt durch eine höhere instrumentelle Auflösung des D2B gegenüber dem D1A, festgestellt. Daher wurde in einem zweiten Verfeinerungszyklus die Partikelgröße der rein kubischen Eisphase in der bevorzugten [111]-Stapelrichtung isotrop verfeinert, gleichbedeutend mit einem isotropen topotaktischen Wachstum der kubischen Eis Ic-Körner. In einem weiteren Zyklus wurde der hexagonale Phasenanteil in die RietveldModelle eingebaut und hinsichtlich der Gitterkonstanten, des Phasenanteiles, der Partikelgröße als auch einer Stapelfehlordnung (Verfeinerung der Peakprofilparameter, vergl. Kapitel 2.2.1) in bevorzugter [002]-Stapelrichtung des gestörten hexagonalen Anteils in der [100]- / [010]-Kristallgitterebene verfeinert. Die Verfeinerung von Vorzugsorientierungen des hexagonalen Phasenanteiles führte in jedem Falle zu einer Divergenz der RietveldVerfeinerung. Die freie Verfeinerung der Reflexprofilparameter für die isotrope Partikelgröße des kubischen Phasenanteiles zeigte sich im Gegensatz zur Verfeinerung der Größe der Stapelfehlordnungsbereiche des gestörten hexagonalen Phasenanteiles als unkritisch. Für den reinen kubischen Phasenanteil zeigten sich die Verfeinerungen als stabil und konvergent. Im Gegensatz dazu führte eine Verfeinerung der atomaren Parameter (Atompositionen des Sauerstoffes, Deuteriums, sowie der ADP's) des als hexagonal angenommenen Phasenanteiles zu einer Divergenz der Rietveld-Modelle. Zumeist divergierten die Verfeinerungen bei dem Versuch die thermischen Auslenkungsparameter sowie die Reflexprofilparameter für die Partikelgrößen sowie den Stapelfehlordnungsparameter des als „hexagonal“ angenommenen Phasenanteiles zu verfeinern bzw. anzupassen. 
Die thermischen Auslenkungsparameter waren mit den Stapelfehlordnungs- sowie anisotropen Partikelgrößenparameter des hexagonalen Phasenanteiles hoch korreliert. Nach der manuellen Festlegung der thermischen Auslenkungsparameter des hexagonalen Phasenanteiles konnte zumeist der mit der Größe der Stapelfehlordnungen korrelierte Reflexprofilparameter sowie die Reflexprofilparameter für die Bestimmung der anisotropen hexagonalen Partikelgrößen frei verfeinert werden. Die Peakprofilparameter aus denen sowohl die Partikelgrößen als auch die Größe der Stapelfehlordnungen bestimmbar sind zeigten sich gegenüber den Temperaturfaktoren und damit der Anpassung des Reflexprofiles gegenüber als sehr empfindlich. Als Peakprofilfunktion in den Verfeinerungen wurden die Simpson-Rules-Profilfunktionen verwendet (Howard (1982)) (vergl. Kapitel 2.2.1).

Die als Basis für die Verfeinerungen nötigen Peakprofilparameter sowie der weiteren instrumentellen Parameter (z.B. Wellenlänge) wurden durch eine Rietveld-Verfeinerung eines vor den eigentlichen Strukturuntersuchungen des kubischen Eis Ic gemessenen Siliziumstandards festgelegt. Die Berechnung der richtungsabhängigen Partikelgrößen senkrecht (S) sowie parallel (P) zur Stapelrichtung sowie die Größe der in [002]-Richtung liegenden Stapelfehlerbereiche (SF) wurden aus den verfeinerten Peakprofilparametern der Profilfunktionen nach Cagliotti et al. (1958) berechnet (vergl. Kapitel 2.2.1). Neben den Rietveld-Verfeinerungen wurden die Diffraktogramme durch Einzelreflexfits angepasst um eine qualitative Bestimmung zumindest der Phasenanteile zu ermöglichen (vergl. Anhang 8.3.3.1.4). Sowohl die Rietveld-Modelle als auch die Verfeinerungsstrategie waren für alle Verfeinerungen äquivalent. Die Verfeinerungen wurden für die kubischen Eisphasen Ic (XII), Ic (V), Ic (II), Ic (IX/III) und Ic (HDA) mit manuell festgelegten thermischen Auslenkungsparametern des hexagonalen Phasenanteiles und der beschriebenen Verfeinerungsstrategie konsistent als ein stark partikelgrößen- sowie stapelfehlordnungsbeeinflusster hexagonaler Phasenanteil verfeinert. Es ergaben sich Rietveld-Anpassungen der gemessenen Diffraktogramme mit Rp-Werten von etwa 6-13\%, sowie wRp-Werten um die 8-13\%. Die größten Abweichungen ergaben sich erwartungsgemäß für alle Verfeinerungen in dem Winkelbereich von $20^{\circ}-30^{\circ}$ in $2 \theta$ für den als hexagonal angenommenen [100]-Reflex sowie des Intensitätsverlaufes des kubischen [111]Reflexes. 


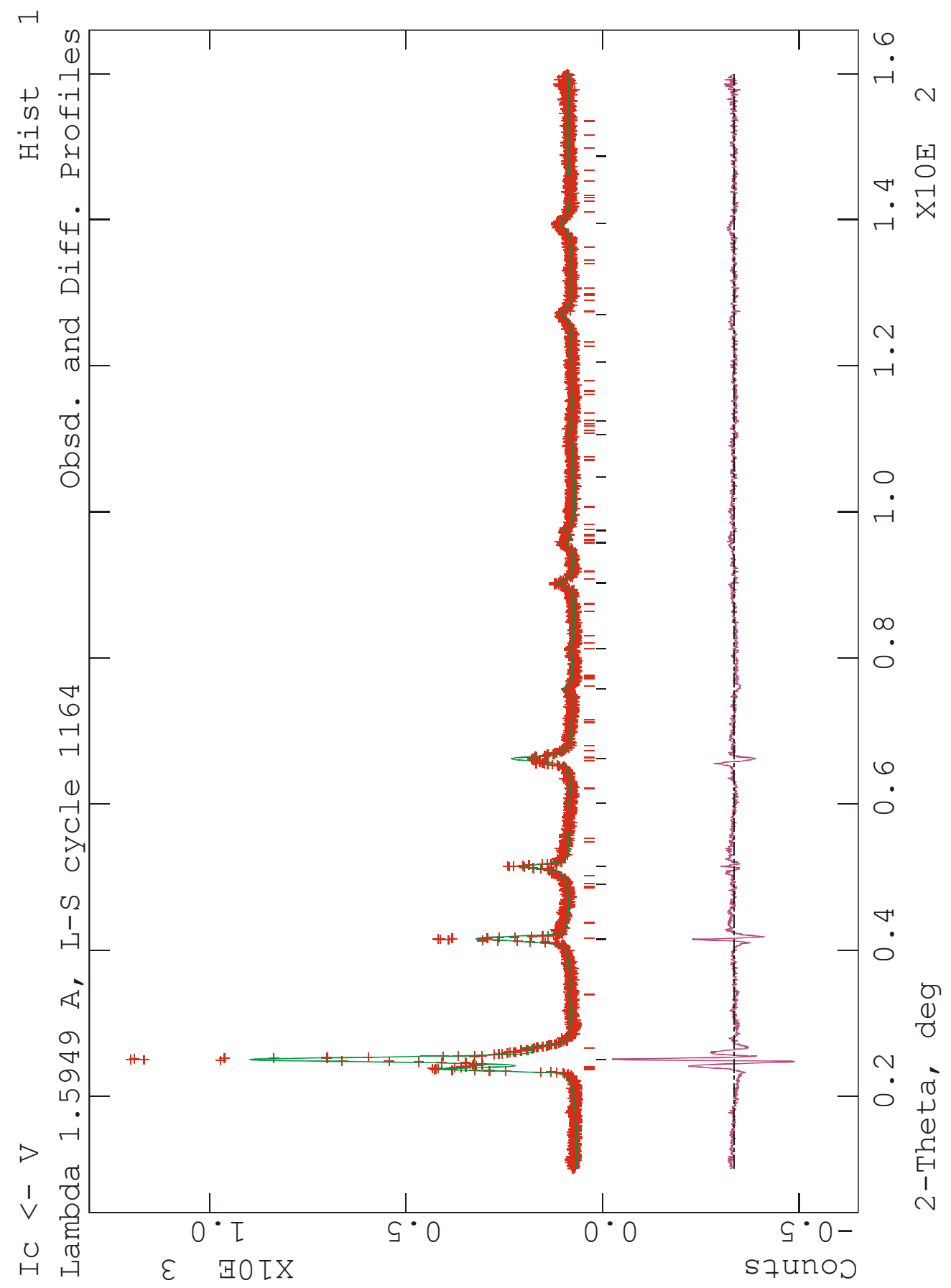

Abbildung 3.8: Diffraktogramm der Verfeinerung von Ic, hergestellt aus Eis $\mathbf{V}$ bei einer Temperatur von T 180K und bei Umgebungsdruck mit für den kubischen Phasenanteil frei verfeinerten sowie für den hexagonalen Phasenanteil angepassten thermischen Auslenkungsparametern. Signifikante Abweichungen der Reflexintensitäten sind für den kubischen [111]-Reflex sowie den kubischen [331]-Reflex zu verzeichnen. Während die zu niedrige Anpassung des kubischen [111]-Reflexes auf zu hoch gewählte thermische Auslenkungsparameter deutet, weist die zu hohe Anpassung des kubischen [331]-Reflexes auf zu klein gewählte thermische Auslenkungsparameter im Zuge dieser Verfeinerung hin. Die Intensitätsabweichungen dürften aber eher durch die nur ungenügende Modellierung der möglichen Arten von Stapelfehlern sowie den Häufigkeiten mit denen diese auftreten, begründet sein. Es ist daher davon auszugehen, dass die Abweichungen durch diese mäßige Modellierung der Struktur und damit durch eine ungenügende Anpassung der Phasenanteile, vornehmlich des hexagonalen Phasenanteiles, bedingt sind (vergl. Reflexlagen: I : Eis Ih, I : Eis Ic). 
Die vorherige Abbildung 3.8 zeigt die Anpassung des Rietveld-Modells an das gemessene Diffraktogramm von kubischem Eis Ic, hergestellt aus der Hochdruckeisphase V. Die mittels Rietveld-Verfahren angepassten Diffraktogramme des aus den weiteren Hochdruckeisphasen hergestellten kubischen Eis Ic sind im Anhang 8.3.3.1.2, Abbildung 8.3.3.1.2.a - d, dargestellt. In Tabelle 3.2 sind die Ergebnisse der Rietveld-Verfeinerungen der aus den verschiedenen Hochdruckeisphasen hergestellten kubischen Eisphasen in einem Vergleich dargestellt. Die wesentlichsten Abweichungen zeigten sich in der Anpassung des WarrenProfils, d.h. den Intensitäten und den Peakprofilen des [100]-, [111]- und des [101]-Reflexes sowie der Anpassung der Intensitäten der verbleibenden höheren kubischen sowie hexagonalen Reflexe durch die isotropen Temperaturfaktoren.

\begin{tabular}{|c|c|c|c|c|c|}
\hline \multicolumn{5}{|c|}{ Eis Ic aus Ausgangsphase } & \multirow{2}{*}{\begin{tabular}{|c|} 
Parameter \\
Gitterkonstante $[\AA]$
\end{tabular}} \\
\hline HDA & $\mathbf{V}$ & XII & III/IX & II & \\
\hline $6.3597(18)$ & $6.3594(15)$ & $6.3638(18)$ & $6.3528(21)$ & $6.3502(21)$ & a ( Ic) \\
\hline $4.4849(33)$ & $4.4886(18)$ & $4.4794(21)$ & $4.4916(18)$ & $4.4812(30)$ & a (Ih) \\
\hline $7.6289(135)$ & $7.6447(93)$ & $7.3395(33)$ & $7.6619(153)$ & $7.4674(692)$ & c (Ih) \\
\hline 1.7010 & 1.7031 & 1.6385 & 1.7058 & 1.6664 & c/a-Verhältnis \\
\hline & & & & & Phasenanteil [\%] \\
\hline $79(5)$ & $74(6)$ & $70(6)$ & $49(6)$ & $53(2)$ & Ic \\
\hline 76 & 73 & 74 & 47 & 51 & Ic* \\
\hline & & & & & Partikelgröße d $[\AA]]$ \\
\hline 144(7) & $162(8)$ & 121(5) & $261(25)$ & 189(18) & Ic ** \\
\hline $58(3)$ & $262(59)$ & $201(30)$ & 167(39) & $29(9)$ & (P) Ih \\
\hline $35(3)$ & $602(139)$ & $500(55)$ & $1906(898)$ & $283(51)$ & (S) Ih \\
\hline 0.6 & 2.30 & 2.48 & 11.7 & 9.75 & S/P-Verhältnis \\
\hline $95(64)$ & $65(16)$ & $420(80)$ & $65(12)$ & $47(11)$ & SF \\
\hline & & & & & Dichte $\rho\left[\mathrm{gcm}^{-1}\right]$ \\
\hline 1.034 & 1.034 & 1.043 & 1.038 & 1.039 & Ic \\
\hline 1.001 & 0.997 & 1.032 & 0.994 & 1.024 & Ih \\
\hline 8.01 & 6.93 & 6.19 & 8.09 & 8.71 & $\mathbf{R p}$ \\
\hline 10.72 & 9.26 & 8.51 & 12.01 & 11.08 & WRp \\
\hline- & $-915(4)$ & - & $-340(3)$ & $-54(5)$ & $\Delta \mathbf{H}\left[\mathrm{Jmol}^{-1}\right] \mathrm{HD} \rightarrow \mathbf{I c}$ \\
\hline
\end{tabular}

Tabelle 3.2: Vergleich der aus den Rietveld-Modellen verfeinerten Ergebnisse des zwei Phasengemisches, * : Die kubischen Phasenanteile wurden durch Einzelfit-Anpassungen bestimmt (vergl. Anhang 8.3.3.1.4, Tabelle 8.3.3.1.4.3) $* *$ : im kubischen System gibt es nur eine isotrope Partikelgröße, da nur eine ausgezeichnete Stapel-richtung, in [111]-Richtung existiert, - : Daten nicht verfügbar. Durch RietveldAnpassung bestimmte Peakprofilparameter zur Berechnung der Partikelgrößen vergl. Anhang 8.3.3.1.3. 
Ein Vergleich der aus den Rietveld-Modellen berechneten und als ungestört angenommenen kubischen Eisphasenanteils zeigt, dass diese im Rahmen des Fehlers eine recht gute Übereinstimmung zu den Ergebnissen der Einzel-Reflexanpassungen zeigen. Der rein kubisch geordnete Phasenanteil wurde, z.B. im Falle des Eis V zu 74(6)\% bestimmt, zeigte sich jedoch wie bei den anderen Rietveld-Modellen im Laufe der weiteren Verfeinerungen stabil gegenüber der Freigabe der Peakprofilparameter der Reflexprofile sowie der atomaren Parameter der jeweiligen Phasenanteile. Für HDA ergab die Verfeinerung einen Phasenanteil von 79(5)\% im Vergleich zu der Einzelfit-Auswertung von $76 \%$ und liegt somit im Rahmen des Fehlers der Rietveld-Verfeinerungen.

Für kubisches Eis Ic, hergestellt aus HDA, mit vermutlich nur kurzweitig lokal angeordneten kohärenten Domänen, ergab sich in den beiden unterschiedlichen Auswertungen der höchste Anteil an reinem ungestörtem kubischen Eis. Die aus den dichteren Eisphasen, Eis XII und Eis V, hergestellten kubischen Eisphasenanteile liegen im Rahmen ihres Fehlers in derselben Größenordnung als für die aus den weniger dichten Phasen, Eis III / IX und Eis II, hergestellten kubischen Eisphasen, die den geringsten rein kubischen Phasenanteil aufweisen.

Für die protonengeordneten Ausgangsphasen Eis IX/III sowie Eis II ergeben sich in den Einzelreflexanpassungen sowie aus den Verfeinerungen zu den anderen Eisphasen konsistent die geringsten Anteile an reinem kubischen Eis. Auffällig ist, dass für die aus den HDEisphasen HDA, V, XII hergestellten kubischen Eisphasen im Rahmen der Fehler nur

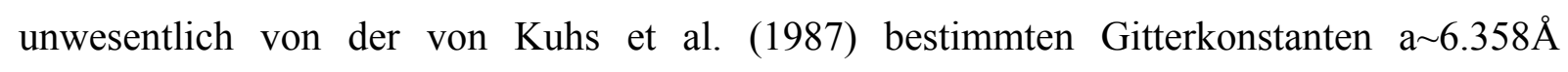
abweichen. Hingegen weisen die aus den Eisphasen III/IX sowie II hergestellte kubische Eisphase Ic eine Abweichung in der Gitterkonstante a auf. Für alle hexagonalen Phasenanteile ist eine deutliche Verschiebung des Gitterkonstantenverhältnisse c/a (vergl. Kapitel 5.5.1) zu höheren Werten zu verzeichnen. Dies könnte gleichbedeutend mit einer Aufweitung des gestörten Kristallgitters in c, d.h. der [001]-Stapelrichtung gegenüber der Gitterkonstanten a in der senkrechten [100]- / [010]-Kristallgitterebene sein. Für alle kubischen Eisphasen würde sich dies als Verzerrung des Gitters durch Gitterfehler, verursacht durch vorhandene interne Spannungen in den Kristalliten vermuten lassen. Für das Eis Ic hergestellt aus den HDEisphasen III/IX und II sowie in allen freien Verfeinerungen der jeweiligen kubischen Eisphase zeigen sich Abweichungen der simulierten Reflexlagen und der bestimmbaren Reflexlagen durch Anpassungen der Gitterkonstanten a für das kubische Eis Ic sowie des als 
hexagonal, fehlgeordneten vermuteten Phasenanteiles, der durch die Anpassung der hexagonalen Gitterkonstanten gegeben ist. Aufgrund der starken Überlappung der hexagonalen ([002]-Reflex) und der kubischen ([111]-Reflex) Reflexe bleibt die eindeutige Anpassung der Rietveld-Parameter an die gemessenen Reflexprofile durch die nur ungenau beschriebenen Strukturmodelle sowie der teilweise manuellen Anpassung der RietveldParameter, sowohl des kubischen als auch vornehmlich des als gestört angenommenen hexagonalen Phasenanteiles aber zweifelhaft. Es ist davon auszugehen, dass die Abweichungen in den Gitterkonstanten der für Ic (III/IX) und Ic(II) sowohl für die hexagonalen als auch für die kubischen Phasenanteile nicht signifikant durch eine Verzerrung der kubischen und der als hexagonal vermuteten kohärenten Domänen verursacht sind, bedingt durch interne Spannungen im Gefüge der Kristallite. Die Existenz von internen Spannungen, die zu strukturellen Verzerrungen innerhalb der Kristallite führen können, die zudem bei äquivalenter Besetzung der Einheitszelle und unter Berücksichtigung der unterschiedlichen Phasenanteile Abweichungen zu den Dichten von Eis Ic (König (1943)) sind jedoch nicht auszuschließen. Diese möglichen Verzerrungen des Gefüges dürften jedoch durch die größeren Varianzen in der Reflexprofil-Anpassung nur einen geringen Einfluss auf die Bestimmung der Gitterkonstanten haben, so dass davon auszugehen ist, dass die Abweichungen keine Signifikanzen in Bezug zu strukturellen Änderungen aufweisen. Die kubischen Partikelgrößen für Eis Ic (IX) und Eis Ic (II), d.h. den weniger dichten Ausgangsphasen sind im Rahmen der aus den Rietveld-Verfeinerungen ermittelten Fehler größer als für die dichteren Ausgangsphasen Eis Ic (XII) und Eis Ic (V) sowie für Eis Ic (HDA) aus dem quasikristallinem, nahgeordnetem Eis HDA. Für die aus den weniger dichten Eisphasen, dem Eis III/IX sowie Eis II hergestellten kubischen Eisphasen sind die hexagonalen Partikelgrößen parallel in der als bevorzugt angenommenen Stapelrichtung wesentlich kleiner als senkrecht zur hexagonalen Stapelrichtung. Dies ist aus den signifikant abweichenden S/P-Verhältnissen der hexagonalen Partikelgrößen senkrecht sowie parallel zur [002]-Stapelrichtung zu ersehen. Die Partikelgrößen senkrecht zur Stapelrichtung scheinen zwei Extrema zu ergeben. Während die (P)-Partikelgrößen für Eis Ic (XII), Eis Ic (V) und Eis Ic (IX) im Rahmen der Fehler in nahezu derselben Größenordnung liegen, weichen signifikant die für Eis Ic (HDA) sowie für Eis Ic (II) zu wesentlich kleineren Werten hin ab. Dies ist ebenso für die (S)-Partikelgrößen, d.h. senkrecht zur hexagonalen Stapelrichtung gegeben. Jedoch sind eindeutige Korrelationen von kubischen und hexagonalen Partikelgrößen der 
angenommenen hexagonalen Strukturen, mit dem Phasenanteil von rein kubischem Eis Ic für die aus verschiedenen HD-Eisphasen hergestellten Proben nicht festzustellen.

Unter der Annahme der Existenz von hexagonal basalen Strukturen oder von hexagonalen Sequenzen der Art .....ABCABCAB.ABABABABAB.ABCABCABC..... , die zwischen bzw. in den kubischen Stapelschichten liegen, könnten die Abweichung in den Gitterkonstanten und der Änderungen in den Reflexlagen sowie der Reflexasymmetrie ein Indiz für interne Spannungen der Kristallite, aber auch ein $\mathrm{Maß}$ für die lateralen Fehlordnungen sein. Neben dem aus den Reflexprofilparametern zu verfeinernde Stapelfehlordnungsgrößen sind die Partikelgrößen, ein Maß für die Größe der kohärenten und ungestörten Domänen. Mit dem Phasenanteil korreliert ist dies ein Maß für die Anzahl und die Häufigkeit mit der die Stapelfehlordnungen in der kubischen Matrix auftreten. Abgesehen von dem aus dem kubischen Eis XII hergestellten kubischen Eis Ic, weichen die Größen der Stapelfehlordnungsbereiche der aus den unterschiedlichen Hochdruckeisphasen hergestellten kubischen Eisphasen im Rahmen der Fehler nicht signifikant voneinander ab. Für Eis Ic (HDA), Eis Ic (II) sowie Eis Ic (III/IX) ergibt sich aufgrund der zur Stapelrichtung kleineren Partikelgröße eine „schnellere“ Abfolge von kubisch gestapelten sowie fehlgestapelten Schichtfolgen kohärenter Domänen. Hingegen wechselt für Eis Ic(XII) und Eis Ic(V) die Abfolge in Stapelrichtung nicht so häufig. Die Partikelgrößen senkrecht zur Stapelrichtung weisen deutliche Varianzen auf. Während hingegen für Eis Ic(HDA) wiederum eine kleine Partikelgröße gegeben ist, sind diese für Eis Ic(V) sowie Eis Ic(XII) im Rahmen der ermittelten Fehler gleich. Deutlich im Rahmen des Fehlers abweichend ist die Partikelgröße von Eis Ic(III/IX). Das Verhältnis der Partikelgrößen S/P zeigt jedoch, dass diese für Eis Ic(IX/III) gefolgt von Eis Ic(II) am größten sind und mehr auf eine Schichtstruktur hindeuten. Das Verhältnis der Partikelgrößen S/P von Eis Ic(V) sowie Eis (XII) sind mit einem Faktor von $\sim 2.5$ sowie für Eis Ic(HDA) mit einem Faktor deutlich $\leq 1$ signifikant abweichend (vergl. Tabelle 3.2), d.h. auf ein mehr isotropes, räumlich begrenztes Wachstum von hexagonalen Bereichen hindeutend. In jedem Falle ergibt sich, dass für die aus den jeweiligen Ausgangsphasen hergestellten kubischen Eisphasen unterschiedliche Wechselfolgen ungestörter kubischer und gestörter hexagonaler Schichten gegeben sind. Die aus den angepassten Reflexprofilen berechneten isotropen kubischen, anisotropen hexagonalen Partikelgrößen sowie die Größe der Stapelfehlordnungsbereiche deuten auf unterschiedliche Wachstumsprozesse beim Phasenübergang der gequenchten HD-Eisphasen in kubisches Eis 
Ic hin. Die unterschiedlichen Partikelgrößen in und senkrecht zur Stapelrichtung können dahin interpretiert werden, dass das topotaktische Wachstum der kubischen Kristallite in der Ausgangsphase in ungestörte und ungeordnete kohärente Bereiche während der Phasenumwandlung je nach Ausgangsphase, räumlich begrenzt ist. Diese kubisch kohärenten Bereiche sind umgeben von hexagonal stapelfehlgeordnetem Eis Ih, dass zudem je nach Ausgangsphase unterschiedlich topotaktisch gewachsen ist. Geht man von der Interpretation von Kuhs et al. (1987) aus, so sind die Stapelfehlordnungen für kubisches Eis Ic, hergestellt aus protonengeordnetem Eis II sowie für Eis IX, der protonengeordneten Variante des Eis III, sehr viel häufiger vorhanden als für Eis Ic hergestellt aus den protonenungeordneten und dichteren Eisphasen XII,V sowie der nur kurzreichweitig geordneten Eisphase HDA.

Eine Einteilung in die Kategorien aus protonengeordnetem/partiell protonengeordnetem Eis II, IX/III sowie aus den dichteren Eisphasen dem protonenungeordnetem Eis XII sowie dem partiell protonengeordnetem Eis V lässt sich über die Partikelgrößenverhältnisse aber auch ebenso über die Verhältnisse der Partikelgrößen, der bestimmten Phasenanteile sowie der kubischen Gitterkonstanten treffen. Erfahrungen aus mehreren, auch früheren Experimenten belegen, dass kubisches Eis Ic, hergestellt aus der HD-Eisphase V, in Übereinstimmung zu den Experimenten von Arnold (1968) als auch Kuhs (pers. Mitteilung, 2001) nahezu dieselben Diffraktogramme und damit vergleichbare Strukturen aufweisen. Für Ausgangsphasen, die im Gegensatz zu Eis V starke Vorzugsorientierungen aufweisen können, z.B. Eis II oder Eis III, ergeben sich nach der Phasenumwandlung deutlich verschiedene Streubilder, je nach Güte der Ausgangsphase. Mehr als nur zu vermuten ist daher, dass die Streubilder des kubischen Eis Ic maßgeblich durch die Struktur der Ausgangsphase hinsichtlich vorhandener Vorzugsorientierungen bestimmt.

Eine weitergehende Interpretation ist aufgrund einer vorhandenen Korrelation von den aus den Verfeinerungen bestimmten Partikelgrößen zu den weiteren bestimmten Parametern aufgrund der mäßigen Anpassung der Intensitätsprofile durch die in GSAS vorhandenen Modelle für Partikelgrößeneffekte, Stapelfehlordnungen sowie die Möglichkeit existierender interner Spannungen als auch die eventuelle Existenz von Vorzugsorientierungen für die hexagonale Phase stark eingeschränkt. 


\subsubsection{Kinetik der Phasenübergänge in kubisches Eis Ic}

Neben den strukturellen Unterschieden der aus den verschiedenen Hochdruckeisphasen XII, V, IX/III und II hergestellten kubischen Eis Ic zeigten sich zudem Unterschiede in dem Phasenumwandlungsverhalten. Diese Phasenübergange wurden in situ am D1B des ILL sowie am BW5 des HASYLAB verfolgt.

Verwendung zur Bestimmung bzw. Untersuchung der Umwandlungskinetik fanden jedoch nur Eispulver der jeweiligen Eisphasen, die durch einen Vergleich der Reflexintensitäten nur eine geringe Ausprägung von Vorzugsorientierungen und damit eine gute Pulverstatistik mit Abweichungen $\leq 3 \sigma$ in den Reflexintensitäten aufwiesen. Für Eis XII sowie für das Eis V ergab sich durch den Vergleich der Intensitäten eine zumeist gute Pulverstatistik, während hingegen für die Eisphasen IX/III und Eis II diese in meisten Fällen starke Vorzugsorientierungen aufwiesen und die Pulvergüten somit für Messungen mit einem eindimensionalem Detektor nicht hinreichend waren.

In einer Vielzahl von Herstellungsversuchen ergab sich für Eis II lediglich eine Probe mit einer qualitativ hinreichenden Probengüte. Aufgrund einer $\mathrm{zu}$ hoch gewählten Umwandlungstemperatur von T 143K vollzog sich der Phasenübergang der „recoverten” Eis II Probe in kubisches Eis innerhalb von $\mathrm{t} \sim 35$ Minuten und machte daher eine sinnvolle Bestimmung der Kinetik unmöglich. Bei den Phasenübergangen von „recovertem” Eis IX/III in das kubische Eis Ic zeigte sich in den meisten Fällen der Effekt einer Stagnation, sogar eines völligen Stillstandes der Phasenumwandlung. In einem von fünf Fällen konnte lediglich eine nahezu vollständige Umwandlung von „recovertem“ Eis IX/III in kubisches Eis Ic beobachtet werden. Diese Phasenumwandlung zeigte jedoch eher einen unregelmäßigen Verlauf bei der Umwandlung, die sich durch einen nicht sehr kontinuierlichen Verlauf der Reflexintensitäten bemerkbar machte.

Zwei weitere Proben wurden für ein kinetisches Experiment am D20 des ILL in Grenoble, das erst zu einem späteren Zeitpunkt zur Verfügung stand, präpariert und hinsichtlich der Kinetik der Phasenumwandlung von Eis IX in kubisches Eis Ic verfolgt. Die Ergebnisse des D20Experimentes zeigen auch zum Ende der Phasenumwandlung eine Stagnation an (Kuhs, persönliche Mitteilung (2001)). 
Die Phasenübergange von Eis II und IX/III in Eis Ic wurden bei weiteren Messungen am BW5 des HASYLAB an einer weiteren Eis II-Probe sowie an drei weiteren Eis IX/III-Proben durchgeführt. Aufgrund technischer Probleme (thermische Instabilitäten des Kryostaten, „Beam losses“) konnten die Umwandlungen von Eis II in kubisches Eis Ic sowie die Umwandlungen von Eis IX/III jedoch nicht komplett verfolgt werden.

In den Auswertungen wurden die Phasenanteile für kubisches Eis Ic aufgrund des sowohl nur ungenau bekannten kubischen als auch dem völlig unbekannten, stapelfehlgeordnetem hexagonalen Eisstrukturmodell über die Bestimmung der Intensitätsprofile festgelegt. Als problematisch stellte sich die Bestimmung der integrierten Reflexintensitäten der kubischen Eisphase aufgrund der schlechteren Meßstatistik am D1B heraus. Die zeitlichen Intensitätsverläufe der Ausgangsphasen ließen sich hingegen zu denen des kubischen Eises durch eine einfachere Einzelpeakfitanpassung genauer bestimmen. Zur Bestimmung der Kinetik wurden daher nur die zeitlichen Intensitätsverläufe von nicht mit kubischen Reflexen überlappenden Reflexintensitäten der sich umwandelnden Ausgangsphasen betrachtet. (vergl. Anhang 8.3.3.2, Abbildung 8.3.3.2.a bis Abbildung 8.3.3.2.d).

Um eine Vergleichbarkeit der Reflexintensitäten der jeweiligen Eisphasenanteile zu gewährleisten wurden diese nach der Festlegung und Subtraktion des Untergrundes durch die Anpassung der integrierten Reflexintensitäten für die Ausgangsphasen durch Reflexprofilanpassungen, d.h. durch Fitten des komplexen Intensitätsprofils mittels einer Überlagerung von Gauß- sowie Lorentzpeakprofilen, bestimmt (vergl. Anhang 8.3.3.1.4). Die Intensitätsverläufe wurden jeweils für die Ausgangsphase auf die Reflexintensitäten des Ausgangszustandes bzw. für die kubische Eisphase auf die Intensitäten des Endzustandes normiert. Die Abbildung 3.9.1 zeigt die aus den gemessenen Diffraktogrammen bestimmten zeitlichen Verläufe der Phasenumwandlungen der Eisphasen XII und V in das kubische Eis Ic. In den Abbildungen 3.9.2 und Abbildung 3.9.3 ist das zeitliche Verhalten für ausgezeichnete Peaks bei den Phasenumwandlungen dargestellt. Aus dem zeitlichen Verlauf der normierten Integralintensitäten von Eis XII und Eis V zeigt sich bei einer vergleichbaren Umwandlungstemperatur von $\mathrm{T} \sim 143 \mathrm{~K}$ ein Unterschied in den Umwandlungszeiten und damit in den Zeitkonstanten. Geht man von den Intensitätsverläufen der ursprünglichen Phasen aus, so ist bei Eis XII etwa $50 \%$ der Probe nach $\mathrm{t} \sim 55$ Minuten umgewandelt worden. Die 
Umwandlung von Eis XII in kubisches Eis begann nach der Equilibrierung, d.h. dem Erreichen einer konstanten Temperatur von T 143K nach t 200 Minuten.

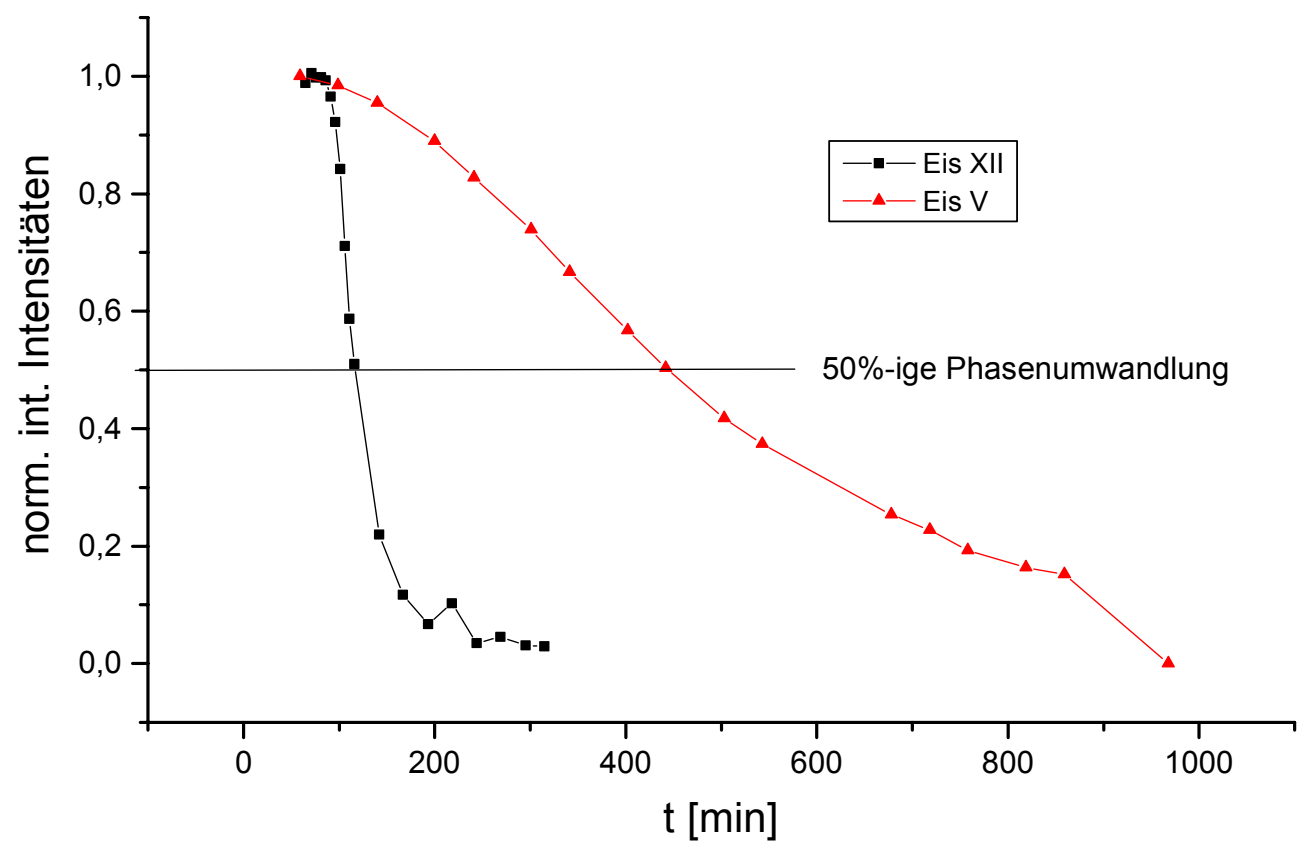

\begin{abstract}
Abbildung 3.9.1: Zeitlicher Intensitätsverlauf der Phasenumwandlung der Hochdruckeisphasen Eis XII und Eis V in kubisches Eis Ic, gemessen am D1B des ILL, Umwandlungstemperatur T 143K; Eis XII, Eis V : zeitlicher Verlauf des arithmetischen Mittels dreier integrierter Reflexprofile, d.h. unterschiedlicher kristallographischer Richtungen. Der jeweilige Phasenumwandlungverlauf einzelner Reflexe ist in den folgenden Abbildung 3.9.2 sowie Abbildung 3.9.3 dargestellt.
\end{abstract}

Für Eis V setzte die Umwandlung ca. $\mathrm{t} \sim 20$ Minuten nach der Equlibrierung bei $\mathrm{T} \sim 143 \mathrm{~K}$ ein. Nach $\mathrm{t} \sim 6$ Stunden wurden 50\% der Ausgangsphase in kubisches Eis umgewandelt. Die 100\%ige Phasenumwandlung der ,recoverten” Hochdruckeisphasen in kubisches Eis Ic erfolgte für Eis XII nach $t \sim 4$ Stunden und für Eis V nach etwa $t \sim 16$ Stunden. Für beide Eisphasen ist ein zumeist kontinuierlicher Verlauf der Intensitäten für die Ausgangsphase XII und V als Funktion der Zeit abzulesen. Für Eis XII ist für die Umwandlung bei etwa 10\% Ausgangsphase sowie für Eis V in der Endphase der Umwandlung ein unruhiger Verlauf zu erkennen. Die Intensitäten im Falle von Eis XII zeigen am Ende der Phasenumwandlung eine außerhalb der Messstatistik liegende Überhöhung des Phasenanteils von ca. $8 \%$ auf 10\% innerhalb von $t \sim 15$ Minuten an, die jedoch im weiteren Verlauf der Phasenumwandlung wieder verschwindet. 


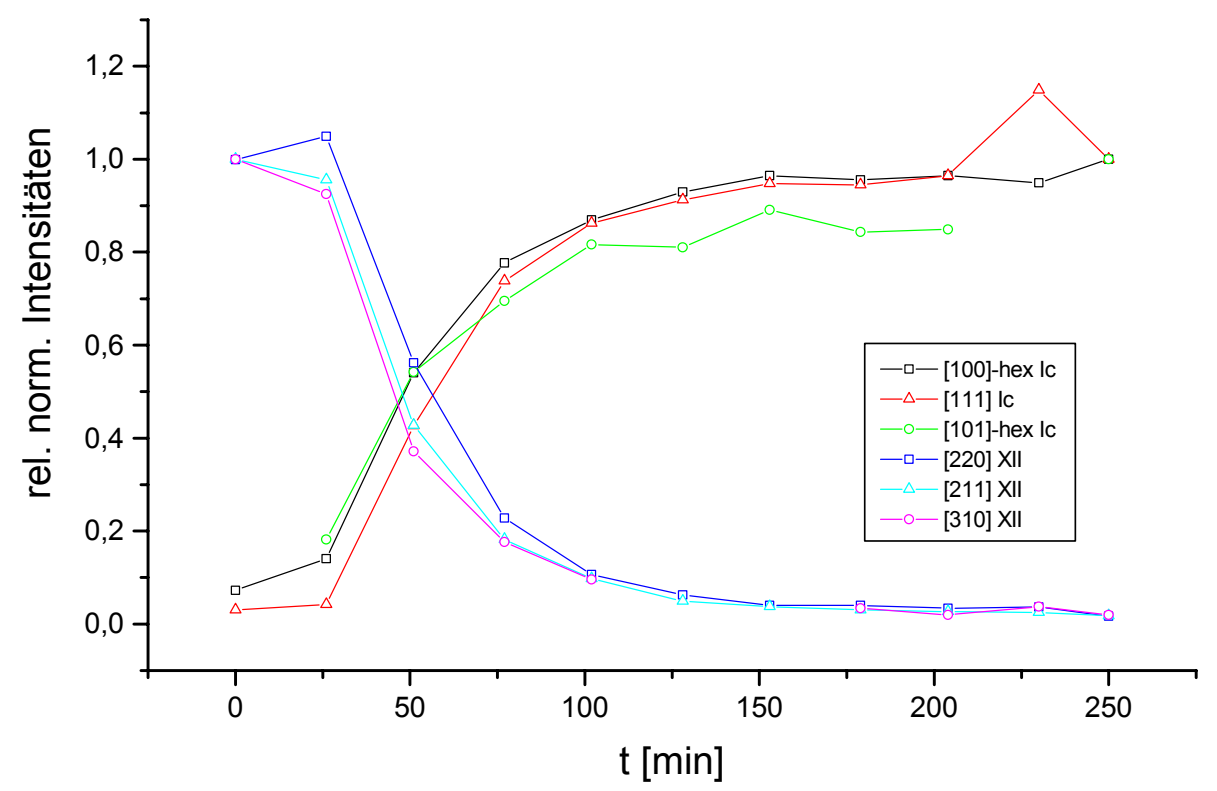

Abbildung 3.9.2: zeitlicher Verlauf der Phasenumwandlung von Eis XII $\rightarrow$ Eis Ic. Der Abbau der integrierten Reflexintensitäten des [220]-, des [211]- sowie des [310]-Eis XII Reflexes vollzieht sich nahezu äquivalent und deutet auf eine isotrope Umwandlung hin. [101]-hex Ic entspricht dem Streubeitrag des Warrenanteils des [111]-kubischen Reflexes. Diffraktogramme der Phasenumwandlung (vergl. Anhang 8.3.3.2, Abbildung 8.3.3.2.b).

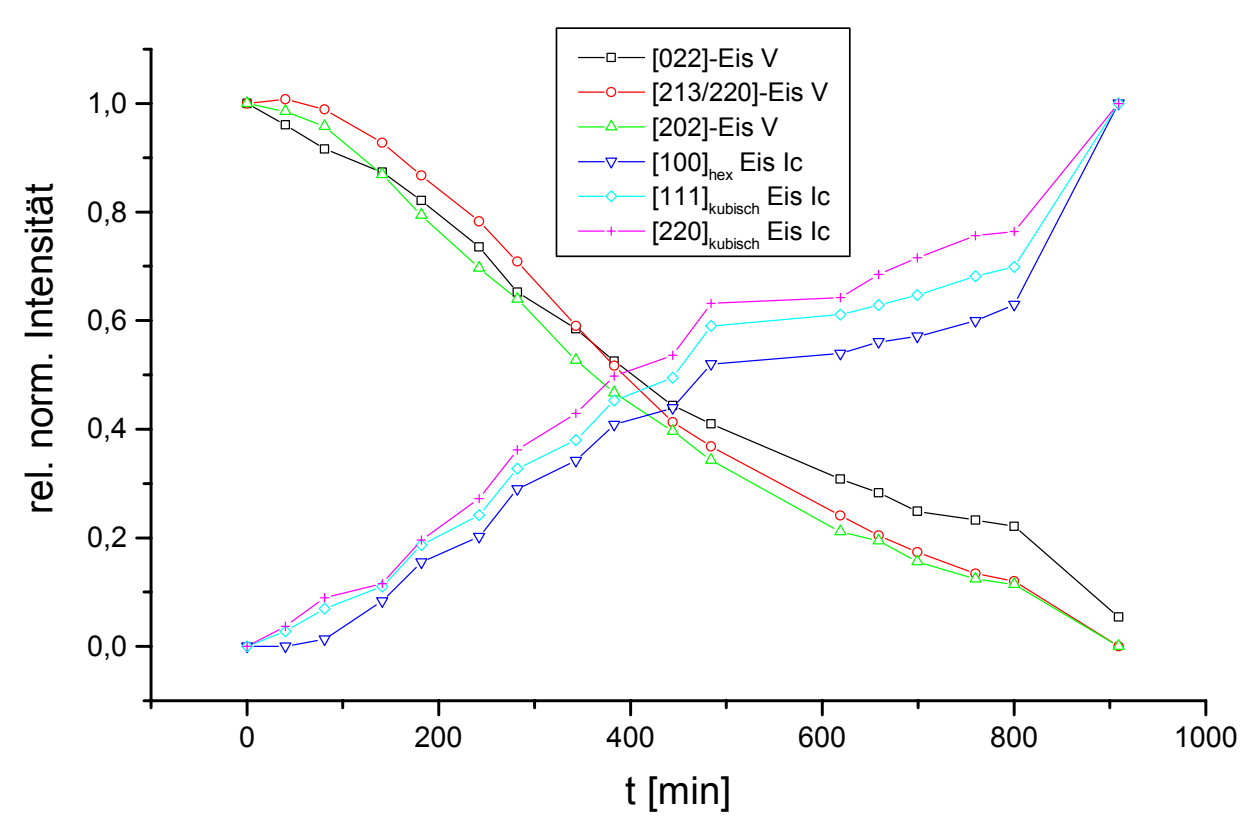

Abbildung 3.9.3: zeitlicher Verlauf der Phasenumwandlung von Eis $\mathrm{V} \rightarrow$ kubisches Eis Ic. Der Abbau der integrierten Reflexintensitäten des [022]-, des [213/220]- sowie des [202]-Eis V Reflexes vollzieht sich nahezu äquivalent und deutet ebenso auf eine kontinuierliche nahezu isotrope Umwandlung hin, während hingegen beim Wachstum des kubischen Eis Ic Unterschiede deutlich werden. Diffraktogramme der Phasenumwandlung (vergl. Anhang 8.3.3.2, Abbildung 8.3.3.2.a). 
Für Eis V fällt am Ende der Phasenumwandlung die Intensität gegenüber der gesamten Umwandlungszeit innerhalb des Zeitraumes von $\mathrm{t} \sim 1 \mathrm{~h}$ von $\sim 17 \%$ auf nahezu $\sim 0 \%$ ab.

Der unruhige Verlauf könnte auf ein Problem hindeuten, dass durch eine inhomogene Korngrößenverteilung bedingt ist. Größere Körner könnten sich für das Eis XII in Reflexionsstellung, für das Eis $\mathrm{V}$ aus der Reflexionsstellung drehen und damit zu einer schnellen und sprunghaften Änderung der Intensitäten führen. Diese größeren Körner können durch Rekristallisationen der Ausgangsphase vor und während der Phasenumwandlung gebildet werden. Eine andere Interpretation ist, dass vorwiegend kleinere Kristallite in einer homogenen Korngrößenverteilung aufgrund der höheren Oberflächenenergien bevorzugt und schneller in der neuen Struktur rekristallisieren. Die größeren Kristallite der Ausgangsphase hingegen könnten sich gegenüber einer Rekristallisation stabiler zeigen. Beispiele für solche Rekristallisationseffekte, die während einer Phasenumwandlung stattfinden und die Kinetik durch Korngrößeneffekte beeinflussen, wurden von Vandermeer und Gordon (1962) in durchgeführten Rekristallisationsexperimenten an Aluminium-Kupferproben beschrieben. Zudem ist nicht auszuschließen, dass an Eis und eisverwandten Strukturen im Gegensatz zu Aluminium-Kupferproben weitere Effekte, z.B. sowohl durch Diffusion als auch Sublimation sowie Kondensation aufgrund des hohen Dampfdruckes dieser Strukturen, eine Rolle hinsichtlich der Bildung von Nukleationskeimen sowie des strukturellen Umbaus spielen dürften und auch hier wiederum die Sublimation von kleinen Körnern in der Probe bevorzugt stattfinden würde.

Der zeitliche Verlauf der Phasenumwandlung wurde für die Ausgangsphasen Eis XII und Eis V aus den Intensitätsverläufen der nicht mit kubischen Reflexen überlappenden [220]-, [211]und [310]-Reflexe für Eis XII (vergl. Anhang 8.3.3.2, Abbildung 8.3.3.2.b) sowie der [022]-, [213/220]- und des [202]-Reflexe des Eis V (vergl. Anhang 8.3.3.2, Abbildung 8.3.3.2.a) bestimmt. Aus den Abbildungen 3.9.2 sowie Abbildung 3.9.3 ist zu ersehen, dass die zeitlichen Verläufe der gewählten Intensitäten für die Eisphase V sowie für das Eis XII annähernd äquivalent verläuft. Die Umwandlung von Eis IX/III in kubisches Eis Ic zeigt sich hingegen durch den zeitlichen Verlauf der gewählten Reflexe nicht kontinuierlich (vergl. Abbildung 3.10.1 sowie Abbildung 3.10.2). 


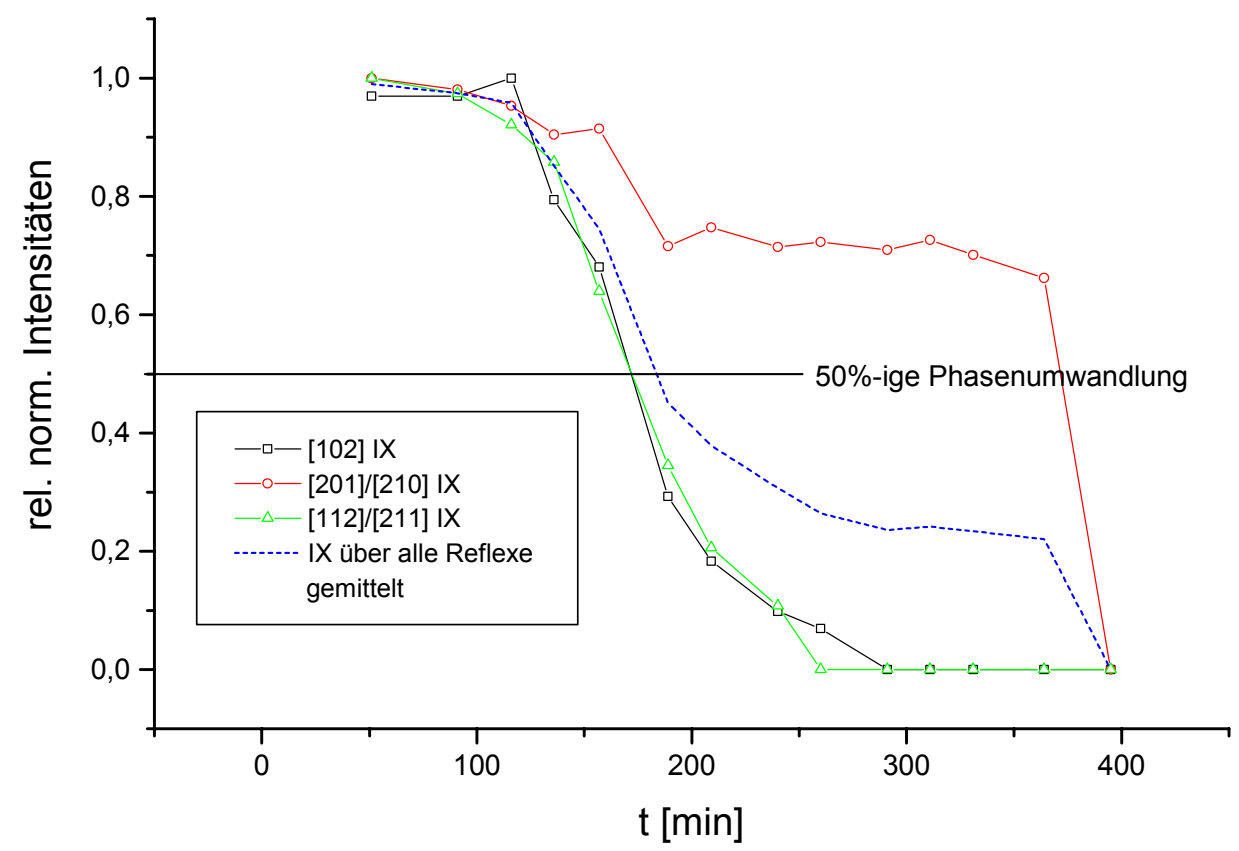

Abbildung 3.10.1: Zeitlicher Verlauf der Phasenumwandlung der „recoverten“ HD-Phase Eis IX/III $\rightarrow$ kubisches Eis Ic mit Einzelpeak-Kinetik und arithmetischem Mittel dreier integrierter Reflexprofile. Deutlich ist der anisotrope Verlauf der integrierten Reflexprofile als Funktion der Zeit. Diffraktogramme der Phasenumwandlung (vergl. Anhang 8.3.3.2, Abbildung 8.3.3.2.c)

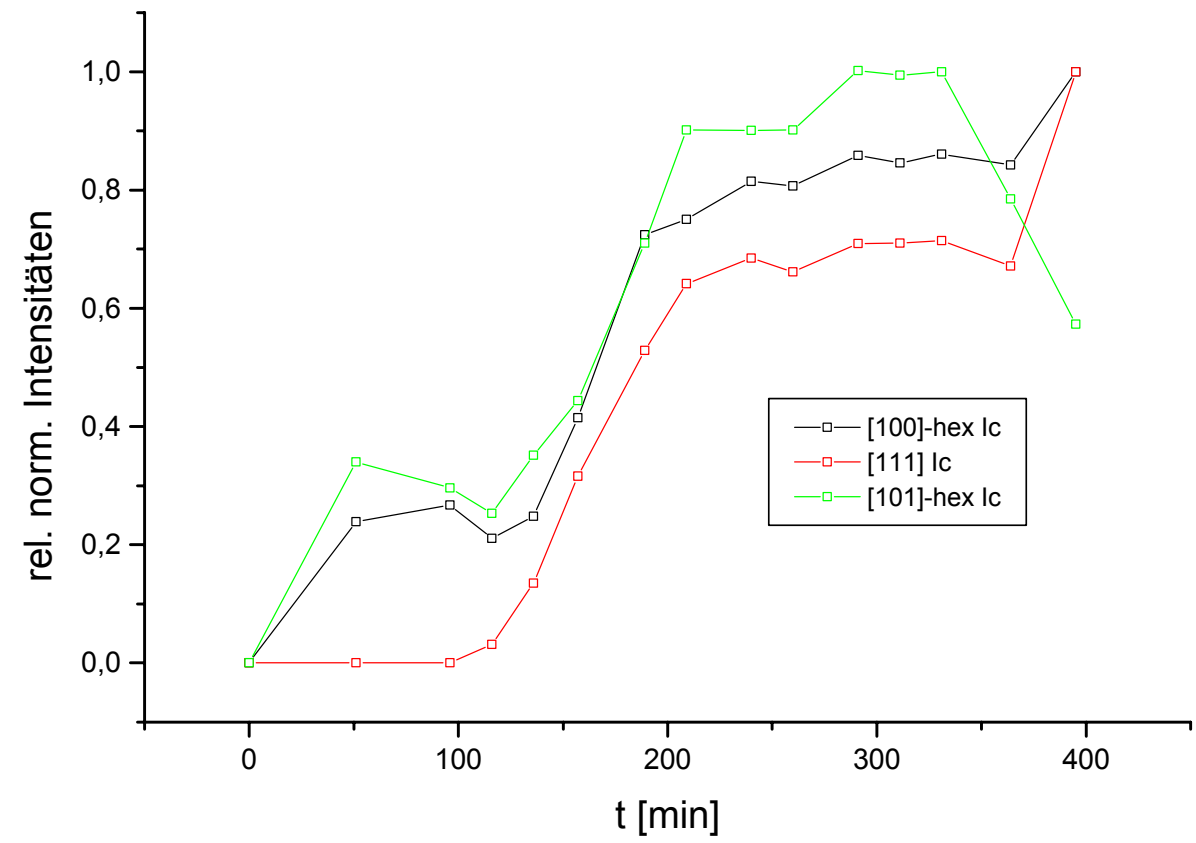

Abbildung 3.10.2: Zeitlicher Verlauf der Phasenumwandlung der „recoverten“ HD-Phase Eis IX/III mit Einzelpeak-Kinetik der kubischen Reflexe. [101]-hex Ic entspricht dem Warrenanteil des [111]-kubischen Reflexes. Zu erkennen ist, dass zuerst hexagonale bzw. gestörte hexagonale Streubeiträge im Diffraktogramm gebildet werden. Diffraktogramme der Phasenumwandlung (vergl. Anhang 8.3.3.2, Abbildung 8.3.3.2.c). 
Die Abbildung 3.10.1 zeigt das Phasenumwandlungsverhalten der [102]-, [201/210]- und [112/211]-Reflexe der Eis IX/III- Probe als Funktion der Zeit. Die Verläufe der Peakintensitäten der Ausgangsphase verlaufen für den [102] sowie den [112/211]-Reflex annähernd gleich. Für diese beiden Reflexe ist ein nahezu 50\%-iger Umbau in kubisches Eis Ic nach $t \sim 2.9$ Stunden erfolgt. Lediglich der Verlauf des [201/210]-Reflexes zeigt ein abweichendes Verhalten. Nach etwa $\mathrm{t} \sim 6$ Stunden scheint der Phasenanteil der Ausgangsphase Eis IX/III auf 66\% abgefallen zu sein. Die Reflexintensitäten der beiden anderen Eis IX/IIIReflexe sind nach einer Zeit von $\mathrm{t} \sim 4.8$ Stunden auf Null abgesunken. Interssanterweise findet man, dass die Peaks der hexagonalen Anteile von kubischem Eis Ic, des hexagonalen [100]und des hexagonalen [101]-Peaks, zuerst ansteigende Streubeiträge aufweisen. Der dem rein kubischen Eis Ic zugehörige [111]-Peak bzw. der bei einem möglichen hexagonalen Anteil überlappende hexagonale [002]-Reflex zeigt bis zu $t \sim 1.5$ Stunden nach Beginn der Phasenumwandlung keinen Intensitätszuwachs. Erst nach ca. $t \sim 1.5$ Stunden steigt die Intensität an. Die Intensitäten der hexagonalen Anteile des kubischen Eises Ic sind während und bis zu diesem Zeitpunkt bis auf etwa 34\% für den [100]-Reflex und etwa 27\% für den [101]-Reflex angewachsen (vergl. Abbildung 3.10.2). Mit dem Intensitätsanstieg des [111]Reflexes des kubischen Eises Ic stagniert die Umwandlung der Eisphase IX in die hexagonalen Anteile. Nach ca. $\mathrm{t} \sim 2$ Stunden entwickeln sich die Intensitäten des kubischen Eis Ic-Reflexes sowie die der hexagonalen Anteile nahezu identisch. Die Wachstumsgeschwindigkeit des [111]-Reflexes bleibt jedoch leicht hinter dem der hexagonalen Anteile zurück, so dass bei einer 50\%-igen Umwandlung der Ausgangsphase Eis IX/III nach den Intensitäten des [102]- sowie des [112/211]-Reflexes für das Eis Ic nach dem [111]-Reflex ein Phasenanteil von 44\% vorliegt. Der Phasenanteil der als hexagonalen Anteile bezeichneten Streubeiträge beträgt im Gegensatz dazu etwa $60 \%$.

In dem Zeitraum von $\mathrm{t} \sim 3.3$ Stunden bis etwa $\mathrm{t} \sim 6.6$ Stunden nach Beginn der Phasenumwandlung sind die Intensitätsverläufe der hexagonalen Anteile und der kubischen Anteile annähernd gleich (vergl. Abbildung 3.10.2). Die wesentlich geringere Steigung der Intensitätsverläufe der Peaks für Eis IX/III sowie des kubischen Eises und der damit korrelierten Phasenanteile deutet auf einen sich verlangsamenden Umbau hin.

Nach sechs Stunden zeigt sich eine sprunghafte Änderung des [201/210]-Reflexes des Eis IX sowie der Intensität des [101]-Reflexes des hexagonalen Anteils der kubischen Phase an. Ein 
äquivalentes Verhalten ist für den [111]-Reflex der kubischen Eisphase als auch für den [100]-Reflex des hexagonalen Anteils zu erkennen.

Die Intensitätsverläufe der gewählten und nicht mit den kubischen Reflexen überlappenden Eis IX/III-Reflexe lassen während der Phasenumwandlung auf eine evtl. Ausbildung einer Vorzugsorientierung in [201/210]-Richtung oder eines gleich orientierten größeren Kornes schließen, die zudem mit der sich bildenden neuen kubischen Phase über einen weiten Zeitraum hinweg koexistiert. Die Stagnation der Phasenumwandlung kann durch mit einer temporären Ausheilung der sich neubildenden kubischen Eisphase mit der Ausgangsphase einhergehen. Dies wird durch die wesentlich geringeren Zuwächse der Intensitäten angedeutet.

Denkbar wäre zudem, dass die Stagnation durch Protonenumordnungseffekte für das sich in diesem Temperaturbereich umwandelnde partiell protonengeordnete Eis IX/III handelt. Eine Aussage diesbezüglich ist jedoch nicht definitiv zutreffen. Ein ähnlicher Stagnationseffekt ist bei der Phasenumwandlung der partiell protonengeordnete Eisphase $\mathrm{V}$ in kubisches Eis Ic nicht festzustellen.

Aufgrund der anfänglich unklaren Zuordnung von hexagonalen und kubischen Streubeiträgen fehlenden [111]-kubischen oder überlappenden [002]-hexagonalen Eispeaks ist aus den Intensitätsverhältnissen ebenso nicht $\mathrm{zu}$ bestimmen, ob zuerst bei der Umwandlung in geringen, jedoch stetig ansteigenden Phasenanteilen ,gestörtes hexagonales Eis $\mathrm{Ih}^{\text {“ }}$ gebildet wird, dass sich im weiteren Verlaufe in kubisches Eis Ic umwandelt. Aussagen hinsichtlich der verursachenden Mechanismen oder welcher Art die physikalischen Effekte sind, die zu einem solchen Verhalten führen, sind aufgrund der zur Verfügung stehenden apparativen Möglichkeiten aus diesen Messungen nicht zu klären. Eine Diskussion der Kinetik der Phasenumwandlung von Eis IX/III in kubisches Eis Ic mittels der JMAK-Theorie ist aufgrund der ungeklärten Verläufe nicht sinnvoll. Die gemessenen Diffraktogramme der Phasenumwandlung von Eis II in kubisches Eis Ic sind im Anhang 8.3.3.2, Abbildung 8.3.3.2.d dargelegt. 


\title{
3.3.3 JMAK-Fits für die Phasenübergänge XII $\rightarrow$ Ic , V $\rightarrow$ Ic
}

Die für die Ausgangseisphasen Eis XII und Eis V aus den experimentell ermittelten zeitlichen Verläufen dargestellten JMAK-Plots sind in der Abbildung 3.11 dargestellt.

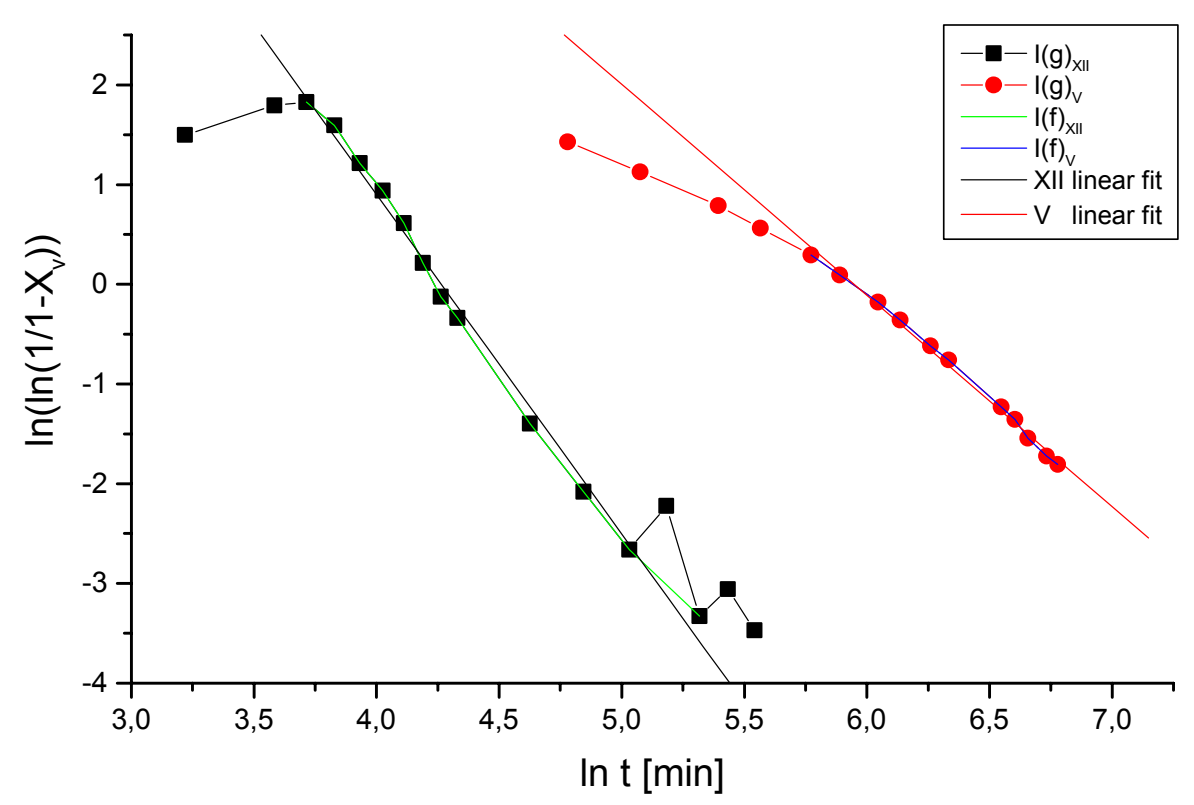

\begin{abstract}
Abbildung 3.11: JMAK-Plots der Phasenumwandlungen der Eisphasen XII und V in kubisches Eis Ic@ T 143K; I(g) : gemittelte Intensitäten, I(f) : zur Bestimmung des Avrami-Exponenten verwendete Intensitätswerte, linear fit : lineare Fits der verwendeten I(f). Die Steigungen, die in dieser Darstellung den Avrami-Exponenten repräsentieren, wurden durch einen linearen Fit der Intensitätsverläufe bestimmt. Abweichungen von dem JMAK-Verhalten sind am Anfang von Eis V sowie am Anfang und am Ende der Phasenumwandlung für Eis XII festzustellen, die durch Intensitätsänderungen der gewählten Peakintensitäten (Referenzparameter) von Eis XII und Eis V repräsentiert sind. Ob es sich hier um einen Rekristallisationseffekt, z.B. um eine kinetische Hemmung bei der Phasenumwandlung oder aber um ein „Hineindrehen“ eines verbleibenden größeren Kornes, z.B. Eis XII-Kornes in Reflexionsrichtung handelt, ist nicht zu klären.
\end{abstract}

Für das kinetische Verhalten der Umwandlung von Eis XII ergibt sich aufgrund des Fits ein Avrami-Exponent von $\mathrm{n} \sim 3$ bei einer Zeitkonstanten von $\tau \sim 55$ Minuten. Koza (persönliche Mitteilung (1999)) bestimmte in einer vergleichbaren Messung der Rekristallisation an Eis XII in kubsches Eis Ic bei einer Temperatur von T 150K den Avrami-Exponenten ebenso zu $\mathrm{n} \sim 3$. Die Zeitkonstante wurde im Gegensatz zu dem übereinstimmenden Avrami-Exponenten zu $\tau \sim 36$ Minuten bestimmt. Geht man für beide Messungen von einer konstanten Nukleationsrate aus, so ergibt sich aufgrund der bestimmten Avrami-Exponenten für Eis XII ein zweidimensionales, d.h. flächiges Wachstum der sich bildenden Eis IcKristallisationskeime bzw. der sich abbauenden Eis XII-Struktur. Die Abweichungen der Zeitkonstanten sind durch unterschiedliche Temperaturen, bei denen die Rekristallisationen 
durchgeführt wurden, bedingt. Neben der Temperatur können jedoch auch Korngrenzflächeneffekte (z.B. Vorzugsorientierungen und Korngrenzenenergien) als mögliche Ursache für eine kinetische Hemmung der Rekristallisation angeführt werden. Aussagen bezüglich der Kinetik als Funktion der Temperatur können aufgrund der wenigen systematischen Messungen und der strukturellen Eigenschaften der Probe nicht getroffen werden. Für Eis V ergibt sich aus dem JMAK-plot ein anfänglich nicht lineares Verhalten, das sich im Laufe der fortschreitenden Rekristallisation einem linearem Verlauf stark annähert. Lässt man die Punkte mit der größten Abweichung außer acht und fittet die verbleibenden, so ergibt sich für Eis V ein Avrami-Exponent von $n=2$. Ein Fit unter Berücksichtigung der außeracht gelassenen Punkte ergab einen Avrami-Exponenten von $\mathrm{n} \sim 1.7 \approx 2$. Unter der Annahmen der JMAK-Theorie, d.h. bei einer konstanten Keimbildungsrate ergibt sich für Eis V ein "eindimensionales" Kornwachstum bzw. ein Kornwachstum mit einer ausgeprägten Anisotropie. Dies ist jedoch im Vergleich zu dem nahezu äquivalenten Verläufen der integrierten Reflexintensitäten nicht schlüssig. Es ist daher davon auszugehen, dass der Keimbildungsprozess bei der Phasenumwandlung in Eis V nicht den Annahmen der JMAKTheorie folgt. Der unterschiedlich dimensionierte Abbau der Ausgangsphasen bzw. der Aufbau der sich bildenden Phase ist durch die verschiedenen strukturellen Eigenschaften der Kristallite gegeben. Je nach Struktur der Phase bzw. zugehöriger Raumgruppe ergeben sich für das Eis XII sowie das Eis V verschiedene Kristallwachstumsflächen, die in unterschiedlichen Anbaumöglichkeiten von Wassermolekülen in der kubischen Struktur resultieren. Diese Wassermoleküle bzw. nanoskopische Ringstrukturen (Cluster) der ursprünglichen Eis XII- sowie der Eis V-Kristallite werden während der Phasenumwandlung aus dem Kristallgefüge gelöst und in die neue Struktur eingebaut. Je nach Struktur (Raumgruppe) und kristallographischer Orientierung der Ausgangsstruktur können die Keime der neu zu bildenden kubischen Eisphase unterschiedliche Wachstumsmöglichkeiten haben und können so zu einem unterschiedlichen topotaktischen Wachstumsvorgang mit begrenztem isotropen bis stark anisotropem Wachstum führen, die unter Berücksichtigung von Keimgrößenunterschieden und evtl. vorhandenen Texturen nicht zu einem der JMAK-Theorie entsprechendem Verhalten führen kann. Anzumerken ist jedoch, dass das Eis V aufgrund der am D1B im Winkelbereich von $\sim 20^{\circ} \leq 2 \theta \leq \sim 80^{\circ}$ bestimmten Reflexverhältnisse im eigentlichen Sinn als polykristallines Eis angesehen wurde, so dass das Phasenumwandlungsverhalten von Eis $\mathrm{V}$ in kubisches Eis Ic sowie die verursachenden Gründe für die Abweichung von JMAK-Verhalten als offene Frage bezeichnet werden muss. 


\subsection{Der Phasenübergang von kubischem Eis Ic in Eis Ih}

Als Grundlage für die Experimente der Strukturuntersuchungen an Eis Ih in Wechselwirkung mit den Gasen Argon, Helium und Neon als Funktion des Druckes und der Temperatur war gutes Eis Ih-Pulver mit hinreichender Pulvergüte als Ausgangsmaterial notwendig. Dieses wurde über die in Kapitel 3.2 beschriebene Hochdruckpräparationsroute hergestellt. Die Pulverqualität wurde am Anfang der eigentlichen Experimente durch Intensitätsvergleiche einzelner Reflexe als Funktion des Drehwinkels der Eisproben in $\omega$, jedoch nicht über das Reflexverhältnis untereinander verifiziert. Die Rietveld-Verfeinerungen der aus den verschiedenen Experimenten gewonnenen Datensätze ergab, dass das so hergestellte Eispulver „Fehlordnungen” aufwies. Diese zeigten sich erst durch einen späteren Vergleich der Intensitätsabweichungen der hexagonalen [100]-, [002]- und [101]-Reflexe des Eis Ih als Funktion der Temperatur und sind als Überbleibsel oder Relikte der Präparation aus der kubischen Eisphase anzusehen. Sie haben einen wesentlichen Einfluss auf die Auswertbarkeit und Aussagefähigkeit in Rietveld-Verfeinerungen (vergl. Kapitel 5.). Der Existenzbereich von kubischem Eis Ic wurde experimentell unabhängig von mehreren Autoren in dem Bereich von $\mathrm{T} \sim 120 \mathrm{~K}$ bis $\mathrm{T} \sim 160 \mathrm{~K}$ bei Drücken $\leq$ Umgebungsdruck bestimmt. Systematische Untersuchungen hinsichtlich der Phasentransformation von kubischem Eis Ic in das hexagonale Pendant wurden von Dowell \& Rinfret (1960), Handa et al. (1988) sowie Londono et al. (1989) durchgeführt. Diese Experimente belegen eindeutig, dass die Phasenumwandlung von kubischem Eis Ic in hexagonales Eis $\mathrm{Ih}$ in mehreren Phasenumwandlungsschritten erfolgt. Londono et al. (1989) bestimmten diffraktrometrisch über einen Temperaturbereich von $\mathrm{T} \sim 100 \mathrm{~K}$ bis $\mathrm{T} \sim 210 \mathrm{~K}$, dass der Phasenübergang von aus der Hochdruckeisphase Eis II hergestelltem kubischen Eis Ic in Eis Ih in mehreren Schritten vollzogen wurde. Diese Aussage leiteten sie durch die Bestimmung der Halbwertsbreiten des kubischen [111]- /hexagonalen [002]-Reflexes ab. Ab T 100K bis T 160K blieb die relative Halbwertsbreite des kubischen [111]-/hexagonalen [002]-Reflexes nahezu konstant, während in dem Temperaturbereich zwischen $\mathrm{T} \sim 160 \mathrm{~K}$ und $\mathrm{T} \sim 175 \mathrm{~K}$ diese auf die Hälfte abfällt. $\mathrm{Ab}$ einer Temperatur von $\mathrm{T} \sim 175 \mathrm{~K}$ bis $\mathrm{T} \sim 210 \mathrm{~K}$ fällt diese nochmals auf etwa $25 \%$ des ursprünglichen Wertes $\mathrm{ab}$. Aussagen bezüglich des Intensitätsverhaltens wurden nicht getroffen. Der weitere Verlauf oder die Angabe der Intensitätsverhältnisse sind nicht weiter dokumentiert. 
In diesem Verlauf ist bei einer Temperatur zwischen $\mathrm{T} \sim 205 \mathrm{~K}$ bis $\mathrm{T} \sim 210 \mathrm{~K}$ eine Steigungsänderung der relativen Halbwertsbreiten angedeutet und könnte auf einen weiteren Phasenumwandlungsschritt hindeuten. Hinweise auf einen weiter ausgedehnten Temperaturbereich der Phasenumwandlung bis zu T 237K wurden von Mayer \& Hallbrucker (1987) bei einer Phasenumwandlung eines aus einer „amorphen Eisphase“ hergestellten kubischen Eis Ic in hexagonales Eis Ih gefunden. Handa et al. (1988) fanden bei kalorimetrischen Messungen von Phasenübergängen von kubischem Eis Ic, hergestellt aus den Hochdruckeisphasen II, IX sowie V hinsichtlich der Phasenumwandlungstemperaturen sowie dem Phasenumwandlungsverhalten Unterschiede. Aus den Phasenumwandlungskurven, d.h. den Verläufen der Enthalpieänderungen zeigte sich, dass diese Phasenumwandlungen über einen weiten Temperaturbereich stattfanden und unterschiedliche Enthalpieänderungen aufwiesen. Die von Handa et al. (1988) bestimmten Temperaturbereiche sowie die kalorimetrisch bestimmten Enthalpieänderungen sind in Tabelle 3.3 sowie Tabelle 3.4 dargestellt.

\begin{tabular}{|c|c|c|c|c|}
\hline & Ic(II) & Ic(V) & Ic(IX) & Ic(VI)* (D2B) \\
\cline { 1 - 3 } $\mathbf{T}_{\mathbf{1}}[\mathbf{K}]^{+}$ & $186.2(3.7)$ & $178.9(3.6)$ & $178.9(3.6)$ & \multirow{2}{*}{$195 \mathrm{~K} \pm 3.5 \mathrm{~K}$} \\
\hline $\mathbf{T}_{\mathbf{2}}[\mathbf{K}]^{+}$ & $226.5(4.5)$ & $217.4(4.4)$ & $229.9(4.6)$ & \\
\hline
\end{tabular}

Tabelle 3.3: Phasenumwandlungstemperaturen von Eis Ic, gewonnen aus HD-Eis in Eis Ih, $T_{1}:$ Beginn der Phasenumwandlung, $T_{2}$ : Ende der Phasenumwandlung; * : Beginn der Phasenumwandlung von Ic(VI) am D2B in situ bestimmt, durch Änderungen der Reflexverhältnisse und dem Erscheinen des hexagonalen [101]-Reflexes

\begin{tabular}{|c|c|c|c|c|c|}
\hline Ih aus Ic aus & LDA $^{*}$ & V & XII & IX & II \\
\hline$\Delta \mathbf{H}\left[\mathbf{J} / \mathbf{m o l}^{-1}\right]$ & $-35(4)$ & $-50(1)$ & - & $-13(4)$ & $-36(4)$ \\
\hline
\end{tabular}

Tabelle 3.4: Vergleich der Enthalpieänderungen von kubischem Eis Ic, hergestellt aus unterschiedlichen Hochdruckeisphasen, in hexagonales Eis Ih, $\Delta \mathrm{H}$ : Enthalpieänderung beim Übergang kubisches Eis Ic in Eis Ih (Handa et al. (1988)), LDA ist dem HDA die am nächsten verwandte Phase, es wird zudem vermutet, dass HDA bei dem Übergang zu Ic erst eine Phasentransformation zu LDA vollzieht

Das für die Herstellung von Eis Ih benötigte Eis Ic wurde für die beiden Eis-/GasExperimente (Grundlage für die Untersuchungen in Kapitel 5.) aus „recovertem” Eis V gewonnen, das zuvor aus der flüssigen Phase bei einem Druck von $\mathrm{p} \sim 5 \mathrm{kbar}$ nukleiert wurde. Die Phasenumwandlung von Eis V in kubisches Eis Ic begann bei etwa T 153K und war bei $\mathrm{T} \sim 157 \mathrm{~K}$ nach $\mathrm{t} \sim 40$ Minuten nahezu abgeschlossen. Diese Phasenumwandlungstemperatur ist im Rahmen des Fehlers von $\Delta \mathrm{T} \sim \pm 3.5 \mathrm{~K}$ mit der von Handa et al. (1988) konsistent. Die weitere Umwandlung von kubischem Eis Ic in Eis Ih wurde durch eine stetige Temperaturerhöhung in Schritten von $\Delta \mathrm{T} \sim 5-10 \mathrm{~K}$ durchgeführt (vergl. Abbildung 3.12). Im 
Gegensatz zu Handa et al.(1988) wurde der Phasenübergang Eis Ic(V) in Eis Ih nicht durch kalorimetrische Messungen sondern durch diffraktometrische Methoden, d.h. über den Vergleich der Intensitäten als Funktion der Zeit festgestellt. Der Phasenübergang, der nur in dem Temperaturbereich von $\mathrm{T} \sim 180 \mathrm{~K}$ bis $\mathrm{T} \sim 210 \mathrm{~K}$ beobachtet werden konnte, liegt mit einem transient gemessenem Beginn der Phasenumwandlung bei einer Temperatur von T 195K (bei einer Heizrate $\sim 0.5 \mathrm{~K} / \mathrm{s}$ ) höher. Die durch Diffraktion bestimmte Umwandlungstemperatur entspricht im Rahmen des Fehlers dem Mittelwert der Temperaturen am Beginn und Ende des von Handa et al. (1988) bestimmten Phasenübergangsbereiches.

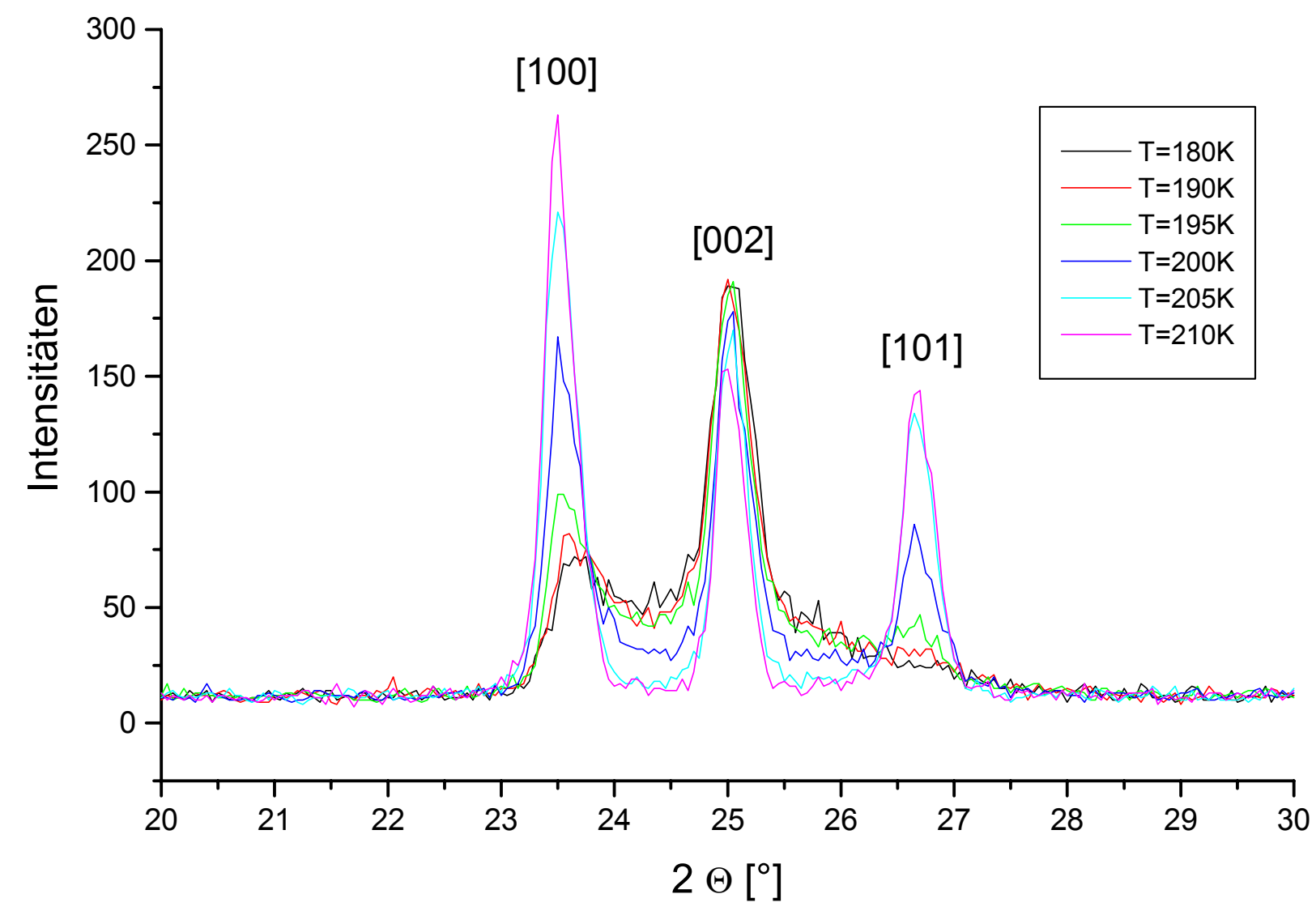

Abbildung 3.12: Intensitätsverläufe der [100]-, [002]- und [101]-Reflexe beim Übergang von kubischem Eis Ic in Eis Ih als Funktion der Temperatur, gemessen am D2B im Temperaturintervall von T 180K bis T 210K. Bei den Temperaturen von $\mathrm{T} \sim 180 \mathrm{~K}$ bis $\mathrm{T} \sim 190 \mathrm{~K}$ zeigen die Diffraktogramme die für kubisches Eis Ic vorhandenen Reflexe als auch die durch hexagoanle Fehlordnungen bedingten asymmetrische Reflexformen. Ebenso zeigt sich bei diesen Temperaturen das Fehlen des hexagonalen [101]-Reflexes, der erst bei Temperaturen $T>190 \mathrm{~K}$ deutlich außerhalb der Untergrundschwankung zu erkennen ist. 


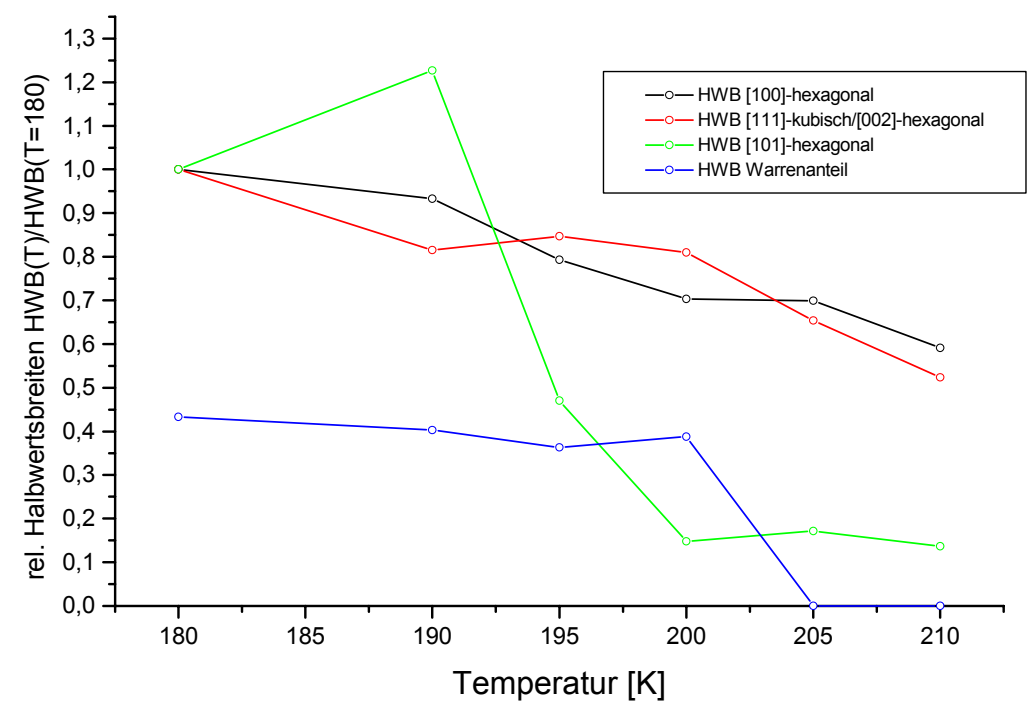

Abbildung 3.13: (oben) : Durch Mehrfach-Fit bestimmte relative Halbwertsbreiten des hexagonalen [100]Reflexes, des Warren- Anteiles, Überlagerung des kubischen [111]/hexagonalen [002]-Reflexes und eines Anfangs unscharfen Untergrund-Reflexes mit einem scharfen hexagonalen [101]- Reflex. Die Fehler der in den Abbildungen 3.13 und 3.14 dargestellten Verläufen sind durch Maximalfehlerabschätzung bestimmt worden und können auf etwa $10 \%$ der Funktionswerte beziffert werden. Die Verläufe sind jeweils auf den Ausgangszustand bei einer Temperatur von $T \sim 180 \mathrm{~K}$ normiert.

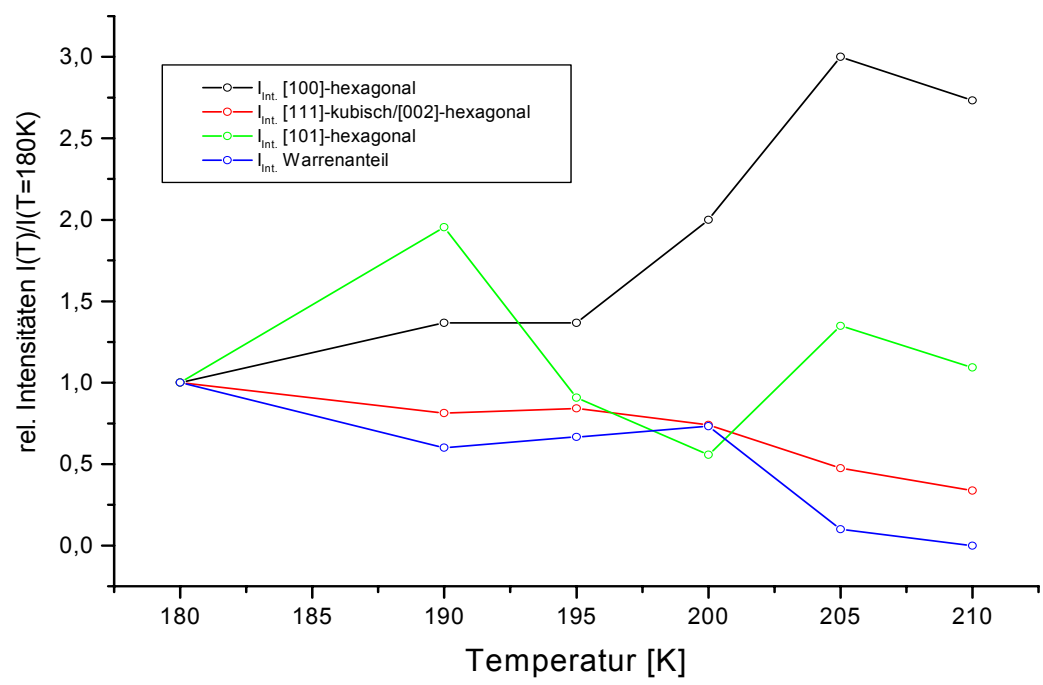

Abbildung 3.14: (unten) : Durch Mehrfach-Fit bestimmte integrierte Intensitäten des hexagonalen [100]Reflexes, des Warren-Anteiles, Überlagerung des kubischen [111]/ hexagonalen [002]-Reflexes und eines Anfangs unscharfen Untergrund- Reflexes mit einem scharfen hexagonalen [101]-Reflex

Die Bestimmung der Intensitätsverläufe in den Abbildungen 3.13 sowie 3.14 wurden über Einzel-Reflexfits durch Überlagerungen von Gauß- und Lorentzpeaks bestimmt (vergl. hierzu 
Anhang 8.3.3.1.4). Für den Phasenübergang wurden mittels eines Mehrfach-Fits von Gaußund Lorentzfunktionen die Reflexparameter als Funktion der Temperatur bestimmt. Die gefitteten Reflexparameter wurden zuerst durch manuelles Anpassen der Fit-Funktionen und durch ein darauffolgendes Minimierungsverfahren ( $\mathrm{Chi}^{2}$-Minimierung) bestimmt. Die Reflexparameter als Funktion der Temperatur sind in den vorherigen Abbildungen 3.13 und 3.14 dargestellt.

Der Umbau aus dem kubischen in das hexagonale Eis bzw. einer Verringerung der nun als „kubischen Stapelfehlordnungen” zu betrachtenden Phasenanteile in ein „durch Stapelfehlordnungen nahezu ungestörtes" Eis Ih erfolgt nicht isotrop. In einem qualitativen Vergleich der Halbwertsbreiten sowie der integrierten Intensitäten, im speziellen der hexagonalen [100]- und der kubischen [111]-/hexagonalen [002]- und hexagonalen [101]Reflexparameter, zeigt sich im Rahmen des Fehlers ein unterschiedlicher Verlauf, der auf einen schrittweisen Umbau der fehlgeordneten Strukturen hindeutet. Aufgrund der ReflexÜberlappung des kubischen [111]- sowie des hexagonalen [002]-Reflexes ist eine differenzierte Betrachtung im Hinblick auf Wachstums- und Abbaugeschwindigkeit sowie der zeitlichen Entwicklung von Partikelgrößen der beiden Phasen nicht möglich. Hinweise auf einen schrittweisen Umbau dieser Strukturen wurden schon durch Experimente von Londono et al. (1989) ebenso gegeben wie durch die von Handa et al. (1988) experimentell ermittelten unterschiedlichen Umwandlungstemperaturen und Enthalpieverläufe. Die Intensitäten des [111]-kubischen/[002]-hexagonalen Reflexes zeigen in dem Temperaturbereich von $\mathrm{T} \sim 180 \mathrm{~K}$ bis etwa $\mathrm{T} \sim 210 \mathrm{~K}$ eine stetigen und kontinuierlichen Abfall. Im Experiment von Londono et al. (1989) fällt die relative Halbwertsbreite über einen Temperaturbereich von $\mathrm{T} \sim 180 \mathrm{~K}$ bis $\mathrm{T} \sim 210 \mathrm{~K}$ auf etwa $50 \%$ ab. Ein detaillierter Vergleich kann aufgrund der aus verschiedenen Hochdruckeisphasen hergestellten kubischen Eisphasen sowie der unterschiedlichen Umwandlungstemperaturen nicht getroffen werden. Die höhere Phasenumwandlungstemperatur kann auf eine kinetische Hemmung der Ausheilung bzw. Reorganisation der Fehlordnungen der im Kubischen vorhandenen hexagonalen Stapelfehler hindeuten. Für die Intensitäten des hexagonalen [100]-Reflexes zeigt sich ab der Temperatur von $\mathrm{T} \sim 180 \mathrm{~K}$ ein stetiger Anstieg, der zu einer Erhöhung der Intensität um $\sim 25 \%$ führt. Im weiteren Verlauf erhöht sich die Intensität mit einem wesentlich größeren Anstieg (vergl. Abbildung 3.14, ab T 195K). Die Halbwertsbreite des hexagonalen [100]-Peaks zeigt im Rahmen des Fehlers ein zum kubischen [111]-/ hexagonalen [002]-Reflex analoges Verhalten. 
Für die hexagonalen [101]-Reflexparameter und die Warren-Reflexparameter ist der Verlauf deutlich verschieden. Während die Halbwertsbreite des Warren-Reflexes bis zu Temperaturen von etwa T 200K im Rahmen des Fehlers annähernd konstant ist, zeigt die Halbwertsbreite des [101]-Reflexes die größte Abhängigkeit als Funktion der Temperatur. Die Warren-Anteile als Schulterstreubeiträge des hexagonalen [100]-Reflexes weisen in diesem Temperaturbereich eine nur geringere Variation hinsichtlich der Intensität und der Halbwertsbreite auf und deuten auf einen Erhalt des in lateral fehlgeordneten Strukturen enthaltenen streuenden Volumens hin. Ein Umbau erscheint vielmehr zwischen den kubischen und hexagonalen Domänen stattzufinden. Ab einer Temperatur $\mathrm{T}>200 \mathrm{~K}-205 \mathrm{~K}$ fällt der Streubeitrag der Warren-Anteile deutlich sprunghaft auf einen Wert nahezu null ab und deutet auf eine spontane Reorganisation hin. Für den hexagonalen [101]-Reflex ist das zeitliche Verhalten deutlich verschieden. Bei einer Temperatur von T 190K zeichnet sich die Bildung eines hexagonalen [101]-Reflexes ab (vergl. Abbildung 3.12). Das Maximum der relativen Halbwertsbreite und der Peakintensitäten bei $\mathrm{T} \sim 190 \mathrm{~K}$ für diesen Reflex ist aufgrund des Fitverfahrens gegeben und beinhaltet zum einen den Streubeitrag des Untergrundes sowie des hexagonalen [101]-Reflexes. Die Trennung in einen Untergrundanteil und in einen [101]Reflex erwies sich als problematisch (vergl. Anhang 8.3.3.1.4). Bis zu dieser Temperatur ist daher der Peak durch den Untergrund beeinflusst. Erst bei einer Temperatur von T 205K (vergl. Abbildung 3.12) ist der Untergrund unter dem hexagonalen [101]-Reflex nahezu verschwunden, eine Beeinflussung der Reflexparameter findet nicht mehr statt.

Der Streubeitrag des hexagonalen [100]- und des [101]-Reflexes zeigt in dem Temperaturbereich von $\mathrm{T} \sim 200 \mathrm{~K}$ bis $\mathrm{T} \sim 210 \mathrm{~K}$ einen nahezu kontinuierlich Verlauf, wobei der Streuanteil des [100]-Reflexes deutlich stärker anwächst, wie aus der mittleren Steigung der relativen Intensitäten zu ersehen ist. Die in Abbildung 3.12 dargestellten Diffraktogramme in dem Winkelbereich von $20^{\circ} \leq 2 \theta \leq 30^{\circ}$ zeigen bei $\mathrm{T} \sim 180 \mathrm{~K}$ sowie $\mathrm{T} \sim 190 \mathrm{~K}$ das von König (1943) zuerst und von Kuhs et al. (1987) detailliert beschriebene typische Streubild für kubisches Eis Ic mit dem fehlenden hexagonalen [101]-Reflex. Erst bei Temperaturen $\mathrm{T} \geq 190 \mathrm{~K} \sim 195 \mathrm{~K}$ zeichnet sich der hexgonale [101]-Reflex deutlich signifikant vom Untergrund ab. Im Streubild ist dies ein Argument dafür, dass die Phasenumwandlung mit einem strukturellen Umbau von kubischem Eis Ic in hexgonales Eis Ih erst bei höheren Temperaturen stattfindet als bei der von Handa et al. (1988) festgestellten Übergangstemperatur von $\mathrm{T} \sim 178 \mathrm{~K}$. Die aus dem zeitlichen Verlauf der Diffraktogramme 
bestimmte Phasenübergangstemperatur von $\mathrm{T} \sim 195 \mathrm{~K}$ liegt um etwa $\Delta \mathrm{T} \sim 17 \mathrm{~K}$ höher, entspricht jedoch in etwa dem Mittelwert von T 198K der von Handa et al. (1988) angegebenen Temperaturen des Phasenumwandlungsbereiches.

Aus dem Wachstumsverhalten der Streubeiträge und den aus den Reflexhalbwertbreiten abgeleiteten Partikel- oder Bereichsgrößen ist festzustellen, dass es sich um einen komplexen, mehrstufigen Vorgang handelt, der sich zudem über einen weiteren Temperaturbereich hin ausdehnt. Aussagen sowohl hinsichtlich des zeitlichen Verlaufes der Reflexintensitäten als auch der Reflexprofile sind äquivalent zu denen Londono et al. (1989) sowie von Handa et al. (1988) quantitativ zutreffen. Eine detaillierte Interpretation konnte aus zeitlichen Gründen nicht mehr durchgeführt werden. Durch das Anwachsen der Intensität und der Halbwertsbreite der [111]-Reflexschulter wird der Umbau der kubischen Ausgangsphase in ungeordnete Bereiche angedeutet. Die Warren- Anteile als Schulterbeiträge des [100]Reflexes verbleiben über einen weiten Temperaturbereich als nahezu konstanter Phasenanteil, fallen dann jedoch bei T 205K schnell ab. In diesem Zuge steigen die Reflexintensitäten des [100]- und [101]-Reflexes mit annähernd gleicher Wachstumsgeschwindigkeit schnell an. Die Bereichsgrößen der an dem Warren-Anteil beteiligten Strukturen scheinen wenig veränderlich. Im Gegensatz dazu scheinen sich die Bereichsgrößen der Phasenanteile der an dem [111/002]-Schulterbeitrag beteiligten Strukturen durch die anwachsende Halbwertsbreite zu verkleinern. Die kontinuierlich abfallende Halbwertsbreite des [111/002]-Reflexes weist auf eine kontinuierliche Partikel- oder Bereichsvergrößerung hin und könnte daher einen kontinuierlichen Abbau der kubischen Stapelfolge in die hexagonale Stapelfolge andeuten.

Eine weiterführende Diskussion der Kinetik mit der JMAK-Theorie sowie des strukturellen Umbaus (Ordnung der Stapelfehler) können aufgrund der kontinuierlichen Temperaturerhöhung und der nicht vollständig verfolgten Umwandlung nicht getroffen werden. Der strukturelle Umbau scheint aufgrund der bestimmten Intensitätsänderungen im Streubild deutlich erst bei höheren Temperaturen und zudem über weitgehend verbleibende ungeordnete Bereiche vollzogen $\mathrm{zu}$ werden. Eine weiterführende Diskussion und Interpretation der verfolgten Intensitätsverläufe gestaltet sich aufgrund der letztendlich nur annähernd beschreibbaren Struktur des kubischen Eises hinsichtlich der Stapelfehlordnungen und der bei der Phasentransformation stattfindenden Umordnung derzeitig als nicht möglich. 


\subsubsection{Imperfektionen im Eis Ih}

Im Zuge der Auswertungen der Eis Ih-Experimente mit verschiedenen Gasen, sowohl der Eis Ih-Datensätze unter der Verwendung von Argongas aus dem ersten als auch der Eis IhDatensätze unter der Verwendung von Neongas in dem zweiten Experimentes konnte durch Rietveld-Verfeinerungen nachgewiesen werden, dass Eis Ih Pulver, welches durch die Herstellung aus kubischem Eis Ic aus der Hochdruckeisphase V gewonnen wurde, Imperfektionen aufwies. Diese äußerten sich in einer systematischen Intensitätsabweichung der relevanten Eis Ih-Peaks, dem [100]-, [002] und dem [101]-Reflex. Die Reflexe wiesen über einen weiten Druck- und Temperaturbereich deutliche Abweichungen gegenüber den Intensitäten eines ideal polykristallinen Eis Ih auf. Der [002]-Reflex wies im Experiment gegenüber dem [100]- und dem [101]-Reflex eine deutliche Überhöhung auf. Diese wurde in den ersten Verfeinerungszyklen unter der Annahme einer [001]-Vorzugsorientierung verfeinert. Es zeigte sich jedoch, dass durch diese Anpassung das Reflexprofil im allgemeinen eine Verschlechterung erfuhr, so dass von einer Vorzugsorientierung in [001] nicht ausgegangen werden konnte. Verfeinerungen unter der Annahme anderer Vorzugsorientierungen, z.B. [100]- sowie [101]-Vorzugsorientierungen, verschlechterten ebenfalls das Verfeinerungsergebnis, so dass eine Vorzugsorientierung der Proben-Kristallite mit großer Wahrscheinlichkeit ausgeschlossen werden konnte.

Die Rietveld-Verfeinerungen der mit Neongas gemessenen Datensätze bei T 140K, T 215K und $\mathrm{T} \sim 250 \mathrm{~K}$ wurden daher systematisch verglichen. Eine Änderung der Reflexintensitäten der Eis Ih-Reflexe, bedingt durch eine druck- und temperaturabhängige Lösung der verwendeten Gase Argon, Neon und Helium konnte nicht festgestellt werden. Es zeigt sich kein siginifikanter Gang der Intensitätsverschiebungen der [100]-, [002]-, [101]-Reflexe mit dem Druck bei den Temperaturen von T 140K und T 215K. Die Rietveld-Verfeinerungen der bei den verschiedenen Temperaturen und höheren Drücken gemessenen Datensätze, bestätigten damit zum einen, dass es sich um keine ausgebildete Vorzugsorientierung handelte, zum anderen, dass die systematische Intensitätsabweichung keine Funktion des Druckes war. Es konnte festgestellt werden, das die Intensitätsabweichungen bei Temperaturen unterhalb von $\mathrm{T} \sim 215 \mathrm{~K}$ über den Zeitraum der Messung von $\mathrm{t} \sim 2$ Tagen erhalten blieben. Erst bei Datensätzen, deren Temperatur oberhalb von $\mathrm{T} \geq 250 \mathrm{~K}$ lag, zeigte sich eine 
Annäherung der Reflexintensitäten an die simulierten Intensitätsverhältnisse des ,idealen Eises Ih " (vergl. Abbildung 3.15).

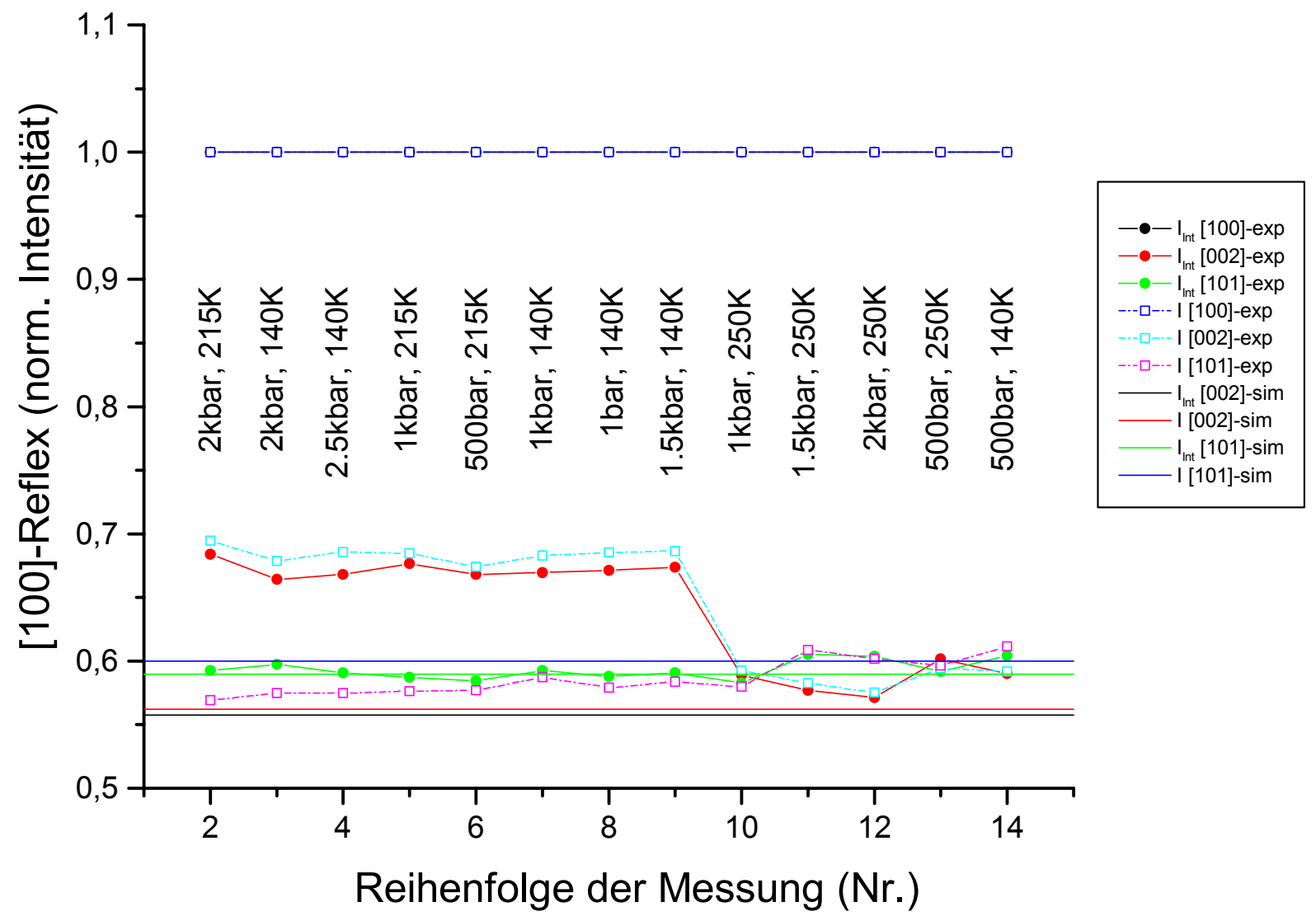

\begin{abstract}
Abbildung 3.15 : Intensitätsverhältnisse der hexagonalen [100]-, [002]-, [101]-Reflexe für Neon-Eis Ih in der Reihenfolge der Messung. Eindeutig ist die Temperaturabhängigkeit zwischen T 215K und T 250K zu erkennen, Die Messungen sind in chronologischer Reihenfolge aufgeführt, ohne in diesem Fall eine konkrete zeitliche Aussage zu machen. Die bei den Messungen herrschenden Umgebungsbedingungen, Druck und Temperatur sind an den jeweiligen Punkten angetragen. - exp : Intensitäten aus experimentellen Daten, -sim : Intensitäten aus simulierten Daten. Diffraktogramme mit den Intensitätsabweichungen (vergl. Kapitel 5.2.1, Abbildung 5.2 sowie Abbildung 5.3).
\end{abstract}

Die Abbildung 3.15 verdeutlicht die Intensitätsänderungen der ersten drei Eis Ih-Reflexe. Die sprunghafte Änderung deutet auf ein Rekristallisations- bzw. Ordnungsphänomen innerhalb der Eisstruktur im Bereich von T 215K bis T 250K hin.

Mayer \& Hallbrucker (1988) fanden durch kalorimetrische Messungen bei der Umwandlung von kubischem Eis Ic aus amorphem Eis erste Hinweise auf einen wesentlich weiter ausgedehnten Phasenumwandlungsbereich bis $\mathrm{zu} \mathrm{T} \sim 240 \mathrm{~K}$, entgegen den bisherigen experimentellen Befunden von Handa et al. (1988), der die Phasenumwandlung von kubischem Eis aus Eis V bis T 220K verfolgte. Weitere Experimentelle Hinweise auf einen 
weiter ausgedehnten Phasenumwandlungsbereich der kubischen Eisphasen (aus anderen Eisphasen hergestellt) wurden in der Literatur nicht beschrieben.

Die hier gefundenen Hinweise deuten in Übereinstimmung zu dem Experiment von Mayer \& Hallbrucker (1988) auf einen weiter ausgedehnten Phasenumwandlungsbereich hin, als bisher für kubisches Eis Ic aus Eis V angenommen wurde. Diese Ausweitung, dargelegt durch die festgestellten Intensitätsabweichungen, scheint durch Ordnungsphänomene in dem hexagonalen Eis Ih in dem Temperaturbereich zwischen T 200K und T 240K verursacht zu sein und deutet auf verbleibende Fehlordnungen insbesondere verbleibende kubische Stapelfolgen bei der Umwandlung von kubischem Eis Ic in hexagonales Eis Ih hin.

Eine weitere mögliche Interpretation ist der Verbleib größerer kubischer Kristallite in der Eis Ih-Matrix, die sich bei der Phasenumwandlung thermodynamisch stabiler zeigen und erst bei Temperaturen weit oberhalb der eigentlichen Phasenumwandlungstemperatur in hexagonales Eis Ih umgewandelt werden. Diese kubischen Kerne dienen für die sich umwandelnde kubische Phase als Keimorte für das topotaktische Aufwachsen von hexagonalen Kristallstrukturen. Eine äquivalente Annahme ist, dass wie bei der Bildung von hexagonalen Schneekristallen anfänglich kubische Kristallisationskeime gebildet werden. Diese stabileren kubischen Keime dienen zum Umwachsen bzw. zur Kristallbildung von freien sowie in Clustern angeordneten Wassermolekülen in der hexagonalen Struktur. Diese kubischen Kristallisationskeime müssten aber sehr klein sein, wie es für kubisches Eis Ic in kleinen Strukturen, z.B. in Form von Kristallisationskeimen von Schneekristallen, vermutet wird (Kobayashi \& Kuroda (1987)). Hier ist jedoch anzumerken, dass sicherlich ein Unterschied zwischen den freigewachsenen Schneekristallen und den im Bulk gewachsenen Kristallisationskeimen besteht.

Aufgrund der nicht genau bekannten Natur dieser hexagonalen Defektstrukturen war ihre Modellierung in den Rietveld-Verfeinerungen nicht möglich. Die Nichtberücksichtigung der Defekte beeinflusste jedoch das Verfeinerungsergebnis hinsichtlich aller Strukturparameter (vergl. Kapitel 5). Probleme mit Defekten waren dagegen für die mit Neongas gemessenen Datensätzen bei T 250K nicht erkennbar. Mit diesen Datensätzen konnte die Bestimmung der Gasinhalte und auch die Bestimmung der mit Gas wechselwirkenden Eisstruktur als Funktion des Druckes und der Temperatur versucht werden. 


\begin{tabular}{|c|c|c|c|c|}
\hline Reflex & $\mathbf{2 \Theta}\left[{ }^{\circ}\right]$ & $\begin{array}{c}\mathbf{I}_{\text {int }} \\
\text { Int. Peakintens. }\end{array}$ & $\begin{array}{c}\mathbf{I} \\
\text { Peakintens. }\end{array}$ & $\begin{array}{c}\text { HWB } \\
\text { Halbwertsbreite }\end{array}$ \\
\hline$[\mathbf{1 0 0}]$ & 23.568 & 1.000 & 1.000 & 0.4509 \\
\hline$[\mathbf{0 0 2}]$ & 25.062 & 0.558 & 0.562 & 0.4476 \\
\hline$[\mathbf{1 0 1}]$ & 26.473 & 0.589 & 0.600 & 0.4432 \\
\hline
\end{tabular}

Tabelle 3.5: Eis Ih, simuliert bei Umgebungsdruck von p 1bar und einer Temperatur von T 223K, Kuhs und Lehmann (1983).

\begin{tabular}{|c|c|c|c|}
\hline Reflex & $\mathbf{I}_{[002]} / \mathbf{I}_{[100]}$ & $\mathbf{I}_{[101]} / \mathbf{I}_{[100]}$ & Scan-Nr. \\
\hline 1bar, 237K & 0.69 & 0.67 & 21 \\
\hline $\mathbf{0 . 5 k b a r , ~ 2 3 7 K ~}$ & 0.67 & 0.65 & 24 \\
\hline 1kbar, 237K & 0.66 & 0.67 & 23 \\
\hline 1.5kbar, 237K & 0.68 & 0.68 & 20 \\
\hline 2kbar, 237K & 0.69 & 0.66 & 19 \\
\hline 2kbar, 215K & 0.65 & 0.68 & $101]-R e f l e x e$ \\
\hline
\end{tabular}

Tabelle 3.6: Vergleich der integrierten Peakintensitäten der [100]-, [002]-, [101]-Reflexe von Eis Ih mit Argon als Druckmedium bei Temperaturen $\mathbf{T}<250 \mathrm{~K}$.

Die Existenz von Fehlordnungen der mit Argongas gemessenen Datensätze aus dem ersten Experiment konnte aus den Rietveld-Verfeinerungen nicht eindeutig verifiziert werden. Ein Vergleich der Intensitätsverhältnisse der [100]-, [002]-, [101]-Reflexe zeigt, dass keine signifikante Abhängigkeit als Funktion des Druckes und der Temperatur gegeben ist und es sich daher lediglich um Intensitätsänderungen durch z.B. Vorzugsorientierungen handelt (vergl. Tabelle 3.5 und Tabelle 3.6). Bei den Helium-Datensätzen von T 265K, T 255K und T 237K zeigte sich der Effekt der Intensitätsunterschiede für die [100]-, [002]-, [101]Reflexe nicht. Bevor diese Probe mit Helium als Drucküberträgermedium gemessen wurde, wurde die Probe zuvor mit Argongas als Drucküberträgermedium bei Temperaturen oberhalb von T 237K über mehrere Stunden gemessen. Die Temperaturen der gemessenen Datensätze lagen gemäß der Tabelle 5.10, Kapitel 5.3.2 bei $\mathrm{T} \sim 250 \mathrm{~K}$ und $\mathrm{T} \sim 265 \mathrm{~K}$ maximal. Es kann daher davon ausgegangen werden, dass die bei den mit Argongas gemessenen Datensätzen evtl. verbliebenen Fehlordnungen im Laufe der Messungen ausgeheilt sind. 


\subsection{Herstellung der Eisphasen Eis II, Eis III}

Die Herstellung von polykristallinem Eis II bzw. Eis III zur Strukturanalyse als auch von „recovertem” Eis II und Eis IX (Tieftemperaturphase von Eis III) als Ausgangsmaterial für Phasenumwandlungen in das kubische Eis Ic gestaltete sich schwierig. Mittels der pVTApparatur wurden mehrere Präparationsrouten aus der Literatur überprüft, die eine gezielte Herstellung erlauben sollten. Die Überprüfung durch Schmelzkurvenexperimente für Eis II sowie Eis III war aufgrund der annähernd gleichen Dichten im Rahmen der Fehler der verwendeten Drucksensoren nicht möglich. Ebenso war die Genauigkeit der ProbenTemperatursensoren in dem gesamten System durch stetig auftretende lokale Wärmeflüsse nicht ausreichend, um die durch die fest-fest-Phasenumwandlung zwischen Eis II und Eis III bedingten Temperaturänderungen mit hinreichender Genauigkeit zu erfassen. Es wurden daher Proben mit der pVT-Apparatur über die aus der Literatur bekannten Präparationsrouten hergestellt und durch Strukturbestimmungsmethoden an Großgeräten wie z.B. dem D2B des ILL in situ überprüft. Der Transport von Göttingen zum ILL erfolgte bei währenden Druckund Temperaturbedingungen. Die Pulvergüten der über die verschiedenen Präparationsrouten hergestellten Eisphasen wurden am BW5 überprüft. Diese Proben wurden in Göttingen wiedergewonnen und unter Stickstofftemperaturen und Umgebungsdruck zum BW5 transportiert. Als einfachste Herstellungsmethode von Eis III ergab sich die Kompression von Eis Ih bei Temperaturen von $\mathrm{T} \sim-25^{\circ}(\mathrm{T} \sim 248 \mathrm{~K})$ bis $\mathrm{T} \sim-30^{\circ} \mathrm{C}(\mathrm{T} \sim 243 \mathrm{~K})$ auf Drücke oberhalb von etwa $\mathrm{p} \sim 2.5 \mathrm{kbar}$ bis $\mathrm{p} \sim 3 \mathrm{kbar}$ Gasdruck oder durch Nukleation, d.h. Kühlen von flüssigem Wasser in dem genannten Druckbereich (vergl. Abbildung 3.1).

An dieser Stelle ist jedoch darauf hinzuweisen, dass bei der Verwendung von Gasen mit kleineren Atomradien als Argon diese in die Eis Ih-Struktur eingebaut/gelöst werden können. Der Einbau bewirkt eine Vergrößerung des Existenzbereichs zu höheren Drücken und Temperaturen. Dieser Effekt wurde von Londono et al. (1992) und Dyadin et al. (1994) für Helium und Wasserstoff sowie im Rahmen dieser Arbeit für Neon beobachtet (vergl. Kapitel 4). Unter Verwendung dieser Gase als Drucküberträgermedium konnte Eis III in einigen Fällen nicht durch Kompression von Eis Ih, in einigen Fällen nur als transiente Phase hergestellt werden. In dem eigentlichen Existenzbereich von Eis III bildete sich ein dem Eis II verwandtes Gas-Hydrat. Die Effekte der Gaslöslichkeit in Eisstrukturen ist Gegenstand von Untersuchungen, denen im Rahmen eigener Experimente (vergl. Kapitel 4. ff. und Kapitel 5. 
ff.) nachgegangen wurde. Für die Mehrzahl der Herstellungsversuche von Eis II sowie Eis III wurde jedoch Argon als Drucküberträgermedium verwendet, das sich erwiesenermaßen nicht in den Eisstrukturen löst (Namiot und Bukhgalter (1965)).

Am BW5 des HASYLAB wurden zudem Streuexperimente hinsichtlich der Pulvergüte an verschieden hergestellten Eisproben durchgeführt. Diese bestätigten, dass die über Nukleation aus der Flüssigkeit hergestellten Eis III-Proben sowohl eine beträchtliche Vorzugsorientierung als auch eine in den Debye-Scherrer-Ringen feststellbare inhomogene Korngrößenverteilung aufwiesen. Die Pulvergüte ist somit von minderer Qualität und für präzise Pulverdiffraktionsmessungen mit einem eindimensionalen Detektor, wie z.B. dem D2B, nur eingeschränkt zu verwenden. Systematische Untersuchungen hinsichtlich der Ausbildung von Vorzugsorientierungen sowie Texturen an Eis ("Texturvererbungen" beim Phasenübergang) waren nicht Ziel und im Rahmen dieser Arbeit nicht durchzuführen. Eine homogenere Korngrößenverteilung mit geringerer Vorzugsorientierung und damit verbundener besserer Pulvergüte ergab sich bei der Kompression von polykristallinem Eis Ih (vergl. Kapitel 3.2) mit Kompressionsraten $10-15 \mathrm{bar} / \mathrm{s}$ bei Temperaturen um $\mathrm{T} \sim-25^{\circ} \mathrm{C}(\sim 248 \mathrm{~K})$ bis $\mathrm{T} \sim-30^{\circ} \mathrm{C}$ $(\sim 243 \mathrm{~K})$. Für systematische Untersuchungen an „recovertem” Eis III / Eis IX sowie der Untersuchung des Phasenüberganges von Eis III/IX in das kubische Eis Ic war es nötig, dieses auf flüssige Stickstofftemperatur abzuschrecken. Durch strukturelle Kontrolle mittels Neutronenstreuexperimenten am D1B und D2B konnte gezeigt werden, dass sich Eis III in vier von vier Abkühlversuchen in Eis II umwandelte. Experimentelle Hinweise auf einen Einfluss der Kühlgeschwindigkeit wurden von Londono et al. (1993) gegeben. Diese gaben an, dass lediglich in einem kleinen Druck- und Temperaturkorridor Eis III bzw. die bei tiefen Temperaturen existierende protonengeordnete Variante Eis IX bis zu tiefen Temperaturen zu erhalten sei. Die dafür benötigten Kühlraten wurden mit $1-2 \mathrm{~K} / \mathrm{s}$ bei Drücken von $\mathrm{p} \sim 2.7 \mathrm{kbar}$ p 3.2kbar angegeben. Aus technischen Gründen verwendeten Londono et al. (1993) HDZellen mit geringerer Masse sowie geringeren Probenvolumina (Kuhs pers. Mitteilung, 2001). Mit den hier verwendeten HD-Zellentypen aus Remanit, Aluminium, Titan-Zirkon sowie V2A-Stahl konnten die angegeben Kühlraten anfänglich, d.h. bis zu Temperaturen $\mathrm{T} \sim-50^{\circ} \mathrm{C}$ $(\sim 223 \mathrm{~K})$, nicht erreicht werden. $\mathrm{Zu}$ tieferen Temperaturen hin lag die Kühlrate bei $\sim 1.5 \mathrm{~K} / \mathrm{s}$. Im Zuge der Herstellungsversuche von Eis II sowie Eis III ergab sich, dass bei der Kompression von feinkristallinem Eis Ih bei Temperaturen von etwa T $-60^{\circ} \mathrm{C}(\sim 213 \mathrm{~K})$, d.h. in dem Temperaturbereich zur Herstellung von Eis II, ebenso feinkristallines Eis III 
hergestellt werden konnte, Eis III somit im Existenzbereich von Eis II zufinden ist. Es ist davon auszugehen, dass dieses Eis III gegenüber dem Eis II metastabil ist, da weitere Temperatur- bzw. Druckänderungen zu einer Phasenumwandlung in Eis II führten. Dieses Eis III-Herstellungsverfahren ist konsistent $\mathrm{zu}$ der von Bertie et al. (1963) erstmals beschriebenen Präparation. Die Erfolgsquote für diese Eis III-Präparationsroute lag für fünf hergestellte Proben bei $\sim 100 \%$. Ebenso gelang es das bei diesen tieferen Temperaturen hergestellte Eis III in drei von drei Fällen ohne Phasenumwandlung in Eis II auf T 77K abzuschrecken. Die Bildung von Eis III scheint bei den angegebenen Druck- und Temperaturbedingungen, d.h. in dem Stabilitätsbereich von Eis II kinetisch bevorzugt zu sein, obgleich das Eis III unter diesen Bedingungen metastabil ist. Erste Hinweise hinsichtlich einer druck- und temperaturabhängigen Bildungskinetik von Eis II und Eis III wurden durch Experimente von Tammann (1903, 1909) gegeben. Tammann (1903, 1909) stellte fest, dass sich die Phasengrenzen von Eis II und Eis III bei den dort herrschenden Druckbedingungen als Funktion der Temperatur mehrfach überschnitten. Kinetische Effekte als Ursachen für dieses Verhalten wurden soweit bekannt nicht diskutiert und waren seit der Arbeit von Tammann $(1903,1909)$ auch nicht mehr im Interesse einer experimentellen Untersuchung.

Die Herstellung von polykristallinem Eis II gestaltete sich aufgrund der oben beschriebenen experimentellen Befunde als sehr viel schwieriger und führte daher nur zu wenigen in situ Strukturbestimmungen an Eis II. Eis II kann, soweit bekannt und in der Literatur von Kamb \& Prakash (1968) sowie von Wilson et al. (1965) beschrieben ist, nicht aus der flüssigen Phase nukleiert werden. Bei starker Unterkühlung der Flüssigkeit bei Drücken um p 3kbar und kühlen dieser auf Temperaturen von $\mathrm{T}=\sim-43^{\circ} \mathrm{C}(\sim 230 \mathrm{~K})$ bildete sich stets Eis III. In ersten Versuchen wurde das Eis II durch Abkühlung von nukleiertem Eis III bei Gasdrücken $\mathrm{p} \sim 2.5 \mathrm{kbar}$ bis $\mathrm{p} \sim 3 \mathrm{kbar}$ oder durch Kompression von Eis $\mathrm{Ih}$ bei $\mathrm{T}=-40^{\circ} \mathrm{C}(\sim 233 \mathrm{~K})$ auf $\mathrm{p} \sim 2.5 \mathrm{kbar}$ bis $\mathrm{p} \sim 3 \mathrm{kbar}$ hergestellt. Bei der Verwendung von aus der flüssigen Phase nukleierten Eis III-Proben wurden nicht vernachlässigbare Vorzugsorientierungen festgestellt. Die Pulvergüte von über den Phasenübergang $\mathrm{Ih} \rightarrow \mathrm{III} \rightarrow \mathrm{II}$ gewonnenem Eis II zeigte für annähernd alle Proben ausgeprägte Vorzugsorientierungen. Diese machten sich durch eine mehr als $5 \sigma$ betragende Intensitätsabweichung von den ideal simulierten Eis IIDiffraktogrammen bemerkbar. Ebenso wie für die Bildung von Eis III wurden im Rahmen dieser Arbeit keine systematischen Untersuchungen hinsichtlich der Ausbildung von Vorzugsorientierungen oder Ausbildung von Texturen durchgeführt. Aussagefähige 
Untersuchungen hinsichtlich Texturverwandtschaften sowie von Texturvererbungen wurden unter anderem von Stern et al (1997) und Bennett et al. (1997) an dem Phasenübergang von Eis Ih zu Eis II durchgeführt. Um hier weitere Aussagen treffen zu können wären jedoch systematische Experimente nötig, welche die Untersuchung der Auswirkung von temperaturabhängigen Kompressionsraten zum Ziel haben könnten, um so einen Aufschluss über die kinetischen Verhältnisse zu erhalten.

\subsection{Herstellung der Eisphasen Eis V und Eis VI}

Die Herstellung von Eis V zeigte sich im Gegensatz zur Herstellung von Eis II, Eis III sowie die noch im weiteren zu diskutierende Herstellung der metastabilen Eisphasen IV und XII als unproblematisch. Am einfachsten konnte das Eis V aus der flüssigen Phase durch Nukleation gewonnen werden. Flüssiges Wasser wurde in der HD-Zelle unter Hochdruck auf p 5.6kbar bis p 6kbar gesetzt und dann durch ausfrieren in der HD-Zelle in dem Flüssigkeitsbad nukleiert. Die Nukleation von Eis V aus der flüssigen Wasserphase setzte zumeist bei Temperaturen von $\mathrm{T} \sim-12.5^{\circ} \mathrm{C}$ bis $\mathrm{T} \sim-15^{\circ} \mathrm{C}(\sim 260 \mathrm{~K}$ bis $255 \mathrm{~K})$ unterhalb des bei dem gewählten Druck gegebenen Schmelzpunktes ein.

Festgestellt werden konnte, dass eine „frische Probe“, d.h. neue präparierte Probe bis zur Nukleation wesentlich stärker unterkühlt werden musste als eine ,aufgetaute“ und erneut nukleierte Probe. Dieser Effekt wurde erstmals von Evans (1967) als eine Art „Gedächtnisfunktion“ beschrieben und durch systematische Untersuchungen hinsichtlich der Nukleationsbedingungen und des sog. Memory-Effektes verifiziert. In situ Strukturuntersuchungen von nukleiertem Eis V zeigten in drei von drei Fällen, dass das auf diesem Wege hergestellte Eis $\mathrm{V}$ relativ wenig Vorzugsorientierungen aufwies. Die Abweichung in den Intensitäten war kleiner als $2 \sigma$. Für die Probe kann somit von einer hinreichend guten Pulverqualität ausgegangen werden. Auch wurde im Zuge von Experimenten an wiedergewonnenen Hochdruckeisphasen Eis $\mathrm{V}$ über eine fest-fest Präparationsroute, z.B. Eis $\mathrm{Ih} \rightarrow$ Eis $\quad \mathrm{III} \rightarrow$ Eis $\mathrm{V}$ hergestellt. Ausgehend von einem polykristallinen Eis Ih wies das über die fest-fest-Phasenumwandlungen gewonnene Eis V eine im Rahmen der durch in situ Neutronenstreuung bestimmten Messstatistik ebenso gute Pulverstatistik aus. Ein Vergleich der stärksten Eis V-Reflexintensitäten zeigte keine wesentlichen Unterschiede des durch Nukleation gewonnenen Eis V zu dem über die fest- 
fest-Route präparierten Eis V auf. Diese Aussage ist mit den bisherigen, aus RietveldVerfeinerungen gewonnenen Erfahrungen konsistent (Lobban, pers. Mitteilung (2000); Kuhs, pers. Mitteilung (2001)).

Die Herstellung von Eis VI gestaltete sich aufgrund des zur Verwendung stehenden maximalen Gasdruckes sowie des zur Verfügung stehenden Gasvolumens der Versuchsapparatur sehr schwierig. Der Existenzbereich von Eis VI sowohl für $\mathrm{H}_{2} \mathrm{O}$ als auch für $\mathrm{D}_{2} \mathrm{O}$ beginnt bei Drücken oberhalb von $\mathrm{p} \sim 6 \mathrm{kbar}$ bis $\mathrm{p} \sim 6.2 \mathrm{kbar}$.

Bei einem zur Verfügung stehenden Druck von $\mathrm{p} \sim 6.5 \mathrm{kbar}$ (Dichtigkeitsprobleme der $\mathrm{pVT}$ Apparatur ab p 6.3kbar) war eine Umwandlung von flüssigem Wasser in Eis VI oder eine Phasenumwandlung zu Eis VI aus der Eisphase V und einem damit verbundenen Druckabfall, hervorgerufen durch die Volumenänderung, nicht in einem Schritt zu vollziehen. Der Gasdruck musste daher während der Phasenumwandlung ständig nachgefahren werden. Am ILL sowie am ISIS des RAL konnten aufgrund von limitierenden apparativen und sicherheitstechnischen Aspekten keine Eis VI-Proben hergestellt werden.

Im Zuge der Ausarbeitung von Präparationsrouten für die metastabilen Eisphasen IV und XII konnte Eis VI jedoch auch unterhalb des eigentlichen Stabilitätsbereiches als metastabile Phase in dem Stabilitätsbereich von Eis V bei Drücken um die $\mathrm{p} \geq 5.2 \mathrm{kbar}$ bis $\mathrm{p} \sim 5.6 \mathrm{kbar}$ in einem einzigen Fall aus der flüssigen Phase durch langsames Kühlen bei den genannten Drücken nukleiert werden und sowohl über die Bestimmung der Schmelzkurve als auch durch die Dichteänderung zur flüssigen Phase identifiziert werden. Systematische strukturelle Analysen und Untersuchungen hinsichtlich der Pulvergüte von Eis VI konnten aber aufgrund der apparativen Limits nicht durchgeführt werden.

\subsection{Herstellung der metastabilen Eisphasen IV, XII}

In dem Stabilitätsbereich von Eis V sowie Eis VI konnte durch Experimente von Bridgman (1912) die Existenz einer metastabilen Eisphase, das Eis IV, durch Bestimmung der Schmelzkurve nachgewiesen werden. Metastabil heißt in diesem Sinn, dass die Eisphase nur einen irreversiblen Phasenübergang in eine stabile Eisphase vollzieht. Lobban et al. (1998) konnten in dem Stabilitätsbereich von Eis V eine weitere metastabile Eisphase, Eis XII, durch 
in situ Strukturanalysen entdecken, so dass die Existenz zweier metastabiler Eisphasen zweifelsfrei nachgewiesen ist. Lobban et al. (1998) gelang für diese Eisphase XII die Bestimmung einiger Schmelzpunkte (vergl. Kapitel 4.3.4). In den beiden Arbeiten wurde berichtet, dass die Herstellung dieser metastabilen Eisphasen schwierig ist und diese nicht gezielt, d.h. beliebig reproduzierbar hergestellt werden konnten, so dass die strukturellen Untersuchungsmöglichkeiten dieser metastabilen Eisphasen im wesentlichen durch die limitierte Möglichkeit der Herstellung begrenzt waren. Bridgman (1912) konnte die Eisphase IV durch Dekompression von Eis VI gewinnen. Der Phasenübergang von Eis VI in Eis IV fand in dem Druckbereich von $\mathrm{p} \sim 5.3 \mathrm{kbar}$ bis $\mathrm{p} \sim 5 \mathrm{kbar}$ sowie in dem Temperaturbereich von $\mathrm{T} \sim 258.4 \mathrm{~K}$ bis $\mathrm{T} \sim 259.4 \mathrm{~K}$ statt.

Bridgman (1912) bestimmte die Schmelzkurve von Eis IV in dem Druckbereich von p 4kbar bis $\mathrm{p} \sim 5.5 \mathrm{kbar}$ und konnte diese durch den Vergleich der Schmelzkurven der bis dahin bekannten Eisphasen als neue Eisphase identifizieren. Evans (1967) konnte Eis IV über Nukleation aus der flüssigen Wasserphase unter Verwendung eines Nukleators TCPA (Tetrachlorophthalicanhydrid) gewinnen. Er fand, dass das mittels des Nukleators hergestellte Eis IV zwischen $\mathrm{p} \sim 3.2 \mathrm{kbar}$ bis $\mathrm{p} \sim 4 \mathrm{kbar}$ existierte und bestimmte den Verlauf der Schmelzkurve. Nishibata (1972) sowie Engelhardt \& Whalley (1972) gelang unabhängig voneinander die Herstellung von Eis IV. Nishibata (1972) gelang die Erzeugung von Eis IV durch die von Bridgman (1912) etablierte Präparationsroute mittels Dekompression von Eis VI. Der Phasenübergang von Eis VI in Eis IV fand bei nahezu identischen Druck- und Temperaturbedingungen ( $\mathrm{p} \sim 5.35 \mathrm{kbar}$ bis $\sim 5 \mathrm{kbar}$ sowie $\mathrm{T} \sim 258.4 \mathrm{~K}$ bis $\mathrm{T} \sim 259.4 \mathrm{~K}$ ) statt. Engelhardt \& Whalley (1972) gelang, ebenso wie Evans (1967), die Nukleation von Eis IV aus der flüssigen Phase unter Zuhilfenahme von TCPA. Sie fanden jedoch die Eisphase IV in einem höheren Druckbereich von $\mathrm{p} \sim 4.2 \mathrm{kbar}$ bis $\mathrm{p} \sim 5.9 \mathrm{kbar}$ und identifizierten diese ebenso über die Bestimmung der Schmelzkurve.

Engelhardt \& Whalley (1978) sowie Engelhardt \& Kamb (1981) gelang es, das Eis IV zu nukleieren und zu „recovern”. Sie ermittelten die Struktur des "recoverten“ Eis IV mittels Röntgendiffraktion. Die Autoren berichten, dass die Probengröße einen wesentlichen Einfluss auf die Nukleationswahrscheinlichkeit von Eis IV hat. In über 60 Versuchen konnte bei einer Probengröße von $1.5 \mathrm{ml} \leq \mathrm{V} \geq 0.8 \mathrm{ml}$ kein Eis IV nukleiert werden. Bei einem Probenvolumen von $\mathrm{V} \sim 0.2 \mathrm{ml}$ ergab sich lediglich eine $10 \%$-ige Nukleationswahrscheinlichkeit. Lobban et al. 
(1998) konnten Eis IV bei p 5.8kbar und einer Temperatur von T 261K (unter Zuhilfenahme von Quarzwolle als Nukleator) aus der flüssigen Wasserphase nukleieren. Das Eis IV konnte in einem Orange-Kryostaten erzeugt werden, in dem die flüssige Probe mit $\sim 0.3 \mathrm{~K} / \mathrm{min}$ von T $\sim 268 \mathrm{~K}$ auf T $\sim 260 \mathrm{~K}$ nicht isobar gekühlt wurde. Nachdem die Endtemperatur von T 260K erreicht wurde, ist der durch die Kühlung abgefallene Druck wiederum auf p 5.8kbar erhöht worden. Die Phasentransformation von der flüssigen Phase in Eis IV setzte sofort nach dem Erreichen des Druckes von p 5.8kbar ein (Lobban (1998)). Trotz mehrerer Versuche, die unter äquivalenten Experimentbedingungen durchgeführt wurden, gelang die Nukleation von Eis IV in nur einem Versuch.

Im Zuge der Eis IV-Nukleationsversuche konnte Lobban (1998) die in diesem Druck- und Temperaturbereich bis dahin unbekannte metastabile Eisphase XII aus der flüssigen Wasserphase unter annähernd äquivalenten Versuchsbedingungen nukleieren und strukturell als neue Eisphase identifizieren. Die Nukleation erfolgte bei einem Druck von etwa p 5.5kbar während der Kühlung der wäßrigen Phase von T 270K auf T 260K, d.h. bei annähernd gleichen Nukleationsbedingungen von Eis IV. Die mittlere Kühlrate betrug jedoch nur $\sim 0.04 \mathrm{~K} / \mathrm{min}$. Die Nukleation von Eis XII setzte bei einer Temperatur von T 261K und bei einem Druck von p 5.5kbar ein. Lobban et al. (1998) führten mehrere in situ Experimente zur Nukleation von Eis XII durch. Die Erfolgschance unter Berücksichtigung aller Versuche betrug ca. 20\%. In weiteren Experimenten wurde von Lobban (1998) ein Hinweis auf eine weitere, strukturell nicht identifizierte Phase, die sog. „Yellow Phase“ gefunden. Diese wurde durch nicht isobares Kühlen der flüssigen Probe bei p 5.5kbar von Raumtemperatur auf $\mathrm{T} \sim 263 \mathrm{~K}$ mit einer Kühlrate von $\sim 0.16 \mathrm{~K} / \mathrm{min}$ nukleiert.

Im Falle der metastabilen Eisphasen IV und XII belegen die Nukleationsexperimente, dass die Präparation aus der flüssigen Phase, die prinzipiell nicht unterschiedlich zu denen der als stabil bezeichneten Eisphasen III oder V ist, signifikante Unterschiede aufweist. Aus den bisher in der Literatur beschriebenen Experimenten kann abgeleitet werden, dass Druckänderungen sowie Kühlraten bei nicht isobarem Kühlen einen Einfluss auf die Herstellungswahrscheinlichkeit der metastabilen Eisphasen haben. Ebenso ist die Verwendung von Nukleatoren in einigen Fällen, z.B. bei der Herstellung aus der flüssigen Phase, von Bedeutung. 
Koza et al. (1999) konnten das Eis XII auch über die feste Phase durch schnelle Kompression von Eis Ih bei Temperaturen von $\mathrm{T} \sim 77 \mathrm{~K}$ von $\mathrm{p} \sim 1$ bar auf bis $\mathrm{zu} p \sim 20 \mathrm{kbar}$ mit Kompressionsraten von $>1-2 \mathrm{kbar} / \mathrm{min}$ erzeugen. Die untere Grenze, bei der das Eis XII über diese Art der Präparation erzeugt werden konnte, lag bei p 12kbar (Koza, pers. Mitteilung (1999)). Die metastabilen Eisphasen IV und XII existieren im wesentlichen in demselben Druck- und Temperaturbereich wie Eis V und Eis VI.

Die bisherigen Erfahrungen zeigen auch wenn sie zeitlich weit auseinander liegen und in der Literatur wenig präzise beschrieben sind, dass die Herstellung der metastabilen Eisphasen nicht beliebig reproduzierbar ist. Eine Identifizierung mittels der pVT-Apparatur war nur zum einen über die bei der Phasenumwandlung stattfindende Volumen- und der damit korrelierten Druckänderung, zum anderen über die Bestimmung der Schmelzkurve möglich. Für eine strukturelle Bestätigung der beiden metastabilen Eisphasen musste jedoch zum einen ein Verfahren zur gezielten Nukleation erarbeitet und zum anderen unter diffraktometrischer Kontrolle die mit der pVT-Apparatur bestimmten Schmelzkurven der vermuteten metastabilen Eisphasen verifiziert werden.

Basierend auf den Präparationsrouten von Lobban et al. (1998), Evans (1967) sowie von Engelhardt \& Whalley (1972) wurden daher Experimente zur Herstellung der metastabilen Eisphasen IV und XII durchgeführt. Für die von den Autoren beschriebenen Präparationsverfahren ergaben sich in je 20 Versuchen kein Hinweis auf die Herstellung der metastabilen Eisphasen IV/XII. Die nukleierten Eisphasen wurden über Schmelzkurvenbestimmung als zumeist ( 95\%) Eis V bzw. bei Drücken oberhalb von p 5.6kbar in einigen Fällen $(\sim 5 \%)$ als Eis VI identifiziert. Das aus der flüssigen Phase nukleierte Eis VI lag unter diesen Druckbedingungen metastabil in dem Stabilitätsbereich von Eis V vor. Die Druckänderungen bei der Nukleation von Eis V lagen typischerweise in der Größenordnung von $\Delta p \sim 230 \pm 30$ bar, für das Eis VI $\Delta p \sim 420 \pm 45$ bar.

Aufgrund der Tatsache, dass die in der Literatur beschriebenen Herstellungsverfahren nicht zu der gewünschten häufigen Nukleation von Eis IV bzw. Eis XII führten, wurden diese hinsichtlich Ausgangsdruck, Druckänderungsraten bei Dekompression/Kompression, Ausgangstemperatur sowie Temperaturänderungsraten, Masse der Probe sowie Massenprozent des verwendeten Nukleators (TCPA, aber auch Quarzwolle) variiert. 
Aufgrund der schlechten Kontrollierbarkeit der Dekompressionsraten durch die manuelle Einstellung der Druckablassventile, d.h. durch die nur ungenügende Möglichkeit variable Druckänderungen mit einer hinreichenden Genauigkeit zu realisieren, wurde die Präparation über die flüssige Phase unter Abkühlung bevorzugt verwendet. Als problematisch stellte sich hier auch die Realisierung variabler, hoher Kühlraten heraus. Die Realisierung dieser war aber aufgrund der Hinweise auf unterschiedliche Kühlraten zur Herstellung von Eis IV, von Eis XII (Lobban Thesis (1998)) von essentieller Bedeutung (vergl. Kapitel 2.1).

In einem von ca. 90 durchgeführten Nukleationsversuchen mit unterschiedlichen Ausgangsbedingungen hinsichtlich der o.g. Parameter, in denen zudem Druck- und Temperaturprofile (Variation des Druckes zwischen p 4.5Kbar bis p 6.5kbar, Badendtemperaturvariation zwischen $\mathrm{T} \sim-15^{\circ} \mathrm{C}$ bis $\mathrm{T} \sim-40^{\circ} \mathrm{C}$ ) variiert wurden, konnte bei einem Druck von $\mathrm{p} \sim 5.9 \mathrm{kbar}$ und einer voreingestellten Temperatur des Flüssigkeitsbades von $\mathrm{T} \sim-22.6^{\circ} \mathrm{C}$ kurzzeitig eine Eisphase nukleiert werden, die über die höhere Druckänderung von $\Delta \mathrm{p} \sim 330 \pm 30$ bar zumindest nicht als Eis V oder Eis VI identifiziert werden konnte. Nach etwa einer Minute wandelte sich diese Phase, angezeigt durch einen Druckanstieg von $\Delta \mathrm{p} \sim 96 \mathrm{bar}$, wieder um. Diese Umwandlung wurde durch das weitere Kühlen der Probe auf Temperaturen $\mathrm{T}<-23^{\circ} \mathrm{C}$ verursacht. Diese Eisphase konnte nach der Umwandlung durch die darauffolgende Schmelzkurvenbestimmung als Eis V identifiziert werden. Bei diesem Versuch wurde die HD-Zelle mit $\mathrm{V} \sim 1.5 \mathrm{ml}_{2} \mathrm{O}$ und einem Massenanteil des Nukleators TCPA von $\sim 5 \%$ befüllt und der Anfangsdruck auf die flüssige Probe auf $\mathrm{p} \sim 6.2 \mathrm{kbar}$ eingestellt. Danach wurde der HD-Stick in das auf T $\sim 22.6^{\circ} \mathrm{C}$ vorgekühlte Flüssigkeitsbad gestellt. Bei dem nicht isobaren Kühlen der Probe von Raumtemperatur auf die eingestellte Badtemperatur erfolgte dann die Nukleation einer nicht identifizierten, jedoch aufgrund der Druckänderungen zu vermutenden Eisphase XII oder IV.

In vier von vier weiteren Versuchen konnte die als Eis XII / IV vermutete Phase nukleiert werden, wobei die Ausgangsbedingungen, wie Druck, Temperaturen (Bad, Raumtemperatur), Massenanteil des Nukleators TCPA und Füllmenge an $\mathrm{D}_{2} \mathrm{O}$, annähernd vergleichbar waren. In diesen Versuchen zeigte sich, dass die flüssige Wasserprobe mit einem mittleren Flüssigkeitsvolumen von $\mathrm{V} \sim 1.5 \mathrm{ml}$ bis maximal $\mathrm{V} \sim 2 \mathrm{ml}$ bei einem Druck um die $\mathrm{p} \sim 5.6 \mathrm{kbar}$ bis p 6.2kbar Gasdruck mit einer wohldefinierten Kühlrate asymptotisch von Raumtemperatur auf eine Endtemperatur von $\mathrm{T} \sim-22.5^{\circ} \mathrm{C}$ gekühlt werden musste. Nach Erreichen der 
Endtemperatur erfolgte die Nukleation zumeist nach $t \sim 15$ bis $t \sim 20$ Minuten. Langsameres Abkühlen, gekoppelt durch eine niedriger eingestellte Badtemperatur, forcierte in den durchgeführten Experimenten die Bildung von Eis V. Bei höheren Ausgangsdrücken von $\mathrm{p} \geq 6.5 \mathrm{kbar}$ und einem Unterschreiten der Temperatur von $\mathrm{T} \sim-22.6^{\circ} \mathrm{C}$ konnte in einzelnen Fällen Eis VI nukleiert werden (zum Abkühlungsverfahren vergl. Kapitel 2.1.1).

Nachdem die Phase mehrmals gezielt nukleiert werden konnte, wurde mit diesen Proben die Schmelzkurve in einem weiten Temperaturbereich vermessen, so dass diese Phase über einen Zeitraum von mehreren Stunden stabil blieb. Auch konnte bei diesen Experimenten zu tieferen Drücken hin eine fest-fest-Phasengrenze, vermutlich Eis IV / XII zu Eis V, bestimmt werden (vergl. Kapitel 4.3.3, Abbildung 4.6). Ebenso konnte festgestellt werden, dass sich diese Phase bei Drücken von p 5.0kbar bis p 5.3kbar bei weiterem Kühlen unter Temperaturen von $\mathrm{T} \sim 260 \mathrm{~K}$ in Eis V umwandelt. Ausgehend von diesen Ergebnissen, wurden weitere Versuche durchgeführt um dieses Verfahren weiter zu verifizieren. Die Auswertung sowohl der in Göttingen als auch der am D2B des ILL sowie am GEM des ISIS durchgeführten Nukleationsexperimente $(\mathrm{n} \sim 35)$, aus denen die Druck- sowie Temperaturänderungsraten $\frac{d p}{d t}, \frac{d T}{d t}$ bestimmt wurden, ergaben im Rahmen der bestimmten Fehlergrenzen (bedingt durch Probenprärationen mit unterschiedlichen Probenvolumina, unterschiedlichen Gesamtvolumina der Druckerzeugersysteme, Unterschiede im möglichen Ausgangsdruck der Gasdruckapparaturen sowie durch unterschiedliche Ausgangsdrücke durch Raumtemperaturschwankungen) keinen deutlich signifikanten Zusammenhang der Druck- und Temperaturänderungen. Als systematisch ergab sich jedoch für die Nukleation von Eis IV/XII ein unterer Grenzdruckbereich sowie eine untere Grenztemperatur, bei der diese Phase oberhalb dieser Grenzen mit hoher Wahrscheinlichkeit nukleiert werden konnte. Der Grenzdruckbereich konnte aus den Experimenten zu etwa p 5.2kbar bis minimal $\mathrm{p} \sim 4.9 \mathrm{kbar}$ nach der vollzogenen Phasenumwandlung sowie die Endtemperatur $\mathrm{zu} \mathrm{T} \sim-22.6^{\circ} \mathrm{C}$ bestimmt werden. Bei einem Unterschreiten der empirisch ermittelten Druck- und Temperaturwerte erfolgte nach einigen Sekunden bis maximal zwei Minuten eine Umwandlung in das bekannte Eis V. Als die wesentlichsten Parameter, die in diesen Experimenten verifziert und bestimmt werden konnten, ergaben sich die apparativ an die Badtemperatur gekoppelte Kühlrate sowie der maximale Endruck nach Erreichen des thermischen Gleichgewichtes nach vollzogener Phasenumwandlung. 
Basierend auf diesem ausgearbeiteten Präparationsverfahren wurden Nukleationsversuche am D2B des ILL in Grenoble sowie am GEM, ISIS des RAL in Didcot durchgeführt. Am D2B konnte die nukleierte Eisphase in sechs von sechs Versuchen als das von Lobban et al. (1998) entdeckte Eis XII identifiziert werden. Die mittlere Masse der Proben lag bei $\mathrm{V} \sim 2 \mathrm{ml} \mathrm{D}_{2} \mathrm{O}$ mit einem massenprozentigen Anteil des TCPA-Nukleators von 5-10\%. Die Nukleationszeiten lagen nach Erreichen der Endtemperatur zwischen $\mathrm{t} \sim 10$ Minuten und maximal $\mathrm{t} \sim 45$ Minuten. Die Nukleationsversuche am GEM (ISIS) waren im Gegensatz dazu nicht in diesem Maße erfolgreich. Als Ursache sind apparative Unterschiede in den verwendeten Druckzellen sowie der Druckerzeugeranlage anzuführen. Zum einen ergaben sich durch andere Druckzellenabmessungen und damit unterschiedlicher Masse der Zellen unterschiedliche Kühlraten, die während des Experimentes durch Änderungen in der Badtemperatur angepasst werden mussten. Ebenso konnte aus Sicherheitstechnischen Aspekten lediglich ein Maximaldruck von knapp über p 5.2kbar realisiert werden, der nach dem Erreichen der Endtemperatur unter dem notwendigen unteren Grenzdruck von $\mathrm{p} \sim 4.9 \mathrm{kbar}-5.2 \mathrm{kbar}$ lag. Als wesentlicher Faktor stellte sich hier das Verhältnis von Umwandlungsvolumen der Probe zum Totvolumen des HD-Sticks heraus. Um das für die Phasenumwandlung benötigte Gasvolumen zu realisieren wurde beim Abkühlvorgang der Druck ständig nachgefahren, um diesen bei über p 5.2kbar minimal, d.h. oberhalb des Grenzdruckes, zu halten. Durch die Druckerhöhung sowie die abgeänderte Badtemperatur auf etwa $\mathrm{T} \sim-20.5^{\circ} \mathrm{C}$ konnte die Eisphase XII am GEM (ISIS) mit einer Nukleationswahrscheinlichkeit von 4 aus 12 Versuchen hergestellt werden. Die mittlere Masse der Probe lag wie beim D2B bei V 1.5ml bis $\mathrm{V} \sim 2 \mathrm{ml}$ und einem Nukleatoranteil von 5-10\% an der Masse. Auch wurde in einem Fall die Probenmasse von $\mathrm{V} \sim 1.5 \mathrm{ml}$ auf etwa $\mathrm{V} \sim 0.64 \mathrm{ml} \mathrm{D}_{2} \mathrm{O}$ verringert, damit das Totvolumen, d.h. das im Stick befindliche Gasvolumen erhöht wird; der Massenanteil des Nukleators wurde angepasst. In diesem Falle konnte durch die geringe Masse unter Verwendung des ausgearbeiteten Präparationsverfahren durch nicht isobares Kühlen der Probe von p 5.2kbar bei einer eingestellten Badtemperatur von $\mathrm{T} \sim-20.5^{\circ} \mathrm{C}$ in einem einzigen Fall Eis IV nukleiert werden. Das Eis IV konnte in situ bei p $\sim 5$.2kbar und T $\sim 260.5 \mathrm{~K}$ beobachtet werden. Bei dem Versuch einige Schmelzpunkte der überraschend nukleierten Eisphase IV (bei Erwärmung) zu bestimmen, vollzog das Eis IV eine Phasenumwandlung in Eis XII, das sich nach einigen wenigen Minuten in Eis V umwandelte. Ein Masseneinfluss auf die Bildungswahrscheinlichkeit von Eis IV, wie von Engelhardt \& Whalley (1972) beschrieben, scheint durch diesen Versuch bestätigt. 


\section{Stabilitätsgrenzen der Eisphasen}

Wasser in seiner flüssigen und kristallinen Form kann mit Gasen auf vielfältige Weise wechselwirken. Neben den unter Normaldrücken sowie bis zu hohen Drücken auftretenden Gaslöslichkeiten in Wasser, werden aufgrund der Wasser- und Gas-Wechselwirkungen bei hohen Drücken Ausbildungen von neuen Van der Waals-Verbindungen vermutet. Von van Hinsberg (1994, 1999) wird eine derartige neue Struktur im System Wasser und Stickstoff, von Voss et al. (1993) ebenso im System Wasserstoff und Wasser vermutet.

Ebenso ist der Einbau von Gasen in kristalline Wasserstrukturen, wie z.B. bei Eis Ih oder die Ausbildung eisverwandter Strukturen wie den Gas-Hydraten möglich. Diese lassen sich in zwei Gruppen, die Clathrat-Hydrate und die Gas-Hydrate einordnen. Clathrat-Hydrate entstehen durch den Einbau von Gasen in eine abgeschlossene Struktur aus Wasserkäfigen, in denen je nach Gasfugazität Gasatome oder Moleküle von einer Wasserwirtsstruktur umbaut werden. Im Gegensatz dazu werden bei Gas-Hydraten die Gasatome oder Moleküle nicht von einer Wasserstruktur eingebaut, sondern können entsprechend ihrer Gasfugazität bei gegebenen Druck- und Temperaturbedingungen in der Wasserwirtsstruktur adsorbiert oder desorbiert werden, ohne dass es notwendig ist, die eisverwandte Wirtsstruktur zu zerstören. Die Gase sind vielmehr in einer Wasserwirtsstruktur eingebunden und gelöst, in die sie durch Diffussionsprozesse gelangen. Der Unterschied zwischen Gas-Hydraten und ClathratHydraten besteht prinzipiell in der Möglichkeit eines freien Gasaustausches in der Struktur (Kuhs, persönliche Mitteilung (2000)).

Erste Hinweise bzw. experimentelle Beweise von Wechselwirkungen von Gasen mit Eisphasen, d.h. der Ausbildung von Clathrat-Hydraten wurden von Davy (1810) geführt. Dieser entdeckte ein Chlor-Hydrat, dass sich aus Chlorgas und Wasser bildete. De Forcrand \& Villard (1888) wiesen erstmals nach, dass sich diese neue Verbindung auch in Wechselwirkung von Wasser mit Kohlenwasserstoffen wie Methan und Äthan bildete. Die Untersuchung dieser Verbindungen, insbesondere die Bildungsbedingungen sowie die Bildungspartner wurden von Deaton \& Frost (1939) mit Wasser und verschiedenen Reinstgasen sowie Erdgasen durchgeführt. 
Ergebnis dieser Experimente war, dass Atmosphärengase mit Wasser bzw. Eis unter wohl definierten Druck- und Temperaturbedingungen in Wechselwirkung treten und eine bis dahin unbekannte, neue Verbindung, das Gas-Hydrat (Clathrat-Hydrat) bildeten. Erst von Stackelberg (1954) konnte die Gas-Hydrate mittels röntgenographischer Beugungsmethoden in zwei kristallographisch unterschiedliche Strukturen, in Clathrat-Hydrat vom Typ I und Typ II, unterscheiden (vergl. Abbildung 4.1, Kapitel 4.1.1).

\subsubsection{Eisverwandte Strukturen : Clathrat-Hydrate}

Clathrat-Hydrate vom Typ I, Raumgruppe $\operatorname{Pm} \overline{3} \mathrm{n}$, besitzen eine kubische Elementarzelle, die aus zwei kleinen Käfigen sowie sechs großen Käfigen aufgebaut ist. Die kleinen Käfige bestehen aus jeweils zwölf pentagonalen, die großen Käfige bestehen aus zwölf pentagonalen und zwei hexagonalen Oberflächen. Die Elementarzelle für Clathrat-Hydrat Typ I enthält 46 Wassermoleküle, die Gitterkonstante beträgt $\mathrm{a}_{0} \sim 12 \AA$. Bei der Bildung dieser Käfige werden die Gasmoleküle in die sich aufbauenden Wasserkäfige eingelagert. Wie der Aufbau der Clathrat-Hydratstruktur in einer Eismatrix vonstatten geht, ist im Detail nicht geklärt, jedoch scheinen bei dem topologischen Umbau Diffusions-, Sublimations- sowie Kondensationsprozesse von Wassermolekülen, vorzugsweise an Strukturdefekten der EisAusgangsphase ansetzend, eine Rolle zu spielen (Arbeiten hierzu: Sloan and Fleyfel (1992), Salamantin et al. (1998), Kuhs et al. (1999), Kuhs et al. (2000)). Durch den Umbau ergibt sich so ein eisverwandtes Netzwerk aus Wasserkäfigen, in denen die Gasmoleküle eingelagert sind, die diese Struktur thermodynamisch erst stabilisieren. Gasmoleküle, die in der ClathratHydratstruktur eingebaut werden, sind in dem kleinen Käfig von $n=20$ und in dem großen Käfig von $n=24$ Wassermolekülen umgeben. Die Typ I-Struktur hat für den kleinen Käfig einen freien Durchmesser im Innern des Käfigs von etwa d 5.03 und für den großen Käfig von $\mathrm{d} \sim 5.86 \AA$. Das Clathrat-Hydrat vom Typ II, Raumgruppe $\mathrm{Fd} \overline{3} \mathrm{~m}$ besitzt ebenfalls eine kubische Struktur mit einer Gitterkonstanten von $\mathrm{a}_{0} \sim 17.2 \AA$, besteht jedoch aus $\mathrm{n}=16$ kleinen sowie acht großen Käfigen mit zwölf pentagonalen und vier hexagonalen Oberflächen. In der Elementarzelle vom Typ II befinden sich n=136 Wassermoleküle. Die Koordinationszahl für den kleinen Käfig ist wie beim Typ I n=20, für den großen Käfig jedoch $n=28$. Die freie

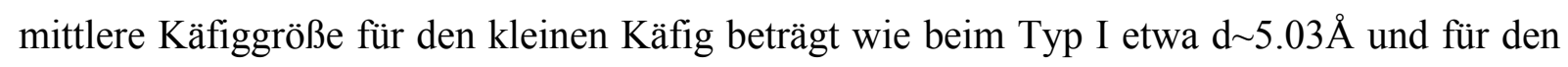
großen Käfig d 6.57Å. Nach Davidson et al. (1978) sowie Ripmeester et al. (1994) gehören 
Clathrat-Hydrate $\mathrm{zu}$ den wahren Einschlussverbindungen, bei denen das eingelagerte Gasmolekül oder Atom keine chemische Verbindung mit der Wasserstruktur eingeht.

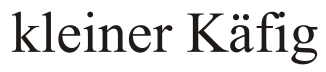

Struktur I

Struktur II
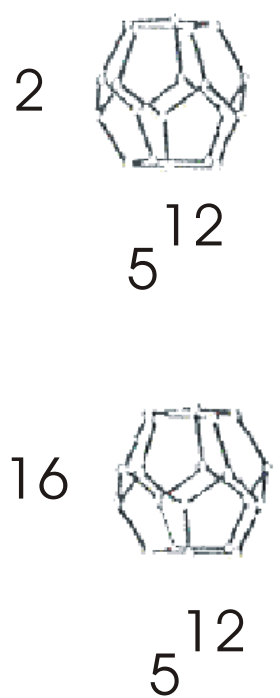

$+8$

großer Käfig
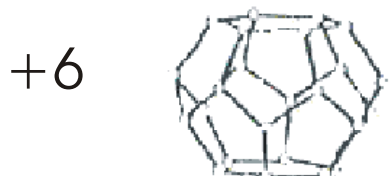

$5^{12} 6^{2}$

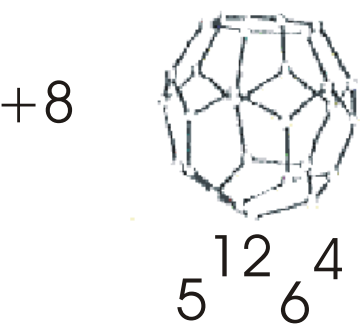

\begin{abstract}
Abbildung 4.1: Beide Clathrat-Hydratstrukturen, Typ I und Typ II, bestehen jeweils aus dem kleinen Käfig, bestehend aus jeweils 12 pentagonalen Flächen sowie den großen Käfig für Struktur I aus 12 pentagonalen sowie 2 hexagonalen, für Struktur II aus 12 pentagonalen und vier hexagonalen Oberflächen. Die Käfige bzw. die begrenzenden Oberflächen der Käfige werden durch Wassermoleküle, die 4-fach durch Wasserstoffbrückenbindungen in der Struktur gebunden sind, gebildet. Für beide Strukturtypen sind die Käfige in einer kubischen Einheitszelle angeordnet.
\end{abstract}

Die Gase $\mathrm{N}_{2}, \mathrm{O}_{2}$ sowie Ar bilden ein Clathrat-Hydrat vom Strukturtyp II, die Gase $\mathrm{CH}_{4}, \mathrm{CO}_{2}$ sowie Xe bilden ein Clathrat-Hydrat vom Strukturtyp I.

Untersuchungen der Besetzung der Käfige haben gezeigt, dass die Clathrat-Hydrate nichtstöchiometrische Verbindungen sind und die Besetzung der Käfige zum einen von der Größenrelation der Käfige zu dem des Gasatoms oder Gasmoleküls sowie den Druck- und Temperaturbedingungen bei der Bildung des Clathrat-Hydrates abhängt. Einen Überblick über die Größenverhältnisse von Gasen sowie den Clathrat-Hydrattypen I und II wird in der Arbeit von Nixdorf (1996) und in dem Buch von E.D.Sloan (1997) gegeben. Eine statistisch thermodynamische Theorie der Clathratbildung wurde erstmals durch Van der Waals \& Platteeuw (1959) gegeben, in dem die thermodynamischen Eigenschaften und die Bildung der Clathrat-Hydrate durch ein einfaches festes Lösungsmodell beschrieben werden. Dieses liefert als Funktion der Fugazität des verwendeten Gases den Füllgrad der kleinen und großen Käfige. Der Füllgrad, d.h. die Anzahl der Moleküle in den Clathrat-Hydratkäfigen für den 
jeweiligen Käfig, wird, basierend auf der Theorie von Van der Waals \& Platteeuw (1959) durch die Langmuir-Konstante $\mathrm{C}_{\mathrm{i}}$ beschrieben:

$$
y=\frac{C_{i} \cdot p}{\left(1+C_{i} \cdot p\right)}
$$

Bei Kenntnis der Wechselwirkungspotenziale von Wasser und dem verwendeten Gas kann die Langmuir-Konstante errechnet werden. Aufgrund des experimentell bestimmbaren Füllgrades von Clathrat-Hydraten ist somit ein Vergleich der Füllgrade gegeben und stellt einen kritischen Test für die verwendeten molekularen Wechselwirkungspotenziale dar. Systematische Arbeiten an Clathrat-Hydraten hinsichtlich der Struktur, des Füllgrades sowie der Synthesebedingungen wurden von Kuhs et al. (1997), Klapproth et al. (1999) sowie Chazallon (1999) an Stickstoff und Sauerstoff-Clathrat-Hydraten sowie durch Lobban et al. (1996) an Argon-Clathrat-Hydraten durchgeführt.

\begin{tabular}{|c|c|c|c|c|}
\hline $\mathrm{p}[\mathrm{bar}]$ & $\mathrm{f}[\mathrm{bar}]$ & $\mathrm{y}_{\mathrm{sc}} /[\%]$ & $\mathrm{y}_{\mathrm{lc}}[\%]$ & $\mathrm{y}_{\text {ges. }}[\%]$ \\
\hline 148 & 142 & $84.6(6)$ & $97.8(6)$ & $89.0(8)$ \\
\hline 160 & 154 & $81.9(6)$ & $98.4(6)$ & $87.4(8)$ \\
\hline 200 & 193 & $82.2(3)$ & $99.6(6)$ & $88.0(7)$ \\
\hline 300 & 297 & $86.4(3)$ & $102.6(6)$ & $91.8(7)$ \\
\hline 543 & 631 & $91.5(3)$ & $107.4(6)$ & $96.8(7)$ \\
\hline 750 & 1041 & $96.0(3)$ & $111.6(6)$ & $101.2(7)$ \\
\hline 1000 & 1797 & $96.3(3)$ & $117.0(6)$ & $103.2(7)$ \\
\hline
\end{tabular}

Tabelle 4.1: Mittels Rietveld-Verfeinerungen aus synthetisierten $\mathbf{N}_{2}$-Clathrat-Hydraten bestimmte Besetzungen y des kleinen $\left(y_{s c}\right)$ und den großen Käfigs $\left(y_{\mathrm{lc}}\right)$ (Chazallon (1999)). Die Besetzung des kleinen und des großen Käfigs als Funktion des Druckes für Stickstoff-Clathrat-Hydrat verlaufen unterschiedlich.

Es zeigte sich in Messungen von synthetisierten Stickstoff-Clathrat-Hydraten mittels Neutronenstreuexperimenten, dass die Füllgrade bis zu Drücken von p 1kbar eine gute Übereinstimmung mit dem Langmuir-Verhalten auf der Grundlage der Theorie von van der Waals \& Platteeuw (1959) zeigen, im einzelnen aber Hinweise auf signifikante Abweichungen gegeben sind (vergl. Tabelle 4.1). Die Abweichungen zeigten für den großen Käfig eine signifikante Korrelation mit steigendem Gasdruck. Für Drücke oberhalb p 200bar zeigten sich für mit Stickstoff synthetisierten Clathrat-Hydrat Abweichungen, resultierend in einem erhöhten Füllgrad als von den theoretischen Vorhersagen des Modells beschrieben (vergl. Kuhs et al. (1998)). Ursache hierfür ist eine Doppelbesetzung des großen Clathrat- 
Hydrat-Käfigs, die durch Rietveld-Strukturanalysen der Neutronendaten nachgewiesen wurde und die im Widerspruch zu der von Van der Waals \& Platteeuw (1959) ursprünglich formulierten Theorie steht. Diese lässt pro Käfig nur die Besetzung mit einem Gasmolekül zu. Der experimentell beobachtete Füllgrad kann aber durch die Einführung und empirische Anpassung einer weiteren Langmuir-Konstanten $\mathrm{C}_{\mathrm{ii}}$ für die doppelte Besetzung, beschrieben werden (Kuhs et al. (1997)).

$$
y_{i i}=\frac{C_{i} \cdot p}{\left(1+C_{i} \cdot p+C_{i} \cdot C_{i i} \cdot p^{2}\right)}
$$

Die Beschreibung über eine weitere Langmuir-Konstante für den Fall von KäfigDoppelbesetzungen trägt aber in keiner Weise der druckabhängigen Änderung der Käfiggeometrie Rechnung. Der mit der Fugazitätszunahme verbundene mechanische Druck dürfte aber zu einer Verkleinerung der Gitterkonstanten und damit der Käfige führen, die dem Langmuir-Modell einer Druck unabhängigen Geometrie der Sorptionsplätze widerspricht. Der zunehmende Einbau von Gasmolekülen insbesondere im Fall einer Doppelbesetzung wirkt dem entgegen. Es kann daher davon ausgegangen werden, dass die Größenänderungen für den kleinen Käfig als Funktion des steigenden Druckes (oder fallender Temperatur) schneller erfolgt als für die großen Clathrat-Hydratkäfige (vergl. Tabelle 4.1). Der Effekt der Druckabhängigkeit der Käfigdurchmesser wurde von Chazallon et al. (1999) für StickstoffClathrat-Hydrat für den Druckbereich $\mathrm{p}<1 \mathrm{kbar}$ nachgewiesen. Eine anisotrope druckmechanische Verformung der Käfigstrukturen ist die Folge. Ein analoges Verhalten ist für Argongas aufgrund der Experimente von Lobban et al. (1999) ebenfalls zu erwarten. Die Clathrat-Hydrat-Synthesen wurden für Argon im Druckbereich von p 90bar bis maximal p 4.8kbar (Lobban, pers. Mitteilung, (1999)) sowie für Stickstoff von p 160bar bis maximal p 2.8kbar (Chazallon et al. (1999)) durchgeführt. Weitergehende Kristallstrukturanalysen der mit Argon- und Stickstoffgas synthetisierten Clathrat-Hydrat Datensätze in dem Druckbereich $\mathrm{p} \geq 1 \mathrm{kbar}$ sind nicht durchgeführt worden, da ein geeignetes Modell für den Besetzungsgrad sowie für die Position der Gasmoleküle bzw. Gasatome im großen Käfig noch nicht durch MD-Simulationen (Klapproth et al. (1999)) oder durch Rietveld-Verfeinerungen guter experimentell erfasster Datensätze verifiziert ist. Ein Vorschlag für ein Strukturmodell im Falle der Doppel-Besetzung des großen Käfigs mit den Gasen Ar und $\mathrm{N}_{2}$ existiert jedoch und wird in den Verfeinerungen des Argon-Clathrat-Hydrates verwendet. Nur wenige Arbeiten (z.B. Van Hinsberg (1999); Dyadin et al. (1991); Hirai et al. (2000)) beschäftigten sich bisher 
mit der systematischen Untersuchung von Gas-Hydraten bei Drücken oberhalb von $\mathrm{p} \sim 1 \mathrm{kbar}$. Angaben oder Details zur Struktur oder zum Füllgrad wurden in diesen Arbeiten nicht veröffentlicht.

\subsubsection{Eisverwandte Strukturen : Gas-Hydrate}

Experimentelle Hinweise auf die Ausbildung von Gas-Hydraten, mit Gasen, die einen kleinen Wechselwirkungsradius (z.B. molekularer Wasserstoff $\mathrm{H}_{2}$, Helium $\mathrm{He}$ sowie Neon $\mathrm{Ne}$ ) besitzen, ergaben sich durch Schmelzkurvenexperimente von Namiot \& Bukhgalter (1965). Dyadin et al. (1994) konnte unter Verwendung dieser Gase den Einfluss auf die Lage der Schmelzkurve von Eis Ih als Funktion des Druckes bestimmen. Die Schmelzkurven wurden von Dyadin et al. (1994) bis zu Drücken bis etwa p 2.5kbar Gasdruck verfolgt und zeigen eine deutliche Verschiebung der festen Phase zu höheren Drücken und Temperaturen (vergl. Kapitel 4.3.1, Abbildung 4.5). Londono et al. (1989, 1994) konnte durch Neutronendiffraktionsexperimente an Eis II unter der Verwendung von Helium als Drucküberträger einen signifikanten Einfluss auf die Eisstruktur als Funktion der Gasbesetzung feststellen. In der Abbildung 4.2 ist die von Londono et al. bestimmte Eis IIHydratstruktur dargestellt.
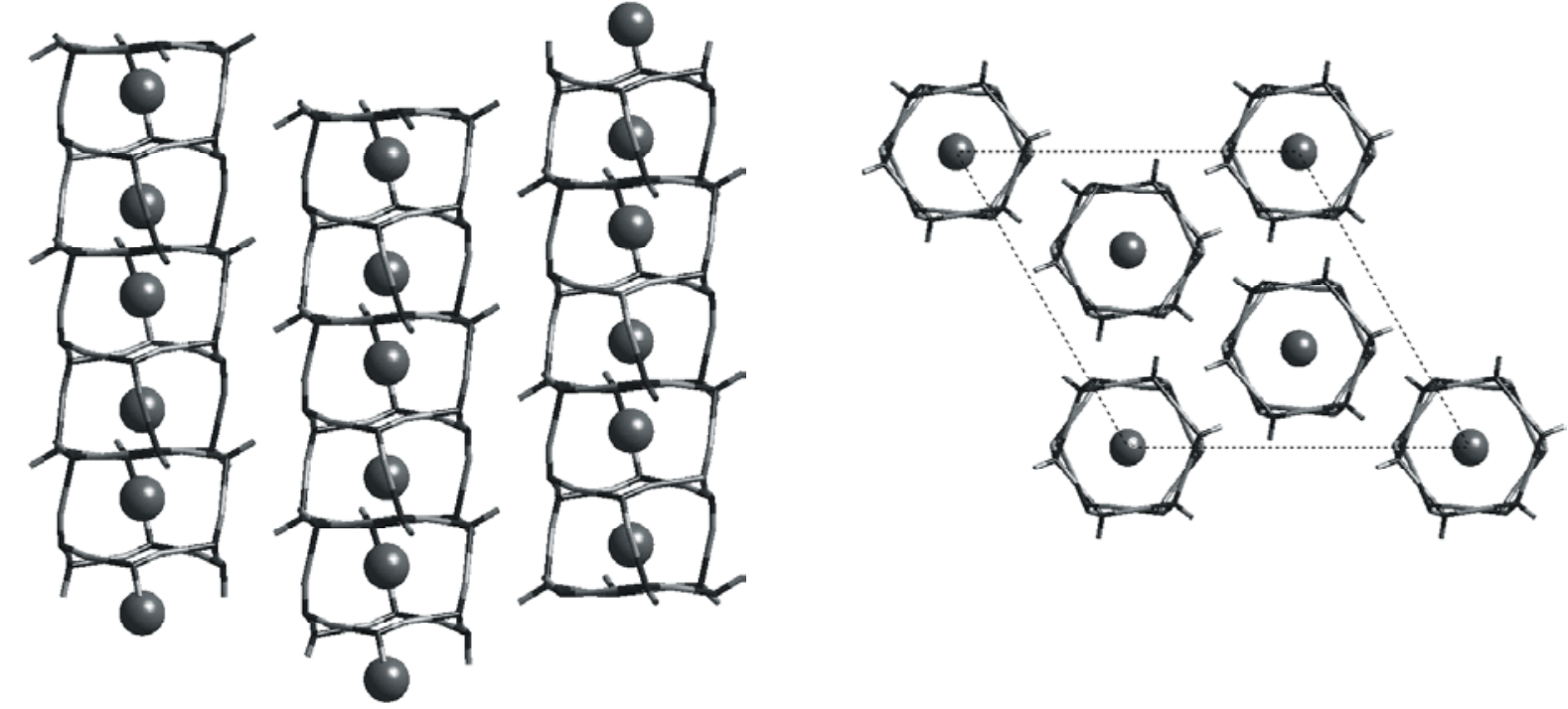

Abbildung 4.2 : Eis II-Hydratstruktur nach Londono et al. (1994), (links) : hexagonale c-Kanäle, aufgebaut aus jeweils wechselnden ,puckered rings“ und „flat rings“, (rechts) : Projektion der hexagonalen Kanäle entlang der c-Achse, Kugeln : eingebaute Gasatome, die sich jeweils ober- und unterhalb eines Wassermolekülringes befinden. Der Übersichthalber sind die Wasserstoffbrücken zwischen den Kanälen nicht dargestellt. Aus Lobban (1998). 
Lobban (1998) konnte unter der Verwendung von Argongas als Drucküberträgermedium keine Gasbesetzung in der Eis II-Struktur feststellen. Der strukturelle Unterschied von Eis II zu dem Eis II-(He)-Hydrat ist schematisch in der Abbildung 4.3 dargestellt.
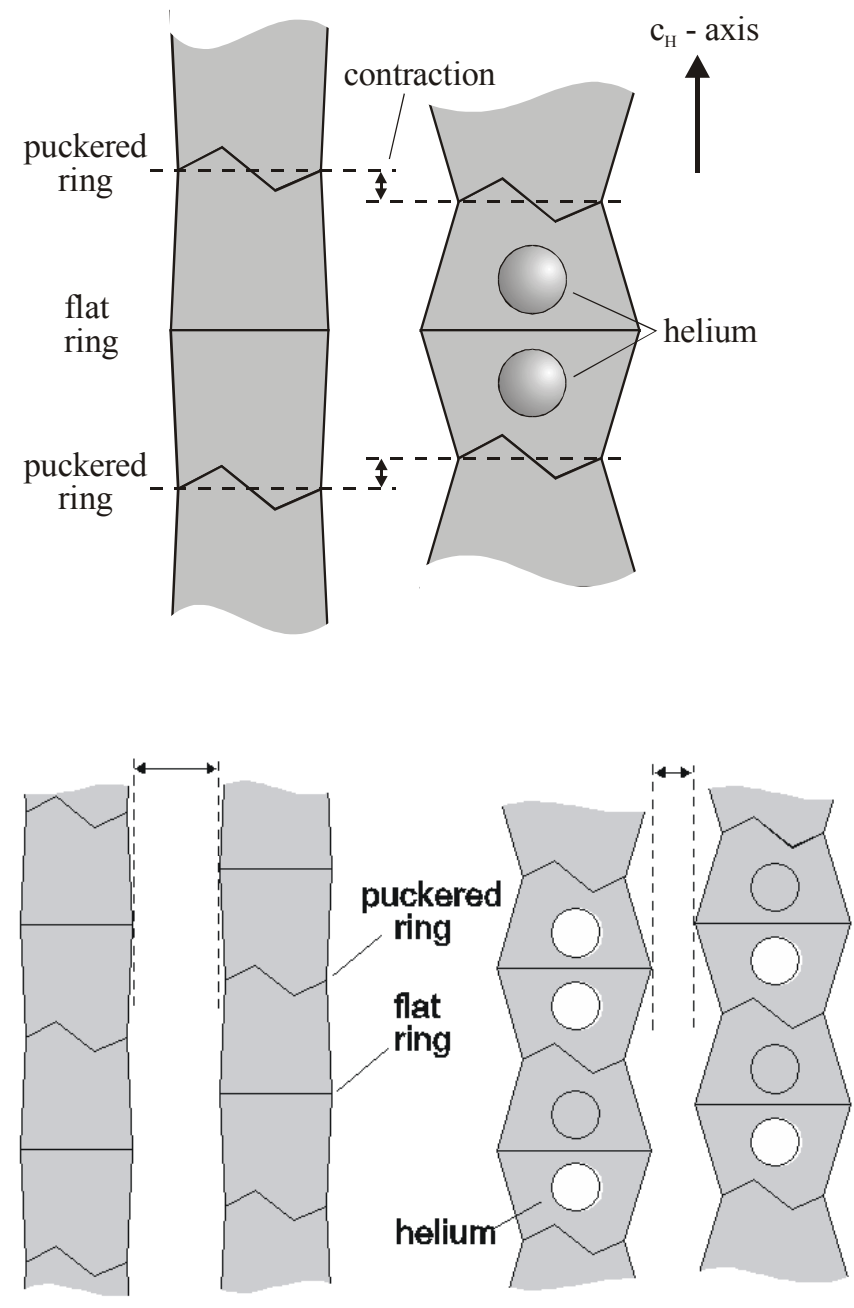

\begin{abstract}
Abbildung 4.3: (oben): Aufweitung der sechseckigen, parallel zur c-Achse verlaufenden Kanäle im Eis II bei Heliumgasbesetzung. In der Abbildung (oben) sind zwei parallele benachbarte Kanäle dargestellt, oben links ohne Helium, oben rechts mit Heliumeinschluss. Die Kanäle werden bei Gaseinschluss ungleichmäßig aufgeweitet und nähern sich dabei aneinander an, wobei jedoch die Gitterkonstante a, b eine nur geringe Änderung zeigt. (unten) : durch Gasbesetzung findet eine Kontraktion der Kanäle in cAchsenrichtung von Eis II bei Heliumeinschluss statt. (links unten): Kanal ohne Heliumbesetzung, (rechts unten) : die Verkürzung der c-Achse ist mit großer Wahrscheinlichkeit durch die zusätzlichen $\mathrm{He}^{-} \mathrm{H}_{2} \mathrm{O}$ Wechselwirkungen zurückzuführen. Die Wasserstoffbrückenbindungen zwischen den Kanälen sind der Übersicht halber nicht dargestellt. Aus Lobban (1998).
\end{abstract}

Äquivalente Strukturuntersuchungen an Eis Ih unter Verwendung der Gase $\mathrm{H}_{2}$, Helium sowie Neon mit dem Ziel, Kenntnisse über strukturelle Änderungen sowie der Gasbesetzung als Funktion des Druckes und der Temperatur experimentell zu verifizieren, sind soweit bekannt nicht durchgeführt worden. Ebenso wurden bisher nur wenige Arbeiten, die sich mit Gasdiffusionsprozessen an Eis beschäftigen und Aussagen über die Gasbesetzung geben 
könnten, durchgeführt und in der Literatur beschrieben worden. Bei der Verwendung von Gasen, die einen größeren Wechselwirkungsradius besitzen (z.B. Argon Ar, Stickstoff $\mathrm{N}_{2}$, Sauerstoff $\mathrm{O}_{2}$ ) ist aufgrund der Größe der Gase ein Einbau in die kristallinen Eisstrukturen von Eis Ih sowie Eis II nicht möglich.

\subsection{Das Phasendiagramm von Eis in Wechselwirkungen mit Gasen}

Beginnend bei Tammann (1900) und Bridgman (1912) wurden zur Bestimmung der Phasengrenzen mechanische Druckerzeugersysteme (Flüssigkeitsdruckzellen, PistonZylinder, Diamantstempelzellen) verwendet. Mit diesen lassen sich zwar sehr hohe Drücke bis zu mehr als $\mathrm{p} \sim 1 \mathrm{Mbar}$ realisieren, jedoch ist konstruktionsbedingt die Genauigkeit gerade im Druckbereich bis zu p 7kbar, bei denen viele Eisphasen existieren, of nicht gut genug. Gerade im Hinblick auf unterschiedliche Messgenauigkeiten hinsichtlich der Bestimmung von Drücken und Temperaturen ist die Identifizierung einer Eisphase über die etablierten Phasengrenzen mit Unsicherheiten behaftet. In dem Stabilitätsbereich von Eis V sowie im unteren Stabilitätsbereich von Eis VI werden aufgrund durchgeführter Schmelzkurvenexperimente weitere, teils nur über die Schmelzkurve identifizierte neue Eisphasen vermutet. Chou et al. (1998) konnten über einen weiten Druck- und Temperaturbereich eine Schmelzkurve einer bis dahin unbekannten Eisphase bestimmen. Hinweise auf zwei weitere unbekannte Eisphasen wurden durch die Bestimmung der Schmelzkurven von Mishima \& Stanley (1998) gegeben. Auch konnte in einem in situ Neutronenstreuexperiment in Gegenwart von Argongas als Drucküberträgermedium von Lobban (1998) eine neue Phase ("yellow Phase") bei p 5.4kbar und T 263K aus der flüssigen Phase nukleiert und strukturell identifiziert werden. Jedoch konnten keine Aussagen über fest-fest Phasengrenzen getroffen werden. Ebenso wurden auch schon frühere Hinweise auf eventuelle neue, bisher unbekannte Phasen gegeben. Bizhigitov \& Sirota (1986) fanden bei Drücken von $\mathrm{p} \sim 1 \mathrm{kbar}$ bis $\mathrm{p} \sim 2 \mathrm{kbar}$ bei Temperaturen von $\mathrm{T} \sim 100 \mathrm{~K}$ bis $\mathrm{T} \sim 120 \mathrm{~K}$ Hinweise auf einen Phasenübergang. Ein Überblick über die Schmelzkurven der bisher bestimmten bzw. vermuteten Eisphasen in dem Druckbereich von $\mathrm{p} \sim 2 \mathrm{kbar}$ bis $\mathrm{zu} \mathrm{p} \sim 12 \mathrm{kbar}$ ist in der Abbildung 4.4 gegeben. Im Vergleich zum Eisphasendiagramm aus Kapitel 1.3, Abbildung 1.5 ist $\mathrm{zu}$ ersehen, dass je nach Autor, die Schmelzkurven unterschiedliche Verläufe aufweisen. Eine detaillierte Diskussion dieser Unterschiede kann aufgrund der zumeist nicht angegebenen absoluten Kalibrierungen der Versuchsaufbauten und der nicht zu 
verifizierenden apparativen Fehlern, nur eingeschränkt geführt werden. Auch ist anzumerken, dass die Phasendiagramme von $\mathrm{H}_{2} \mathrm{O}$ und $\mathrm{D}_{2} \mathrm{O}$ unterschiedlich sind, mit oft nicht bekannten Verschiebungen der Existenzbereiche der verschiedenen Eisphasen. Für den Fall von Eis V stimmt die V-L $\mathrm{H}_{2} \mathrm{O}-$ Schmelzkurve von Bridgman(1912) mit der von Engelhardt \& Whalley (1977) $\mathrm{V}-\mathrm{L} \mathrm{H}_{2} \mathrm{O}$ sehr gut überein. Im Gegensatz dazu zeigen die V-L $\mathrm{H}_{2} \mathrm{O}$ - Schmelzkurven von Evans (1967) sowie Mishima \& Stanley (1998) wesentliche Abweichungen in dem Schmelzkurvenverläufen auf. $\mathrm{Ob}$ diese z.B. aufgrund einer ungenauen Druck- und Temperaturkalibrierung oder durch andere Effekte zustande kommen, lässt sich aus den Publikationen nicht mehr rekonstruieren. Abweichungen in den Schmelzkurven von Evans (1967), der erstmals eine organische Substanz als Nukleator (TCPA = Tetrachlorophthalicanhydrid) verwendete sowie Mishima \& Stanley (1998), der in einer Öllösung emulgierte $\mathrm{H}_{2} \mathrm{O}$-Kristallite verwendete, sind experimentell nachgewiesen. Sowohl Evans (1967) als auch Nishibata (1972) belegten experimentell, dass bei der Verwendung von organisch/chemischen Nukleatoren die Lage sowie der Verlauf der Phasengrenzen signifikant beeinflusst werden. Als Ursache für diesen Effekt ist die Schmelzpunkterniedrigung an Phasengemischen, verursacht durch Lösungseffekte anzuführen. Evans (1967) bestimmte z.B. die Schmelzkurve von Eis Ih als Funktion des Druckes und der Temperatur unter Verwendung von TCPA und konnte die Phasengrenze von $\mathrm{p} \sim 1$ bar bis ca. $\mathrm{p} \sim 3.5 \mathrm{kbar}$, oberhalb des von Tammann (1900) bestimmten Existenzbereiches verfolgen. Extrapoliert man die V-L $\mathrm{H}_{2} \mathrm{O}$ - Schmelzkurven von Chou et al. (1998) und von Engelhardt \& Whalley (1972), die in verschiedenen Druckbereichen gemessen wurden, so ist in dem Verlauf als Funktion des Druckes und der Temperatur ein Unterschied zu erkennen. Da bei diesen Messungen keine organischen Nukleatoren verwendet wurden, ist von einem apparativen Einfluss, z.B. durch Kalibrierungs-, Auflösungs- und Genauigkeitsunterschiede, auszugehen. Eine gute Übereinstimmung ergibt sich für die mit verschiedenen experimentellen Aufbauten bestimmte V-L $\mathrm{H}_{2} \mathrm{O}$-Schmelzkurve von Engelhardt \& Whalley (1972) mit der V-L BridgmanSchmelzkurve. Diese Schmelzkurve diente für die hier eigens durchgeführten Experimente als Referenzkurve (vergl. Abbildung 4.5 in Kapitel 4.3.1 sowie Abbildung 4.4). 


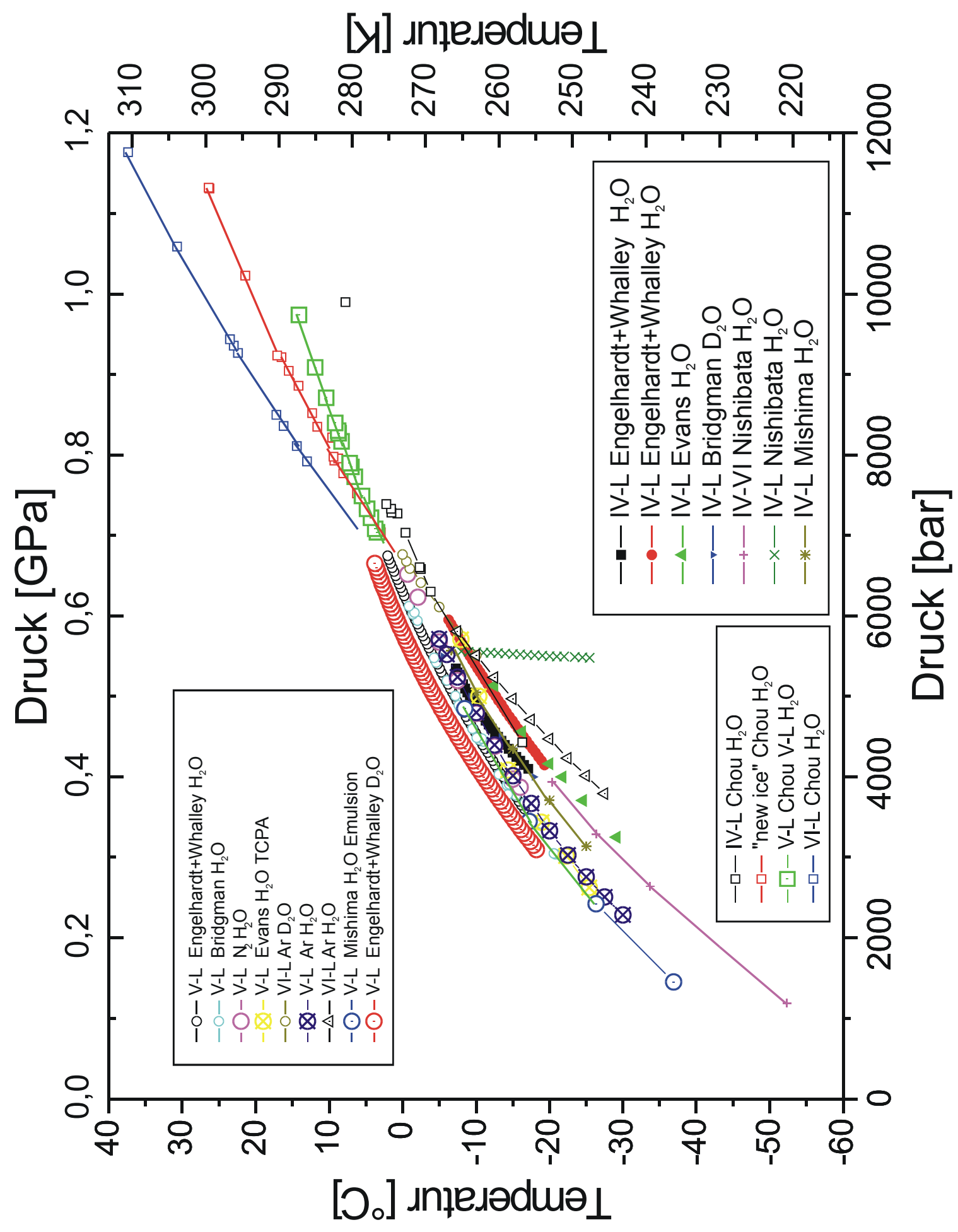

Abbildung 4.4: Übersicht über die nach derzeitigem Stand in der Literatur beschriebenen Schmelzkurven in dem Temperatur- und Druckbereich von $\mathrm{T} \sim 40^{\circ} \mathrm{C}$ bis $\mathrm{zu} \sim-60^{\circ} \mathrm{C}$ sowie in dem Druckbereich von p 1bar bis zu p 12kbar (Literaturhinweise vergl. Kapitel 1.3).

Die im allgemeinen als Gas-Hydrate bezeichneten Clathrat-Hydrate koexistieren über einen weiten Druckbereich mit den Hochdruck-Eisphasen. Zudem existieren in dem Stabilitätsbereich von Eis V sowie Eis VI metastabile Eisphasen. Eine Überprüfung der 
Existenzbereiche im Hinblick auf die Bildung der metastabilen Eisphasen, der Koexistenz von Eis und Gas-Hydraten sowie Eis und Clathrat-Hydraten nahe der Schmelzkurve, war daher angebracht. Im Zuge der Untersuchung und der Verifizierung von Eisphasen und deren Herstellungsrouten war eine detaillierte Überprüfung der Phasengrenzen unter dem Einfluss der verwendeten Gase unbedingt notwendig. Für einen Vergleich von Schmelzkurvenverläufen war daher die Bestimmung der jeweiligen Phasengrenzen einzelner Eisstrukturen, im besonderen der Schmelzkurven zur Herstellung für die spätere in situ Strukturuntersuchung, von besonderer Bedeutung.

\subsection{Experimentelle Untersuchung von Schmelzkurven im System Eis und Gas bis zu Gasdrücken von $p \sim 6.5 \mathrm{kbar}$}

Im folgenden sind die experimentellen Ergebnisse der Schmelzkurvenexperimente sowie der Nukleationsexperimente in Gegenwart der verschiedenen, als Drucküberträgermedium verwendeten Gase dargelegt. Diese experimentellen Untersuchungen hatten das Ziel neben der Bestimmung der Schmelzkurven auch die Gas-Eis-Wechselwirkungen sowie die Synthesebedingungen von Gas-Hydraten als Funktion von Druck und Temperatur unter Verwendung verschiedener Gase zu untersuchen. Diese Experimente wurden mit der in Göttingen aufgebauten pVT-Versuchsapparatur (vergl. Kapitel 2) sowie in weiteren Experimenten mit der am ISIS sowie am ILL vorhandenen Ausrüstung durchgeführt bzw. verifiziert. Die Identifikation der hergestellten Eisphasen erfolgte über die Bestimmung der Schmelzkurven sowie der bei einem Phasenübergang stattfindende Druckänderung, die durch Dichteänderungen der Probe hervorgerufen wird. Die strukturelle Verifizierung konnte aufgrund der in Kapitel 2 beschrieben Probleme hinsichtlich des „Recoverns“ der an der pVTApparatur hergestellten Proben nicht in Göttingen durchgeführt werden; sie erfolgte jeweils im Zuge der Nukleations- und Schmelzkurvenexperimente am ISIS sowie am ILL mittels Neutronendiffraktion.

Im Zuge der Untersuchung der Eisphasen und der Verifizierung der Herstellungsrouten war eine Überprüfung der Phasengrenzen unter dem Einfluß der verwendeten Gase unbedingt notwendig. Die Ergebnisse hatten auch Bedeutung für die Optimierung der Synthesebedingungen von Clathrathydraten unter Verwendung verschiedener atmosphärischer Gase. 
Mit der pVT-Apparatur wurden mit den Gasen Argon, Stickstoff und Neon die Eisphasengrenzen für die Eisphasen Ih, III und V bestimmt. Es erfolgte die Bestimmung der sowohl fest-flüssig- als auch eine teilweise Bestimmung der fest-fest-Phasengrenzen. Um den Einfluß von Gasen und eventuelle systematische Fehler der Apparatur zu überprüfen, wurde in einem Serienversuch die flüssig-fest-Phasengrenze von Eis $V$ mit einem Flüssigkeitsdruckerzeugersystem, mit Silikon-Öl als nichtkompressiblem Drucküberträgermedium, vermessen und sowohl mit den Literaturwerten als auch den mit Gasen vermessenen Phasengrenzen verglichen. Zweck dieser Messungen war es, zum einen Aussagen über die Genauigkeit und Reproduzierbarkeit der Messergebnisse mit der verwendeten Apparatur im Vergleich zu den Literaturverläufen zu erhalten, und zum anderen den Einfluss der Gase zu bestimmen. Durch einen Vergleich zu den von Tammann (1900) sowie den von Bridgman (1912) bestimmten, nicht gasbeeinflussten Phasengrenzen sollte ein Maß für die Beeinflussung infolge der Löslichkeit der Gase in Wasser gefunden werden. Die Versuche wurden sowohl mit $\mathrm{D}_{2} \mathrm{O}$ als auch mit $\mathrm{H}_{2} \mathrm{O}$ durchgeführt, wobei vorrangig meist $\mathrm{D}_{2} \mathrm{O}$ verwendet wurde, da unter dem Gesichtspunkt der reproduzierbaren Phasenherstellung und der Ausarbeitung von Präparationsrouten für polykristalline Eisphasen diese mittels Neutronendiffraktion an $\mathrm{D}_{2} \mathrm{O}$-Proben am ILL verifiziert werden sollten. Einem Vergleich der aus den Experimenten resultierenden Verläufen der Phasengrenzen mit den experimentellen Ergebnissen von Tammann (1900), Bridgman (1912) sowie den weiteren in der Literatur angegebenen Verläufe wurde nachgegangen.

\subsubsection{Schmelzkurvenexperimente an Eis Ih}

Die Lage und der Verlauf der Schmelzkurve von Eis Ih sowie die fest-fest-Phasengrenze zu Eis II und Eis III wurden mit den Gasen Argon, Stickstoff und Neon experimentell bestimmt und verglichen. Die Bestimmung der fest-fest-Phasengrenzen von Eis Ih zu Eis III bzw. zu Eis II unter Verwendung der Gase Argon und Stickstoff ergab im Rahmen der bei der Phasenumwandlung auftretenden Hysterese-Effekte als Funktion der Temperatur im Rahmen der Fehler der Apparatur keine signifikanten Änderungen in der Lage der bestimmten Eisphasengrenzen von Ih / Eis II bzw. Eis Ih / Eis III zu den als Referenz dienenden Verläufen in der Literatur (Kell \& Whalley (1968)). 
Die aktuellste Arbeit, die sich mit der Phasengrenze von Eis Ih zu der flüssigen Wasserphase beschäftigt, ist von Kishimoto \& Maruyama (1998) durchgeführt worden. Mit einer hohen Messpunktdichte bestimmten sie sehr detailliert die $\mathrm{H}_{2} \mathrm{O}$-Eis Ih-Schmelzkurve in dem Druckbereich von $\mathrm{p} \sim 1$ bar bis $\mathrm{p} \sim 2$ 2.5kbar. Kishimoto \& Maruyama (1998) verwendeten zur Bestimmung der Phasengrenzen ein mechanisches Druckerzeugersystem. Einflüsse auf die Schmelzkurve durch Löslichkeitseffekte von Gasen in der Eisstruktur, z.B. an der Phasengrenze von Eis Ih zu Eis II, können durch die mechanische Druckübertragung ausgeschlossen werden. Die Abbildung 4.5 zeigt die mit den Gasen Argon und Stickstoff für $\mathrm{H}_{2} \mathrm{O}$ sowie für $\mathrm{D}_{2} \mathrm{O}$ mit Neon als Drucküberträger bestimmten Schmelzkurven von Eis Ih im Vergleich zu den von Evans (1967) und Kishimoto \& Maruyama (1998) mit an $\mathrm{H}_{2} \mathrm{O}$ sowie von Bridgman (1912) mit $\mathrm{D}_{2} \mathrm{O}$ bestimmten Schmelzkurven. Bei den eigenen bestimmten Schmelzkurven handelt es sich um die druckrekalibrierten sowie die temperaturkorrigierten Kurven (vergl. Kapitel 2).

Im Rahmen der Genauigkeit der Druckmesssensoren und der einstellbaren Temperaturen ist für die Gase Argon und Stickstoff für den Verlauf der $\mathrm{H}_{2} \mathrm{O}$-Eis Ih-Schmelzkurve keine signifikante Abweichung im Vergleich zu den Schmelzkurven von Bridgman (1912) oder Kishimoto \& Maruyama (1998) zu erkennen. Die resultierende Genauigkeit, d.h. der Vertrauensbereich dieser Werte, ist durch die in der Abbildung 4.5 dargestellten Punktgrößen in etwa verdeutlicht und entspricht ca. dem 1.5fachen Standardfehler im Druck p und in der Temperatur $\Delta \mathrm{T} \sim \pm 0.5 \mathrm{~K}$. Die Proben, die zur Messung dieser Schmelzkurven verwendet wurden, sind zum Teil durch Ausfrieren unter Druck aus der flüssigen Phase nukleiert worden. Eine Abhängigkeit in dem Verlauf der Schmelzkurve als Funktion des bei der Nukleation herrschenden Druckes oder der Konzentration der in der flüssigen Phase gelösten Gase als Funktion des Druckes und der Zeit konnte in vielen durchgeführten Experimenten nicht festgestellt werden, wurden jedoch auch nicht hinsichtlich dieser Fragen konkret verfolgt oder dokumentiert. Ebenso zeigte die Schmelzkurve von Eis Ih-Proben, bei Umgebungsdruck hergestellt und dann unter Druck gesetzt, keine Abweichung zu dem in Abbildung 4.5 dargestellten Verhalten. Es kann daher davon ausgegangen werden, dass die Gase Argon (Namiot \& Bukhgalter (1965)) sowie Stickstoff nicht in der Eis Ih-Struktur gelöst oder eingebaut werden. Die unter der Verwendung der Gase $\mathrm{H}_{2}$, Helium sowie Neon bestimmten Schmelzkurven wurden von Dyadin et al. (1994) bis zu Drücken von Umgebungsdruck bis etwa $\mathrm{p} \sim 2.5 \mathrm{kbar}$ 


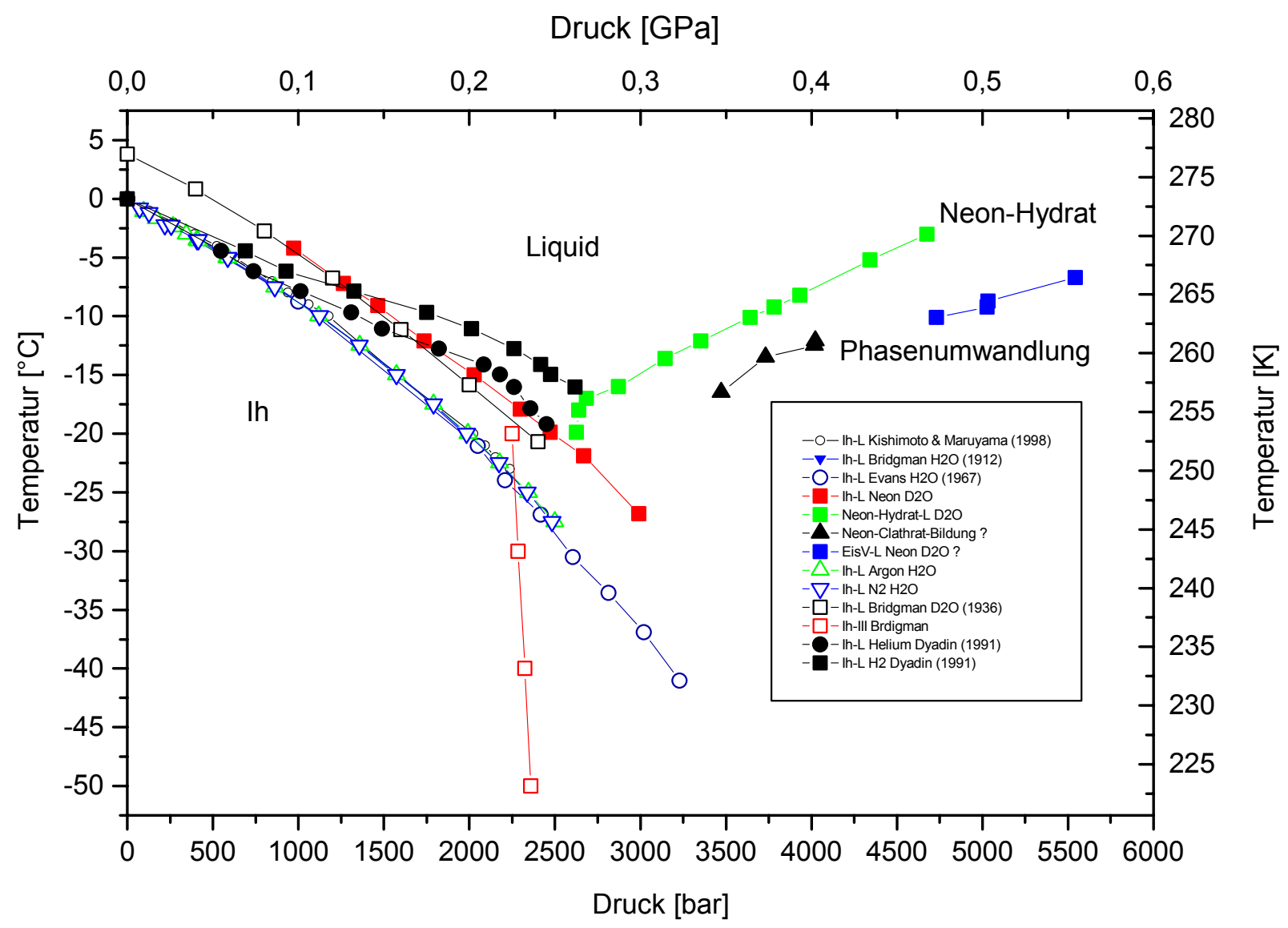

Abbildung 4.5: Eisphasendiagramm im Druckbereich p 1bar bis p 6kbar mit den Gasen Argon, Stickstoff und Neon als Drucküberträgermedium gemessen.

Gasdruck verfolgt und zeigen eine deutliche Verschiebung zu höheren Temperaturen. Beide Schmelzkurvenverläufe deuten auf eine hohe Löslichkeit von $\mathrm{H}_{2}$ sowie Helium in Eis Ih hin, wobei die Abweichung in Gegenwart von $\mathrm{H}_{2}$ größer ist als unter der Verwendung von Heliumgas. Zu kleiner werdenden Drücken nähern sich, wie von Dyadin et al. beschrieben, die beiden Verläufe an den "normalen" Verlauf der Eis Ih-Schmelzkurve an.

Die Gasbesetzung der Eismatrix dürfte, bedingt durch Diffusion der Gase über die Korngrenzflächen in das Volumen des Eis (Kanäle und Ringstrukturen) im allgemeinen eine Funktion des Gasdruckes der Temperatur sein wobei die Besetzung zusätzlich von der druckund temperaturabhängigen Kristallstruktur abhängt. 
Ebenso ist bisher nicht bekannt bzw. untersucht worden, welchen Einfluss das von Furukawa \& Nada (1997) durch Ellipsometrie experimentell nachgewiesene Quasi-Liquid-Layer (QLL) auf Gasdiffusionsprozesse in Eisstrukturen sowie die absolute Besetzung der Eismatrix haben. Denkbar wären Unterschiede in der Löslichkeit des QLL sowie des "Bulks" in Eis bei Temperaturen unterhalb des Schmelzkurvenverlaufes. Furukawa \& Nada (1997) berichteten,

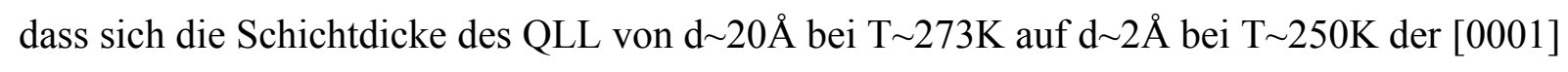
Eis- Oberfläche reduziert. Satoh et al. (1996) bestimmten für die Gase Argon, Helium und Neon in einem Temperaturbereich von $\mathrm{T} \sim 258.2 \mathrm{~K}$ bis $\mathrm{T} \sim 268.2 \mathrm{~K}$ und einem verwendeten maximalen Gasdruck von p 7Mpa (p 70bar) die Diffusionskoeffizienten in der Größenordnung für Helium $\mathrm{D}=10^{-9} \mathrm{~m}^{2} \mathrm{~s}^{-1}$, Neon $\mathrm{D}=10^{-10} \mathrm{~m}^{2} \mathrm{~s}^{-1}$ und Argon $\mathrm{D}=10^{-11} \mathrm{~m}^{2} \mathrm{~s}^{-1}$. Molare Besetzungen der Gase Helium und Neon wurden in der Größenordnung $\mathrm{n} \sim 10^{-6}, \mathrm{n} \sim 10^{-7}$ sowie $\mathrm{n} \sim 10^{-11}$ gefunden. Weitere Experimentelle Werte von molaren Gasbesetzungen bei Drücken $\mathrm{p} \geq 70$ bar werden nicht angegeben. Lediglich Dyadin et al. (1994) gaben an, dass eine Eis Ih-Probe bei einem Druck von $\mathrm{p} \sim 2.5 \mathrm{kbar}$ und einer Temperatur von $\mathrm{T} \sim-25^{\circ} \mathrm{C}$ $(\mathrm{T} \sim 248.15 \mathrm{~K})$ in einem Volumen von $\mathrm{V}=1 \mathrm{ml}$ Eis ein Heliumgasvolumen von $\mathrm{V} \sim 12 \mathrm{ml}$ gelöst hat, was einer molaren Gasbesetzung von $\mathrm{n} \sim 10^{-2}$ bei Standardbedingungen entsprechen würde, einem Wert der wesentlich unter den oben genannten für höhere Temperaturen bestimmten Werten liegt.

Anzumerken ist jedoch, dass die aufgenommene Gasmenge nach der Druckreduzierung auf Umgebungsdruck gemessen wurde und damit wahrscheinlich einen methodischen Fehler zu geringeren Gasbesetzungen aufweisen dürfte (Kahane et al. (1969), Haas et al. (1971)). Namiot \& Bukhgalter (1965) gaben als Ergebnis von Gefrierpunktsvergleichen, die relativen Löslichkeiten der Gase $\mathrm{H}_{2}$ (0.64), He (2.0) und Neon (0.9) bei $\mathrm{T} \sim 0^{\circ} \mathrm{C}$ und $\mathrm{p} \sim 300 \mathrm{bar}$ von Eis zu flüssigem Wasser an. Dies ist konsistent zu der Aussage, dass Gase mit kleinerem Wechselwirkungsradius aufgrund der Kinetik sowohl leichter in die Eistruktur eindiffundieren können als auch aufgrund der Thermodynamik sich bei vergleichbaren Drücken höhere molare Gasbesetzungen ergeben. Für $\mathrm{H}_{2}$ ist die angegebene relative Löslichkeit jedoch inkonsistent zu den von Dyadin et al. (1994) bestimmten Schmelzkurvenverläufen sowie den bestimmten molaren Gasbesetzungen. Dies kann darauf zurückzuführen sein, dass das anisotrope $\mathrm{H}_{2}$-Molekül in der Größenordnung zwischen dem kleineren Heliumatom und dem größeren Neonatom liegt und sich somit nach der statistisch gleichverteilten MolekülOrientierung in den Kanälen eine geringere molare Besetzung der Eisstruktur ergibt. 
Bei der Verwendung von Neon zeigte sich in den eigenen Experimenten eine Abweichung von der gas-unbeeinflussten Eis Ih-Schmelzkurve zu höheren Temperaturen hin. An dieser Stelle ist anzumerken, dass im Hinblick auf die in situ Strukturuntersuchung mittels Neutronendiffraktion die Schmelzkurve mit $\mathrm{D}_{2} \mathrm{O}$ gemessen wurde. Als Referenzkurve wird daher die $\mathrm{D}_{2} \mathrm{O}$ Ih-Schmelzkurve von Bridgman (1912) angeführt.

Aufgrund der Messgenauigkeit, die zum einen durch die Genauigkeit der verwendeten Druckmesssensoren sowie dem apparativen Aufbau (Verhältnis Totvolumen / Probenvolumen vergl. Kapitel 2) gegeben ist, konnte der Verlauf der Eis Ih-Schmelzkurve lediglich bis hinunter zu Drücken von p 600bar mit hinreichender Genauigkeit verfolgt werden (vergl. Abbildung 4.5).

Die Abweichung der mit Neongas gemessenen Eis Ih-Schmelzkurve von der BridgmanReferenzkurve zeigt eine eindeutige Verschiebung zu höheren Temperaturen mit steigendem Druck (vergl. Abbildung 4.5). Zu niedrigeren Drücken findet ebenso wie von Dyadin et al. (1994) festgestellt, eine Annäherung an die Vergleichskurve statt und entspricht damit dem Druckverhalten der molaren Gasbesetzungen bei p 2.5kbar sowie p 70bar Gasdruck. Ein Fit des Schmelzkurvenverlaufes zeigte im Rahmen des Fehlers keine signifikante Abweichung bei $\mathrm{p} \sim 1$ bar Umgebungsdruck zum Schmelzpunkt von $\mathrm{D}_{2} \mathrm{O}$ unter Standardbedingungen. Die Abweichungen von Dyadin et al. (1994) an $\mathrm{H}_{2}$ und Helium sowie die eigenen Experimente an Neon mit Eis Ih, konsistent zu Namiot \& Bukhgalter (1965), belegen eine als Funktion von Druck und Temperatur bestimmte Löslichkeit des Neons in der Struktur von Eis Ih.

Aus der Abweichung der mit Gas gemessenen Eis Ih-Schmelzkurven zu den Referenzkurven ist feststellbar, dass die Gasbesetzung für die Gase $\mathrm{H}_{2}$ und Helium für $\mathrm{H}_{2} \mathrm{O}$-Eis Ih wesentlich höher ist als bei der Verwendung von Neongas für $\mathrm{D}_{2} \mathrm{O}$-Eis Ih. Diese Aussage stimmt jedoch mit den von Namiot \& Bukhgalter (1965) bestimmten Werten nicht überein. Der Frage der Gasbesetzungen wurde im Rahmen von in situ Beugungsexperimenten an Eis Ih mit Helium und Neon und der Auswertung mittels Rietveld-Verfeinerungen nachgegangen. Die experimentellen Befunde sowie eine Interpretation werden im Kapitel 5.5ff. gegeben und diskutiert. 
Bei der Verwendung von Neongas als Drucküberträgermedium konnte in dem Druckbereich von $\mathrm{p} \sim 2.75 \mathrm{kbar}$ bis hin $\mathrm{zu}$ Drücken von $\mathrm{p} \sim 5 \mathrm{kbar}$ durch die Bestimmung des Schmelzkurvenverlauf eine neue Phase (grüne Meßpunkte, vergl. Abbildung 4.5) entdeckt werden. Der Verlauf zeigte keine Übereinstimmung mit der $\mathrm{D}_{2} \mathrm{O}$-Eis III-L-Schmelzkurve von Bridgman (1912) oder der V-L D 2 O-Schmelzkurve von Engelhardt \& Whalley (1972).

Durch in situ Neutronendiffraktion am ILL konnte diese bis dato unbekannte Phase als NeonEis II-Hydrat identifiziert werden (vergl. Kapitel 5.2.3). In den weiteren Experimenten konnte an der pVT-Apparatur in Göttingen für das Eis II-(Neon)-Hydrat über einen weiten Druckbereich, von $\mathrm{p} \sim 2.5 \mathrm{kbar}$ bis $\mathrm{p} \sim 4.5 \mathrm{kbar}$, die Schmelzkurve bestimmt werden. Der Tripelpunkt von Eis Ih-(Neon)-/Eis II-(Neon)-Hydrat/L konnte aus den Schmelzkurvenverläufen für $\mathrm{D}_{2} \mathrm{O}$ zu p$=2.40 \mathrm{kbar} \pm 48 \mathrm{bar}, \mathrm{T}=254.1 \mathrm{~K} \pm 0.5 \mathrm{~K}$ bestimmt werden.

Im Zuge dieser Schmelzkurvenexperimente zeigte sich auch, dass in dem Stabilitätsbereich dieser neuen, als Neon-Hydrat identifizierten Phase, über einen weiten Druckbereich langsame Druckänderungen zu systematisch niedrigeren Drücken festgestellt werden konnten. Der Druckbereich, in dem diese Druckänderungen auftraten, konnte in einigen wenigen Experimenten zu p $\sim 3.3 \mathrm{kbar}$ bis $\mathrm{p} \sim 4 \mathrm{kbar}$ sowie $\mathrm{p} \sim 4.75 \mathrm{kbar}$ bis $\mathrm{p} \sim 5.5 \mathrm{kbar}$ festgestellt werden. Diese Druckerniedrigung deutet auf die Bildung einer dichteren, aus den Göttinger Schmelzkurvenexperimenten bisher nicht identifizierbaren Phase hin.

$\mathrm{Ob}$ es sich um eine kinetisch gehemmte Umwandlung in Eis V oder aber um eine andere, weitere unbekannte Phase handelt, ist aus den Experimenten nicht auszusagen gewesen. Es konnten lediglich einige wenige Messpunkte verfolgt werden, da die Druckänderungen mit fortschreitender Zeit kleiner wurden und diese zu einer signifikanten Bestimmung der Phasengrenze nicht verwendet werden konnten. Bei einer Verifizierung durch in situ Neutronenstreuexperimente am ILL an Eis Ih mit Neon als Drucküberträger, zeigte sich unter äquivalenten Bedingungen, d.h. bei Drücken $a b p \sim \geq 1 \mathrm{kbar}$ und Temperaturen von $\mathrm{T} \sim 215 \mathrm{~K}$, die Bildung eines neuen Clathrat-Hydrates, Neon-Clathrat-Hydrat (vergl. Kapitel 5.2.1).

$\mathrm{Ob}$ es sich bei der in den Schmelzkurvenexperimenten nicht identifizierbare Phase um NeonClathrat-Hydrat handelt, ist durch neuere Versuche (vergl. Kapitel 5.2.1) eher auszuschließen. Geht man von der Voraussetzung aus, dass es sich hierbei um die Bildung von Neon-Clathrat- 
Hydrat in dem Stabilitätsbereich des Neon-Hydrates handelt (vergl. Abbildung 4.5), so würde dies auf eine Umwandlung von Neon-Hydrat in Neon-Clathrat-Hydrat hinweisen. Als Schlussfolgerung ist davon auszugehen, dass das Neon-Clathrat-Hydrat bei den dortigen Druck- und Temperaturbedingungen die thermodynamisch stabilere Phase ist.

\subsubsection{Schmelzkurvenexperimente an Eis im Druckbereich von $p \sim 2.5 \mathrm{kbar}$ bis $\mathrm{p} \sim 6.5 \mathrm{kbar}$}

Neben den Schmelzkurven von Eis V, Eis VI unter der Verwendung verschiedener Gase wie Argon, Stickstoff und Neon sowie eines fluiden, mechanischen Drucküberträgermediums zur Verifizierung der Messungenauigkeiten und systematischer Fehler konnte die Schmelzkurve der metastabilen Eisphase von Eis XII in einem weiten Druck- und Temperaturbereich mit der pVT-Apparatur in Göttingen bestimmt und in situ mittels Neutronendiffraktion punktuell verifiziert werden. Aufgrund der bestimmten systematischen Fehler und Messungenauigkeiten ist eine Diskussion der Ergebnisse für die verschiedenen Eisphasen relativ untereinander möglich. Ein Vergleich einzelner Ergebnisse der druck- und temperaturkorrigierten Werte mit den Literaturangaben wird im folgenden in der Abbildung 4.6 dargestellt und diskutiert.

\subsubsection{Eis V und Eis VI}

Für Eis V konnte die Schmelzkurve in weitem Druckbereich bestimmt werden. Für $\mathrm{D}_{2} \mathrm{O}$-Eis VI konnte diese in dem Druckbereich von $\mathrm{p} \sim 5.5 \mathrm{kbar}$ bis $\mathrm{p} \sim 6.5 \mathrm{kbar}$ bestimmt werden, während für $\mathrm{H}_{2} \mathrm{O}$-Eis $\mathrm{VI}$ die Schmelzkurve von $\mathrm{p} \sim 6.5 \mathrm{kbar}$ in dem eigentlichen Stabilitätsbereich von Eis V bis hinunter zu p 3.75kbar vermessen werden konnte. Eine in situ-Verifikation konnte aufgrund der apparativen Grenzen am ILL sowie am ISIS für Eis VI nicht durchgeführt werden. Aufgrund der bei der Nukleation bestimmten Druckänderung lässt sich jedoch aufgrund der bisherigen Erfahrungswerte davon ausgehen, dass es sich mit hoher wahrscheinlich in beiden Fällen um Eis VI handelt. Untersuchungen der Eis VISchmelzkurven mit Stickstoff wurden nicht durchgeführt. Der Frage, ob bei anderen Eisphasen aufgrund der Wechselwirkungen von Eis und Gas bzw. Wasser und Gas systematische Verschiebungen auftreten, z.B. durch Schmelzpunkterniedrigung, wurde am Beispiel der Eisphase V untersucht. Geht man von rein strukturellen Aspekten aus, so ist z.B. 


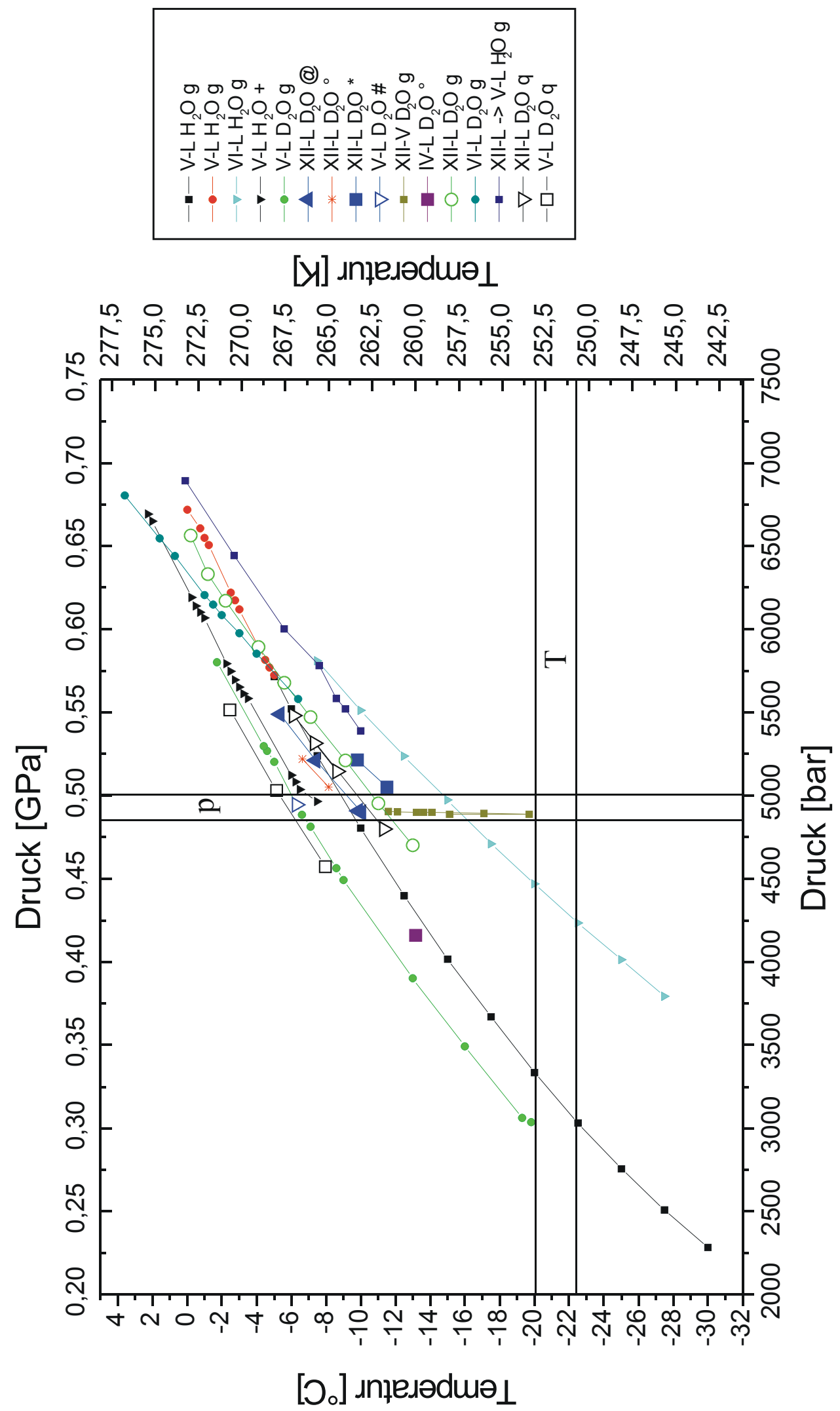

Abbildung 4.6: Experimentell ermittelte Schmelzkurven in dem Druckbereich von p 2kbar bis p 6.8kbar. g: Apparatur in Göttingen, + : fluides Drucküberträgermedium Silikon-Öl, @: in situ verifizierte Messpunkte am ILL, ${ }^{\circ}$ : in situ verifizierte Messpunkte von Lobban et al. (1998), *: Auszug unkorrigierter Messpunkte (vergl. Kapitel 2.), \#: Verwendung von TCPA mit einem Massenanteil von max. $10 \%$ der Probe, q: in situ verifizierte Messpunkte am ISIS, außer bei + wurde Argongas als Drucküberträgermedium verwendet. Die Eis V-L Stickstoffgas-Schmelzkurve stimmt im Rahmen der Fehler mit V-L $\mathrm{H}_{2} \mathrm{O}$ g überein und ist daher nicht dargestellt. p: Drucklimit, T: Temperaturlimit 
für Eis V als geschlossene Struktur eine Löslichkeit wie bei Eis Ih als sehr offener Struktur nicht möglich (vergl. Kapitel 5). Im Zuge der Experimente wurde ein eventueller Gaseinfluss durch die Vermessung der Eis V-L-Schmelzkurve mit Argon und Stickstoff durchgeführt. Die Abweichungen zwischen der Argon- und der Stickstoff-Schmelzkurve zeigten eine innerhalb des Fehlers von $\sim 2 \%$ im Druck liegende systematische Abweichungen und sind daher unter diesen apparativen Bedingungen als nicht signifikant zu betrachten.

Der Tripelpunkt von V-VI-L unter Verwendung der beiden Gase Stickstoff und Argon wurde für $\mathrm{D}_{2} \mathrm{O} \mathrm{zu} \mathrm{p}=6.64 \mathrm{kbar} \pm 132 \mathrm{bar}, \mathrm{T}=275.47 \mathrm{~K} \pm 2.5 \mathrm{~K}$ und für $\mathrm{H}_{2} \mathrm{O}$ zu $\mathrm{p}=6.88 \mathrm{kbar} \pm 137 \mathrm{bar}$, $\mathrm{T}=269.5 \pm 2.5 \mathrm{~K}$ bestimmt. Über den Druckbereich von $\mathrm{p} \sim 2.25 \mathrm{kbar}$ bis $\mathrm{p} \sim 6.5 \mathrm{kbar}$ konnte die Schmelzkurve von $\mathrm{H}_{2} \mathrm{O}$-Eis V (Bezeichnung V-L $\mathrm{H}_{2} \mathrm{O}$ g) sowie für $\mathrm{D}_{2} \mathrm{O}$-Eis V (Bezeichnung $\mathrm{V}-\mathrm{L} \mathrm{D}_{2} \mathrm{O} \mathrm{g}$ ) in dem Druckbereich von $\mathrm{p} \sim 3 \mathrm{kbar}$ bis $\mathrm{p} \sim 5.5 \mathrm{kbar}$ vermessen werden. Bei der Bestimmung dieser Kurven wurde kein TCPA als Nukleator verwendet.

Aus den Verläufen der beiden Kurven ist ein Unterschied in der Krümmung als Funktion des Druckes und der Temperatur gegeben. Für einen systematischen Vergleich von Schmelzkurven aus verschiedenen Experimenten ist daher die Überprüfung der jeweiligen Phasengrenzen für $\mathrm{H}_{2} \mathrm{O}$ sowie $\mathrm{D}_{2} \mathrm{O}$ absolut notwendig. Eine in der Literatur als Abschätzung der Existenzbereiche verwendete Re-Skalierung der von $\mathrm{H}_{2} \mathrm{O}$ - und $\mathrm{D}_{2} \mathrm{O}$-Phasengrenzen über die Druck- und Temperaturverschiebungen der jeweiligen Tripelpunkte ist daher nicht tragbar; dies zeigte sich ebenso an den $\mathrm{H}_{2} \mathrm{O}$ - sowie $\mathrm{D}_{2} \mathrm{O}$ - Schmelzkurven von Eis VI. Die $\mathrm{H}_{2} \mathrm{O}$-Eis VI Schmelzkurve konnte in einem ersten Experiment von $\mathrm{p} \sim 3.75 \mathrm{kbar}$ bis $\mathrm{zu}$ p 5.75kbar verfolgt werden. Ebenso wie beim Eis V wurde hier kein Nukleator verwendet. In einem zweiten Experiment wurde die Schmelzkurve von Eis V mit einem Massenanteil von $\sim 5 \%$ TCPA in dem Druckbereich von $\mathrm{p} \sim 5.75 \mathrm{kbar}$ bis zu p $6.75 \mathrm{kbar}$ vermessen .

Die Verläufe der mit und ohne TCPA vermessenen $\mathrm{H}_{2} \mathrm{O}$-VI-L-Schmelzkurve sowie von punktuell bestimmten Eis V-L-Schmelzpunkten mit TCPA (vergl. offenes großes blaues Dreieck auf Eis V-D $\mathrm{D}_{2} \mathrm{O}-(\mathrm{g})$ Schmelzkurve) lässt den Schluss zu, dass bei einem verwendetem TCPA-Massenanteil der Probe von maximal 10\% im Rahmen der erreichbaren Messgenauigkeit kein Einfluss des Nukleators auf die Schmelzkurven festzustellen ist. 
Die Proben, die zur Bestimmung der Schmelzkurven von Eis V sowie Eis VI dienten, wurden in den ersten Experimenten mit der pVT-Apparatur über die fest-fest-Präparationsroute $\mathrm{L} \rightarrow \mathrm{Ih} \rightarrow \mathrm{II} / \mathrm{III} \rightarrow \mathrm{V} \rightarrow \mathrm{VI}$ hergestellt, um die eventuelle Nukleation einer metastabilen Phase aus der flüssigen Phase auszuschließen.

Die Eis V- und die Eis VI-Proben wurden nach der Bestimmung der Schmelzkurven komplett geschmolzen, um bei gegebenem Druck und Temperatur die Dichte- und damit die Gasdruckänderung im System festzustellen. Die Druckänderungen lagen bei Verwendung von $\mathrm{H}_{2} \mathrm{O}$ sowie für $\mathrm{D}_{2} \mathrm{O}$ für Eis $\mathrm{V}$ bei etwa $\Delta \mathrm{p} \sim 230 \mathrm{bar} \pm 30 \mathrm{bar}$, für Eis IV/XII bei $\Delta \mathrm{p} \sim 340 \mathrm{bar} \pm 40$ bar und für das in diesem Druck- und Temperaturbereich metastabile Eis VI bei $\Delta \mathrm{p} \sim 420 \pm 45$ bar. Massennormiert ergaben sich für den Druck vor und nach der Phasenumwandlung Verhältniswerte von 1.041(4) für V-L und 1.18(2) für L-VI. Für Eis XII ergab die Verhältniszahl von L-IV/XII 1.11(5). Diese Verhältniszahlen zur Identifizierung der nukleierten Eisphase zeigten sich jedoch sehr sensibel gegenüber unterschiedlichen Phasenübergangsbedingungen (Anfangsdruck bei der Phasenumwandlung sowie Temperatur, vergl. auch Kapitel 3.7). Es wurde daher versucht, den Phasenübergang zur Identifikation der Proben bei annähernd vergleichbaren Druckbedingungen durchzuführen.

\subsubsection{Metastabiles Eis XII und Eis IV}

Aufgrund der nur begrenzt zur Verfügung stehenden Neutronen-Messzeit konnte die Schmelzkurve von Eis XII nur teilweise in situ vermessen werden. Durch die Experimente am D2B des ILL sowie am GEM am ISIS des RAL konnte jedoch mehrfach in situ nachgewiesen werden, dass mit dem an der Göttinger Versuchsapparatur ausgearbeiteten Herstellungsverfahren die metastabile Eisphase XII mit hoher Wahrscheinlichkeit gezielt und reproduzierbar hergestellt werden konnte. Die Druckänderungen, die bei dem Phasenübergang von der flüssigen Phase in die feste Eisphase bestimmt wurden, zeigten sich am GEM nach der Korrektur hinsichtlich des Verhältnisses von Probevolumen zu Stickvolumen konsistent zu den Druckänderungen, die sich an der Göttinger pVT-Apparatur ergaben.

Am D2B konnten die Druckdifferenzen aus apparativen Gründen (zu großes Totvolumen der Druckerzeugereinheit) nicht gemessen werden. Für die Beurteilung, ob es sich um die 
gesuchte metastabile Eisphase XII oder IV (aufgrund der nahezu gleichen Dichten nicht zu verifizieren) handelt, wurde neben dem Kriterium der Druckänderung letztendlich die Schmelzkurve als Funktion des Druckes bestimmt und über deren Verlauf die Eisphase identifiziert.

Aufgrund der hohen, in situ verifizierten Eis XII-Nukleationswahrscheinlichkeit sowie der Unterscheidungsmöglichkeit aufgrund der Dichteänderungen kann mit hoher Wahrscheinlichkeit die Aussage getroffen werden, dass die mittels der Göttinger pVTApparatur bestimmte und nicht als Eis V oder Eis VI identifizierte und in der Abbildung 4.6 dargestellte Schmelzkurve (XII-L $\mathrm{D}_{2} \mathrm{O}$ g), der Schmelzkurve von Eis XII von Lobban et al. (1998) entspricht. Zu den in der Abbildung 4.7 dargestellten in situ bestimmten Eis XIISchmelzpunkten zeigt sich, dass die am ILL sowie am ISIS bestimmten Eis XIISchmelzpunkte eine Abweichung in der Temperatur bei vergleichbaren Drücken zeigten. Die Abweichung zwischen den ISIS- und den Göttinger Schmelztemperaturen kann aufgrund der druckkorrigierten sowie der am ISIS präziser bestimmten Temperatur $\mathrm{zu} \Delta \mathrm{T} \sim 1 \mathrm{~K}$ angegeben werden. Die Bestimmung der Temperaturabweichung zwischen den pVT-Daten sowie den in situ Daten erfolgte über einen Vergleich einer am GEM über einen Druckbereich von $\mathrm{p} \sim 1 \mathrm{kbar}$ in situ bestimmten Eis V-Schmelzkurve zu der druckkorrigierten Eis V-Schmelzkurve gemessen mit der pVT-Apparatur.

Unter Verwendung des in Kapitel 3.7 beschriebenen in situ verifiziertem Eis XIIHerstellungsverfahrens konnte aus der flüssigen Phase mehrmals in Göttingen eine Phase nukleiert werden, die durch ihre Schmelzkurve nicht als Eis V oder VI identifiziert werden konnte. In einem Druckbereich von $\mathrm{p} \sim 4.5 \mathrm{kbar}$ bis $\mathrm{p} \sim 6.5 \mathrm{kbar}$ konnte die $\mathrm{D}_{2} \mathrm{O}$-Schmelzkurve dieser als Eis XII zu vermutenden Eisphase vermessen werden (vergl. Abbildung 4.7).

Berücksichtigt man die am ISIS bestimmte absolute Temperaturabweichung so kann diese Schmelzkurve über den korrigierten Temperaturoffset mit hoher Wahrscheinlichkeit als die der Eisphase XII identifiziert werden. Auch stimmen die in situ bestimmten Schmelzpunkte am GEM (ISIS) sowie am D2B (ILL) im Rahmen der Genauigkeiten mit dem in Göttingen bestimmten Schmelzkurvenverlauf überein. 


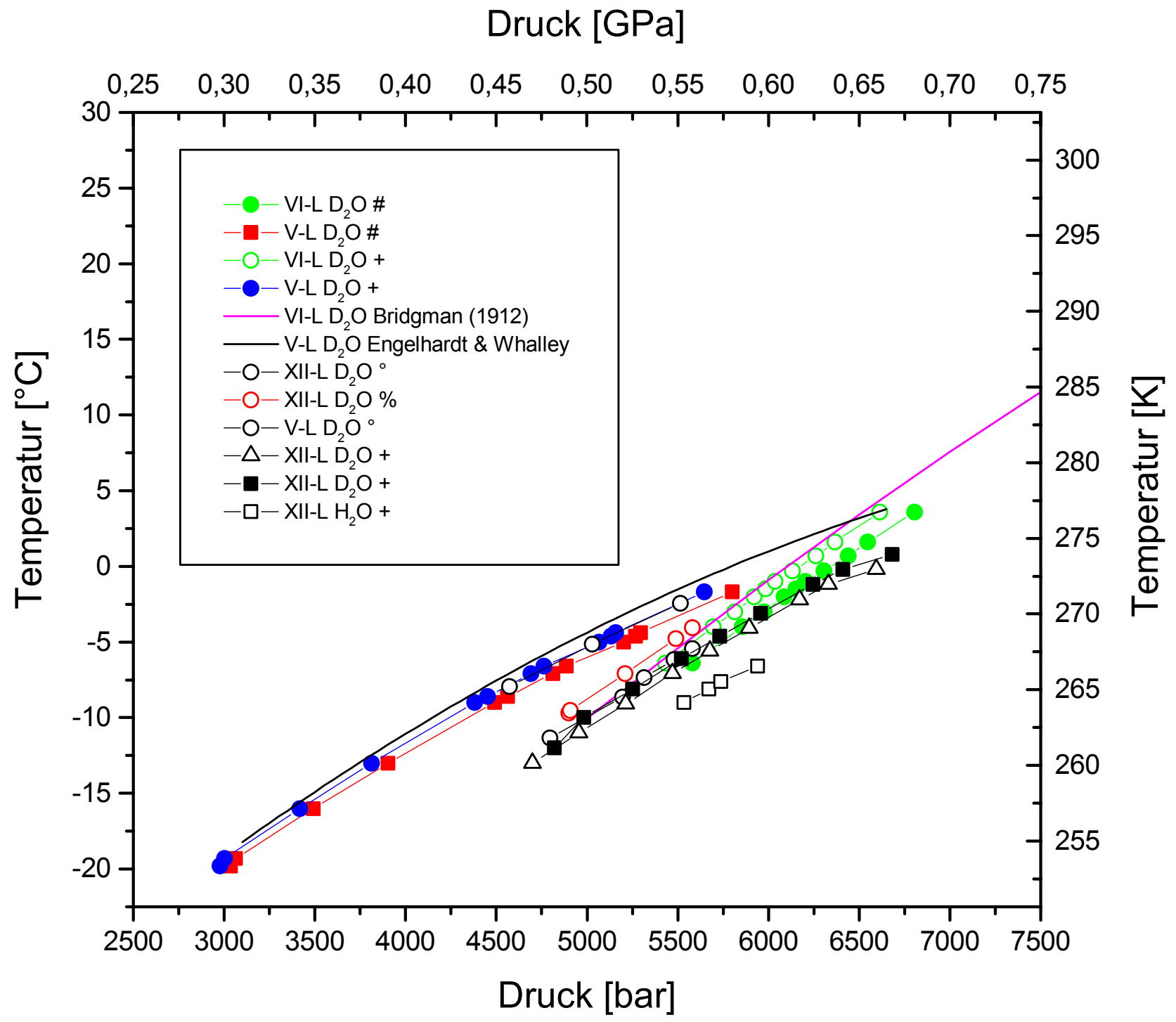

Abbildung 4.7 : Gemessene Schmelzkurven in dem Stabilitätsbereich von Eis V im Vergleich zu den Referenzkurven von Bridgman (1912) sowie Engelhardt \& Whalley (1972). Als Drucküberträgermedium für die Messungen wurde an der pVT-Apparatur sowie am ISIS und am ILL Argongas verwendet. \# : Rohdaten der an der pVT-Apparatur bestimmten, + : Druck-(ILL) in situ verifizerte Schmelzpunkte

Die für die Bestimmung der Eis XII-Schmelzkurve verwendeten Proben waren mit dem TCPA Nukleator mit einem Massenanteil von 5-10\% hergestellt worden. Um der Frage, ob TCPA für die Nukleation der Eisphase XII notwendig ist, nachzugehen, wurde ein weiteres Nukleationsexperiment mit einer $\mathrm{H}_{2} \mathrm{O}$-Probe ohne Zusatz von TCPA durchgeführt. Die Probenmasse betrug ebenso wie bei dem D2O-Experiment $\mathrm{V} \sim 1.5 \mathrm{ml}$ bei $\mathrm{p} \sim 5.9 \mathrm{kbar}$ und $\mathrm{T} \sim-$ $22.5^{\circ} \mathrm{C}$. Nach der zu vermutenden Eis XII/ IV-Nukleation, durch die Druckänderung bei $\Delta \mathrm{p} \sim 354$ bar identifiziert, wurde dann in dem Druckbereich $\mathrm{p} \sim 5.2 \mathrm{kbar}$ bis $\mathrm{p} \sim 5.75 \mathrm{kbar}$ teilweise 
eine Schmelzkurve vermessen. Diese zeigte ebenfalls keine Übereinstimmung mit der $\mathrm{H}_{2} \mathrm{O}-\mathrm{V}-$ L-Schmelzkurve oder mit der $\mathrm{H}_{2} \mathrm{O}$ - VI-L- Schmelzkurve. Es kann daher davon ausgegangen werden, dass diese Schmelzkurve der von $\mathrm{H}_{2} \mathrm{O}$-Eis XII/ IV, die zudem ohne Verwendung des Nukleators hergestellt werden konnte, entspricht (vergl. Abbildung 4.7).

Die Verwendung des Nukleators scheint daher zumindest für die $\mathrm{H}_{2} \mathrm{O}$-Eis XII-Nukleation nicht notwendig. Für die Herstellung von $\mathrm{D}_{2} \mathrm{O}$-Eis XII/IV wurde dies nicht verifiziert. Im Zuge der Schmelzkurvenbestimmung an dem $\mathrm{H}_{2} \mathrm{O}$-Eis XII zeigte sich bei $\mathrm{p} \sim 5.75 \mathrm{kbar}$ ein Phasenübergang von $\mathrm{H}_{2} \mathrm{O}$-Eis XII/ IV vermutlich in Eis VI durch den Übergang der Eis XII/ IV-Schmelzkurve in die Verlängerung der Eis VI-Schmelzkurve an (Abbildung 4.6, XII$\mathrm{L} \rightarrow \mathrm{VI}-\mathrm{L} \mathrm{H}_{2} \mathrm{O}$ ). In situ konnten am ISIS mehrere Phasenübergänge von $\mathrm{D}_{2} \mathrm{O}$ - Eis XII in Eis VI verfolgt werden. Der Phasenübergang vollzog sich bei einem Druck von $\mathrm{p} \sim 5.5 \mathrm{kbar}$ und aufgrund einer Probenerwärmung in einem Temperaturintervall von $\mathrm{T} \sim 260 \mathrm{~K}$ bis $\mathrm{T} \sim 267 \mathrm{~K}$. Die mit der Göttinger pVT-Apparatur bei p 4.8kbar gemessenen nahezu senkrechten Verläufe (vergl. Abbildung 4.6, XII-V $\quad \mathrm{D}_{2} \mathrm{O} \quad \mathrm{g}$ ), verdeutlichen das in den Herstellungsversuchen gefundene Druck- und Temperaturlimit, bei dem Eis XII nach der Nukleation erhalten werden konnte, sich aber bei Erwärmung zur Schmelzkurvenbestimmung mit großer Wahrscheinlichkeit in Eis V umgewandelt hat. Dies konnte durch die Bestimmung einzelner Schmelzpunkte bestätigt werden.

Am ISIS konnte unter struktureller Kontrolle in einem Druckbereich von $\mathrm{p} \sim 4.75 \mathrm{kbar}$ bis $\mathrm{p} \sim 5.5 \mathrm{kbar}$ und Temperaturen bis $\mathrm{T} \sim 257.5 \mathrm{~K}$ Eis XII in situ beobachtet werden, wobei der Druck von $\mathrm{p} \sim 4.75 \mathrm{kbar}$ nur wenig unterhalb des aus den bisherigen Erfahrungen ermittelten Druck-Nukleationslimits liegt (vergl. Kapitel 3.7 sowie Kapitel 4.3.3, Abbildung 4.6). In der vorherigen Abbildung 4.7 ist der Druckbereich von $\mathrm{p} \sim 3 \mathrm{kbar}$ bis $\mathrm{p} \sim 7 \mathrm{kbar}$ in dem die Eis XIISchmelzkurve vermessen werden konnte, dargestellt.

In der Abbildung 4.6 sind zum einen die in situ bestimmten Schmelzkurven von Eis XII und die an der Göttinger pVT-Apparatur bestimmte Schmelzkurve, zum anderen auf experimentellen Befunden basierenden Druck- und Temperaturlimits der Eis XII-Herstellung sowie die Druck- und Temperaturbedingungen an denen Eis XII in situ am ISIS beobachtet werden konnte, dargestellt. 
Bei einem Druck von $\mathrm{p} \sim 4.7 \mathrm{kbar}$ in dem Temperaturbereich von $\mathrm{T} \sim 265 \mathrm{~K}$ bis $\mathrm{T} \sim 257.5 \mathrm{~K}$ zeigte sich konsistent $\mathrm{zu}$ den Eis XII-Nukleationsversuchen, dass sich das Eis XII $\left(\mathrm{D}_{2} \mathrm{O}\right)$ in Eis V umwandelt. Es handelt sich wohl aller Wahrscheinlichkeit nach um die Eis XII/Eis V festfest-Phasengrenze (vergl. Kapitel 4.3.3, Abbildung 4.6). Bisherige Erfahrungen haben gezeigt, dass Eis XII sensibel auf starke Druck- und Temperaturänderungen reagiert und sich bei solchen spontan in zumeist Eis V umwandelt.

Wie weit der Existenzbereich von Eis XII ausgedehnt ist, ist von großem Interesse hinsichtlich der Frage, ob es sich bei dem Eis XII um eine stabile Phase handeln kann, die unter den bisherigen genannten Druck- und Temperaturbedingungen lediglich metastabil in dem Stabilitätsbereich von Eis V gewonnen werde konnte. Geht man von einer hypothetisch stabilen Eisphase XII aus, so sollte der korrigierte $\mathrm{D}_{2} \mathrm{O}$-Eis XII-Schmelzkurvenverlauf bei höheren Drücken oberhalb des Verlaufs der bestimmten $\mathrm{D}_{2} \mathrm{O}$-Eis VI-L-Phasengrenze liegen. Geht man von den bisherigen bestimmten $\mathrm{D}_{2} \mathrm{O}-\mathrm{V}-\mathrm{L}$ - und $\mathrm{D}_{2} \mathrm{O}-\mathrm{VI}-\mathrm{L}-\mathrm{Schmelzkurven}$ aus, so lässt sich allerdings die Aussage treffen, dass es sich bei Eis XII mit hoher Wahrscheinlichkeit um eine zu den Eisphasen V und VI metastabile Eisphase handelt, da die Eis XIISchmelzkurve unterhalb des V-VI-L-Tripelpunkts liegt.

Eine Diskussion hinsichtlich der Vergleichbarkeit von Messungen ist aus einem Vergleich der in Abbildung 4.7 dargestellten Schmelzkurvenverläufen von Engelhardt \& Whalley (1972) sowie Bridgman (1912) als Referenzkurven mit eigenen experimentellen Befunden angebracht. Wie im Kapitel 2 beschrieben, ergeben sich trotz der Druck- und Temperaturkorrektur durch sekundäre Standards (interne Drucksensor-Kalibrierung durch Heisemanometer) Abweichungen zu den Schmelzkurven von Engelhardt \& Whalley (1972) und erschweren so die Aussage ob es sich bei diesen Abweichungen um apparative Effekte oder aber um probenbedingte Effekte, z.B. durch Schmelzpunkterniedrigung aufgrund von Wasser-Gas-Wechselwirkungen, handelt. Literaturangaben zu Wasser- oder Gaslöslichkeiten in diesem Druck- und Temperaturbereich konnten nicht gefunden werden.

In einem weiteren Eis XII-Experiment am D2B des ILL in Grenoble wurde der Frage nach der Möglichkeit des "recoverns" von Eis XII nachgegangen, um eine Aussage über die beiden gefundenen Existenzbereiche treffen zu können (Lobban et al. (1998), Koza et al. (1999) (vergl. Kapitel 3.7). Die Frage ist, ob die beiden Existenzbereiche zusammenhängend sind, 
oder aber ob es sich um zwei Existenzenklaven handelt. Im Zuge der Eis XIIPräparationsversuche am ILL zeigte sich, dass übermäßiges Kühlen der Probe bei isobarem Druck auf Temperaturen unter $\mathrm{T} \sim \leq-15.6^{\circ} \mathrm{C}$ (z.B. $\mathrm{T} \leq 257.5 \mathrm{~K}$ bei $\mathrm{p} \sim 5 \mathrm{kbar}$ ) unterhalb der Schmelzkurve zu einer Phasenumwandlung, zumeist in Eis V, führte. Phasenumwandlungen in Eis VI wurden anhand der Volumenänderungen ebenso festgestellt, waren jedoch unter Berücksichtigung aller Versuche lediglich mit $5 \% \mathrm{zu}$ verzeichnen. Das in mehreren Versuchen hergestellte Eis XII wurde nach der in situ Identifikation in der ILL HD-Zelle mit flüssigem Stickstoff auf $\mathrm{T} \sim 77 \mathrm{~K}$ abgeschreckt. Die mittlere Kühlrate lag wie bei den in Göttingen verwendeten HD-Zellen bei $\sim 1.5 \mathrm{~K} / \mathrm{s}$. Es zeigte sich nach dem Erreichen von T 77K und der anschließenden in situ Strukturkontrolle, dass sich Eis XII in fünf von fünf Versuchen in Eis V umgewandelt hat. Wann und bei welcher Temperatur diese Umwandlung stattfand, konnte aufgrund der während des Quenchens nur ungenau bestimmbaren Parameter Druck und Temperatur nicht verifiziert werden.

Fraglich ist, ob die Umwandlung von Eis XII durch zu langsames Kühlen auf StickstoffTemperaturen die Phasenumwandlung forciert. Hinweise auf Kühlungseffekte wurden von Londono et al. (1993) an Quenchversuchen von Eis III in die Tieftemperaturphase zu Eis IX gefunden. Von Koza et al. (1999) oder anderen Autoren ist nicht bekannt, dass versucht wurde, das mit einem piston-cylinder hergestellte Eis XII aus dem zweiten Existenzbereich in den ersten durch Reduzierung des mechanischen Druckes auf etwa $\mathrm{p} \sim 5 \mathrm{~kb}$ ar bis $\mathrm{p} \sim 6 \mathrm{kbar}$ und der Erwärmung von Temperaturen von $\mathrm{T} \sim 77 \mathrm{~K}$ auf Temperaturen oberhalb von $\mathrm{T} \sim 257.5 \mathrm{~K} \mathrm{zu}$ überführen. Möglich war es jedoch, im Zuge der Untersuchungen des Phasenüberganges von Eis XII in kubisches Eis Ic, das über das Herstellungsverfahren von Koza et al. (1999) bei $\mathrm{T} \sim 77 \mathrm{~K}$ und Umgebungsdruck, d.h. im „recoverten“ Zustand über einen längeren Zeitraum (einige Tage bis zu einer Woche) zu erhalten. Der Grund für den Erhalt sowohl dieser als auch anderer „recoverter“ Eisphasen ist die bei diesen Temperaturen und Umgebungsdruck anzuführende geringe Mobilität der Wassermoleküle. Erst bei Temperaturen von T $120 \mathrm{~K}$ ist diese wiederum groß genug, um eine Phasenumwandlung in beobachtbaren Zeiträumen zu ermöglichen. Eine Aussage hinsichtlich des Zusammenhängens der zwei Existenzbereiche von Eis XII kann somit derzeit nicht getroffen werden. 


\section{Strukturuntersuchungen}

\subsection{Argon-Clathrat-Hydrat}

Im Zuge der Durchführung der Eis Ih-Experimente erfolgte sobald der Synthesedruck für Argongas von p 90bar bei einer Temperatur von $\mathrm{T} \sim 0^{\circ} \mathrm{C}$ überschritten wurde, die kontinuierliche Argon-Clathrat-Hydrat-Synthese. Die Proben lagen daher für einen großen Teil der Messungen als Phasengemisch mit variablem Massenanteil von Eis Ih und ArgonClathrat-Hydrat vor. Eine Berücksichtigung und Beschreibung der Clathrat-Hydratstrukturen sowie des Phasenanteils des Clathrat-Hydrates erschien daher geboten. Hinsichtlich der Rietveld-Verfeinerungen ergaben sich aufgrund der nur ungenügenden kristallographischen Beschreibung des Clathrat-Hydrates Probleme, die eine genaue Strukturbestimmung der Eisphase wesentlich erschwerte. An dieser Stelle ist anzumerken, dass die strukturelle Untersuchung der Clathrat-Hydrate, im speziellen von Argon-Clathrat-Hydraten, nicht im eigentlichen Gegenstand dieser Arbeit waren. Es stellte sich jedoch im Nachhinein heraus, dass die Modellierung des Argon-Clathrat-Hydrates in dem Phasengemisch aus Eis und Clathrat-Hydrat für die Rietveld-Strukturverfeinerungen der in den Experimenten gemessenen Eisprobe wesentlichen Einfluss auf das Verfeinerungsergebnis hatte (vergl. Kapitel 5.4).

Bei den Eis Ih Experimenten mit Argongas ist festzustellen, dass sich mit zunehmender Messzeit der Massenanteil des Clathrat-Hydrates erwartungsgemäß von anfänglich $0 \%$ für den ersten gemessenen Datensatz bei $\mathrm{p} \sim 1$ bar und $\mathrm{T} \sim 237 \mathrm{~K}$ bis auf ca. $70-80 \%$ bei dem zuletzt gemessenen Datensatz von p 1kbar und T 265K nach ca. $\mathrm{t}=3-5$ Tagen Messzeit erhöhte. Die Synthese erfolgte um so schneller, je höher der Gasdruck und je näher die Temperatur an der Schmelzkurve von Eis Ih war. Die Rietveld-Verfeinerung des Phasengemisches von Eis Ih und Argon-Clathrat-Hydrat bereitete aufgrund der nur annähernd kristallographisch beschriebenen Struktur des Argon-Clathrat-Hydrates als Funktion des Druckes und der Temperatur Probleme. Aus früheren experimentellen Arbeiten (Lobban et al. (1996)) ergaben sich abhängig vom Synthesedruck unterschiedliche kristallographische Modelle mit denen diese Verfeinerungen durchgeführt wurden. Für die Wasserstruktur war das kristallographische Modell in beiden Fällen gleich. Das Gasatom wurde im ersten Fall in dem kleinen und großen Käfig auf eine Lage bzw. im zweiten Fall in dem großen Käfig auf eine gesplittete Lage gesetzt (Lobban, pers. Mitteilung, (2000)). Im Zuge der Verfeinerungen 
stellte sich die beste Übereinstimmung des Strukturfits und der gemessenen Clathrat-HydratPeaks bei dem in der Tabelle 5.1 beschriebenen Modell mit je einer Gaslage pro Käfig heraus.

\begin{tabular}{|c|c|c|c|c|c|c|}
\hline Nr. & Atomtyp & $\mathbf{x}$ & $\mathbf{y}$ & $\mathbf{z}$ & frac & Uiso \\
\hline $\mathbf{1}$ & $\mathrm{O}$ & 0.8750 & 0.8750 & 0.8750 & 1 & 0.054 \\
\hline $\mathbf{2}$ & $\mathrm{O}$ & 0.7828 & 0.7828 & 0.7828 & 1 & 0.061 \\
\hline $\mathbf{3}$ & $\mathrm{O}$ & 0.8189 & 0.8189 & 0.6300 & 1 & 0.058 \\
\hline $\mathbf{4}$ & $\mathrm{D}$ & 0.8412 & 0.8412 & 0.8412 & 0.5 & 0.068 \\
\hline $\mathbf{5}$ & $\mathrm{D}$ & 0.8174 & 0.8174 & 0.8174 & 0.5 & 0.071 \\
\hline $\mathbf{6}$ & $\mathrm{D}$ & 0.7946 & 0.7946 & 0.7275 & 0.5 & 0.05 \\
\hline $\mathbf{7}$ & $\mathrm{D}$ & 0.8038 & 0.8038 & 0.6816 & 0.5 & 0.053 \\
\hline $\mathbf{8}$ & $\mathrm{D}$ & 0.8589 & 0.8589 & 0.6292 & 0.5 & 0.068 \\
\hline $\mathbf{9}$ & $\mathrm{D}$ & 0.7302 & 0.8550 & 0.5854 & 0.5 & 0.058 \\
\hline $\mathbf{1 0}$ & $\mathrm{Ar}$ & 0 & 0 & 0 & 0.99 & 0.085 \\
\hline $\mathbf{1 1}$ & $\mathrm{Ar}$ & 0.4137 & 0.4317 & 0.4317 & 0.2 & $0.25 *$ \\
\hline
\end{tabular}

Tabelle 5.1: Verwendetes Argon-Clathrat-Hydrat modell bei T 273K und p 2.5kbar; x,y,z: fraktionelle Koordinaten, frac: fraktionelle Besetzung auf der kristallographischen Lage, Uiso: isotrope ADP's (vergl. Kapitel 2), * : Der hohe Wert des Parameter Uiso deutet in dem verwendeten Modell auf eine ausgeschmierte, d.h. in dem grossen Käfig auf eine stark delokalisierte Lage der der Argonatome hin, der auf diesem Wert festgehalten wurde. Der Füllgrad gibt an, in welchem Maß der Clathratkäfig mit Gasmolekülen besetzt ist (vergl. Kapitel 4.1.1). Der Parameter frac in den Rietvled-Verfeinerungen entspricht im allgemeinen der Besetzung der angegebenen kristallographischen Lage in dem RietveldModell. Aus diesem lassen sich unter Berücksichtigung der Multiplizität der kristallographischen Lage der Füllgrad der Käfige angeben.

Eine freie Verfeinerung der Füllgrade der an der Clathrat-Hydratstruktur beteiligten Deuterium- und Sauerstoffatome sowie der isotropen ADP-Parameter war nicht möglich. Die Modellparameter wurden daher manuell variiert. Die manuelle Anpassung beschränkte sich, nach einigen Versuchen bei denen die Strukturverfeinerung divergierte, auf den Parameter frac und die ADP's der Gasatome in der Struktur. Die beste Anpassung wurde mit dem im oben genannten Modell beschriebenen fraktionellen Besetzungen erreicht. Dies entspricht einer Besetzung des kleinen Käfigs zu nahezu 100\% sowie des großen Käfigs zu 80\%. 


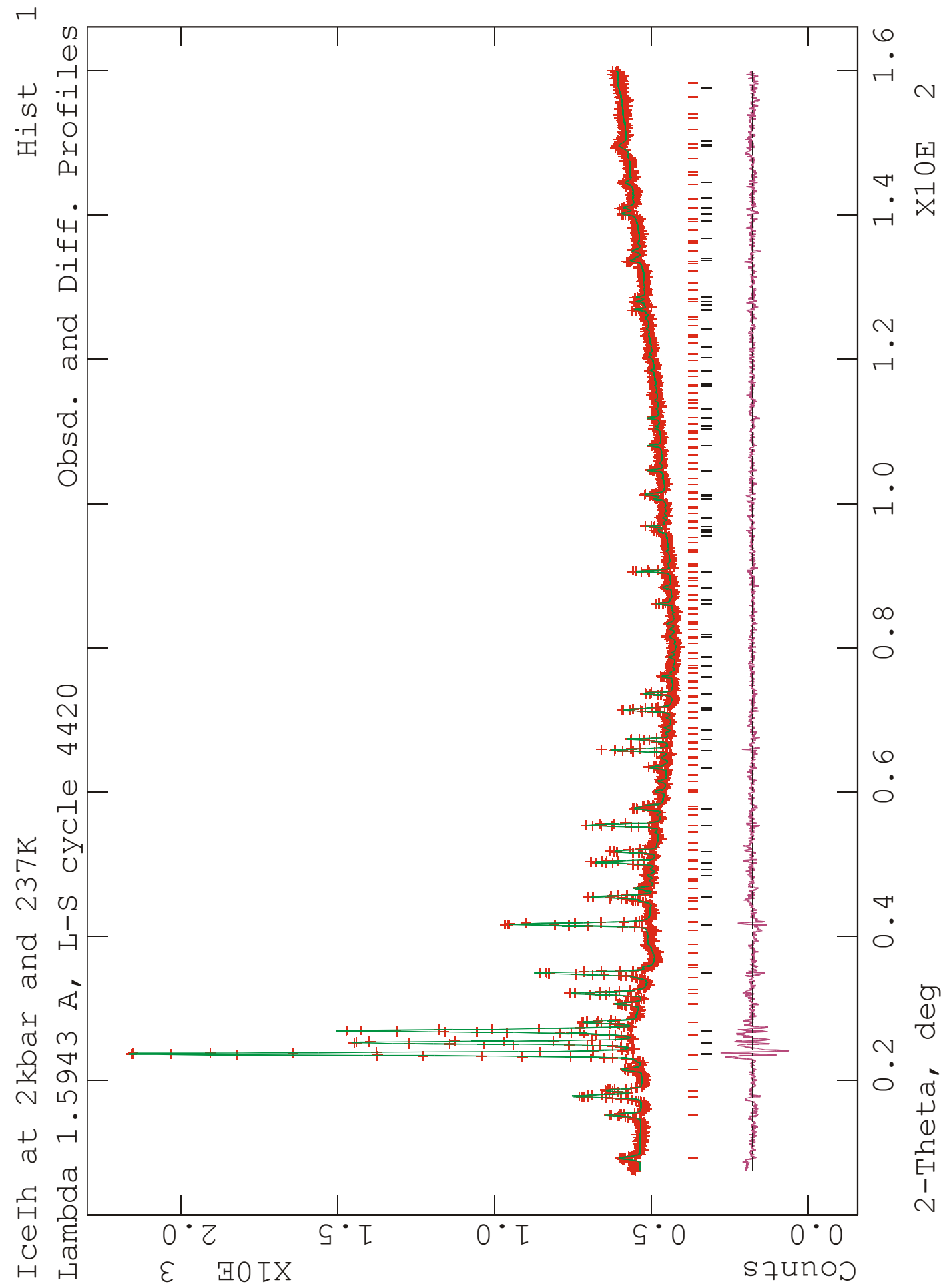

Abbildung 5.1: Rietveld-Strukturfit von Eis Ih und Argon-Clathrat-Hydrat bei T 237K und p 2kbar. Phasenanteil des Argon-Clathrat-Hydrates an der Probe beläuft sich auf n 22\%.

Dieses Modell wurde für die Rietveld-Anpassung für das bei $\mathrm{T} \sim 237 \mathrm{~K}$ und $\mathrm{p} \sim 2 \mathrm{kbar}$ synthetisiertem Argon-Clathrat-Hydrat (Abbildung 5.1) verwendet. Um das während der Messung synthetisierte Clathrat-Hydrat zu zersetzen wurde während des gesamten Experimentes nach dem Ende einer jeweiligen Messung der Druck verringert bzw. auf 
Umgebungsdruck gesenkt und einige Minuten bis zu einer halben Stunde abgewartet. In den meisten Datensätzen zeigten sich nach Druckentlastung Intensitätsveränderungen der Clathrat-Peaks zu den Eis-Peakintensitäten durch die Dissoziation von Argon-ClathratHydrat. Die Fehler der aus den Rietveld-Verfeinerungen berechneten Größen, wie z.B. die Gitterkonstanten sind für Argon-Clathrat-Hydrat mindestens um den Faktor zehn größer als die bei diesen Experimenten bestimmten Gitterparameter von Eis Ih. Eine Aussage bezüglich der Clathrat-Hydratstruktur ist daher nicht möglich, eine Diskussion der Ergebnisse der Verfeinerungen für das Clathrat-Hydrat soll aufgrund dieser Befunde nicht geführt werden.

\subsection{Neue Phasen im System Wasser und Neon}

In den eigenen Experimenten zur Schmelzkurven- und Phasengrenzenbestimmung von Eisphasen (vergl. Kapitel 4.3.1) wurden konsistent zu den Experimenten von Namiot \& Bukhgalter (1965) Hinweise auf eine Löslichkeit von Neon in Eis Ih gefunden. Es konnte festgestellt werden, dass die Löslichkeit von Neongas in der Eisstruktur wie bei Helium zu einer Ausweitung des Eis Ih-Stabilitätsbereiches führt. Im Zuge der mit Neongas durchgeführten Experimente konnten an der Göttinger pVT-Apparatur Hinweise erarbeitet werden, die neben der Existenz des Eis Ih-Neon-Hydrates auf die Existenz weiterer Phasen schließen ließen. Durch in situ Verifizierung mittels Neutronendiffraktion konnte der experimentelle Nachweis zweier, bis dato unbekannter Phasen, zum einen das Neon-ClathratHydrat vom Stackelberg Typ II, zum anderen ein dem Eis II verwandtes Neon-Hydrat, äquivalent zu dem in der Literatur beschriebenem Helium-Eis II-Hydrat in situ entdeckt sowie deren Struktur beschrieben werden. Die Schmelzkurven von Eis Ih mit Neon sowie von Eis II-Neon-Hydrat konnten jeweils im Druckbereich von $\mathrm{p} \sim 1 \mathrm{kbar}$ bis $\mathrm{p} \sim 2.75 \mathrm{kbar}$ sowie von $\mathrm{p} \sim 2.75 \mathrm{kbar}$ bis $\mathrm{p} \sim 4.7 \mathrm{kbar}$ verfolgt werden.

Die Existenz von Neon-Clathrat-Hydrat in der Literatur als unwahrscheinlich bezeichnet (z.B. De Forcrand (1923), Van der Waals \& Platteeuw (1959)). Lediglich der Einbau von Neon als zusätzliche Komponente in Doppel-Hydraten (Barrer \& Ruzicka (1962)) oder in einige topologisch verwandte Silikatstrukturen war bislang bekannt (Quarz und Christobalit, Barrer \& Vaughan (1967), Sodalith und Cancrinit, Barrer \& Vaughan (1971)). 


\subsubsection{Neon-Clathrat-Hydrat}

In dem ersten Experiment zur Bestimmung der strukturellen Eigenschaften des Eis Ih mit Argongas als Drucküberträgermedium ergaben sich Probleme sowohl bei der RietveldVerfeinerung der Eis Ih-Phase durch die ständig fortschreitende Synthese von ArgonClathrat-Hydrat als auch durch die zur Zeit nur ungenügende kristallographische Beschreibung dieser Phase. Für die Bildung von Clathrat-Hydraten ist neben Druck, Temperatur und den molekularen Wechselwirkungspotenzialen, die Größe des Gasmoleküls im Verhältnis zu den Käfiggrößen ein entscheidender Faktor. In einem zweiten Experiment zur Struktur des Eis Ih wurde Neongas als Drucküberträger verwendet. Bis zu diesem Experiment wurde davon ausgegangen, dass Neon sich zwar ähnlich dem Helium, in der Eisstruktur lösen kann, jedoch sowohl die Gasbesetzung als auch die Wechselwirkungen zu gering sind, um diffraktometrisch nachweisbare strukturelle Änderungen der Eis Ih-Struktur $\mathrm{zu}$ bewirken. Ebenso wurde davon ausgegangen, dass Neonatome zu klein sind um die Bildung von Neon-Clathrat-Hydrat zu ermöglichen. Im Zuge der hier durchgeführten Experimente konnte erstmals die Bildung von Neon-Clathrat-Hydrat nachgewiesen werden. Erste vage Hinweise auf die Ausbildung dieser neuen unbekannten Struktur hatten sich bereits während der Durchführung von Schmelzkurvenexperimenten an Eis Ih ergeben (vergl. Kapitel 4.3.1, Abbildung 4.5). Die Identifizierung als Neon-Clathrat-Hydrat erfolgte durch ein stetig an Intensität gewinnendes Beugungsdiagramm. Aus den Intensitätslagen konnte das Neon-Clathrat-Hydrat als Stackelberg Strukturtyp II identifiziert werden. Erste Anzeichen für das Neon-Clathrat-Hydrat zeigten sich bei Drücken von $\mathrm{p} \sim 1 \mathrm{kbar}$ und Temperaturen um T 215K. Anzumerken ist, dass die Eis Ih-Probe in dem das Neon-Clathrat-Wachstum stattfand keine „frische Probe gewesen ist“ sondern vielmehr eine Probe, die zuvor mit Argongas als Drucküberträgermedium bis zu Drücken von $\mathrm{p} \sim 2.5 \mathrm{kbar}$ gemessen worden ist. Das während dieser Messungen synthetisierte Argon-Clathrat-Hydrat wurde jedoch vor dem Einsatz von Neongas als Druckübertägermedium durch aufheizen bei Umgebungsdruck vollständig zersetzt. Die vollständige Zersetzung wurde an dem Beugungsbild nachgewiesen. Ebenso wurde das gesamte Druckerzeugersystem mehrmals mit Neongas gespült. Die nachfolgende Tabelle 5.2 stellt die bei einem Druck von $\mathrm{p} \sim 2 \mathrm{kbar}$ und der Temperatur von $\mathrm{T} \sim 215 \mathrm{~K}$ durch Rietveld-Verfeinerungen bestimmten Strukturparameter und die folgende Abbildung 5.2 die Anpassung der Verfeinerung an das gemessene Diffraktogramm dar. Für diesen Datensatz wurde der Phasenanteil an Neon-Clathrat-Hydrat zu 7.89\% bestimmt. 
Sowohl die Atompositionen als auch die ADP's konnten nicht frei verfeinert werden und wurden deshalb auf den Argon-Clathrat-Hydratwerten (vergl. Tabelle 5.1) festgehalten. Eine manuelle Variation der isotropen ADP's erbrachte keine wesentliche Verbesserung des Strukturfits. Daher wurden lediglich die fraktionellen Besetzungen und damit die Füllgrade des großen und kleinen Käfigs verfeinert.

\begin{tabular}{|c|c|c|c|c|c|c|}
\hline Nr. & Atomtyp & $\mathbf{x}$ & $\mathbf{y}$ & $\mathbf{z}$ & frac & Uiso \\
\hline $\mathbf{1}$ & $\mathrm{O}$ & 0.8750 & 0.8750 & 0.8750 & 1 & 0.054 \\
\hline $\mathbf{2}$ & $\mathrm{O}$ & 0.7828 & 0.7828 & 0.7828 & 1 & 0.061 \\
\hline $\mathbf{3}$ & $\mathrm{O}$ & 0.8189 & 0.8189 & 0.6300 & 1 & 0.058 \\
\hline $\mathbf{4}$ & $\mathrm{D}$ & 0.8412 & 0.8412 & 0.8412 & 0.5 & 0.068 \\
\hline $\mathbf{5}$ & $\mathrm{D}$ & 0.8174 & 0.8174 & 0.8174 & 0.5 & 0.071 \\
\hline $\mathbf{6}$ & $\mathrm{D}$ & 0.7946 & 0.7946 & 0.7275 & 0.5 & 0.05 \\
\hline $\mathbf{7}$ & $\mathrm{D}$ & 0.8038 & 0.8038 & 0.6816 & 0.5 & 0.053 \\
\hline $\mathbf{8}$ & $\mathrm{D}$ & 0.8589 & 0.8589 & 0.6292 & 0.5 & 0.068 \\
\hline $\mathbf{9}$ & $\mathrm{D}$ & 0.7302 & 0.8550 & 0.5854 & 0.5 & 0.058 \\
\hline $\mathbf{1 0}$ & $\mathrm{Ne}$ & 0 & 0 & 0 & 1.210 & 0.1 \\
\hline $\mathbf{1 1}$ & $\mathrm{Ne}$ & 0.418 & 0.418 & 0.418 & 0.486 & 0.25 \\
\hline
\end{tabular}

Tabelle 5.2: Strukturparameter der Rietveld-Verfeinerung für das Neon-Clathrat-Hydrat-Modell bei einem Druck von $p \sim 2 k b a r$ und einer Temperatur von T 215K; $x, y, z$ : fraktionelle Koordinaten, frac: Besetzung, Uiso: ADP's (Defintion vergl. Kapitel 2.)

Die Füllgrade konnten durch Rietveld-Verfeinerungen bei den gegebenen Bedingungen zu 121(11)\% für den kleinen Käfig (frac Ne10, Multiplizität 1) sowie 194(20 )\% für den großen Käfig (frac Ne11, Multiplizität 4) bestimmt werden und lassen eine Doppelbesetzung für den großen und kleinen Käfig vermuten. Die Gitterkonstante beträgt bei diesen Bedingungen $\mathrm{a}_{0}=17.0993 \AA$. Es ist jedoch darauf hinzuweisen, dass die Intensitätsanpassung der reflexüberlappenden Eis Ih-Peaks, des [100]-, [002]- und [101]-Peaks sowie der hkl-ClathratHydratpeaks bei tieferen Temperaturen $(\mathrm{T} \leq 215 \mathrm{~K})$ relativ hohe Abweichungen aufzeigen. Im Gegensatz dazu sind die Peakanpassungen für Winkel in $2 \Theta>30^{\circ}$ als gut zu bezeichnen. Im Kapitel 3.4.1 wurden diese Abweichungen, die durch vermutete Fehlordnungen der Eis IhStruktur verursacht werden, eingehender diskutiert. Aus den Rietveld-Verfeinerungen konnten die mit Neongas gemessenen Datensätze bei einer Temperatur von T 250K bestimmt werden, die zum einen die geringste Beeinflussung durch Neon-Clathrat-Hydrat sowie zum anderen die geringste Reflexänderung durch die Imperfektionen im Eis Ih aufwiesen. Für den 
Datensatz bei einem Druck von $\mathrm{p} \sim 2 \mathrm{kbar}$ und $\mathrm{T} \sim 250 \mathrm{~K}$ lag der Phasenanteil des ClathratHydrates bei etwa 8.1(7)\%.

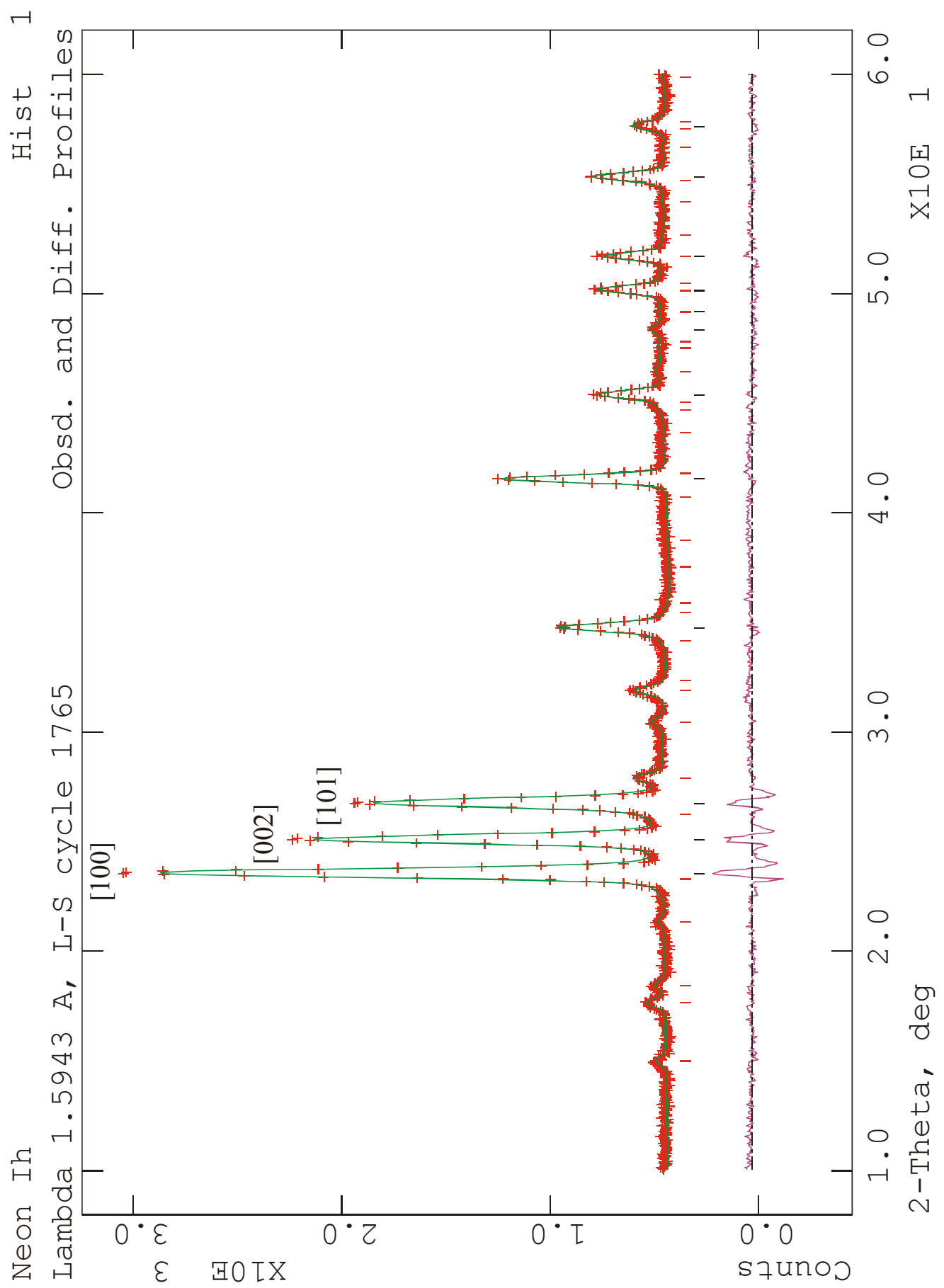

Abbildung 5.2: Rietveld-Strukturfit von Eis Ih bei Umgebungsbedingungen von p 2kbar und T 215K. In der Abbildung: (schwarz) Intensitätslagen der Eis Ih-Struktur, (rot) Intensitätslagen des Neon-ClathratHydrates vom Stackelberg Strukturtyp II. In dieser Abbildung ist zudem die durch verbliebene Imperfektionen des Eis Ih verursachte Abweichung des [100]- sowie des [002]-Reflexintensität zur [101]Reflexintensität im Diffraktogramm zu erkennen (vergl. simulierte Intensitätsverhältnisse in Abbildung 3.15, Kapitel 3.4.1). 

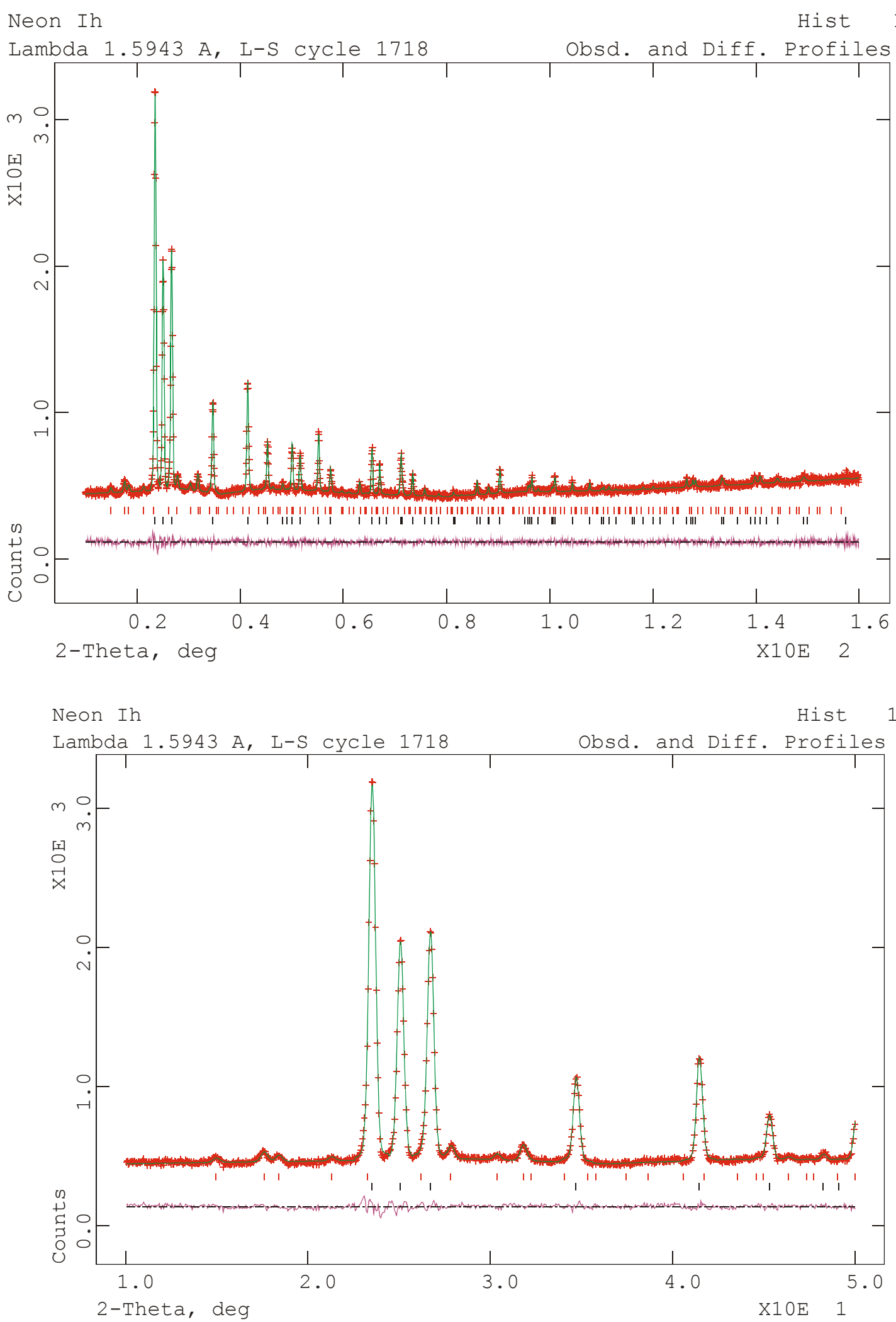

Abbildung 5.3: Rietveld-Strukturfits von Neon-Clathrat-Hydrat bei p 2kbar und T 250K; (oben): Diffraktogramm im Bereich $2 \Theta=10^{\circ}-160^{\circ}$, (unten): Diffraktogramm zur Indentifizierung des NeonClathrat-Hydrates relevanten Bereiches von $2 \Theta=10^{\circ}-50^{\circ}$. Die Intensitätsverhältnisse der [100]- und der [002]- sowie der [101]-Reflexintensität entsprechen annähernd denen von simuliertem polykristallinen Eis Ih gemäß Kuhs und Lehmann (1983) (vergl. Intensitätsverhältnisse in Tabelle 3.5 sowie Tabelle 3.6, Kapitel 3.4.1 als auch Abbildung 5.2). 
Die bestimmten Füllgrade des kleinen und großen Käfigs, äquivalent der Struktur des ArgonClathrat-Hydrates bei vergleichbaren Umgebungsbedingungen, ergaben wesentlich höhere Besetzungen für das Neon-Clathrat-Hydrat. In der Differenzkurve sind keine signifikanten Abweichungen zu erkennen und deuten ebenso wie der $w R p=2.25 \%$ und der $\mathrm{R} p=1.76 \%$ Wert auf eine gute Qualität und Übereinstimmung des Rietveld-Strukturfits hin. Problematisch ist neben dem geringen Phasenanteil die Peaküberlappung der stärksten Eis Ih-Reflexe, dem [100]- und dem [101]-Reflex. Aus den Verfeinerungen der Datensätze, die Neon-ClathratHydrat enthielten, konnte im Verlauf der Verfeinerungen eine starke Korrelation zwischen der "preferred orientation", d.h. einer Vorzugsorientierung der Eiskristallite der Eisphase, und des Füllgrades des großen Neon-Clathrat-Käfigs festgestellt werden. Auch zeigte sich eine Korrelation zwischen dem Phasenanteil des Neon-Clathrates-Hydrates mit den fraktionellen Besetzungen des Neons in der Eisstruktur. Ohne wohlbegründete Annahmen hinsichtlich der strukturellen Eigenschaften, die durch weitere experimentelle Befunde erarbeitet werden müssen, sind die Aussagen hinsichtlich der Gasbesetzung der neuen Phasen als nicht eindeutig gesichert anzunehmen. Lässt man jedoch eine Diskussion des Strukturfits des mit Neongas gemessenen Datensatzes bei $\mathrm{T} \sim 250 \mathrm{~K}$ und $\mathrm{p} \sim 2 \mathrm{kbar} z \mathrm{z}$, so ergibt sich für den kleinen Käfig eine nahezu vollständige Besetzung von 94\%. Für den großen Käfig ergibt sich eine zu einem vergleichbaren Argongas-Füllgrad beträchtliche Abweichung im Füllgrad. Dieser beträgt für das Neon-Clathrat-Hydrat $\sim 330(35) \%$. Dies entspricht einer mehr als dreifachen Besetzung der großen Käfige (vergl. Abbildung 5.3, Strukturfits für Neon-Clathrat-Hydrat bei p 2kbar und T 250K). Geht man von den Größenverhältnissen der Käfigstruktur, mit einer dem Argon-Clathrat-Hydrat annähernd gleichen Gitterkonstanten von $\mathrm{d}_{0}=6.57 \AA$ für den großen Käfig aus, so ließen sich rein von geometrischen Aspekten geleitet folgende Anordnungen und Füllgrade erwarten: Für Argonatome mit einem Wechselwirkungsdurchmesser von etwa d 3.76 A könnte jeweils nur ein Atom in den Käfig mit $\mathrm{d}_{0}=6.57 \AA$ eingebaut werden. Für Neonatome würde sich aufgrund des geringeren Wechselwirkungdurchmessers von $\mathrm{d} \sim 3.08 \AA$ bei verschiedenen Anordnungen Besetzungen von einem Neonatom bis zu fünf bei trigonaler dipyramidaler Anordnung oder sechs Neonatomen bei oktaedrischer Anordnung ergeben. Grundlage für diese Überlegungen ist jedoch, dass sich die Struktur der Käfige durch die Einlagerung nicht verändert. Ausgehend von diesem statischen, rein geometrischem Modell ohne Berücksichtigung thermischer Auslenkungen der Gasatome, erscheint die durch Rietveld-Verfeinerung bestimmte 3.3-fache Besetzung des großen Käfigs durchaus möglich. Die Messungen bei denen Neon-Clathrat- 
Hydrat beobachtet und die Anpassungen der fraktionellen Besetzungen aus den RietveldVerfeinerungen verifiziert werden konnten, sind in der folgenden Tabelle 5.4 in der Reihenfolge der Messungen aufgelistet.

\begin{tabular}{|c|c|c|c|c|c|c|c|c|}
\hline $\mathrm{Nr}$ & p [ bar] & $T[K]$ & $\%$ Neon & \multicolumn{2}{|c|}{ Fraktionelle Besetzung Frac } & $\mathbf{a}[\AA \AA]$ & wRp/Rp & \multirow{2}{*}{ Ih p.o. in [001] } \\
\hline \multicolumn{4}{|c|}{ SC : kleiner Käfig (small cage) } & SC & LC & \multicolumn{2}{|c|}{ LC : großer Käfig (large Cage) } & \\
\hline 1 & 1500 & 215 & $<0.1$ & - & - & - & $2.54 / 1.97$ & - \\
\hline 2 & 2000 & 215 & 7.89 & $1.211(143)$ & $0.486(120)$ & $17.0970(102)$ & $2.79 / 2.04$ & $0.9231(22)$ \\
\hline 3 & 2000 & 140 & 9.40 & $1.101(110)$ & $0.925(\mathrm{x})$ & $17.0476(69)$ & $2.66 / 2.08$ & $0.9326(47)$ \\
\hline 4 & 2550 & 140 & 9.30 & $0.365(25)$ & $1.006(210)$ & $17.0273(66)$ & $2.10 / 1.50$ & $0.9047(38)$ \\
\hline 5 & 1000 & 215 & 4.30 & $1.1386(\mathrm{x})$ & $1.014(\mathrm{x})$ & 17.1171(159) & $2.40 / 1.85$ & $0.9142(59)$ \\
\hline 6 & 500 & 215 & $<1$ & - & - & - & $2.49 / 1.93$ & - \\
\hline 7 & 1000 & 140 & 0 & - & - & - & $2.73 / 2.08$ & - \\
\hline 8 & 1 & 140 & 0 & - & - & - & $2.97 / 2.31$ & - \\
\hline 9 & 1500 & 140 & 0 & - & - & - & $2.31 / 1.60$ & - \\
\hline 10 & 1000 & 250 & 0 & - & - & - & $2.59 / 1.94$ & - \\
\hline 11 & 1500 & 250 & 0 & - & - & - & $2.41 / 1.84$ & - \\
\hline 12 & 2000 & 250 & 8.09 & $0.940(44)$ & $0.867(36)$ & $17.1288(96)$ & $2.25 / 1.76$ & $0.9789(56)$ \\
\hline 13 & 500 & 250 & 0 & - & - & - & $2.40 / 1.82$ & - \\
\hline 14 & 500 & 140 & 0 & - & - & - & $2.14 / 1.42$ & - \\
\hline
\end{tabular}

Tabelle 5.4: Rietveld-Verfeinerungsergebnisse der einzelnen Neon-Clathrat-Hydrat-Messungen; frac : fraktionelle Besetzung; p.o. : ,preferred orientation“, d.h. Vorzugsorientierung in [001], für p.o.=1 handelt es sich um eine völlig regellose Probe. Diese wurden vor der Verfeinerung der Füllgrade frei verfeinert und dann in den weiteren Verfeinerungszyklen festgehalten.

Durch einen Vergleich der bestimmten Phasenanteile als Funktion der Zeit bzw. der integrierten Intensitäten der ersten Neon-Clathrat-Hydrat-Peaks als Funktion von Druck und Temperatur, die während der Messungen bestimmt wurden, kann der Bereich in dem das Neon-Clathrat-Hydrat vorliegen kann, eingegrenzt werden (vergl. Abbildung 5.4).

Durch einen Vergleich der bestimmten Phasenanteile als Funktion der Zeit bzw. der integrierten Intensitäten der ersten Neon-Clathrat-Hydrat-Peaks als Funktion von Druck und Temperatur, die während der Messungen bestimmt wurden, kann der Bereich in dem das Neon-Clathrat-Hydrat vorliegen kann, eingegrenzt werden (vergl. Abbildung 5.4).

In jedem Falle zeigen die am D2B in situ durchgeführten Experimente an Neon-ClathratHydraten, dass das bei einer Temperatur von $\mathrm{T} \sim 250 \mathrm{~K}$ und bei Drücken von $\mathrm{p} \sim 2.2 \mathrm{kbar}$ synthetisierte Neon-Clathrat-Hydrat über einen Zeitraum von mehreren Stunden ( $\mathrm{t} \sim 16 \mathrm{~h}$ ) stabil blieb. Auch bei weiterer isothermer Druckerhöhung, bei einer konstanten Temperatur von $\mathrm{T} \sim 250 \mathrm{~K}$, zeigte sich das Neon-Clathrat-Hydrat über einen Zeitraum von jeweils $\mathrm{t} \sim 2 \mathrm{~h}$ bei 
Drücken von $\mathrm{p} \sim 2.8 \mathrm{kbar}$ sowie $\mathrm{p} \sim 3.5 \mathrm{kbar}$ stabil, so dass das Neon-Clathrat-Hydrat als stabile Phase gelten muss. Bei einer weiteren Druckerhöhung auf $\mathrm{p} \sim 4.2 \mathrm{kbar}$ wandelte sich das NeonClathrat-Hydrat schnell in das Eis II-(Ne)-Hydrat um. Aufgrund dieses experimentellen

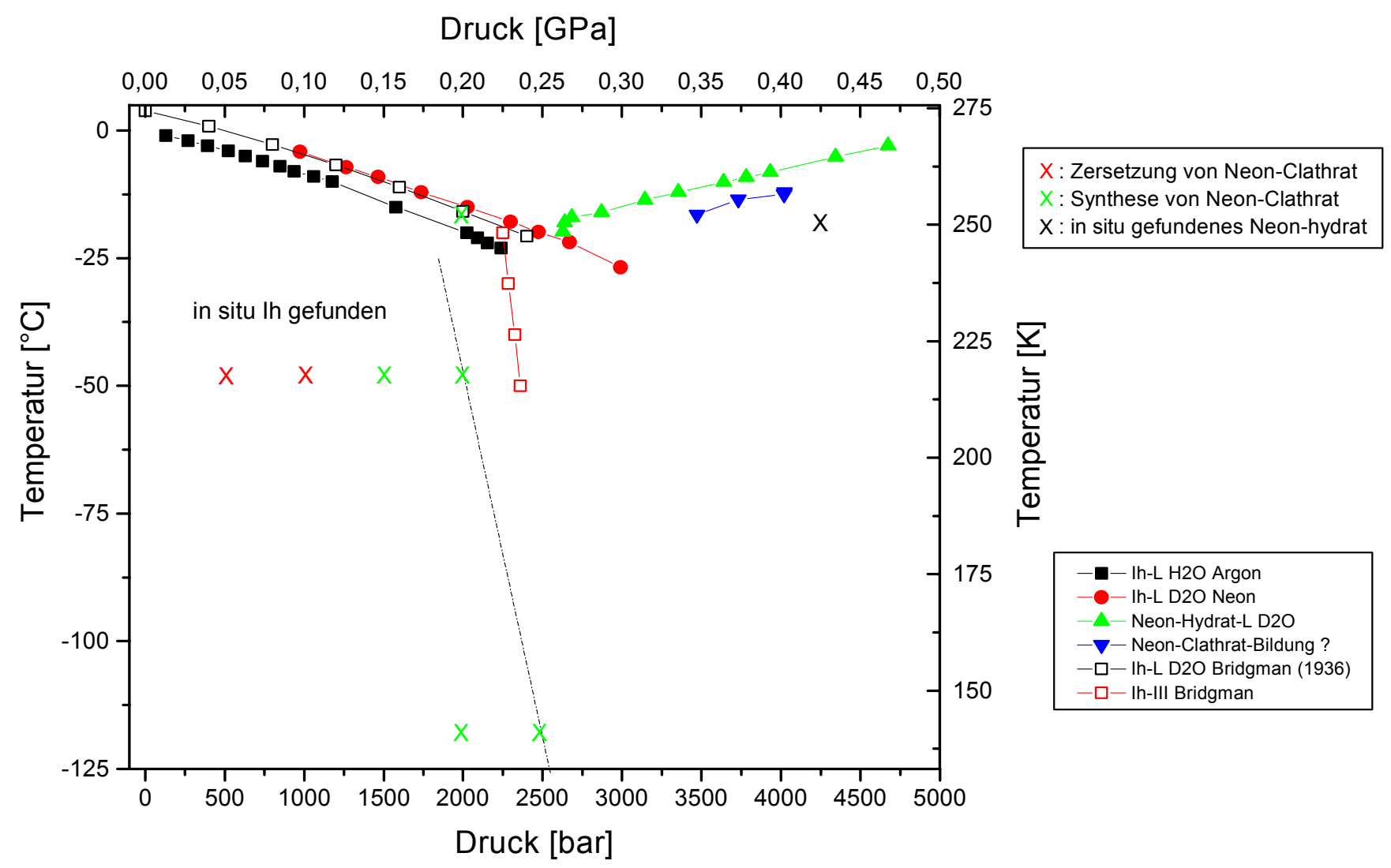

\begin{abstract}
Abbildung 5.4: abgeschätzter Stabilitätsbereich des Neon-Clathrates-Hydrates, bestimmt durch die Veränderung des Phasenanteiles an Neon-Clathrat-Hydrat an der Eis Ih-Probe als Funktion von Druck und Temperatur.
\end{abstract}

Befundes ist zu vermuten, dass das Neon-Clathrat-Hydrat zwischen den Stabilitätsbereichen von Eis Ih-(Ne)-Hydrat sowie von Eis II-(Ne)-Hydrat exitiert. Hinweise auf weitere unidentifizierte Phasenübergänge wurden in den Göttinger pVT-Experimenten gefunden (vergl. Abbildung 4.5, Kapitel 4.3.1). Bei der bestimmten unbekannten Phasengrenze (große blaue Dreiecke) zeigte sich ein langsamer Druckabfall der auf eine Phasenumwandlung in eine dichtere Phase hindeutet. Um eine anfangs vermutete Neon-Clathrat-Hydrat-Bildung scheint es sich nach den neuerlichen Ergebnissen indes nicht zu handeln. Um was für eine Phase es sich handelt, evtl. Eis V, ist im Nachhinein nicht zu verifizieren. In jedem Falle handelt es sich jedoch um eine dichtere Phase, da an den bestimmten Punkten eine deutliche Druckverringerung festgestellt wurde und dies somit die Phasengrenze von Eis II-(Ne)-Hydrat 
in eine dichtere Phase darstellt. Eine punktuelle in situ Verifizierung sowohl der Schmelzkurve als auch des Existenzbereiches konnte aus zeitlichen Gründen während der Messzeit nicht durchgeführt werden.

\subsubsection{Gas-Hydrate}

Im Gegensatz zu den früh entdeckten Clathrat-Hydraten wurde erstmals ein dem Eis II strukturell verwandtes Gas-Hydrat von Londono et al. (1992) bei in situ Neutronendiffraktionsexperimenten von Eis Ih unter Verwendung von Heliumgas als Drucküberträgermedium in dem Stabilitätsbereich von Eis II gefunden. Londono et al. (1992) stellten fest, dass die fest-fest-Phasengrenze von Eis Ih unter Verwendung von Helium zu höheren Drücken verschoben ist und durch Löslichkeit von Helium in der Eisstruktur über den eigentlichen Existenzbereich hinaus stabilisiert wird. Andere Phasen wie Eis III bzw. Eis V konnten bei Verwendung von Heliumgas in ihrem Stabilitätsbereich nicht oder nur transient hergestellt werden. Durch Arbeiten von Haas (1971) wurden Gaslöslichkeiten von Helium in normalem Eis Ih mittels Diffusionsmessungen experimentell belegt. Ebenso ergaben sich bei Schmelzkurvenexperimenten von Dyadin et al. (1994) unter Verwendung von Gasen wie Wasserstoff und Helium Verschiebungen der Schmelzkurven (vergl. Kapitel 4.3.1). In Neutronendiffraktionsexperimenten von Lobban et al. (1998) konnte ein Einfluss des als Drucküberträgermedium verwendeten Argongases auf die Eis Ih-Struktur sowie die Struktur von Eis II ausgeschlossen werden. Diese Befunde belegen eindeutig, dass Gase, deren Wechselwirkungsradien klein sind, wie im Falle des Wasserstoffs und Heliums, in Eisstrukturen wie Eis Ih und Eis II gelöst werden und diese dadurch über ihren eigentlichen Existenzbereich hinaus stabilisiert werden.

\subsubsection{Eis II-Neon-Hydrat}

In ersten pVT-Experimenten mit Neongas zeigte sich schon früh eine Phase oberhalb von Drücken $\mathrm{p}>2.5 \mathrm{kbar}$, deren Schmelzkurve nicht mit der von Eis III oder Eis II übereinstimmte. Eine in situ Bestimmung der strukturelle Parameter und die in situ Verifizierung einiger Schmelzpunkte dieser unbekannten, jedoch als Eis II-(Ne)-Hydrat vermuteten Phase, war daher geboten. Um dieser Frage nachzugehen, wurde die Probe bei $\mathrm{p} \sim 1$ bar Umgebungsdruck mit dem synthetisierten Neon-Clathrat-Hydrat auf T 250K erwärmt um das während der 
Messungen gebildete Neon-Clathrat-Hydrat zu zersetzen und anschließend aufgeschmolzen,. Danach wurde der Gasdruck auf das Wasser in der Probe von $\mathrm{p} \sim 1$ bar an in einem Schritt auf einen Druck von p 2.5kbar erhöht. In weiteren Schritten wurde dann der Druck um jeweils $\Delta \mathrm{p} \sim 500$ bar erhöht. Bei einer Temperatur von $\mathrm{T} \sim 250 \mathrm{~K}$ und einem Druck von $\mathrm{p} \sim 4.4 \mathrm{kbar}$ nukleierte aus der flüssigen Wasserphase eine dem Eis II von den Reflexlagen äquivalente und in den Reflexintensitäten ähnliche kristalline Phase. Bei einem Druck von $p \sim 4.2 \mathrm{kbar}$ und einer Temperatur von T 250K wurden dann zwei Datensätze gemessen. Anschließend wurde der Druck auf die Probe auf einen Druck von $p \sim 3 k b a r$ reduziert und die Probe in Schritten von $\Delta \mathrm{T} \sim 1 \mathrm{~K}$ erwärmt, um einen Schmelzpunkt in situ zu bestimmen. Weitere Meßpunkte oder die Bestimmung mehrerer Punkte der Schmelzkurve konnten aus zeitlichen Gründen nicht mehr durchgeführt werden. Grundlage für die Verfeinerung der gemessenen Datensätze ist das von Londono et al. (1989) für Eis II-(He)-Hydrat bestimmte kristallographische Modell. Dieser bestimmte bei annähernd äquivalenten Druck- und Temperaturbedingungen von $\mathrm{p} \sim 4.1 \mathrm{kbar}$ und $\mathrm{T} \sim 250 \mathrm{~K}$ ein dem Eis II verwandtes Eis II-(Helium)-Hydrat in einer rhomboedrischen Elementarzelle mit den Gitterkonstanten $\mathrm{a}_{\mathrm{H}}=12.9538(89) \AA$ und $\mathrm{c}_{\mathrm{H}}=6.2057(30) \AA$ in der Raumgruppe $\mathrm{R} \overline{3}$. Um die Struktur des Eis II-(Ne)-Hydrates durch Rietveld-Verfeinerungen zu verifizieren, wurde lediglich für das Startmodell an Stelle des Heliums Neon in das kristallographische Modell eingebaut. Tabelle 5.5 zeigt die Atompositionen und die jeweilige fraktionelle Besetzung (frac) für $\mathrm{O}$ und $\mathrm{D}$ auf.

\begin{tabular}{|c|c|c|c|c|c|c|c|}
\hline & $\mathbf{O ( 1 )}$ & $\mathbf{O ( 2 )}$ & $\mathbf{D}(\mathbf{3})$ & $\mathbf{D}(\mathbf{4})$ & $\mathbf{D}(\mathbf{5})$ & $\mathbf{D}(\mathbf{6})$ & $\mathbf{N e}$ \\
\hline $\mathbf{x}$ & $0.2138(17)$ & $0.1868(13)$ & $0.1581(15)$ & $0.2193(12)$ & $0.1032(14)$ & $0.2413(11)$ & 0 \\
\hline $\mathbf{y}$ & $0.1965(18)$ & $0.2339(10)$ & $0.1959(11)$ & $0.2079(11)$ & $0.2185(13)$ & $0.3094(15)$ & 0 \\
\hline $\mathbf{z}$ & $0.0580(31)$ & $0.4672(28)$ & $-0.0504(31)$ & $0.2143(22)$ & $0.4802(24)$ & $0.5671(36)$ & 0.297 \\
\hline frac* $^{*}$ & 1 & 1 & 1 & 1 & 1 & 1 & $\mathbf{x}_{\mathbf{N e}}{ }^{\#}$ \\
\hline
\end{tabular}

Tabelle 5.5: Strukturparameter der EisII-(Ne)-Hydrat Wasserstruktur, basierend auf Londono et al. (1992), * : frac : fraktionelle Besetzung der kristallographischen Lagen, $\mathbf{x}_{\mathrm{Ne}}$ : aus den RietveldVerfeinerungen zu bestimmende fraktionelle Gasbesetzung der Eis II-(Ne)-Hydratstruktur, \# : Bei dieser Atomposition handelt es sich um eine allgemeine Punktlage mit der Multiplizität von 6, so dass die bei einem frac $=1$ die Elementarzelle mit maximal 6 Gasatomen (100\%-Strukturbesetzung) besetzt sein kann.

\begin{tabular}{|c|c|c|c|}
\hline & Eis II & Eis II-(He)-Hydrat & Eis II-(Ne)-Hydrat \\
\hline $\mathbf{a}_{\mathbf{H}}[\AA]$ & $12.93239(34)$ & $12.9538(89)$ & $12.9331(43)$ \\
\hline $\mathbf{c}_{\mathbf{H}}[\AA]$ & $6.22339(35)$ & $6.2057(30)$ & $6.2071(32)$ \\
\hline $\mathbf{c}_{\mathbf{H}} / \mathbf{a}_{\mathbf{H}}$ & $0.4812(3)$ & $0.4791(4)$ & $0.4799(6)$ \\
\hline
\end{tabular}

Tabelle 5.6.1 : Vergleich der Eis II- zu den Eis II-(Gas)-Hydratstrukturen bei Umgebungsbedingungen von Eis II und Eis II-(Ne)-Hydrat @ p 4.2kbar und T 250K, sowie für Eis II-(Helium)-Hydrat bei $p \sim 4.77 \mathrm{kbar}$ und $\mathrm{T} \sim 195 \mathrm{~K}$ 


\begin{tabular}{|c|c|c|c|c|c|c|}
\hline \multicolumn{7}{|c|}{ Eis II bei p 4.2kbar und T 250K @ } \\
\hline & $\mathbf{O ( 1 )}$ & $\mathbf{O ( 2 )}$ & $\mathbf{D ( 3 )}$ & $\mathbf{D}(4)$ & $\mathbf{D ( 5 )}$ & $\mathbf{D}(6)$ \\
\hline $\mathbf{x}$ & $0.2138(17)$ & $0.1868(13)$ & $0.1581(15)$ & $0.2193(12)$ & $0.1032(14)$ & $0.2413(11)$ \\
\hline $\mathbf{y}$ & $0.1965(18)$ & $0.2339(10)$ & $0.1959(11)$ & $0.2079(11)$ & $0.2185(13)$ & $0.3094(15)$ \\
\hline$\overline{\mathbf{Z}}$ & $0.0580(31)$ & $0.4672(28)$ & $-0.0504(31)$ & $0.2143(22)$ & $0.4802(24)$ & $0.5671(36)$ \\
\hline $\mathbf{U}_{\text {iso }}$ & $0.029(2)$ & $0.020(1)$ & $0.046(1)$ & $0.036(1)$ & $0.037(1)$ & $0.034(1)$ \\
\hline \multicolumn{7}{|c|}{ Eis II-(Neon)-Hydrat bei $\mathrm{p} \sim 4.2 \mathrm{kbar}$ und $\mathrm{T} \sim 250 \mathrm{~K} \#$} \\
\hline $\mathbf{x}$ & $0.2253(57)$ & $0.1872(57)$ & $0.1514(57)$ & $0.2209(56)$ & $0.1087(58)$ & $0.2372(47)$ \\
\hline $\mathbf{y}$ & $0.2002(57)$ & $0.2320(50)$ & $0.1963(42)$ & $0.2128(59)$ & $0.2148(68)$ & $0.3059(49)$ \\
\hline $\mathbf{z}$ & $0.05393(123)$ & $0.4834(134)$ & $0.0003(423)$ & $0.1998(113)$ & $0.4806(111)$ & $0.5664(127)$ \\
\hline $\mathbf{U}_{\text {iso }}$ & $0.0371(17)$ & $0.0405(18)$ & $0.0613(17)$ & $0.0503(164)$ & $0.0504(174)$ & $0.0541(178)$ \\
\hline \multicolumn{7}{|c|}{ Eis II-(Helium)-Hydrat bei p $\sim 4.77 \mathrm{kbar}$ und $\mathrm{T} \sim 195 \mathrm{~K} *$} \\
\hline $\mathbf{x}$ & $0.2195(6)$ & $0.1896(5)$ & $0.1517(4)$ & $0.2248(4)$ & $0.1089(6)$ & $0.2380(4)$ \\
\hline $\mathbf{y}$ & $0.1965(6)$ & $0.2310(4)$ & $0.2010(4)$ & $0.2168(4)$ & $0.2162(6)$ & $0.3053(4)$ \\
\hline $\mathbf{z}$ & $0.048(1)$ & $0.483(1)$ & $-0.011(1)$ & $0.2003(8)$ & $0.4855(9)$ & $0.5591(8)$ \\
\hline$U_{\text {iso }}$ & $0.0282^{+}$ & $0.0282^{+}$ & $0.0373^{+}$ & $0.0373^{+}$ & $0.0373^{+}$ & $0.0373^{+}$ \\
\hline
\end{tabular}

Tabelle 5.6.2 : Eis II-Hydratstrukturen im Vergleich : @ Eis II (Lobban et al. 1998) unter Verwendung von Argon als Drucküberträgermedium, \# EisII-(Neon)-Hydratstruktur (diese Arbeit) bei annähernd

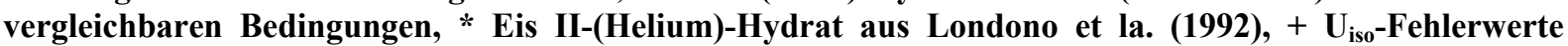
berechnet aus Londono et al. (1992). Als problematisch zeigte sich die Verfeinerung des mit Parameters $\mathbf{U}_{\text {iso }}$ korrelierten Füllgrades $x_{\mathrm{Ne}}$ der Gasbesetzung der Neonatome auf den kristallographischen Lagen gemäß Tabelle 5.5.

\begin{tabular}{|c|c|c|c|c|c|}
\hline & $\mathbf{x}$ & $\mathbf{y}$ & $\mathbf{z}$ & frac & $\mathbf{U}_{\text {ISo }}$ \\
\hline Neon $^{1}$ & 0 & 0 & $0.2824(120)$ & $0.144(5)$ & $0.3(2)$ \\
\hline Neon $^{2}$ & 0 & 0 & $0.2726(142)$ & $0.389(17)$ & $0.126^{\#}$ \\
\hline Helium * & 0 & 0 & $0.277(6)$ & 0.170() & $0.126^{+}$ \\
\hline
\end{tabular}

Tabelle 5.7 : Vergleich der Helium und Neon Gaspositionen in Eis II-Hydrat sowie der fraktionellen Besetzung der in Abb. 4.2 dargestellten Gaslagen in den Kanälen der Eis II-Hydratstruktur. Die x- sowie die y-Koordinate zeigen an, dass sich das Gasatom in dem Rietveld-Modell in der Mitte der Kanäle aufhält, lediglich die z-Positionen sind verschieden, +: keine Fehlerangabe von Londono et al. (1992)*. Die Verfeinerung der fraktionellen Koordinaten, des Besetzungsparameters frac sowie die thermischen Auslenkungsparameter ADP wurden sequentiell durchgeführt.

Das Neonatom wurde auf die Position $x=0, y=0$ und $z=0.297$ gesetzt und befindet sich damit zwischen einem "puckered ring" und einem "flat ring” der Eis II ähnlichen Struktur (vergl. Abbildung 4.2, Kapitel 4.1.2). Aus den Rietveld-Verfeinerungen ergibt sich bei Umgebungsbedingungen von $\mathrm{p} \sim 4.2 \mathrm{kbar}$ und $\mathrm{T} \sim 250 \mathrm{~K}$ für das Eis II-(Neon)-Hydrat folgende verfeinerten Strukturparameter (Gitterkonstanten, Atompositionen, Gasbesetzungen) im Vergleich zum Eis II-(Helium)-Hydrat (Londono et al. (1989) sowie zum gasunbeeinflussten Eis II (Lobban et al. (1998)). 
In der vorherigen Tabelle 5.6.1 sowie Tabelle 5.6.2 ist das Ergebnis der Verfeinerung der Atompositionen für Eis II-(Neon)-Hydrat von zwei gemessenen und verfeinerten Datensätzen dargestellt. Signifikante Unterschiede von Eis II-(Neon)- und Eis II-(Helium)-Hydrat sind in den Atompositionen der Eis II-Hydratstruktur festzustellen. Die Gaspositionen sowie die c/aVerhältnisse der Gitterkonstanten zeigen dagegen keine signifikanten Unterschiede ab.

Für die allgemeine Punktlage der Neonposition in der Eis II-Hydrat-Elementarzelle ergibt sich gemäß Tabelle 5.7 eine Besetzung der Elementarzelle mit Neonatomen zu $n=38.9(1.7) \%$ bei einem festgesetztem Parameter $\mathrm{U}_{\text {iso }}=0.126$ mit $\mathrm{wRP}=3.02 \%$ sowie $\mathrm{Rp}=2.22 \%$ für die Güte der Verfeinerung. Von Londono et al. (1992) wurde bei vergleichbaren Bedingungen, d.h. einem Druck von $\mathrm{p} \sim 4.77 \mathrm{kbar}$ und einer Temperatur von $\mathrm{T} \sim 250 \mathrm{~K}$ für die Heliumatome ein thermischer Parameter von $\mathrm{U}_{\text {iso }}=0.126$ bei einer Helium-Besetzung in der Eisstruktur von $\mathrm{n}=102 \%$ bestimmt. Eine gleichzeitige Verfeinerung der fraktionellen Koordinaten sowie des thermischen Parameters $U_{\text {iso }}$ für die Neonatome war nicht möglich, wurde daher manuell

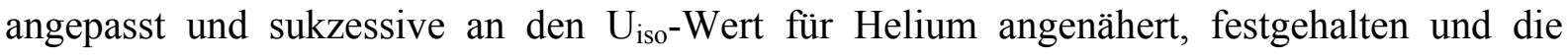
Struktur mit variablen fraktionellen Koordinaten verfeinert.

Die Gasbesetzung der Eis II-(Ne)-Hydratstruktur zeigte sich sensibel gegenüber der Verfeinerung sowie der Verfeinerungstrategie hinsichtlich der Reihenfolge der Verfeinerungsparameter. Im Falle der Besetzung scheint sich hier die unterschiedliche Größe des Gasatoms bemerkbar zu machen, die zu einer geringeren Besetzung der Hydratstruktur mit Neon führt. Die nachfolgende Abbildung 5.5 zeigt das Verfeinerungsergebnis des Eis II(Neon)-Hydrat Datensatzes. In der Differenzkurve sind jedoch gerade im hinteren Bereich des Diffraktogramms Abweichungen festzustellen. Vermutungen, es könnte sich um festes, auskristallisiertes Neon handeln, ließen sich jedoch auf Basis der Arbeiten von Mills und Grilly (1955) nicht bestätigen.

Wahrscheinlicher ist, dass es sich um eine Vorzugsorientierung handelt, die wesentlichen Einfluss auf das Verfeinerungsergebnis haben. Von einer Vorzugsorientierung kann ausgegangen werden, da die Neon-Hydrat-Proben nicht durch die Kompression von polykristallinem Eis Ih gewonnen wurden, sondern diese aus der flüssigen Phase bei einem Druck von $\mathrm{p} \sim 5 \mathrm{kbar}$ und einer Temperatur von $\mathrm{T} \sim 250 \mathrm{~K}$ nukleiert wurden. 


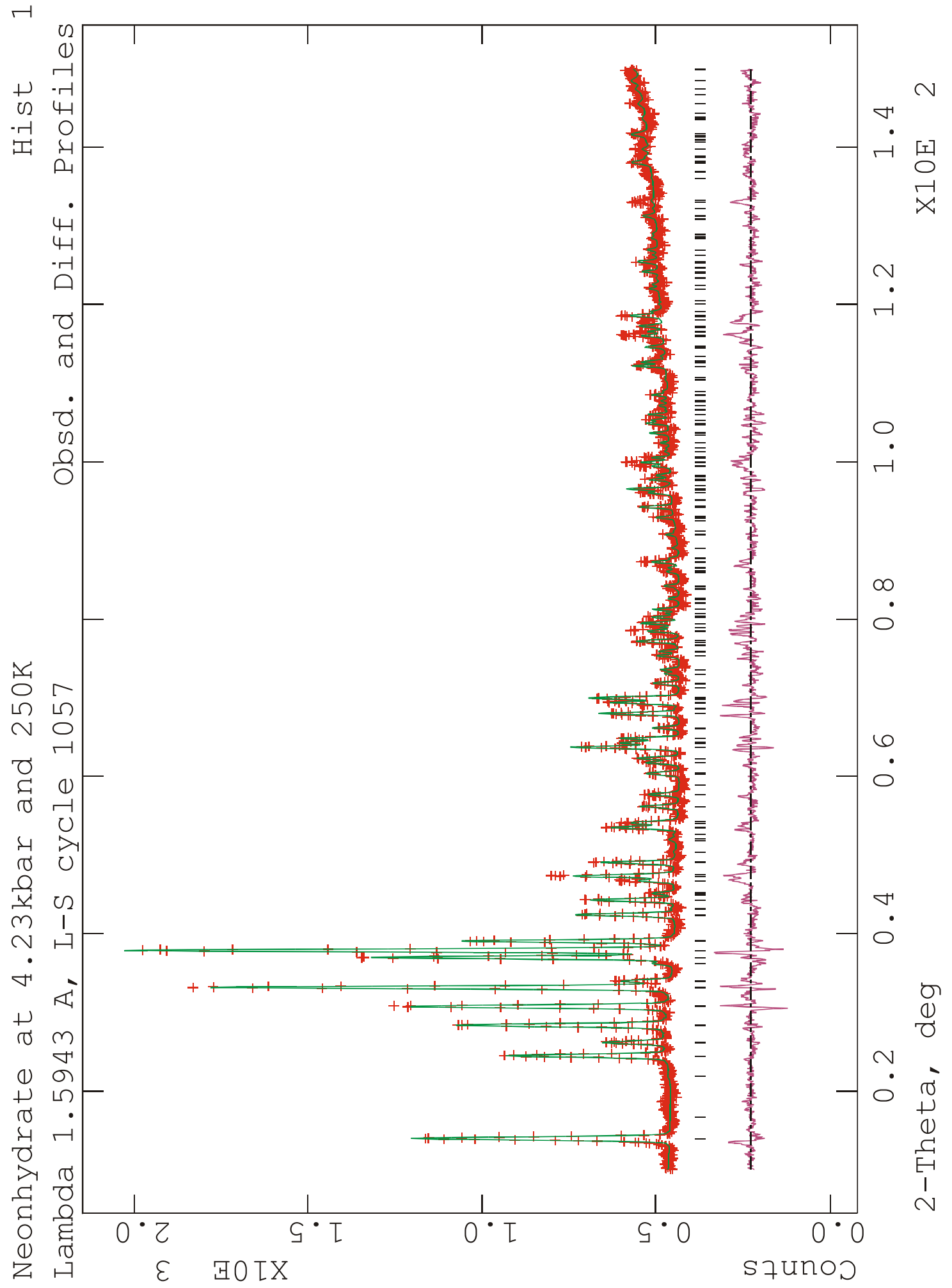

Abbildung 5.5: Rietveld-Strukturfit von Eis II-(Ne)-Hydrat bei p 4.23kbar und T 250K mit einer freiverfeinerten Gasbesetzung von $n=38.9(1.7) \%$ bei einem thermischen Parameter für Neon von Uiso=0.3(2). Im hinteren Diffraktogramm sind Abweichungen in der Rietveld-Anpassung zu erkennen . 
Der Aufbau der Eis II-Struktur in die Hydratstruktur wird durch den Einbau der Gase Neon und Helium signifikant geändert. Die Änderungen in den Gitterkonstanten bei dem Einbau von Neon oder Helium zeigen ebenso wie die Änderungen der atomaren Positionen der Eis IIHydratstruktur keine im Rahmen der Fehler wesentlichen Abweichungen, so dass durch den Einbau von Neon statt Helium in die Eis II-Hydratstruktur der Aufbau der Ringstrukturen eine nur unwesentliche Änderung erfährt.

Durch in situ Experimente am D2B des ILL zeigte sich, dass bei einer Präparation im System Eis Ih und unter Verwendung von Neongas als Drucküberträgermedium bei Drücken von $\mathrm{p} \sim 2.1 \mathrm{kbar}$ sowie $\mathrm{p} \sim 2.4 \mathrm{kbar}$ Eis III reproduzierbar in mehreren Versuchen gefunden werden konnte.

Aufgrund einer schlechten Pulverqualität, bedingt durch eine ausgeprägte Vorzugsorientierung war, eine Rietveld-Verfeinerung hinsichtlich der Struktur jedoch nicht möglich. Die Präparation erfolgte über die Kompression von Eis Ih bei T 250K auf einen Druck von $\mathrm{p} \sim 2.5 \mathrm{kbar}$. Bei weiterer Druckerhöhung zeigte sich, dass das Eis III bis zu Drücken von $\mathrm{p} \sim 3.5 \mathrm{kbar}$ stabil war. Bei weiterer Druckerhöhung erfolgte dann eine Phasentransformation in Eis II-(Ne)-Hydrat. Aufgrund dieses experimentellen Befundes lässt sich vermuten, dass die in Göttingen mit der pVT-Apparatur bestimmte Eis II-(Ne)-HydratSchmelzkurve, die punktuell am D2B des ILL in-situ verifiziert wurde, im oberen Bereich der Eis II-(Ne)-Hydrat-Schmelzkurve entspricht.

Bei einem Druck von p 3.75kbar zeigt sich in der gemessenen Schmelzkurve ein „KINK“, der sich bei näherer Betrachtung durch Schmelzkurvenfits in einer deutlichen Änderung der Krümmung bemerkbar macht (vergl. Abbildung 4.5, Kapitel 4.3.1 und Abbildung 5.4, Kapitel 5.2.1 zur folgenden Abbildung 5.6). Es scheint nicht ausgeschlossen, dass es sich bei diesem Punkt, der im Schnittpunkt zweier, an den gemessenen Kurvenverläufen gefitteten Funktionen liegt, um einen Phasenübergang von EisII-(Ne)-Hydrat in Eis III handelt. Im System Helium und Eis wurden bei der Kompression von Eis Ih auf Drücke p $>2.5$ kbar zu Eis II-(He)-Hydrat, Eis III als transiente Phase gefunden (Kuhs, Gotthardt, Moretzki, pers. Mitteilung 2001). 
Es ist daher nicht auszuschließen, dass die in den pVT-Experimenten in dem Druckbereich von $\mathrm{p} \sim 3.75 \mathrm{kbar}$ bis $\mathrm{p} \sim 2.7 \mathrm{kbar}$ bestimmte Eis II-(Ne)-Hydrat-Schmelzkurve derjenigen von in situ gefundenem Eis III entspricht.

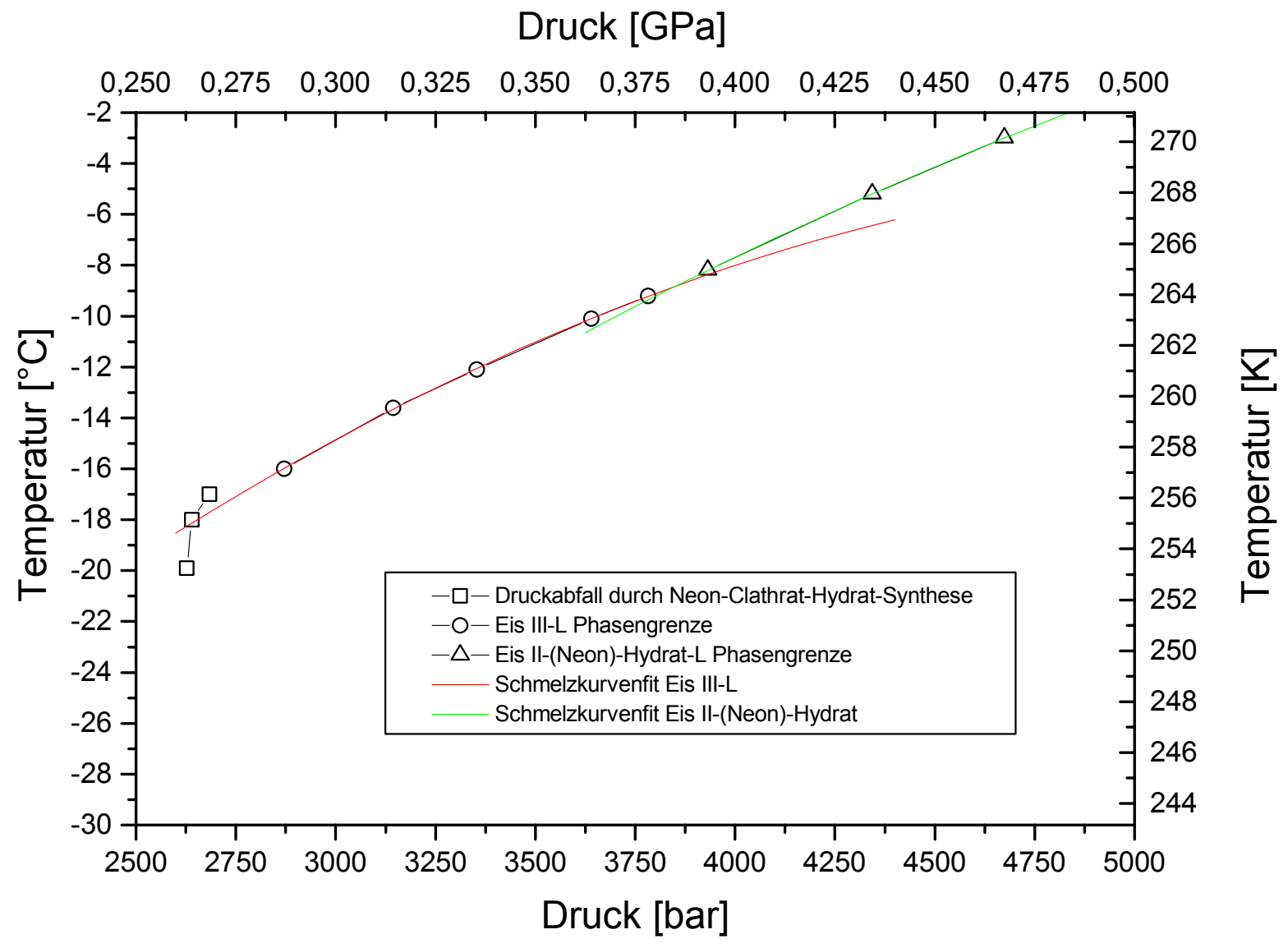

\footnotetext{
Abbildung 5.6: „Kink“ in der Schmelzkurve bei p 3.75kbar, eventueller Tripel-Punkt der Phasen Eis III(Ne)-Hydrat, Eis II-(Ne)-Hydrat und flüssiger Phase $L$ bei einem Druck p 3.85kbar $\pm 140 \mathrm{bar}$ und einer Temperatur $T \sim 8.89 \pm 0.5^{\circ} \mathrm{C}(264.25 \mathrm{~K} \pm 0.5 \mathrm{~K})$, im System $\mathrm{D}_{2} \mathrm{O}$.
} 


\subsection{Strukturelle Untersuchungen an Eis Ih}

\subsubsection{Die mittlere Struktur von Eis Ih}

In der Elementarzelle des Eis Ih sind vier Wassermoleküle enthalten, die über Wasserstoffbrücken miteinander verbunden sind. Entlang einer Wasserstoffbrückenbindung befinden sich zwei Wasserstoffe mit je einer halben Besetzung auf der Bindung. Die mittlere Eisstruktur genügt der hexagonalen Raumgruppe $\mathrm{P}_{3} / \mathrm{mmc}$. Die Struktur von Eis Ih wurde von Kuhs \& Lehmann (1986) an Eis Ih-Einkristallen bestimmt. Bei diesen Messungen wurden die Atompositionen und die Gitterkonstanten bestimmt. Unter der Berücksichtigung der Symmetrieoperationen der Raumgruppe kann die Elementarzelle und die Kristallstruktur durch das folgende kristallographische Modell in Tabelle 5.8 beschrieben werden.

\begin{tabular}{|c|c|c|c|c|c|}
\hline Nr. & Atomtyp & $\mathbf{x}^{\mathbf{1}}$ & $\mathbf{y}^{\mathbf{1}}$ & $\mathbf{z}^{\mathbf{1}}$ & frac $^{2}$ \\
\hline $\mathbf{1}$ & O & 0.3333 & 0.6667 & 0.0621 & 1 \\
\hline $\mathbf{2}$ & D & 0.3333 & 0.6667 & 0.1983 & 0.5 \\
\hline $\mathbf{3}$ & D & 0.4539 & 0.9075 & 0.0171 & 0.5 \\
\hline
\end{tabular}

Tabelle 5.8: Eis Ih Atompositionen, ${ }^{1}: x, y, z$ entsprechen den fraktionellen Koordinaten, frac : fraktionelle Besetzung der kristallographischen Lage. Atompositionen bei Umgebungsdruck p 1bar und einer Temperatur von T 223K aus Kuhs \& Lehmann (1986) with revisions Kuhs (1996, pers. Mitteilung)

Die vier Positionen der O-Atome in der Elementarzelle sind durch die Koordinaten

$$
1 / 3,2 / 3, z_{0} \quad 1 / 3,2 / 3,1 / 2-z_{0} \quad 2 / 3,1 / 3,1 / 2+z_{0} \quad 2 / 3,1 / 3,1-z_{0}
$$

gegeben, wobei $z_{0}$ eine freie Koordinate dieser Lage ist. Aufgrund der Besetzung einer Wasserstoffbrücke mit je einem halben Wasserstoff (Deuterium) ist ein Sauerstoff von je vier halbbesetzten Wasserstoffen umgeben, wobei diese in zwei Bindungstypen innerhalb der Eisstruktur unterschieden werden können. Je vier halbbesetzte Wasserstoffe vom Typ H1 befinden sich in den Bindungen in c-Richtung, deren Koordinaten sind durch 1/3, 2/3, $\mathbf{z}_{\mathbf{h} \mathbf{1}}$ gegeben. Weiterhin existieren 12 je halbbesetzte Wasserstoffe vom Typ H2, deren

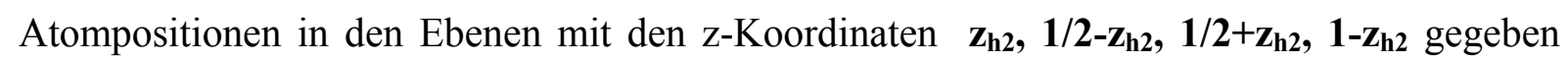
sind durch

$$
\mathrm{x}_{\mathrm{h} 2}, 1-\mathrm{x}_{\mathrm{h} 2}, \mathrm{z}_{\mathrm{h} 2} \quad \mathrm{x}_{\mathrm{h} 2}, 2 \mathrm{x}_{\mathrm{h} 2}, \mathrm{z}_{\mathrm{h} 2} \quad 1-2 \mathrm{x}_{\mathrm{h} 2}, 1-\mathrm{x}_{\mathrm{h} 2}, \mathrm{z}_{\mathrm{h} 2}
$$


Die Bindungsabstände und die Bindungswinkel der O-Atome sowie der D-O-DBindungswinkel untereinander sind bei dem oben angegebenen Datensatz sowie bei den weiteren Temperaturen in der nachfolgenden Tabelle 5.9 angegeben.

\begin{tabular}{|c|c|c|c|}
\hline $\mathbf{T}[\mathbf{K}]$ & $\mathbf{2 2 3}$ & $\mathbf{1 2 3}$ & $\mathbf{6 0}$ \\
\hline $\mathbf{O 1 - O 2}[\AA \boldsymbol{\AA}]$ & $2.752(8)$ & $2.755(1)$ & $2.754(1)$ \\
\hline $\mathbf{O 2 - O 3}[\AA]$ & $2.765(1)$ & $2.755(1)$ & $2.753(1)$ \\
\hline $\mathbf{O 1 - O 2 - O 5}\left[^{\circ}\right]$ & $109.55(15)$ & $109.32(1)$ & $109.55(15)$ \\
\hline $\mathbf{O 2 - O 3 - O 1 2}\left[^{\circ}\right]$ & $109.40(2)$ & $109.62(1)$ & $109.40(2)$ \\
\hline $\mathbf{D 1 - O 1 - D 2}\left[^{\circ}\right]$ & $109.10(30)$ & $109.30(6)$ & $109.31(6)$ \\
\hline $\mathbf{D 2 - O 1 - D 2}\left[^{\circ}\right]$ & $109.86(26)$ & $109.65(6)$ & $109.63(6)$ \\
\hline
\end{tabular}

Tabelle 5.9 Bindungswinkel und Bindungsabstände in Eis Ih, *.) aus Kuhs (1996), bei Umgebungsdruck von $p \sim 1 b a r$

Eine signifikante Temperaturabhängigkeit der Atompositionen und damit der Bindungslängen und Bindungswinkel ist unter Berücksichtigung sowohl der in Tabelle 5.9 dargestellten als auch der angepassten thermischen Auslenkungen aus den Verfeinerungen nicht zu erkennen. Die Bindungswinkel variieren im Rahmen des Fehlers um den idealen Tetraederwinkel von $109.47^{\circ}$.

Die Sauerstoffatome in der Eisstruktur sind in hexagonalen Ringen zu je sechs Sauerstoffatomen angeordnet und liegen mit der Ringnormalen in Richtung der c-Achse sowie senkrecht zu dieser (vergl. Abbildung 5.7). Die Ringe sind nicht planar und in einer schichtförmigen Struktur jeweils in der Stapelfolge ...ABABAB... in der Kristallstruktur angeordnet, so dass sich durch die Stapelung der Ringe in c- und senkrecht dazu in aRichtung eine kanalförmige, offene Struktur ergibt. Die Sauerstoffatome des in Richtung der c-Achse orientierten Ringes sind in einem sogenannten „chair ring“ angeordnet und liegen in der Kristallstruktur im Abstand $\mathrm{z}_{\mathrm{h}}$ etwas ober- und unterhalb der Basis-Ebenen.

Die Folge, mit der die Sauerstoffe im Layer A angeordnet sind folgen, angefangen bei dem Atom O1, der Sequenz +, -, +, -,+, - . Im Layer B ist die Folge entsprechend umgekehrt. Sowohl der Bindungsabstand als auch der Bindungswinkel der O-Atome in dem c-Ring (,chair ring“) sind aufgrund der Symmetrie der Raumgruppe gleich. Der Durchmessser des Kanals in c-Richtung kann aus den aus den tabellierten zu d $\sim 5.283 \AA$ angegeben werden. 


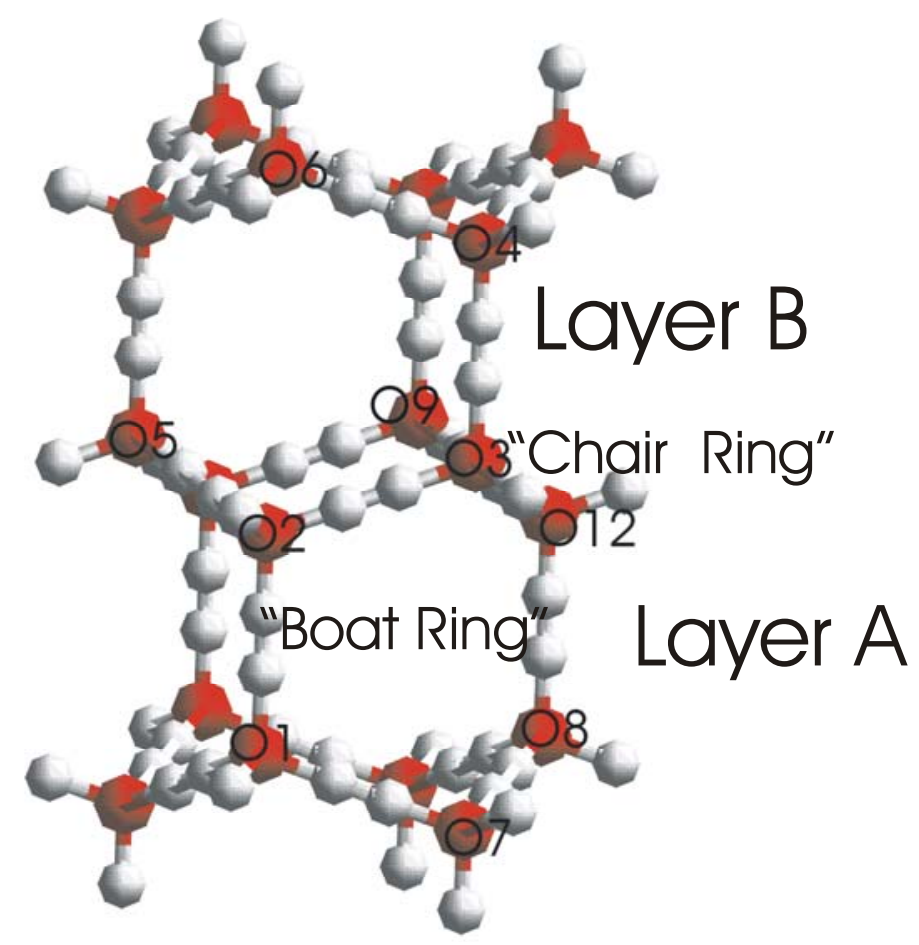

Abbildung 5.7: Eisstruktur, aufgebaut aus senkrecht zueinander stehenden Wassermolekülringen, aus dem „boat ring“ sowie dem „chair ring“. Die c-Gitterachse liegt parallel zu der Wasserstoffbrückenbindung der Sauerstoffe O8-O12 in dem „Boat ring“, senkrecht dazu in der aGitterachse, d.h. parallel zur Wasserstoffbrückenbindung O2-O12 liegende der, „chair ring““.

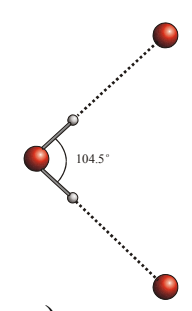

a.)

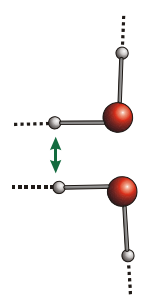

c.)

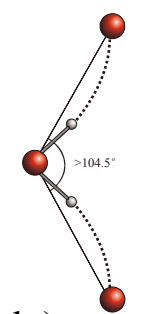

b.)

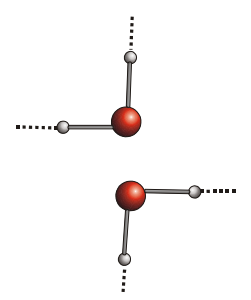

d.)

Abbildung 5.8: a.) unbeeinflusste Wasserstoffbrücke, b.) Verbiegung bzw. Verwindung der Wasserstoffbrücke; c.) und d.) Ausbildung von repulsiven O-O, D-D-Wechselwirkungskontakten durch einen äußeren Einfluss 
Die Ringe senkrecht zur c-Achse (,boat rings“) sind einfacher aufgebaut. Die Bindungsabstände innerhalb der ,chair- und der „,boat“-Ringe sind aufgrund der Symmetrie

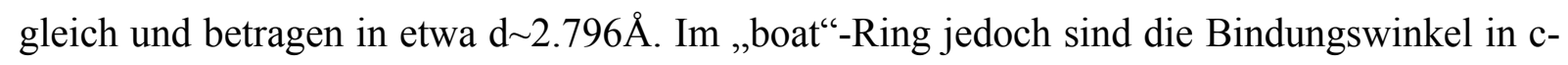
Richtung leicht vergrößert (vergl. Tabelle 5.9).

Eis Ih zeigt wie das flüssige Wasser eine Dichteanomalie, die unterhalb der Temperaturen von $\mathrm{T} \sim 73 \mathrm{~K}$ zu einer negativen thermischen Expansivität führt. Die Mechanismen, die zu diesem physikalischem Verhalten führen, sind für Wasser und Eis auf molekularer Ebene noch nicht gänzlich verstanden. Die Vermutung ist, dass die physikalische Ursache durch die Wasserstoffbrückenbindungen der in der Eisstruktur angeordneten Wassermoleküle bedingt ist (vergl. Abbildung 5.8).

Durch die Anordnung der Wassermoleküle und die Ausbildung der Wasserstoffbrückenbindungen ergeben sich unter den in der Struktur befindlichen Molekülen attraktive sowie repulsive Wechselwirkungen. Diese sind durch die attraktiven Wechselwirkungen O-D...O sowie die repulsiven Wechselwirkungen durch D...D und O...O dargestellt. Anzumerken ist, dass derzeitig kein universelles Wasserpotenzial existiert, das alle physikalischen Eigenschaften der flüssigen Wasserphase sowie die strukturellen und dynamischen Eigenschaften der kristallinen Eisphasen beschreiben kann (Guillot and Guissani (2001).

Für Aussagen über die strukturbestimmenden molekularen Wechselwirkungen ist die Kenntnis der strukturellen Parameter als Funktion des Druckes und der Temperatur über einen weiten Bereich hinweg sehr nützlich. Aufgrund des größeren Van der Waals'schenWechselwirkungsradius von Sauerstoff gegenüber denen von Deuterium oder Wasserstoff wird vermutet, dass die weitreichenden repulsiven Wechselwirkungen der Sauerstoffe untereinander bei äußeren höheren Drücken und tieferen Temperaturen einen größeren Einfluss auf die Ausbildung der Eisstruktur haben als im Vergleich zu den repulsiven D-D $(\mathrm{H}-\mathrm{H})$ sowie den attraktiven D-O (H-O)-Wechselwirkungen. Betrachtungen zur Ausbildung und dem Verhalten von repulsiven Wechselwirkungskontakten von molekular gebundenen Wasserstoff- und Sauerstoffatomen wurden z.B. von Savage \& Finney (1986) in Eis sowie in kristallinen Hydratstrukturen durchgeführt. 
Ziel war es daher, experimentelle Daten über die strukturellen Eigenschaften von Eis Ih in einem möglichst weiten Druck- und Temperaturbereich zu erfassen. Aus RietveldVerfeinerungen sollten strukturelle Informationen gewonnen werden, die Aussagen über die Ausbildung und den Einfluss von sich ergebenden attraktiven bzw. repulsiven Wechselwirkungen innerhalb der Eisstruktur erlauben.

Temperaturabhängige Synchrotronmessungen der Gitterkonstanten wurden von Röttger et al. (1994) an Eis Ih-Pulver durchgeführt und von Line \& Whitworth (1996) experimentell bestätigt. Die Messungen wurden bei Umgebungsdruck von etwa $p \sim 1$ bar in einem Temperaturbereich von $\mathrm{T} \sim 140 \mathrm{~K}$ bis $\mathrm{T} \sim 265 \mathrm{~K}$ durchgeführt. Ergebnis dieser Experimente ist, dass in dem vermessenen Temperaturbereich die thermische Expansiviät isotrop verläuft. Das c/a-Verhältnis der Gitterkonstanten wurde für $\mathrm{H}_{2} \mathrm{O}$ zu c/a=1.62806 und für $\mathrm{D}_{2} \mathrm{O} \mathrm{zu}$ $\mathrm{c} / \mathrm{a}=1.62828$ bestimmt und zeigte sich als Funktion der Temperatur als Konstante. Das experimentell verifzierte c/a-Verhältnis der Gitterkonstanten liegt etwas unterhalb des Wertes einer homogenen idealen Packung von Wassermolekülen, dessen theoretischer Wert $\mathrm{c} / \mathrm{a}=1.63299$ beträgt.

Die durch Synchrotrondiffraktionsexperimente gewonnenen temperaturabhängigen Strukturdaten von Eis Ih legen nahe, dass die vom Idealwert abweichenden c/a-Verhältnisse mit einer Abweichung der O...O...O-Bindungswinkel korreliert sind.

\subsubsection{Strukturelle Untersuchungen an $\mathrm{D}_{2} \mathrm{O}$-Eis Ih in Wechselwirkung mit Gasen}

Um zum einen die isothermen Kompressibilitäten von Eis Ih zu bestimmen, zum anderen den Fragen und der Bedeutung der sich bei äußeren Einflüssen ausbildenden attraktiven und repulsiven Wechselwirkungskontakten nachzugehen, wurden mittels Neutronenstrahlung Diffraktionsmessungen am D2B des ILL in Grenoble an $\mathrm{D}_{2} \mathrm{O}$-Eis Ih-Pulver als Funktion des Druckes und der Temperatur durchgeführt.

In zwei zeitlich getrennten Experimenten wurden die strukturellen Eigenschaften von Eis Ih, auch unter Verwendung verschiedener Gase bestimmt. In dem ersten Experiment wurde zunächst Argongas als Drucküberträgermedium verwendet. 
Da Argongas sich nicht in der Struktur löst, sollte dieses Experiment Aufschluss über die temperatur- und druckabhängigen Eigenschaften der Wasserstoffbrückenbindungen in der Eis Ih-Struktur geben. Nachdem gemäß Tabelle 5.10 die Datensätze von Eis Ih als Funktion des Druckes und der Temperatur mit Argongas gemessen wurden, ist in dem zweiten Teil des Experimentes Eis Ih unter Verwendung von Heliumgas als Druckübertägermedium gemessen worden. In einem weiteren späteren Experiment wurde Neongas als Druckübertägermedium verwendet und ebenso gemäß der Tabelle 5.10 die strukturellen Eigenschaften von Eis Ih unter dem Einfluss des Gases Neon bestimmt.

\begin{tabular}{|c|c|c|c|c|c|c|c|}
\hline \multicolumn{7}{|c|}{ p [bar] } \\
\hline $\mathbf{T}[\mathbf{K}]$ & $\mathbf{1}$ & $\mathbf{1 0 0}$ & $\mathbf{5 0 0}$ & $\mathbf{1 0 0 0}$ & $\mathbf{1 5 0 0}$ & $\mathbf{2 0 0 0}$ & $\mathbf{2 5 0 0}$ \\
\hline $\mathbf{1 4 0}$ & $\mathrm{Ar} ; \mathrm{Ne}$ & - & $\mathrm{Ar} ; \mathrm{Ne}$ & $\mathrm{Ar} ; \mathrm{Ne}$ & $\mathrm{Ar}, \mathrm{Ne}$ & $\mathrm{Ar}, \mathrm{Ne}$ & $\mathrm{Ar}, \mathrm{Ne}$ \\
\hline $\mathbf{1 6 9}$ & $\mathrm{Ar}$ & - & - & - & - & $\mathrm{Ar}$ & - \\
\hline $\mathbf{1 9 5}$ & $\mathrm{Ar}$ & - & - & - & - & $\mathrm{Ar}$ & - \\
\hline $\mathbf{2 1 5}$ & - & - & $\mathrm{Ne}$ & $\mathrm{Ne}$ & $\mathrm{Ne}$ & $\mathrm{Ar}, \mathrm{Ne}$ & - \\
\hline $\mathbf{2 3 5}$ & - & $\mathrm{Ar}$ & - & - & - & - & - \\
\hline $\mathbf{2 3 7}$ & $\mathrm{Ar}, \mathrm{He}$ & - & $\mathrm{Ar}$ & $\mathrm{Ar}$ & $\mathrm{Ar}$ & $\mathrm{Ar}, \mathrm{He}$ & - \\
\hline $\mathbf{2 5 0}$ & - & - & $\mathrm{Ne}$ & $\mathrm{Ne}$ & $\mathrm{Ne}$ & $\mathrm{Ne}$ & $\mathrm{Ne}$ \\
\hline $\mathbf{2 5 5}$ & $\mathrm{Ar}$ & - & $\mathrm{Ar}$ & $\mathrm{Ar}$ & $\mathrm{Ar}$ & $\mathrm{Ar}, \mathrm{He}$ & - \\
\hline $\mathbf{2 6 5}$ & $\mathrm{Ar}$ & - & $\mathrm{He}$ & $\mathrm{Ar}, \mathrm{He}$ & $\mathrm{Ar}$ & $\mathrm{Ar}$ & - \\
\hline
\end{tabular}

Tabelle 5.10: Überblick über die mit den Gasen Argon (Ar), Helium (He) und Neon (Ne) als Funktion von Druck und Temperatur gemessenen Datensätze

Die durch diese Experimente gewonnenen Erkenntnisse sollten Aufschluss über die Gas- und Wasserwechselwirkungen sowie über die Beeinflussung der Eisstruktur durch Gaslöslichkeiten bzw. Einbau von Gasen in die Struktur geben und damit zu vermutende strukturelle Änderungen verifiziert werden. Wechselwirkungen von Eis Ih und Gasen, die sogar zu einem völligen Umbau der Eisstruktur führen, sind durch die Bildung von im allgemeinen als Gas-Hydrat beschriebene Strukturen bekannt (vergl. Kapitel 5.2.1 sowie Kapitel 5.2.3).

Die strukturellen Eigenschaften von Eis Ih unter der Verwendung der Gase Argon, Helium sowie Neon wurden gemäß der Tabelle 5.10 in dem Druckbereich von $p \sim 1$ bar bis $p \sim 2.5 \mathrm{kbar}$ sowie in dem Temperaturbereich von $\mathrm{T} \sim 265 \mathrm{~K}$ bis hinunter zu T $140 \mathrm{~K}$ bestimmt. 
Das für die Messungen verwendete Eis Ih-Pulver wurde in beiden Fällen über die Hochdruckeisphase V hergestellt. Nähere Erläuterungen zur Präparation und der auftretenden Problematik sind in Kapitel $3 \mathrm{zu}$ finden. Zur Überprüfung der Pulvergüte wurden einzelne Reflexe aus dem Eis Ih-Diffraktogramm als Funktion der Orientierung der Eisprobe vermessen. Die maximale Abweichung in den Intensitäten des hexagonalen [101]-Reflexes bei dem Argon-Experiment konnte $\mathrm{zu}$ dem theoretisch berechneten Intensitätswert (Simulation eines idealen, regellosen Pulvers) kleiner als $3 \sigma$ festgestellt werden. Es wurde daher während der Experimente von einer hinreichend guten Pulvergüte ausgegangen. Eine Überprüfung der Reflexintensitäten untereinander wurde nicht durchgeführt. Während der Messungen von Eis Ih mit Argon wurde bei Drücken, die über dem Synthesedruck von Argon-Clathrat-Hydrat ( $\mathrm{p} \sim 90 \mathrm{bar}, \mathrm{T} \sim 2.5^{\circ} \mathrm{C}$ für $\mathrm{D}_{2} \mathrm{O}$ ) lagen, fortwährend Argon-ClathratHydrat synthetisisert. Nach jeder Einzelmessung wurde daher der Gasdruck auf $\mathrm{p} \sim 1$ bar Umgebungsdruck reduziert und einige Zeit gewartet, um das Clathrat-Hydrat zu zersetzen. Es zeigte sich jedoch, dass das Clathrat-Hydrat in diesen Phasen der Messung zwar erwartungsgemäß zerfiel, jedoch konnte dies nur bis zu einem gewissen Grad beobachtet werden. Nach zumeist einigen Minuten stagnierte die Zersetzung des Clathrat-Hydrates, der Phasenanteil blieb dann bei den eingestellten Temperaturbedingungen stabil. Eine starke Argon-Clathrat-Hydratbildung konnte oberhalb der Temperaturen von T 237K und Drücken oberhalb von $\mathrm{p} \sim 500$ bar festgestellt werden. Zwar wurde nach jeder Messung der Druck auf Umgebungsdruck reduziert und die Probe einige Zeit (Minutenskala) stehengelassen, um die gebildeten Clathrate-Hydrate zu zersetzen, jedoch erhöhte sich der Phasenanteil während der Messung weiter.

Nachdem die Messungen der Datensätze mit Argongas durchgeführt waren, wurde die Probe auf Temperaturen von $\mathrm{T} \sim 265 \mathrm{~K}$ erwärmt, der Gasdruck auf $\mathrm{p} \sim 1$ bar Umgebungsdruck reduziert und die Eis Ih-Probe für die Dauer von $t \sim 3 h$ stehengelassen, um das während den Messungen synthetisierte Argon-Clathrat-Hydrat zu zersetzen. Der Phasenteil betrug zu diesem Zeitpunkt bereits $\mathrm{n} \approx 70-80 \%$ an dem Probenvolumen. Für den zweiten Teil der Messung wurde das Druckerzeugersystem sowie die Probe danach mehrmals mit Heliumgas mit einigen hundert bar Druck gespült und die gemäß der Tabelle 5.10 angegebenen Datensätze von Eis Ih mit Heliumgas als Drucküberträgermedium als Funktion von Druck und Temperatur vermessen. 
In Anbetracht eines als sehr gering bezifferten verbliebenen Argon-Clathrat-HydratPhasenanteiles $(\mathrm{n} \sim 5 \%)$ und aufgrund apparativer und daraus resultierender zeitlicher Probleme konnte und musste auf eine Neu-Präparation der Probe verzichtet werden.

In dem späteren Experiment wurde eine zu dem ersten Experiment vergleichbare Eis Ih-Probe hergestellt und mit Neongas als Drucküberträgermedium gemäß Tabelle 5.10 untersucht. Die Probe wurde über diesselbe Hochdruckpräpationsroute hergestellt und zeigte eine zur ArgonProbe vergleichbare Güte hinsichtlich verfizierter Einzelintensitäten.

Bei den Experimenten mit Argon- und Heliumgas, sowie in dem zweiten Experiment mit Neongas, wurden vor den druck- und temperaturabhängigen Messungen an Eis Ih jeweils Messungen mit Siliziumpulver sowie Nullpunktscans des Neutronenstrahls ohne Probe durchgeführt. Aus diesen wurden dann über Rietveld-Verfeinerungen die für die jeweiligen Messungen verwendete Neutronenwellenlänge sowie der $2 \theta$-Nullpunkt als Basis für die Eis Ih-Rietveld-Modellierungen bestimmt. Für das Silizium wurde gemäß NBS-Standard eine Gitterkonstante von $\mathrm{a}=5.43088(4) \AA\left(\mathrm{T}=25^{\circ} \mathrm{C}\right)$ angenommen. Für das erste Experiment mit Argon- und Heliumgas wurde die Wellenlänge zu $\lambda=1.59428(1) \AA$, für das zweite Experiment mit Neongas zu $\lambda=1.59433(6) \AA ̊$ bestimmt.

\subsection{Verfeinerungsstrategie und Verfeinerungsproblematiken der Strukturbestimmung und des Eis Ih-Gasinhaltes}

Die gemäß Tabelle 5.10 gemessenen Datensätze wurden mittels der Rietveld-Methodik ausgewertet. Ausgehend von dem kristallographischen Modell von Eis Ih (Kuhs \& Lehmann (1986)) wurde die Eistruktur in mehreren Verfeinerungszyklen sukzessive verfeinert und angepasst, wobei auf die Konsistenz der in der Rietveld-Verfeinerung freigegebenen Parameter in allen Datensätzen, d. h. den Messungen mit Argon-, Helium- und Neongas, geachtet wurde. Zuerst wurde die Eisstruktur modelliert und verfeinert. In den ersten Verfeinerungszyklen wurden neben den instrumentellen Parametern, die Gitterkonstanten, Clathrat-Hydrat-Phasenanteile sowie Vorzugsorientierungen in der bevorzugten [001]Rekristallisationsrichtung der Probe frei verfeinert. Andere Vorzugsorientierungen wie z.B. die [100]- und die [101]-Richtung wurden ebenso verfeinert, jedoch ergaben sich hinsichtlich der 
Intensitätsanpassungen in dem Diffraktogramm deutlich höhere Abweichungen in den Verfeinerungen der mit Argon- und Helium- sowie mit Neongas gemessenen Datensätzen.

Im zweiten Verfeinerungszyklus wurden die Atompositionen (fraktionelle Koordinaten) der Sauerstoffe und der Deuteriumatome sowie die thermischen Auslenkungsparameter (ADP's) verfeinert, um sowohl die durch die Kompression als auch die durch Eis-GasWechselwirkungen bedingten Einflüsse auf die Eistruktur als Funktion von Druck und Temperatur, im speziellen die Verschiebung der Sauerstoffatome, festzustellen.

Für die Frage der Gasbesetzung der Eisstruktur bei Gegenwart Helium- und Neongas als Drucküberträgermedium wurden zwei Verfeinerungen parallel, mit und ohne in die Kristallstruktur eingebauten Gasatomen, durchgeführt. Um eine innere Konsistenz der Eisstruktur-Verfeinerungen zu gewährleisten, wurde in einem ersten Schritt die Gasbesetzung innerhalb der Struktur auf null und in einem weiteren Verfeinerungszyklus ungleich null gesetzt. Die „gasfreie“ Struktur als auch die gasbesetzte Eisstruktur wurde zunächst sowohl isotrop als auch anisotrop verfeinert.

Die Rietveld-Modellierung der Gasinhalte, für die im ersten Experiment mit Heliumgas sowie in dem zweiten Experiment mit Neongas gemessenen Datensätze, stellte sich hinsichtlich der zu verfeinernden Anzahl der Gasatome in der Struktur als auch deren kristallographischen Lagen als problematisch dar.

Aufgrund der Annahme von verschieden starker Wechselwirkungen der Gasatome mit den in der Eisstruktur befindlichen Wassermolekülen wurde angenommen, dass sich die Gasatome innerhalb der Struktur aufgrund des Größenverhältnisse der Eiskanäle zu den gasatomaren Durchmessern an unterschiedlichen kristallographischen Positionen befinden können. Ausschlaggebend sind hierfür die van der Waals'schen-Wechselwirkungsradien und die durch die lokalen Strukturen bedingte Beschaffenheit der van der Waals-Oberflächen in der Struktur. Durch diese ausgeprägten van der Waals'schen Wechselwirkungen können die Gasatome lokal an den Kanalwänden gebunden oder in ungebundener Form in der Mitte der Kanäle vorkommen. Aus einem Durchmesservergleich (,rigid body“) der Ringstrukturen mit einem Durchmesser von $\mathrm{d} \sim 5.283 \AA \mathrm{zu}$ dem Gasdurchmesser, für Helium $\mathrm{d} \sim 2.8 \AA$ und für Neon 
d 3.08 ^, würde ein maximaler Gasinhalt pro Eiskäfig von n 4 Gasmolekülen Neon ergeben, berechnet aus dem maximal möglichen freien Kugelvolumen in der Ringstruktur (unter Berücksichtigung der van der Waals'schen Radien). Aufgrund der Gitterdynamik der Eisstruktur und der thermischen Dynamik der Gasatome dürfte dieser Wert von $\mathrm{n} \sim 4$ zu hoch sein.

Für Helium ließe sich aufgrund des geringeren atomaren Wechselwirkungsradius ein höherer Besetzungsgrad der Eisstruktur erwarten. In den Kanälen können sich die Gasatome daher auf nahezu beliebigen Positionen befinden. Aufgrund dieser Tatsachen dürfte sich für adsorbiertes Heliumgas ebenso wie für adsorbiertes Neongas eine verschmierte Gasverteilung in der Struktur ergeben, deren Bestimmung durch diffraktometrische Methoden nicht zu erfassen ist. Weiterhin ist anzumerken, dass neben diesen $\mathrm{zu}$ erwartenden mess- und verfeinerungstechnischen Problemen das Streuvermögen der verwendeten Gase (Streulänge von Helium: 3.263(4) fm; Neon: 4.547(1) fm) kleiner als die von Sauerstoff (5.803(4) fm) sowie Deuterium (6.671(4) fm) ist. Die Gasatome werden daher nur einen relativ geringen Streubeitrag liefern. Aufgrund der Struktur der Elementarzelle wurden einzelne Lagen für die Gasatome als sehr wahrscheinlich angenommen und in die Modellierung der RietveldVerfeinerungen als stark ausgeschmierte kristallographische Lagen übernommen. Die Lagen der Gasatome für Helium und Neon wurden in fraktionellen Koordinaten der Elementarzelle angenommen $\mathrm{zu}$ :

1. $\mathrm{x}=0.5 ; \mathrm{y}=0.5 ; \mathrm{z}=0.25$, beschreibt die Lage in der Mitte des „Chair-Ringes“, $\mathrm{x}=0 ; \mathrm{y}=0 ; \mathrm{z}=0.25$, beschreibt die Lage der Gasatome in der Mitte des „Boat-Ringes“ und $\mathrm{x}=0, \mathrm{y}=0, \mathrm{z}=0$, beschreibt die Schwerpunktslage mitten in dem durch die Ringe aufgebauten Eiskäfigs.

Neben den Betrachtungen zu der Probengüte und den Rietveld-Modellierungen der Struktureffekte der Proben wurde zuzüglich das Streuvermögen der als Drucküberträgermedium verwendeten und mit der Eistruktur wechselwirkenden Gase Helium und Neon sowie deren ausgeschmierte kristallographische Lage mittels Rietveld-Verfahren untersucht. Die ersten Reflexe von Eis Ih, die zudem für gutes, nicht texturiertes Pulver die höchsten Intensitäten aufweisen, liegen in dem Winkelbereich in $2 \theta \approx 23^{\circ}-40^{\circ}$. 
Um eine Aussage über die Streuanteile der verwendeten Gase als Funktion der Besetzung sowie der kristallographischen Lagen zu ermöglichen, wurden Simulationen der Streubeiträge für Neon- sowie Heliumgas an den zu vermutenden kristallographischen Lagen (vergl. Anhang 8.5.4) als Funktion des thermischen Auslenkungsparameters $U_{\text {iso }}$ durchgeführt. $\mathrm{Zu}$ diesem Zweck wurden in den Rietveld-Modellen die Eisstrukturen unter Verwendung von Neongas bei Temperaturen von $\mathrm{T} \sim 140 \mathrm{~K}$ und einem Druck von $\mathrm{p} \sim 2.5 \mathrm{kbar}$ sowie Heliumgas bei einem Druck von p 2kbar und einer Temperatur von T 237K aus den RietveldVerfeinerungen gelöscht und die Gasatome auf oben genannten kristallographischen Lagen mit der fraktionellen Besetzung von frac $=1$, d.h. ein Gasatom pro kristallographischer Lage und einem isotropen thermischen Auslenkungsparameter (ADP-Wert) von $\mathrm{U}_{\text {iso }} \sim 0.4$ gesetzt. Dieser Wert stellt die obere mögliche Grenze, die in dem verwendeten RietveldVerfeinerungsprogramm (GSAS) angegeben werden kann dar und entspricht bei einem isotropen Auslenkungsparameter einer Verschiebung bzw. Delokalisierung des Atoms von der kristallographischen Lage von $\mathrm{d} \sim 0.6 \AA$. Es wurden daher Modellierungs- sowie Verfeinerungsversuche durchgeführt, bei denen der Eiskäfig mit Gasatomen auf allgemeinen kristallographischen Lagen besetzt wurde. Die Rietveld-Modelle wurden isotrop als auch anisotrop verfeinert, teils durch Freigabe der Parameter, teils mit vorgegebenen Werten für die fraktionelle Besetzung, den Positionen der Gasatome sowie deren thermischen Auslenkungsparametern. Es zeigte sich jedoch keine eindeutige Lösung. Für den Großteil der Versuche divergierten die Verfeinerungen. Für die gewählten Modelle ergab sich, dass die Streubeiträge von Helium und Neon schon bei Winkel $2 \theta<30^{\circ}$ im Rauschen des Untergrundes verschwinden. Bei einer Besetzung jeder angegebenen Lage mit einem Gasatom und einem isotropen thermischen Auslenkungsparameter von $\mathrm{U}_{\text {iso }} \sim 0.25$ ergab sich, dass die Streubeiträge bei einem Winkel von $2 \theta \approx 57.5^{\circ}$ für Heliumgas und Neongas ebenso im Untergrund der Messung verschwinden.

In dem zweiten Verfeinerungszyklus wurden die Gasatome an die angenommenen kristallographischen Lagen mit der fraktionellen Besetzung von eins gesetzt, sowie die aus dem vorherigen Verfeinerungszyklus bestimmten Atompositionen und die isotropen ADP's der Wasserstruktur als Startparameter verwendet. Die fraktionelle Besetzung der Gaslagen, die Atompositionen sowie die ADP's der Wasserstruktur wurden zuerst isotrop frei verfeinert. 
Die zunächst isotropen ADP-Parameter der Gasatome wurden bei der freien Verfeinerung auf den Mittelwert 0.2 der verfeinerungstechnisch möglichen oberen (0.4) und unteren Grenze (0) als Startwert festgelegt.

In den Verfeinerungen zeigte sich, dass die Atompositionen sowie die thermischen Auslenkungsparameter der Eisstruktur, im speziellen die der Sauerstoffe, stark mit dem Parameter des Phasenanteiles an Argon-Clathrat-Hydrat korreliert waren. Eine quantitative Überprüfung der gemessenen Datensätze hinsichtlich der Phasenanteile an Argon-ClathratHydrat sowie der Vorzugsorientierung als Funktion der Zeit in der Reigenfolge der zeitlichen Messung war daher notwendig. Im Zuge dieser Verfeinerungen zeigte sich zudem, dass die Besetzung der Gaslagen stark mit den thermischen Auslenkungsparameter, den ADP's der Gasatome sowie einer Vorzugsorientierung des Eises korreliert ist. Aufgrund der Elementarzelle und der damit wahrscheinlichsten Kristallwachstumsrichtung wurde in allen Verfeinerungen zuerst von einer [001]-Vorzugsorientierung ausgegangen. Die Bestimmung der Eisstruktur unter Verwendung von Argon-, Helium- und Neongas gestaltete sich aufgrund mehrerer verfeinerungstechnischer sowie experimenteller Probleme schwierig. Hauptsächliches Problem war, dass bei den Eis Ih-Datensätzen unter Verwendung von Argongas, Heliumgas und sogar Neongas ein Phasengemisch aus Eisprobe und ClathratHydrat vom Stackelberg Typ II vorlag. Die Strukturmodelle, sowohl für die Clathrat-Hydrate, als auch für die gasbeeinflusste Eis Ih-Struktur sind derzeitig nur ungenau beschrieben. Die Anpassung sich überlappender Reflexe der beiden Phasen in dem Gemisch und damit die Bestimmung strukturrelevanter Parameter ist daher problematisch. Die Reflexüberlagerungen des Argon-Clathrat-Hydrates bzw. Neon-Clathrat-Hydrates mit dem hexagonalen [100]- und [101]-Reflex der Eisstruktur ergaben verfeinerungstechnische Probleme hinsichtlich der Anpassung der Profilparameter und der damit verbundenen Korrelationen bei der Bestimmung der Atompositionen und der thermischen Auslenkungsparameters. Die nur ungenügende Beschreibung des in diesem Druckbereich vorliegenden Clathrat-Hydrat-Typs in den Rietveld-Modellen hatte einen wesentlichen Einfluss auf die Verfeinerungsergebnisse der Eisstruktur sowie des Gasinhaltes. Zwar wurde versucht das Argon-Clathrat-Hydrat zu zersetzen, jedoch scheint dies über einen längeren Zeitraum hinweg stabil in der Probe zu verbleiben. Effekt könnte hier sein, dass sich anfangs oberflächennahes Clathrat-Hydrat in Gas und Eis zersetzt und so einen „Eispanzer“ um die verbleibenden Clathrat-Hydratbereiche legt und so zu einer Verzögerung des Zerfalls führt. Ebenso ergaben sich Probleme bei der 
Anpassung Reflexintensitäten durch die verbliebenen Imperfektionen im Eis (vergl. Kapitel 3.4.1). Es wurde versucht diese Relikte mit den in GSAS vorgesehenen Stapelfehlerbeschreibungen nach Howard (1982) (vergl. auch Kapitel 2.2.1) durch die Anpassung der Reflexprofilparameter zu berücksichtigen, jedoch ohne eine wesentliche Verbesserung der Anpassung mit dem gewählten Modell zu erreichen; eine geeignete Beschreibung der Stapelfehler in GSAS konnte nicht gefunden werden. Näherungsweise konnten diese Defekte zum einen als Vorzugsorientierung, eine anisotrope thermische Auslenkung aber auch als eine starke Verschiebung der Sauerstoffpositionen verfeinert werden. Um diese als systematischen Fehler der Rietveld-Verfeinerungen zufassen, wurde die anisotropen thermischen Auslenkungsparameter sowie die Atompositionen, im speziellen für die Sauerstoffatome, frei verfeinert. Es ergab sich zwar zumeist eine Konvergenz der Rietveld-Modelle, jedoch stellte sich, sowohl bedingt durch die verbliebenen Imperfektionen als auch durch den verbliebenen Clathrat-Hydrat-Phasenanteil, das Ergebnis als physikalisch unwahrscheinlich heraus (z.B. aus den Rietveld-Verfeinerungen bestimmte Wasserstoffbrückenbindungsabstände von O-D...O mit d $>2.99 \AA$ ). Ebenso hatten diese GasModellierungen einen geringen Einfluss auf die Bestimmung und die Verfeinerung der mit dem Gas wechselwirkenden Eisstruktur. Die absolute Gasbesetzung, die Verläufe der Gasbesetzungen sowie die Bestimmung der Eisstruktur als Funktion des Druckes und der Temperatur gestaltete sich aufgrund der beschriebenen Probleme hinsichtlich der RietveldModellierung und Verfeinerung der einzelnen, jedoch korrelierten Parameter als schwierig und trotz der Konvergenz der Verfeinerungen als nicht eindeutig. Bedingt durch die Verfeinerungsproblematiken wurden die Datensätze daher lediglich isotrop verfeinert. Ebenso wurde die Probe durch Verifikation mittels Rietveld-Verfahren auf Kontamination der $\mathrm{D}_{2} \mathrm{O}$ Eisproben mit $\mathrm{H}_{2} \mathrm{O}$ untersucht. Die fraktionelle Besetzung der Deuteriumatome $\left(0.5^{\wedge} 100 \%\right)$ zeigte im Rahmen der Verfeinerung der fraktionellen Besetzung des Eisphasenanteiles eine Änderung von maximal 2\% für die beiden Deuteriumatome D1 und D2 in der Struktur, so dass eine größere Verunreinigung mit $\mathrm{H}_{2} \mathrm{O}$ ausgeschlossen werden kann. Auch kann im Rahmen des Fehlers aufgrund der verfeinerten nahezu gleichen fraktionellen Besetzungen der Deuteriumatome D1 und D2 eine partielle Protonenordnung, die nicht einer völligen Protonenunordnung der Struktur entspricht, ausgeschlossen werden. Demzufolge ist keine Korrelation der Gasbesetzung der Struktur mit der fraktionellen Besetzung der Deuteriumatome als Protonenordnungsparameter zu vermuten. 
Im Gegensatz $\mathrm{zu}$ den Lageparametern der Atome in der Einheitszelle zeigten sich die Gitterkonstanten in den Rietveld-Verfeinerungen als sehr stabil; für verschiedene Strukturmodelle lagen die Werte der Gitterkonstanten durchgehend für alle Datensätze im Rahmen des dreifachen des angegebenen Standardfehlers der Rietveld-Verfeinerungen.

\subsection{Experimentelle Ergebnisse}

\subsubsection{Gitterkonstanten von Eis Ih unter Gaswechselwirkung als Funktion von Druck und Temperatur}

Aus den Messungen sollten durch Rietveld-Verfeinerungen die Gitterkonstanten und weitere strukturelle Informationen, wie fraktionelle Koordinaten der Atome in der Einheitszelle bestimmt und aus diesen die Wechselwirkungsabstände sowie Bindungswinkel der Wassermoleküle als Funktion des Druckes und der Temperatur, auch unter der Wechselwirkung der unterschiedlichen Gase bestimmt werden. Die folgende Abbildung 5.9 zeigt einen Vergleich der in den Experimenten bestimmten Gitterkonstanten bei Umgebungsdruck von Eis Ih in dem Temperaturbereich von $\mathrm{T} \sim 140 \mathrm{~K}$ bis $\mathrm{T} \sim 265 \mathrm{~K}$ mit denen von Röttger et al. (1994). Die durch Neutronendiffraktionsmessungen bestimmten Gitterkonstanten unter Verwendung von Argongas stimmen in dem angenommenen Vertrauensbereich mit denen von Röttger et al. (1994) angegebenen Werten überein. Der Vertrauensbereich der Neutronendaten wurde als dreifacher Wert des aus den RietveldVerfeinerungen bestimmten Standardfehlers angenommen. Diese Annahme ist durch eine Reihe von durchgeführten Verfeinerungen einiger gemessener Datensätze begründet. Für die Verfeinerungen wurden jeweils unterschiedliche Startwerte als auch unterschiedliche Verfeinerungsstrategien verwendet. Bei einer vergleichbaren Güte der unterschiedlichen Rietveld-Fits ergab sich ein Fehler, der innerhalb des Vertrauensbereichs von etwa $3 \sigma$ liegt. Aus der Abbildung 5.9 ist ersichtlich, dass bei der Verwendung von Argongas als Drucküberträgermedium die als Funktion der Temperatur bestimmten Gitterkonstanten bei Umgebungsdruck p 1bar im Rahmen des angegebenen Fehlers mit den Synchrotrondaten von Röttger et al. (1994) übereinstimmen. Ein systematischer Fehler in den Experimenten bzw. den mit Argongas modellierten Verfeinerungen ist nicht wahrscheinlich. 


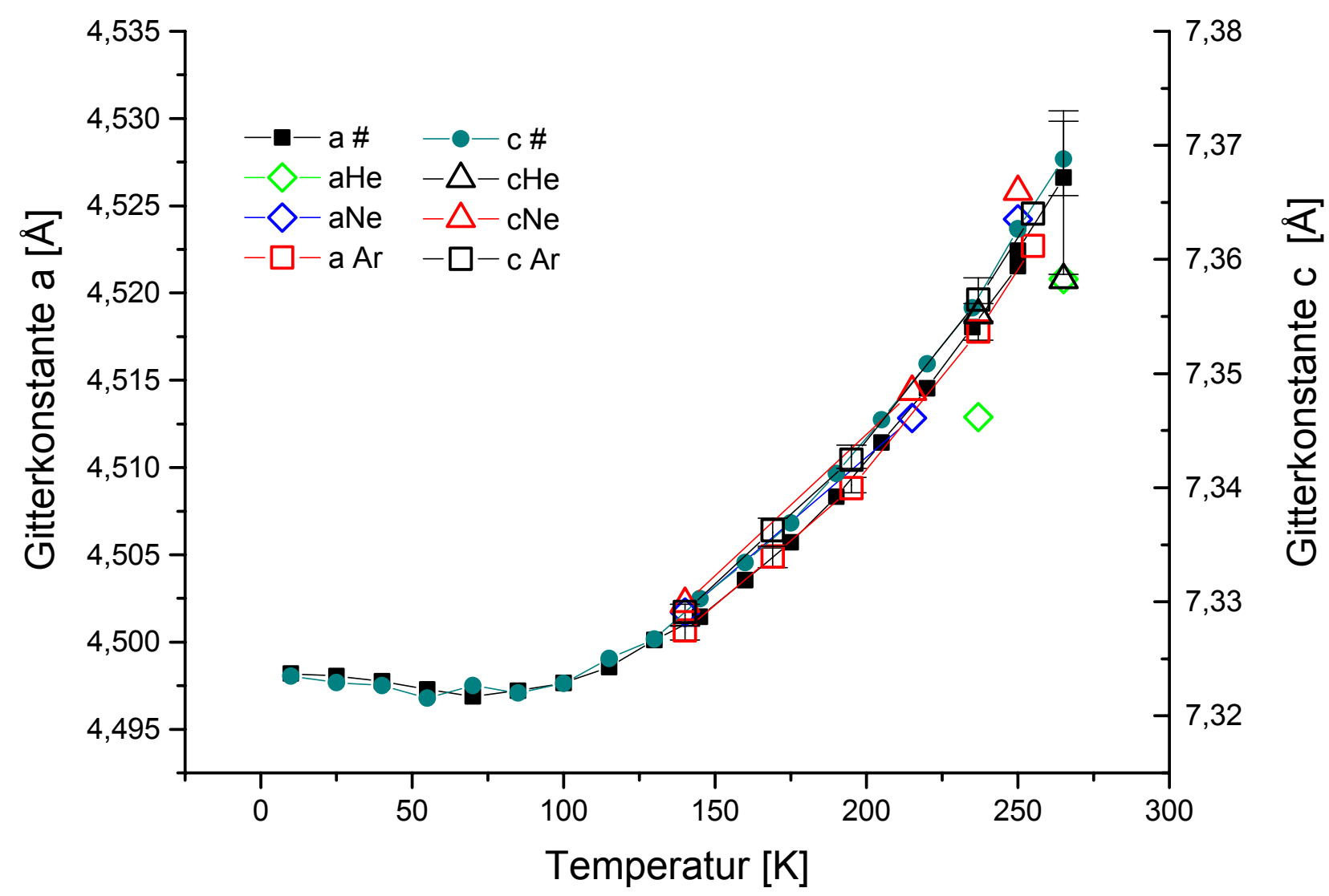

\begin{abstract}
Abbildung 5.9: Die unter der Verwendung von Argon-, Helium- und Neongas aus den Daten ermittelten und Gitterkonstanten sowie als Funktion der Temperatur bestimmten Gitterkonstantenverläufen von $\mathrm{D}_{2} \mathrm{O}$-Eis Ih bei p 1bar als Funktion der Temperatur im Vergleich zu den Referenzdaten von Röttger et al. (1994) ( \#). Größe der Symbole entspricht der Fehlertoleranz bzw. dem Vertrauensbereich der aus den Rietveld-Verfeinerungen bestimmten Fehlern.
\end{abstract}

Die Abbildungen 5.10 und 5.11 zeigen die durch Rietveld-Verfeinerungen bestimmten Gitterkonstanten a und c der gemessenen Eis Ih-Datensätze mit den verwendeten Gasen Helium, Neon und Argon als Funktion von Druck und Temperatur im Vergleich. Die Gitterkonstanten für das Experiment mit Neon bei Umgebungsdruck p 1bar zeigen im Rahmen des Fehlers einen äquivalenten Verlauf in a und c, ähnlich den Gitterkonstanten unter Verwendung von Argongas. Bei den Temperaturen von T 140K und T 215K stimmen a und c im Rahmen der angegebenen Fehler mit den Synchrotron-Gitterkonstanten überein, ebenso die Gitterkonstante a für den T 250K-Datensatz. 


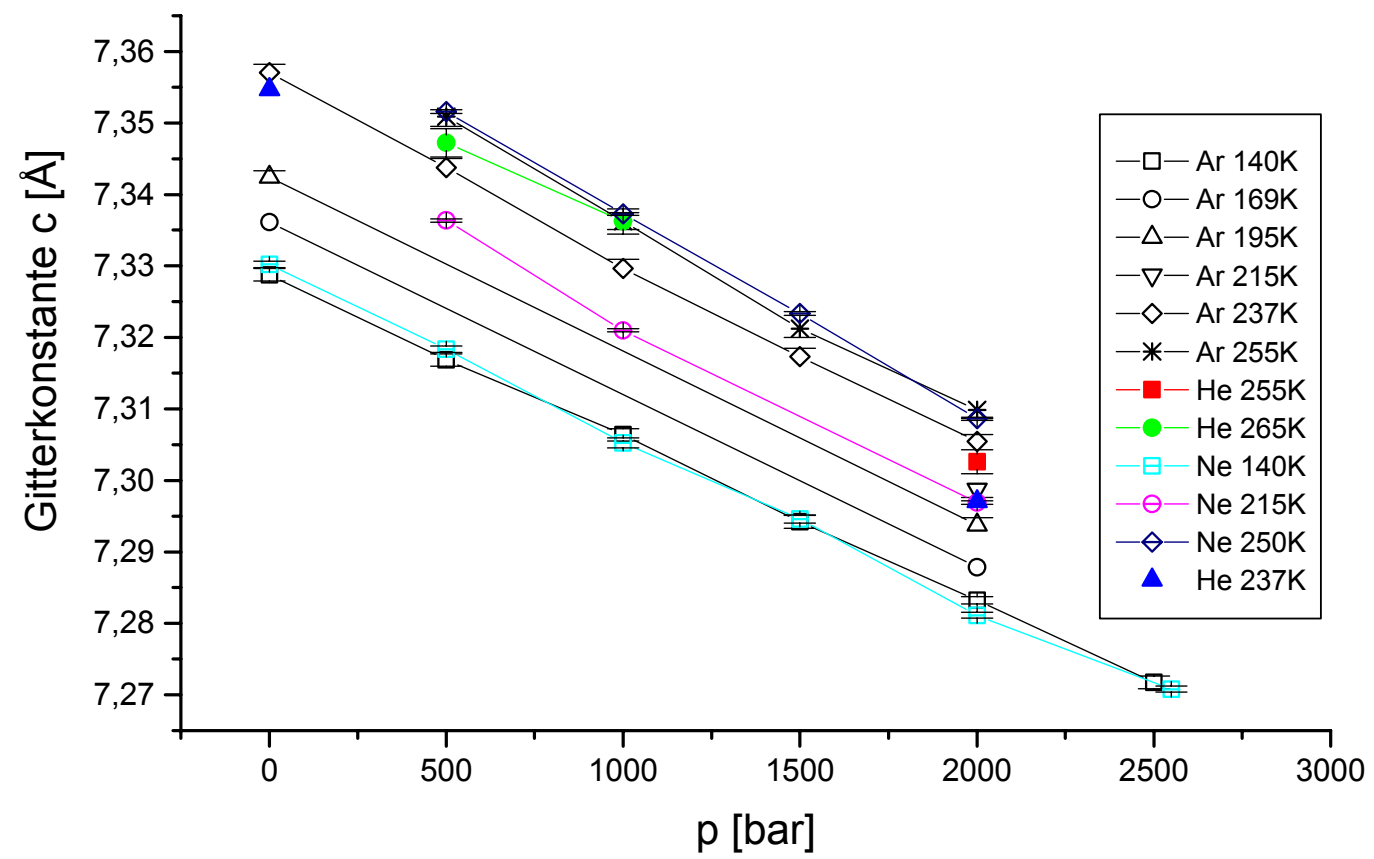

Abbildung 5.10: Gitterkonstante c als Funktion des Druckes und der Temperatur unter Verwendung der Gase Helium (He), Neon (Ne) und Argon (Ar).

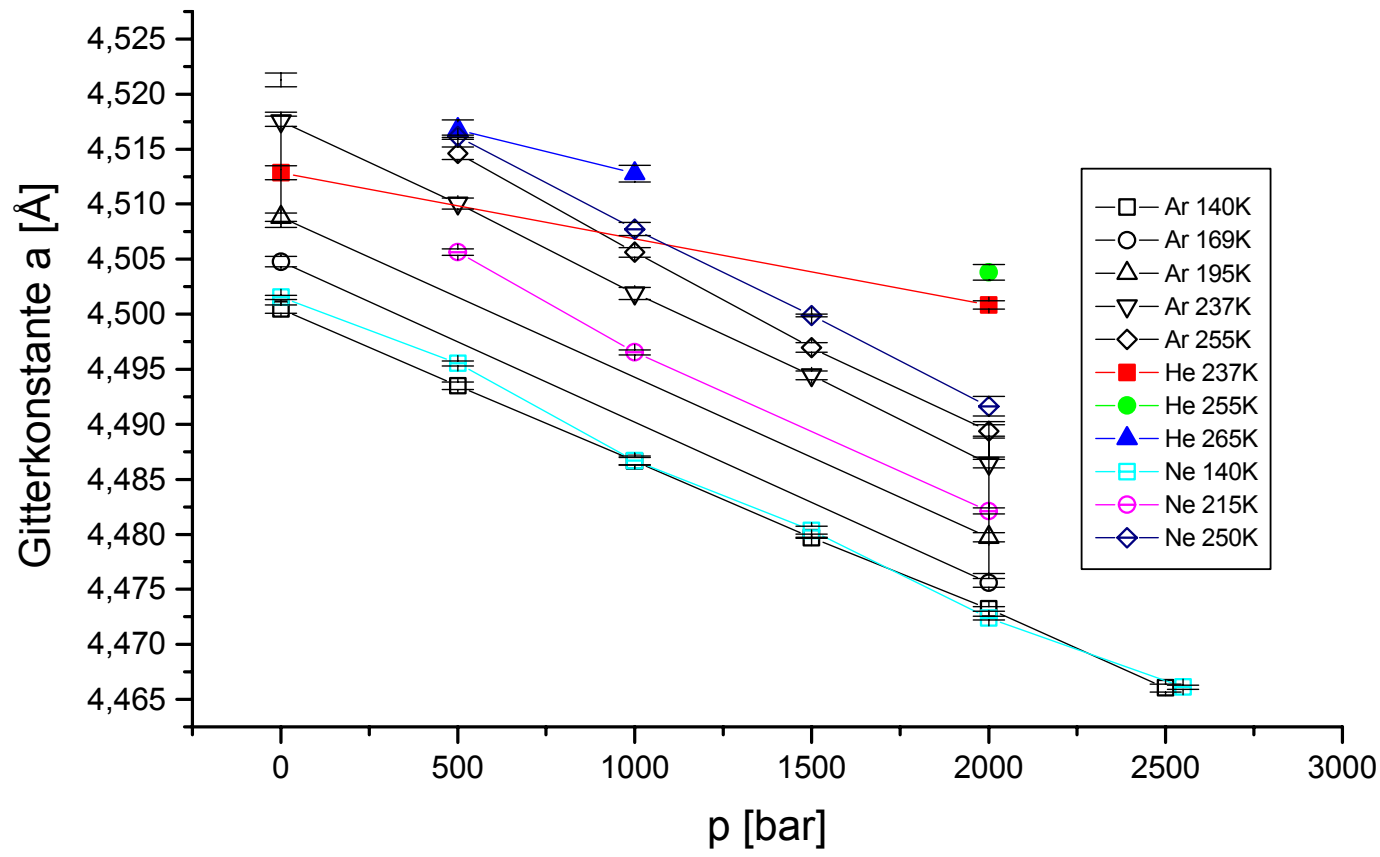

Abbildung 5.11: Gitterkonstante a als Funktion des Druckes und der Temperatur unter Verwendung der Gase Helium (He), Neon (Ne) und Argon (Ar). 
Die Gitterkonstante c bei $\mathrm{T} \sim 250 \mathrm{~K}$ ist im Rahmen des Fehlers um etwa $0.04 \%$ größer. Anzumerken ist, dass die Gitterkonstanten in Gegenwart sowohl von Neongas bei T 215K und T $250 \mathrm{~K}$ als auch von Heliumgas bei $\mathrm{T} \sim 265 \mathrm{~K}$ und Umgebungsdruck von $\mathrm{p} \sim 1$ bar über den Verlauf der Gitterkonstanten angepasste Werte sind. $\mathrm{Zu}$ bemerken ist, dass die unter Verwendung von Heliumgas bestimmten Gitterkonstanten als Funktion der Temperatur in a und in $\mathrm{c}$ im Vergleich $\mathrm{zu}$ den in Gegenwart von Argon- und Neongas bestimmten Gitterkonstanten bei Umgebungsdruck $\mathrm{p} \sim 1$ bar und einer Temperatur von $\mathrm{T} \sim 265 \mathrm{~K}$ signifikant zu kleineren Werten hin.

Für die unter Verwendung von Argongas gemessenen Datensätze zeigen die Gitterkonstanten a und c im Rahmen der Fehler einen linearen Verlauf als Funktion des Druckes. Die linear approximierbaren Gitterkonstantensteigungen werden aufgrund der Anharmonizität der Wechselwirkungspotenziale mit steigender Temperatur steiler und zeigen somit die zu erwartende größere Kompressibilität bei höheren Temperaturen.

Bei Verwendung der Gase Helium und Neon als Drucküberträgermedium verhalten sich die Gitterkonstanten als Funktion des Druckes und der Temperatur ebenso wie zu erwarten, proportional zur Temperatur sowie reziprok zum Druck. Während die Gitterkonstantenverläufe der mit Neongas gemessenen Datensätze mit geringen Abweichungen nahezu äquivalent zu denen unter Verwendung von Argongas gemessenen Datensätze verlaufen, weichen die für die mit Heliumgas gemessenen Datensätze bestimmten Gitterkonstantenverläufe signifikant ab. Auch liegen die Gitterkonstanten a und c für die mit Helium- aber auch mit Neongas gemessenen Datensätze bei äquivalenten Temperaturen, z.B. bei $\mathrm{T} \sim 255 \mathrm{~K}$, deutlich unterhalb der vergleichbaren Gitterkonstanten der mit Argon als Drucküberträgermedium gemessenen Datensätze. Die signifikanten Änderungen der Gitterkonstanten zeigen damit deutlich auf eine Beeinflussung der Eisstruktur durch Lösung von Gasen hin, ebenso wie auf eine geringere Kompressibilität bei höheren Temperaturen infolge einer Gasbesetzung der Eisstruktur. Tabelle 5.11 zeigt die aus den Gitterkonstantenverläufen berechneten Kompressibilitäten der gasbeeinflussten Eis IhStruktur. Für Eis Ih mit den Gasen Argon, Helium und Neon als Drucküberträger ergeben sich die in dem Druckbereich $\mathrm{p} \sim 1$ bar bis $\mathrm{p} \sim 2.55 \mathrm{kbar}$ aus linear gefitteten Gitterkonstanten errechneten isothermen Kompressibilitäten $\kappa$ als Funktion der Temperatur. 


\begin{tabular}{|c|c|c|c|}
\hline $\mathbf{T}[\mathbf{K}]$ & \multicolumn{3}{|c|}{$\mathrm{K}^{\left.\mathbf{M} \mathbf{M b a r}^{-1}\right]}$} \\
\hline & Argon & Neon & Helium \\
\hline $\mathbf{1 4 0}$ & $9.1(1)$ & $9.4(2)$ & - \\
\hline $\mathbf{1 6 9}$ & $9.4(1)$ & - & - \\
\hline $\mathbf{1 9 5}$ & $9.7(1)$ & - & - \\
\hline $\mathbf{2 1 5}$ & - & $10.3(1)$ & $6.5(1)$ \\
\hline $\mathbf{2 3 7}$ & $10.3(1)$ & - & - \\
\hline $\mathbf{2 5 0}$ & & $10.9(1)$ & $6.5(1)$ \\
\hline $\mathbf{2 5 5}$ & $10.8(1)$ & - & - \\
\hline $\mathbf{2 6 5}$ & - & - & \\
\hline
\end{tabular}

Tabelle 5.11: Eis Ih-Kompressibilitäten mit Argon, Neon und Helium als Drucküberträgermedium, bestimmt in dem Temperaturbereich von T 140K bis T 265K, (vergl. Abbildung 5.12).

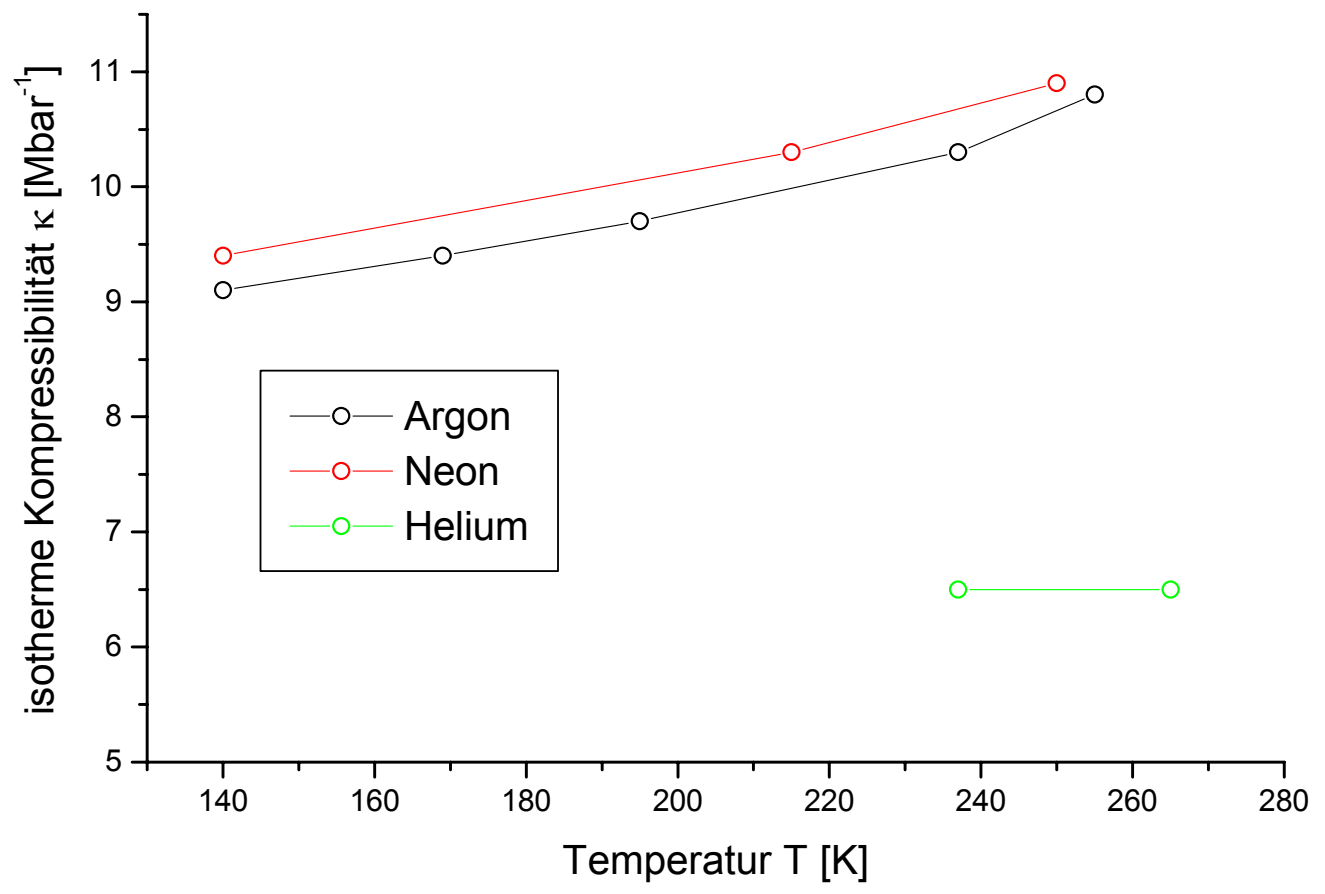

Abbildung 5.12: Funktionenverläufe der isothermen Kompressibilitäten der Eis Ih-Struktur unter der Verwendung der Gase Argon, Neon und Helium als Funktion der Temperatur. Die Größe der Punkte entspricht dem angegebenen Fehler. Zur Berechnung der isothermen Kompressibilitäten vergl. Anhang 8.5.5.1

Für Eis Ih mit Argongas als Druckübertägermedium nimmt die Kompressibilität zu tieferen Temperaturen erwartungsgemäß stetig ab. Für die Gase Neon und Helium nehmen die Kompressibilitäten ebenso reziprok proportional zur Temperatur ab, jedoch ist die Temperaturabhängigkeit unterschiedlich. 
Die Kompressibilität von Neon ist bei annähernd vergleichbaren Temperaturen von T 250K bis $\mathrm{T} \sim 255 \mathrm{~K}$ im Rahmen des Fehlers gleich der Kompressibilität zu Argon, zu tieferen Temperaturen hin sind die errechneten Kompressibilitäten von Eis Ih in Gegenwart von Neongas leicht erhöht. Unter Verwendung von Heliumgas ergibt sich aus den linear approximierten Steigungen eine für $\mathrm{T} \sim 265 \mathrm{~K}$ und $\mathrm{T} \sim 237 \mathrm{~K}$ im Rahmen der Fehlers vergleichbare Kompressibilität.

Die Kompressibilität von Eis Ih unter Einfluss von Heliumgas scheint bei den angegebenen Temperaturen von $\mathrm{T} \sim 237 \mathrm{~K}$ bis $\mathrm{T} \sim 265 \mathrm{~K}$ unabhängig von der Temperatur und weicht zudem signifikant mit einem um den Faktor $\sim 1.6$ zu einer kleineren Kompressibilität als unter der Verwendung der Gase Argon und Neon ab. Weitergehende Aussagen über die isothermen Kompressibilitäten bei tieferen Temperaturen bei der Verwendung von Helium können aufgrund der fehlenden Datensätze nicht getroffen werden. Aus den isothermen Kompressibilitäten, bei denen es sich um die Kompressibilitäten von Eis Ih und dem in der Eis Ih-Struktur adsorbierten Gases handelt, ist abzulesen, dass bei der Verwendung von Argongas, Heliumgas und Neongas die Kompressibilitäten im Rahmen des Fehlers im Vergleich zueinander signifikant abweichen. Die isotherme Kompressibilität bei der Verwendung von Neongas weicht bei einer Temperatur von $\mathrm{T} \sim 140 \mathrm{~K}$ im Vergleich zur Kompressibilität in Gegenwart von Argongas um $~ 3 \%$ ab, während hingegen bei einer Temperatur von $\mathrm{T} \sim 250 \mathrm{~K}$ die Abweichung in den isothermen Kompressibilitäten $\sim 2.5 \%$ beträgt.

Bei tieferen Temperaturen ist keine eindeutige Annäherung der Kompressibilitätsverläufe bei der Verwendung von Neongas zu Argongas ab zulesen, während bei höheren Temperaturen eine Annäherung der Kompressibilitäten extrapolierbar ist. Dies ist gleichbedeutend mit der Aussage anzunehmen, dass die Gasbesetzung der Eis Ih-Strukur bei hohen Temperaturen gering zu sein scheint. Unklar ist, warum eine gasbesetzte Struktur, wenn eine auch nur geringfügig höhere Kompressibilität zu tieferen Temperaturen hin aufweißt als zu einer gasunbeeinflussten Struktur. Die deutliche und signifikante Abweichung der isothermen Kompressibilität bei der Verwendung von Heliumgas lässt hingegen deutlich auf eine höhere Gasbesetzung der Eis Ih-Struktur bei höheren Temperaturen als bei der Verwendung von Neongas schließen. 
Es ist daher anzunehmen, dass die Gasbesetzung der Eis Ih-Struktur mit Heliumgas, aufgrund des kleineren Van der Waals'schen Wechselwirkungsradius über einen weiteren Druck- und Temperaturbereich vorliegt.

Das Verhalten der aus den Gitterkonstanten berechneten Kompressibilitäten wirft daher die Frage auf, inwieweit bei Änderungen der Umgebungsbedingungen (Druck- und Temperatur) ein Gasaustausch durch die offenen Kanäle möglich ist und der mit der Verformung der Eisstruktur den Besetzungsgrad für die verschiedenen Gase unter Berücksichtigung der Gasfugazitäten bei äquivalenten Umgebungsbedingungen bestimmt. Es wurde versucht, dieser Frage durch einen quantitativen Vergleich der Gitterkonstanten nachzukommen, um neben den problematischen Rietveld-Verfeinerungen Aussagen bezüglich. der Gasinhalte als auch der Strukturparameter als Funktion von Druck und Temperatur, nachzukommen. In der folgenden Tabelle 5.12 sind für die Temperatur von $\mathrm{T} \sim 237 \mathrm{~K}$ und einem Druck von $\mathrm{p} \sim 1$ bar die Gitterkonstanten aus den Experimenten mit Argon- und Heliumgas als Drucküberträgermedium zu denen von Röttger et al. (1994) quantitativ verglichen.

\begin{tabular}{|c|c|c|c|c|c|c|c|}
\hline & He237K & Ar 237K & 237K * & $\begin{array}{c}\text { Abweichung } \\
{[\%]}\end{array}$ & He 237K & $\begin{array}{c}\text { Ar 237K } \\
\text { Abweichung } \\
{[\%]}\end{array}$ \\
\hline $\mathbf{p}[\mathbf{b a r}]$ & 1 & 1 & 1 & 1 & 2000 & 2000 & 2000 \\
\hline $\mathbf{a}[\AA]$ & $4.5180(4)$ & $4.5234(18)$ & $4.5236(4)$ & -0.12 in a & $4.5129(6)$ & $4.4864(4)$ & 0.59 in a \\
\hline $\mathbf{c}[\AA \mathbf{\AA}]$ & $7.3536(40)$ & $7.3636(40)$ & $7.3688(13)$ & $(-0.13$ in c) & $7.2971(9)$ & $7.3045(10)$ & -0.10 in c \\
\hline $\mathbf{c} / \mathbf{a}$ & $1.6276(17)$ & $1.6285(25)$ & $1.6283(5)$ & -0.06 & $1.6169(7)$ & $1.6281(8)$ & -0.69 \\
\hline
\end{tabular}

Tabelle 5.12: Vergleich der Gitterkonstanten von Argon, Helium und Neon zu den Referenzwerten von Röttger et al. (1994) bei $T \sim 237 \mathrm{~K}$ und p 1bar sowie T 237K und p 2kbar, * Referenz: Röttger et al. (1994). Die prozentualen Abweichungen des Datensatzes mit Helium bei $T \sim 237 \mathrm{~K}$ sind auf den Datensatz mit Argon bei T 237K, bei einem Druck von p 1bar bzw. p 2000bar bezogen.

Die c/a-Verhältnisse unter Verwendung von Argongas bei einer Temperatur von T 237K und dem Röttger et al. (1994) stimmen im Rahmen ihrer Fehlerbereiche überein.

Für Eis Ih mit Helium als Drucküberträgermedium liegt sowohl das c/a-Verhältnis als auch die Gitterkonstanten in a und $\mathrm{c}$ bei Temperaturen von $\mathrm{T} \sim 237 \mathrm{~K}$ und bei einem Umgebungsdruck von $\mathrm{p} \sim 1 \mathrm{bar}$ unterhalb derer von Eis Ih mit Argongas gemessen. Gleichbedeutend ist dies mit einer nahezu isotropen Kontraktion der Eis Ih-Struktur in den Kristallachsen a $(-0.12 \%)$ und c $(-0.13 \%)$ der Elementarzelle. 
Bei einem Heliumgasdruck von p 2kbar weicht das c/a-Verhältnis, im Vergleich zu den bei vergleichbaren Umgebungsbedingungen gemessenen Datensätzen mit Argongas, um $\sim 0.69 \%$ weit deutlicher zu kleineren Werten ab. Festzustellen ist, dass sich die Abweichungen in den Achsen der Elementarzelle jedoch nicht gleich verhalten. Für die Gitterkonstante a ist bei Verwendung von Helium bei Temperaturen von T $237 \mathrm{~K}$ und einem Druck von $\mathrm{p} \sim 2 \mathrm{kbar}$ eine Vergrößerung im Vergleich zu Argon $(\sim 0.59 \%)$ festzustellen. Hingegen ist für die Gitterkonstante c eine Verringerung, d.h. Kontraktion (-0.1\%) im Vergleich zu den mit Argongas gemessenen Datensätzen festzustellen. Ein Verlauf in den Gitterkonstanten oder dem c/a-Verhältnis kann aufgrund der wenigen gemessenen Datensätze bei Drücken von $\mathrm{p} \sim 1$ bar und $\mathrm{p} \sim 2 \mathrm{~kb}$ ar und einer Temperatur von $\mathrm{T} \sim 237 \mathrm{~K}$ nicht angegeben werden. Bei einer Temperatur von $\mathrm{T} \sim 255 \mathrm{~K}$ ist die Vergleichbarkeit und damit Aussagefähigkeit lediglich bei einem Druck von $\mathrm{p} \sim 2 \mathrm{kbar}$ gegeben (vergl. Tabelle 5.13).

\begin{tabular}{|c|c|c|c|}
\hline & He 255K & Ar 255K & Abweichung [\%] \\
\hline $\mathbf{p}[\mathbf{k b a r}]$ & 2 & 2 & 0.32 in a \\
\hline $\mathbf{a}[\AA]$ & $4.5038(7)$ & $4.4894(6)$ & -0.1 in c \\
\hline $\mathbf{c}[\AA]$ & $7.3026(4)$ & $7.3099(12)$ & -0.42 \\
\hline $\mathbf{c} / \mathbf{a}$ & $1.6214(5)$ & $1.6283(5)$ & \\
\hline
\end{tabular}

Tabelle 5.13: Gitterkonstanten von Eis Ih unter Verwendung von Helium- und Argongas bei Umgebungsbedingungen von $\mathbf{T} \sim 255 \mathrm{k}$ und $\mathbf{p} \sim 2 \mathrm{kbar}$ im Vergleich. Die prozentualen Abweichungen des Datensatzes He255K bei $\mathrm{T} \sim 255 \mathrm{~K}$ und $\mathrm{p} \sim 2 \mathrm{kbar}$ sind auf den Datensatz von Argon (Ar255K) bei einer Temperatur von $\mathrm{T} \sim 255 \mathrm{~K}, \mathrm{p} \sim 2 \mathrm{kbar}$ bezogen.

\begin{tabular}{|c|c|c|c|}
\hline & He 265K & He 265K & Abweichung [\%] \\
\hline $\mathbf{p}[\mathbf{b a r}]$ & 500 & 1000 & He500bar-1kbar \\
\hline $\mathbf{a}[\AA \mathbf{A}]$ & $4.5168(9)$ & $4.5128(8)$ & -0.09 \\
\hline $\mathbf{c}[\AA \mathbf{\AA}]$ & $7.3472(20)$ & $7.3362(18)$ & -0.15 \\
\hline $\mathbf{c} / \mathbf{a}$ & $1.6266(5)$ & $1.6257(8)$ & -0.06 \\
\hline
\end{tabular}

Tabelle 5.14: Gitterkonstanten der mit Helium gemessenen Datensätze als Funktion des Druckes. Die proz. Abweichungen des Datensatzes He265K bei einem Druck vonp 1kbar und einer Temperatur von T 265K sind auf den Datensatz He265K bei einem Druck von p 500bar und äquivalenter Temperatur bezogen.

Äquivalent zu den mit Heliumgas gemessenen Datensätzen bei T 237K ist eine Aufweitung in a $(0.32 \%)$ sowie in c eine geringe Kontraktion (-0.1\%) festzustellen. Die Aufweitung als auch die Kontraktion gegenüber den mit Argongas gemessenen Datensätzen verlaufen, soweit aus den zwei zu vergleichenden Temperaturen bei $\mathrm{T} \sim 237 \mathrm{~K}$ und $\mathrm{T} \sim 255 \mathrm{~K}$ ersichtlich ist, 
reziprok zu der Temperatur. Ein Vergleich der Gitterkonstanten und der c/a-Verhältnisse der mit Heliumgas gemessenen Datensätze bei $\mathrm{T} \sim 265 \mathrm{~K}$ ist in der nachfolgenden Tabelle 5.14 gegeben.

Bei der Verwendung von Helium zeigt sich bei einer Temperatur von $\mathrm{T} \sim 265 \mathrm{~K}$ eine Kontraktion der Gitterkonstanten in der a- sowie in der c-Achse, die eine proportionale Korrelation als Funktion des Druckes aufweist (vergl. Tabelle 5.14).

Um eine bessere Übersicht über die Gitterverformung über die Gitterkonstanten zu erhalten, wurde versucht die Verläufe für die beiden mit Heliumgas gemessenen Datensätze bei T $\sim 265 \mathrm{~K}$ sowie bei $\mathrm{T} \sim 237 \mathrm{~K}$ als Funktion des Druckes sowie als Funktion der Temperatur isobar bis $\mathrm{zu}$ einem Druck von $\mathrm{p} \sim 2 \mathrm{kbar}$ zu fitten bzw. $\mathrm{zu}$ extrapolieren, um eine Vergleichbarkeit zu den mit Argongas gemessenen Datensätzen herzustellen. Die per Gauß'schem Fehlerfortpflanzungsgesetz ermittelten Varianzen lagen jedoch deutlich über dem durch den Effekt der Aufweitung bzw. Kontraktion der Gitterkonstanten erwarteten Abweichungen.

Die in dem zweiten Experiment mit Neongas als Drucküberträger gemessenen Datensätze wurden im Vergleich zu den mit Helium- sowie mit den Argongas gemessenen Datensätzen zumeist bei anderen Temperaturen gemessen. Um einen Vergleich der Gitterkonstanten für die mit Neongas sowie für die mit Argongas gemessenen Datensätze durchzuführen und so Aussagen über Druck und Temperatur abhängige Strukturänderungen zu ermöglichen, wurden die experimentell ermittelten Gitterkonstantenverläufe in dem Druck- und Temperaturbereich gemäß Tabelle 5.10 gefittet bzw. für beide Datensätze bei äquivalenten Umgebungsbedingungen inter- bzw. extrapoliert. Die Gitterkonstantenverläufe wurden aufgrund einer höheren Messpunktdichte durch Polynomfits 2. Grades bestimmt. Für die Funktionenfits wurden für die mit Neon gemessenen Datensätze in dem Druckbereich von $\mathrm{p} \sim 1$ bar bis $\mathrm{p} \sim 2 \mathrm{~kb}$ ar bei einer Temperatur von $\mathrm{T} \sim 140$ als auch bei Drücken von $\mathrm{p} \sim 500$ bar bis $\mathrm{p} \sim 2 \mathrm{kbar}$, sowohl bei einer Temperatur von $\mathrm{T} \sim 215 \mathrm{~K}$ als auch bei einer Temperatur von T $250 \mathrm{~K}$ verwendet. Als Basis der Funktionenfits für die unter Verwendung von Argongas experimentell ermittelten Gitterkonstanten dienten die Datensätze in dem Druckbereich von $\mathrm{p} \sim 1$ bar bis $\mathrm{p} \sim 2 \mathrm{~kb}$ ar sowohl bei einer Temperatur von $\mathrm{T} \sim 140 \mathrm{~K}$ als auch bei $\mathrm{T} \sim 237 \mathrm{~K}$ als auch in dem äquivalenten Druckbereich bei einer Temperatur von T 255K. 
Ein direkter Vergleich sowohl der unter Verwendung von Argon- sowie Neongas experimentell bestimmten als auch durch Fitverfahren festgelegten Gitterkonstanten sollte für die Datensätze bei $\mathrm{T} \sim 140 \mathrm{~K}$ in dem Druckbereich von $\mathrm{p} \sim 1$ bar bis $\mathrm{p} \sim 2 \mathrm{kbar}$, bei einer Temperatur von $\mathrm{T} \sim 215 \mathrm{~K}$ bei $\mathrm{p} \sim 1$ bar sowie $\mathrm{p} \sim 2 \mathrm{kbar}$ sowie bei einer Temperatur von $\mathrm{T} \sim 250 \mathrm{~K}$ bei Drücken von $\mathrm{p} \sim 1$ bar sowie $\mathrm{p} \sim 2 \mathrm{kbar}$ durchgeführt werden.

In der Tabelle 5.15.a sind sowohl die experimentellen als auch die per fit ermittelten Gitterkonstanten für die mit Argon und Neon gemessenen Datensätze im Vergleich für Drücke von $\mathrm{p} \sim 1$ bar und $\mathrm{p} \sim 2 \mathrm{kbar}$ als direkt vergleichbare Umgebungsbedingungen bei Temperaturen von $\mathrm{T} \sim 140 \mathrm{~K}, \mathrm{~T} \sim 215 \mathrm{~K}$ sowie $\mathrm{T} \sim 250 \mathrm{~K}$ dargestellt. In der Tabelle 5.15.b sind die sowohl aus den experimentell als auch den durch Anpassung ermittelten Gitterkonstantenabweichungen unter Verwendung der Gase Argon, Neon zu den Referenzwerten von Röttger et. (1994) dargelegt.

\begin{tabular}{|c|c|c|c|c|c|c|c|}
\hline & & \multicolumn{2}{|c|}{ Röttger et al. (1994) } & \multicolumn{2}{c|}{ Argongas } & \multicolumn{2}{c|}{ Neongas } \\
\hline $\mathbf{T}[\mathbf{K}]$ & $\mathbf{p}[\mathbf{b a r}]$ & $\mathbf{a}[\AA \mathbf{\AA}]$ & $\mathbf{c}[\boldsymbol{\AA}]$ & $\mathbf{a}[\AA]$ & $\mathbf{c}[\AA]$ & $\mathbf{a}[\AA]$ & $\mathbf{c}[\AA]$ \\
\hline $\mathbf{2 5 0}$ & $\mathbf{1}$ & $4.5215(1) \mathrm{p}$ & $7.3627(3) \mathrm{p}$ & $4.5213(6) \mathrm{p}$ & $7.3620(5) \mathrm{p}$ & $4.5245(6) \mathrm{p}$ & $7.3654(7) \mathrm{p}$ \\
\hline & $\mathbf{2 0 0 0}$ & - & - & $4.4886(2) \mathrm{p}$ & $7.3082(63) \mathrm{p}$ & $4.4916(9) \mathrm{e}$ & $7.3086(3) \mathrm{e}$ \\
\hline $\mathbf{2 1 5}$ & $\mathbf{1}$ & $4.5135(9) \mathrm{p}$ & $7.34921(15) \mathrm{p}$ & $4.5215(15) \mathrm{p}$ & $7.3622(6) \mathrm{p}$ & $4.5160(15) \mathrm{p}$ & $7.3540(22) \mathrm{p}$ \\
\hline & $\mathbf{2 0 0 0}$ & - & - & $4.4885(6) \mathrm{p}$ & $7.3085(3) \mathrm{p}$ & $4.4821(\mathrm{e})$ & $7.2969(3) \mathrm{e}$ \\
\hline $\mathbf{1 4 0}$ & $\mathbf{1}$ & $4.5010(9) \mathrm{p}$ & $7.3291(15) \mathrm{p}$ & $4.5008(2) \mathrm{e}$ & $7.3285(5) \mathrm{e}$ & $4.5015(2) \mathrm{e}$ & $7.3302(5) \mathrm{e}$ \\
\hline & $\mathbf{2 0 0 0}$ & - & - & $4.4660(4) \mathrm{e}$ & $7.2717(9) \mathrm{e}$ & $4.4724(2) \mathrm{e}$ & $7.2811(4) \mathrm{e}$ \\
\hline
\end{tabular}

Tabelle 5.15.a: Gefittete Gitterkonstanten für die mit Argon- und Neongas gemessenen Datensätze im Vergleich bei den Temperaturen $T \sim 250 \mathrm{~K}, \mathrm{~T} \sim 215 \mathrm{~K}$ und $\mathrm{T} \sim 140 \mathrm{~K}$ sowie bei den Gasdrücken von $\mathrm{p} \sim 1 \mathrm{bar}$ sowie p 2kbar. * Referenzwerte von Röttger et al. (1994). Anmerkung: Die mit p gekennzeichneten Gitterkonstanten wurden auf Basis der experimentell bestimmten Gitterkonstanten bei den in der Tabelle angegebenen Umgebungsbedingungen einen Polynomfit 2. Grades bestimmt. Die Kennzeichnung e bezeichnet die aus den experimentellen Daten durch Rietveld-Verfeinerungen bestimmten Gitterkonstanten.

Es zeigt sich, dass die angepassten Gitterkonstanten für die mit Argongas gemessenen Datensätze zumindest bei den Temperaturen von $\mathrm{T} \sim 250 \mathrm{~K}$ sowie $\mathrm{T} \sim 140 \mathrm{~K}$ und einem Druck von p 1bar im Rahmen der Fehler zu den Referenzwerten von Röttger et al. (1994) übereinstimmen. Bei der Temperatur von $\mathrm{T} \sim 215 \mathrm{~K}$ ist die Abweichung der angepassten Gitterkonstanten für die mit Argongas gemessenen Datensätze durch die Polynom-Anpassung deutlich oberhalb der angegebenen Fehler. 


\begin{tabular}{|c|c|c|c|c|c|c|c|}
\hline $\begin{array}{c}\mathbf{T} \\
{[\mathbf{K}]}\end{array}$ & $\begin{array}{c}\mathbf{p} \\
\text { [bar] }\end{array}$ & $\begin{array}{c}\Delta \mathbf{a} / \mathbf{a} \\
{[\%]}\end{array}$ & $\begin{array}{l}\Delta \mathrm{c} / \mathrm{c} \\
{[\%]}\end{array}$ & \multicolumn{4}{|c|}{ c/a-Vergleich } \\
\hline \multirow[t]{2}{*}{250} & 1 & $<0.01$ & $<0.01$ & \multirow{14}{*}{$1.6283(5)^{*}$} & $1.6283(3)$ & \multirow{4}{*}{$\begin{array}{l}\text { Röttger et al. } \\
\quad(1994)^{*}\end{array}$} & Argongas \\
\hline & 2000 & -0.73 & -0.95 & & $1.6282(2)$ & & Argongas \\
\hline \multirow[t]{2}{*}{250} & 1 & 0.07 & 0.04 & & $1.6279(3)$ & & Neongas \\
\hline & 2000 & -0.66 & -0.73 & & $1.6272(3)$ & & Neongas \\
\hline \multirow[t]{2}{*}{250} & 1 & 0.07 & 0.04 & & $1.6279(3)$ & \multirow[t]{2}{*}{ Argongas } & Neongas \\
\hline & 2000 & 0.07 & $<0.01$ & & $1.6272(3)$ & & Neongas \\
\hline \multirow[t]{2}{*}{215} & 1 & 0.055 & 0.065 & & $1.6284(4)$ & \multirow{6}{*}{$\begin{array}{l}\text { Röttger et al. } \\
\quad(1994)^{*}\end{array}$} & Neongas \\
\hline & 2000 & -0.69 & -0.16 & & $1.6280(3)$ & & Neongas \\
\hline \multirow[t]{2}{*}{140} & 1 & $<0.01$ & $<0.01$ & & $1.6282(2)$ & & Argongas \\
\hline & 2000 & -0.77 & -0.78 & & $1.6280(3)$ & & Argongas \\
\hline \multirow[t]{2}{*}{140} & 1 & $<0.01$ & 0.02 & & $1.6284(3)$ & & Neongas \\
\hline & 2000 & -64 & -65 & & $1.6280(3)$ & & Neongas \\
\hline \multirow[t]{2}{*}{140} & 1 & 0.015 & 0.023 & & $1.6284(3)$ & \multirow[t]{2}{*}{ Argongas } & Neongas \\
\hline & 2000 & 0.14 & 0.13 & & $1.6280(5)$ & & Neongas \\
\hline
\end{tabular}

Tabelle 5.15.b: Vergleich der aus den Gitterkonstanten berechneten c/a-Verhältnisse und der berechneten prozentualen Gitterkonstantenänderungen als Funktion von Druck und Temperatur sowie der verwendeten Gasart zu den Referenzwerten von Röttger et al. (1994) sowie unter Verwendung der Gase Argon und Neon.

Geht man von den Ergebnissen der experimentellen Neutronendaten und den daraus gefitteten Werten aus (vergl. Tabelle 5.15.a sowie Tabelle 5.15.b), so deutet sich im Rahmen der angegebenen Fehler bei einer vergleichbaren Temperatur von $\mathrm{T} \sim 250 \mathrm{~K}$ bei Verwendung von Neon bei einem Druck von $\mathrm{p} \sim 1$ bar eine Aufweitung in den Kristallachsen a und c gegenüber Argon als Drucküberträgermedium an, die in der a-Achse etwa $0.07 \%$ sowie in der c-Achse $0.04 \%$ beträgt. Aus diesen bestimmten Gitterkonstanten und den daraus berechneten c/aVerhältnissen bei der Verwendung von Neongas (bei T $250 \mathrm{~K}$ und einem Druck von $\mathrm{p} \sim 1$ bar) kann gefolgert werden, dass das Kristallgitter im Vergleich zu den mit Argongas durchgeführten Messungen eine geringe Aufweitung durch eine geringe Gasbesetzung bei Umgebungsdruck erfährt. Ein Vergleich des von Röttger et al. (1994) bestimmten c/aVerhältnis (bei einem atmophärischen Druck von p 1bar) von c/a=1.6283(5) zu dem berechneten c/a-Verhältnis zeigt im Rahmen der bestimmbaren Fehlergrenzen eine geringe isotrope Aufweitung. Bei einem Druck von p 2kbar zeigt sich, dass für die unter Neoneinwirkung experimentell bestimmten Gitterkonstanten zu denen der in Gegenwart von Argongas bestimmten, in der a-Achse eine geringe Aufweitung festzustellen ist, während die c-Achse hingegen im Rahmen des angegebenen Fehlers gleich ist. Das c/a-Verhältnis liegt deutlich sowohl unter dem Wert von Röttger et al. (1994) als auch von dem in Gegenwart von Argongas bestimmten c/a-Verhältnis und deutet somit auf eine anisotrope Kompression der Eisstruktur hin (vergl. auch Abbildung 5.13). Bei der Temperatur von T 215K und einem Druck von $\mathrm{p} \sim 1$ bar ist in den Achsen a und c aus dem Vergleich der gefitteten sowie den 
experimentell bestimmten Gitterkonstanten eine geringe isotrope Aufweitung zu den Gitterkonstanten von Röttger et al. (1994) bei Umgebungsdruck festzustellen. Bei einem Druck von p 2kbar ist die durch den Druck bedingte Kompression des Eisgitters, die sich zu den Werten von Röttger et al. (1994) als stark anisotrope Kontraktion darstellt, festzustellen. Die Abweichungen sind im Rahmen der Gitterkonstantenfehler sowohl für die a als auch die c-Achse signifikant, wobei in der a-Achse eine deutlicherere Kontraktion stattfindet. Bei einer Temperatur von $\mathrm{T} \sim 140 \mathrm{~K}$ und einem Druck von $\mathrm{p} \sim 1$ bar stimmen die Gitterkonstanten sowohl für die mit Argongas als auch für die mit Neongas gemessenen Datensätze im Vergleich in den Kristallachsen a und c im Rahmen des Fehlers zu den Werten von Röttger et al. (1994) überein. Die Aufweitung der Gitterkonstanten der im Vergleich der mit Argongas und Neongas gemessenen Datensätze ist unter Berücksichtigung der c/a-Verhältnisse nicht signifikant. Eine deutliche Aufweitung der Gitterkonstanten unter Verwendung von Neon im Vergleich zur Verwendung von Argon ist nicht feststellbar. Bei einem Druck von p 2kbar lässt sich jedoch in der a-Achse eine Aufweitung von $\sim 0.14 \%$ und in der c-Achse eine Aufweitung um $0.13 \%$ feststellen. Die Aufweitung ist isotrop. Bei der Verwendung von Neongas zeigt sich im Gegensatz sowohl unter der Verwendung von Argongas als auch zu den Referenzwerten von Röttger et al. (1994), dass für die Gitterkonstantenänderungen $\Delta \mathrm{a} / \mathrm{a}$ sowie $\Delta \mathrm{c} / \mathrm{c}$ bei vergleichbaren Umgebungsbedingungen (vergl. $\Delta$ a/a sowie $\Delta \mathrm{c} / \mathrm{c}$ bei $\mathrm{T} \sim 250 \mathrm{~K}$, Tabelle 5. 15.b) kleinere Werte gegeben sind, die auf eine geringere Kompression der Eisstruktur durch die Gegenwart von Neongas hindeuten. Eine eindeutige Funktion der im Vergleich angeführten experimentell und durch Anpassung ermittelten Gitterkonstantenänderungen ist anhand der in Tabelle 5.15.b dargestellten Werte als Funktion des Druckes und der Temperatur nicht abzulesen. Ebenso kann keine eindeutige Aussage bzgl. einer Gitterdeformation durch Gegenwart von Neongas bei Umgebungsbedingungen von $\mathrm{p} \sim 1$ bar gefolgert werden. Bei der Verwendung von Helium scheint eine eindeutige Tendenz der Gitterkonstantenänderungen gegeben zu sein. Bei einem Druck von $\mathrm{p} \sim 1$ bar und einer Temperatur von $\mathrm{T} \sim 237 \mathrm{~K}$ ist eine Kontraktion der Gitterkonstanten in den Achsen a und c festzustellen, die sich bei steigendem Druck und ansteigender Temperatur zumindest in der Gitterkonstante a außerhalb des Fehlers in eine Aufweitung gegenüber der Verwendung von Argon übergeht. Dieser Effekt könnte durch eine zum Druck proportionale sowie zur Temperatur reziprok proportionale Gasbesetzung der Struktur bedingt sein. Bei der Verwendung von Neongas sind diese Effekte als Funktion der Temperatur, d.h. bei den Temperaturen von $\mathrm{T} \sim 140 \mathrm{~K}, \mathrm{~T} \sim 215 \mathrm{~K}$ sowie $\mathrm{T} \sim 250 \mathrm{~K}$ nicht sehr deutlich ausgeprägt. Zudem 
scheinen die Änderungen in den Gitterkonstanten von a und c als Funktion des Druckes und der Temperatur inkonsistent zu sein. Aufgrund der sich zwischen den Gasatomen und der Eisstruktur ausgebildeten attraktiven Van der Waals'chen Wechselwirkungen ist eher von einer Kontraktion der Eisstruktur auszugehen. Diese könnte, je nach Quantität der Gasatome sowie der möglichen Gaspositionen innerhalb der Struktur, zu einer isotropen sowie anisotropen Kontraktion der „Boat“- sowie „Chair“-Ringe führen. Bei einer genügenden Kompression der Eisstruktur ist zudem denkbar, dass ein in einem Käfig befindliches Gasatom in repulsiver Wechselwirkung mit der Wasserstruktur steht. Diese könnten dann in einer anisotropen Verzerrung oder gar Aufweitung der Gitterkonstanten gegenüber einer gasunbeinflussten Struktur resultieren. Sorptionssimulationen, die zum Ziel hatten die Gasbesetzung sowie die Positionen der Gasatome zu bestimmen (vergl. Kapitel 5.6) zeigten jedoch, dass sich die Gaslagen mit den stärksten Wechselwirkungen in der Mitte des Eiskäfigs, jedoch vereinzelt auch Gaslagen in den Ringen befinden.

Als signifikantester Nachweis der Eis- und Gaswechselwirkungen sind daher die c/aVerhältnisse (vergl. Abbildung 5.13) anzuführen, deren Verläufe als Funktion des Druckes und der Temperatur für die Gase Helium und Neon im Vergleich zu dem Verlauf von mit Argon gemessenen Datensätzen signifikante Abweichungen zeigen. Die druck- und temperaturabhängigen Änderungen lassen auf eine unterschiedliche Gasbesetzung der Eisstruktur sowie eine stark unterschiedliche Verzerrung der Struktur in den Gitterkonstanten schließen. Von Röttger et al. (1994) wurde das c/a-Verhältnis von Eis Ih im Temperaturbereich von $\mathrm{T} \sim 60 \mathrm{~K}$ bis $\mathrm{T} \sim 265 \mathrm{~K}$ und bei Umgebungsdruck von $\mathrm{p} \sim 1 \mathrm{bar}$ als temperaturunabhängig und $\mathrm{zu}$ einem Wert von $\mathrm{c} / \mathrm{a}=1.6283(5)$ bestimmt. Die aus den Gitterkonstanten berechneten c/a-Verhältnisse sind in der folgenden Abbildung 5.13 dargestellt. Die berechneten Werte stimmen für die mit Argongas gemessenen Datensätze mit einer Abweichung von unter 0.01\% mit dem von Röttger et al. (1994) bestimmten Wert über den vermessenen Druck- und Temperaturbereich überein. Für die mit Argon gemessenen Datensätze zeigt das c/a-Verhältnis im Rahmen des Fehlers keine eindeutige abweichende Tendenz als Funktion des Druckes und der Temperatur. Eine Maximalfehlerabschätzung der c/a-Verhältnisse wurde mittels Gauß`scher Fehlerfortpflanzung durchgeführt und ergab einen Fehler von $\sim 0.02 \%$. Es kann somit von einer Konstanz des c/a-Verhältnisses als Funktion des Druckes und der Temperatur für die mit Argon gemessenen Datensätze im Rahmen des Fehlers ausgegangen werden. 


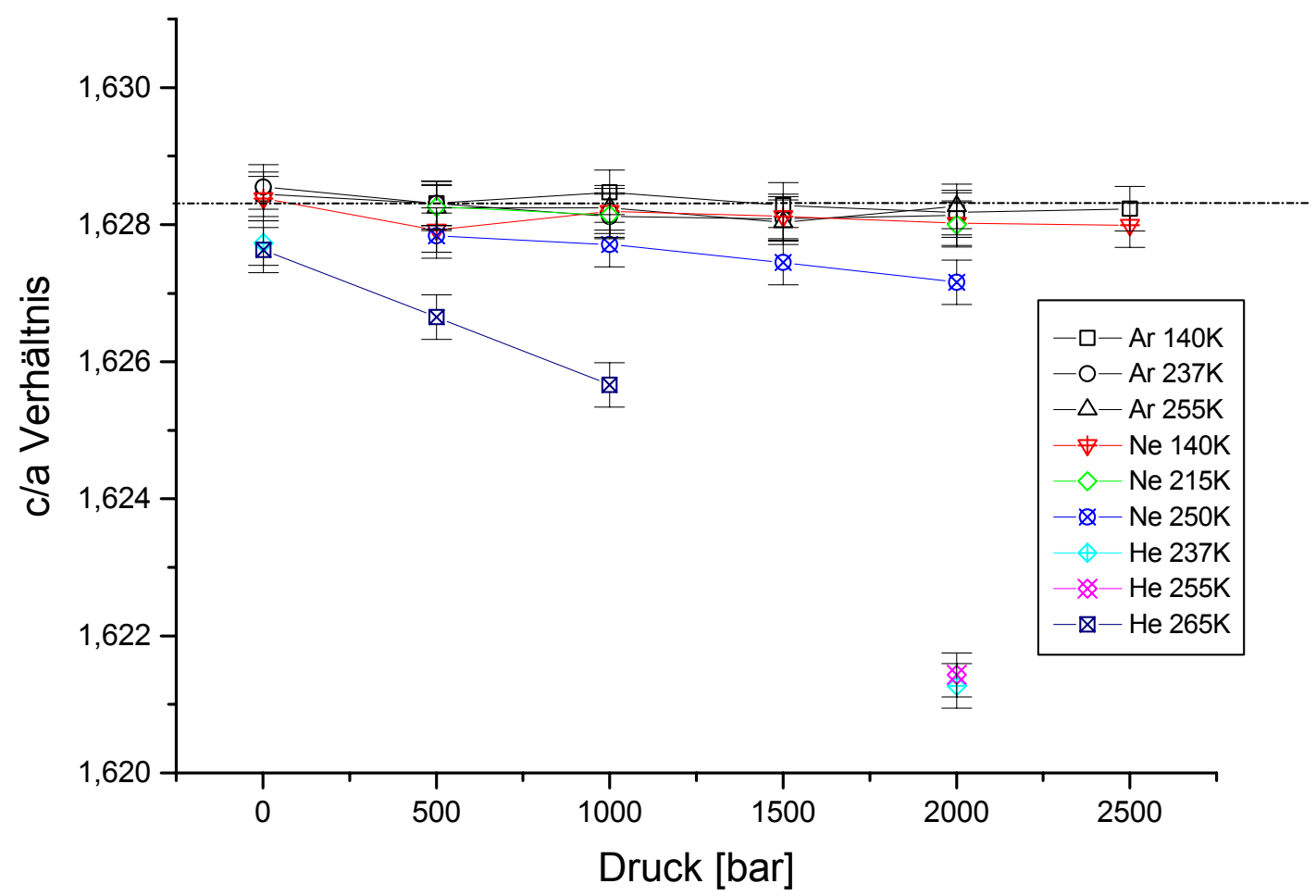

\begin{abstract}
Abbildung 5.13: Vergleich der aus den Gitterkonstanten berechneten c/a-Gitterkonstantenverhältnisse als Funktion der Temperatur, berechnet für Argon bei T 140K, T 237K und T 255K, für Helium bei T 237K, T 255K und T 265K sowie für Neon bei $T \sim 140 K, T \sim 215 K$ und $T \sim 250 K$ als Funktion des Druckes. Die gestrichelte Linie zeigt das von Röttger et al. (1994) bestimmte c/a-Verhältnis bei Umgebungsdruck p 1bar in dem Temperaturbereich von $\mathrm{T} \sim 60 \mathrm{~K}$ bis $\mathrm{T} \sim 265 \mathrm{~K}$.
\end{abstract}

Dieser experimentelle Befund deutet daraufhin, dass die Kompression des Eisgitters in dem angegebenen Druck- und Temperaturbereichen bis zu Temperaturen von $\mathrm{T} \sim 140 \mathrm{~K}$ und einem Druck von p 2.5kbar in den Kristallachsen unabhängig ist. Die Struktur ist somit unter der Verwendung eines nicht in der Eistruktur adorbierbaren Gases in den Gitterkonstanten a und c isokompressibel. Die c/a-Verhältnisse der Gitterkonstanten bei der Verwendung der Gase Helium und Neon zeigen im Gegensatz zu denen von mit Argongas bestimmten c/aVerhältnissen signifikante Änderungen. Bei der Verwendung dieser Gase zeigt sich eine deutliche Abhängigkeit des c/a-Verhältnisses als Funktion des Druckes und der Temperatur. Mit zunehmendem Druck zeigen sich größere Abweichungen von dem c/a-Verhältnis von Röttger et al. (1994). In Gegenwart von Neongas nähern sich die c/a-Verhältnisse sich im Rahmen der Fehler an das c/a-Verhältnis von Eis Ih unter Verwendung von Neongas deutlich zu tieferen Temperaturen an das c/a-Verhältnis von Röttger et al. (1994) an (vergl. Abbildung 5.13, Datensätze von Neongas bei Temperaturen von T 215K sowie T 140K). 
Diese Abweichungen sind zudem reziprok proportional als Funktion der Temperatur. Am deutlichsten ist eine Abweichung für den Datensatz bei $\mathrm{T} \sim 250 \mathrm{~K}$ zu beobachten, während hingegen bei den tieferen Temperaturen von $\mathrm{T} \sim 215 \mathrm{~K}$ sowie $\mathrm{T} \sim 140 \mathrm{~K}$ keine signifikanten oder nur geringe Abweichungen festzustellen sind.

Bei der Verwendung von Heliumgas zeigt sich äquivalent eine Abweichung des c/aVerhältnis zu dem von Röttger et al. (1994), das proportional zum Druck zu kleineren c/aVerhältnissen abweicht. Eine reziprok proportionale Temperaturabhängigkeit des c/aVerhältnisses als Funktion der Temperatur zeigt sich äquivalent zu den mit Neongas bestimmten Gitterkonstantenverhältnissen sowohl im Rahmen der Fehler als auch bedingt durch die geringeren Temperaturunterschiede nicht. Mit steigenden Temperaturen werden die c/a-Verhältnisse bei der Verwendung von Heliumgas nicht wesentlich größer (hierzu vergleiche Abbildung 5.13, Datensätze He237K zu He255K bei einem Druck von p 2000bar sowie den extrapolierten Verlauf des c/a-Verhältnisses des Datensatzes He265K bis zu einem Druck von $\mathrm{p} \sim 2000 \mathrm{bar})$.

\subsubsection{Eis Ih-Struktur als Funktion des Druckes und der Temperatur}

Aus den Gitterkonstantenverhältnissen ergibt sich, dass die Eisstruktur in Wechselwirkung mit den Gasen Helium und Neon zu der mit Argongas unbeeinflussten Struktur deutlichen strukturellen Änderungen unterworfen ist. Durch die Gitterkonstantenverläufe konnte eine eindeutige Aussage bezüglich des druck- und temperaturabhängigen Verhaltens des Gitterkonstantenverhältnisses getätigt werden. Eindeutige Aussagen bezüglich einer isotropen oder anisotropen Gitterbeeinflussung durch die Änderungen der Gitterkonstanten a und c der Eisstruktur bei Ungebungsbedingungen zeigen keine wesentliche Signifikanz. Neben der Bestimmung der Gitterkonstanten wurde daher versucht Aussagen über die strukturellen Parameter der Elementarzelle der gemessenen Eis Ih-Datensätzen zu gewinnen. In den Rietveld-Verfeinerungen wurden die thermischen Auslenkungsparameter (ADP's) sowie die Atompositionen (fraktionellen Koordinaten) bestimmt, aus denen die Bindungsabstände sowie die Bindungswinkel der Sauerstoffe in der Eisstruktur berechnet wurden. Neben der Bestimmung der Bindungsabstände sowie der Bindungswinkel der attraktiven Wechselwirkungen vom Typ O...D (Wasserstoffbrückenbindungen) sind die repulsiven Wechselwirkungen vom Typ D...D sowie die repulsiven Wechselwirkungen der 
weitreichenden O...O-Wechselwirkungen von Interesse. Bei im allgemeinen als Funktion des Druckes und der Temperatur sich ausbildenden Wechselwirkungen, wird den weitreichenden repulsiven O...O-Wechselwirkungskontakten ein größerer und strukturbestimmender Einfluss zugeschrieben (Savage (1985), Savage \& Finney (1986). Zu Beginn der Untersuchungen war nicht bekannt, dass die Aussagefähigkeit der durchgeführten Experimente durch die verbliebenen Imperfektionen, bedingt durch die Art der Herstellung (vergl. Kapitel 3.4.1) sowie durch den Clathrat-Hydratphasenanteil, drastisch eingeschränkt ist.

Im folgenden werden die strukturellen Parameter der Elementarzelle, die Atompositionen für die Datensätze bei den Temperaturen von T 140K, T 237K und T $255 \mathrm{~K}$ sowie bei Drücken von $\mathrm{p} \sim 2 \mathrm{kbar}, \mathrm{p} \sim 500 \mathrm{bar}$ und $\mathrm{p} \sim 1$ bar dargestellt und diskutiert. An dieser Stelle ist jedoch anzumerken, dass lediglich die Datensätze bei der Verwendung von Argongas bei den Umgebungsbedingungen von $\mathrm{p} \sim 2 \mathrm{~kb}$ ar und $\mathrm{T} \sim 250 \mathrm{~K}$, $\mathrm{p} \sim 2 \mathrm{kbar}$ und $\mathrm{T} \sim 237 \mathrm{~K}$ und $\mathrm{p} \sim 2 \mathrm{kbar}$ und $\mathrm{T} \sim 140 \mathrm{~K}$, bei der Verwendung von Heliumgas bei $\mathrm{p} \sim 2 \mathrm{kbar}$ und $\mathrm{T} \sim 237 \mathrm{~K}$ sowie bei der Verwendung von Neongas bei den Umgebungsbedingungen von $\mathrm{p} \sim 1$ bar und $\mathrm{T} \sim 140 \mathrm{~K}$, $\mathrm{p} \sim 1.5 \mathrm{kbar}$ und $\mathrm{T} \sim 140 \mathrm{~K}$ und $\mathrm{p} \sim 2.0 \mathrm{kbar}$ und $\mathrm{T} \sim 140 \mathrm{~K}$ länger als $\mathrm{t} \sim 5 \mathrm{~h}$ gemessen wurden und nur diese über eine ausreichende Meßstatistik zur Bestimmung der Elementarzellenparameter verfügen.

\subsubsection{Eis Ih-Struktur unter Einfluss von Argongas}

Die folgenden Abbildungen 5.14.a, 5.14.b, 5.14.c sowie 5.14.d zeigen exemplarisch die bei der Temperatur von T 140K als Funktion des Druckes Rietveld-verfeinerten Datensätze von Eis Ih unter der Verwendung von Argongas als Drucküberträgermedium. Die mittels Rietveld-Verfeinerungen als Funktion des Druckes bestimmten Strukturparameter bei den Temperaturen von $\mathrm{T} \sim 140 \mathrm{~K}, \mathrm{~T} \sim 237 \mathrm{~K}$ und $\mathrm{T} \sim 255 \mathrm{~K}$ sind in der Tabelle 5.16 zusammengefasst. In den Verfeinerungen wurden die Atompositionen der in der Elementarzelle befindlichen Atome sowie deren isotropen Temperaturfaktoren bestimmt. 


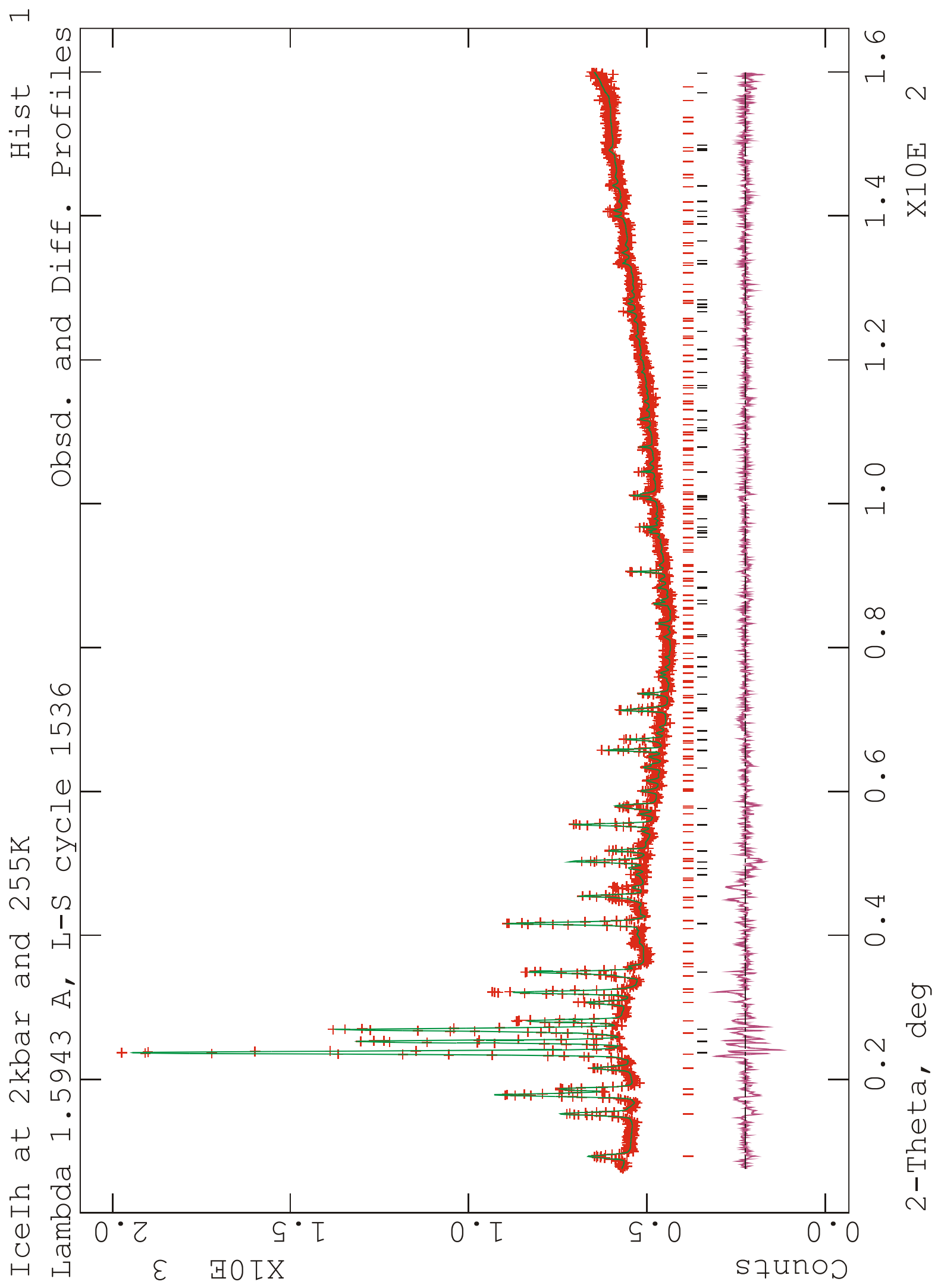

Abbildung 5.14.a: Rietveld-Anpassung des Diffraktogramms von Eis Ih, gemessen bei einer Temperatur von T 255K und einem Druck von p 2kbar. Argon-Clathrat-Hydrat-Phasenanteil n 21\%. 


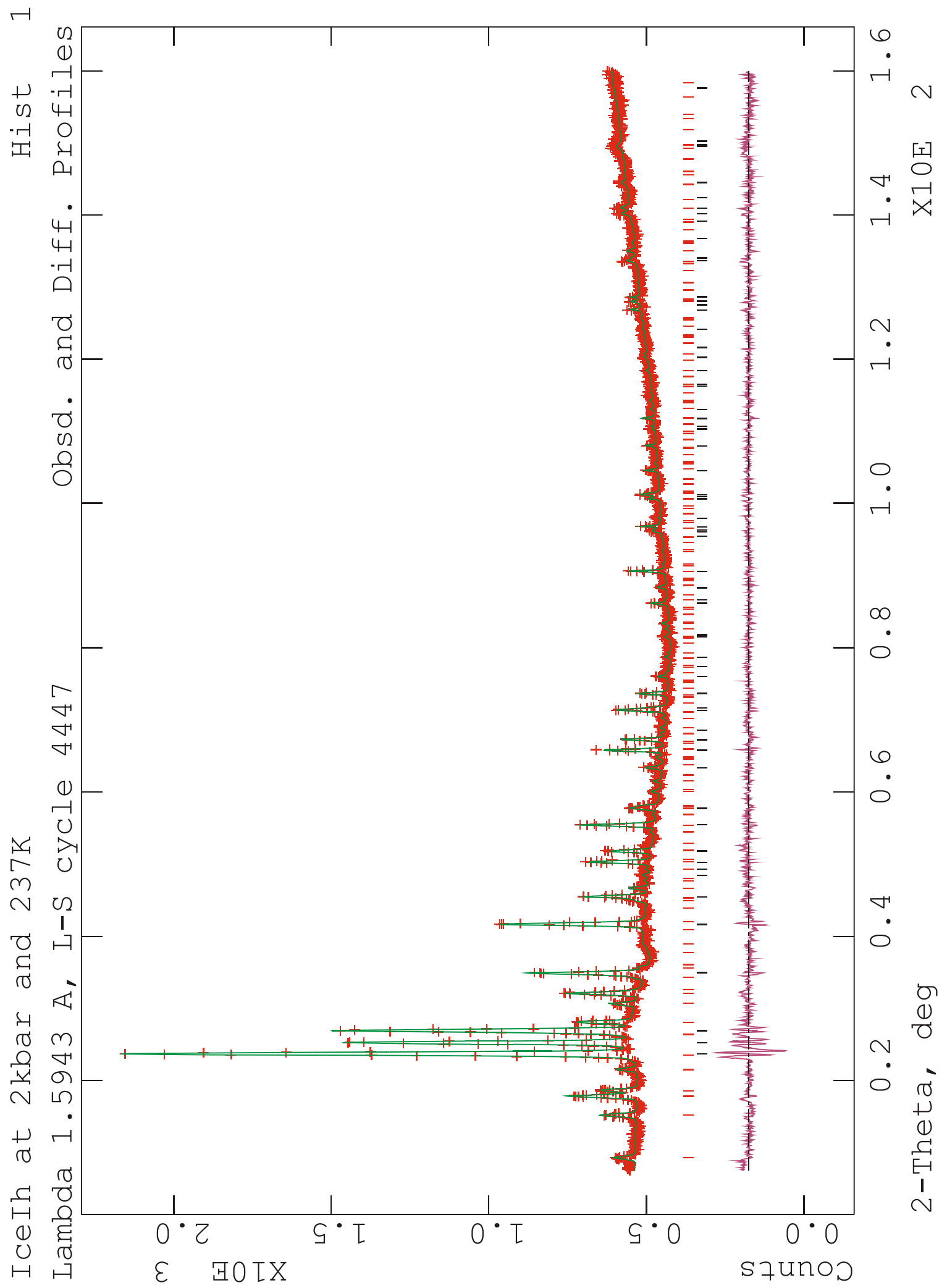

Abbildung 5.14.b: Rietveld-Anpassung des Diffraktogramms von Eis Ih, gemessen bei einer Temperatur von $\mathbf{T} \sim 237 \mathrm{~K}$ und einem Druck von $\mathbf{p} \sim 2 \mathrm{kbar}$. Argon-Clathrat-Hydrat-Phasenanteil $\mathbf{n} \sim 22 \%$. Zum Vergleich Abbildung 5.8.d zeigt das Verfeinerungsergebnis von Eis Ih mit Argon als Drucküberträgermedium bei einer Temperatur von $\mathrm{T} \sim 237 \mathrm{~K}$ und einem Druck von $\mathrm{p} \sim 1.5 \mathrm{kbar}$. 


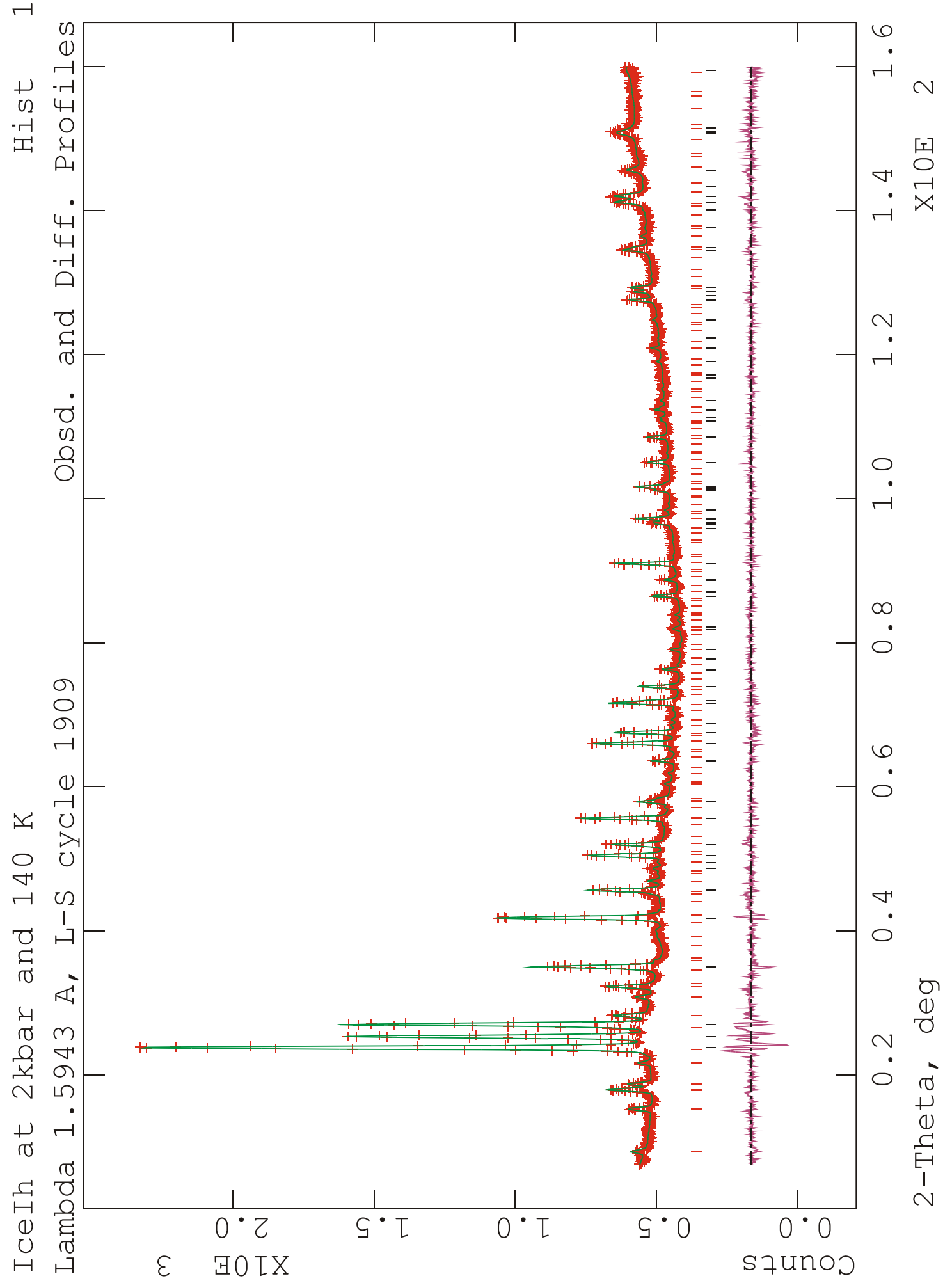

Abbildung 5.14.c: Rietveld-Anpassung des verfeinerten Diffaktogramms von Eis Ih unter Verwendung von Argon als Drucküberträgermedium bei einem Druck von p 2kbar und einer Temperatur von T 140K. Argon-Clathrat-Hydrat-Phasenanteil n 15.8\%. 


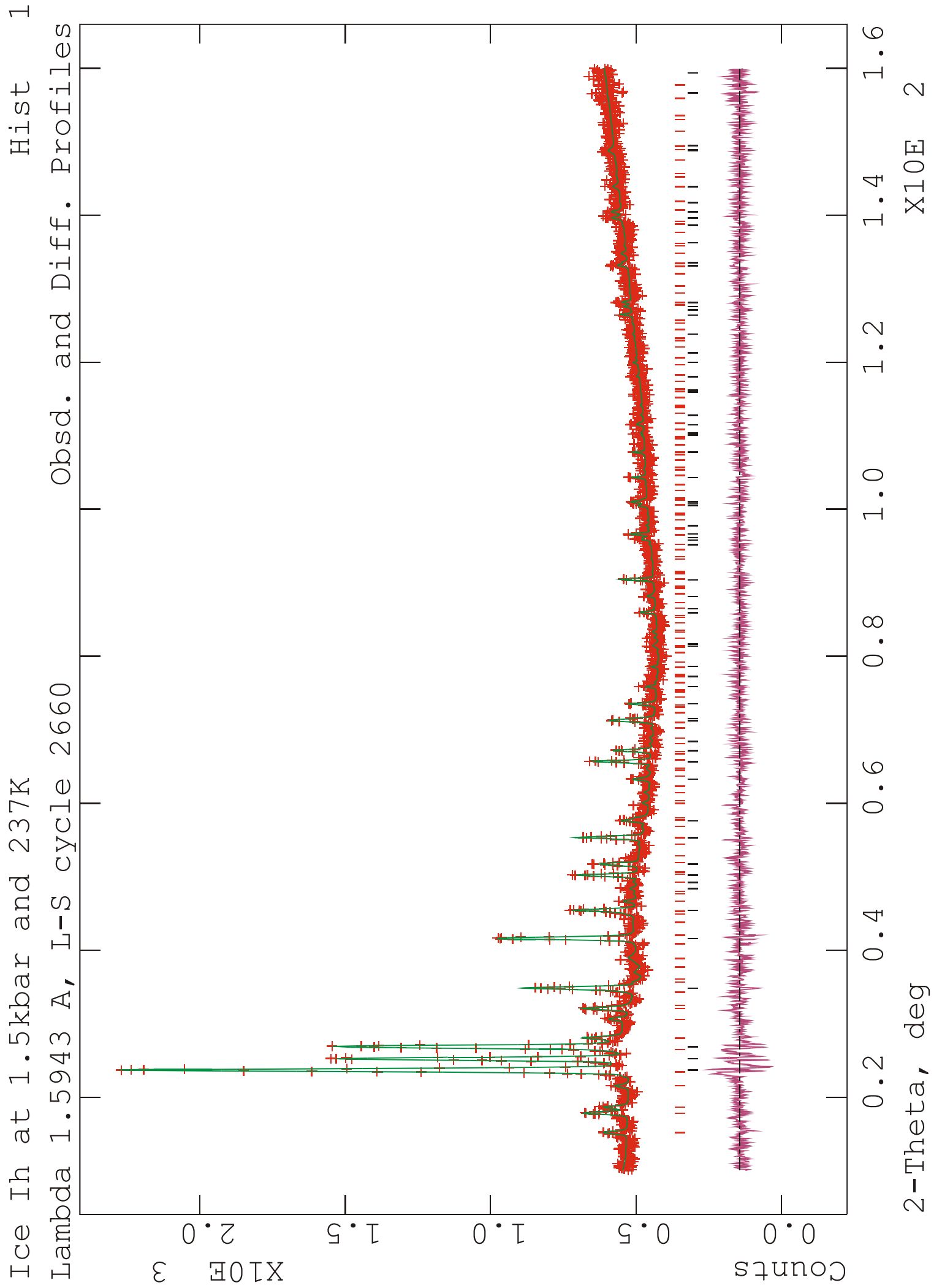

Abbildung 5.14.d: Rietveld-Anpassung des verfeinerten Diffaktogramms von Eis Ih unter Verwendung von Argon als Drucküberträgermedium bei einem Druck von p 1.5kbar und einer Temperatur von T 237K. Argon-Clathrat-Hydrat-Phasenanteil n 16.2\%. 


\begin{tabular}{|c|c|c|c|c|c|c|c|}
\hline p [bar] & 1 & 500 & 1000 & 1500 & 2000 & 2500 & $\mathbf{T}[\mathrm{K}]$ \\
\hline $\mathbf{O}_{\mathrm{Z}}$ & $0.06189(116)$ & $0.06201(120)$ & $0.06207(130)$ & $0.06256(129)$ & $0.06223(744)$ & $0.06172(119)$ & \multirow{7}{*}{140} \\
\hline$\overline{D 1} 1_{z}$ & $0.19625(188)$ & $0.19864(186)$ & $0.19914(196)$ & $0.19924(198)$ & $0.19886(113)$ & $0.19911(184)$ & \\
\hline D2 ${ }_{X}$ & $0.45420(134)$ & $0.45277(125)$ & $0.45443(149)$ & $0.45456(142)$ & $0.45450(834)$ & $0.45526(133)$ & \\
\hline $\mathbf{D 2}_{\mathrm{Z}}$ & $0.01646(149)$ & $0.01534(137)$ & $0.01508(155)$ & $0.01533(150)$ & $0.01527(894)$ & $0.01423(146)$ & \\
\hline Uiso 0 & $0.02604(190)$ & $0.02532(170)$ & $0.02586(177)$ & $0.02585(177)$ & $0.02683(111)$ & $0.02687(177)$ & \\
\hline Uiso D1 & $0.03649(389)$ & $0.03709(380)$ & $0.03800(405)$ & $0.03907(414)$ & $0.03590(234)$ & $0.03773(381)$ & \\
\hline Uiso D2 & $0.03985(345)$ & $0.04910(330)$ & $0.04390(366)$ & $0.04285(354)$ & $0.04366(219)$ & $0.04418(348)$ & \\
\hline $\mathbf{O}_{\mathrm{Z}}$ & $0.06225(165)$ & $0.06084(181)$ & $0.06056(193)$ & $0.06118(188)$ & $0.06247(128)$ & $\mathrm{x}$ & \multirow{7}{*}{237} \\
\hline$\overline{D 1} 1_{z}$ & $0.19841(251)$ & $0.19556(249)$ & $0.19923(261)$ & $0.19721(273)$ & $0.19970(186)$ & $\mathrm{x}$ & \\
\hline D2 ${ }_{X}$ & $0.45356(170)$ & $0.45508(183)$ & $0.45577(200)$ & $0.45499(194)$ & $0.45531(133)$ & $\mathrm{x}$ & \\
\hline $\mathbf{D} 2_{\mathrm{Z}}$ & $0.01585(195)$ & $0.01666(211)$ & $0.01591(233)$ & $0.01586(224)$ & $0.01657(147)$ & $\mathrm{x}$ & \\
\hline Uiso $O$ & $0.04671(315)$ & $0.04748(324)$ & $0.05149(357)$ & $0.04932(336)$ & $0.04919(225)$ & $\mathrm{x}$ & \\
\hline Uiso D1 & $0.06419(663)$ & $0.05300(612)$ & $0.05677(651)$ & $0.06080(675)$ & $0.05906(453)$ & $\mathrm{x}$ & \\
\hline Uiso D2 & $0.06640(531)$ & $0.06421(543)$ & $0.06281(576)$ & $0.06578(564)$ & $0.06360(369)$ & $\mathrm{x}$ & \\
\hline $\mathbf{O}_{\mathrm{Z}}$ & $\mathrm{x}$ & $0.06194(209)$ & $0.06249(199)$ & $0.06315(273)$ & $0.06276(172)$ & $\mathrm{x}$ & \multirow{7}{*}{255} \\
\hline $\mathrm{D1}_{\mathrm{Z}}$ & $\mathrm{x}$ & $0.19735(304)$ & $0.19895(295)$ & $0.20203(387)$ & $0.19995(268)$ & $\mathrm{x}$ & \\
\hline D2 ${ }_{X}$ & $\mathrm{x}$ & $0.45315(200)$ & $0.45416(197)$ & $0.45676(293)$ & $0.45525(171)$ & $\mathrm{x}$ & \\
\hline $\mathbf{D 2}_{\mathrm{Z}}$ & $\mathrm{x}$ & $0.01673(236)$ & $0.01784(226)$ & $0.01510(349)$ & $0.01788(197)$ & $\mathrm{x}$ & \\
\hline Uiso 0 & $\mathrm{x}$ & $0.05127(384)$ & $0.05029(372)$ & $0.05560(540)$ & $0.05381(324)$ & $\mathrm{x}$ & \\
\hline Uiso D1 & $\mathrm{x}$ & $0.06394(774)$ & $0.06285(759)$ & $0.05939(984)$ & $0.06983(693)$ & $\mathrm{x}$ & \\
\hline Uiso D2 & $\mathrm{x}$ & $0.06568(236)$ & $0.06328(579)$ & $0.07213(780)$ & $0.06491(489)$ & $\mathrm{x}$ & \\
\hline
\end{tabular}

Tabelle 5.16: Durch Rietveld-Verfeinerungen bestimmte fraktionelle Koordinaten der in der Eis Ih-Einheitszelle modellierten Atome sowie deren isotropen thermischen Auslekungsparametern als Funktion des Druckes und der Temperatur der Langzeit-Messungen $(t \geq 5 \mathrm{~h})$. Zum Vergleich der bei einem Umgebungsdruck von p 1bar bestimmten Strukturparameter von Kuhs \& Lehmann (1986) (vergl. Tabelle 5.17), x: keine experimentellen Daten vorhanden.

\begin{tabular}{|c|c|c|c|c|}
\hline \multicolumn{5}{|c|}{ Kuhs \& Lehmann (1986), p 1 bar } \\
\hline $\mathbf{T}[\mathbf{K}]$ & $\mathbf{O}_{\mathbf{Z}}$ & $\mathbf{D 1}_{\mathbf{Z}}$ & $\mathbf{D 2}_{\mathbf{X}}$ & $\mathbf{D 2}_{\mathbf{Z}}$ \\
\hline $\mathbf{1 5}$ & $0.06212(3)$ & $0.19898(7)$ & $0.454449(4)$ & $0.01695(5)$ \\
\hline $\mathbf{6 0}$ & $0.06210(4)$ & $0.19891(10)$ & $0.45439(6)$ & $0.01697(6)$ \\
\hline $\mathbf{1 2 3}$ & $0.06218(7)$ & $0.19874(14)$ & $0.45436(7)$ & $0.01698(9)$ \\
\hline $\mathbf{2 2 3}$ & $0.06211(23)$ & $0.19833(18)$ & $0.45395(8)$ & $0.01706(8)$ \\
\hline
\end{tabular}

Tabelle 5.17: Fraktionelle Atompositionen als Funktion der Temperatur isobar, Referenzwerte von Kuhs \& Lehmann (1986), with revisions Kuhs (1996). 
Im Rahmen der hohen Fehler, die als dreifache Fehler der Rietveld-Standardfehler angenommen werden, sind keine signifikanten Änderungen für die $\mathrm{O}_{\mathrm{Z}}$-Positionen des Sauerstoffs, der $\mathrm{D} 1_{\mathrm{X}^{-}}$sowie $\mathrm{D} 2_{\mathrm{Y}^{-}}$als auch $\mathrm{D} 2_{\mathrm{Z}^{-}}$Positionen als Funktion des Druckes feststellbar. Ebenso wenig konnte eine signifikante Abhängigkeit der aufgeführten Atompositionen im Rahmen der Fehler als Funktion der Temperatur festgestellt werden. Lediglich für die isotropen thermischen Auslenkungsparameter ist erwartungsgemäß eine zur Temperatur proportionale Abhängigkeit festzustellen.

Die bei steigendem Druck bedingte Kompression der Eisstruktur (Verkürzung der Gitterkonstanten) und die damit verbundene höhere Dichte ist in erster Linie durch eine Verkürzung der O...O-Abstände bzw. durch Änderung der O...O...O-Winkel bedingt. Die Korrelation von Bindungsabständen $\mathrm{O} . . \mathrm{O} \mathrm{zu}$ Bindungswinkeln O...O...O zeigte ebenso aufgrund der hohen Fehler keine mit Druck oder Temperatur signifikante Änderung. Ob eine strukturelle Änderung in der Elementarzelle als Funktion des Druckes und der Temperatur stattfindet, läßt sich im Rahmen der Fehlergrenzen nicht feststellen. Auch stimmen die Werte im Vergleich zu den von Kuhs \& Lehmann (1986), with revisions Kuhs (1996) festgestellten im Fehlerbereich, bei vergleichbaren Temperaturen und Drücken bei $\mathrm{p} \sim 2 \mathrm{kbar}$, überein. Daher sind im Rahmen der Fehler keine signifikanten Änderungen als Funktion der Temperatur und des Druckes für die Atompositionen in der Eisstruktur festzustellen.

\subsubsection{Eis Ih-Hydrat. Wechselwirkung von Eis Ih mit Helium und Neon}

Durch die Schmelzkurvenexperimente an Eis Ih sowie dem Gitterkonstanten- und Kompressibilitätenvergleich konnte konsistent zu Dyadin et al. (1994) gezeigt werden, dass in der Eisstruktur von Eis Ih sowohl Heliumgas als auch Neongas adsorbiert werden und einen Einfluss auf die Struktur ausüben. Durchgeführte Rietveld-Verfeinerungen, in denen die Eis Ih-Struktur mit eingebautem Argongas modelliert wurden, zeigten erwartungsgemäß nur einen geringen Argongasinhalt von unter $0.06 \%$. Als ein Beleg für die Nichtlöslichkeit von Argongas kann dies jedoch nicht gelten. Eine mögliche diffuse Besetzung der Eis Ih-Struktur mit Argonatomen ist nicht auszuschließen. 
In Bezugnahme auf die Schmelzkurvenexperimenten kann jedoch ausgesagt werden, dass im Rahmen der experimentellen Fehler kein wesentlicher Einfluss des als sehr gering anzunehmenden adsorbierten Argongasinhaltes feststellbar ist. Die mit dem Druck proportional ansteigenden Abweichungen der Schmelzkurven von Eis Ih mit Neongas (eigene Experimente) sowie mit Heliumgas (Dyadin et al., 1994) hingegen belegen eine mit dem Druck ansteigende Stabilisierung des Eisgitters durch die Besetzung mit Gasatomen. Bei einer Betrachtung der Gitterkonstanten von Eis Ih ist dieser Effekt durch die unterschiedlichen Änderungen der Gitterkonstanten in a und c sowohl für Heliumgas als auch für Neongas als Funktion des Druckes und der Temperatur feststellbar. Dies lässt auf eine maßgebliche Beeinflussung des Eisgitters als Funktion der Besetzung schließen. Das Eis Ih stellt daher im eigentlichen Sinn, gemäß der Definition eines freien Gasaustausches in der Struktur, ein Eis Ih-Gashydrat dar (vergl. Kapitel 4.1.2).

Ziel der Verfeinerungen war es daher neben dem Gasinhalt, die durch die van der Waals'schen Wechselwirkungen des Gasinhaltes und des Eisgitters verursachten strukturellen Änderungen als Funktion des Druckes und der Temperatur zu bestimmen.

\subsubsection{Gasinhalt in Eis Ih-Hydrat für Helium- und Neongas}

\subsubsection{Neon}

Das c/a-Verhältnis der Eis Ih-Struktur unter der Verwendung von Neon (vergl. Abbildung 5.13, Kapitel 5.5.1) zeigt deutlich, dass die stärkste Beeinflussung durch das verwendete Neongas bei einer Temperatur von $\mathrm{T} \sim 250 \mathrm{~K}$ gegeben ist. Im Vergleich zu den anderen Temperaturen ist daher aufgrund der Eis Ih-Struktur- / Gas-Wechselwirkungen von einer maximalen Besetzung dieser auszugehen. Zur Bestimmung des Gasinhaltes wurden daher für die bei einer Temperatur von T $250 \mathrm{~K}$ mit Neon gemessenen Datensätze verwendet. In der Tabelle 5.18 sind die Ergebnisse der, isotrop, mit und ohne in die Rietveld-Verfeinerungen einmodellierten Neongases, frei verfeinerten Datensätze dargestellt. 


\begin{tabular}{|c|c|c|c|c|}
\hline p [bar] & $\mathbf{5 0 0}$ & $\mathbf{1 0 0 0}$ & $\mathbf{1 5 0 0}$ & $\mathbf{2 0 0 0}$ \\
\hline \multicolumn{5}{|c|}{ mit Gas } \\
\hline Ne4 & $0.053(11)$ & $0.028(12)$ & $0.003(12)$ & $0.060(11)$ \\
\hline Ne5 & $0.053(7)$ & $0.033(9)$ & $0.014(08)$ & $0.030(7)$ \\
\hline Ne6 & X & X & X & X \\
\hline wRp & 2.87 & 2.60 & 2.28 & 2.74 \\
\hline Rp & 2.26 & 1.94 & 1.77 & 2.16 \\
\hline wRp & 2.81 & ohne Gas & 2.86 & 2.72 \\
\hline Rp & 2.24 & 1.95 & 2.26 & 2.15 \\
\hline
\end{tabular}

Tabelle 5.18: durch Rietveld-Verfahren bestimmte Gasbesetzung der Eis Ih-Struktur mit Neon bei einer Temperatur von $\mathrm{T} \sim 250 \mathrm{~K}$ als Funktion des Druckes. Die in der Tabelle mit $X$ gekennzeichneten Einträge bedeuten, dass im Zuge der Verfeinerungen sich für diese Gaslagen negative Besetzungen ergeben und diese dann auf null gesetzt wurden.

Die Abbildungen 5.15.a sowie 5.15.b zeigen exemplarisch einen Vergleich von angepassten Diffraktogrammen als Verfeinerungsergebnis der Eis Ih-Struktur unter Verwendung von Neongas bei einem Druck von $\mathrm{p} \sim 500$ bar und einer Temperatur von $\mathrm{T} \sim 250 \mathrm{~K}$ mit und ohne Gas.

Betrachtet man die Differenzkurven für die Verfeinerung der Eis Ih-Struktur mit und ohne Neongas, so ergeben sich keine signifikanten Unterschiede. Ebenso ist die Güte der Verfeinerungen mit und ohne in den Rietveld-Modellen eingebautem Neongas vergleichbar, so dass die Verfeinerungen daher von vergleichbarer Qualität und hinreichend gut angepasst sind (vergl. Abbildung 5.15.a sowie Abbildung 5.15.b).

Die aus den Verfeinerungen bestimmten marginal signifikanten Besetzungszahlen geben für sich genommen nur einen schwachen Beweis für eine Neon-Besetzung der Kanäle in Eis Ih. Bei einer Temperatur von $\mathrm{T} \sim 250 \mathrm{~K}$ und einem Druck von $\mathrm{p} \sim 500$ bar würde sich eine Neonbesetzung von $\mathrm{n} \sim 2.904$ Gasmolekülen ergeben, wohin gegen sich bei einem Druck von $\mathrm{p} \sim 2 \mathrm{kbar}$ nur eine Besetzung von $\mathrm{n} \sim 0.3$ Gasmolekülen errechnen lässt (vergl. Tabelle 5.18). Um Aussagen bezüglich des als Funktion des Druckes und der Temperatur bestimmten Gasinhaltes der Eisstruktur treffen zu können, wurden diesbezüglich Simulationsrechungen durchgeführt. Diese werden an späterer Stelle erläutert und diskutiert (vergl. Kapitel 5.6). 


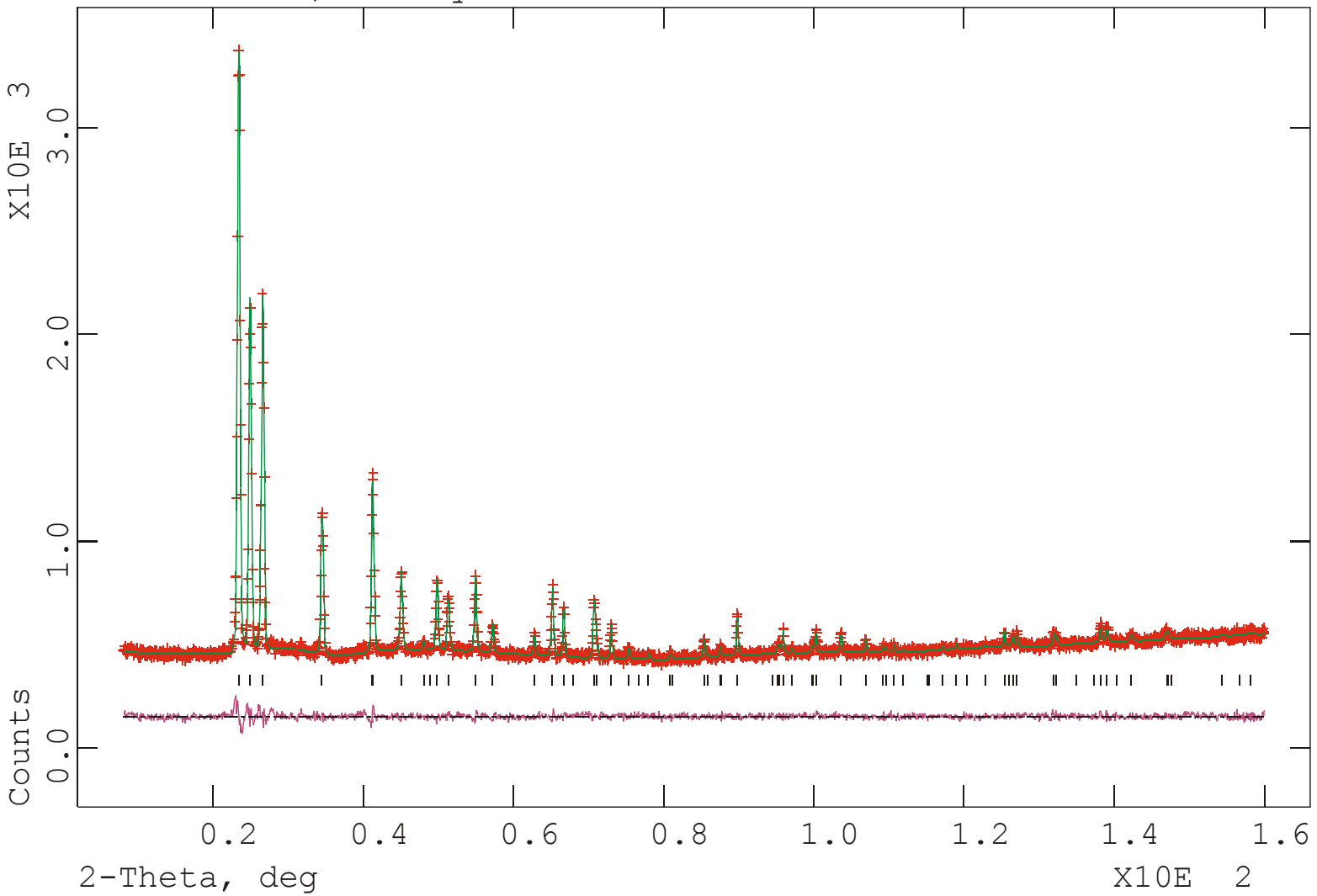

Ih with Neon at 500bar and $250 \mathrm{~K}$

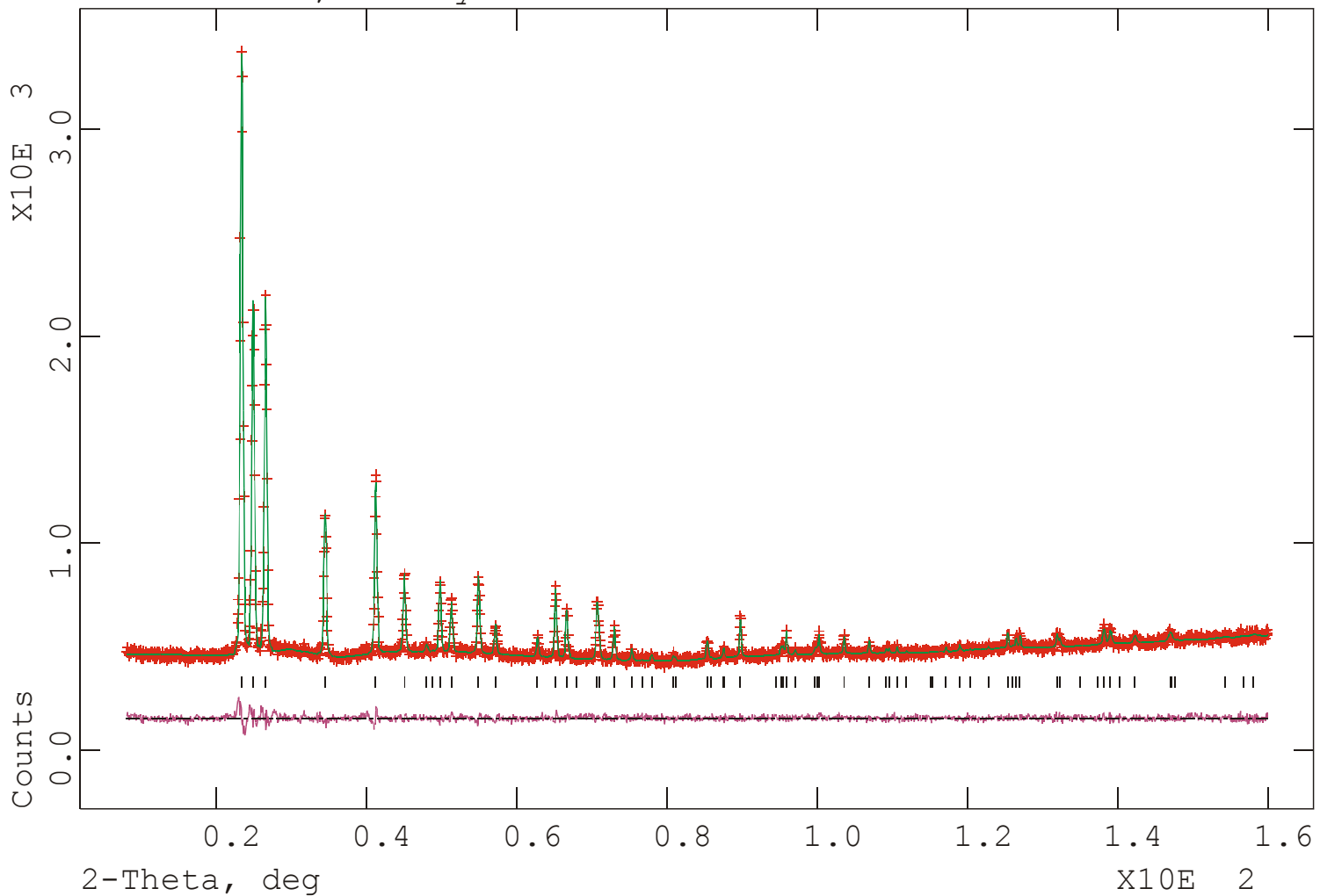

Abbildung 5.15.a (oben), Abbildung 5.15.b (unten): Rietveld-Verfeinerungen bei einer Temperatur von T 250K und einem Druck von p 500bar, (oben) mit Neongas, (unten) ohne Neongas in der RietveldVerfeinerung. 
Als Ursache neben dem geringen Streuvermögen der Gasatome ist eine ausgeschmierte Verteilung der Gasatome in der Struktur, die sowohl eine Bestimmung der Gaslagen als auch der Gasbesetzung sehr erschweren, anzuführen. Wesentlich hierfür dürfte die Frage sein, in welchen Anteilen das Gas durch van der Waal'sche Wechselwirkungen in der Struktur oder vielmehr an einzelnen Wassermolekülen gebunden sind oder wie die räumliche Bewegungsfreiheit der Gasatome in den Kanälen der Eisstruktur ist. In den folgenden Tabellen 5.19 sowie 5.20 sind die Atompositionen sowie die isotropen thermischen Auslenkungsparameter der bei einer Temperatur von $\mathrm{T} \sim 250 \mathrm{~K}$ und mit Neongas als Drucküberträgermedium gemessenen Datensätze als Funktion des Druckes im Vergleich der Verfeinerungen mit und ohne Neongas dargestellt.

\begin{tabular}{|c|c|c|c|c|}
\hline $\mathbf{p}[\mathbf{b a r}]$ & $\mathbf{5 0 0}$ & $\mathbf{1 0 0 0}$ & $\mathbf{1 5 0 0}$ & $\mathbf{2 0 0 0}$ \\
\hline $\mathbf{O z}$ & $0.06341(117)$ & $0.06158(117)$ & $0.06290(113)$ & $0.06280(125)$ \\
\hline $\mathbf{D}_{\mathbf{1}} \mathbf{z}$ & $0.19960(156)$ & $0.19829(150)$ & $0.20235(158)$ & $0.20237(196)$ \\
\hline $\mathbf{D}_{\mathbf{2}} \mathbf{x}$ & $0.45404(115)$ & $0.45436(115)$ & $0.45521(134)$ & $0.45540(117)$ \\
\hline $\mathbf{D}_{\mathbf{2}} \mathbf{z}$ & $0.01750(130)$ & $0.01661(117)$ & $0.01729(120)$ & $0.01779(140)$ \\
\hline Uiso O & $0.05296(196)$ & $0.05284(196)$ & $0.05326(186)$ & $0.05378(142)$ \\
\hline Uiso D1 & $0.05697(364)$ & $0.05730(392)$ & $0.06063(411)$ & $0.06097(134)$ \\
\hline Uiso D2 & $0.06192(246)$ & $0.06355(285)$ & $0.06222(273)$ & $0.06152(196)$ \\
\hline po-ratio & $0.97724(294)$ & $0.98144(318)$ & $0.97868(302)$ & $0.98062(299)$ \\
\hline
\end{tabular}

Tabelle 5.19: Eis Ih-Struktur mit Neongas isotrop verfeinert, *.) po-ratio : Vorzugsorientierung der Kristallite in Richtung der angegebenen hkl, der po-ratio beträgt für eine völlig regellose Probe 1. Gasinhalt gemäß der Tabelle 5.18

\begin{tabular}{|c|c|c|c|c|}
\hline $\mathbf{p}[\mathbf{b a r}]$ & $\mathbf{5 0 0}$ & $\mathbf{1 0 0 0}$ & $\mathbf{1 5 0 0}$ & $\mathbf{2 0 0 0}$ \\
\hline $\mathbf{O z}$ & $0.06330(144)$ & $0.06123(193)$ & $0.06387(120)$ & $0.06354(120)$ \\
\hline $\mathbf{D}_{\mathbf{1}} \mathbf{z}$ & $0.20005(156)$ & $0.19858(156)$ & $0.20166(168)$ & $0.20322(175)$ \\
\hline $\mathbf{D}_{\mathbf{2}} \mathbf{x}$ & $0.45436(111)$ & $0.45358(129)$ & $0.45437(144)$ & $0.45539(117)$ \\
\hline $\mathbf{D}_{\mathbf{2}} \mathbf{z}$ & $0.01765(123)$ & $0.01629(186)$ & $0.01765(129)$ & $0.01782(132)$ \\
\hline Uiso O & $0.05310(146)$ & $0.05055(183)$ & $0.05390(240)$ & $0.05386(237)$ \\
\hline Uiso D1 & $0.05826(399)$ & $0.05834(339)$ & $0.05686(420)$ & $0.06020(149)$ \\
\hline Uiso D2 & $0.06364(318)$ & $0.06381(186)$ & $0.06071(324)$ & $0.06218(111)$ \\
\hline po-ratio & $0.97142(610)$ & $0.977230(657)$ & $0.96009(618)$ & $0.95087(298)$ \\
\hline
\end{tabular}

Tabelle 5.20: Eis Ih-Struktur ohne Neongas, isotrop verfeinert, *.) po-ratio : Vorzugsorientierung der Kristallite in Richtung der angegebenen hkl, der po-ratio beträgt für eine völlig regellose Probe 1.

Es zeigt sich, dass die in der Eisstruktur mit und ohne Neongas verfeinerten Atompositionen im Rahmen des angegebenen Fehlers, dem dreifachen Standardfehler der Verfeinerung, nicht wesentlich voneinander abweichen. Dieser Befund zeigt sich durchgängig bei den 
Datensätzen mit und ohne Neongas verfeinert bei Drücken von $\mathrm{p} \sim 500 \mathrm{bar}, \mathrm{p} \sim 1 \mathrm{kbar}, \mathrm{p} \sim 1.5 \mathrm{kbar}$ und p 2kbar bei der Temperatur von T 250K. Die festgestellten Änderungen in den Atompositionen sind bezogen auf die dreifachen Standard-Fehlergrenzen nicht signifikant.

An dieser Stelle ist anzumerken, dass es sich bei den Verschiebungen in den Atompositionen um sehr kleine Effekte handelt. Für den Datensatz bei einem Druck von p 500bar ergibt sich für die Sauerstoffe bei einer Differenz von $\Delta \mathrm{z} \sim 0.00011$ (144) für $\mathrm{O}_{z}$ in fraktionellen Koordinaten bei der Verfeinerung mit und ohne Gas eine Verschiebung der Sauerstoffe in cRichtung des Kristallgitters, die deutlich innerhalb der angegebenen Fehlergrenzen liegt. Der Einfluss des Gases auf die Eisstruktur ist somit aufgrund der kleinen Effekte und der im Vergleich dazu großen Fehler nicht zu verifizieren.

Eine Aussage bezüglich der Beanspruchung der Wasserstoffbrückenbindung ist daher nicht zutreffen. Für die Eisstruktur mit Neon als Drucküberträger ergeben sich im Rahmen des Fehlers keine nennenswerten Änderungen aufgrund der Lösung von Neon in Eis Ih. Für die weiteren gemessenen Datensätze unter der Verwendung von Neon bei den Temperaturen von $\mathrm{T} \sim 215 \mathrm{~K}$ sowie $\mathrm{T} \sim 140 \mathrm{~K}$ sind die Atompositionen zwar durch Rietveld-Verfeinerungen bestimmt worden, jedoch sind diese aufgrund der geringeren Probengüte bedingt durch die in Kapitel 3.4.1 beschriebenen Imperfektionen durch die Probenherstellung im Rahmen der Fehler identisch mit den Werten in Tabelle 5.19 und 5.20. Eine Aussage über diese Datensätze ist daher am wenigsten zu treffen. 


\subsubsection{Helium}

Äquivalent zur der in Kapitel 5.4 beschriebenen Verfeinerungsstrategie wurden die Datensätze, gemessen unter der Verwendung von Heliumgas, mit isotropen thermischen Auslenkungsparametern frei verfeinert. Die Ergebnisse der Verfeinerungen mit und ohne in die Eis Ih-Struktur eingebautem Heliumgas sind in den folgenden Tabellen 5.21, 5.22 sowie 5.23 dargestellt.

\begin{tabular}{|c|c|c|c|c|c|}
\hline $\mathbf{T}[\mathrm{K}]$ & \multicolumn{2}{|c|}{265} & 255 & \multicolumn{2}{|c|}{237} \\
\hline \multicolumn{6}{|c|}{ mit Heliumgas } \\
\hline p [bar] & 500 & 1000 & 2000 & 1 & 2000 \\
\hline He4 & $\mathrm{X}$ & $\mathrm{X}$ & $\mathrm{X}$ & $\mathrm{X}$ & $\mathrm{X}$ \\
\hline He5 & $0.133(38)$ & $0.98(26)$ & $0.049(27)$ & $0.112(24)$ & $0.065(16)$ \\
\hline He6 & $0.163(38)$ & $0.98(36)$ & $0.186(36)$ & $0.179(33)$ & $0.139(21)$ \\
\hline WRp & 3.40 & 3.29 & 3.52 & 3.49 & 2.12 \\
\hline $\mathbf{R p}$ & 2.69 & 2.63 & 2.77 & 2.75 & 1.53 \\
\hline \multicolumn{6}{|c|}{ ohne Heliumgas } \\
\hline wRp & 3.42 & 3.29 & 3.53 & 3.49 & 2.12 \\
\hline $\mathbf{R p}$ & 2.71 & 2.63 & 2.77 & 2.75 & 1.53 \\
\hline
\end{tabular}

Tabelle 5.21: durch Rietveld-Verfahren bestimmte Gasbesetzung der Eis Ih-Struktur mit Helium bei den in der Tabelle aufgeführten Umgebungsbedingungen. Die in der Tabelle mit X gekennzeichneten Einträge bedeuten, dass im Zuge der Verfeinerungen sich für diese Gaslagen negative Besetzungen ergeben und diese dann auf null gesetzt wurden.

Bei den Gasbesetzungen wurden Unterschiede zwischen den Neon- und Helium-Eis IhStrukturen festgestellt. Im Gegensatz zu den Gaslagen von Neon Ne4 sowie Ne5 ließen sich bei der Verfeinerung der mit Helium gemessenen Datensätze die Gaslagen He5 sowie He6 verfeinern. Außer dem Größenunterschied der verwendeten Gase ist keine andere physikalische Ursache für die unterschiedlich verfeinerten Gaslagen und Gasbesetzungen ersichtlich. Die folgenden Abbildungen 5.16.a sowie 5.16.b zeigen die Rietveld-Anpassungen der mit und ohne in die Eis Ih-Struktur eingebautem Heliumgas. 


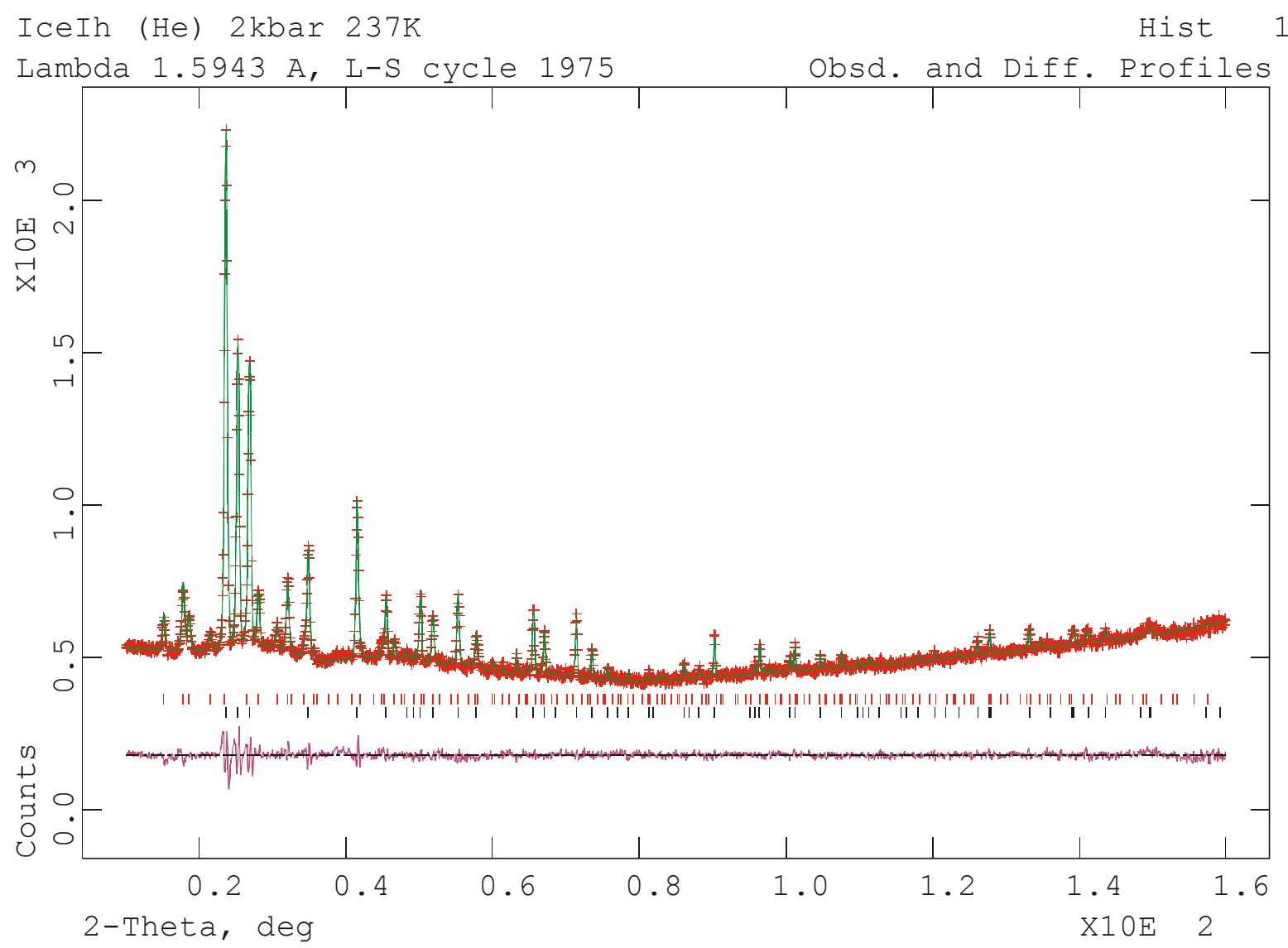

IceIh (He) 2kbar 237K Hist 1 Lambda 1.5943 A, L-S cycle 2009 Obsd. and Diff. Profiles

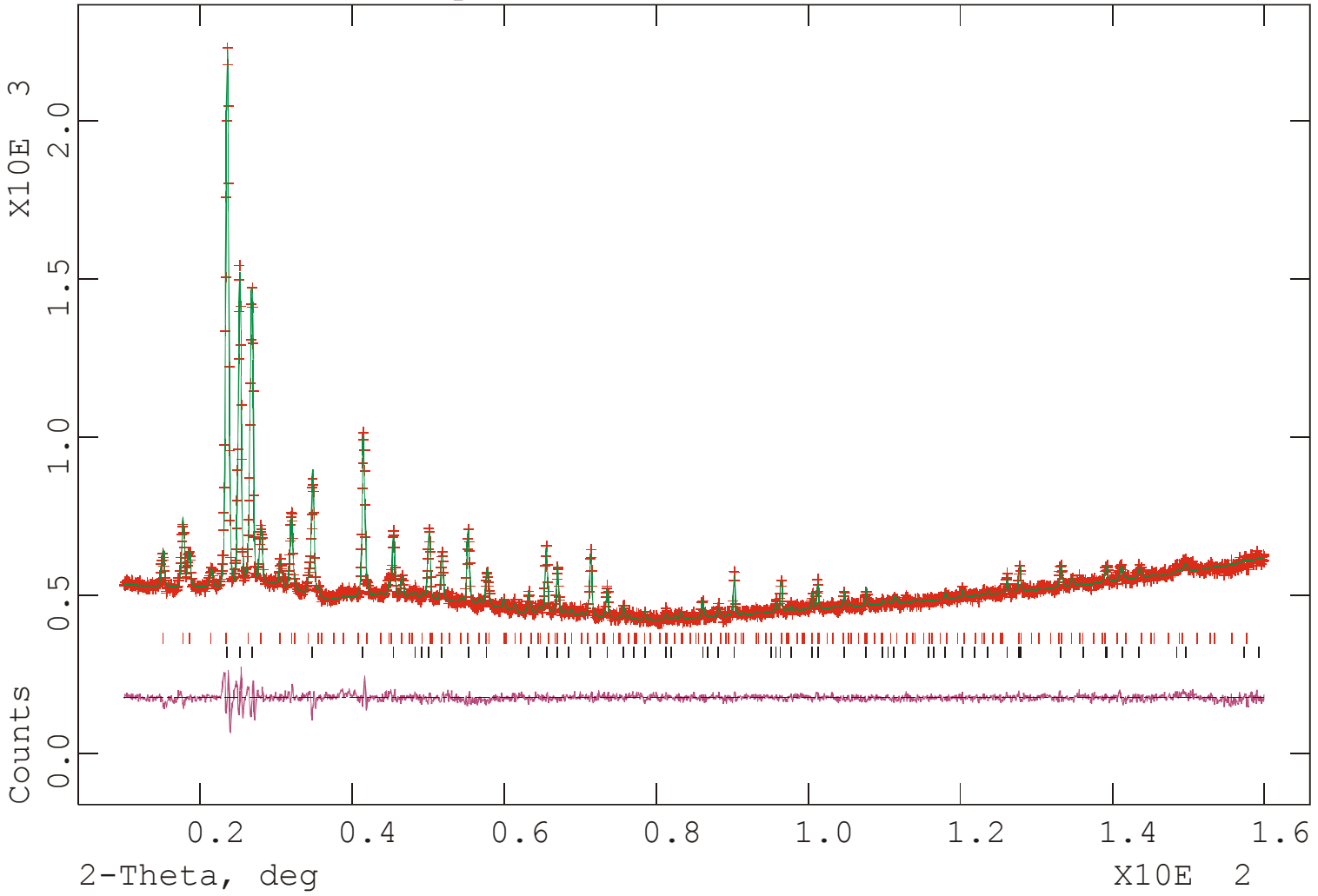

Abbildung 5.16.a (oben), Abbildung 5.16.b (unten): Rietveld-Verfeinerungen bei einer Temperatur von T 237K und einem Druck von p 2kbar, (oben) mit Heliumgas, (unten) ohne Heliumgas in der RietveldVerfeinerung. 
Die Besetzungszahlen für die He5-, He6-Gaslagen liegen um eine Größenordnung höher und zeigen entgegen den bestimmten Besetzungen der Verfeinerungen der mit Neon gemessenen Datensätze keine eindeutige druck- und temperaturabhängige Tendenz. Die bestimmte höhere Gasbesetzung könnte durch den geringeren atomaren Durchmessers des Heliums bedingt sein, eine erhöhte Besetzung erscheint daher möglich. Bei einer Multiplizität der Gaslagen He4, He5, He6 von 2, 6, 2 ergibt sich somit eine Besetzung von 2.8(1) Helium-Atomen pro Elementarzelle bei einer Temperatur von $\mathrm{T} \sim 265 \mathrm{~K}$ und einem Druck von $\mathrm{p} \sim 500 \mathrm{bar}$. Bei einem Druck von $\mathrm{p} \sim 1 \mathrm{kbar}$ sowie einer Temperatur von $\mathrm{T} \sim 265 \mathrm{~K}$ würden sich 0.984(40) HeliumAtome ergeben.

Bisherige Arbeiten (Haas et al 1971, Dyadin et al. 1994) die den Gasinhalt von Helium in Eis Ih experimentell bestimmt haben, fanden bei Standardbedingungen (d.h. Gasdruckerhöhung auf die Probe, danach Reduktion auf Umgebungsdruck) ein Verhältnis von lediglich einem Helium-Gasmolekül auf etwa 400 Wassermoleküle bei einem Druck von p 1bar (molare Gasbesetzung $\mathrm{n} \sim 10^{-3}$ ). Dyadin et al. (1994) gaben an, dass bei einer Temperatur von $\mathrm{T} \sim-25^{\circ} \mathrm{C}$ und einem Druck von $\mathrm{p} \sim 2.5 \mathrm{kbar}$ bei einem Volumen von $\mathrm{V} \sim 1 \mathrm{ml}$ Eis eine Gasvolumenadsorption von $\mathrm{V} \sim 12 \mathrm{ml}$ Heliumgas bei Standardbedingungen festgestellt wurde (molare Gasbesetzung $\mathrm{n} \sim 10^{-2}$ ), d.h. eine mit dem Druck korrelierte Besetzung der Eisstruktur $\mathrm{zu}$ erwarten ist.

Durch die mit fortschreitendem Druck stattfindende Kompression der Eisstruktur wird der freie Raum in den Käfigen für die Gasatome stetig verkleinert, so dass die Gasbesetzung gegen einen maximalen Wert konvergiert und dies so zu einer Stagnation der Gasbesetzung zum steigenden Druck bei gegebener Umgebungstemperatur führt. Zudem ist vorstellbar, dass bei einer umgebungsbedingten maximalen Gasbesetzung bei weiter ansteigendem Druck / sinkender Temperatur und damit Kompression / Kontraktion der Eisstruktur ein Verringerung der Gasbesetzung durch die Verringerung des freien Volumens der Eisstruktur denkbar ist. Dies würde zumindest die mit steigendem Druck reziprok korrelierte Gasbesetzung sowohl bei der Verwendung von Neongas als auch von Heliumgas erklären können. Ebenso wie bei der als Funktion des Druckes bestimmten Neongasbesetzung ist daher für die Heliumbesetzung davon auszugehen, dass die entgegen der erster Erwartung gefundene Druckabhängigkeit eher ein Ergebnis der recht hohen Fehlertoleranzen der Verfeinerungen 
sind. Aufgrund des höheren van der Waals'schen Radius des Neon ist aber davon auszugehen, dass das Neon bei Kompression eher aus der Eis-Struktur gedrängt werden kann.

\begin{tabular}{|c|c|c|c|c|c|}
\hline T[K] & \multicolumn{2}{|c|}{265} & 255 & \multicolumn{2}{c|}{237} \\
\hline $\mathbf{p}[\mathbf{b a r}]$ & 500 & 1000 & 2000 & 1 & 2000 \\
\hline $\mathbf{O z}$ & $0.05968(301)$ & $0.05946(275)$ & $0.05912(164)$ & $0.05869(225)$ & $0.06096(132)$ \\
\hline $\mathbf{D}_{\mathbf{2}} \mathbf{z}$ & $0.19579(375)$ & $0.19416(338)$ & $0.19757(393)$ & $0.19405(309)$ & $0.19683(201)$ \\
\hline $\mathbf{D}_{\mathbf{3}} \mathbf{x}$ & $0.45643(315)$ & $0.45624(334)$ & $0.45574(304)$ & $0.45454(210)$ & $0.45403(138)$ \\
\hline $\mathbf{D}_{\mathbf{3}} \mathbf{z}$ & $0.01297(333)$ & $0.015269(300)$ & $0.01302(364)$ & $0.01422(274)$ & $0.01297(164)$ \\
\hline Uiso O & $0.05527(459)$ & $0.05399(429)$ & $0.05184(412)$ & $0.04904(366)$ & $0.04379(195)$ \\
\hline Uiso D1 & $0.06671(990)$ & $0.06255(948)$ & $0.06985(970)$ & $0.05928(804)$ & $0.05935(531)$ \\
\hline Uiso D2 & $0.08097(850)$ & $0.07656(777)$ & $0.06863(741)$ & $0.06227(582)$ & $0.06286(393)$ \\
\hline po-ratio & $0.96775(180)$ & $0.97894(718)$ & $0.99989(713)$ & $0.98946(632)$ & $0.99141(404)$ \\
\hline \% & 24.1 & 33.1 & 20.3 & 21.0 & 21.2 \\
\hline
\end{tabular}

Tabelle 5.22: Eis Ih-Struktur mit Heliumgas frei und mit isotropen thermischen Auslenkungsparametern verfeinert, \%: verbliebener Phasenanteil an Argon-Clathrat-Hydrat nach dem Gaswechsel von Argongas zu Heliumgas. Helium-Gasinhalt gemäß der Tabelle 5.21.

\begin{tabular}{|c|c|c|c|c|c|}
\hline T[K] & \multicolumn{2}{|c|}{265} & 255 & \multicolumn{2}{c|}{$\mathbf{2 3 7}$} \\
\hline $\mathbf{p}[\mathbf{b a r}]$ & 500 & 1000 & 2000 & 1 & 2000 \\
\hline $\mathbf{O z}$ & $0.05951(303)$ & $0.05934(273)$ & $0.05923(304)$ & $0.05878(226)$ & $0.06089(135)$ \\
\hline $\mathbf{D}_{\mathbf{2}} \mathbf{z}$ & $0.19556(375)$ & $0.19403(338)$ & $0.19752(399)$ & $0.19409(300)$ & $0.19682(204)$ \\
\hline $\mathbf{D}_{\mathbf{3}} \mathbf{x}$ & $0.45667(321)$ & $0.45643(288)$ & $0.45581(284)$ & $0.45461(210)$ & $0.45406(238)$ \\
\hline $\mathbf{D}_{\mathbf{3}} \mathbf{Z}$ & $0.01292(336)$ & $0.01526(303)$ & $0.01282(298)$ & $0.01407(240)$ & $0.01279(156)$ \\
\hline Uiso O & $0.05545(459)$ & $0.05418(432)$ & $0.05192(402)$ & $0.04900(366)$ & $0.04389(195)$ \\
\hline Uiso D1 & $0.0671(108)$ & $0.06286(954)$ & $0.06917(966)$ & $0.05860(732)$ & $0.05943(534)$ \\
\hline Uiso D2 & $0.08152(870)$ & $0.07702(783)$ & $0.06941(750)$ & $0.06288(588)$ & $0.06317(396)$ \\
\hline po-ratio & $0.97367(776)$ & $0.97988(721)$ & $0.99992(716)$ & $0.98943(635)$ & $0.99157(405)$ \\
\hline \% & 24.4 & 32.1 & 21.3 & 20.9 & 21.2 \\
\hline
\end{tabular}

Tabelle 5.23: Eis Ih-Struktur ohne Heliumgas frei und mit isotropen thermischen Auslenkungsparametern verfeinert, \% : verbliebener Phasenanteil an Argon-Clathrat-Hydrat nach dem Gaswechsel von Argongas zu Heliumgas

Signifikante Unterschiede in den Differenzkurven in den mit Helium und ohne Helium angepassten Diffraktogrammen sind wie bei den mit Neonatomen durchgeführten Verfeinerungen nicht festzustellen. Ebenso wie bei den mit Neongas gemessenen Datensätze sind sowohl der Gasinhalt als auch die Gaslagen für die mit Heliumgas gemessenen Datensätze in der Eistruktur durch die Rietveld-Verfeinerungen nicht aus den gemessenen Diffraktrogrammen zu verifizieren (vergl. Abbildung 5.16.a sowie Abbildung 5.16.b). In den vorherigen Tabellen, Tabelle 5.22 sowie Tabelle 5.23, sind die aus den Rietveld- 
Verfeinerungen bestimmten Atompositionen der Eis Ih-Struktur für alle mit Heliumgas als Drucküberträgermedium gemessenen Datensätze dargestellt. Im Rahmen des aus den Rietveld-Verfeinerungen angenommenen dreifachen Standardfehlers sind die Atompositionen, der Phasenanteile sowie die Vorzugsorientierungen („po“: prefered orientation) für die Verfeinerung mit Helium- / ohne Heliumgas im Rahmen ihres Fehlerbereiches nicht zu unterscheiden. Eine signifikante und systematische Änderung in den Atompositionen als Funktion des Druckes und der Temperatur ist ebenso nicht festzustellen. Ein Einfluss des Gases auf die Eisstruktur ist damit wie bei den verfeinerten Datensätzen unter der Verwendung von Neongas nicht $\mathrm{zu}$ beobachten. Festzustellen ist, dass die verfeinerten Parameter (Atompositionen) der in Gegenwart von Heliumgas gemessenen Datensätze zumeist einen erheblich höheren Fehler aufweisen. Ursache hiefür ist der bei allen gemessenen mit Heliumgas gemessenen Datensätzen verbliebene Argon-Clathrat-HydratPhasenanteil von $\mathrm{n} \sim 20.3 \%$ bis $\mathrm{n} \sim 33.1 \%$. Das synthetisierte Argon-Clathrat-Hydrat ist ein Überbleibsel der vorherigen Messung, bei dem die Eisstruktur mit Argongas als Drucküberträger als Funktion von Druck und Temperatur gemessen wurde.

Aufgrund der aus den experimentellen Daten und mittels Rietveld-Verfahren bestimmten Strukturinformationen kann lediglich die Aussage getroffen werden, dass Neon und damit vermutlich auch Helium in der Eisstruktur mittels diffraktometrischer Methoden in diesem Druck und Temperaturbereich nicht gut erfasst werden können.

\subsubsection{Struktur von Eis Ih und Eis Ih-Hydrat. Experimentelle Ergebnisse im Vergleich.}

Aus dem Vergleich sowohl der in den Abbildung 5.10 sowie Abbildung 5.11, Kapitel 5.5.1 dargestellten Gitterkonstantenverläufe der Gitterkonstanten a und c als auch dem daraus berechnetem Gitterkonstantenverhältnis c/a (vergl. Abbildung 5.13, Kapitel 5.5.1) lässt sich im Gegensatz zu den verfeinerten Atompositionen der gemessenen Datensätze ein Einfluss auf die Eisstruktur durch die Verwendung der Gase Helium und Neon als Funktion des Druckes und der Temperatur eindeutig belegen. Im Gegensatz zum Gas Argon, bei dem sich das Eis Ih über den gesamten gemessenen Druck- und Temperaturbereich isokompressibel gezeigt hat, ist das c/a Verhältnis bei den Gasen Helium und Neon deutlich verschieden. Für Neon- sowie sehr viel stärker ausgeprägt für Heliumgas, ist eine signifikante Abweichung von dem isokompressiblem Gitterkonstantenverhältnis festzustellen. Signifikante Unterschiede in 
den Parametern der Eisstruktur als Funktion des Druckes und der Temperatur sind im Rahmen der hohen Fehler jedoch nicht zu verifizieren. Ursache hierfür sind die im Strukturmodell nicht berücksichtigten Stapelfehler, die zu systematischen Verfälschungen der verfeinerbaren Parameter für alle nicht oberhalb der Temperatur von $\mathrm{T} \sim 240 \mathrm{~K}$ ausgeheilten Proben führt. Ebenso haben die wenig aussagefähigen Verfeinerungsergebnisse ihre Ursache in der kristallographisch nur ungenügend beschriebenen Clathrat-Hydrat-Struktur vom Stackelberg Typ II, dem Argon-Clathrat- sowie dem neu entdeckten Neon-Clathrat-Hydrat (vergl. Kapitel 5.2.1). Im Falle der mit Heliumgas durchgeführten in situ Experimente verblieb Argon-Clathrat-Hydrat als Überbleibsel der mit Argongas durchgeführten Messungen. Auch in den mit Neongas durchgeführten in situ Neutronenstreuexperimenten zeigte sich durch die nur ungenaue Modellierung des Neon-Clathrat-Hydrates ein Einfluss auf die Verfeinerungsergebnisse. Im Falle der mit Argon- und Heliumgas durchgeführten Verfeinerungen ist der Einfluß des Argon-Clathrat-Phasenanteiles nicht zu vernachlässigen. Anzumerken ist zudem, dass die Messzeiten für einen einzelnen Datensatz aufgrund der begrenzten in situ Meßzeit für die meisten Datensätze eingeschränkt werden mußte und nur vereinzelt längere Meßzeiten $\mathrm{t} \geq 5$ Stunden realisiert werden konnten. Diese längeren Messungen wurden im Falle der Verwendung von Neongas bei tiefen Temperaturen durchgeführt, um den Einfluss thermischer Effekte, d.h. den Temperaturschwingungen der Atome zu begrenzen. Aufgrund der nicht modellierten Stapelfehlordnungen für die unter Verwendung von Neongas durchgeführten Messungen sind diese Datensätze nur eingeschränkt zu verwenden. Geht man von den Datensätzen aus, bei denen die eben erwähnten möglichen Fehlordnungseffekte entweder durch die Art Probenherstellung oder durch die Gas-Clathrat-Hydratsynthese eine nur untergeordnete Rolle spielen, so sind die Datensätze unter der Verwendung von Argongas bei der Temperatur von T $140 \mathrm{~K}$, unter Verwendung von Neon bei einer Temperatur von $\mathrm{T} \sim 250 \mathrm{~K}$ sowie mit Heliumgas als Drucküberträgermedium bei einer Temperatur von $\mathrm{T} \sim 237 \mathrm{~K}$ am vertrauenswürdigsten.

Ein direkter Vergleich dieser Datensätze untereinander sowie mit den Literaturangaben von Kuhs \& Lehmann (1986), with revisions Kuhs (1996) ist in der folgenden Tabelle 5.24 gegeben. Der Vergleich der in Tabelle 5.24 sowie Tabelle 5.25 bestimmten atomaren Positionen zeigt, dass im Rahmen der hohen Fehler keine signifikante Änderung als Funktion der Temperatur, des Druckes oder der Verwendung der im Eis löslichen Gase Helium und 
Neon festzustellen gegenüber der von Kuhs \& Lehmann (1986), with revisions Kuhs (1996) bestimmten Struktur von Eis Ih bei Umgebungsdruck von $\mathrm{p} \sim 1$ bar festzustellen ist.

\begin{tabular}{|c|c|c|c|c|}
\hline \multicolumn{5}{|c|}{ Argongas, $p \sim 2 k b a r$} \\
\hline $\mathbf{T}[\mathbf{K}]$ & $\overline{\mathbf{O z}}$ & $\overline{\mathbf{D}_{1} \mathbf{z}}$ & $\mathbf{D}_{2} \mathbf{x}$ & $\mathbf{D}_{2} \mathbf{z}$ \\
\hline 140 & $0.06223(744)$ & $0.19886(113)$ & $0.45450(834)$ & $0.01527(894)$ \\
\hline \multicolumn{5}{|c|}{ Neongas, $\mathbf{p} \sim 2 \mathrm{kbar}$ (gas ${ }^{1}$ und gasfrei $^{2}$ Struktur gemittelt) } \\
\hline 250 & $0.06280(125)^{1}$ & $0.20237(196)^{1}$ & $0.45540(117)^{1}$ & $0.01779(140)^{1}$ \\
\hline & $0.06354(120)^{2}$ & $0.20322(175)^{2}$ & $0.45539(117)^{2}$ & $0.01782(132)^{2}$ \\
\hline \multicolumn{5}{|c|}{ Heliumgas, p 2kbar (gas ${ }^{1}$ und gasfreie $^{2}$ Struktur gemittelt) } \\
\hline 237 & $0.05912(164)^{1}$ & $0.19757(393)^{1}$ & $0.45574(304)^{1}$ & $0.01302(364)^{1}$ \\
\hline & $0.05923(304)^{2}$ & $0.19732(399)^{2}$ & $0.45581(284)^{2}$ & $0.01282(298)^{2}$ \\
\hline \multicolumn{5}{|c|}{ Kuhs und Lehmann (1986), p 1bar, with revisions Kuhs (1996) } \\
\hline 15 & $0.06212(3)$ & $0.19898(7)$ & $0.454449(4)$ & $0.01695(5)$ \\
\hline 60 & $0.06210(4)$ & $0.19891(10)$ & $0.45439(6)$ & $0.01697(6)$ \\
\hline 123 & $0.06218(7)$ & $0.19874(14)$ & $0.45436(7)$ & $0.01698(9)$ \\
\hline 223 & $0.06211(23)$ & $0.19833(18)$ & $0.45395(8)$ & $0.01706(8)$ \\
\hline
\end{tabular}

Tabelle 5.24: Vergleich der Atompositionen der Eis Ih Elementarzelle unter Verwendung der Gase Argon, Neon und Helium. Referenzwerte von Kuhs \& Lehmann (1986), with revisions Kuhs (1996) wurden an Eis Ih-Einkristallen bestimmt.

\begin{tabular}{|c|c|c|}
\hline \multicolumn{3}{|c|}{ Argon, 2kbar } \\
\hline $\mathbf{T}[\mathbf{K}]$ & O1-O2 [̊̊] & O2-O3[Å] \\
\hline 140 & $2.7400(15)$ & $2.7250(50)$ \\
\hline \multicolumn{3}{|c|}{ Neon, 2kbar (gas und gasfrei Struktur gemittelt) } \\
\hline \multirow[t]{2}{*}{250} & $2.7310(150)^{1}$ & $2.7505(48)^{1}$ \\
\hline & $2.7325(148)^{2}$ & $2.7508(48)^{2}$ \\
\hline \multicolumn{3}{|c|}{ Helium, 2kbar (gas ${ }^{1}$ und gasfreie ${ }^{2}$ Struktur gemittelt) } \\
\hline \multirow[t]{2}{*}{237} & $2.7471(63)^{1}$ & $2.7590(180)^{1}$ \\
\hline & $2.7461(63)^{2}$ & $2.7600(21)^{2}$ \\
\hline \multicolumn{3}{|c|}{ Kuhs und Lehmann (1986), Revision (Kuhs 1996),1bar } \\
\hline 15 & $2.7539(4)$ & $2.7530(1)$ \\
\hline 60 & $2.7543(7)$ & $2.7532(2)$ \\
\hline 123 & $2.7531(10)$ & $2.7533(3)$ \\
\hline 223 & $2.7593(12)$ & $2.7605(4)$ \\
\hline
\end{tabular}

Tabelle 5.25: Bindungsabstände von Eis Ih im Vergleich zu den Referenzwerten von Kuhs \& Lehmann (1986), with revisions Kuhs (1996).

Es zeigt sich erwartungsgemäß, dass die Eisstruktur unter den Gasdrücken eine Kompression erleidet. Für Argon ist durch das Verhältnis der Bindungsabstände O1-O2 sowie O2-O3 wiederum eine Bestätigung der Isokompressibilität bei Umgebungsdruck als auch hohen Drücken gegeben. Für die Gase Neon und Helium ist durch die höheren Fehler keine Aussage 
zu treffen. Für eine Betrachtung der mittleren O...O-Abstände genügen die Gitterkonstanten und deren Änderungen. Für die mit Helium gemessenen Datensätze bei einer Temperatur von $\mathrm{T} \sim 237 \mathrm{~K}$ und einem Druck von $\mathrm{p} \sim 2 \mathrm{kbar}$ sowie unter der Verwendung von Neon bei einer Temperatur von $\mathrm{T} \sim 250 \mathrm{~K}$ und einem Druck $\mathrm{p} \sim 2 \mathrm{kbar}$ ist jeweils in der Gitterkonstanten a eine Aufweitung sowie in c eine annähernde Konstanz der Gitterkonstanten zu finden (vergl. Tabelle 5.4). Der Bindungsabstand der Sauerstoffatome in c, die Bindung (O1-O2) ist kleiner als der O2-O3-Bindungabstand in a-Richtung des Kristallgitters. Dies gilt gleichermaßen für die mit Helium- als auch Neongas gemessenen Datensätze, bei denen eine Aufweitung der Gitterkonstanten in a und eine geringe Kotraktion (für Helium) sowie eine Konstanz in c (für Neon) bei gleichen Druck- und Temperaturbedingungen gegeben ist. Diese Verhalten der Aufweitung in der Gitterkonstanten a sowie einer Konstanz bzw. Kontraktion der Gitterkonstanten in c entspricht dem Verhalten von Eis II-Hydrat unter Verwendung von Heliumgas (Lobban (1998)) oder von Neon (vergl. Kapitel 5.2.3).

Aufgrund der aus den experimentellen Daten und mittels Rietveld-Verfahren bestimmten Strukturinformationen kann lediglich die Aussage getroffen werden, dass durch die Besetzung der Gase Neon und Helium die Eisstruktur strukturellen Änderungen erfährt. Ebenso kann die Aussage getroffen werden, dass die verwendeten Gase Neon und damit vermutlich auch Helium mittels diffraktometrischer Methoden in diesem Druck und Temperaturbereich nicht erfasst werden können.

\subsection{Sorptionssimulationen von Helium- und Neongas in Eis Ih-Hydrat}

Aufgrund der Schwierigkeiten mittels Rietveld-Verfeinerungen aus den experimentellen Daten die absolute Gasbesetzung zu bestimmen, wurde versucht einen Überblick über den druck- und temperaturabhängigen Verlauf sowie die Gaslagen der Gasatome mittels molekularer Computersimulation (Monte-Carlo-Methoden) $\mathrm{zu}$ erhalten. Für die Sorptionsrechnungen wurden daher die ohne Gas verfeinerten Eisstrukturen der Datensätze bei den Temperaturen von $\mathrm{T} \sim 140 \mathrm{~K}, \mathrm{~T} \sim 215 \mathrm{~K}$ und $\mathrm{T} \sim 250 \mathrm{~K}$ für die mit Neon gemessenen Datensätze sowie die Datensätze unter Verwendung von Helium bei den Temperaturen von $\mathrm{T} \sim 237 \mathrm{~K}, \mathrm{~T} \sim 255 \mathrm{~K}$ und $\mathrm{T} \sim 265 \mathrm{~K}$ als Grundlage der Sorptionsrechnungen verwendet. 
Mit dem Programm Cerius der Fa. Molecular Simulations wurden aus den Rietveldverfeinerten kristallographischen Modellen Eismodelle mit $\mathrm{n}=480$ Atomen bzw. $\mathrm{n}=96$ Wassermolekülen erstellt. Die Wassermoleküle waren in einer würfelförmigen Anordnung mit Kantenlängen von $\mathrm{a} \sim 15 \AA$ in $\mathrm{x}, \mathrm{b} \sim 13 \AA$ in y sowie $\mathrm{c} \sim 14 \AA$ in $\mathrm{z}$ angeordnet. Diese wurde entsprechend den „Eisregeln“ mit einem Programm „order2“ von Lobban (1997) erstellt. Die möglichen Positionen entsprechend der tetraedrischen Konfigurationen der Protonen um die Sauerstoffatome wurden durch Permutation aller Deuteriumpositionen gesucht und durch die Minimierung des resultierenden Diplmomentes der Eisstruktur wurde eine geeignete Konfiguration ausgewählt. Die maximal zugelassene Abweichung des Dipolmomentes für eine geeignete protonenungeordnete Struktur mit der angegebenen Anzahl von $\mathrm{n}=96$ Wassermolekülen wurde $\mathrm{zu} \mathrm{m}= \pm 0.03$ Debye festgelegt. Als weitere Bedingung wurden periodische Randbedingungen der entstehenden Anordnung eingefügt. Die Beschreibung der Wechselwirkungspotenziale für die Sorptionsrechnungen erfolgte über 12-6 Lennard-JonesPotenziale für die verwendeten Atome.

Für die Helium- und Neonatome, die in diesen Simulationsrechnungen als Sorbat dienten, wurden die Cerius-Standard-Potentiale $(\mathrm{He} 4+4$ sowie Ne4+4, definiert durch das UniversalForce-Field (Rappé et al. (1992), festgelegt. Die Sorptionsrechungen wurden mit bekannten Wasser-Potenzialmodellen durchgeführt, bei denen zum Teil die Potentialparameter aufgrund früherer Erfahrungen geändert wurden. Zum Einsatz kamen das SPC-Potenzialmodell von Berendsen (1981), das SC-Potentialmodell von Stillinger\&Rahman (1974), sowie Abwandlungen derselben. In dem SPC-Wassermodell wurden in den Sorptionsrechnungen lediglich die Wechselwirkungen der O-O-Atome in der Struktur betrachtet, während in dem SC-Wassermodell die O-O- sowie die O-D- als auch D-D-Wechselwirkungen mit einbezogen wurden. Die Gassorption sowie die im weiteren nicht diskutierten Sorptionsenergien wurden durch Berücksichtigung aller durch die Potenziale gegebenen Wechselwirkungen in einer bis 8Å-umfassenden Sphäre innerhalb des Sorptionsmodells errechnet.

Eine Konvergenz der mittleren Wechselwirkungsenergien der MC-Simulation wurde durchgehend nach $\sim 1.5$ bis 2 Millionen Simulationsschritten erreicht. In der folgenden Tabelle 5.26 sind die verwendeten Potenziale sowie deren Potenzialparameter aufgelistet. 


\begin{tabular}{|c|c|c|c|c|}
\hline & Do [kcal/mol] & Ro $[\AA]$ & Do $[\mathbf{k c a l} / \mathbf{m o l}]$ & Ro $[\AA]$ \\
\hline & \multicolumn{2}{|c|}{ L-J-Potenzial O } & \multicolumn{2}{|c|}{ L-J-Potenzial D } \\
\hline SPC $^{*}$ & 0.1553 & 3.5537 & $X$ & $X$ \\
\hline SPC-1 $^{+}$ & 0.0560 & 2.3620 & $\mathrm{X}$ & $\mathrm{X}$ \\
\hline SPC-2 $^{\#}$ & 0.1953 & 3.1537 & $\mathrm{X}$ & $\mathrm{X}$ \\
\hline SC $^{*}$ & 0.1553 & 3.5537 & 0.0560 & 2.6320 \\
\hline SC-1 $^{+}$ & 0.0560 & 2.3260 & 0.0560 & 2.6320 \\
\hline SC-2 $^{\#}$ & 0.1953 & 3.1537 & 0.0560 & 2.6320 \\
\hline
\end{tabular}

Tabelle 5.26: L-J-Potenzialparameter für $\mathrm{H}_{2} \mathrm{O}$-Eis Ih-Struktur, -X: O-D- sowie D-D-Wechselwirkungen wurden in den Sorptionsrechnungen nicht berücksichtigt. *: Potentialparameter gemäß Literaturangaben, +: Potentialparameter gemäß Universal-Force-Field, Cerius Standard-Potenzial Defintion, \#: Potentialparameter gemäß Klapproth (pers. Mitteilung, 2000). Die Potenzialbezeichnungen sind durch die chronologische Verwendung entstanden und nicht von weiterer systematischer Bedeutung.

Die Sorptionsrechnungen wurden standardisiert mit dem SC-Potential für Helium- sowie Neongas für die gemäß Tabelle 5.10, Kapitel 5.3.2, experimentell verifizierten Datensätze durchgeführt. Zudem wurden mit ausgesuchten Datensätzen sowohl die Variation der Gasbesetzungen als auch der Gasbesetzungsverläufe als Funktion des Druckes mit unterschiedlichen Potenzialen simuliert, um so Aufschluß über den Einfluss der verwendeten unterschiedlichen Potenzialparameter erhalten zu können.

\subsubsection{Ergebnisse der Sorptionssimulationen}

Sowohl für Helium- als auch Neongas ergaben sich mit dem verwendeten SC-1 Potenzialmodell eine als Funktion des Druckes und der Temperatur gegebene Abhängigkeit der Gasbesetzungen. Die Abhängigkeit ist im allgemeinen eine zum Druck nicht linear proportionale Funktion, die gegen eine maximale Gasbesetzung bei definierten Umgebungsbedingungen konvergiert. Die aus den Simulationsergebnissen ermittelten Gasbesetzungsverläufe entsprechen eher der intuitiven Anschauung als den aus den Beugungsdaten ermittelten Gasbesetzungen.

Die aus den Sorptionsrechnungen bestimmten absoluten Gasbesetzungen für das Neongas zeigen bei verschiedenen Temperaturen nahezu parallele Verläufe mit einem steilen Anstieg bis zu einem Druck von p 500bar und einer Stagnation der Besetzung bis zum maximalen Druck von $\mathrm{p} \sim 2.5 \mathrm{kbar}$ für Neongas, $\mathrm{p} \sim 2 \mathrm{kbar}$ für Heliumgas. Die maximalen Gasbesetzungen sind jedoch durchaus unterschiedlich. Die höchste Gasbesetzung ergibt sich für Neongas bei einer Temperatur von $\mathrm{T} \sim 140 \mathrm{~K}$. Bei den Temperaturen von $\mathrm{T} \sim 215 \mathrm{~K}$ sowie $\mathrm{T} \sim 250 \mathrm{~K}$ liegt die 
Gasbesetzung deutlich unterhalb dieser Maximalbesetzung. Die mit steigendem Druck ansteigende Gasbesetzung ist der Temperatur reziprok proportional (vergl. Abbildung 5.17). In Gegenwart von Neongas entspricht dieses Temperaturverhalten der Gaslöslichkeit von atmosphärischen Gasen in flüssigem Wasser und steht der durch die Adsorption von Neongas verursachten Abweichungen der c/a-Gitterkonstantenverhältnisse von Eis Ih entgegen (vergl. Kapitel 5.5.1, Abbildung 5.13). In Gegenwart von Heliumgas ist dieses Verhalten nicht aus den Abbildungen 5.17 sowie der Abbildung 5.12, Kapitel 5.5.1 sowie letztendlich aufgrund der wenigen mit Heliumgas bestimmten Datensätze zu erkennen. Vielmehr ist die konstante Kompressibilität in Gegenwart von Heliumgas durch eine größenordnungsmäßig und nahezu vergleichbare Gasbesetzung der Eisstruktur als Funktion der Temperatur unter den angegebenen Druck- und Temperaturbedingungen gegeben.

Die Gasbesetzung für Heliumgas liegt bei annähernd vergleichbaren Druck- und Temperaturbedingungen von $\mathrm{T} \sim 250 \mathrm{~K}$ und $\mathrm{T} \sim 255 \mathrm{~K}$ und einem Druck von $\mathrm{p} \sim 2 \mathrm{kbar}$ um den Faktor 65 höher. Als Ursache für diese drastisch erhöhte Gasbesetzung dürfte hier sicherlich das Größenverhältnis des wechselwirkenden Gaspartner Helium und den offenen Kanälen der Eisstruktur zu nennen sein, so dass bei äquivalenten Umgebungsbedingungen diese mehr mit dem kleineren Heliumgas besetzt werden können. Ebenso entspricht die maximale Gasbesetzung als Funktion der Temperatur nicht der experimentell bestimmten Abweichung des c/a-Gitterkonstantenverhältnisses, von der angenommen wird, dass sie mit der Gasbesetzung korreliert.

Die Simulationsergebnisse deuten an, dass bei steigendem Druck die Besetzung des Eisgitters, zumindest für Neongas gegen einen vom gewählten Potenzial abhängigen, Besetzungsgrad konvergiert, jedoch den experimentell bestimmten Abweichungen des c/aGitterkonstantenverhältnis von der gasfreien Eisphase als Funktion der Temperatur nicht entsprechend ist. Bei einer Temperatur von T 140K ist z.B. nach einem steilen Anstieg im unteren Druckbereich für Neon die Stagnation der Gasbesetzung bei einem Druck von p 2kbar schon annähernd erreicht. Eine weitere Druckerhöhung dürfte nach Maßgabe der Sorptionssimulationen zu keiner weiteren Erhöhung der Gasbesetzung führen, da sich bei steigendem Druck die Kompression des Eisgitters erhöht und das Gasvolumen in der Struktur begrenzt. Eine stetig ansteigende Besetzung ist daher aufgrund der van der Waals'schen Radien der Eisstruktur sowie der wechselwirkenden Gaspartner nicht möglich. Nach 
Abbildung 5.17 scheint die aus den Simulationsergebnissen verifizierte Gasbesetzung für Heliumgas in dem äquivalenten Druckintervall steiler anzusteigen, was auf eine höhere Besetzung der Struktur mit Helium im Vergleich zum Neon bei vergleichbaren Umgebungsbedingungen schließen lässt.

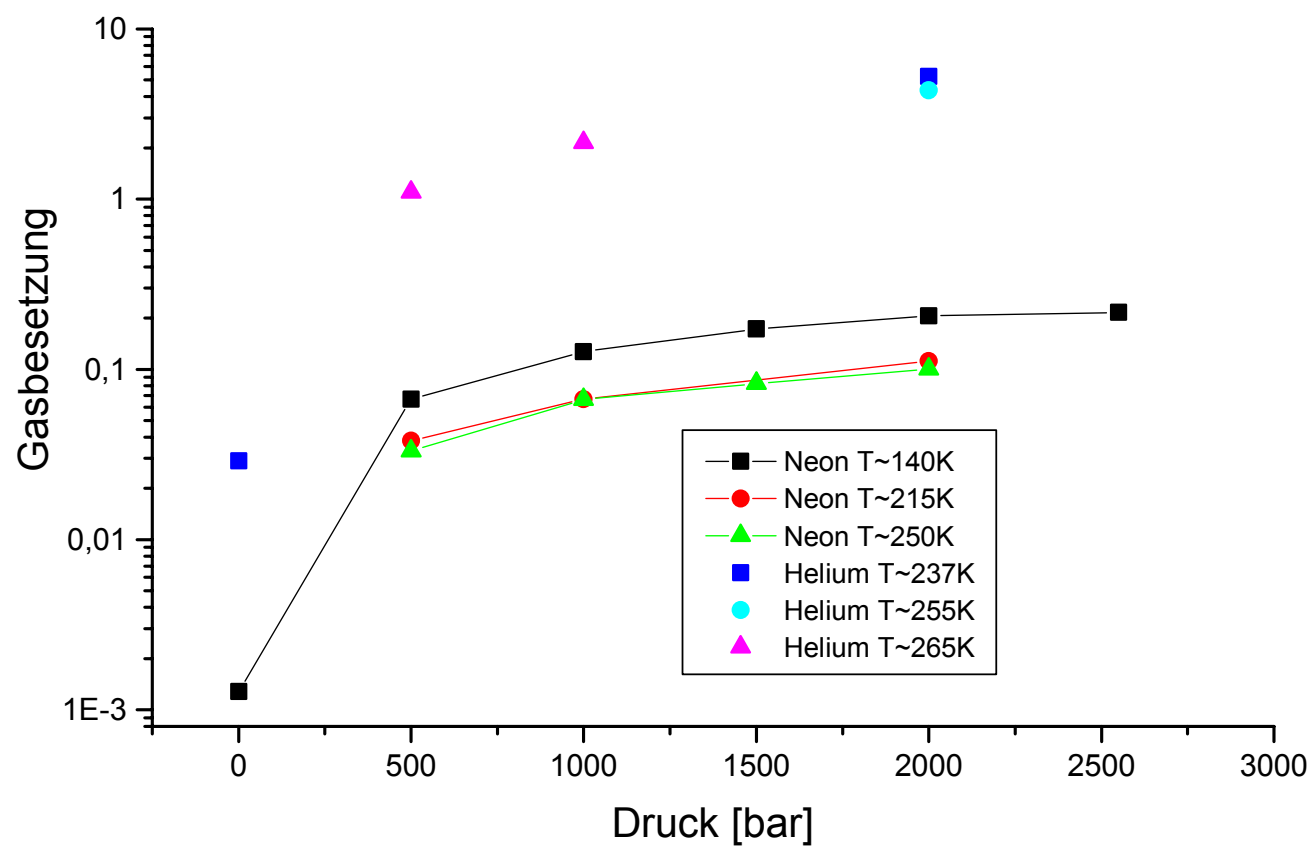

\section{Abbildung 5.17: Gasbesetzung der ,rigid body“ Eis Ih-Struktur als Funktion des Druckes für die Gase Helium und Neon bei der Verwendung des SC1-Potenzialmodells}

$\mathrm{Ab}$ einem Druck von $\mathrm{p} \sim 2 \mathrm{kbar}$ ist jedoch ebenso so schnell eine Konvergenz des Besetzungsgrades erreicht. Um den Einfluss der Potenzialparameter auf die Gasbesetzungen als Funktion des Druckes und der Temperatur zu bestimmen, wurde die Gasbesetzung für Neongas bei einer Temperatur von $\mathrm{T} \sim 250 \mathrm{~K}$ und einem Druck von $\mathrm{p} \sim 2 \mathrm{kbar}$ mit dem SPCPotenzial und dessen Parametervariation gemäß der Tabelle 5.26 simuliert. Die folgende Abbildung 5.18 zeigt einen Vergleich der normalisierten Gasbesetzungsverläufe in Abhängigkeit des verwendeten Potenzials gemäß der Tabelle 5.26.

Die simulierten Neon-Gasbesetzungsverläufe für die unterschiedlichen Potenziale zeigen einen annähernd vergleichbaren, zum Druck proportionalen Verlauf. Unterschiede ergeben sich jedoch hinsichtlich der absoluten Gasbesetzung (vergl. Abbildung 5.18). Gerade im Druckbereich von p 500bar bis p 1kbar zeigen sich für die unterschiedlichen Potenzialparameter des SPC-Potenzialmodells sowie des SC-Potenzialmodells signifikante 
Abweichungen. Die größte Abweichung ist für das SPC-2 Potenzial zu verzeichnen (Klapproth, pers. Mitteilung, 2000), das ausgehend von einem SPC-Wassermodell an die experimentellen Gasbesetzungen eines Stickstoff-Clathrat-Hydrat-Systems durch sukzessive Verfeinerung der Potenzialparameter angepasst wurde. Die Tabelle 5.27 gibt einen Überblick über die Abweichungen der normierten Gasbesetzungen in dem simulierten Druckbereich.

\begin{tabular}{|c|c|c|c|c|}
\hline \%Abweich. & p [bar] & SPC-1 & SPC-2 & SC \\
\hline \multirow{3}{*}{ SPC } & 500 & $-35,7$ & $+407,8$ & $-1,08$ \\
\cline { 2 - 5 } & 1000 & $-13,1$ & $+425,5$ & $-2,47$ \\
\cline { 2 - 5 } & 1500 & $-5,29$ & $+453,3$ & $-3,77$ \\
\hline
\end{tabular}

Tabelle 5.27: Abweichungen der normierten Gasbesetzungen vom SPC-Potenzialmodell als Funktion des Druckes bei einer Temperatur von $\mathbf{T} \sim 250 \mathrm{~K}$ mit Neongas.

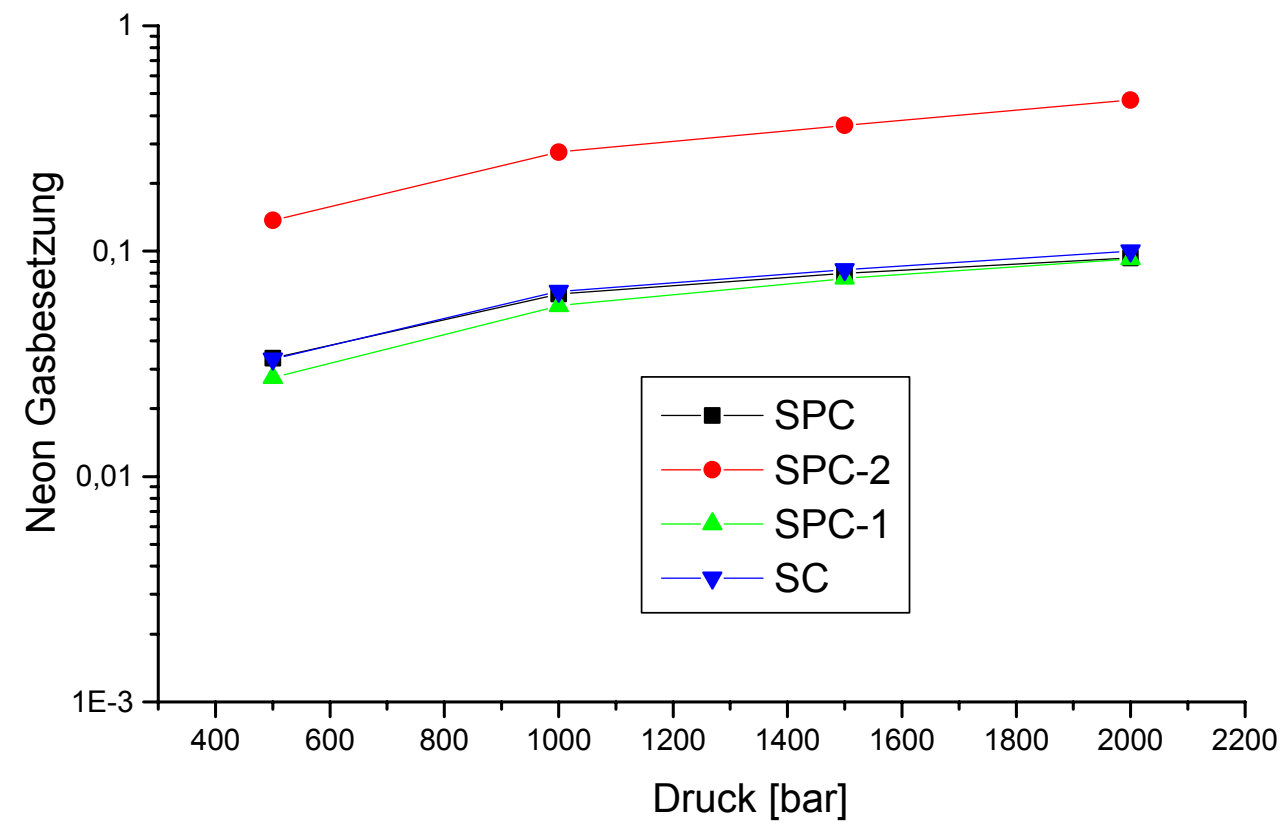

Abbildung 5.18: simulierte Gasbesetzungsverläufe für Neongas als Funktion des Druckes bei einer Temperatur von $\mathbf{T} \sim 250 \mathrm{~K}$ unter Verwendung der gemäß Tabelle 5.26 definierten Lennard-JonesPotenziale.

Je nach Wahl des Potenzials- und dessen Potenzialparameter fallen die Abweichungen der Gasbesetzungen als Funktion des Druckes unterschiedlich aus. Während die Abweichungen des SPC-Potenzials zum SC-Potenzial über den Druckbereich von p 500bar bis p 1500bar nur gering ausfallen, sind die Abweichungen in den Gasbesetzungen zum SPC-1 sowie zum SPC-2 deutlich größer. Zum Beispiel ist bei einer Temperatur von T 140K die Besetzung von Neongas in der Eisstruktur für das Potential SPC-2 von Klapproth (pers. Mitteilung, 2000) um 
den Faktor 8 höher. Die Gasbesetzungen können im einzelnen nicht diskutiert werden, da eine Aussage über die Gasbesetzungsverläufe als Funktion des Druckes im wesentlichen von dem verwendeten Potenzial und damit von der Art und Anzahl der zugelassenen Wechselwirkungen abhängt. Die mittels Sorptionssimulationen errechneten Verläufe der Gasbesetzungen können somit als Verifizierungsmöglichkeit der experimentellen, durch Neutronendiffraktion bestimmten Gasbesetzung, nicht herangezogen werden. Die folgende Abbildungen 5.19 zeigt im Vergleich die in den Sorptionsrechnungen bestimmte Anzahl der Neon- und Heliumgasatome als Funktion des Druckes.

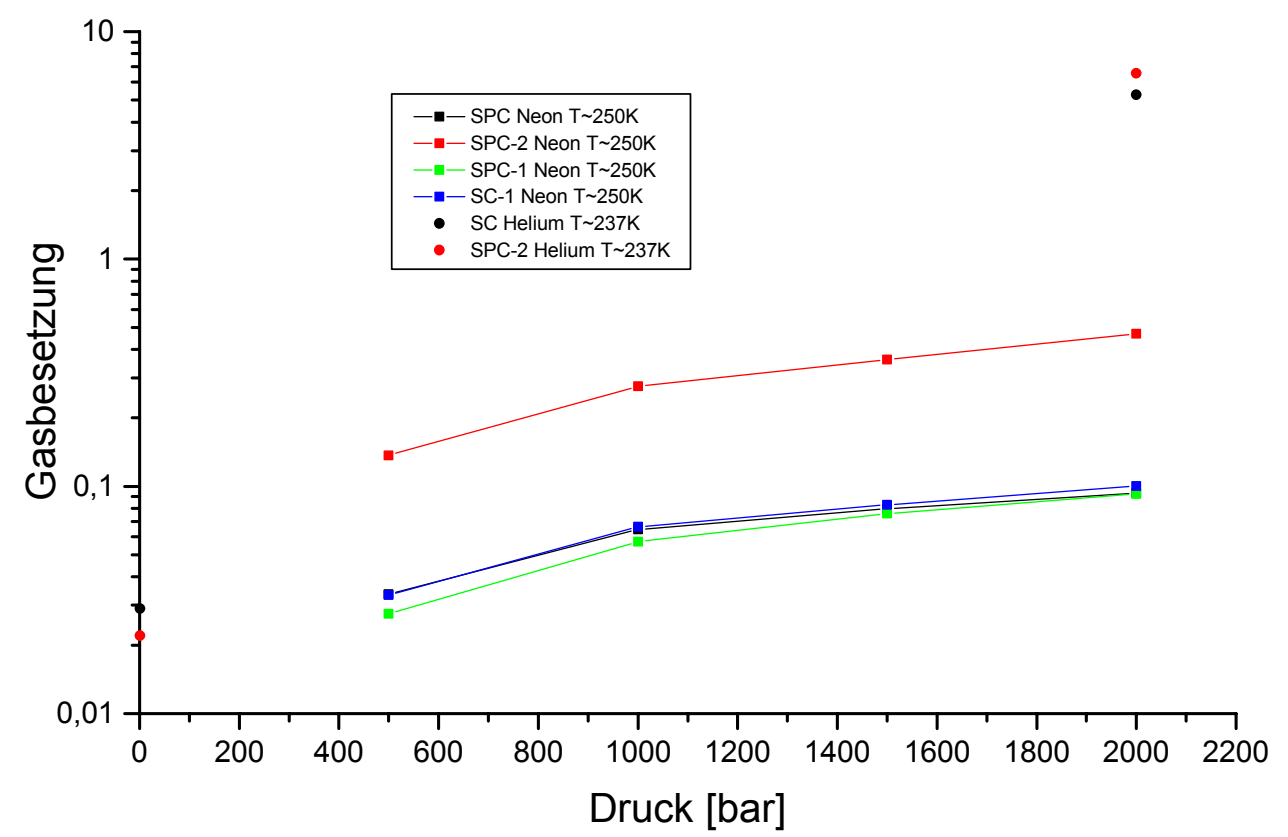

\begin{abstract}
Abbildung 5.19: Gasbesetzung der Eis Ih-Struktur mit Neongas bei einer Temperatur von T 250K sowie für Heliumgas bei einer Temperatur von $\mathbf{T} \sim 237 \mathrm{~K}$ als Funktion des Druckes unter Verwendung verschiedener Potenzialmodelle und Potenzialparameter.
\end{abstract}

Die größten Abweichungen in den Besetzungen der Sorptionsmodelle, sowohl für Neongas als auch für Heliumgas, ergeben sich im Vergleich für das von Klapproth (pers. Mitteilung, 2000) an Stickstoff-Clathraten-Hydraten optimierten SPC-2 Potenzial. Die Besetzung der Eis Ih-Struktur mit Neongas ist im Vergleich zu den anderen verwendeten Potenzialen um den Faktor 4.7 bei einer Temperatur von $\mathrm{T} \sim 250 \mathrm{~K}$ und einem Druck von $\mathrm{p} \sim 2 \mathrm{kbar}$ größer und liegt somit über der simulierten Gasbesetzung bei einer Temperatur von $\mathrm{T} \sim 215 \mathrm{~K}$ sowie $\mathrm{T} \sim 140 \mathrm{~K}$ bei der Verwendung des SC-1 Potenzials. Für Heliumgas ist die Besetzung bei einer Temperatur von $\mathrm{T} \sim 237 \mathrm{~K}$ und einem Druck von $\mathrm{p} \sim 2 \mathrm{kbar}$ um den Faktor 1.24 größer. Bei der Temperatur von $\mathrm{T} \sim 250 \mathrm{~K}$ ergab sich im Mittel bei Umgebungsdruck von $\mathrm{p} \sim 1$ bar eine 
Gasbesetzung von $\mathrm{n}=0.0127(30)$ Neongasatomen pro 96 Wassermoleküle. Geht man von einem linearen Verhältnisfaktor (basierend auf dem Größenunterschied der Gase) von 4.7 aus so würde die Besetzung für Helium bei einer Temperatur $\mathrm{T} \sim 250 \mathrm{~K}$ in etwa $\mathrm{n} \sim 0.5969$ Heliumatome pro 96 Wassermoleküle betragen. Bei einer Temperatur von T 140K ergab sich für die Neongasbesetzung durch die Polynomfits eine Besetzung von nahezu 0 Neonatomen. Auf die Berechnung der Gasbesetzungen über die Langmuir-Isothermen aus den Sorptionsenergien wurde aufgrund des Einflusses der gewählten Potenziale und der Potenzialparameter auf die Gasbesetzungen verzichtet. Aus den Sorptionsrechnungen würde sich für Neongas bei Umgebungsdruck von $\mathrm{p} \sim 1$ bar bei einer Temperatur von $\mathrm{T} \sim 140 \mathrm{~K}$ eine Konzentration von $\mathrm{c}_{\mathrm{Neon}}(\mathrm{T} \sim 140 \mathrm{~K}) \sim 0$, bei einer Temperatur von $\mathrm{T} \sim 250 \mathrm{~K}$ eine Konzentration von $\mathrm{c}_{\mathrm{Neon}}(\mathrm{T} \sim 250 \mathrm{~K}) \sim 1.23 * 10^{-4}$ sowie für Helium eine Gaskonzentration von $\mathrm{c}_{\text {Helium }}(\mathrm{T} \sim 250 \mathrm{~K}) \sim 6.2 * 10^{-3}$ ergeben. Dieser Wert liegt um den Faktor zehn niedriger als die von Dyadin et al. (1994) experimentell ermittelte molare Gasbesetzung von $\mathrm{c}_{\mathrm{Helium}} \sim 10^{-2}$ bei Umgebungsdruck von $\mathrm{p} \sim 1$ bar und einer Temperatur von T 248K (vergl. Kapitel 4.3.1). Gemäß der Abbildung 5.19 würde sich bei einem Druck von p 2kbar größenordnungsmäßig die folgenden Gasbesetzungen unter Verwendung der verschiedenen Potenzialparameter ergeben.

\begin{tabular}{|c|c|c|c|c|}
\hline & Heliumatome & Neonatome & $\mathbf{c}_{\text {Helium }}{ }^{*}$ & $\mathbf{c}_{\text {Neon }}{ }^{*}$ \\
\hline SC & 5.35 & 0.0980 & $5.5 * 10^{-2}$ & $1.02 * 10^{-3}$ \\
\hline SPC & - & 0.0913 & - & $0.95 * 10^{-3}$ \\
\hline SPC-2 & 6.65 & 0.469 & $6.93 * 10^{-2}$ & $4.88 * 10^{-3}$ \\
\hline SPC-1 & - & 0.0985 & - & $1.03 * 10^{-3}$ \\
\hline
\end{tabular}

Tabelle 5.28: durch Sorptionsberechnungen ermittelte Gasbesetzungen der Eis Ih-Struktur für Heliumsowie Neongas bei einem Druck von p 2kbar und bei einer Temperatur von T 237K für Helium sowie bei einer Temperatur von $T \sim 250 \mathrm{~K}$ für Neon, *: bezogen auf $\mathrm{n}=96$ Wassermoleküle in der Simulationskonfiguration.

Bei den in Tabelle 5.28 genannten Umgebungsbedingungen bei einem Druck von p 2kbar würde sich bei der Verwendung von Heliumgas eine um den Faktor 10 höhere Gasbesetzung der Eis Ih-Struktur ergeben als vergleichsweise bei der Verwendung von Neongas. Zu der von Dyadin et al. (1994) bei einem Umgebungsdruck von $\mathrm{p} \sim 1$ bar experimentell ermittelten Gasbesetzung liegt die bei einem Druck von $\mathrm{p} \sim 2 \mathrm{kbar}$ durch Sorptionssimulationen bestimmte Gasbesetzung für Heliumgas in derselben Größenordnung. 
Die Ergebnisse der Sorptionsrechnungen zeigen, dass die Gasbesetzungen für Helium und Neon aufgrund der unterschiedlichen van der Waals'schen Wechselwirkungsradien für das größere Neonatom im Vergleich zum Heliumatom erwartungsgemäß niedriger ausfallen. Die Abweichungen in den Gasbesetzungen für die verschiedenen verwendeten Potenziale weisen darauf hin, wie kritisch eine Beschreibung der Wechselwirkungen in Simulationen anhand experimentell bestimmter Besetzungszahlen sein könnte. Aufgrund der komplexen nichtadditiven Wechselwirkungen der Wassermoleküle ist die Bestimmung der Besetzungszahlen als Funktion des Druckes und der Temperatur über Simulationsrechnungen eine große Herausforderung. Geht man von den durch die Sorptionsrechnungen gefundenen mit dem Druck ansteigenden Gasbesetzungsverläufen aus, so ist die durch die Struktur-Verfeinerungen wenig druckabhängige Gasbesetzung der Eisstruktur für Helium- sowie für Neongas zumindest überraschend.

Die Frage nach der absoluten Gasbesetzung der Eisstruktur in Abhängigkeit von Gasart, Druck und Temperatur muss daher weiterhin offen bleiben. 


\section{Diskussion und Ausblick}

Im Rahmen dieser Arbeit konnte einigen wenigen Fragestellungen hinsichtlich der strukturellen Eigenschaft von Eis Ih als Funktion des Druckes und der Temperatur sowie der Wechselwirkungen mit atmosphärischen Gasen nachgegangen werden. Ebenso wurde als Grundlage für die Pulvermessungen der Frage der Herstellung von „gutem“, polykristallinem Eispulver nachgegangen. Bedingt durch die Erfahrungen bei den Versuchen zur reproduzierbaren Herstellung von verschiedenen Eisphasen und nicht zuletzt durch die Ergebnisse der Rietveld-Verfeinerungen an Eis Ih hat sich gezeigt, dass die Aussagefähigkeit der Experimente zum einen durch die Güte und die technischen Möglichkeiten der Apparaturen, zum anderen jedoch aber auch im wesentlichen von den Eigenschaften der Probe bestimmt ist. Gute Beispiele hierfür sind z.B. die Präparationen von Eis Ih-Pulver als Grundlage für die Clathrat-Hydrat-Synthese.

Für die Untersuchung der strukturellen Eigenschaften von Eis Ih als Funktion des Druckes und der Temperatur, zeigte sich die Art der Präparation für ein feinkörniges, nahezu regelloses Pulver ohne gewünschte nenneswerte Vorzugsorientierung zum einen als Vorteil an, zum anderen zeigten sich Relikte der Probenherstellung, die die Auswertungen der gemessenen Strukturen durch RietveldVerfeinerungen wesentlich erschwerten und zum Teil ohne der begründeten Annahme von weiteren, jedoch unbekannten Zwangsbedingungen unmöglich machten.

Im Falle des mit Argon-/Heliumgas sowie des mit Neongas durchgeführten Experimentes zeigten sich, bedingt durch die Probenherstellung, Imperfektionen (Strukturdefekte) im Eis Ih, die bis zu höheren Temperaturen als bisher experimentell beschrieben gefunden wurden. Diese Strukturdefekte, die als verbleibende Stapelfehlordnungen in der hexagonalen Stapelsequenz zu vermuten sind, beeinflussten im wesentlichen die Rietveld-Verfeinerungen der Eis IhElementarzellenparameter, im bedeutendem Maße für die mit Neongas gemessenen Datensätze. Für weitere Experimente ist daher bei gleicher Probenpräparationsroute nicht zwingend von einer vergleichbaren Probengüte auszugehen. Strukturelle Unterschiede am kubischen Eis Ic, hergestellt aus verschiedenen Hochdruckeisphasen, zeigen eindeutige Hinweise auf ein unterschiedliches topotaktisches Wachstum bei der Phasenumwandlung der Hochdruckeisphasen in die kubische Eisphase. Welchen Einfluss strukturelle Unterschiede des kubischen Eis Ic auf die Herstellung sowie die Struktur des Eis Ih haben ist aus den wenigen verfolgten Übergängen letztendlich nicht 
$\mathrm{zu}$ verifizieren.

Die Eis Ih Probengüte, der in den Experimenten verwendeten Eis Ih-Pulver sind im Nachhinein bezüglich der Pulverstatistik (Vorzugsorientierungen, Korngrössen u.s.w) als gut, jedoch hinsichtlich der Strukturbestimmung des Eis Ih durch die verbliebenen strukturellen Fehlordnungen bis zu einer Temperatur von $\mathrm{T} \sim 237 \mathrm{~K}$, als nicht befriedigend $\mathrm{zu}$ bezeichnen. Aufgrund der nicht eindeutig $\mathrm{zu}$ beschreibenden strukturellen Fehlordnungen konnten lediglich die vermessenen Datensätze zur Bestimmung von Gitterkonstanten im Rahmen der durch die RietveldVerfeinerungen bestimmten Fehlertoleranzen herangezogen werden.

Aufgrund der Laborexperimente und der in situ Neutronenstudien haben sich jedoch weitere Fragen, z.B. hinsichtlich von Texturverwandtschaften von Eisphasen, deren Einflüsse auf die Kinetik bei Phasenübergängen, der Keimbildung bei der Nukleation aus der flüssigen Phase oder bei einem fest/fest-Phasenübergang ergeben, die durch die im Rahmen dieser Arbeit durchgeführten Experimente und den experimentellen Möglichkeiten nicht zu beantworten waren.

Die Frage nach einem tieferen Verständnis der Einflüsse sowohl der Kinetik bei Phasenübergängen als auch der Keimbildung bei der Nukleation aus der flüssigen Phase bleibt weiterhin offen und betrifft insbesondere für das gefundene und qualitativ verifizierte Herstellungsverfahren von Eis XII.

Um für zukünftige Experimente bei denen polykristallines Eis Ih als Ausgangsphase dienen soll, reproduzierbar herzustellen, könnten folgende Verfahrensweisen herangezogen werden. Zum Einen müsste das Zermörsern von Eis Ih in einer Tiefsttemperatur-Kugelmühle, die während des Mörservorganges die Temperatur bei annähernd Flüssig-Stickstofftemperaturen hält, stattfinden, zum Anderen könnte das Eis Ih über die Präparationsroute von Bertie et al. (1963) hergestellt werden. Die Imperfektionen sollten dann bei Temperaturen, die oberhalb der bisher beobachteten Temperatur von $\mathrm{T} \sim 237 \mathrm{~K}$, jedoch bei Umgebungsdruck noch weit unterhalb des Schmelzpunktes ausgeheilt werden, um eine Rekristallisation zu minimieren. Diesbezüglich müsste jedoch überprüft werden, ob jeweils bis zu welchen Temperaturen die Defekte in den aus den verschiedenen HDAusgangsphasen hergestellten Eisphasen jeweils verbleiben. Auch sollte für diese Art der Präparation das Rekristallisationsverhalten der Eis Ih-Körner sowie die Ausbildung von 
Rekristallisationstexturen unterhalb des Schmelzpunktes Ziel weiterer Untersuchungen sein.

Im Zuge der Experimente an Eis Ih mit den Gasen Argon und Helium sowie Neon konnte gezeigt werden, dass die Gitterstruktur von Eis Ih in Wechselwirkung mit den Gasen Helium und Neon tritt und sich dies durch eine deutliche Veränderung der Gitterkonstanten manifestiert. Für Argon zeigte sich die Eis-Struktur als Funktion des Druckes und der Temperatur isokompressibel. Bei Neon und Helium hingegen zeigte die Kompression als Funktion von Druck und Temperatur eine Änderung der Gitterkonstantenverhältnisse. Signifikante Aussagen bezüglich der Strukturveränderungen als Funktion von Druck und Temperatur oder des Gasinhaltes konnten aus den experimentellen Befunden aufgrund der hohen Fehler der verfeinerten Parameter nicht getroffen werden. Als Ursache dieser sind für die Messungen unter Verwendung der Gase Argon sowie Helium die während des Experimentes fortschreitende Erhöhung des Clathrat-Hydrat-Phasenanteiles anzuführen. Durch die nur ungenaue kristallographische Beschreibung der Clathrat-Hydrate bei Drücken $\mathrm{p}>1 \mathrm{kbar}$ erwiesen sich die Rietveld-Verfeinerungen der Strukturparameter der Eis IhElementarzelle als beeinflusst durch den Fremdphasenanteil wie auch die Wahl des kristallographischen Modells (z.B. thermische Auslenkungsparameter, Füllgrad, Gaslagen der Gasatome in dem Clathrat-Hydratmodell). Für die mit Argongas sowie die nach dem Gaswechsel mit Heliumgas gemessenen Datensätze galt dies in besonderer Weise.

Die Schmelzkurvenexperimente sowie der experimentelle Befund der veränderten Gitterkonstanten sprechen für eine Löslichkeit der Gase Helium und Neon in Übereinstimmung mit Beschreibungen von Löslichkeiten innerhalb der Eisstruktur in der Literatur. Bezüglich des Gasinhaltes ergaben die Rietveld-Verfeinerungen kein eindeutiges und physikalisch nachvollziehbares Ergebnis. Problematisch zeigte sich hier die Modellierung und Verfeinerung der Gasatome in der Eisstruktur. Aufgrund einer zu vermutenden diffusen, statistischen Verteilung der Gasatome in der Eisstruktur konnte diese nicht ohne weiteres durch die Verfeinerungen bestimmt werden. Eine Aussage bezüglich des Gasinhaltes als Funktion des Druckes und der Temperatur kann somit derzeit nicht getroffen werden. Simulationsrechnungen, die an einem festen Eis-Modell mittels einer molekularen Monte Carlo-Simulation durchgeführt wurden, ergaben eine Abhängigkeit der Löslichkeit von Druck und Temperatur. Bei der Wahl unterschiedlicher Potenzialparameter in den Simulationen ergaben sich Unterschiede hinsichtlich der Gasbesetzungsverläufe, so dass eine absolute Festlegung des Gasinhaltes als Funktion des Druckes und der Temperatur derzeit nicht 
möglich ist.

Um eine Skalierung der experimentell ermittelten Abweichungen der Gitterkonstanten von Eis Ih unter der Verwendung der Gase Helium und Neon zu ermöglichen, wäre die experimentelle Untersuchung nahezu vergleichbarer Strukturen wie z.B. den Eis Ih-Gashydraten oder dem neu entdeckten Neon-Clathrat-Hydrat und der Vergleich $\mathrm{zu}$ den simulierten Strukturen eine Möglichkeit. Über die experimentell ermittelbaren Füllgrade der Neon-Clathrat-Hydratkäfige sowie dem Gasinhalt der Gas-Hydratstrukturen ließe sich durch Anpassung und Optimierung der Potenzialparameter an diesen Systemen die Grundlage zur Berechung des Eis Ih-Gasinhaltes legen.

Aus diesen experimentellen Befunden ist deutlich ersichtlich, dass eine durch pVT-Experimente bestimmte Phasengrenze oder Schmelzkurve unbedingt einer in situ Verifizierung mittels Beugungsmethoden bedarf. Grundvoraussetzung ist hier jedoch auch, dass die Herstellung dieser Phasen für systematische Untersuchungen sowie in situ Verfizierungen an Großgeräten reproduzierbar durchgeführt werden kann und entsprechende Arbeitsvorschriften hierfür entwickelt werden. 


\section{Literaturverzeichnis}

Adamson, A.W., Shirley, F.P. and Kunichika, K.T. (1970), Journal of Colloid and Interface Science 34(3): 461-468.

Adamson, A. W., Dormant, L.M. and Orem, M. (1967), Journal of Colloid and Interface Science 25: 206-217.

Allmann, R., ,,Röntgen-Pulver-Diffraktometrie“, Clausthaler Tektonische Hefte 29, Verlag Sven von Loga, 1994

Anderson, W.A. and Mehl, R.F. (1945), Trans. Metall. Soc. A.I.M.E. 161, 140

Arnold, G. P., Finch, E.D., Rabideau, S.W. and Wenzel, G. (1968). The Journal of Chemical Physics 49(10): 4365-4369.

Avrami, M. (1939). Journal of Chemical Physics 7, 1103

Barnes, W.H. (1929). Proceedings of the Royal Society of London, A125, 670-93

Barrer, R. M. and Ruzicka, D.J. (1962). Trans. Faraday Soc. 58: 2239-2252.

Barrer, R. M. and Ruzicka, D.J. (1962). Trans. Faraday Soc. 58: 2253-2261.

Barrer, R. M. and Ruzicka, D.J. (1962). Trans. Faraday Soc. 58: 2262-2271.

Barrer, R. M. and Vaughan, D.E.W. (1967). Trans. Faraday Soc. 63: 2275-2290.

Barrer, R. M. and Vaughan, D.E.W. (1971). The Journal of Chemical Physics 32: 731-743.

Bellissent-Funel, M.-C. and Bosio, L. (1995). The Journal of Chemical Physics 102(9): 3727-3735.

Benedict, W.S., Gailar, N., and Plyler, E.K. (1956). Journal of Chemical Physics, 24, 1139-65

Bennett, K., Wenk, H-R., Durham, W.B., Stern, L.A. and Kirby, S.H. (1997). Philosophical Magazine A 76(2): 413-435.

Berendsen, H.J.C, Postma, J.P.M., van Gusteren, W.F., and Hermans, J. (1981), ed. Pullman, B., Intermolecular Forces, 331-342

Bertie, J.E., Calvert, L.D. and Whalley, E. (1963). Canadian Journal of Chemistry 42: 1373-1378

Bernal, J.D., and Fowler, R.H. (1933). Journal of Chemical Physics, 1, 515-48

Bizhigitov, T. B. and Sirota, N.N. (1986). JETP Lett. 44(7): 417-419.

Blackmann, M. and Lisgarten, N.D. (1958). Advances in Physics, 7, 189-98

Bopp, P. (1991). Intermolecular Forces, Springer Verlag 
Bragg, W. H., (1922), Proceedings of the Physical Society, 34, 98-103.

Bridgman, P. W. (1911), Water in the liquid and five solid forms under pressure. Harvard University.

Bridgman, P.W., Proceedings of the American Academy of Arts and Sciences, 47, 441, (1912)

Bridgman, P. W. (1935). The Journal of Chemical Physics 3(10): 597-605.

Bridgman, P. W. (1937). The Journal of Chemical Physics 5: 964-966.

Brückel, T.H., Süßenbach, J., Hasylab Annual Report 1995, I-125

Cagliotti, G., Paoletti, A., and Ricci, F.P. (1958), Nucl. Instrum., 3, 223-228

Cahn, J.W., Acta Metall., 4 (1956), 449-459

Chazallon, B., Dissertation Universität Göttingen, 1999

Chazallon, B., Klapproth, A.and Kuhs, W.F., 1999, AIP CP479 74-77, Eds. Johnson, M.E., Kearley, G.J. Büttner, H.G.

Christian, J.W. (1965), The Theory of Transformations in Metals and Alloys, Pergamon, Oxford

Cho, C. H., Singh, S. and Robinson, G.W. (1996). Physical Review Letters 76(10): 1651-1654.

Chou, I.-M., Blank, J.G., Goncharov, A.F., Mao, H-K. and Hemley, R.J. (1998). Science 281.

Chou, I.-M. and Hasselton jr, H.T. (1998). Rev. High Pressure Sci. Technol. 7: 1132-1134.

Circone, S., Stern, L.A., Kirby, S.H., Pinkston, J.C. and Durham, W. B. (1999). Third

International Conference on Gas Hydrates, Salt Lake City, Utah.

Davidson, D. W. and Ripmeester, J.A. (1978). Journal of Glaciology 21(85): 33-49.

Davy, H. (1810). Phil. Trans. Roy. Soc. 101(1)

Day, P., Materials World, October 2000, 8(10)(2000), 25

Deaton, W.M. and Frost, E.M. (1940). Gas 16(6):28-30

De Forcrand, M.R. and Villard, P. (1888). Compt. rend. 106: 1602-1603

Dennison, D.M. (1921), Physical Review, 17, 20-2

De Forcrand, M.R., Comp. Rend. 176, 355-358, 1923

Dowell, L. G. and Rinfret, A.P. (1960). Nature 188(4757): 1144-1148. 
Drits, V. A. and Tchoubar, C. (1990), X-Ray Diffraction by Disordered Lamellar Structures, Springer-Verlag.

Durham, W. B., Kirby, S.H., Heard, H.C., Stern, L.A. and Boro, C.O. (1988). Journal of Geophysical Research 93(B9): 10,191-10,208.

Dyadin, Y. A., Bondaryk, I.V. and Zhurko, F.V. (1991) Inclusion Compounds, J. E. D. D. a. D. D. M. J.L. Attwood, Oxford Science Publications. 5.

Dyadin, Y. A., Aladko, E. Y., Udachin, K.A. and Tkacz, M. (1994). Polish J. Chem. 68: 343-348.

Dyadin, Y. A., Larionov, E.G., Aladko, E.Y., Manakov, A.Y., Zhurko, F.V., Mikina, T.V., Komarov, V.Y. and Grachev, E.V. (1999). Journal of structural chemistry 40(5): 790-795.

Dyke, T. R., Mack, K.M. and Muenter, J.S. (1977). The Journal of Chemical Physics 66: 498-510.

Dyster, J. and Stöckhert, B. (2000). Contributions to Mineralogy and Petrology; Online First.

Elarby-Aquizerat, A., Jal, J.F., Dupuy, J., Schildberg H. and Chieux P. (1987). Journal de Physique 3(48): 465-470.

Engelhardt, H. and Kamb, B. (1978). Journal of Glaciology 21(85): 51-53.

Engelhardt, H. and Kamb, B. (1981). The Journal of Chemical Physics 75(12): 5887-5899.

Engelhardt, H. and Whalley, E. (1972). The Journal of Chemical Physics 56(6): 2678-2684.

Engelhardt, H. and Whalley, E. (1979). Journal of Chemical Physics 71(10): 4050-4051.

Evans, L. F. (1965). Nature 206: 822.

Evans, L. F. (1967). Nature 213: 384-385.

Finch, E. D., Rabideau, S.W., Wenzel, R.G. and Nereson, N.G. (1968). The Journal of Chemical Physics 49(10): 4361-4364.

Frank, F.C. and Nicholas, J.F. (1953). Philosophical Magazine 44: 1213-1235

Furukawa, Y. and Nada, H. (1997). Journal of Chemical Physics B 101: 6167-70.

Gordon, P. (1955), Trans. Metall. Soc. A.I.M.E. 203, 1043

Goto, A., Hondoh , T., Mae, S. (1990). Journal of Chemical Physics, 93, 1412-17

Guillot, B. and Guissani, Y., (2001), Journal of Chemical Physics, 114(15): 6720-6733

Haas, J., Bullemer, B. and Kahane, A. (1971). Solid State Communications 9: 2033-2035. 
Hamilton, W. C., Kamb, B. , LaPlaca, S.J. and Prakash, A. (1969)., eds. B. B. N. R. a. H. Engelhardt, New York, Plenum Press: 45-58.

Handa, Y. P., Klug, D.D. and Whalley, E. (1987). Journal de Physique Colloque C1(3): 435-439.

Handa, Y. P. (1988). Ind. Eng. Chem. Res. 27: 872-874.

Handa, Y. P., Klug, D.D. and Whalley, E. (1988). Can. J. Chem. 66: 919-924.

Hansen, N., Leffers, T. and Kjems, J.K. (1981), Acta Metall. 29, 1523

Hansen, N. and Bay, B. (1981), Acta Metall. 29, 65

Hemley, R. J., Chen, L.C. and Mao, H.K. (1989). Nature 338: 638-640.

Hewat, A.W., Mat. Sci. Forum 9(1986)69-79

Hirai, G.J. et al (2000). J. Chem. Phys. B 104:1429-1433

Hinberg van, M.G.E., Academisch Proefschrift, Universiteit Amsterdam 1994

Hinsberg van, M.G.E., Scheerboom, M. I. M., Schouten, J. A., J. Chem. Phys. 99(1), 752-754, 1999

Howard, C.J., J. Appl. Cryst. 15, 615-20 (1982)

Humphreys, F. J. and Hatherly, M., (1996), Recrystallisation and Related Annealing Phenomen, Pergamon Press.

Hwang, M. J., Wright, D.A., Kapur, A. and Holder, G.D. (1990). Journal of Inclusion Phenomena and Molecular Recognition in Chemistry 8: 103-116.

Ji, J. and Pettitt, B.M. (1994). Molecular Physics 82(1): 67-83.

Johari, G.P. and Whalley, E. (1976). Journal of Chemical Physics 64:4484-4489

Johnson, W.A. and Mehl, R.F. (1939), Trans. Metall. Soc. A.I.M.E. 135, 416

Jorgensen, W.L., Chandrasekhar, J., Madura, J.D. (1983), J. Chem. Phys. 79(2):926-935

Kahane, A., Klinger, J., Philippe, M., (1969), Solid State Communications, 7:1055-1056

Kamb, B. (1964). Acta Cryst. 17: 1437-1449.

Kamb, B. (1965). Science, 150, 205-9

Kamb, B. and Prakash, A. (1968). Acta Crystallographica, B24,1317-24

Kamb, B., Prakash, A. and Knobler, C. (1967). Acta Cryst. 22: 706-715. 
Kamb, B., Hamilton, W.C., LaPlaca, S.J. and Prakash, A. (1971). The Journal of Chemical Physics 55(4): 1934-1945.

Kell, G. S. and Whalley, E. (1968). The Journal of Chemical Physics 48(5): 2359-2361.

Kishimoto, Y. and Maruyama, M. (1998). Rev. High Pressure Sci. Technol. 7: 1144-1146.

Klapproth, A., Chazallon, B. \& Kuhs, W.F., (1999), AIP CP479, 70-73, Eds. Johnson, M.E., Kearley, G.J. Büttner, H.G.

Kobayashi, T., Kuroda, T. (1987), Ed. Sunagawa, I.

König, H. (1943). Zeitschrift für Kristallographie, 105, 279-86

Kolmogorov, A.N. (1937), Izv. Akad. Nauk. USSR-Ser-Matemat. 1(3), 355

Koza, M., Schober, H., Tölle, A., Fujara, F. and Hansen, T. (1999). Nature 397: 660-661.

Kuhs, W. F., Finney, J.L., Vettier, C. and Bliss, D.V. (1984), The Journal of Chemical Physics 81(8): 3612-3623.

Kuhs, W. F., Bliss, D.V. and Finney, J.L. (1987), Journal de Physique 3(48): 631-636.

Kuhs, W. F., Londono, D., Mayer, E., Hallbrucker, A. and Finney, J.L. (1989). Z.

Kristallographie 186: 174-175.

Kuhs, W.F., Dorwarth, R., Londono, D., and Finney, J.L, Eds. Maeno, N. and Hondoh, T, Hokkaido University Press, 1992, Sapporo, pp. 126-130.

Kuhs, W. F., Chazallon, B., Radaelli, P.G. and Pauer, F. (1997). Journal of Inclusion Phenomena an Molecular Recognition in Chemistry. 29: 65-77.

Kuhs, W. F., Gotthardt, F., Chazallon, B., Hensel, E. , and Klapproth, A. (1997). Experimental Report ILL, Experiment No. 5-22-474

Kuhs, W. F., Lobban, C. and Finney, J.L. (1998). Rev. High pressure Sci. Technol. 7: 1141-1143.

Kuhs, W.F, Klapproth, A., Chazallon, B., (1999), Physics of Ice Core Records, Ed. Hondoh, T. Proceedings of PICR-Symposium, Shikokutsekan, 373-392

Kuhs, W. F., Klein, H., Gotthardt, F. and Koza, M. (2000). Experimental Report ILL, Experiment No. 5-24-93.

Kuhs, W.F., Klapproth, A., and Gotthardt, F., Techmer, K., Heinrichs, T. (2000), Geophysical Research Letters, 27(18):2929-2932

Kuhs, W. F. and Lehmann, M.S. (1981). Nature 294: 432-434.

Kuhs, W. F. and Lehmann, M.S. (1983). The Journal of Chemical Physics 87: 4312-4313. 
Kuhs, W.F. and Lehmann, M.S. (1986), Water Science Reviews, 1986, 2, 1

Kuhs, W.F. and Lehmann, M.S. (1986), Water Science Reviews, 1986, 2, 1, with revisions Kuhs (1996)* in Petrenko and Withworth (1999). * with revisions Kuhs (1991), Kuhs, pers. Mitteilung (2001)

Kuhs, W. F. and Lobban, C. (1996). Experimental Report ILL, Experiment No. 5-22-435

Larson, A.C., and Von Dreele, R.B., GSAS General Structure Analysis System Manual 1985-2000, Los Alamos National Laboratory

Levitt, L. S. (1961). The Journal of Chemical Physics 34(4): 1440-1443.

Line, C.M.B. and Whitworth, R.W. (1996). Journal of Chemical Physics 104: 10008-13

Lobban, C., Programm order2, Göttingen unveröffentlicht (1997)

Lobban, C., PhD-Thesis, University College of London (1998)

Lobban, C., Finney, J.L., and Kuhs, W.F. (1998). Nature 391 :268-270

Lobban, C., Kuhs, W.F., Chazallon, B. and Finney, J.L. (1996). Experimental Report ILL, Experiment No. 5-22-461

Lobban, C., Kuhs, W.F., Gotthardt, F. and Finney, J.L. (1999). Experimental Report ILL, Experiment No. 5-24-62.

Lobban, C., Finney, J.L. and Kuhs, W.F. (2000). The Journal of Chemical Physics 112(16): 71697180 .

Londono, J. D. (1989). PhD-Thesis, Faculty of Science, University of London.

Londono, J. D., Finney, J.L. and Kuhs, W.F. (1992). Journal of Chemistry and Physics 97(1): 547552.

Londono, J. D., Kuhs, W.F. and Finney, J.L. (1993). The Journal of Chemical Physics 98(6): 48784888.

Lyon, K. G., Salinger, G.L., Swenson, C.A. and White, G.K. (1977). Journal of Apllied Physics 48(3): 865-868.

Marthinson, K., Furu, T., Nes, E. and Ryum, N. (1990), eds. M. P. A. a. A. D. Rollett, The Minerals, Metals \& Materials Society: 87-101.

Mayer, E. and Hallbrucker, A. (1987). Nature 325(6105):601.602

McCusker, L. B., von Dreele, R.B., Cox, D.E., Louer, D. and Scardi, P. (1999). Journal of Applied Crystallography 32: 36-50. 
Michalak, J.T. and Hibbard, W.R. (1961), Trans. ASM. 53, 331

Mills, R. L. and Grilly, E.R. (1955). Physical Review 99(2): 480-486.

Minceva-Sukarova, B., Slark, G.E. and Sherman, W.F. (1986). Journal of Molecular Structure. 143:87-90

Mishima, O., Calvert, L.D. and Whalley, E. (1984). Nature, 310, 393-5

Mishima, O., Calvert, L.D. and Whalley, E. (1985). Nature, 314, 76-8

Mishima, O. and Stanley, H.E. (1998). Nature 392.

Mishima, O. and Stanley, H.E. (1998). Rev. High Pressure Sci. Technol. 7: 1103-1105.

Morgan, J. and Warren, B.E. (1938). The Journal of Chemical Physics, 6: 666-673

Namiot, A. Y. and Bukhgalter, E.B. (1965). Journal of Structural Chemistry 6: 873-874.

Nishibata, K. (1972). Japanese Journal of Applied Physics 11(11): 1701-1708.

Nishibata, K. and Whalley, E. (1974). The Journal of Chemical Physics 60(8): 3189-3194.

Nixdorf, J. (1996), Dissertation, Fakultät für Chemieingenieurwesen. Karlsruhe, Universität Fridericana.

Odutola, J. A. and Dyke, T.R. (1980). The Journal of Chemical Physics 72: 5062-5070.

Oguro, M. and Withworth, R.W. (1991). J. Phys. Chem. Solids 52(2): 401-403.

O'Keeffe, B. M. (1998). Nature 392: 879.

Pauling, L. (1935). Journal of American Chemical Society, 57, 2680-4

Peterson, S.W. and Levy, H.A. (1957), Acta Crystallagraphica, 10, 70-6

Petrenko, V. F. and Withworth, R. W., Oxford University Press. (1999)

Piermarini, G. J., Munro, R.G. and Block, S. (1984). Mat. Res. Soc. Symp. Proc. 22: 25-28.

Pistorius, C. W. F. T., Rapoport, E. and Clark, J.B. (1968). The Journal of Chemical Physics 48(12): 5509-5514.

Rabideau, S.W., Finch, E.D., and Dennison, A.B. (1968). Journal of Chemical Physics, 49, 4660-5

Rappé, A.K., Casewit, C.J., Colwell, K.S., Goddard III, W.A., and Skiff, W.M. (1992), J. Am. Chem. Soc. 114, 10024-10035

Reiter, S.F. (1952), Trans. Metall. Soc. A.I.M.E. 194, 972 
Rietveld, H.M. (1969), J. Appl. Cryst., 2, 65-71

Röttger, K., Endriss, E., Ihringer, J., Doyle, S. and Kuhs, W.F. (1994). Acta Cryst B50: 644-648.

Rosen, A., Burton, M.S., Smith, G.V. (1964), Trans. Metall. Soc. A.I.M.E. 230, 205

Ripmeester, J.A., Ratcliffe, C. I.,Klug, D. D., Tse, J.S., Proceedings of the 1. International Conference on Natural Gas Hydrates, New Paltz, The New York Academy of Sciences, New York 1994

Salamantin, A.N., Hondoh, T., Uchida, T., Lipenkov, V.Y. (1998), J. Cryst. Growth, 193, 197-218

Satoh, K., Uchida, T., Hondoh, T. and Mae, S. (1996). Proc. NIPR Symp. Polar Meteorol. Glaciol. 10: $73-81$.

Savage, H. (1985). Water Science Review. F. Franks. Cambridge, Cambridge University Press. 2: 67-148.

Savage, H. and Finney, J.L. (1986), Nature 332:717-720

Shaw, G. H. (1986). The Journal of Chemical Physics 84(10): 5862-5668.

Sirota, N.N. and Bizhigitov, T.B. (1987), Sov. Phys. Dokl. 32(12):998-1000

Sirota, N. N. and Bizhigitov, T.B. (1988). Russian Journal of Physical Chemistry 62(2): 134-136.

Sirota, N. N. and Bizhigitov, T.B. (1988). Cryst. Res. Technol. 23(5): 595-603.

Sloan, E.D. (1997). New York, Marcel Dekker

Sloan jr, E. D. and Fleyel, F. (1991). AIChE Journal 37(9): 1281-1292.

Soper, A. K. (1994). The Journal of Chemical Physics 101: 97-112.

Speedy, R. J., Madura, J.D. and Jorgensen, W.L. (1987). The Journal of Chemical Physics 91: 909913.

Stackelberg von, M., Müller, H. R., Zeitschrift für Elektrochemie, Band 58 Nr.1, 1954

Stillinger, F.H. and Rahman, A. (1974), Journal of Chemical Physics 60: 1545-1557

Stern, L. A., Durham, W.B. and Kirby, S.H. (1997). Journal of Geophysical Research 102(B3): 5313-5325.

Strauss, H. L., Chen, Z. and Loong, C-K. (1994). The Journal of Chemical Physics 101(8): 71777180 .

Sukarova, B., Sherman, W.F., and Wilkinson, G.R., J. Mol. Struct. 115, 137 (1984) 
Tajima, Y., Matsuo, T. and Suga, H. (1982). Nature 299: 810-812.

Tammann, G. (1900). Annalen der Physik, Serie 4, 2, 1-31

Tammann, G. (1903), Kristallisieren und Schmelzen, Leipzig, Verlag Von Johann Ambrosius Barth.

Tammann, G. (1909). Z. Anorg. Allgem. Chem. 63: 285-305.

Tammann, G. (1910). Zeitschrift für Physikalische Chemie 72: 609-631.

Tammann, G. (1913). Zeitschrift für Physikalische Chemie 84: 293-312.

Tammann, G. (1913). Zeitschrift für Physikalische Chemie 84: 257-292.

Tammann, G. u. Bandel, G. (1934), Die Schmelz und Umwandlungskurven der Eisarten aus schwerem Wasser, Berlin, Weidmannsche Buchhandlung.

Thompson, P., Cox, D.E., and Hastings, J.B., J. Appl. Cryst. 20, 79 (1987)

Vandermeer, R.A. and Gordon, P. (1963), in Recovery and Recrystallization of Metals ed. Himmel. Interscience. p211.

van der Waals, J. and H., Platteeuw, J.C. (1959), Adv. Chem. Phys. 2, 1-57

Vinet, P., Ferrante, J., Smith, J.R., and Rose, J.H., (1986) J. Phys. C19, L647

Voss, W. L., Finger, L. W., Hemley, R.J. and Mao, H-K. (1993). Physical Review Letters 71: 3150-3153.

Voss, W.L., Finger, L.W., Hemley, R.J., Mao, Ho-kwang, Chemical Phyics Letters 257 (1996) 524530

Warren, B.E. (1941). Physical Review 9:693-698

Whalley, E. and Davidson, D.W. (1965). The Journal of Chemical Physics 43: 2148-2149.

Whalley, E., J.B.R. Heath, and Davidson, D.W., J. Chem. Phys. 48, 2362, (1968)

Williams, W.G., Ibberson, R.M., Day, P. and Enderby, J.E., Physica B, 241-243(1998)234-236

Wilson, G. J., Chan, R.K., Davidson, D.W. and Whalley, E. (1965). The Journal of Chemical Physics 43(7): 2384-2391.

Wollan, E.O., Davidson, W.L. and Shull, C.G. (1949). Physical Review, 75, 1348-52

Young, R. A., Ed. (1995), The Rietveld Method, Oxford University Press. 


\section{Anhang}

8.2.1 Experimentelle Grundlagen - Die pVT-Apparatur : Schematische Zeichnung und Erläuterung des HD-Stick

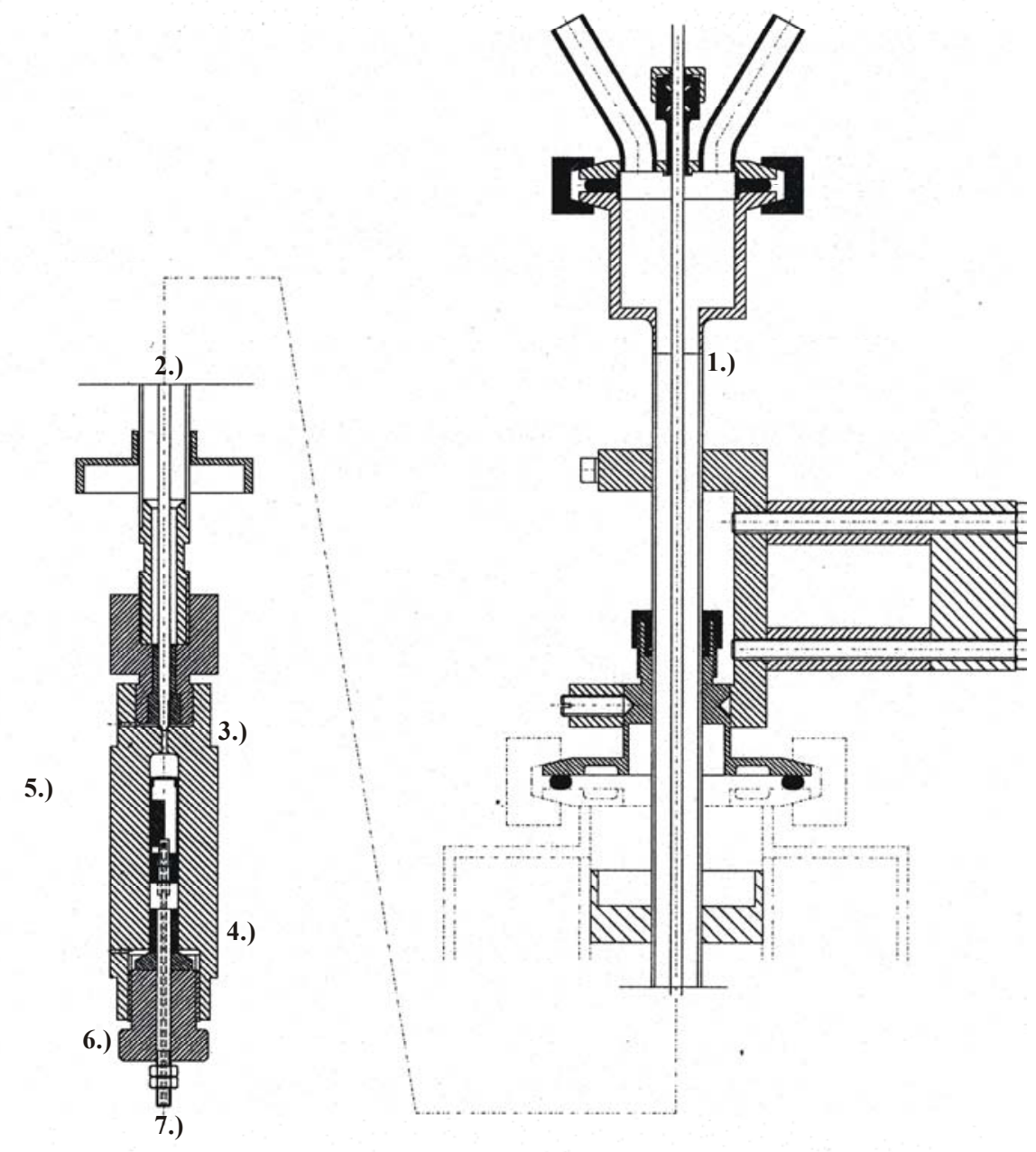

\footnotetext{
1.) Hochdruckstick (HD-Stick inkl. Befestigungaufnahmen und Anschlüssen

2.) Hochdruckrohr zur HD-Zelle

3.) Hochdruckzelle

4.) Dichtpackung Hochdruckstick (HD-Stick inkl. Befestigungs- und

5.) Röngtenprobenhalter

6.) Dichtkonus

7.) Dichtkopf inkl. Bohrung für Temperatursensor
}

Im wesentlichen besteht der HD-Stick aus einem Druckrohr (2.), dass am oberen Ende mit Befestigungselementen und Abstandhaltern zur Halterung im Kryostaten sowie elektrischen A-1 
Anschlüssen für Heizelemente, Temperatursensoren sowie Gasanschlüssen versehen ist (1.) und durch ein äußeres Schutzrohr verläuft. Am Ende des Druckrohres wird die HD-Zelle (3.) mit dem Druckrohr verschraubt (2.). Innerhalb der HD-Zelle befindet sich der Röntgenprobenhalter (5.). Die HD-Zelle ist nach außen bzw. unten mit einem Dichtkopf (7.), auf den eine Dichtpackung (4.) aus einer oder mehreren unterschiedlichen Dichtringen, z.B. aus Kupfer, Polyäthylen, Polypropylen und Teflon aufgeschoben sind, bis zu Gasdrücken von $\mathrm{p} \sim 6.5 \mathrm{kbar}$ abgedichtet. Der Dichtkopf ist mit einer senkrecht durchgehenden Bohrung versehen, die bis in an den Röntgenprobenhalter heranreicht um somit mit einem Mantelthermoelement die Probentemperatur an der Probe zu bestimmen.

\subsection{GEM (General Materials Diffractometer)}

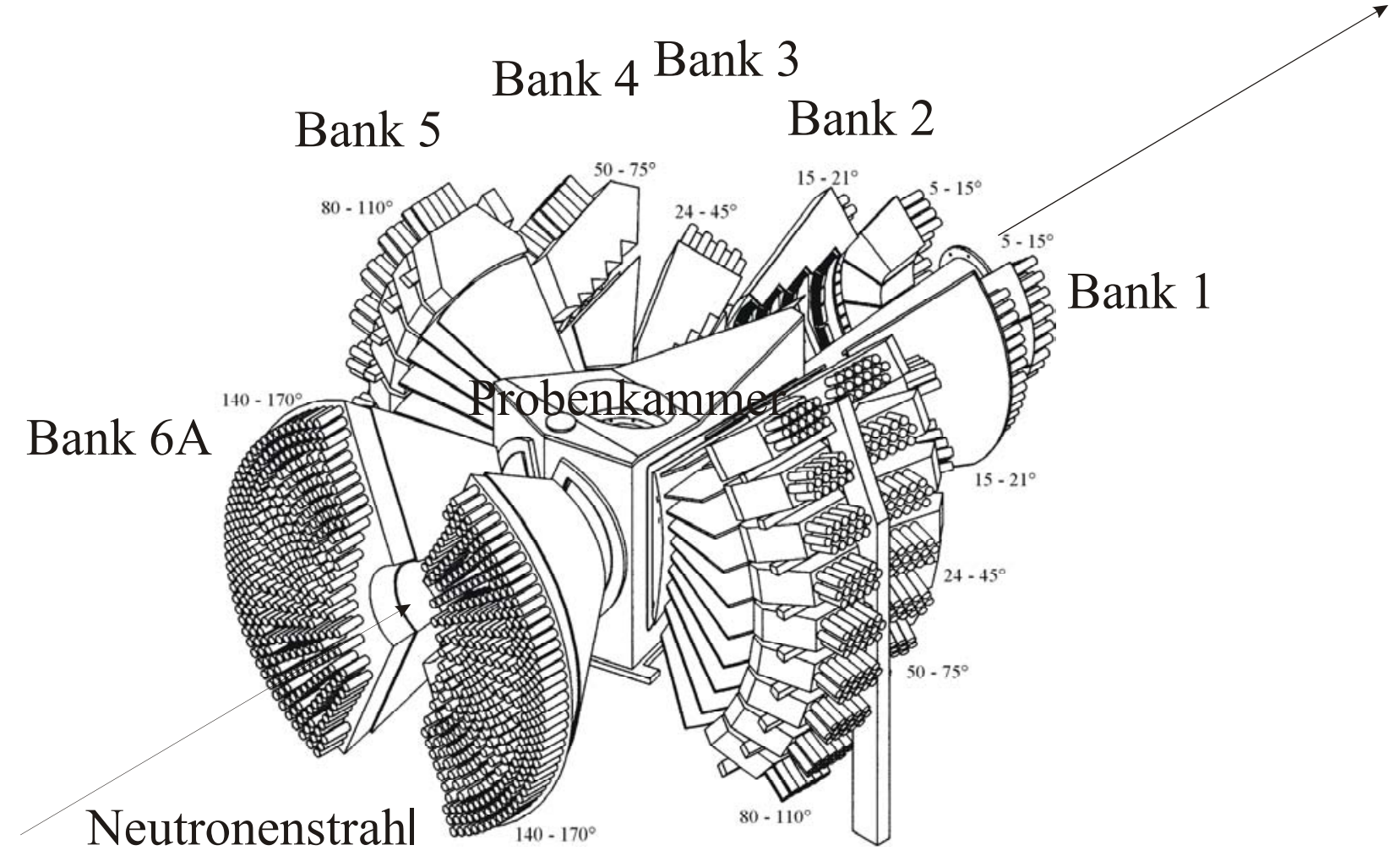

Abbildung 8.2.1.3.1 : Schematische Darstellung des GEM. Die Detektorbänke Bank 1 bis Bank 5 sind für die Detektion von vorwärtsgestreuten Neutronen, die Detektorbänke Bank 6A sowie Bank 6B für rückwärtsgestreute Neutronen ausgelegt. Die Probenkammer ist so ausgelegt, dass ein Orange-Kryostat, ähnlich der Bauform am ILL, in die Probenkammer eingeführt werden kann. In Verbindung mit einer funktionstechnisch äquivalenten Druckerzeugereinheit ist es somit möglich auf der Minutenskala Strukturen mit variabler Winkelauflösung auf der Minutenskala als Funktion von Druck- und Temperatur zu identifizieren, und so insbesondere Phasenübergänge zu verfolgen. 


\subsubsection{Experimentelle und theoretische Grundlagen - Rietveld-Strukturanalyse : Definition des thermischen Auslenkungsparameters U}

Durch thermische Anregung führen die an den Gitterpunkten des Kristallgitters fixierten Kristallgitterbausteine (Atome und Moleküle) Schwingungen um ihre Ruhelage aus, die bei gegebenen Druck- und Temperaturbedingungen der Gleichgewichtslage entsprechen. Diese thermische Auslenkung u eines Atoms oder Moleküls aus der Ruhelage ist proportional zur Temperatur T und entspricht dem Auslenkungsquadrat $<\mathrm{u}^{2}>$. Diese Auslenkung wird in der mathematischen Modellierung des Strukturfaktors durch den Faktor $B_{\mathrm{ij}}$ definiert. Für diesen atomaren Auslenkungsparameter (engl. ADP [Atomic Displacement Parameter]) für das k-te Atom in der Elementarzelle für den gilt:

$$
B_{i j, k}=8 \cdot \pi^{2} \cdot U_{i j, k}
$$

Da jedes, also auch das k-te Atom oder Molekül in dem Kristallgitter drei Translationsfreiheitsgrade hat, weisen das thermische Auslenkungsquadrat $U$ sowie der Faktor B Tensoreigenschaften auf und wird durch eine Fläche 2. Ordnung beschrieben (Schwingungstensor bzw. thermisches Ellipsoid). Ist bei gegebener Temperatur die thermische Auslenkung in allen 3 Freiheitsgraden gleich zur Ausgangslage, so ist die thermische Auslenkung isotrop. In diesem Falle stellt die Fläche gleicher Aufenthaltswahrscheinlichkeit eine Kugel dar. Der isotrope Auslenkungsparameter Uiso entspricht dann:

$$
U_{i s o}=\frac{1}{3} \operatorname{Spur} U_{i j, k}
$$

wobei $\mathrm{U}_{\mathrm{ij}, \mathrm{k}}$ der Tensor der atomaren Auslenkungsquadrate ist. Im allgemeinen ist die thermische Auslenkung jedoch anisotrop, d.h. richtungsabhängig, so dass diese im allgemeinen ein Ellipsoid darstellt. Neben der thermischen Auslenkung wird sowohl der isotrope als auch der anisotrope Verfeinerungsparamter $U$ in den Rietveld-Analysen durch atomare bzw. molekulare Auslenkungen aufgrund von Fehlordnungen und Gitterdefekten beeinflusst. 


\subsubsection{Die Herstellung von Eis Ih : Rekristallistaion von Eis Ih}

Die Abbildung 8.3.2.1 sowie die Abbildung 8.3.2.2 zeigt im Vergleich zur Abbildung 3.5, Kapitel 3.2, gemörsertes $\mathrm{D}_{2} \mathrm{O}$-Eis Ih nach einer Temperzeit bei Umgebungsdruck und einer Temperatur $\mathrm{T}=0^{\circ} \mathrm{C}$ nach $\mathrm{t} \sim 8 \mathrm{~h}$.

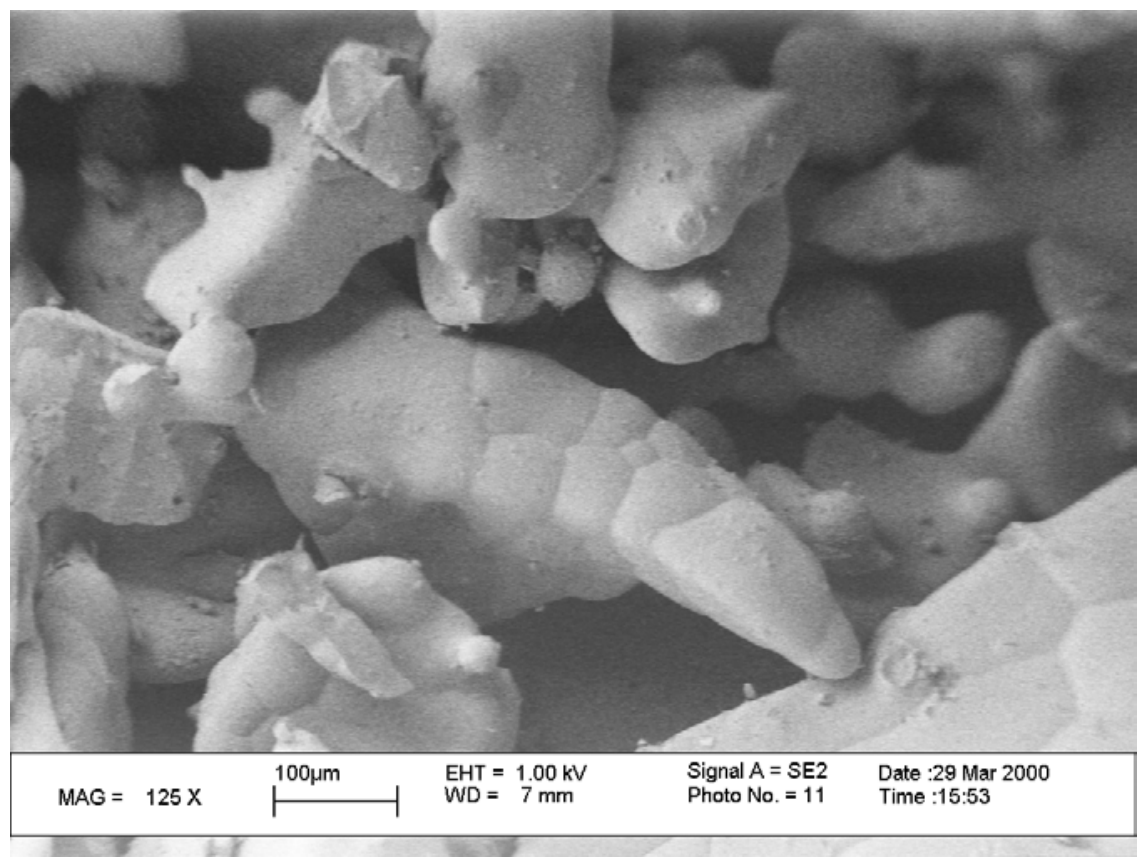

Abbildung 8.3.2.1: mit fortschreitender Zeit ist ein Zusammenwachsen der Eis Ih-Körner zu erkennen.

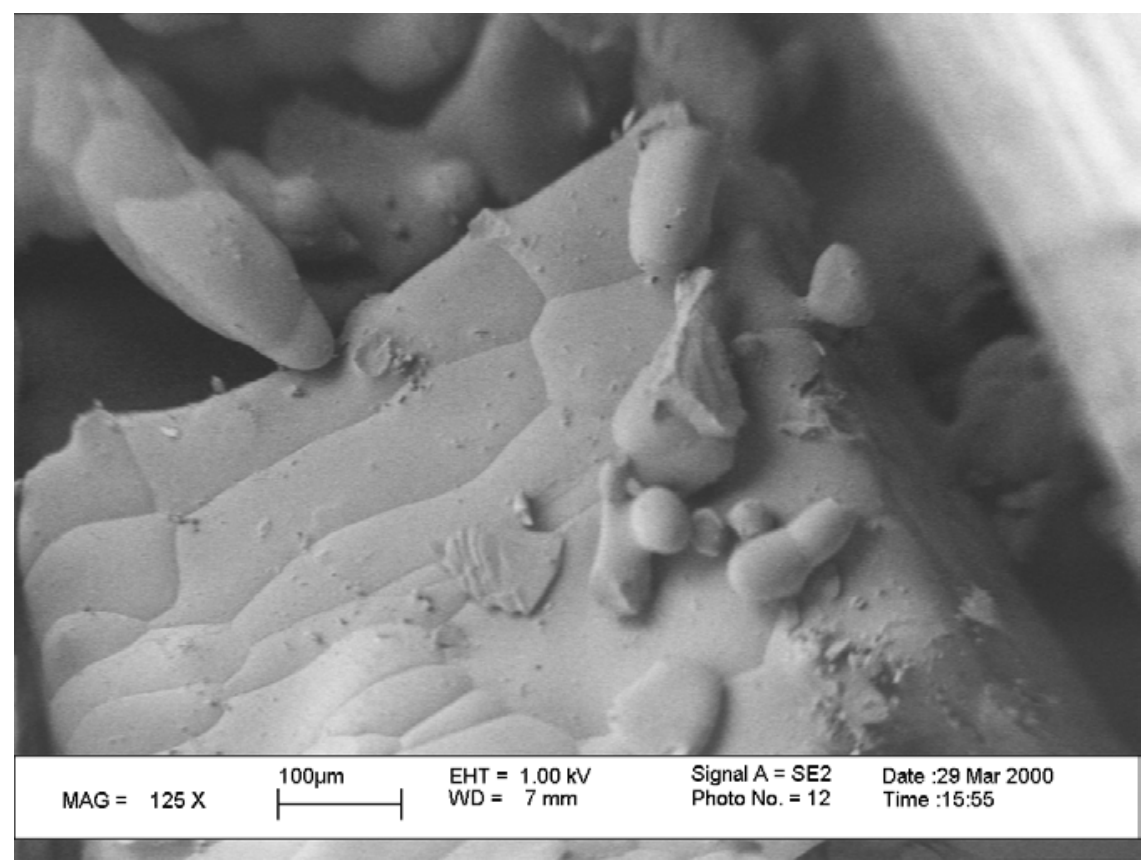

Abbildung 8.3.2.2: deutlich sind die zusammengewachsenen Eis Ih-Körner durch verbliebene Korngrenzen zu erkennen. 
Es zeigt sich in dem angegebenen Maßstab, dass nach $\mathrm{t} \sim 8 \mathrm{~h}$ ein Großteil der in Abbildung 3.5, Kapitel 3.2 erkennbaren Körner mit einer flächigen Ausdehnung $\mathrm{A}<100 \mu \mathrm{m}^{2}$ nahezu verschwunden sind. Die verbleibenden größeren Körner mit flächigen Ausdehnungen wesentlich $>100 \mu \mathrm{m}^{2}$ zeigen Korngrenzenverläufe, die in etwa die Größenverhältnisse der kleineren Körner vor dem Tempern in einem angeschlossenen Gefäß bei Umgebungsdruck von $\mathrm{p} \sim 1$ bar und bei einer Temperatur von $\mathrm{T}=0^{\circ} \mathrm{C}$ wiederspiegeln.

\subsubsection{Phasenübergänge von Hochdruckeisphasen in Eis Ic als transiente Phase : Stapelfehler und lokale Delokationen}

Die Abbildung 8.3.3.1 zeigt im Vergleich die ungestörte kubische Stapelsequenz ...ABCA... (a) und die ungestörte hexagonale Stapelsequenz ...ABAB... (b) in der Projektion der $\{11 \overline{2} 0\}$-Ebene des hexagonalen Gitters.

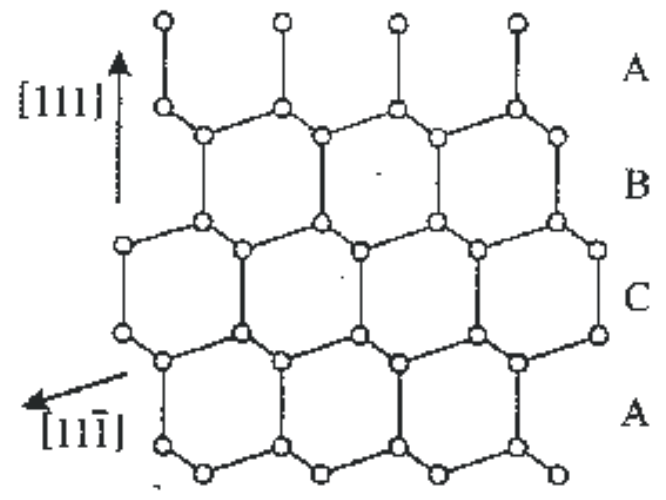

(a)

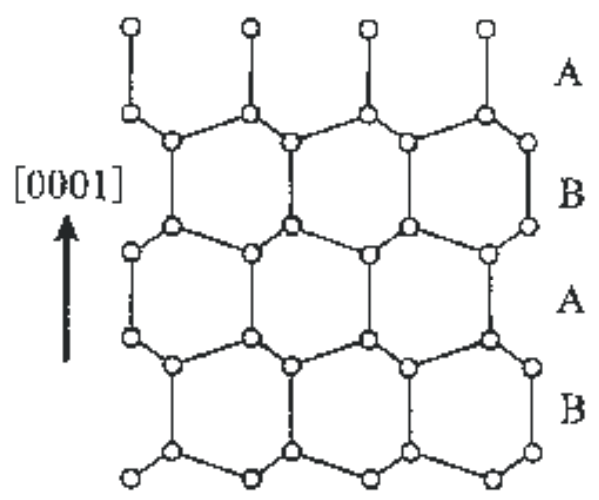

(b)

Abbildung 8.3.3.1 ungestörte Stapelfolgen der kubischen (a) und hexagonalen Sequenzen (b)

Die Abbildungen 8.3.3.2 (a) bis (d) zeigen mögliche Stapelfehler, die durch lateral begrenzte Stapelfehlordnungen mit den zugehörigen Versetzungen verursacht sind. Diese führen zu partiellen Verschiebungen von Gitterbausteinen in der Eisstruktur. In den jeweiligen Abbildungen sind zum Vergleich links die ungestörten Gitterordnungen, rechts deren durch Stapelfehlordnungen fehlgeordnete Gitter dargestellt. Die Abbildungen 8.3.3.2 (a)-(d) zeigen die Strukturen projiziert auf die $\{11 \overline{2} 0\}$-Ebene des hexagonalen Gitters. 


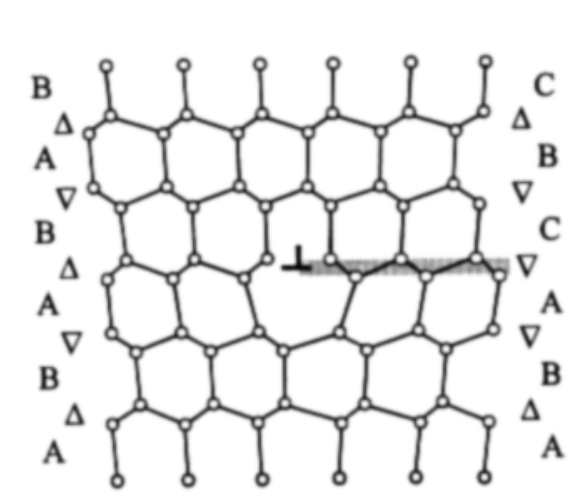

(a)

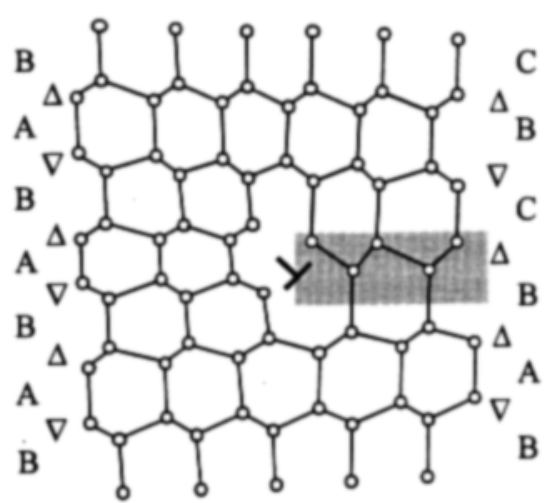

(b)

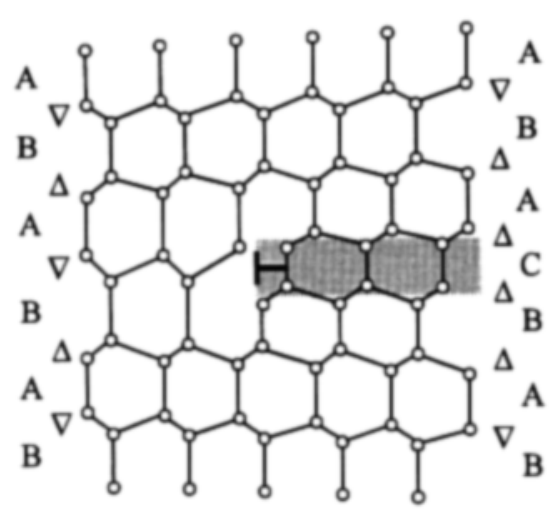

(c)

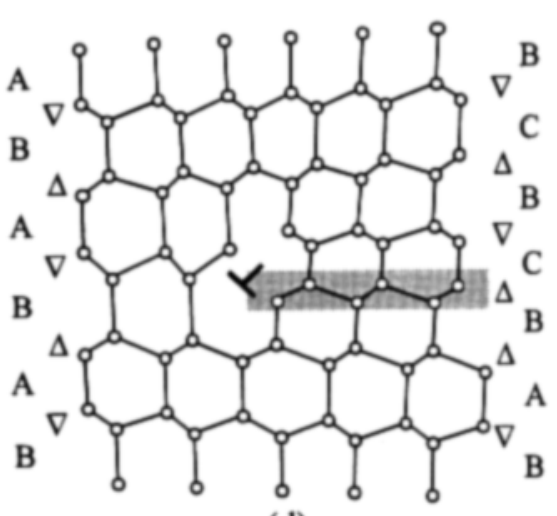

(d)

Abbildung 8.3.3.2: a.) partielle Fehlordnung durch Gleitung einer planaren Ebene (Layers) von B auf C (Shockley-Stapelfeler), (b) Fehlen einer planaren Ebene A mit Scherung zwischen Layer B und C, (c) eingefügte planare Ebene vom Typ $C$, (d) eingefügte planare Ebene vom Typ $C$ mit Scherung zwischen Layer B und C (vergl. Petrenko, V.F und Whitworth, R.W., Seite 176ff).

\subsubsection{Strukturelle Untersuchungen an kubischem Eis Ic}

Die Abbildung 8.3.3.1.1 zeigt überlagerte Diffraktogramme von Eis Ic, hergestellt aus verschiedenen HD-Eisphasen, über den gesamten gemessenen Winkelbereich in $2 \theta$. Aus einem Vergleich der Diffraktogramm-Überlagerungen zeigt sich, dass die im Rahmen der Messgenauigkeit bestimmten Unterschiede in den Intensitäten im wesentlichen in dem Winkelbereich in $2 \theta \sim 7.5^{\circ}$ bis $2 \theta \sim 70^{\circ}$ festzustellen sind. Die Messungen wurden am D2B mit in Göttingen an der pVT-Apparatur hergestellten Proben durchgeführt. Diese wurden in Göttingen wiedergewonnen und unter währenden Flüssigstickstofftemperaturenn in Probenbehältern zum ILL transportiert. Dort wurden die Proben in die Probenbehälter (Vanadiums-caps) des D2B-Kryostaten transferiert. Bei dem Transfer unter flüssiger Stickstoffatmosphäre ging ein Großteil der Eis Ic-Probenmasse, gerade aus Eis II sowie Eis IX/III hergestellt, durch das „,verkochen“ des Stickstoffes und der damit einhergehenden Verwirbelung in dem flüssigen Stickstoff der feinkristallinen Proben, verloren. Dieser 
Massenverlust der Probe war gerade für Eis Ic aus Eis II sowie aus Eis III bedeutend, da bei der Präparation in Göttingen nur je eine Wiedergewinnung der HD-Präparation gelungen ist.

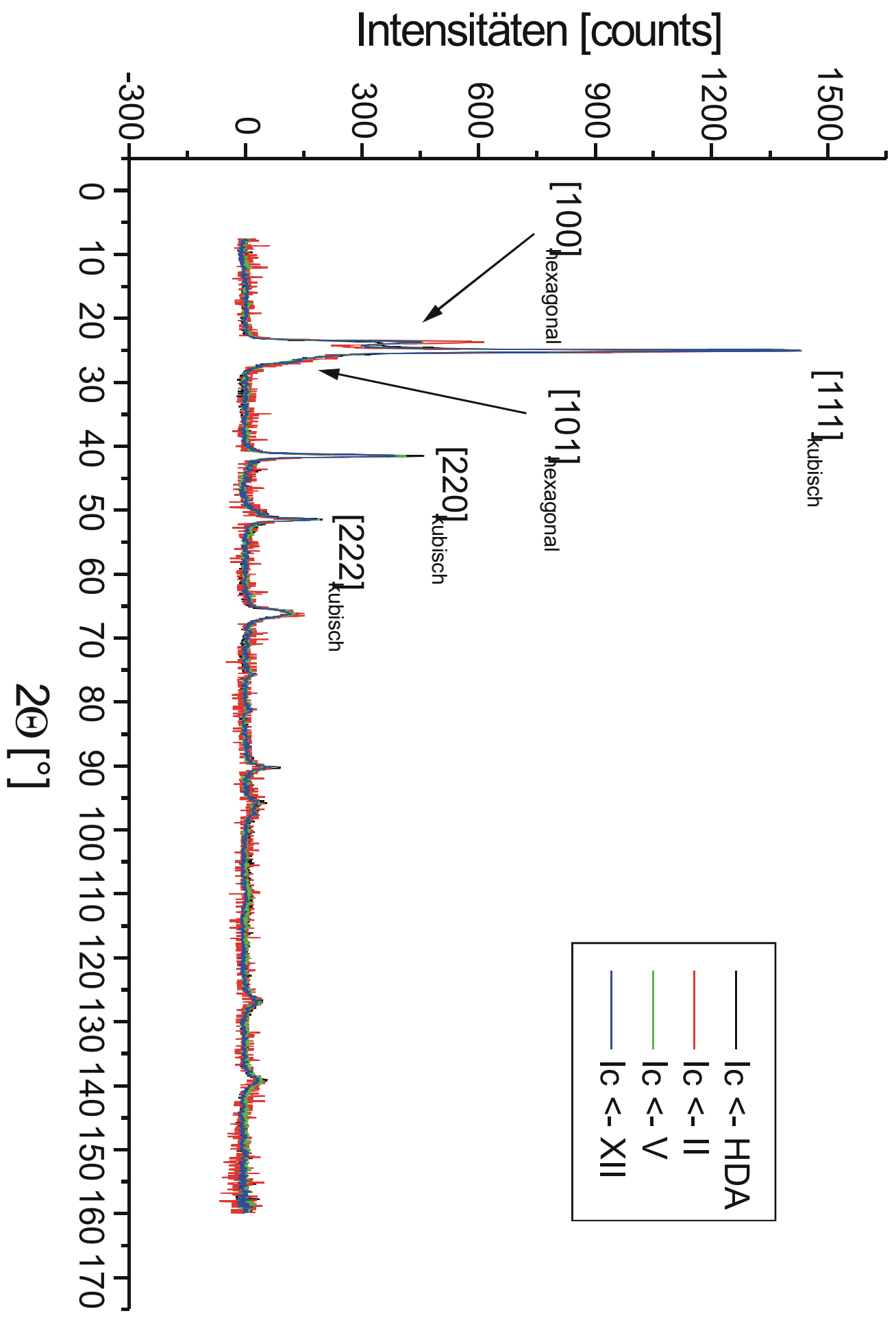

Abbildung 8.3.3.1.1: Überlagerung von Eis Ic-Diffraktogrammen, hergestellt aus den HD-Eisphasen HDA, II, V, XII. Eis Ic aus der Hochdruckeisphase Eis IX ist aufgrund einer um den Faktor 3 größeren Schwankung der Intensitäten nicht in der Abbildung dargestellt. 


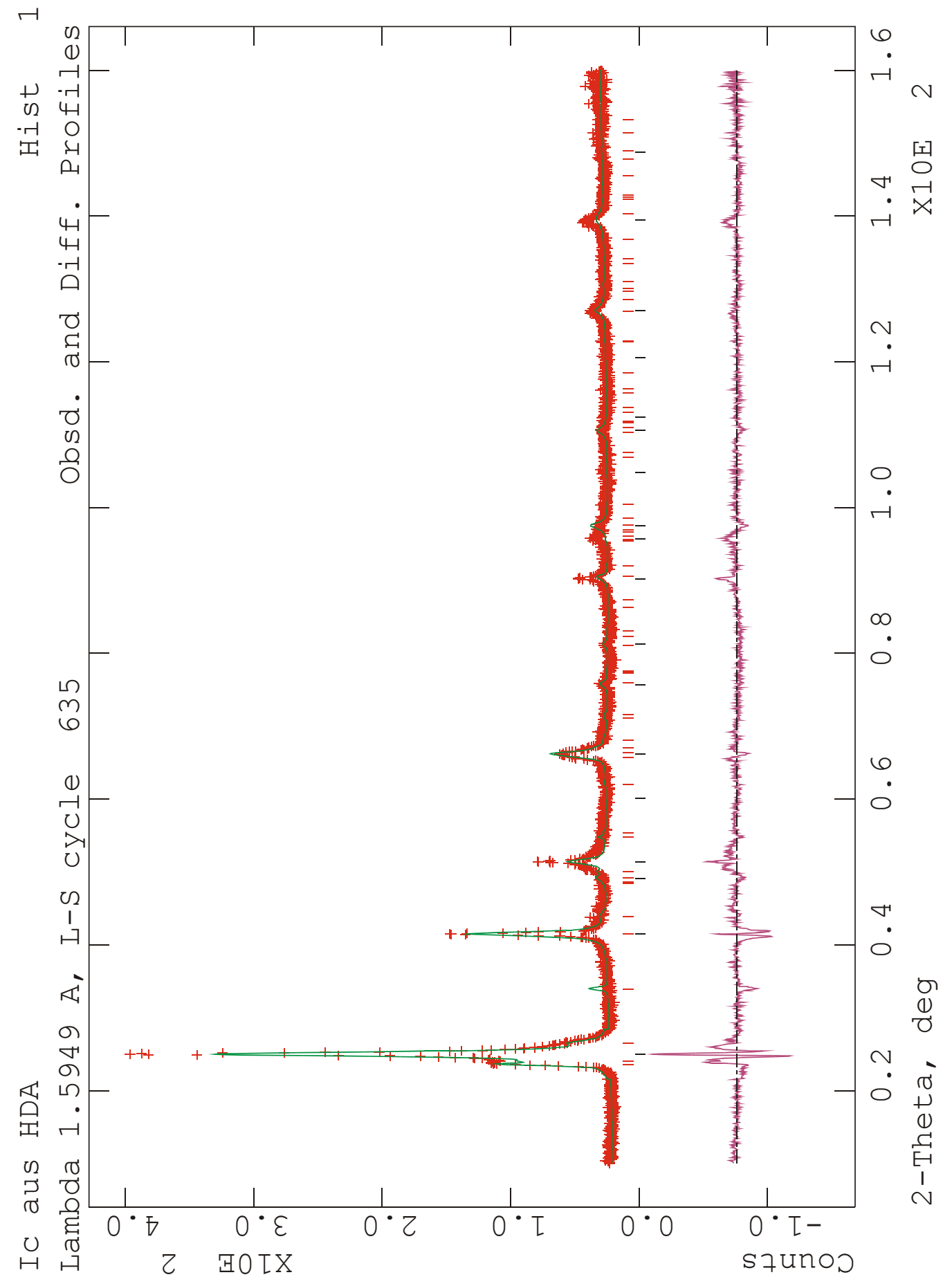

Abbildung 8.3.3.1.2.a: Rietveld-Anpassung des kubischen Eis Ic, hergestellt aus der Hochdruckeisphase HDA bei Umgebungsdruck p 1bar und einer Temperatur von T 180K. I : Reflexmarker Eis IcPhasenanteil, I : Reflexmarker Eis Ih-Phasenanteil. 


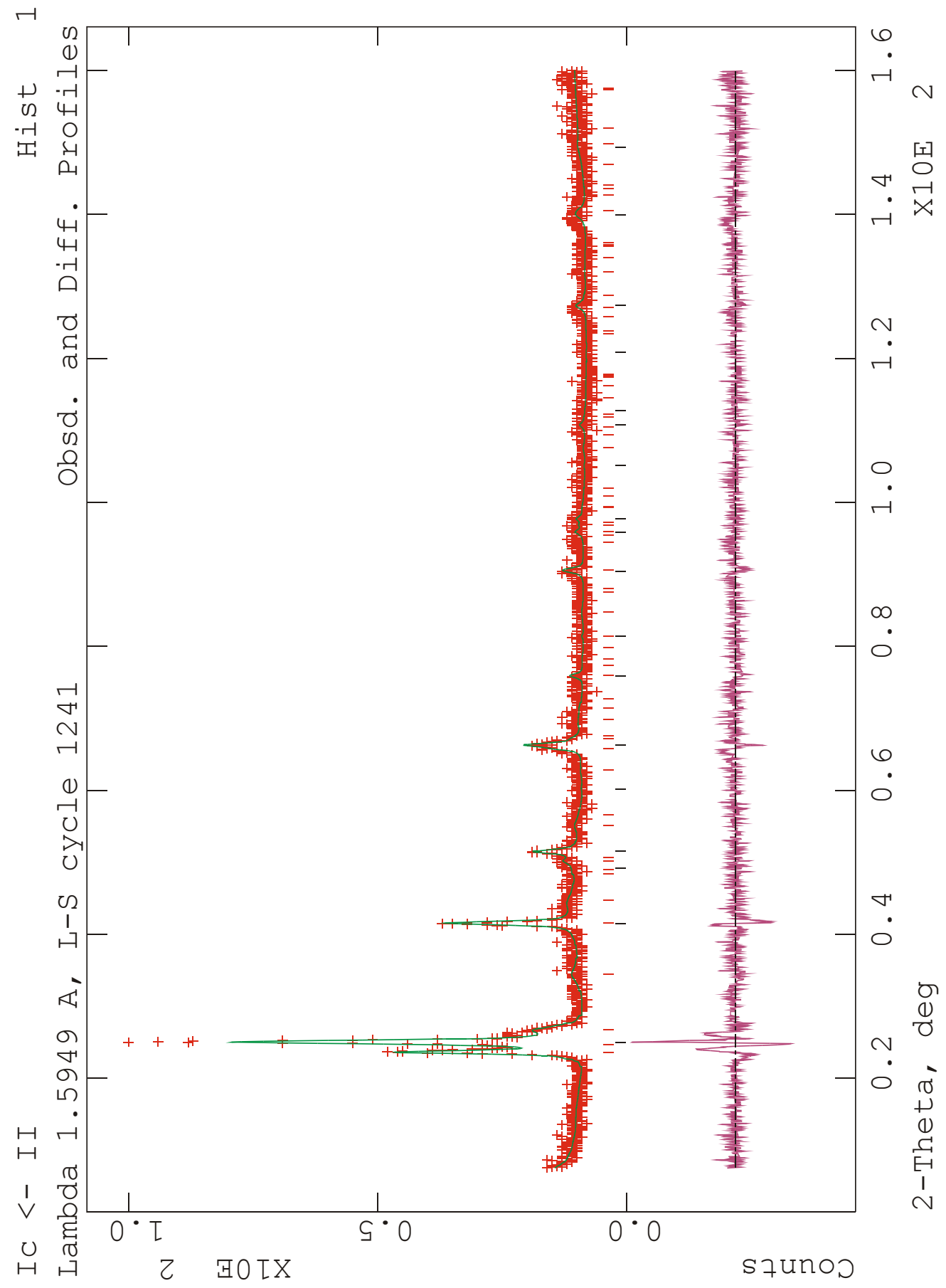

Abbildung 8.3.3.1.2.b: Rietveld-Anpassung des kubischen Eis Ic, hergestellt aus der Hochdruckeisphase Eis II bei Umgebungsdruck p 1bar und einer Temperatur von T 180K. I : Reflexmarker Eis IcPhasenanteil, I : Reflexmarker Eis Ih-Phasenanteil. 


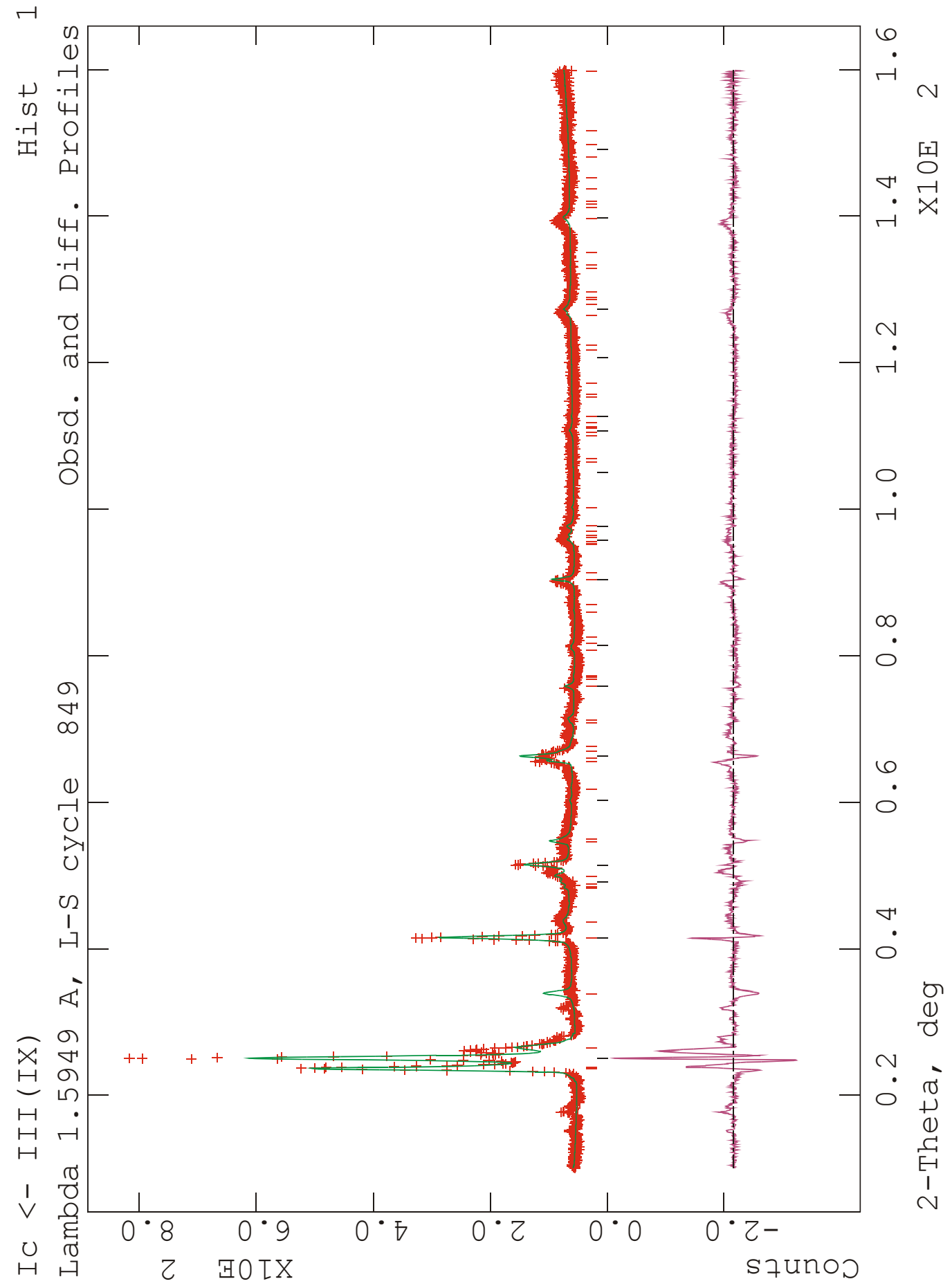

Abbildung 8.3.3.1.2.c : Rietveld-Anpassung des kubischen Eis Ic, hergestellt aus der Hochdruckeisphase Eis III/IX bei Umgebungsdruck p 1bar und einer Temperatur von T 180K. I : Reflexmarker Eis IcPhasenanteil, I : Reflexmarker Eis Ih-Phasenanteil. 


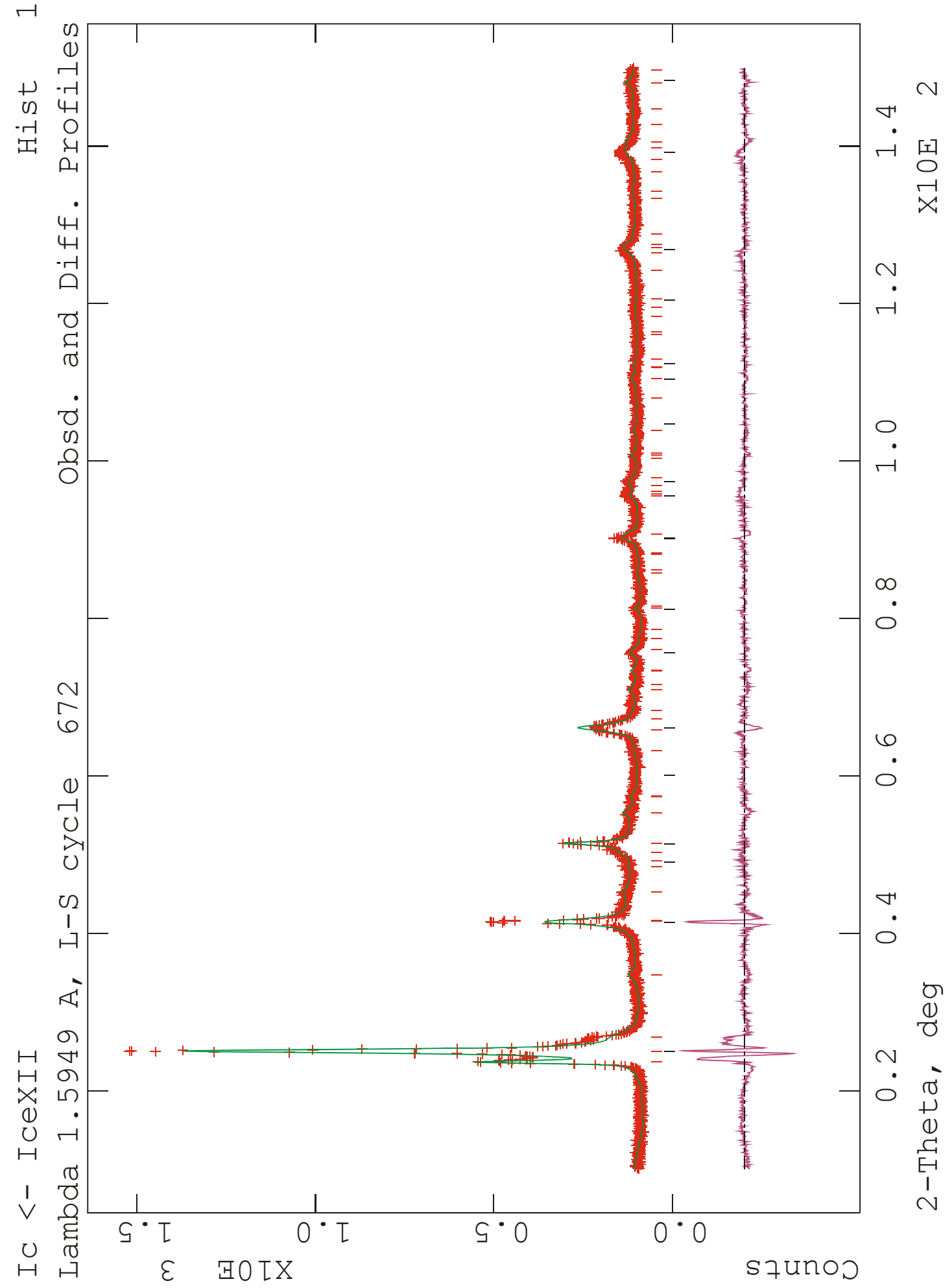

Abbildung 8.3.3.1.2.d: Rietveld-Anpassung des kubischen Eis Ic, hergestellt aus der Hochdruckeisphase Eis XII bei Umgebungsdruck p 1bar und einer Temperatur von T 180K. I : Reflexmarker Eis IcPhasenanteil, I : Reflexmarker Eis Ih-Phasenanteil. 


\subsection{Strukturelle Untersuchungen an kubischem Eis Ic : Peakprofilparameter}

In der folgenden Tabelle 8.3.3.1.3.1 sind die mittels Rietveld-Verfahren bestimmten isotropen thermischen Auslenkungsparameter und die Reflexprofilparameter dargestellt, aus denen über die in Kapitel 2.2.1 angegebenen Gleichungen die Partikelgrößen sowie die Größe der Stapelfehlordnungsbereiche des kubischen sowie des „gestörten hexagonalen“ Phasenanteils berechnet werden.

\begin{tabular}{|c|c|c|c|c|c|c|}
\hline \multirow{4}{*}{ Ic } & & HDA & V & XII & III/IX & II \\
\cline { 2 - 7 } & $\mathrm{X}$ & $57.31(2.98)$ & $50.84(2.52)$ & $67.82(2.86)$ & $31.42(3.38)$ & $43.41(3.86)$ \\
\cline { 2 - 7 } & $\mathrm{Xe}$ & - & - & - & - & - \\
\cline { 2 - 7 } & $\mathrm{Xs}$ & - & - & - & - & - \\
\cline { 2 - 7 } & $\mathrm{O}_{\text {Uiso }}$ & $0.05099(630)$ & $0.05068(528)$ & $0.04501(486)$ & $0.05447(933)$ & $0.03908(620)$ \\
\cline { 2 - 7 } & $\mathrm{D}_{\text {Uiso }}$ & $0.04262(420)$ & $0.05313(510)$ & $0.05338(546)$ & $0.05562(873)$ & $0.05447(580)$ \\
\hline \multirow{5}{*}{ Ih“6 } & $\mathrm{X}$ & $141.80(6.29)$ & $13.65(4.11)$ & $20.41(2.7)$ & $4.14(3.11)$ & $29.05(5.31)$ \\
\cline { 2 - 7 } & $\mathrm{Xe}$ & $94.05(10.12)$ & $17.74(8.47)$ & $20.42(3.2)$ & $44.89(11.58)$ & $252.4(118)$ \\
\cline { 2 - 7 } & $\mathrm{Xs}$ & $86.65(40.05)$ & $126.20(12.01)$ & $16.42(3.20)$ & $126.90(28.14)$ & $175.7(50.1)$ \\
\cline { 2 - 7 } & $\mathrm{O}_{\text {Uiso* }}$ & 0.03965 & 0.025 & 0.025 & 0.08572 & 0.02751 \\
\cline { 2 - 7 } & $\mathrm{D}_{\text {Uiso* }}$ & 0.07102 & 0.05810 & 0.035 & 0.05509 & 0.07151 \\
\cline { 2 - 7 } & $\mathrm{D}_{\text {Uiso* }}$ & 0.07102 & 0.05466 & 0.038 & 0.07141 & 0.06333 \\
\hline
\end{tabular}

Tabelle 8.3.3.1.3.1: verfeinerte Reflexprofilparameter sowie verfeinerte und manuell angepasste thermischeAuslenkungsparameter. Als Basis für die Verfeinerungen der Reflexprofilparameter wurden an einem Silizium-Standard dieWellenlänge $\mathrm{zu} \lambda=1.594939 \AA$ sowie die Reflexprofilparameter $\mathrm{zu} U=67.3$, $V=-227.1, W=229.7$ sowie der Peakasymmetrieparameter $\mathrm{zu}$ asym=2.4643 festgelegt. Die Parameter $X, X e$, Xs entsprechen den Parametern LX, Ptec, Sfec der in der GSAS-Peakprofilfunktion Nr. 2 definierten Parametern. - : Reflexprofilparameter wurden aufgrund des kubischen Eis Ic-Modells nicht verfeinert. * : Verfeinerungsparameter wurden zum Teil frei sowie bei der Divergenz des Modells manuell festgesetzt.

\subsection{Strukturelle Untersuchungen an kubischem Eis Ic: Quantitative Phasenanteile an kubischem Eis Ic mittels Einzelreflexfits}

Um eine quantitative Aussage der kubischen Streubeiträge zu ermöglichen und die Ergebnisse der Rietveld-Verfeinerung hinsichtlich der Phasenanteile an kubischem Eis Ic zu verifizieren, wurde der Gesamtstreubeitrag in dem Winkelbereich von $2 \theta \sim 20^{\circ}$ bis $2 \theta \sim 30^{\circ}$ durch Integration der gemessenen Diffraktogramme bestimmt und in einen prozentualen Zusammenhang zu den bestimmten integrierten Intensitäten des kubischen [111]- Reflexes gesetzt. Für einen quantitativen Vergleich der gemessenen Reflexprofile hinsichtlich der Intensitätsverhältnisse sowie der Reflex-Halbwertsbreiten, der aus den verschiedenen Hochdruckeisphasen gewonnenen kubischen Eisphasen, wurde mit den von Kuhs et al. (1987) bestimmten Eis Ic-Atomparametern die kubische Eisphase mit denselben experimentellen Instrumentparametern des D2B am ILL (Wellenlänge, Nullpunkt, Profilparameter) simuliert. Durch den Vergleich sollten sich zumindest qualitative Rückschlüsse auf die strukturellen 
Unterschiede ziehen lassen. Für einen strukturellen Vergleich des aus unterschiedlichen Hochdruckeisphasen gewonnenen kubischen Phasenanteiles wurden die kubisch indizierbaren Reflexe über einzelne Fit-Anpassungen detailliert bestimmt und mit der Simulation eines Diffraktogramms auf Basis der Strukturparameter von Kuhs et al. (1987) hinsichtlich der Intensitätsverhältnisse sowie den Reflexprofilparametern verglichen. Das zur Strukturbestimmung verwendete Eis Ic wurde von Kuhs et al. (1987) über die von Bertie et al. (1963) angegebene Tieftemperaturroute aus Eis II gewonnen. Mittels Gauß- und Lorentzfits wurden sowohl für die experimentell ermittelten sowie für die simulierten Reflexe die integrierten Intensitäten als auch die Halbwertsbreiten bestimmt. Die simulierten Intensitäten wurden auf den [111]-Reflex normiert. Für die Halbwertsbreiten wurde die Abweichung für den jeweiligen hkl-Wert von den simulierten Halbwertsbreiten bestimmt. Durch die unterschiedlichen Massenanteile, bedingt durch die Präparation und den Transfer der Proben in die in situ Messapparaturen, wurde eine Normierung der Streubeiträge nötig, um z.B. quantitative Aussagen hinsichtlich der Phasenanteile der kubischen Eisphase sowie der als fehlgeordneten hexagonalen Phasenanteile zu ermöglichen. Um die Vergleichbarkeit der experimentell erfassten Reflexintensitäten der Eisphasen zu gewährleisten, wurden diese nach der Festlegung des Untergrundes und dessen Subtraktion auf eine ,massen- und zählratennormalisierte Intensität" normiert. Aufgrund der unterschiedlichen Probenmassen ergeben sich Abweichungen in der Messstatistik, die sich z.B. für Eis IX und Eis II in einer größeren Schwankungsbreite der Intensitäten äußern. Der mittlere Streubeitrag des Winkelbereiches von $20^{\circ}$ bis $30^{\circ}$ in $2 \Theta$ für alle kubischen Eisphasen auf $\sim 50 \%$ bis $\sim 70 \% \mathrm{zu}$ beziffern ist.

Für diese Auswertungen stellte sich die Bestimmung der integrierten Reflexintensitäten der kubischen Eisphase als problematisch heraus. Das Intensitätsprofil für kubisches Eis Ic ergibt sich aus der Überlagerung der Streubeiträge der kubischen Eisphase und durch die von Kuhs et al. (1987) angegebenen fehlgeordneten Phasenanteile.

Um quantitative Aussagen hinsichtlich der Phasenanteile der rein kubischen Phase und den fehlgeordneten Anteilen zu ermöglichen, wurden mehrere Versuche durchgeführt die Intensitätsprofile mit unterschiedlichen Funktionen zu fitten. 
Verwendung fanden hierbei Lorentz- und Gaußfunktionen, mit den Parametern w für die Halbwertsbreite, A als integrierte Intensität, $\mathrm{x}_{\mathrm{c}}$ als Peakposition sowie $\mathrm{y}_{0}$ für den UntergrundOffset:

$$
\begin{gathered}
\text { Lorentz }: y=y_{o}+\frac{2 A}{\pi} \cdot \frac{w}{4\left(x-x_{c}\right)^{2}+w^{2}} \\
\text { Gau } \beta: y=y_{o}+\frac{A}{w \sqrt{\frac{\pi}{2}}} \cdot e^{\frac{-2\left(x-x_{c}\right)^{2}}{w^{2}}}
\end{gathered}
$$

Die Parameter $\mathrm{y}_{0}, \mathrm{~A}, \mathrm{x}_{\mathrm{c}}$ und wurden über $\mathrm{Chi}^{2}$-fits angepasst und gegebenenfalls weiter manuell verfeinert. Aus den Diffraktogrammen (vergl. Abbildung 3.7, Kapitel 3.3.1 sowie den im Anhang 8.3.3.1.2 dargestellten Abbildungen 8.3.3.1.2. (a) - (d) ergibt sich, dass die Basen der Peaks z.B. des [222]- und des [331]-Peaks und damit auch des [111]-Peaks des kubischen Eises verbreitert sind. Für die Peakanpassung der kubischen Peaks wie auch des als hexagonalen [101]-Peak bezeichneten Streubeitrages wurden daher Lorentzfunktionen verwendet.

Die besten Anpassungen der Diffraktogramme ergaben sich durch die Überlagerung von zwei Lorentzfunktionen zur Anpassung des kubischen [111]-Reflexes und des hexagonalen [101]Streubeitrages sowie einer Anpassung des durch Warren-Anteile asymmetrischen hexagonalen [100]-Peak durch zwei Gaußfits. Die Asymmetrie des hexagonalen [100]-Peaks wurde durch einen zusätzlichen Peak, der die lateralen Streubeiträge enthält, beschrieben. An dieser Stelle ist anzumerken, dass ein gleichzeitiges Anpassen aller Peaks durch vier Fitfunktionen mittels ein Least-Squares-Verfahren aufgrund der hohen Parameterzahl. divergierte.

Die Profilanpassungen durch Überlagerung von Gauß- und Lorentzfits stellte sich als sensibel gegenüber der Anzahl der zugelassenen Peaks sowie der Festlegung der Halbwertsbreiten, z.B. des kubischen [111]- Reflexes und der hexagonalen [100]- und [101]- Reflexe heraus. Fitversuche mit einer fünffach-Peaküberlagerung zur besseren Beschreibung des WarrenAnteiles des hexagonalen [100]-Reflexes durch asymmetrische Beta-Funktionen sowie zur Separation des Streubeitrages des als hexagonal [101]- zu vermuteten Reflexes in einen Untergrundanteil und einen rein hexagonalen [101]-Peak, führten zu keinem verwertbaren 
Ergebnis. Es wurden daher zuerst die Lorentzfunktionen in einem ersten Schritt angefittet. In einem zweiten Schritt wurden dann die Gaußfunktionen angepasst. Die Stützstellen für die Anpassung der o.g. Funktionen wurden manuell auf das Intensitätsmaximum sowie die durch die Reflexflanken interpolierbaren Reflexhalbwertsbreiten festgelegt. Das Zweischrittverfahren unter Verwendung des $\mathrm{Chi}^{2}$-Tests führte $\mathrm{zu}$ einer wesentlich stabileren Anpassung an die experimentellen sowie die simulierten Daten und ergab bessere Reflexprofilanpassungen. Bei der Anpassung des hexagonalen [101]-Reflexes handelt es sich daher bei allen Fits um eine Überlagerung mit einem Untergrundanteil. Die folgende Abbildung 8.3.3.1.4.1 zeigt exemplarisch den für Eis Ic (XII) gefitteten Intensitätsverlauf in dem Winkelbereich von $20^{\circ} \leq 2 \Theta \leq 30^{\circ}$.

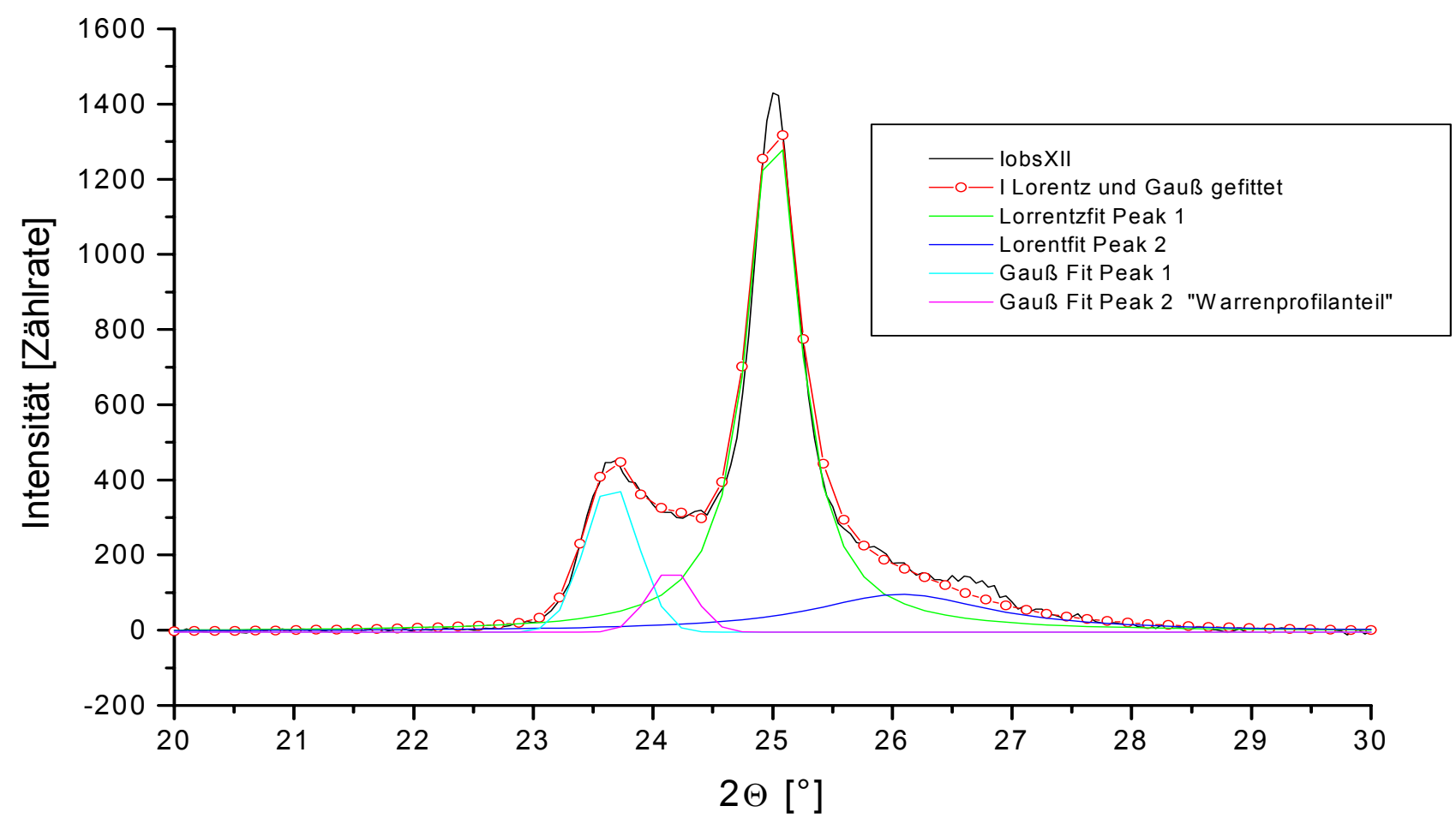

\begin{abstract}
Abbildung 8.3.3.1.4.1 : Darstellung der gefitteten Intensitätsverlaufes durch Überlagerung von Lorentzund Gaußfunktionen von Ic aus Eis XII. Fit besteht aus Überlagerung zweier Gaußfits, Gauß Fit Peak 1 und Gauß Fit Peak 2 (,Warrenprofil-Anteil“), sowie zweier Lorentzfits, Lorentzfit Peak1 und Lorentzfit Peak2. IobsXII : gemessenes Reflexprofil. Die Anpassung der Stützstellen wurde manuell auf das Intensitätsmaximum, der Halbwertsbreitenintensität der Position des jeweiligen angenommenen Peaks festgelegt und nach dem $\mathrm{Chi}^{2}$-Test minimiert. Für die Anpassung der Gauß- und Lorentzfunktionen an die gemessenen Intensitätsprofile wurde das Programm ORIGIN in der Version 5.0 von Fa. Microcal verwendet.
\end{abstract}


Dargestellt ist der gemessene Intensitätsverlauf und die durch Gauß- und Lorentzfunktionen angefitteten überlagerten Intensitätsverläufe. Aus dem Diagramm ist ersichtlich, dass die Fitanpassungen für eine erste quantitative Aussage der reinen sowie der fehlgeordneten Phasenanteile im Rahmen der Messstatistik eine hinreichende Genauigkeit besitzen. Abweichungen zeigen sich hinsichtlich der Peakprofil-Anpassung sowie der Intensität am hexagonalen [100]-, verschobenen hexagonalen [101]- sowie am kubischen [111]-Peak. Die Abweichungen lassen sich auf einen Fehler von $\sim 3 \sigma$ festlegen. Weiterhin wurden aus den Diffraktogrammen der unterschiedlichen kubischen Eisphasen die Peakparameter der [220]-, [222]-, [331]- und des [440]- Reflexes bestimmt. Höher indizierte Reflexe wurden nicht berücksichtigt, da die gefitteten Peakprofile wesentlich größere Fehler aufwiesen. In der Tabelle 8.3.3.1.4.1 sind die gefitteten Reflexparameter des symmetrischen [100]-hexagonalen Peaks, des separierten Warrenanteiles sowie die des mit einem Untergrundpeak überlagerten [101]-hexagonalen Reflexes aufgelistet.

\begin{tabular}{|c|c|c|c|c|}
\hline Reflex & $\begin{array}{c}\text { Ausgangsphase } \\
\text { von } \\
\text { Eis Ic }\end{array}$ & $\begin{array}{c}\text { Intensitätsmaxima } \\
\mathbf{2 \Theta} \text { [ }^{\circ}\end{array}$ & $\begin{array}{c}\text { I int } \\
\text { (gefittete integrale } \\
\text { Intensitäten) }\end{array}$ & $\begin{array}{c}\text { HWB } \\
\text { (Halbwertsbreite, } \\
\text { gefittet) }\end{array}$ \\
\hline$[\mathbf{1 0 0 ]}$ hex & HDA & 23.749 & 56 & 0.511 \\
\hline & V & 23.664 & 207 & 0.417 \\
\hline & XII & 23.653 & 220 & 0.443 \\
\hline & III & 23.654 & 484 & 0.384 \\
\hline W-Profil & II & 23.713 & 825 & 0.400 \\
\hline & VA & 24.263 & 24 & 0.319 \\
\hline & XII & 24.137 & 102 & 0.423 \\
\hline & III & 24.152 & 80 & 0.383 \\
\hline$[\mathbf{1 0 1}]$ hex & II & 24.096 & 128 & 0.374 \\
\hline & HDA & 24.150 & 88 & 0.507 \\
\hline & V & 26.059 & 499 & 2.604 \\
\hline & XII & 26.073 & 274 & 1.300 \\
\hline & III & 26.144 & 523 & 1.800 \\
\hline & II & 26.240 & 339 & 0.870 \\
\hline
\end{tabular}

Anmerkung: Die Fits wurden manuell angepasst, da aufgrund der hohen Parameterzahl eine freie Chi ${ }^{2}-$ Minimierung zu einer Divergenz des Fitverfahrens führte

Tabelle 8.3.3.1.4.1: Profilparameter für die durch Gauß- und Lorentzfits bestimmten Reflexanteile am kubischen [111]-Reflex. [100]-hex : nicht im kubischen zu indizierender Reflex, W-Profil : gefitteter Warren-Anteil, [101]-hex : gefittete Überlagerung eines unscharfen Untergrundpeaks sowie je nach kubischer Eisphase zu vermutenden scharfen hexagonalen [101]-Reflexes. Aufgrund der Überlagerung wird der scharfe Anteil des [101]-Reflexes nicht erfasst. Die integralen Intensitäten des separierten [111]kubischen Reflexes sind in Tabelle 8.3.3.1.4.2 enthalten.

Die gefitteten sowie simulierten Intensitäten sind im Verhältnis zueinander sowie die Halbwertsbreiten und deren Abweichungen in der folgenden Tabelle 8.3.3.1.4.2 aufgelistet. 
Der Vergleich der bestimmten Reflexprofile zeigt, dass keine Korrelation zwischen den bestimmten Parametern wie den Halbwertsbreiten oder den integralen Intensitäten zu erkennen ist.

\begin{tabular}{|c|c|c|c|c|c|c|c|c|}
\hline Reflex & Ic aus & $\begin{array}{c}\text { Int. } \\
\text { I(Fit) }\end{array}$ & $\begin{array}{c}\mathbf{I}_{\mathrm{hkl}} / \mathbf{I}_{111} \\
\text { (simuliert) }\end{array}$ & $\begin{array}{l}\mathbf{I}_{\text {hkl }} / \mathbf{I}_{111} \\
\text { (gefittet) }\end{array}$ & $2 \theta\left[{ }^{\circ}\right]$ & $\begin{array}{c}\text { HWB } \\
\text { (gefittet) }\end{array}$ & $\begin{array}{l}\text { Ic-HWB } \\
\text { D2B-sim. }\end{array}$ & $\triangle H W B$ \\
\hline \multirow{5}{*}{ [111] } & HDA & 1153 & \multirow{5}{*}{1} & 1 & 25.006 & 0.538 & \multirow{5}{*}{$0.39(4)$} & $0.148(4)$ \\
\hline & $\mathrm{V}$ & 1106 & & 1 & 25.017 & 0.506 & & $0.116(4)$ \\
\hline & XII & 1122 & & 1 & 25.009 & 0.514 & & $0.124(4)$ \\
\hline & III & 886 & & 1 & 25.076 & 0.390 & & $0(4)$ \\
\hline & II & 825 & & 1 & 25.067 & 0.400 & & $0.01(4)$ \\
\hline \multirow{5}{*}{ [220] } & HDA & $287(12)$ & \multirow{5}{*}{0.261} & 0.249 & $41.49(1)$ & $0.39(1)$ & \multirow{5}{*}{$0.37(4)$} & $0.02(4)$ \\
\hline & $\mathrm{V}$ & $282(12)$ & & 0.255 & $41.47(1)$ & $0.41(1)$ & & $0.04(4)$ \\
\hline & XII & $288(15)$ & & 0.256 & $41.46(1)$ & $0.45(3)$ & & $0.07(4)$ \\
\hline & III & $436(57)$ & & 0.492 & $41.47(1)$ & $0.33(6)$ & & $-0.04(6)$ \\
\hline & II & $306(27)$ & & 0.379 & $41.49(1)$ & $0.49(9)$ & & $0.12(9)$ \\
\hline \multirow{5}{*}{ [222] } & HDA & $182(18)$ & \multirow{5}{*}{0.147} & 0.157 & $51.41(3)$ & $0.67(9)$ & \multirow{5}{*}{$0.36(9)$} & $0.31(9)$ \\
\hline & $\mathrm{V}$ & $133(16)$ & & 0.120 & $51.43(3)$ & $0.50(6)$ & & $0.14(6)$ \\
\hline & XII & $159(18)$ & & 0.142 & $51.41(1)$ & $0.59(9)$ & & $0.23(9)$ \\
\hline & III & $102(61)$ & & 0.115 & $51.49(6)$ & $0.36(21)$ & & $0(21)$ \\
\hline & II & $134(27)$ & & 0.162 & $51.47(9)$ & $0.64(12)$ & & $0.28(12)$ \\
\hline \multirow{5}{*}{ [331] } & HDA & $282(33)$ & \multirow{5}{*}{0.206} & 0.245 & $66.16(3)$ & $1.27(15)$ & \multirow{5}{*}{$0.33(10)$} & $0.94(15)$ \\
\hline & $\mathrm{V}$ & 291(36) & & 0.263 & $66.11(3)$ & $1.41(18)$ & & $1.08(18)$ \\
\hline & XII & $265(18)$ & & 0.237 & $66.10(3)$ & $1.33(12)$ & & $1.0(12)$ \\
\hline & III & - & & - & - & - & & - \\
\hline & II & $283(33)$ & & 0.343 & $66.20(6)$ & $1.35(21)$ & & $1.023(21)$ \\
\hline \multirow{5}{*}{ [440] } & HDA & $71(12)$ & \multirow{5}{*}{0.0756} & 0.0616 & $90.25(2)$ & $0.54(9)$ & \multirow{5}{*}{$0.29(9)$} & $0.25(9)$ \\
\hline & $\mathrm{V}$ & $83(18)$ & & 0.0750 & $90.19(6)$ & $0.95(21)$ & & $0.66(21)$ \\
\hline & XII & $64(9)$ & & 0.0570 & $90.18(2)$ & $0.70(12)$ & & $0.41(12)$ \\
\hline & III & - & & - & - & - & & - \\
\hline & II & $83(27)$ & & 0.101 & $90.22(1)$ & $1.06(45)$ & & $0.77(45)$ \\
\hline
\end{tabular}

Tabelle 8.3.3.1.4.2: Der [331]- sowie der [440]- Reflex für das aus der Hochdruckeisphase Eis IX/III gewonnenen kubischen Eis Ic konnten aufgrund ihrer geringen Intensitäten nicht mehr mit hinreichender Genauigkeit gefittet werden. $I_{h k l} / I_{111}$ (simuliert) : auf [111]-Reflex normiertes Verhältnis der simulierten integralen Intensitäten. $I_{h k} / I_{111}$ (gefittet) : auf [111]-Reflex normiertes Verhältnis der experimentell bestimmten und gefitteten integralen Intensitäten. $\triangle H W B$ : Differenz der gefitteten Halbwertsbreite zur Halbwertsbreite des jeweiligen simulierten Reflexes 
Die Anpassungen für die kubischen Eisphasen Ic (HDA), Ic (V), Ic (II), Ic (IX) sind von vergleichbarer Qualität, jedoch ergeben sich aufgrund der Normierung für Ic (II) sowie Ic (IX) größere Standardabweichungen. Je nach Ausgangsphase weichen die integrierten Intensitäten der jeweils gefitteten Streubeiträge, die ermittelten Halbwertsbreiten sowie die Lage der Intensitätsmaxima signifikant voneinander ab. Durch Anpassung des asymmetrischen [100]-Peaks durch Gaußfunktionen für einen scharfen symmetrischen sowie einen Warren-Anteil lassen sich sowohl qualitative Aussagen über die unterschiedliche Quantität der fehlgeordneten Bereiche als auch auf eine unterschiedliches Ausdehnung der kohärenten Domänen der Stapelfehler hin treffen. Der jeweilige Anteil der reinen und geordneten kubischen Eisphase ist der Tabelle 8.3.3.1.4.3 zu entnehmen.

Aufgrund der als Funktion des Winkels abfallenden Intensitäten im Verhältnis zur Schwankungsbreite des Untergrundes war es nicht möglich, die höheren Reflexe durch hinreichend genaue Reflexfits in rein kubische sowie fehlgeordnete Anteile zu separieren. Ausgehend von den, auf Basis der Arbeit von Kuhs et al. (1987) simulierten Reflexen der kubischen Eisphase weisen die Halbwertsbreiten sowie die experimentell ermittelten

integrierten Intensitäten der kubisch indizierbaren Reflexe signifikante Variationen auf. Vergleicht man die simulierten Reflexparameter des kubischen Eis Ic, gewonnen aus der Hochdruckeisphase Eis II, mit den in den eigenen Messungen aus derselben Hochdruckeisphase gewonnenen Daten, so zeigen sich hierbei signifikante Abweichungen. Obwohl es sich hier um ein Einzelexperiment mit geringerer statistischer Aussagefähigkeit handelt, lässt sich doch ein Einfluss der strukturellen Eigenschaften und der Historie der Ausgangsphase auf die nach der Phasenumwandlung erhaltene neue Struktur zeigen (vergl. Kapitel 1).

\begin{tabular}{|c|c|c|c|c|c|}
\hline Ausgangseisphase von Eis Ic & HDA & V & XII & IX(III) & II \\
\hline Phasenanteil rein kubisches Eis Ic [\%] & 76 & 73 & 74 & 47 & 51 \\
\hline Enthalpieänderung $\mathbf{\Delta} \mathbf{H}\left[\mathbf{J} / \mathbf{m o l}^{-\mathbf{1}}\right] \mathbf{H D}->\mathbf{I c}$ & - & $-915(4)$ & - & $-340(3)$ & $-54(5)$ \\
\hline
\end{tabular}

Tabelle 8.3.3.1.4.3: Phasenanteile an reinem kubischen Eis Ic, ermittelt durch Reflex-Fits, $\Delta H$ : gemessene Enthalpiedifferenz beim Übergang von kubischem Eis Ic in Eis Ih, Handa et al. (1988), - : keine verfügbare Daten

Eine strukturelle Interpretation der in Tabelle 8.3.3.1.4.2 ermittelten Reflexprofile durch Stapelfehleranteile (Reflexverbreiterung), laterale Fehlordnungen (Warren-Profil, A-18 
Asymmetrie der Reflexe) sowie eventuell vorhandener interner Eigenspannungen der Kristallite (Reflexverschiebungen) als Funktion bzw. Eigenschaft der Ursprungsphase ist aufgrund der vielfältigen Möglichkeiten die gemessenen Streubeiträge durch die oben genannten physikalischen Effekte beschreiben zu können nicht möglich. Die von Handa et al. (1988) für unterschiedlich hergestellte kubische Eisphasen vermuteten strukturellen Abweichungen sind konsistent $\mathrm{zu}$ den durch die in situ Strukturbestimmung bestimmten deutlich feststellbaren Differenzen in den Diffraktogrammen.

Markant ist jedoch, dass die prozentualen Anteile des reinen kubischen Eis Ic der Probe, hergestellt aus den protonengeordneten Hochdruckeisphasen Eis III/IX bzw. Eis II signifikant von den aus den anderen HD-Eisphasen hergestelltem Eis Ic abweichen (vergl. Tabelle 8.3.3.1.4.3).

\subsubsection{Kinetik der Phasenübergange in kubisches Eis Ic}

In den folgenden Abbildungen 8.3.3.2.a bis 8.3.3.2.d sind im Überblick die zeitlichen Verläufe der Phasenübergange aus der jeweiligen Hochdruckeisphase in kubisches Eis Ic bei einer Temperatur von $\mathrm{T} \sim 143 \mathrm{~K}$ zum Zeitpunkt $\mathrm{t}>\mathrm{t}_{0}$ anhand der Diffraktogramme dargestellt. Die zeitliche Abfolge ist farblich kodiert und ist in den Abbildungen von hinten nach vorne zu lesen. $\mathrm{Zu}$ den Intensitäten der einzelnen Diffraktogramme ist in der zeitlichen Abfolge darstellungstechnisch ein Offset dazu addiert. Der zeitliche Verlauf der integralen Intensitäten wurde durch Einzelreflexfits der in den Abbildungen indizierten Reflexe in der zeitlichen Abfolge bestimmt. Die gemäß dem beschriebenem Anpassungsverfahren durch Gauß- sowie Lorentzprofile (vergl. Anhang 8.3.3.1.4) bestimmt. Die verschieden hohen integralen Intensitäten sind bei den verschiedenen Messungen durch unterschiedliche Monitorraten am D1B verursacht. Die zur Bestimmung des Phasenanteiles ausgewählten bzw. verfügbaren Reflexe sind in den Abbildungen dargestellt und indiziert. Im Gegensatz zu den polykristallinen Ausgangsphasen Eis XII und Eis V wiesen die Eisphasen Eis IX/III sowie Eis II ausgeprägte Vorzugsorientierungen aus, die in situ während des Experimentes am D1B durch den Vergleich der Reflexintensitäten der Ausgangsphasen festgestellt werden konnten. 


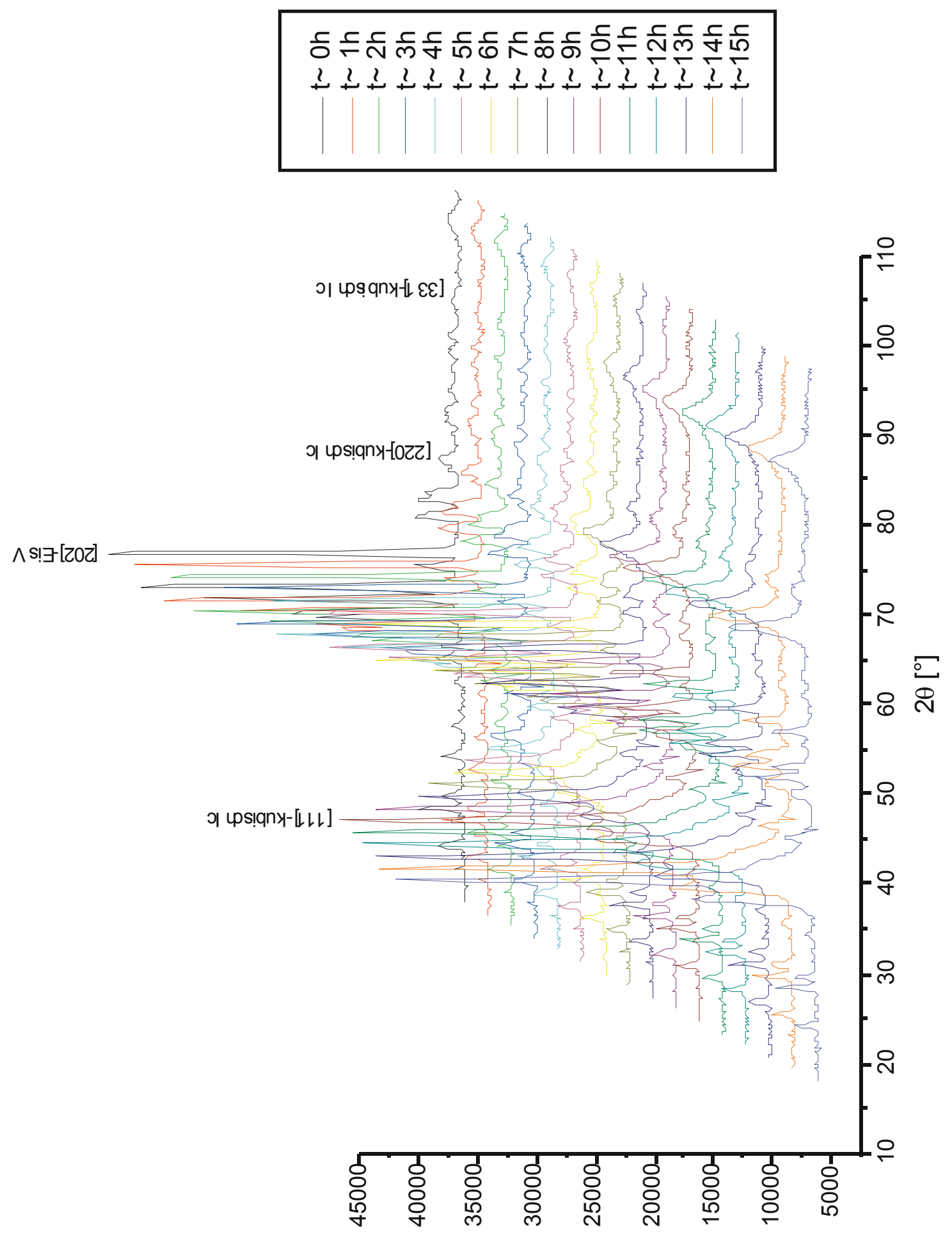

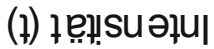

\begin{abstract}
Abbildung 8.3.3.2.a: zeitlicher Verlauf des Phasenüberganges von Eis $V \rightarrow \rightarrow$ Eis Ic bei der Phasenübergangstemperatur von T 143K. Für die Ausgangsphase Eis $V$ ist zur Orientierung der [202]Reflex bei $2 \theta=56.88^{\circ}$ eingezeichnet. Die anderen Eis V-Reflexe, der [022]- sowie der $[213] /[220]-R e f l e x$ sind in dieser Abbildung bei den Winkeln $2 \theta_{[022]}=51.82^{\circ}$ sowie bei $2 \theta_{[213] /[220]}=53.06^{\circ}$ zu finden. Für die kubische Eisphase ist der [111]- sowie der [220]-Reflex dargestellt. Die zusätzlichen ,hexagonalen“ Peaks der kubischen Eisphase sind in diesem Diagramm nicht explizit dargelegt, zur Lage vergl. Abbildung 8.3.3.2.b.
\end{abstract}




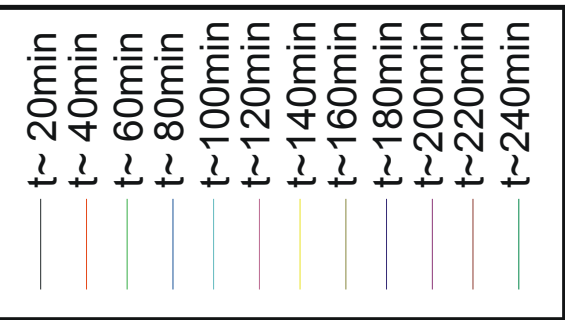

ગ ups!qny-[เE\&]

ગ ups!̣ny-[0zZ]

IIX S! $\exists[0 เ \varepsilon]$

IIX S! $\exists[L L Z]$

IIX s! $\exists[0 Z Z]$

ગ |еuобехәч-[LOL]

ગ ups!qny-[レル]

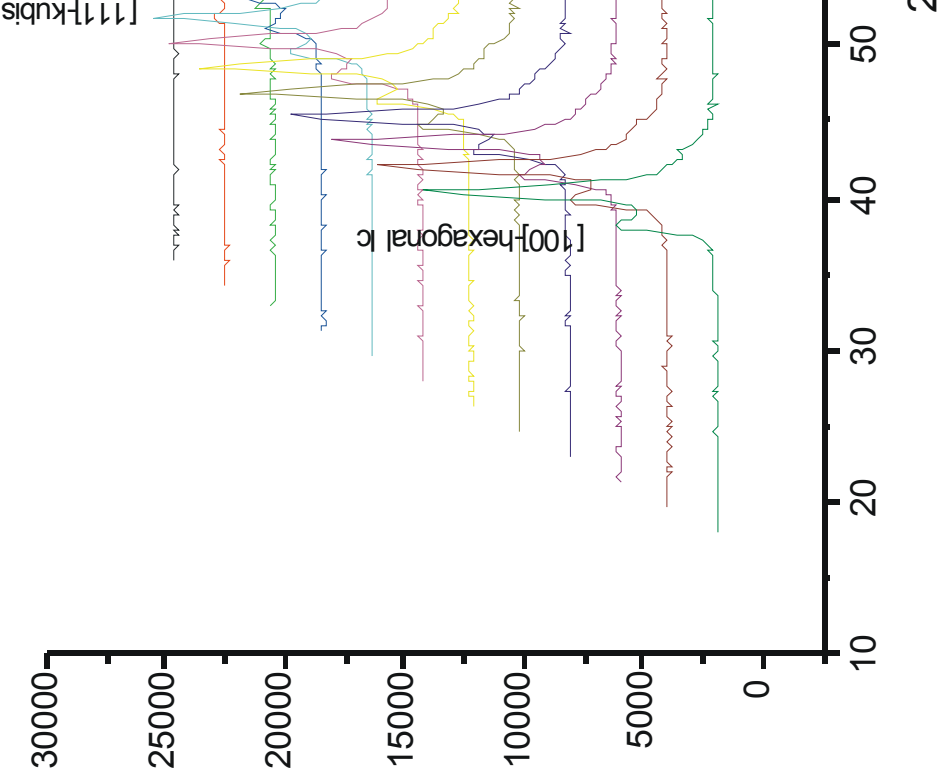

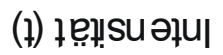

Abbildung 8.3.3.2.b: zeitlicher Verlauf des Phasenüberganges der metastabilen Eisphase Eis XII $\rightarrow$ Eis Ic bei der Phasenübergangstemperatur von T 143K. In der Abbildung sind die kubischen Reflexe, der [100]-hexagonale, der [111]-kubische sowie der [101]-hexagonale Reflex der kubischen Eisphase sowie die Reflexe des Eis XII indiziert und eingezeichnet. Zur Bestimmung des zeitlichen Verlaufes der Ausgangsphase Eis XII wurden der [220]-, der [211]- sowie der [310]-Reflex herangezogen. 


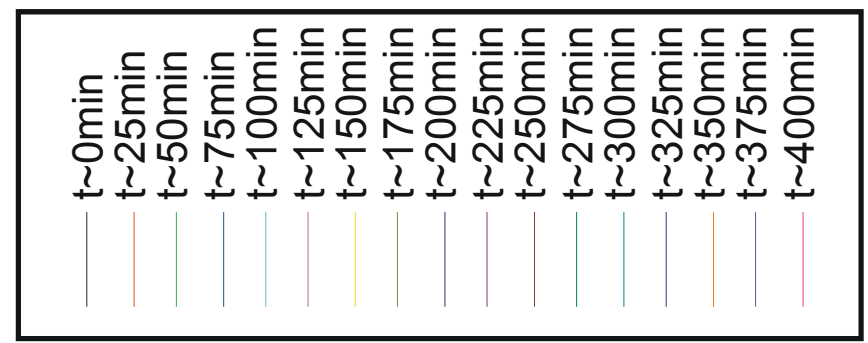

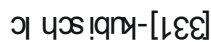

$\mathrm{XI}\|\| \mathrm{S} g-[L / L] /[Z L L]$

ग पऽ !qry-[0zz]

XIA II S马-[0LZ]/[LOZ]

$\mathrm{XI} \Lambda$ II S曰-[ZOL]

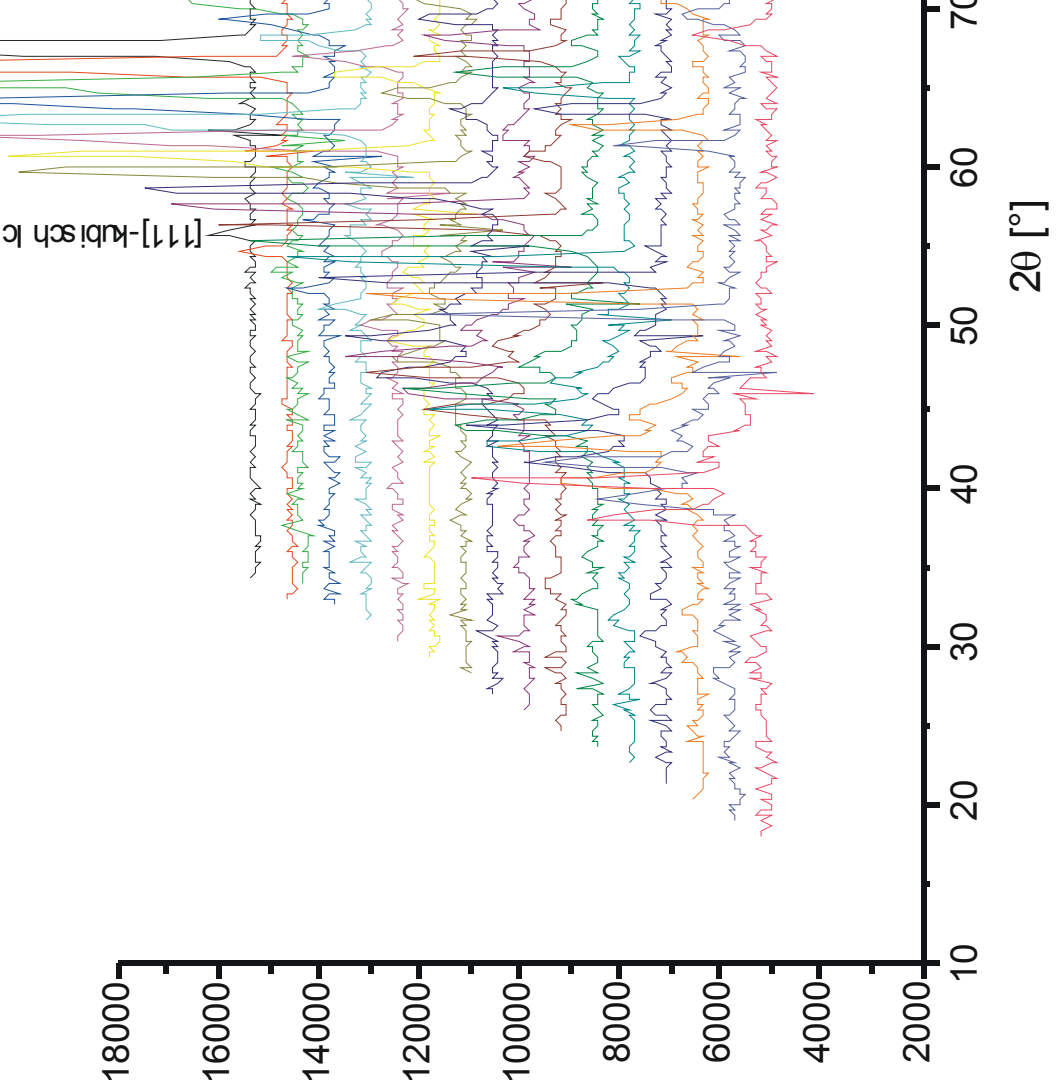

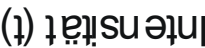

Abbildung 8.3.3.2.c: zeitlicher Verlauf des Phasenüberganges von Eis IX/III $\rightarrow$ in kubisches Eis Ic bei einer Phasenübergangstemperatur von T 143K. Zur Orientierung ist der [111]- sowie der [220]-kubische Reflex für die sich neu bildende kubische Eisphase indiziert und dargestellt. Die Überprüfung der Intensitätsverhältnisse des [102]-, des [201]/210]- sowie des [112]/[211]-Reflexes der Eisphase III während der Messung am D1B ergab, dass diese Probe eine nicht zu vernachlässigende Vorzugsorientierung aufwies. Ob der anisotrope Abbau (vergl. Kapitel 3.3.1, Abbildung 3.10.1) ein Effekt dieser Vorzugsorientierung ist kann nach derzeitigem Erkenntnisstand nicht ausgesagt werden. 


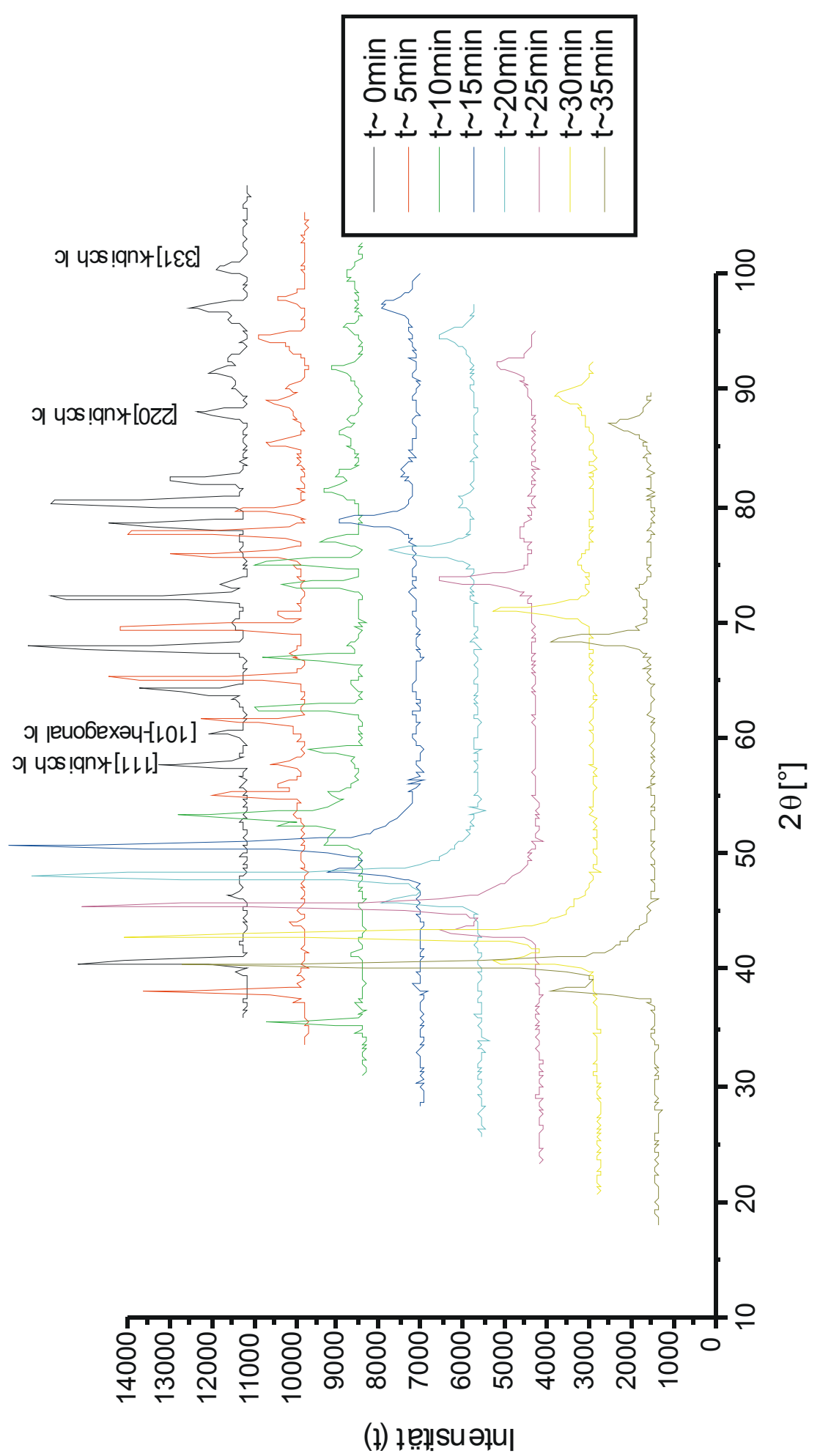

\begin{abstract}
Abbildung 8.3.3.2.d: zeitlicher Verlauf der Phasenumwandlung von Eis II $\rightarrow$ Eis Ic bei der Phasenübergangstemperatur von T 143K. Aufgrund einer für das Eis II zu hoch gewählten Phasenübergangstemperatur von T 143K vollzog sich die Umwandlung von Eis II in kubisches Eis Ic innerhalb von $\mathbf{t} \sim 35 \mathrm{~min}$. Bei dem ersten Datensatz zur Zeit $\mathbf{t} \sim 0 \mathrm{~min}$ ist in der Abbildung schon ein kubischer Phasenanteil von $\sim \mathbf{5 \%}$ festzustellen. Eine Bestimmung mittels JMAK-Theorie wurde nicht durchgeführt. Anzumerken ist, das auch diese Probe eine ausgeprägte Vorzugsorientierung aufwies.
\end{abstract}




\subsubsection{Verfeinerungsstrategie und Verfeinerungsproblematiken der Strukturbestimmung und des Eis Ih-Gasinhaltes}

Die folgenden Abbildungen 8.5.4.1 sowie 8.5.4.2 zeigen die simulierten Streubeiträge von innerhalb der Eis Ih-Struktur adsorbiertem Neon- sowie Heliumgases auf den in Kapitel 5.4 beschriebenen kristallographischen Lagen. Diese befinden sich in den Ringen der Eisstruktur sowie in dem Mittelpunkt des durch die Ringe aufgespannten Volumens Die fraktionelle Besetzung der Gaslagen wurde mit der maximalen Besetzung von frac $=1$ simuliert, so dass jede Position mit je einem Helium- oder Neonatom besetzt ist. Bedingt durch die Festlegung der kristallographischen Lagen sind Reflexlagen für beide Simulationen gleich. Abweichungen ergeben sich durch die Festlegung der Gitterkonstanten in den Streubeitragsimulationen. Die Simulation wurde mit festgelegten Gitterkonstanten von $\mathrm{a}=4.4661 \AA$ sowie $\mathrm{c}=7.2706 \AA$ für Neongas sowie für Heliumgas mit $\mathrm{a}=4.5008 \AA, \mathrm{c}=7.2972$ $\AA$ berechnet. Erwartungsgemäß weichen die Simulationsergebnisse in den Intensitäten aufgrund der unterschiedlichen Streulängen von Helium und Neon ab.

Ein Vergleich der simulierten und auf die Zählraten des Experimentes normierten absoluten Streubeiträge des Gasinhaltes innerhalb der Eisstruktur $\mathrm{zu}$ den gemessenen absoluten Streubeiträgen in den Diffraktogrammen von Eis Ih unter der Wechselwirkung der verwendeten Gase Neon (vergl. Abbildung 5.15 (a) und (b), Kapitel 5.5.6) und Helium (vergl. Abbildung 5.16 (a) und (b), Kapitel 5.5.7) zeigt, dass selbst bei kleiner thermischer Auslenkung, d.h. bei starker Lokalisierung der Gastome in der Eisstruktur und selbst unter der Annahme einer maximal möglichen Gasbesetzung auf allen möglichen kristallographischen Lagen die Streubeiträge so gering sind $(\mathrm{p} \leq 5 \%)$, dass diese innerhalb der Zählstatistik der typischen Schwankungsbreite des Untergrundes der durchgeführten Messungen bzw. der durch Vorzugsorientierungen verfeinerbaren Varianz der Intensitäten in den RietveldModellen liegt und ohne weitere Annahmen von Zwangsbedingungen in den Verfeinerungsmodellen aus diesen der Gasinhalt nicht nachweisbar ist. 

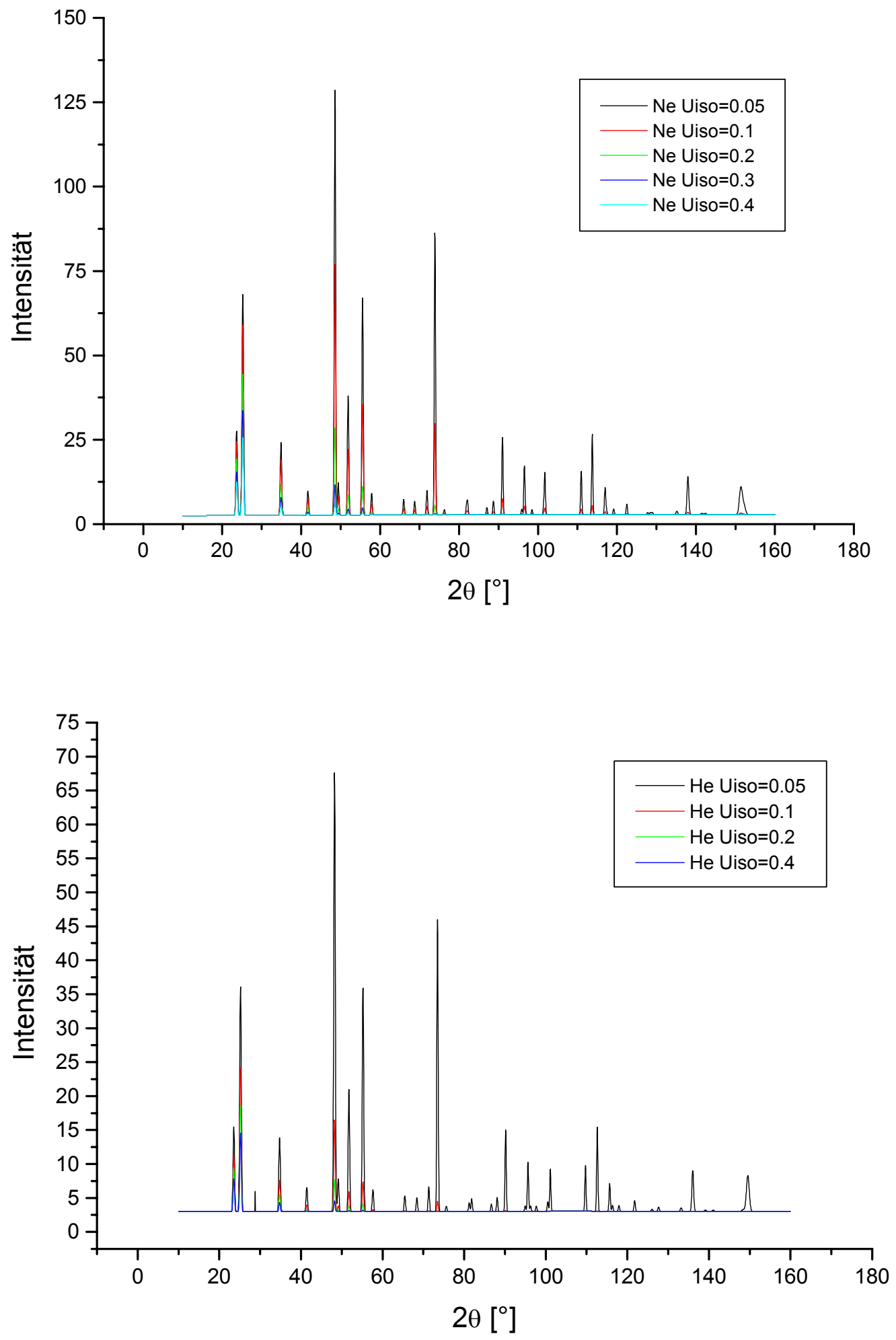

Abbildung 8.5.4.1 für Neongas (oben) und Abbildung 8.5.4.2 für Heliumgas (unten) zeigt die simulierten absoluten Streubeiträge der verwendeten Gase innerhalb der Eis Ih-Struktur als Funktion des thermischen Auslenkungsparameters $\mathbf{U}_{\text {iso. }}$. 


\subsubsection{Experimentelle Ergebnisse: Kompressibilitäten und Expansivitäten von Eis Ih}

Die isothermen Kompressibilitäten sowie die isobaren Expansivitäten eines kristallinen Systems sind durch die folgenden Gleichungen gegeben:

$$
\begin{aligned}
& \text { 1.) Kompressibilität } \kappa(p)=-\frac{1}{V_{p}} \cdot\left(\frac{\partial V}{\partial p}\right)_{T} \\
& \text { 2.) Expansivität } \beta(T)=\frac{1}{V_{T}} \cdot\left(\frac{\partial V}{\partial T}\right)_{p}
\end{aligned}
$$

wobei $\mathrm{V}_{\mathrm{p}}$ und $\mathrm{V}_{\mathrm{T}}$ das Volumen bei den gegebenen Umgebungsbedingungen darstellen. Die Kompressibilität sowie die Expansivität wird durch die Volumen-Druck- sowie VolumenTemperaturrelationen der gemessenen Gitterkonstanten bestimmt. Daher sind sie wesentlich von der Güte und Genauigkeit des Gitterkonstantenfits abhängig. Aufgrund der geringen Messpunktdichte und zudem der teils nicht vermessenen Gitterkonstanten bei einem Druck von $\mathrm{p} \sim 1$ bar als Funktion der Temperatur zeigte sich das Fitten dieser als problematisch. Es wurden daher mehrere Anpassungen der Gitterkonstanten, lineare wie auch Polynom-fits durchgeführt und daraus die Volumina der Elementarzellen berechnet.

Der Vollständigkeithalber sind in der folgenden Abbildung 8.5.5.1.1 die gemessenen und mittels Rietveld-Verfahren bestimmten Gitterkonstanten von Eis Ih unter der Wechselwirkung der verschiedenen Gase als Funktion der Temperatur aufgetragen. Die Kurven der isobaren Gitterkonstanten als Funktion der Temperatur verlaufen im Rahmen ihres Fehlers annähernd gleich. $\mathrm{Zu}$ erkennen ist dies bei einem Vergleich der isobaren Drücke bei $\mathrm{p} \sim 1 \mathrm{kbar}$ und $\mathrm{p} \sim 2 \mathrm{kbar}$ in dem gemessenem Temperaturbereich von $\mathrm{T} \sim 140 \mathrm{~K}$ bis $\mathrm{T} \sim 255 \mathrm{~K}$. Die Gitterkonstantenänderungen in a und in c verlaufen annähernd gleich und zeigen somit die aus den isothermen druckabhängigen Gitterkonstanten bestimmte Isokompressibilität der Eis IhStruktur bei der Verwendung von Argongas als Drucküberträgermedium.

$\mathrm{Zu}$ beobachten ist, dass die thermische Expansiviät bei steigendem Druck erwartungsgemäß abnimmt. Für die isobar bestimmten Gitterkonstanten bei p 2kbar nimmt die Krümmung der Gitterkonstanten über den Temperaturbereich einen weitgehend linear approximierbareren Verlauf an. Bei der Verwendung der Gase Neon und Helium zeigen sich wie bei den isothermen Gitterkonstanten als Funktion des Druckes Änderungen in den Verläufen. 
Eindeutig ist bei der Verwendung von Neongas bei einem Druck von p 1kbar ein Unterschied in dem Verlauf der isobaren Gitterkonstanten als Funktion der Temperatur für die Gitterkonstante a sowie c zu erkennen und ist damit konsistent $\mathrm{zu}$ dem abweichenden c/aVerhältnis der Gitterkonstanten. Die thermische Kontraktion der Eisstruktur bei einem Druck von $\mathrm{p} \sim 1 \mathrm{kbar}$ zeigt, dass diese in der Gitterkonstanten $\mathrm{c}$ stärker ausgeprägt ist. Eine weitergehende Diskussion der thermischen Expansivitäten bei der Verwendung von Helium hinsichtlich des Gitterkonstantenverlaufes ist aufgrund der wenigen festgestellten Messpunkte nicht sinnvoll.

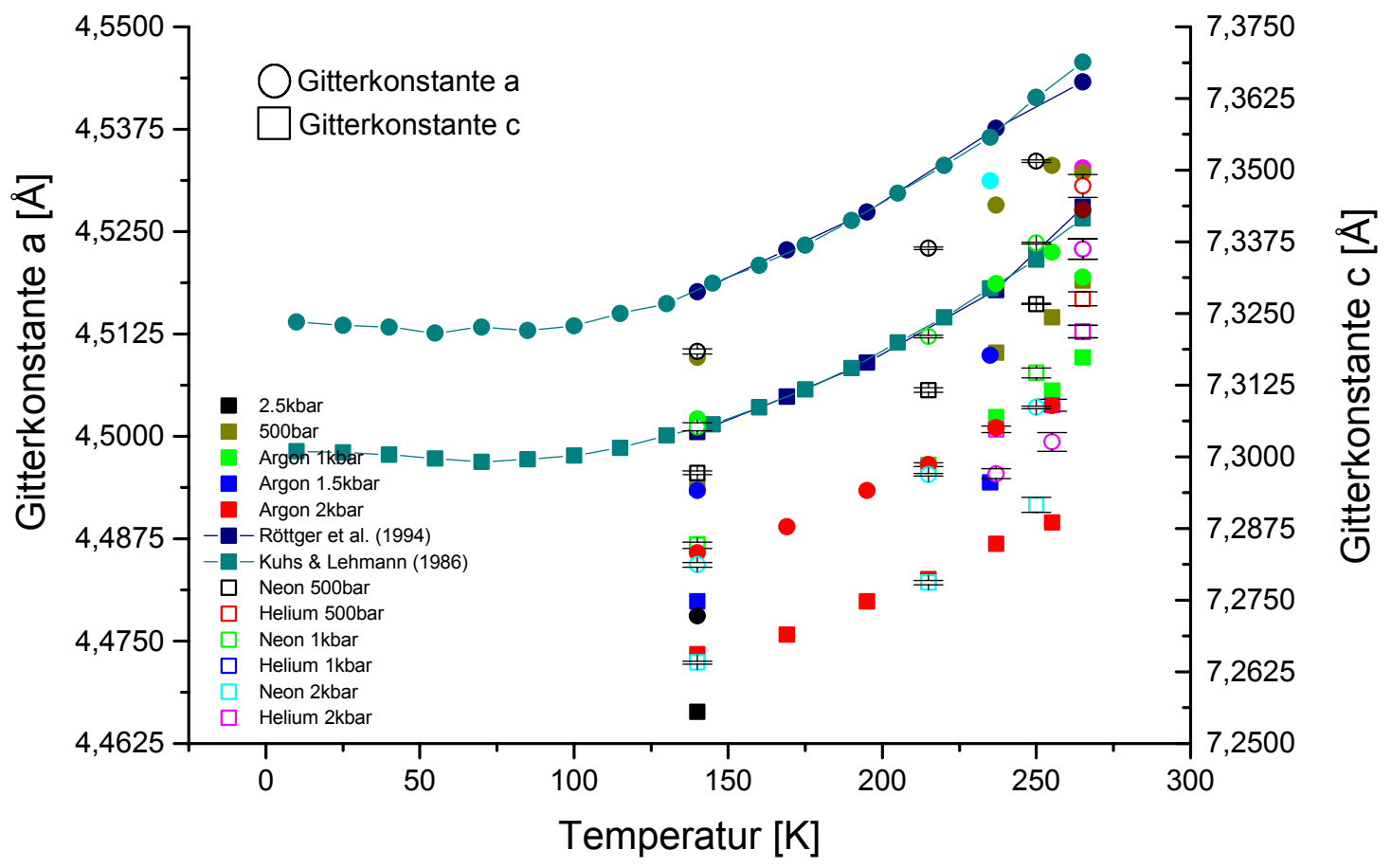

Abbildung 8.5.5.1.1: Auftragung der isobaren Gitterkonstanten mit den Gasen Argon, Helium und Neon. Die Darstellung ist farb-kodiert und gibt die Druckbedingungen an. Kreise - Gitterkonstante a, Quadrate - Gitterkonstante c. 


\section{Danksagung}

Herrn Prof. Dr. W.F. Kuhs möchte ich für die Vergabe diese interessanten Themas und für die Diskussionen, die zum Gelingen dieser Arbeit beigetragen haben, als auch für die Unterstützung während der Promotion danken. Dem ILL in Grenoble, Frankreich möchte ich für die zur Verfügung gestellte Messzeit danken. Besonderen Dank gilt hier Herrn Louis Melesi sowie Peter Cross für die technische Unterstützung während der vielen Experimente.

Dem ISIS des RAL in Didcot, Großbritannien möchte ich für die zur Verfügung gestellte Meßzeit am GEM danken, sowie Herrn Dr. P. Radaelli und Herrn D.W. Dryer für die tatkräftige, technische Unterstützung während des Experimentes. $\mathrm{Zu}$ dem möchte ich Herrn Dr. C. Lobban für die stete Diskussionbereitschaft als auch Herrn Prof. Dr. J.L. Finney für die Betreuung und Diskussion während des Experimentes am ISIS danken. Ein ebensolcher Dank gilt dem HASYLAB für die zur Verfügung gestellte Meßzeit am BW5. Dank gilt hier Herrn Dr. L. Wcislak sowie Herrn Dr. Ing. H. Klein.

Danken möchte ich auch der Werkstatt des Mineralogisch-Kristallographischen-Institutes der Universität Göttingen, im besonderen Maße Herrn Heiner Bartels für seine stetige kompetente und schnelle Hilfe bei technischen Problemen, insbesondere bei anstehenden Messzeiten, sowie Herrn Hensel für seine stetige Bereitschaft sich bei den Proben-Präparationen sowie bei der Organisation der Messreisen zu engagieren.

Herrn Dr. St. Adams, Herrn Dr. E. Matzat und Herrn Dr. K. Mariolacos möchte ich für stete Bereitschaft für konstruktive Diskussionen, vor allem in der Endphase dieser Arbeit danken. Frau Dipl. Chem. Ing. A. Klapproth möchte ich für die Unterstützung bei den Präparationen sowie bei der Erstellung der Simulationsmodelle danken.

Herrn Dr. O. Moretzki und Frau Dipl.-Min. A. Preusser danke ich im besonderen für fachliche und private intensive Diskussionen und der moralischen Unterstützung durch den nachmittäglichen Espresso. Allen anderen Mitarbeitern danke ich für die freundliche Kooperation, insbesondere Herrn K. Häpe, der immer mit Rat und Tat bei der Bewältigung computertechnischer Probleme zur Seite stand. 
Der DFG danke ich für die finanzielle Unterstützung im Rahmen des bewilligten Projektes $\mathrm{Ku}$ $920 / 3$.

Ein ganz besonderer Dank geht an meine Frau Ute, die mir insbesondere in der letzten Zeit der Promotion zur Seite gestanden hat. Wir erwarten in diesen Tagen unser erstes Kind.

\section{Lebenslauf}

Am 3. April 1968 wurde ich, Frank Gotthardt, in Göttingen geboren. Seit Mai 1995 bin ich mit meiner Frau Ute Gotthardt verheiratet.

Von 1975 bis 1979 besuchte ich in Bovenden die Grundschule, von 1979 bis 1985 die Realschule Weende. Nach dem Abschluss der Realschule mit dem erweiterten Sekundarabschluss I erfolgte der Wechsel auf das Fachgymnasium Technik an den Berufbildenden Schulen II in Göttingen. Im Juni 1988 legte ich die Abiturprüfung ab. Von Oktober 1988 bis Oktober 1989 leistete ich den Grundwehrdienst bei der Bundeswehr. Zum Wintersemester 1989 begann ich mein Studium der Physik an der Universität Göttingen. Das Vordiplom bestand ich im SS92.

Von SS95 bis Februar 1997 fertigte ich meine Diplom-Arbeit am IV. Physikalischen Institut der Universität Göttingen an. Das Thema, „Experimentelle Analyse der lokalen Scherbelastung der Zementschicht einer im Human-Becken implantierten Hüftpfannen-Endoprothese“ wurde in der Arbeitsgruppe Biomechanik von Herrn Prof. Dr. Nägerl am IV. Physikalischen Institut angefertigt. Im Februar 1997 bestand ich an der Universität Göttingen die Diplom-Hauptprüfung im Fach Physik. Von März 1997 bis Oktober 1997 beschäftigte ich mich mit weiteren Messungen zum Thema der Diplom-Arbeit.

Von Oktober 1997 bis einschließlich Mai 2001 war ich als wissenschaftlicher Mitarbeiter des Mineralogisch-Kristallographischen Institutes der Universität Göttingen in der Arbeitsgruppe von Herrn Prof. Dr. W.F. Kuhs mit den in dieser Doktorarbeit vorgestellten Arbeiten beschäftigt.

Göttingen, den 12.04.2001 